NISTIR 6253

\title{
Effect of an Obstructed Ceiling on the Activation Time of a Residential Sprinkler
}

Robert L. Veltori

Building and Fire Research Laboratory

National Institute of Standards and Technology

Gaithersburg, MD 20899-8641

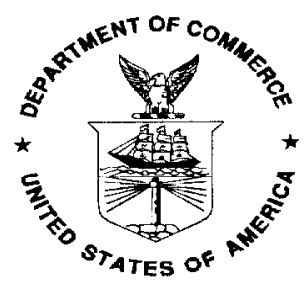

U.S. Department of Commerce

Technology Administration

National Institute of Standards and Technology

Gaithersburg, MD 20899

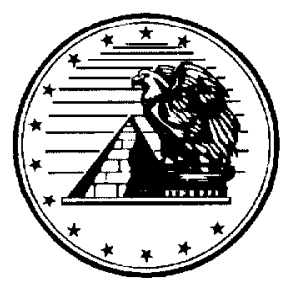

Prepared for:

U.S. General Services Administration Office of Business Performance

Washington, DC 20405 
NISTIR 6253

\section{Effect of an Obstructed Ceiling on the Activation Time of a Residential Sprinkler}

Robert L. Vettori

Building and Fire Research Laboratory

National Institute of Standards and Technology

Gaithersburg, MD 20899-8641

November 1998

\section{U.S. Department of Commerce}

William M. Daley, Secretary

Technology Administration

Gary R. Bachula, Acting Under Secretary for Technology

National Institute of Standards and Technology

Raymond G. Kammer, Director

Gaithersburg, MD 20899
Prepared for:

U.S. General Services Administration

David J. Barram, Administrator

Public Buildings Service

Robert A. Peck, Commissioner

Washington, DC 20405 


\section{Table of Contents}

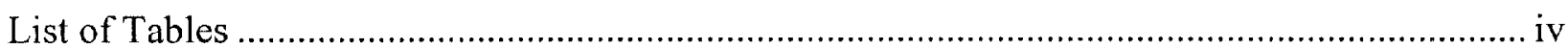

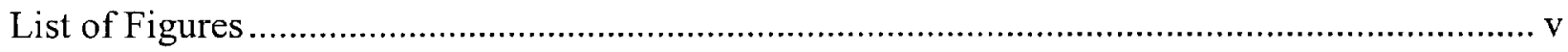

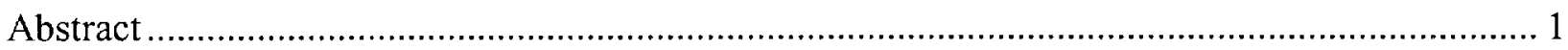

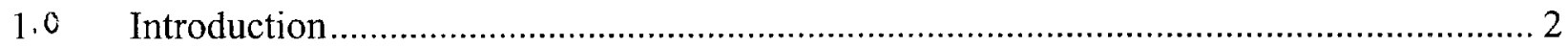

2.0 Experimental Configuration.............................................................................. 2

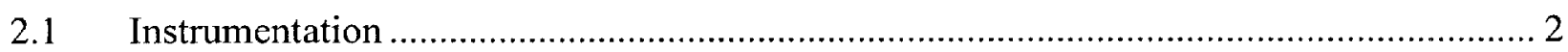

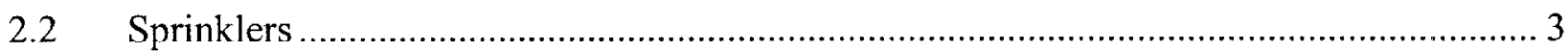

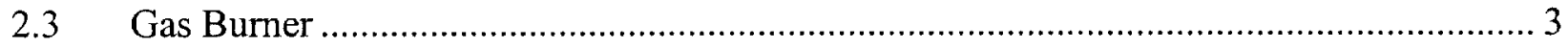

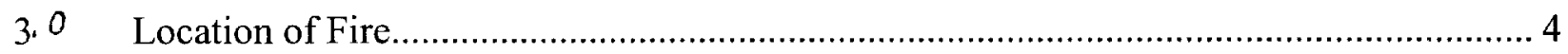

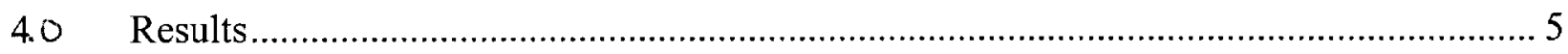

4.1 Effect of Obstructed/Beamed Ceiling on Activation Times ........................................ 5

4.2 Effect of Obstructed/Beamed Ceiling on Ceiling Jet Velocities ..................................... 5

4.3 Effect of Fire Growth on Activation Times ............................................................. 6

4.4 Effect of Burner Position on Activation Times. .................................................... 6

4.5 Effect of Obstructed/Beamed Ceiling on Tempcraturcs ............................................ 7

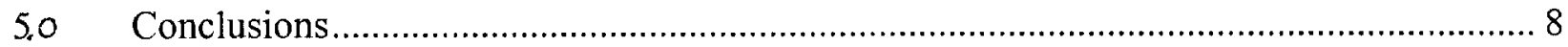

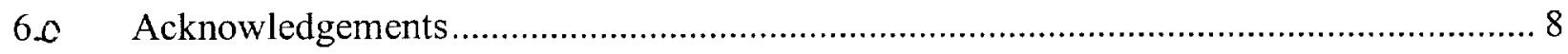

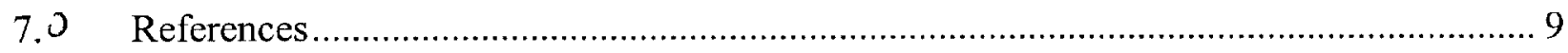

8.3 Appendix A: Determination of the Response Time Index (RTI) and Conductivity (C)

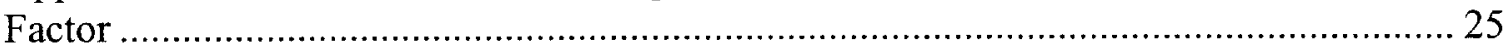

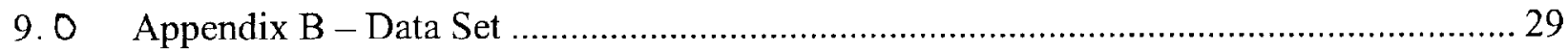




\section{List of Tables}

Table 1 - Comparison of sprinkler activation times.................................................... 10

Table 2 - Average activation times and percent increase in activation times caused by obstructed/beamed ceiling .................................................... 11

Table 3 - Temperature of the ceiling jet at the activated sprinkler at time of activation.

Table 4 Temperature comparisons at the $1.54 \mathrm{~m}(5 \mathrm{ft})$ elevation at the time of

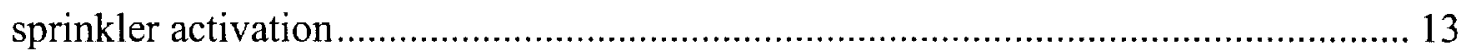

Table 5 - Temperatures comparison at the $1.89 \mathrm{~m}(6 \mathrm{ft})$ elevation at the time of sprinkler activation. 14 


\section{List of Figures}

Figure 1 - Plan view of experimental set up. ................................................................... 15

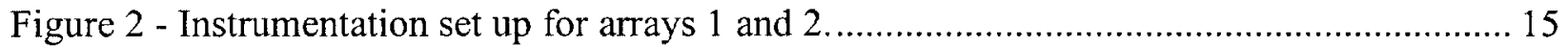

Figure 3 - Fast, medium, and slow fire growth regions................................................. 16

Figure 4 - Calibration data for the rectangular gas burner............................................ 16

Figure 5 - Distances from different burner positions to sprinklers of interest......................... 17

Figure 6 - Position of sprinklers 1 and 4 relative to channels formed by ceiling joists. Location of additional velocity probes, corner burner position, obstructed/beamed ceiling....................................................................... 17

Figure 7 - Ceiling jet velocity comparisons at sprinkler number 1, detached burner position, slow fire growth.

Figure 8 - Ceiling jet velocity comparisons at sprinkler number 1 , detached burner position, medium fire growth.

Figure 9 - Ceiling jet velocity comparisons at sprinkler number 1 , detached burner

position, fast fire growth.

Figure 10 - Ceiling jet velocity comparisons at sprinkler number 1, wall burner position, slow fire growth.

Figure 11 - Ceiling jet velocity comparisons at sprinkler number 1 , wall burner position, medium fire growth.

Figure 12 - Ceiling jet velocity comparisons at sprinkler number 1 , wall burner position, fast fire growth.

Figure 13 - Ceiling jet velocity comparisons at sprinkler number 1, corner burner position, fast firc growth.

Figure 14 - Ceiling jet velocity comparisons for corner burner position, slow fire

growth.

Figure 15 - Ceiling jet velocity comparisons for corner burner position, medium

fire growth.

Figure 16 - Ceiling jet velocity comparisons for corner burner position, fast fire growth. 
Figure 17 - Ceiling jet temperature comparisons at sprinklers 1 and 4 and in the channel above the burner, obstructed/beamed ceiling, burner in corner, slow fire growth.

Figure 18 - Ceiling jet temperature comparisons at sprinklers 1 and 4 and in the channel above the burner, obstructed/beamed ceiling, burner in corner, medium fire growth......

Figure 19 - Ceiling jet temperatures comparisons at sprinklers 1 and 4 and in the channel above the burner, obstructed/beamed ceiling, burner in corner, fast fire growth. 
Effect of an Obstructed Ceiling on the Activation Time of a Residential Sprinkler

Robert L. Vettori

\begin{abstract}
A series of 45 experiments were conducted to compare the effects of two different ceiling configurations on the activation times of a quick response residential pendent sprinkler. The two ceiling configurations consisted of a smooth horizontal unobstructed ceiling and a horizontal ceiling obstructed by parallel beams measuring $0.038 \mathrm{~m}$ (1.5 in) wide by $0.24 \mathrm{~m}$ (9.5 in) deep and spaced $0.41 \mathrm{~m}$ (16 in) on center. For each of the two ceiling configurations, the fire source, a computer controlled methane gas burner, was placed in three different locations within the fire

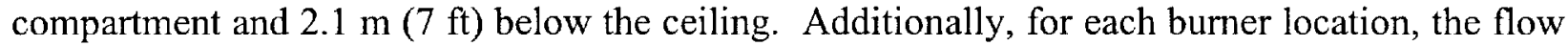
of methane gas to the burner was supplied in such a way as to give three different fire growth scenarios. For the smooth horizontal unobstructed ceiling three experiments were performed for every burner position and fire growth rate. For the horizontal obstructed/beamed ceiling there were two experiments for every burner position and fire growth rate. Measurements taken include the time to sprinkler activation, temperature and velocity of the ceiling jet at the sprinkler of activation, and temperatures and ceiling jet velocities at various other locations and elevations within the fire compartment. The horizontal obstructed/beamed ceiling increased sprinkler activation time by $57 \%$ to $137 \%$ depending on the experimental setup.
\end{abstract}

Key Words: beams; ceiling jets; comers; fire growth; residential sprinklers; sprinkler response; sprinkler systems; tenability limits; wall fires 


\section{Introduction}

There is little quantitative information on the effect beams or other ceiling obstructions have on the activation time of quick response residential sprinklers [1]. Residential sprinklers are only listed for use under smooth horizontal unobstructed ceilings [2,3]. The objective of this study was to determine the effect that a horizontal obstructed/beamed ceiling configuration had on the activation times of a residential sprinkler.

The ceiling jet is usually defined as the gas flow beneath the ceiling surface directed radially away from the fire plume, which is driven by the buoyancy of the hot combustion products. For smooth horizontal unobstructed ceilings, temperature and velocity distributions vary both radially away from the fire center and vertically below the ceiling. Sprinkler response is primarily a function of the physical properties of the sprinkler thermal element, its mounting, and the temperature and velocity of the ceiling jet that flows around it. One of the effects that ceiling obstructions such as beams and joists have is to significantly alter the flow of the ceiling jet.

\section{Experimental Configuration}

These experiments were conducted at the Building and Fire Research Laboratory Annex Building 534. The fire compartment consisted of a room $9.2 \mathrm{~m} \mathrm{x} 5.6 \mathrm{~m}(30 \mathrm{ft} \times 18 \mathrm{ft})$ with a ceiling height of $2.4 \mathrm{~m}(8 \mathrm{ft})$, figure 1. The walls were constructed of a wood frame covered with $12.7 \mathrm{~mm}(0.5 \mathrm{in})$ gypsum board and the floor was concrete. A hollow steel door measuring 0.91 $\mathrm{m} \times 2.1 \mathrm{~m}(3.0 \mathrm{ft} \times 6.8 \mathrm{ft})$ opened to the outside. The air gap under the door measured $25 \mathrm{~mm}$ ( 1 in). This door was closed for all experiments. A stairway of wood construction led to an upper floor that had the same dimensions as the fire compartment. There was no vent from this upper floor. For the smooth horizontal unobstructed ceiling experiments, the ceiling was also covered with $12.7 \mathrm{~mm}(0.5 \mathrm{in})$ gypsum board. For the horizontal obstructed/beamed ceiling, the gypsum board on the ceiling was removed exposing the $0.038 \mathrm{~m} \mathrm{x} 0.24 \mathrm{~m}$ ( 1.5 in $\times 9.5 \mathrm{in})$ wood joists that were spaced $0.41 \mathrm{~m}$ (16 in) on center. These ceiling joists spanned the width of the room; supported in the center by a $0.10 \mathrm{~m} \times 0.18 \mathrm{~m}$ ( 4 in $\times 7$ in) steel I beam that spanned the length of the room. The steel I beam was supported at either end by the wood frame wall and along its length by two steel $0.064 \mathrm{~m}$ (2.5 in) lolly columns. The flooring material for the upper floor

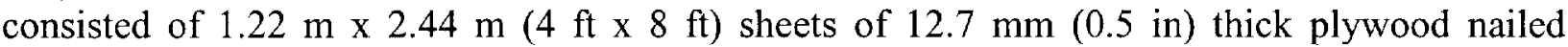
directly to the top of the ceiling joists. Four quick response residential pendent sprinklers were installed on the ceiling in accordance with NFPA 13D, Sprinkler Systems in One and Two Family Dwellings and Manufactured Homes [3]. The location of the four sprinklers, numbered 1 through 4 , is also shown in figure 1.

\subsection{Instrumentation}

Temperature measurements were made with $0.51 \mathrm{~mm}(0.02 \mathrm{in})$ nominal diameter type $\mathrm{K}$ thermocouples. Standard thermocouple instrumentation for all 45 experiments consisted of four vertical arrays of thermocouples, one array located next to each sprinkler, figure 1. Each array was comprised of 12 thermocouples located $0,25,50,75,100,125,150,250,350,450,550$, and $900 \mathrm{~mm}(0,1.0,2.0,3.0,3.9,4.9,5.9,9.8,13.8,17.7,21.7$, and $35.4 \mathrm{in})$ below the ceiling, figure 
2. The standard uncertainty for these temperature measurements is $\pm 2.2^{\circ} \mathrm{C}\left( \pm 4.0^{\circ} \mathrm{F}\right)$ based on manufacturer's data.

Gas velocity measurements were recorded at sprinklers one and two using vertical arrays of bidirectional probes. Each array consisted of four bi-directional probes. The bi-directional probes were located $25,75,125$, and $250 \mathrm{~mm}(1,3,4.9$, and $9.8 \mathrm{in})$ below the ceiling, figure 2 . There were no gas velocity measurements taken at sprinklers three and four. For experiments with the horizontal obstructed/beamed ceiling, additional bi-directional probes were placed within the ceiling joist channels above the corner burner location, figure 6 . The standard uncertainty for the velocity measurements is $\pm 0.1 \mathrm{~m} / \mathrm{s}( \pm 0.3 \mathrm{ft} / \mathrm{s})$ based on manufacturer's data for the differential pressure transducers.

\subsection{Sprinklers}

The sprinklers used throughout these experiments were commercially available quick response residential pendent sprinklers. They were all from the same manufacturer and were all the same model. The sprinklers had glass bulb elements with an activation temperature of $68 \pm 2.4^{\circ} \mathrm{C}$ $\left(154 \pm 5.4^{\circ} \mathrm{F}\right)$. The response time index (RTI) for the sprinklers was $55(\mathrm{~m}-\mathrm{s})^{1 / 2}\left(100(\mathrm{ft}-\mathrm{s})^{1 / 2}\right)$, as determined by an independent testing laboratory [4] $]^{1}$. When mounted under the smooth horizontal unobstructed ceiling, the sprinklers were fully exposed and the center of the glass bulb element was $25 \mathrm{~mm}(1 \mathrm{in})$ below the gypsum cciling. For the experiments in which the gypsum board was removed from the ceiling, the center of the glass bulb element was $37 \mathrm{~mm}\left(1 \frac{112}{2}\right.$ in) below the bottom of the ceiling joists. For each experiment, $25 \mathrm{ml}$ of water was placed in the pipe to which the sprinkler was mounted in order to simulate the water that would normally be present in the sprinkler piping system. The sprinkler system was then pressurized with air to approximately $100 \mathrm{kPa}(15 \mathrm{psi})$. Each sprinkler was connected to a pressure switch that was electronically connected to a timer. The pressure switches were set at $5 \mathrm{kPa}(1 \mathrm{psi})$ so that upon sprinkler activation the drop in pressure would automatically stop the corresponding timer. This provided an activation time to within \pm 0.1 seconds.

\subsection{Gas Burner}

The fire source in this experimental series consisted of a rectangular methane gas burner with a piloted ignition. The burner had dimensions of $0.7 \mathrm{~m} \mathrm{x} 1.0 \mathrm{~m} \mathrm{(28} \mathrm{in} \mathrm{x} 40$ in) and was $0.31 \mathrm{~m} \mathrm{(1}$ $\mathrm{ft}$ ) high. A technical grade of methane was used, which was certified by the supplier to contain at least $98 \%$ methane. The flow of methane gas to the burner was controlled by a computer. The computer was programmed to monitor the flow of methane gas through four mass flow controllers arranged in parallel. With this configuration the heat release rate curve produced by the burner is one that follows the fire growth rates used in the 1993 edition of National Fire Protection Association (NFPA) 72 National Fire Alarm Code Appendix B [5]. The fast, medium and slow fires described by this NFPA standard are based on a wide variety of fires that grow with the square of time and are sometimes referred to as t-squared $\left(t^{2}\right)$ fires. $\Lambda$ fast developing fire is one that would take less than 150 seconds from the time of open burning until the fire reaches a heat release rate of $1055 \mathrm{~kW}$. A medium developing fire is one that would take more

\footnotetext{
${ }^{1}$ Reference 4 is attached as an appendix at the end of this report.
} 
than $150 \mathrm{~s}$, but less then $600 \mathrm{~s}$ from the time of open burning until the fire reaches a heat release rate of $1055 \mathrm{~kW}$. A slow developing fire is one that would take 600 or more seconds from the time of open burning until the fire reaches a heat release rate of $1055 \mathrm{~kW}$. A mathematical representation for these curves is as follows:

Where: $\quad \begin{array}{ll}\dot{Q} & =\text { heat release rate }(\mathrm{kW}) \\ \alpha & =\operatorname{coefficient}\left(\mathrm{kW} / \mathrm{s}^{2}\right) \\ t & =\operatorname{time}(\mathrm{s})\end{array}$

Figure 3 shows the area bounded by the theoretical fast, medium and slow fire growth curves. For the purpose of this experimental series, a fast fire reached $1055 \mathrm{~kW}$ in 150 seconds which corresponds to an $\alpha$ equal to $0.0468 \mathrm{~kW} / \mathrm{s}^{2}$. A medium fire reached $1055 \mathrm{~kW}$ in 300 seconds, which corresponds to an $\alpha$ equal to $0.0117 \mathrm{~kW} / \mathrm{s}^{2}$. A slow fire reached $1055 \mathrm{~kW}$ in 600 seconds, which corresponds to an $\alpha$ equal to $0.00293 \mathrm{~kW} / \mathrm{s}^{2}$. Before the experiments were conducted, the rectangular gas burner was calibrated using the principals of oxygen consumption calorimetry. Figure 4 demonstrates the repeatability obtained with the gas burner during these calibration tests. The three theoretical fire growth curves used in this experimental series are overlaid with data obtained from the respective burner calibration tests. For each fire growth rate, two calibration tests were performed.

\section{$3 \quad$ Location of Fire}

In addition to varying the heat release rate of the fire, the rectangular burner was placed in three different locations within the room. These locations are as follows; in the open or away from any wall (detached experiment), against a wall (wall experiment), and in a corner (corner experiment). Figure 5 shows the distance from the burner to the sprinklers of interest for each burner location. The effect of placing the burner against a wall or in a corner of the room is to restrict the entrainment of air into the plume [6,7]. When the burner is placed away from a wall or what would be considered in the open, cool room air is entrained into the plume from all directions. By placing the burner adjacent to one or more walls, the area over which this cool room air can be entrained into the plume is reduced. One result of this reduction in entrainment is higher flame heights. Since the fuel requires the same amount of air to complete the oxidation process a greater distance is now needed for the fuel vapors to mix with the smaller quantity of air that is entering the plume. With these higher flame heights there is now less distance from the tip of the flame to the bottom of the hot upper layer for the plume to entrain cool air. The higher plume temperatures that result from the reduced cool air entrainment cause similar increases in the upper layer temperatures. 


\section{Results}

\subsection{Effect of Obstructed/Beamed Ceiling on Activation Times}

Table 1 is a comparison of the sprinkler activation times between the smooth horizontal unobstructed ceiling and the horizontal obstructed/beamed ceiling. The burner position and fire growth rate for the 45 experiments are listed. For each burner position and fire growth rate, the location of the first sprinkler to activate and its activation time is also listed. By taking the simple average of the three activation times for the smooth horizontal unobstructed ceiling scenario and the two activation times for the horizontal obstructed/beamed ceiling scenario, table 2 was constructed. Table 2 presents the percent increase in activation time caused by the obstructed/beamed ceiling. $\Lambda$ s can be seen from table 2, the effect of the obstructed/beamed ceiling was to increase the sprinkler activation time for all scenarios. It is interesting to note from table 1 that when the burner was placed in the corner position, under the horizontal obstructed/beamed ceiling configuration, sprinkler number 4 activated first in five out of the six experiments. Figure 5 shows the distance from the burner to both sprinklers 1 and 4 . The radial distance to sprinkler number 4 is twice as far as to sprinkler number 1 . When exposed, the ceiling joists form channels beneath the ceiling. Due to the nature of the construction, the ceiling joists overlap each other on top of the I beam, figure 6 . Although sprinklers 1 and 4 are in the same plane, they are in different channels. Sprinkler number 4 is in the seventh channel away from the burner. Sprinkler number 1 is in the sixth channel. A two zone fire model would not be able to predict this type of phenomenon. In order to predict this type of event one would have to utilize a computer field model such as one being developed at the National Institute of Standards and Technology that uses Large Eddy Simulation techniques for fire [8].

\subsection{Effect of Obstructed/Beamed Ceiling on Ceiling Jet Velocities}

With the removal of the gypsum board from the ceiling, exposing the ceiling joists, the effective height from floor to ceiling now becomes $2.7 \mathrm{~m}$ ( $8 \mathrm{ft} 10 \mathrm{in})$. With the gypsum board removed, the sprinklers are now located $0.27 \mathrm{~m}$ (10.5 in) below this new ceiling height. This alone removes the sprinkler from the hottest part of the ceiling jet as described by Alpert [9] as being in the top $1 \%$ of the floor to ceiling height. Another effect that the ceiling joists had was to slow the ceiling jet velocity dramatically at the sprinkler. Figures 7 through 9 show the effects the obstructed/beamed ceiling had on the ceiling jet velocity for the experiments in which the burner was in the detached position. Figures 10 through 12 show the effects the obstructed/beamed ceiling had on the ceiling jet velocity for the experiments in which the burner was in the wall position. For these figures, 7 through 12, the ceiling jet velocities for the smooth horizontal unobstructed ceiling are the average obtained from the top velocity probe at sprinkler number 1 from the three experiments. The velocities for the horizontal obstructed/beamed ceiling are the average obtained from the top velocity probe at sprinkler number 1 from the two experiments. For the experiments in which the burner was placed in the comer only one figure is shown, figure 13. This is a comparison of the ceiling jet vclocity for a fast growing fire. There are no comparisons for the medium and slow growing fires since at sprinkler number 4 there were no velocity probes. The horizontal obstructed/beamed ceiling velocity in figure 13 is based on the one experiment in which sprinkler number 1 activated. 
When comparing figures 7 through 13, it may be best to describe the "ceiling jet" in the horizontal obstructed/beamed ceiling scenario as having a flow under a beam rather than a traditional ceiling jet as described by Alpert. Because the convective heat transfer is proportional to the square root of the velocity, sprinkler activation is delayed by the reduced ceiling jet velocity.

As stated in the section under instrumentation, the basic measurements for all 45 experiments were the same. In the corner experiments for the obstructed/beamed ceiling scenario, two additional velocity probes were installed in the first two channels formed by the ceiling joists above the burner, figure 6 . These two probes were located in the same plane as sprinkler number 1. They were centered between the joists approximately $10 \mathrm{~cm}$ (4 in) below the top of the ceiling joists. Figures 14 through 16 present data on these additional velocity probes along with data from the velocity probes located at sprinkler number 1 . Comparable to figures 7 through 13 , the effect of the obstructed/beamed ceiling was to reduce the velocity significantly when compared to those found with the smooth horizontal unobstructed ceiling. The effect of the very high velocities produced in the channels as compared with those at sprinkler number 1 may help in explaining why sprinklcr number 4 activated first in 5 out of 6 experiments.

\subsection{Effect of Fire Growth on Activation Times}

The rate of fire growth also had an effect on the time to sprinkler activation. By looking at Table 2 , it can be scen that for all three burner locations and both ceiling configurations that the average time to sprinkler activation increased as the fire growth slowed.

\subsection{Effect of Burner Position on Activation Times.}

For the smooth horizontal unobstructed ceiling, the placement of the burner did not have a pronounced effect on the time to sprinkler activation. Moving the burner from the detached position to the wall position, the effect was hardly noticeable for the fast and medium fires. For the slow growing fire, the average activation time was actually longer. A factor that must be taken into account is that the distance from the center of the burner to the sprinkler is not the same for the different burner positions, figure 5. In the detached position the sprinkler is $2.2 \mathrm{~m}$ ( $7 \mathrm{ft} 3 \mathrm{in}$ ) away from the fire, for the wall position the sprinkler is $2.4 \mathrm{~m}(8 \mathrm{ft}$ ) away. This represents a $10 \%$ increase in distance. The fast and medium fires had slightly faster activation times on average but by looking at the individual experiment results, table 1, it can be seen the results are virtually indistinguishable. For the comer experiments, the activation times are noticeably faster but, in turn, the sprinkler is now closer than in either the detached position or wall position. Another factor that may be taken into account is how the burner was placed against the wall. In describing the effect of a wall on the plume, Zukoski [6] placed a wall across the diameter of a circular burner, which effectively reduced it to a semi circular geometry. By doing this the perimeter over which the plume can entrain air is reduced to $50 \%$ of the original. As can be seen in figure 5, when the burner was placed against the wall, a short side of the rectangular burner was placed up against the wall. The original perimeter of the burner was 3.4 $\mathrm{m}(11 \mathrm{ft})$. By placing a short side of the burner against the wall the perimeter is only reduced by $0.7 \mathrm{~m}$ (28 in), a reduction of only $21 \%$. In this configuration, air was being entrained over $79 \%$ 
of the perimeter of the burner. When the burner was placed in the corner position, $50 \%$ of the burner perimeter is still exposed for the entrainment of air into the plume.

When considering the effect of the burner position in the horizontal obstructed/beamed ceiling configuration, it appears more variables need to be taken into account since it is not always the physically closest sprinkler that activates first.

\subsection{Effect of Obstructed/Beamed Ceiling on Temperatures}

Table 3 gives the ceiling jet temperature at the first sprinkler to activate at the time of activation. All the temperatures measured for the obstructed/beamed ceiling experiments are higher than the smooth horizontal unobstructed ceiling. For the obstructed beamed ceiling configuration, it must be noted that the temperature is that measured $0.27 \mathrm{~m}(10.5 \mathrm{in})$ below the top of the ceiling joists. The temperatures within the channels formed by the ceiling joists would be higher. Figures 17 through 19 compare ceiling jet temperatures at sprinklers 1 and 4 along with the temperatures in the channels above the burner in which the burner was placed in the comer position. The location of the thermocouples within the channels are the same as the location of the additional velocity probes that were added for the obstructed/beamed ceiling, comer burner position, figure 6.

Tables 4 and 5 list temperature readings at the time of sprinkler activation at the $1.54 \mathrm{~m}(5 \mathrm{ft})$ and $1.89 \mathrm{~m}(6 \mathrm{ft})$ elevations respectively. These heights were chosen since they represent possible clcvations that the mouth and eyes of people egressing the room might be located. In each table, the average temperature represents the average of the four thermocouples, one at each sprinkler location, at the specified elevation. The low-high temperature is simply the value of the lowest temperature recorded of the four thermocouples and the highest temperature recorded of the four thermocouples at the time of sprinkler activation. In all cases, for both the $1.54 \mathrm{~m}(5 \mathrm{ft})$ and 1.89 $\mathrm{m}$ (6ft) elevation, the average temperature at sprinkler activation for the horizontal obstructed/beamed ceiling was higher than the smooth horizontal unobstructed ceiling. Additionally, in a large majority of cases, the low temperature measured for the horizontal obstructed/beamed ceiling was higher than the high temperature measures in the smooth horizontal unobstructed ceiling.

As a point of reference, The National Fire Protection Handbook [10] summary of the UL 1626 test criteria [2] states:

The maximum gas or air temperature adjacent to the sprinkler $76.2 \mathrm{~mm}(3 \mathrm{in})$ below the ceiling and $203 \mathrm{~mm}$ ( 8 in) horizontally away from the sprinkler must not exceed $316^{\circ} \mathrm{C}\left(600^{\circ} \mathrm{F}\right)$.

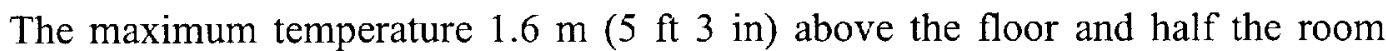
length away from each wall must be less than $93{ }^{\circ} \mathrm{C}\left(200^{\circ} \mathrm{F}\right)$ during the entire test. This temperature must not exceed $54^{\circ} \mathrm{C}\left(130^{\circ} \mathrm{F}\right)$ for more than a two minute period. 


\section{Conclusions}

The effect of this obstructed/beamed ceiling configuration was to increase the activation time for the first sprinkler. This was true for all burner locations and fire growth rates. No effort was made in this experimental series to determine what effect this obstructed/beamed ceiling would have on the activation times of additional sprinklers. Additionally, for the obstructed/beamed ceiling configuration there was an increase in temperature at the $1.54 \mathrm{~m}(5 \mathrm{ft})$ and $1.89 \mathrm{~m}(6 \mathrm{ft})$ elevations creating a potentially greater hazard within the room.

This experimental series was limited to only one depth and spacing of ceiling joists. There are many different sizes and methods for the construction of ceiling joists. Future research needs to be conducted to understand the effects different obstructed/beamed ceilings have on fire growth, suppression, and gas generation.

\section{Acknowledgements}

Appreciation is extended to Dan Madrzykowski, Jay McElroy, Laurean Delauter, Gary Roadarmel and Tamra Belsinger of the Building and Fire Research Laboratory for setting up and conducting these experiments. In addition, appreciation is extended to Henry J. Singer and Stewart Levy of GSA's Office of Business Performance for their support of this project. 


\section{$7 \quad$ References}

1. Bill, R. G., Kung, H. C., Brown, W. R., Hill, E. E., Effects of Cathedral and Beamed Ceiling Construction of Residential Sprinkler Performance. Factory Mutual Research Corporation, International Association of Fire Safety Science, Proceedings June 13-17 1989, P 643-653.

2. Underwriters Laboratory Inc., UL 1626 Standard for Residential Sprinklers for Fire Protection Service, Second Edition. September 30, 1994.

3. National Fire Protection Association, National Fire Protection Association 13D Sprinkler Systems in One and Two Family Dwellings and Manufactured Homes 1993 Edition, National Fire Protection Association, Quincy, MA 02269.

4. Underwriters Laboratory Inc., Determination of the Response Time Index (RTI) and Conductivity (C) Factor. Project Number 96NK9527 April 1996.

5. National Fire Protection Association, National Fire Protection Association 72 Standard National Fire Alarm Code Appendix B. 1993 Edition. National Fire Protection Association, Quincy, MA 02269.

6. Zukoski, E. E., Kubota, T., and Cetegen, B., Entrainment in Fire Plumes. NBS-GCR-80294, National Bureau of Standards, November 1980.

7. Alpert, R. L., and Ward, E. G., Evaluating Unsprinklered Fire Hazards. Factory Mutual Research Corporation, Norwood, MA, SFPE TR 83-02, FMRC J. I. 01836.20, August 1982.

8. McGrattan, K. B., Baum, H. R., Rehm, R. G., Large Eddy Simulations of Smoke Movement, Fire Safety Journal, Vol. 30, p 161-178.

9. Alpert, R. L., Calculations of response Time of Ceiling Mounted Fire Detectors. Fire Technology, Vol. 8, No. 3, pp 181-195, August 1972.

10. National Fire Protection Association, National Fire Protection Association Fire Protection Handbook $18^{\text {th }}$ Edition Section 6 p 191, National Fire Protection Association, Quincy, MA 02269 
Table 1 - Comparison of sprinkler activation times

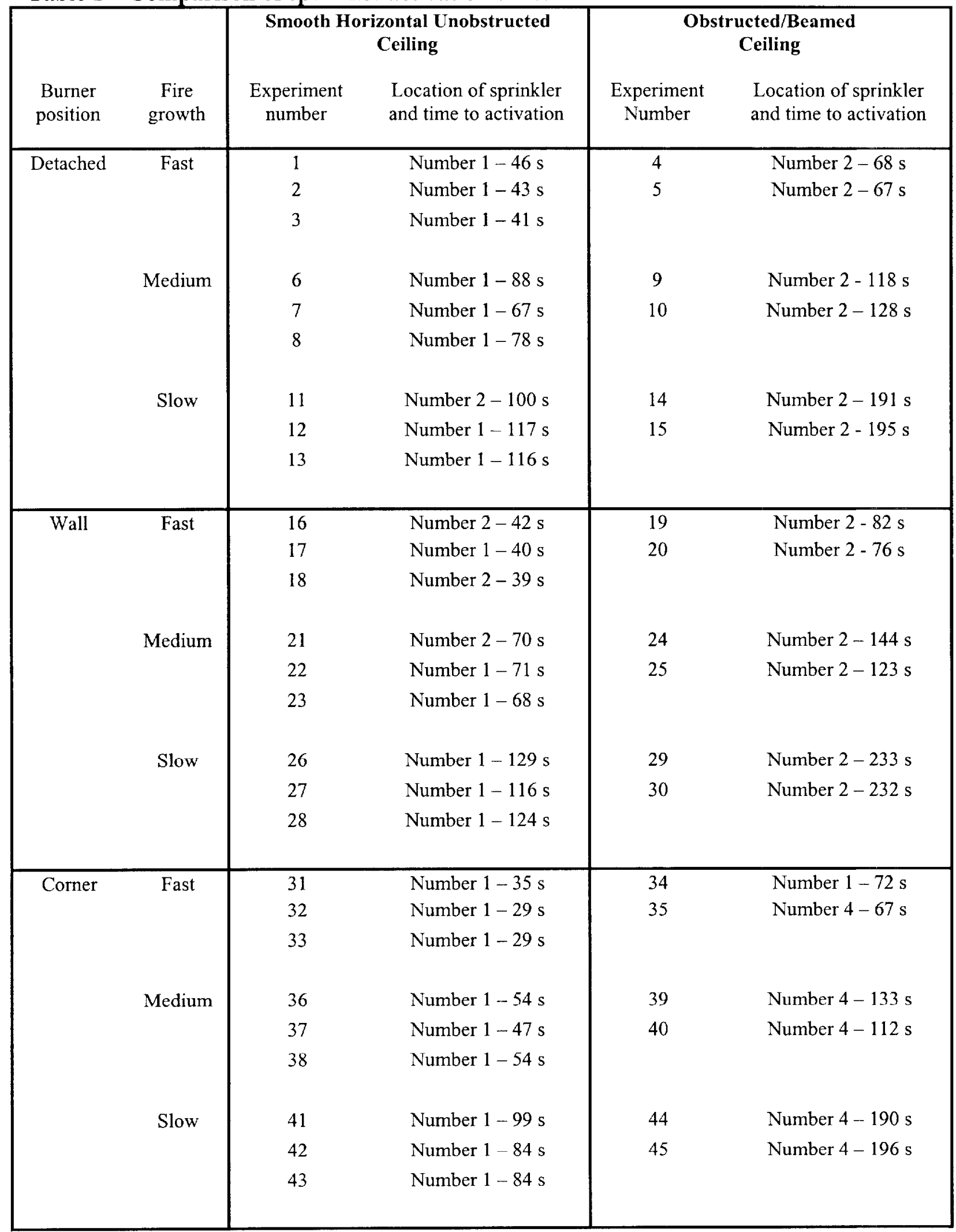


Table 2 - Average activation times and percent increase in activation times caused by obstructed/beamed ceiling

\begin{tabular}{|c|c|c|c|c|}
\hline $\begin{array}{l}\text { Burner } \\
\text { position }\end{array}$ & Fire growth & $\begin{array}{l}\text { Average time to } \\
\text { activation smooth } \\
\text { unobstructed } \\
\text { ceiling } \\
\text { (s) }\end{array}$ & $\begin{array}{l}\text { Average time to } \\
\text { activation } \\
\text { obstructed/beamed } \\
\text { ceiling } \\
\text { (s) }\end{array}$ & $\begin{array}{l}\text { Percent increase in } \\
\text { activation time } \\
\text { caused by } \\
\text { obstructed/beamed } \\
\text { ceiling }\end{array}$ \\
\hline \multirow[t]{3}{*}{ Detached } & Fast & 43 & 68 & 56 \\
\hline & Medium & 78 & 123 & 58 \\
\hline & Slow & 111 & 193 & 74 \\
\hline \multirow[t]{3}{*}{ Wall } & Fast & 40 & 79 & 96 \\
\hline & Medium & 70 & 134 & 92 \\
\hline & Slow & 123 & 233 & 89 \\
\hline \multirow[t]{3}{*}{ Corner } & Fast & 31 & 70 & 124 \\
\hline & Medium & 52 & 123 & 137 \\
\hline & Slow & 89 & 193 & 117 \\
\hline
\end{tabular}


Table 3 - Temperature of the ceiling jet at the activated sprinkler at time of activation

\begin{tabular}{|c|c|c|c|c|c|c|c|}
\hline \multirow{3}{*}{$\begin{array}{c}\text { Burner } \\
\text { position }\end{array}$} & \multirow{3}{*}{$\begin{array}{c}\begin{array}{c}\text { Fire } \\
\text { growth }\end{array} \\
\text { Fast }\end{array}$} & \multirow{2}{*}{\multicolumn{3}{|c|}{$\begin{array}{c}\text { Smooth Horizontal Unobstructed } \\
\text { Ceiling } \\
\begin{array}{c}\text { Experiment } \\
\text { number }\end{array} \\
\begin{array}{c}\text { Location of sprinkler and } \\
\text { ceiling jet temperature } \\
\left({ }^{\circ} \mathrm{C}\right)\end{array} \\
\end{array}$}} & \multicolumn{3}{|c|}{$\begin{array}{c}\text { Obstructed/Bcamed } \\
\text { Ceiling }\end{array}$} \\
\hline & & & & & \multirow{3}{*}{$\begin{array}{c}\text { Experiment } \\
\text { number } \\
4 \\
5\end{array}$} & \multicolumn{2}{|c|}{$\begin{array}{l}\text { Location of sprinkler and } \\
\text { ceiling jet temperature } \\
\left({ }^{\circ} \mathrm{C}\right)\end{array}$} \\
\hline & & 1 & Number 1 & 116 & & Number 2 & 165 \\
\hline & & 2 & Number 1 & 118 & & Number 2 & 153 \\
\hline & & 3 & Number 1 & 108 & & & \\
\hline & Medium & 6 & Number 1 & 102 & 9 & Number 2 & 130 \\
\hline & & 7 & Number 1 & 108 & 10 & Number 2 & 139 \\
\hline & & 8 & Number 1 & 111 & & & \\
\hline & Slow & 11 & Number 2 & 118 & 14 & Number 2 & 136 \\
\hline & & 12 & Number 1 & 100 & 15 & Number 2 & 133 \\
\hline & & 13 & Number 1 & 93 & & & \\
\hline Wall & Fast & 16 & Number 2 & 138 & 19 & Number 2 & 162 \\
\hline & & 17 & Number 1 & 114 & 20 & Number 2 & 165 \\
\hline & & 18 & Number 2 & 118 & & & \\
\hline & Medium & 21 & Number 2 & 114 & 24 & Number 2 & 155 \\
\hline & & 22 & Number 1 & 106 & 25 & Number 2 & 138 \\
\hline & & 23 & Number 1 & 106 & & & \\
\hline & Slow & 26 & Number 1 & 102 & 29 & Number 2 & 129 \\
\hline & & 27 & Number 1 & 99 & 30 & Number 2 & 126 \\
\hline & & 28 & Number 1 & 100 & & & \\
\hline Comer & Fast & 31 & Number 1 & 121 & 34 & Number 1 & 154 \\
\hline & & 32 & Number 1 & 131 & 35 & Number 4 & 148 \\
\hline & & 33 & Number 1 & 143 & & & \\
\hline & Medium & 36 & Number 1 & 124 & 39 & Number 4 & 144 \\
\hline & & 37 & Number 1 & 116 & 40 & Number 4 & 131 \\
\hline & & 38 & Number 1 & 137 & & & \\
\hline & Slow & 41 & Number 1 & 118 & 44 & Number 4 & $129^{*}$ \\
\hline & & 42 & Number 1 & 112 & 45 & Number 4 & 125 \\
\hline & & 43 & Number 1 & 109 & & & \\
\hline
\end{tabular}

*Thermocouple at sprinkler malfunctioned. This value obtained from the thermocouple located $25 \mathrm{~mm}$ ( 1 in) below the sprinkler. 
Table 4-Temperature comparisons at the $1.54 \mathrm{~m}(5 \mathrm{ft})$ elevation at the time of sprinkler activation

\begin{tabular}{|c|c|c|c|c|c|c|c|}
\hline \multirow[b]{2}{*}{$\begin{array}{l}\text { Burner } \\
\text { position }\end{array}$} & \multirow[b]{2}{*}{$\begin{array}{l}\text { Fire } \\
\text { growth }\end{array}$} & \multicolumn{3}{|c|}{$\begin{array}{c}\text { Smooth Horizontal Unobstructed } \\
\text { Ceiling }\end{array}$} & \multicolumn{3}{|c|}{$\begin{array}{l}\text { Obstructed/Beamed } \\
\text { Ceiling }\end{array}$} \\
\hline & & $\begin{array}{l}\text { Experiment } \\
\text { number }\end{array}$ & $\begin{array}{c}\text { Average } \\
\text { temp } \\
\left({ }^{\circ} \mathrm{C}\right) \\
\end{array}$ & $\begin{array}{c}\text { Low }- \text { High } \\
\text { temp } \\
\left({ }^{\circ} \mathrm{C}\right) \\
\end{array}$ & $\begin{array}{l}\text { Experiment } \\
\text { number }\end{array}$ & $\begin{array}{c}\text { Average } \\
\text { temp } \\
\left({ }^{\circ} \mathrm{C}\right) \\
\end{array}$ & $\begin{array}{c}\text { Low ... High } \\
\text { temp } \\
\left({ }^{\circ} \mathrm{C}\right) \\
\end{array}$ \\
\hline \multirow[t]{9}{*}{ Detached } & \multirow[t]{3}{*}{ Fast } & 1 & 41 & $36-48$ & 4 & 61 & $51-76$ \\
\hline & & 2 & 38 & $31-44$ & 5 & 57 & $52-63$ \\
\hline & & 3 & 40 & $31-46$ & & & \\
\hline & \multirow[t]{3}{*}{ Medium } & 6 & 52 & $47-59$ & 9 & 64 & $63-65$ \\
\hline & & 7 & 50 & $46-54$ & 10 & 69 & $67-69$ \\
\hline & & 8 & 53 & $48-57$ & & & \\
\hline & \multirow[t]{3}{*}{ Slow } & 11 & 55 & $47-62$ & 14 & 72 & $68-74$ \\
\hline & & 12 & 52 & $45-60$ & 15 & 70 & $69-71$ \\
\hline & & 13 & 53 & $48-58$ & & & \\
\hline \multirow[t]{9}{*}{ Wall } & \multirow[t]{3}{*}{ Fast } & 16 & 27 & $24-32$ & 19 & 63 & $57-70$ \\
\hline & & 17 & 27 & $22-34$ & 20 & 63 & $61-67$ \\
\hline & & 18 & 25 & $21-31$ & & & \\
\hline & \multirow[t]{3}{*}{ Medium } & 21 & 39 & $36-46$ & 24 & 71 & $66-73$ \\
\hline & & 22 & 39 & $37-44$ & 25 & 67 & $66-68$ \\
\hline & & 23 & 39 & $36-46$ & & & \\
\hline & \multirow[t]{3}{*}{ Slow } & 26 & 49 & $45-54$ & 29 & 68 & $66-71$ \\
\hline & & 27 & 44 & $40-49$ & 30 & 80 & $77-82$ \\
\hline & & 28 & 47 & $43-53$ & & & \\
\hline \multirow[t]{9}{*}{ Corner } & \multirow[t]{3}{*}{ Fast } & 31 & 24 & $22-25$ & 34 & 38 & $28-46$ \\
\hline & & 32 & 21 & $20-22$ & 35 & 37 & $32-42$ \\
\hline & & 33 & 22 & $21-24$ & & & \\
\hline & \multirow[t]{3}{*}{ Medium } & 36 & 24 & $22-25$ & 39 & 53 & $46-62$ \\
\hline & & 37 & 23 & $21-24$ & 40 & 45 & $43-49$ \\
\hline & & 38 & 25 & $22-28$ & & & \\
\hline & \multirow[t]{3}{*}{ Slow } & 41 & 34 & $29-39$ & 44 & 50 & $47-52$ \\
\hline & & 42 & 28 & $24-30$ & 45 & 49 & $47-52$ \\
\hline & & 43 & 26 & $24-29$ & & & \\
\hline
\end{tabular}


Table 5 - Temperatures comparison at the $1.89 \mathrm{~m}(6 \mathrm{ft})$ elevation at the time of sprinkler activation

\begin{tabular}{|c|c|c|c|c|c|c|c|}
\hline \multirow[b]{2}{*}{$\begin{array}{l}\text { Burner } \\
\text { position }\end{array}$} & \multirow[b]{2}{*}{$\begin{array}{l}\text { Fire } \\
\text { growth }\end{array}$} & \multicolumn{3}{|c|}{$\begin{array}{c}\text { Smooth Horizontal Unobstructed } \\
\text { Ceiling }\end{array}$} & \multicolumn{3}{|c|}{$\begin{array}{l}\text { Obstructed/Beamed } \\
\text { Ceiling }\end{array}$} \\
\hline & & $\begin{array}{l}\text { Experiment } \\
\text { number }\end{array}$ & $\begin{array}{c}\text { Average } \\
\text { temp } \\
\left({ }^{\circ} \mathrm{C}\right) \\
\end{array}$ & $\begin{array}{c}\text { Low - High } \\
\text { Temp } \\
\left({ }^{\circ} \mathrm{C}\right)\end{array}$ & $\begin{array}{l}\text { Experiment } \\
\text { number }\end{array}$ & $\begin{array}{c}\text { Average } \\
\text { temp } \\
\left({ }^{\circ} \mathrm{C}\right)\end{array}$ & $\begin{array}{c}\text { Low }- \text { High } \\
\text { temp } \\
\left({ }^{\circ} \mathrm{C}\right) \\
\end{array}$ \\
\hline \multirow[t]{9}{*}{ Detached } & \multirow[t]{3}{*}{ Fast } & 1 & 69 & $59-77$ & 4 & 89 & $82-111$ \\
\hline & & 2 & 68 & $58-80$ & 5 & 89 & $82-106$ \\
\hline & & 3 & 72 & $59-83$ & & & \\
\hline & \multirow[t]{3}{*}{ Medium } & 6 & 77 & $67-85$ & 9 & 87 & $78-100$ \\
\hline & & 7 & 73 & $61-80$ & 10 & 90 & $85-97$ \\
\hline & & 8 & 74 & $65-80$ & & & \\
\hline & \multirow[t]{3}{*}{ Slow } & 11 & 75 & $68-82$ & 14 & 93 & $84-106$ \\
\hline & & 12 & 73 & $65-80$ & 15 & 91 & $84-100$ \\
\hline & & 13 & 71 & $66-76$ & & & \\
\hline \multirow[t]{9}{*}{ Wall } & \multirow[t]{3}{*}{ Fast } & 16 & 52 & $52-53$ & 19 & 103 & $85-129$ \\
\hline & & 17 & 59 & $55-64$ & 20 & 100 & $88-119$ \\
\hline & & 18 & 54 & $46-62$ & & & \\
\hline & \multirow[t]{3}{*}{ Medium } & 21 & 64 & $63-67$ & 24 & 97 & $90-103$ \\
\hline & & 22 & 65 & $62-67$ & 25 & 93 & $91-96$ \\
\hline & & 23 & 67 & $61-76$ & & & \\
\hline & \multirow[t]{3}{*}{ Slow } & 26 & 74 & $69-77$ & 29 & 89 & $84-96$ \\
\hline & & 27 & 67 & $64-71$ & 30 & 101 & $96-112$ \\
\hline & & 28 & 69 & $67-72$ & & & \\
\hline \multirow[t]{9}{*}{ Comer } & \multirow[t]{3}{*}{ Fast } & 31 & 30 & $23-40$ & 34 & 60 & $55-69$ \\
\hline & & 32 & 31 & $21-42$ & 35 & 66 & $60-72$ \\
\hline & & 33 & 32 & $22-44$ & & & \\
\hline & \multirow[t]{3}{*}{ Medium } & 36 & 42 & $35-48$ & 39 & 72 & $69-76$ \\
\hline & & 37 & 38 & $26-45$ & 40 & 63 & $62-65$ \\
\hline & & 38 & 43 & $36-49$ & & & \\
\hline & \multirow[t]{3}{*}{ Slow } & 41 & 56 & $46-65$ & 44 & 63 & $61-68$ \\
\hline & & 42 & 46 & $40-51$ & 45 & 61 & $57-64$ \\
\hline & & 43 & 45 & $41-47$ & & & \\
\hline
\end{tabular}




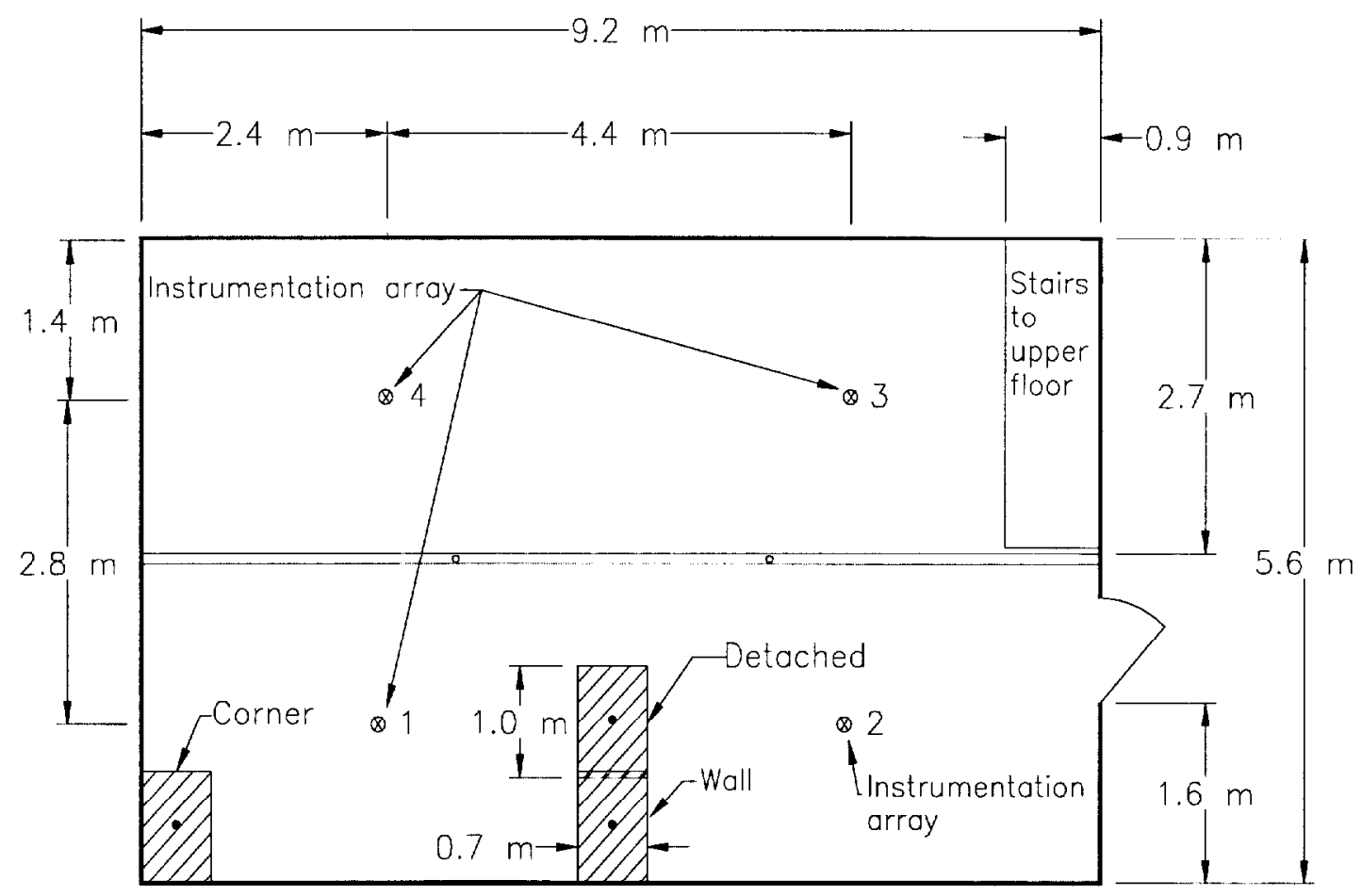

Figure 1 - Plan view of experimental set up.

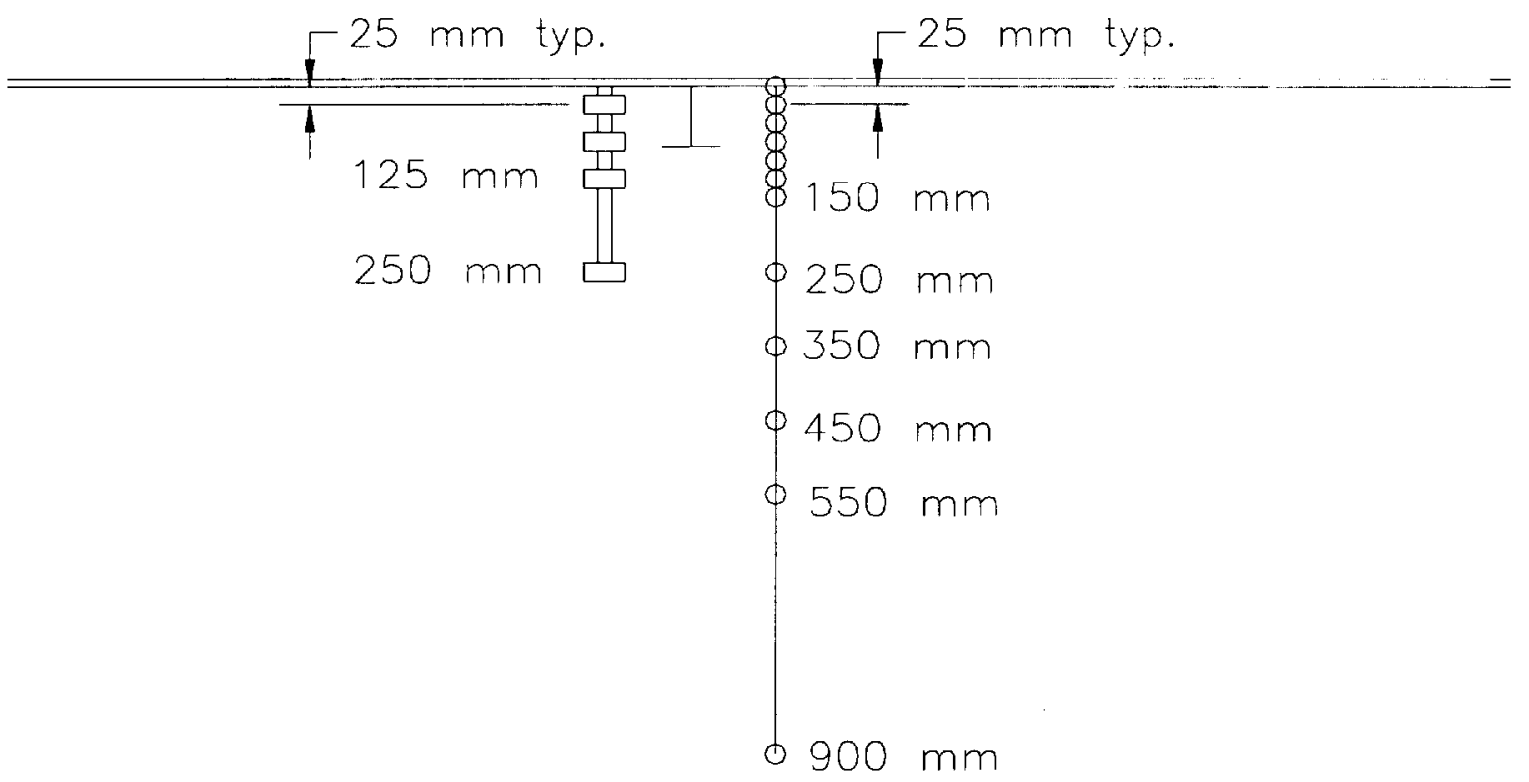

Figure 2 - Instrumentation set up for arrays 1 and 2. 


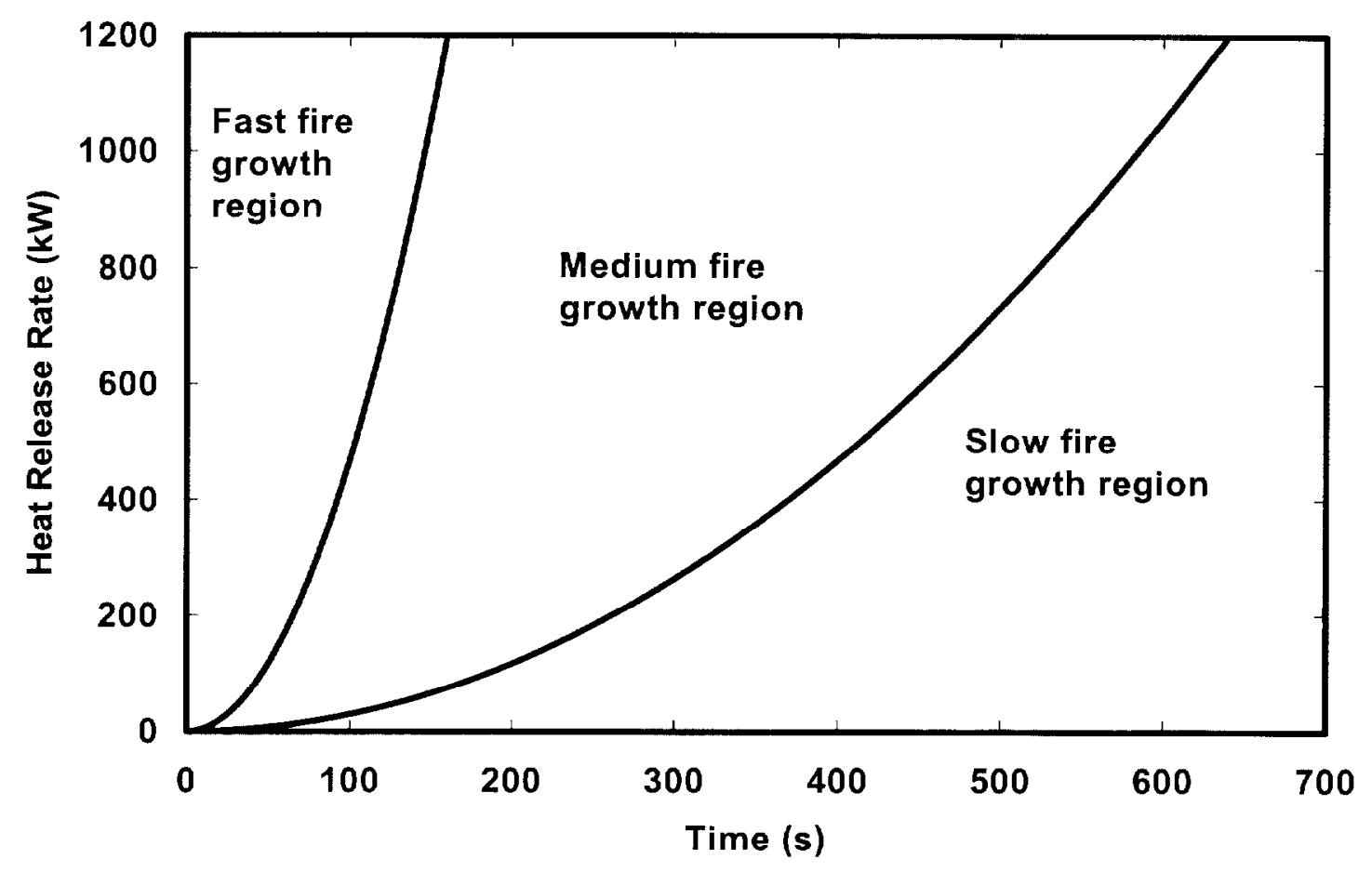

Figure 3 - Fast, medium, and slow fire growth regions.

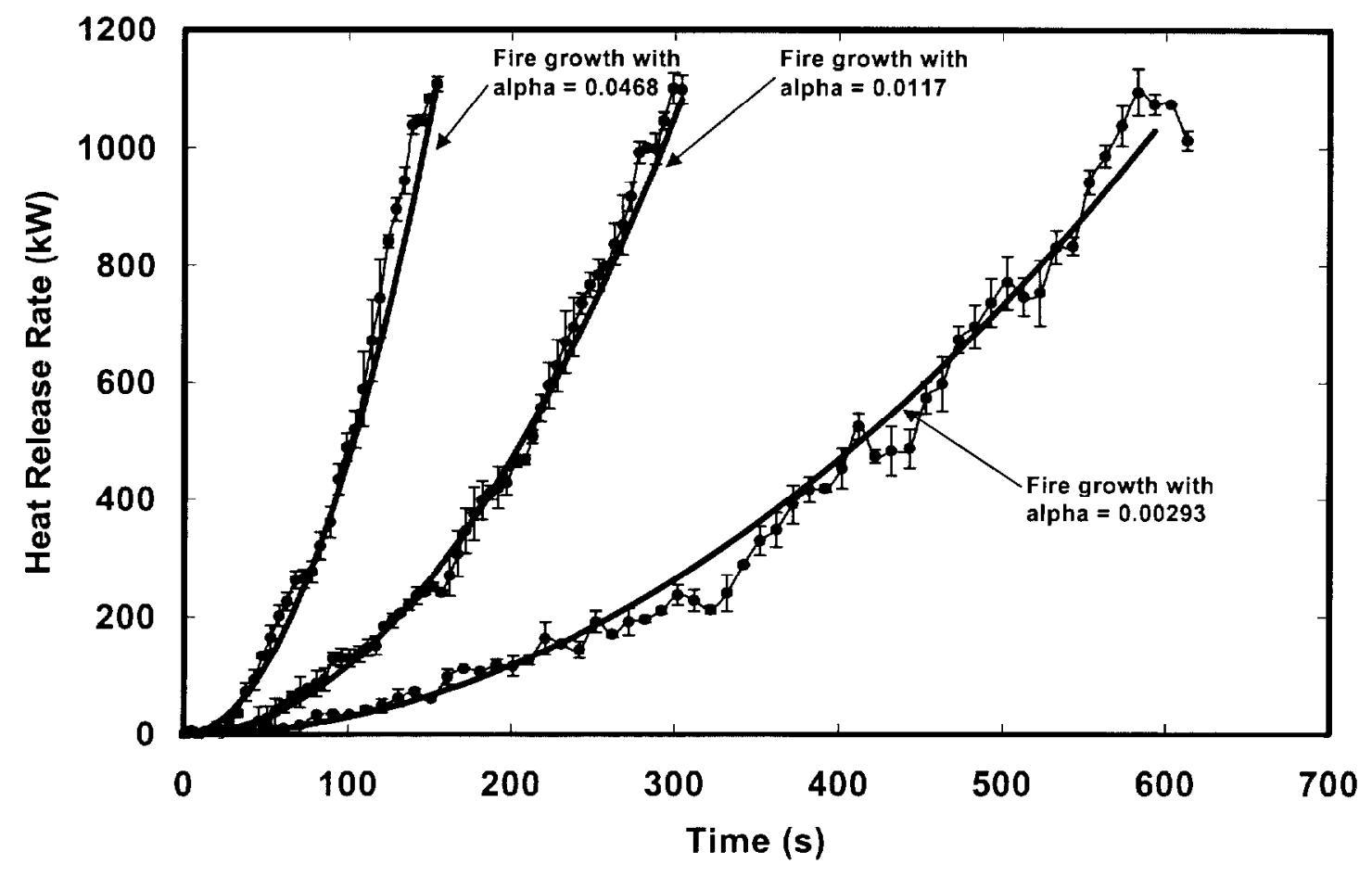

Figure 4 - Calibration data for the rectangular gas burner. 


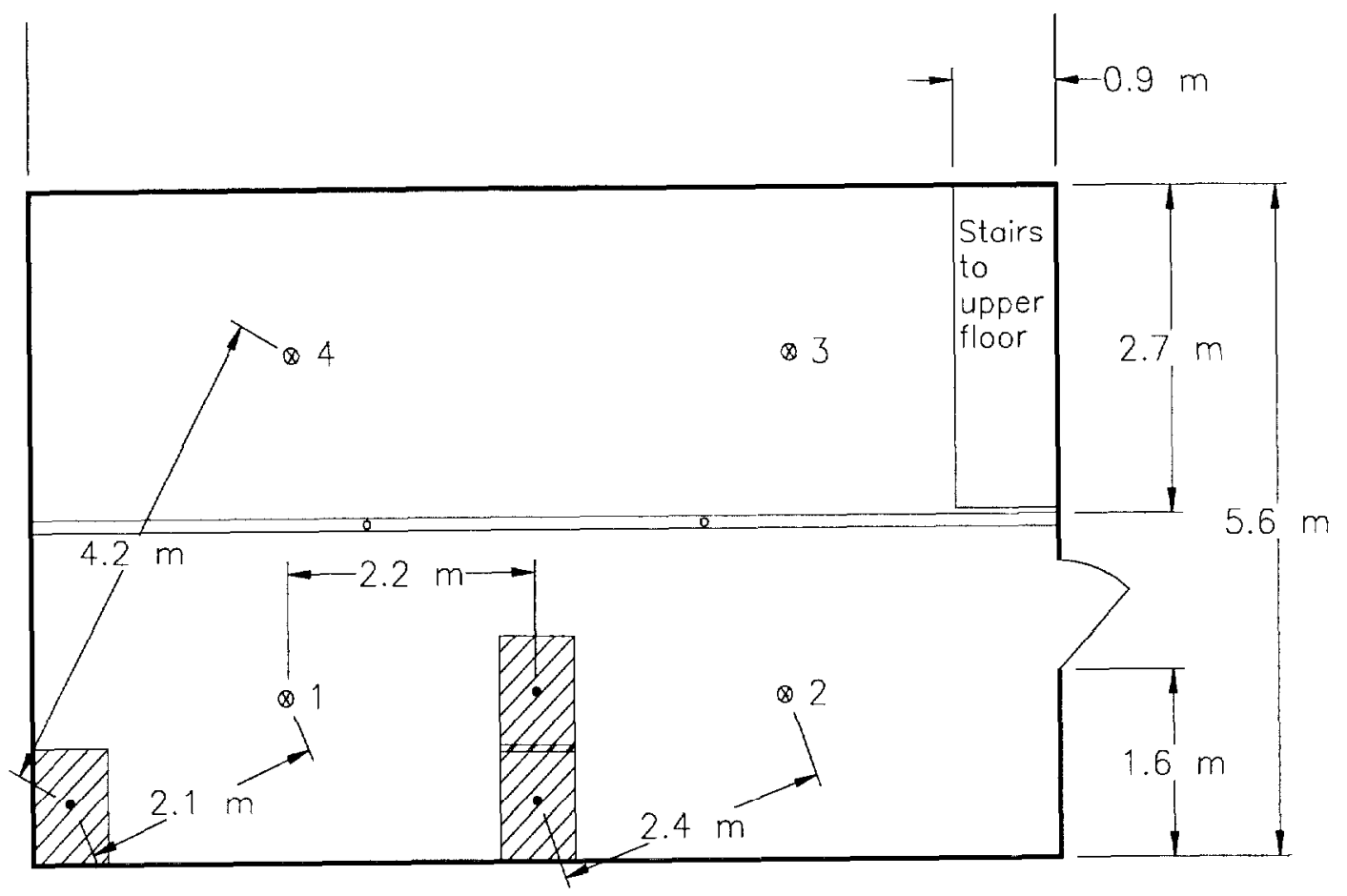

Figure 5 - Distances from different burner positions to sprinklers of interest.

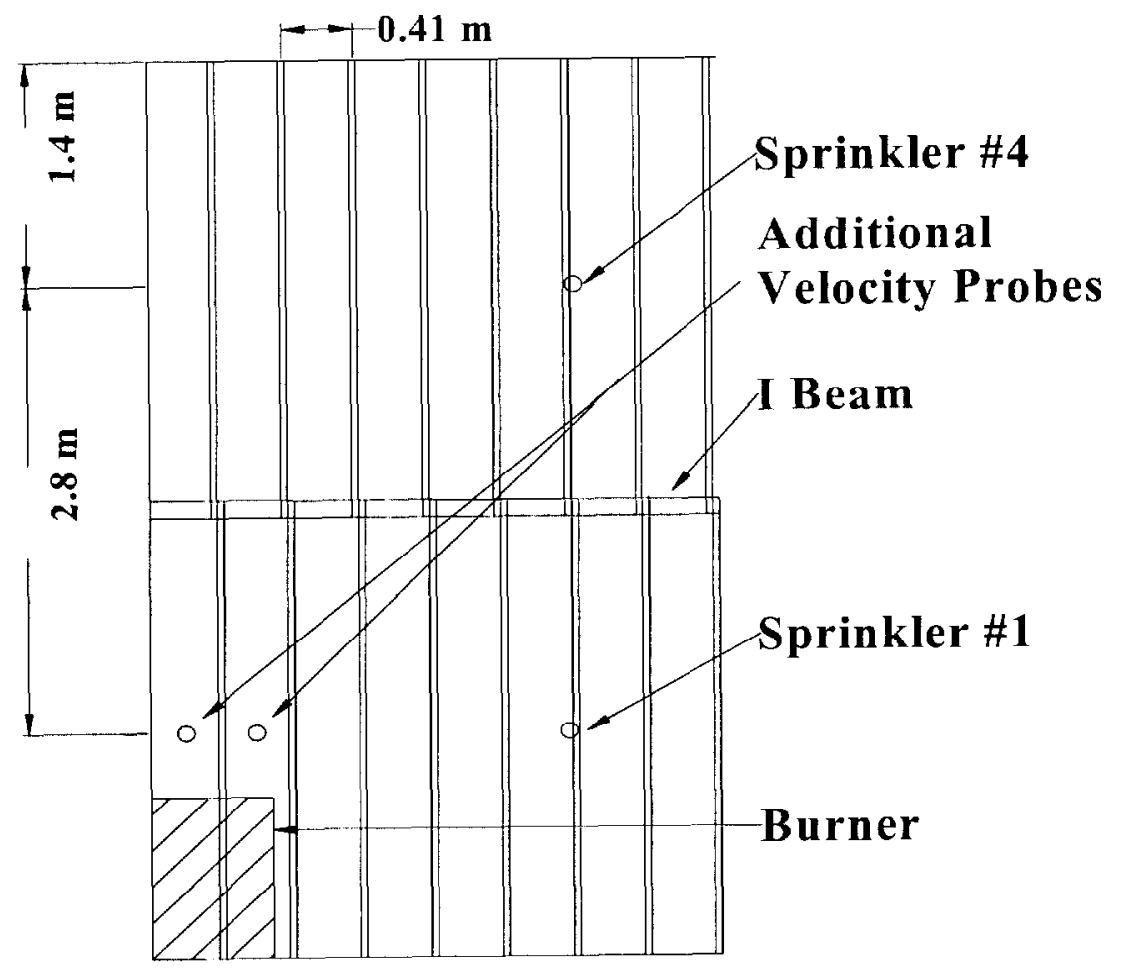

Figure 6 - Position of sprinklers 1 and 4 relative to channels formed by ceiling joists. Location of additional velocity probes, corner burner position, obstructed/beamed ceiling. 


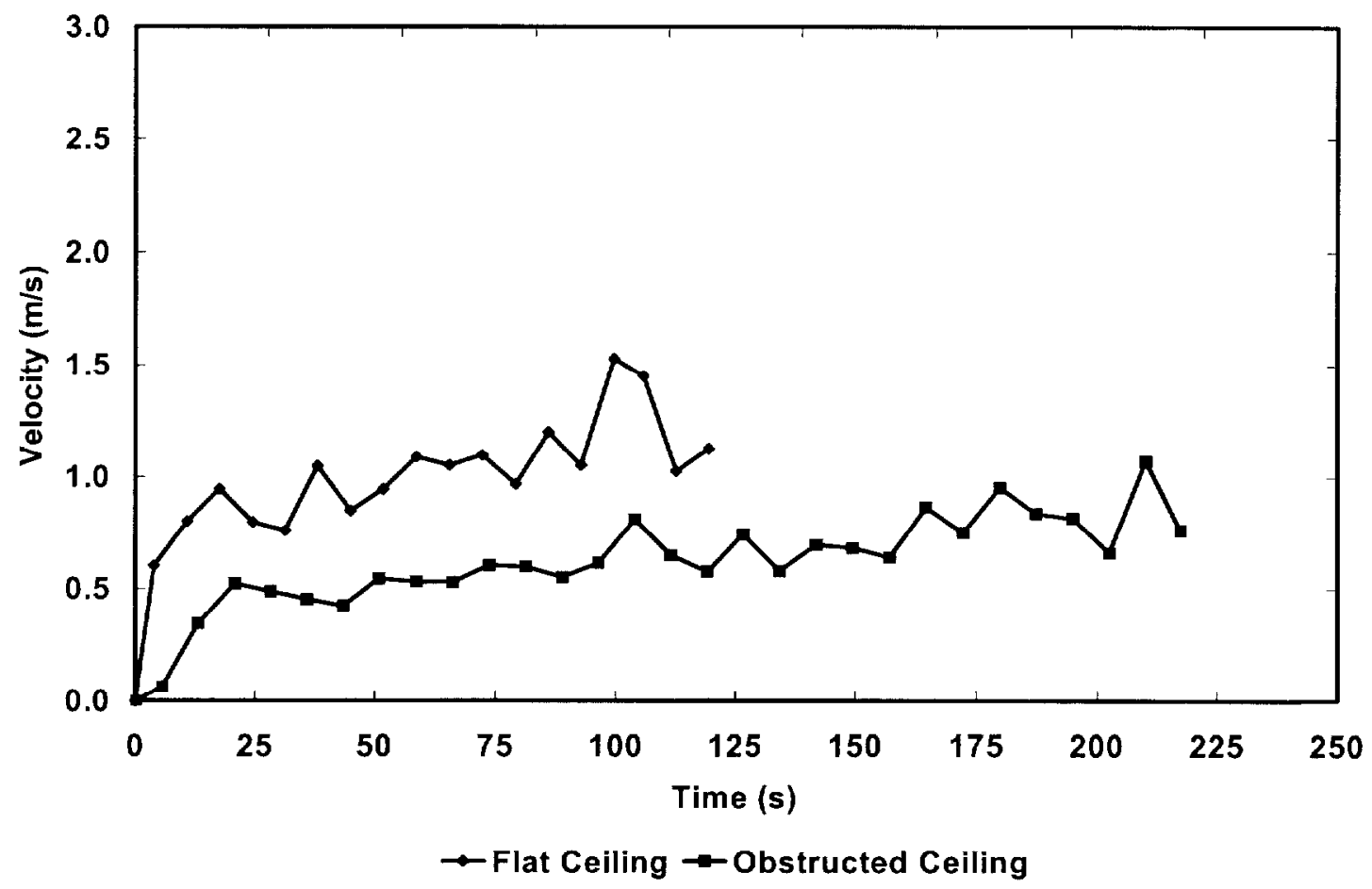

Figure 7 - Ceiling jet velocity comparisons at sprinkler number 1, detached burner position, slow fire growth.

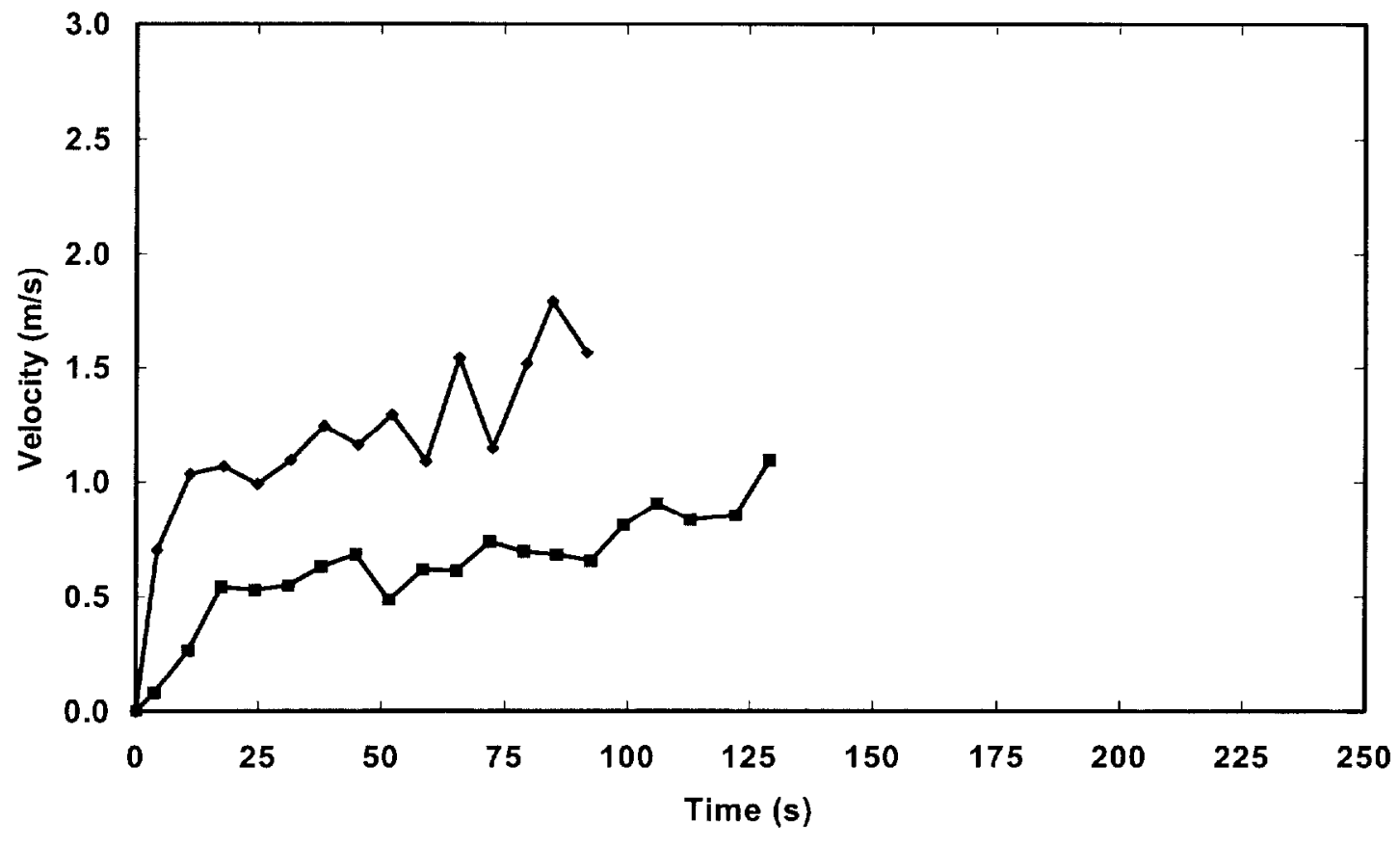

$\rightarrow$ Flat Ceiling $\rightarrow$-Obstructed Ceiling

Figure 8 - Ceiling jet velocity comparisons at sprinkler number 1, detached burner position, medium fire growth. 


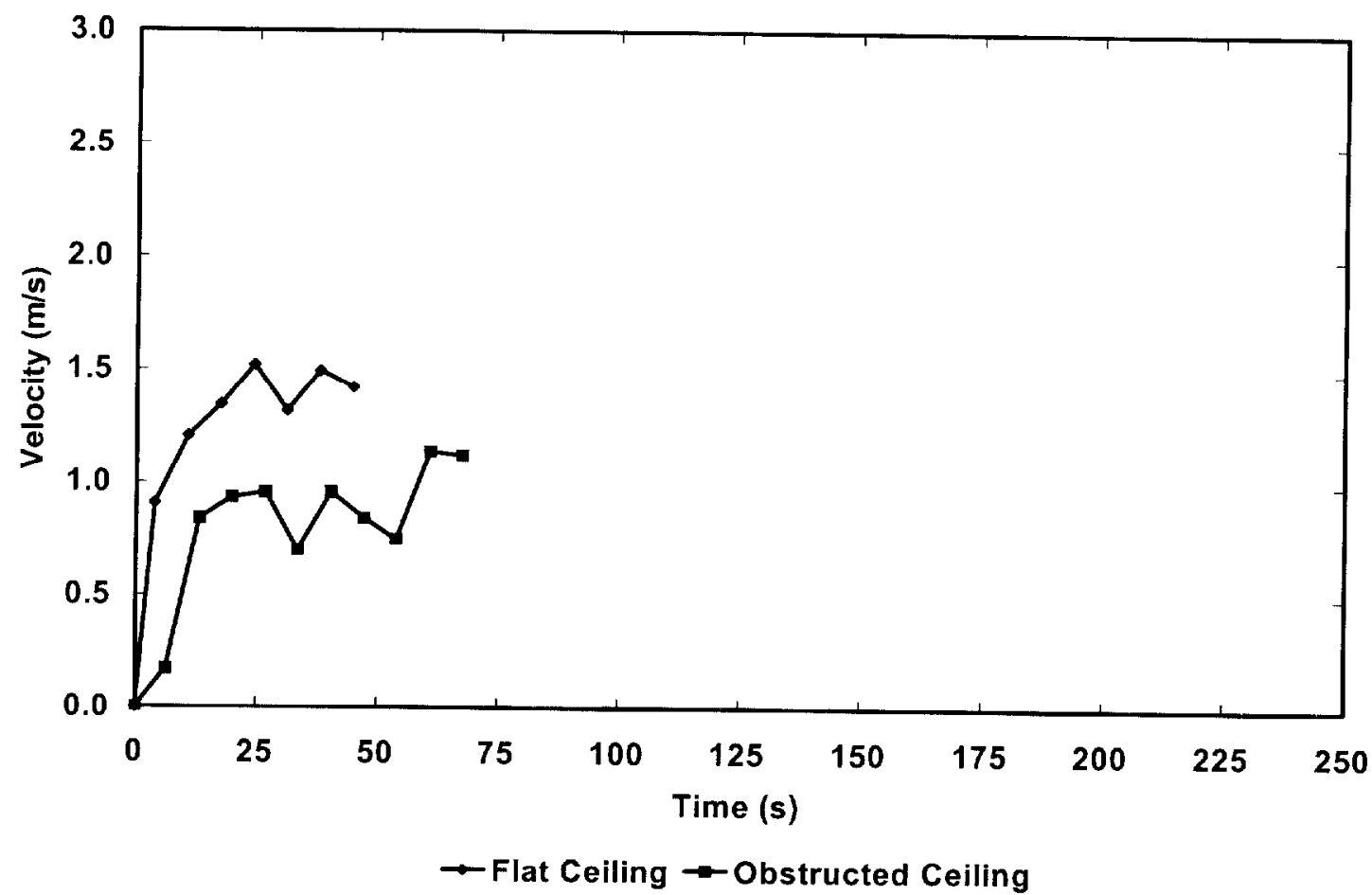

Figure 9 - Ceiling jet velocity comparisons at sprinkler number 1, detached burner position, fast fire growth.

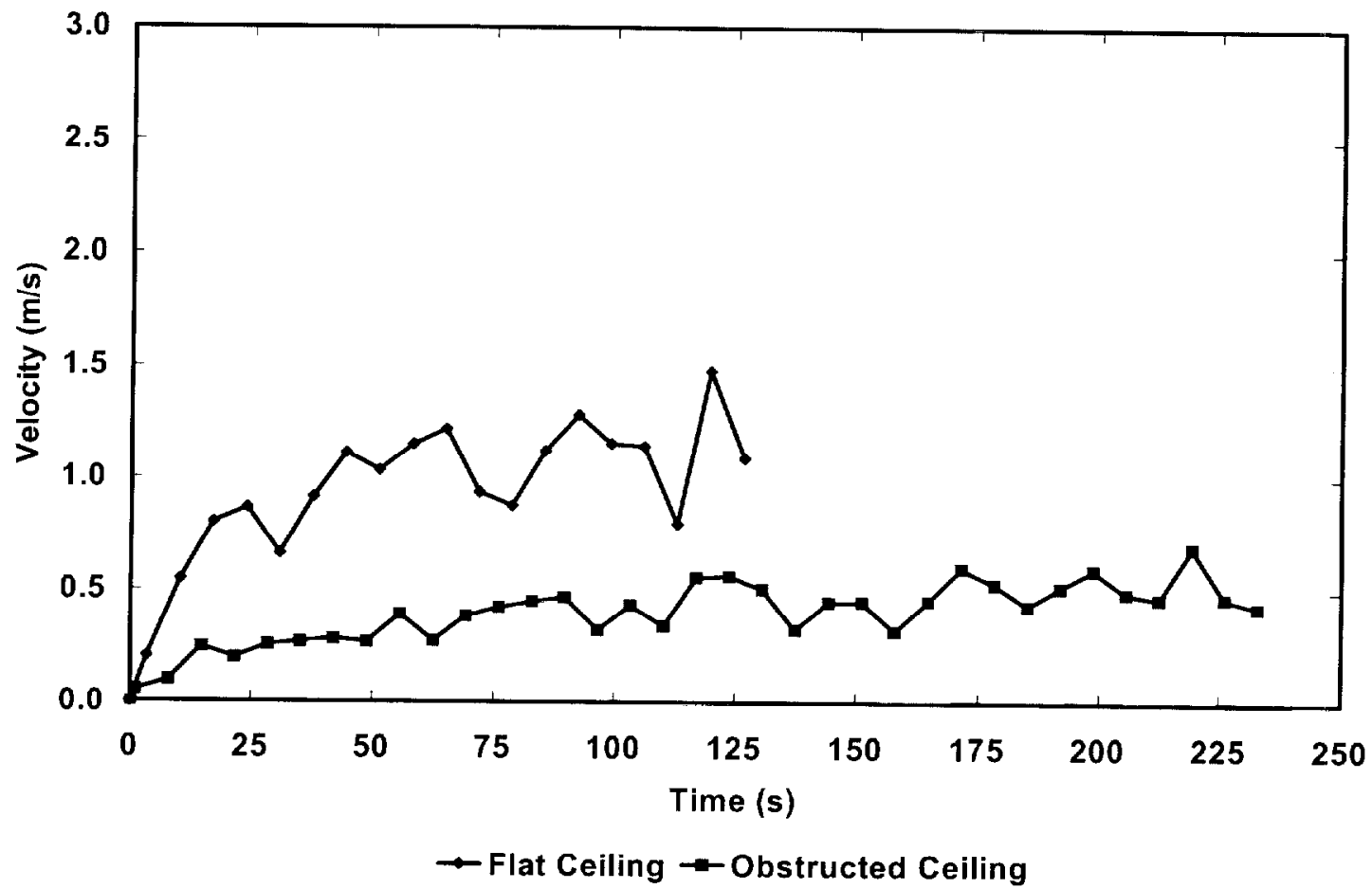

Figure 10 - Ceiling jet velocity comparisons at sprinkler number 1, wall burner position, slow fire growth. 


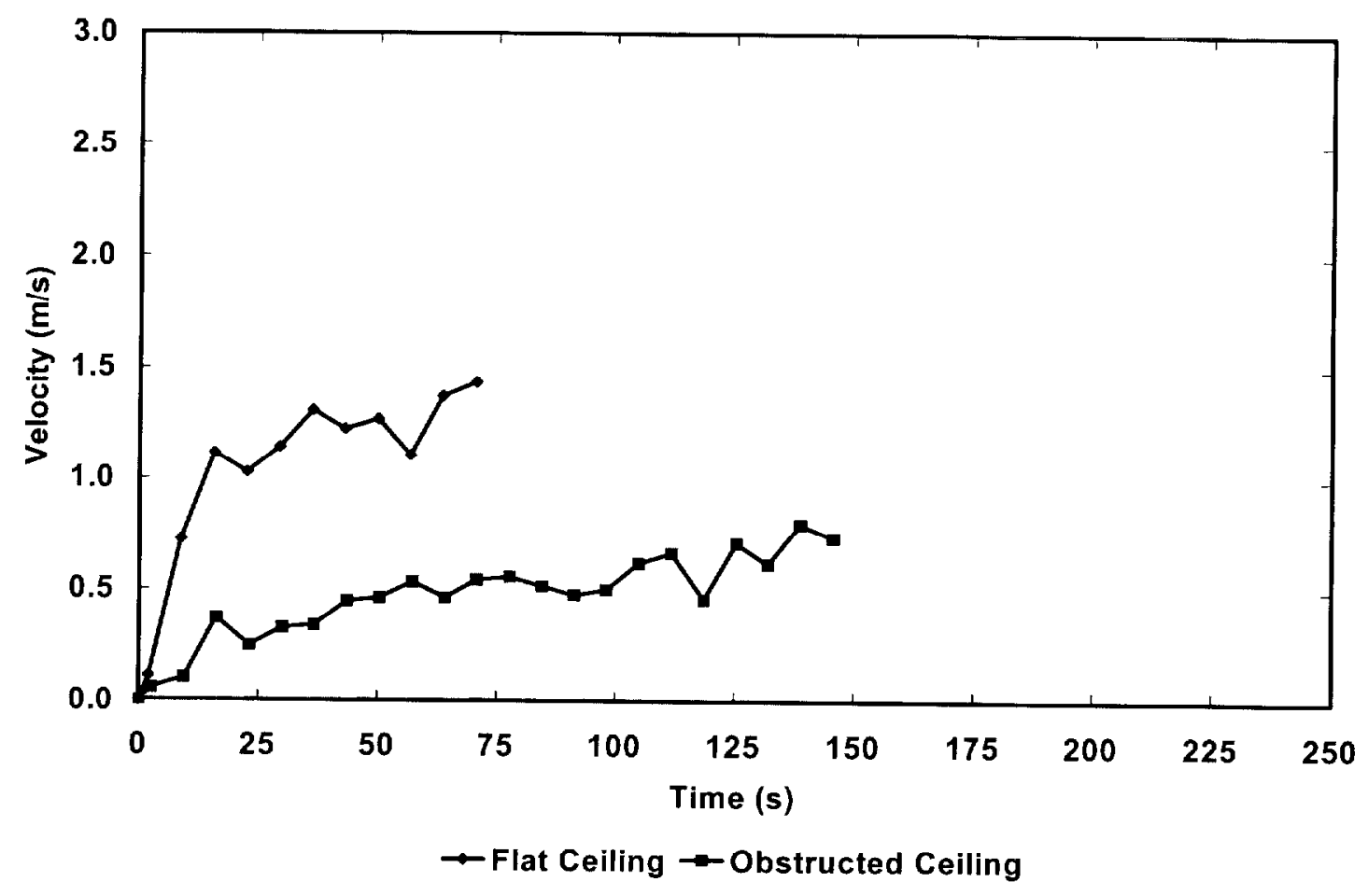

Figure 11 - Ceiling jet velocity comparisons at sprinkler number 1, wall burner position, medium fire growth.

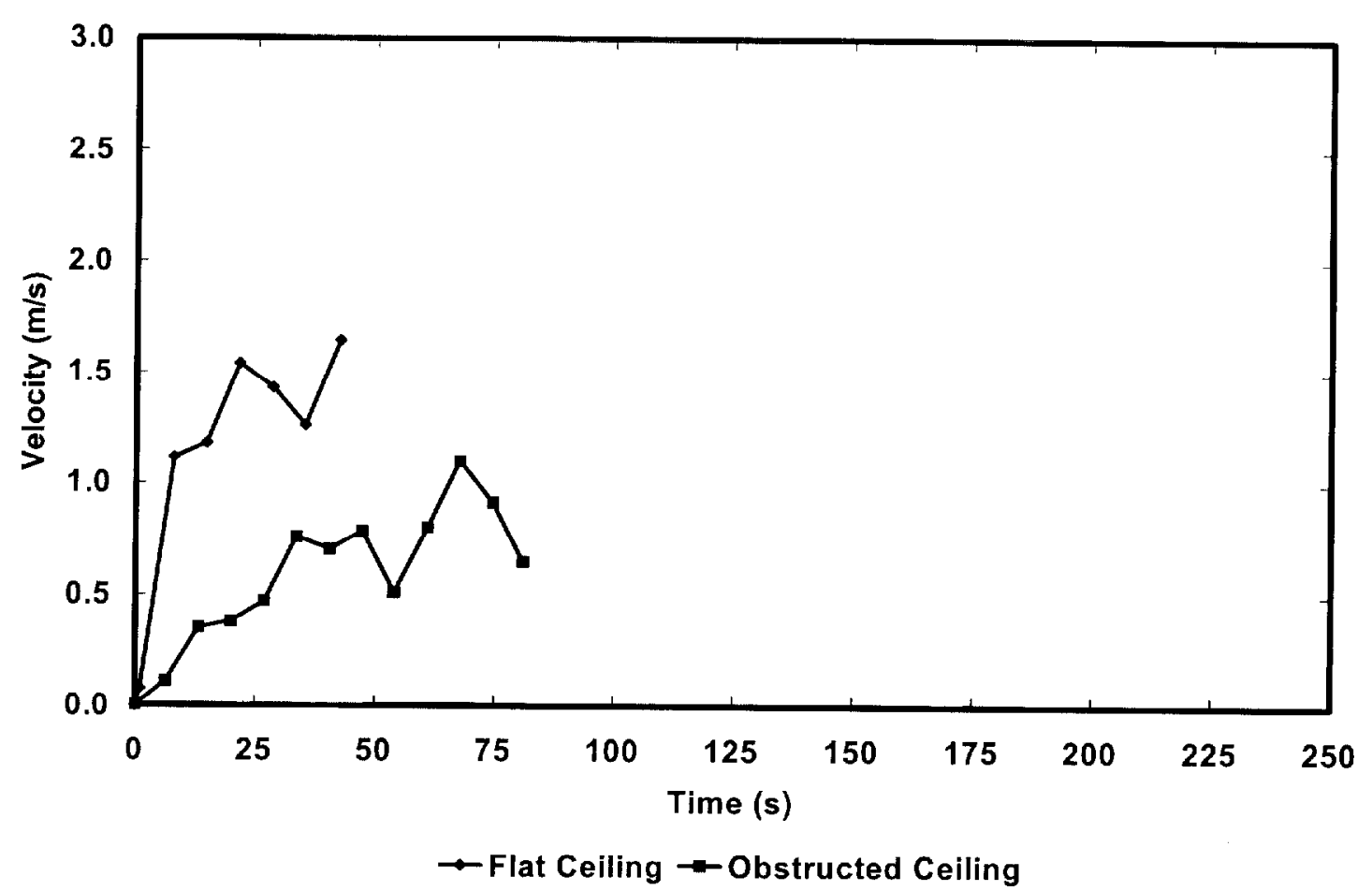

Figure 12 - Ceiling jet velocity comparisons at sprinkler number 1, wall burner position, fast fire growth. 


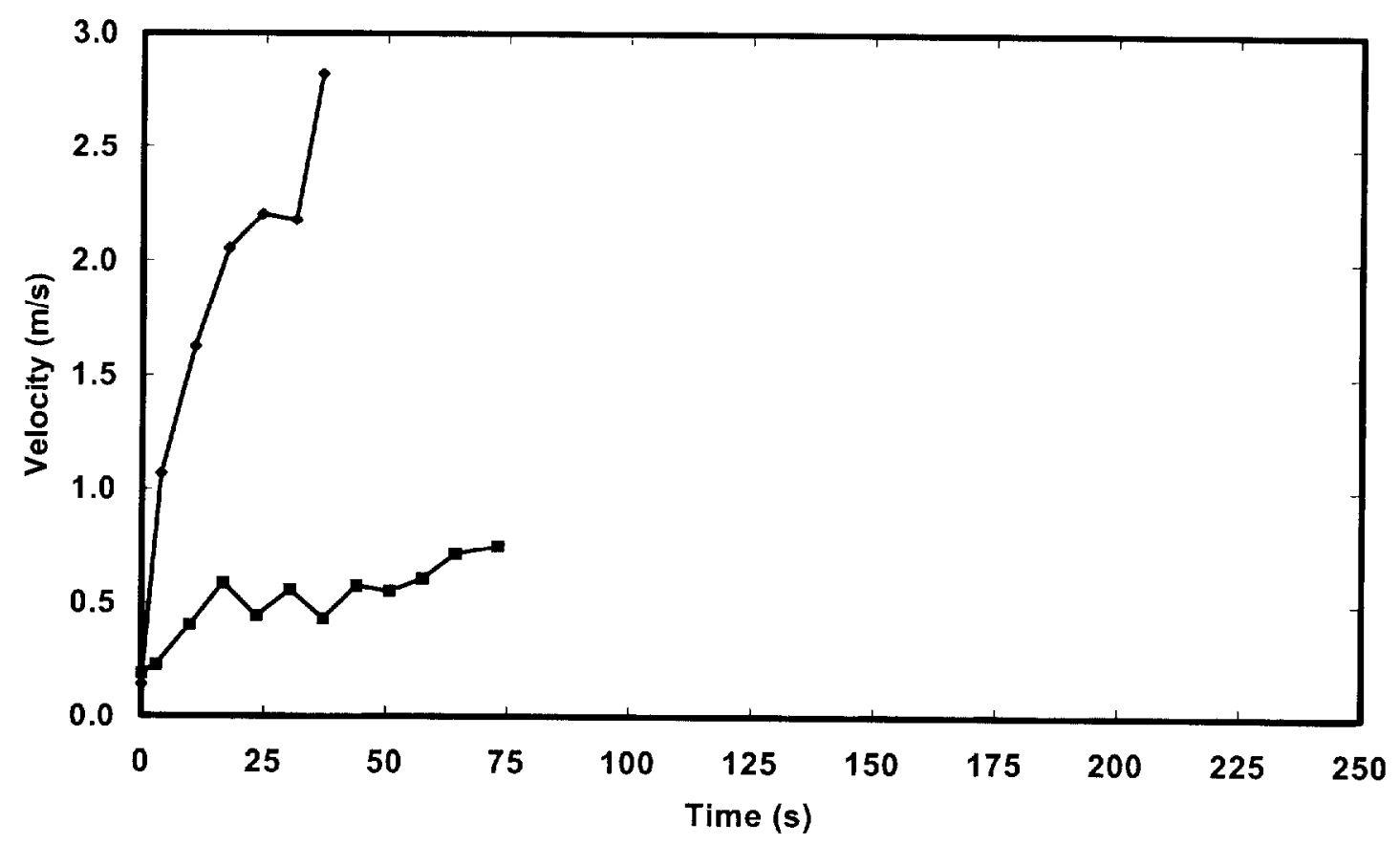

$\rightarrow$ Flat Ceiling $\rightarrow$ Obstructed Ceiling

Figure 13 - Ceiling jet velocity comparisons at sprinkler number 1, corner burner position, fast fire growth.

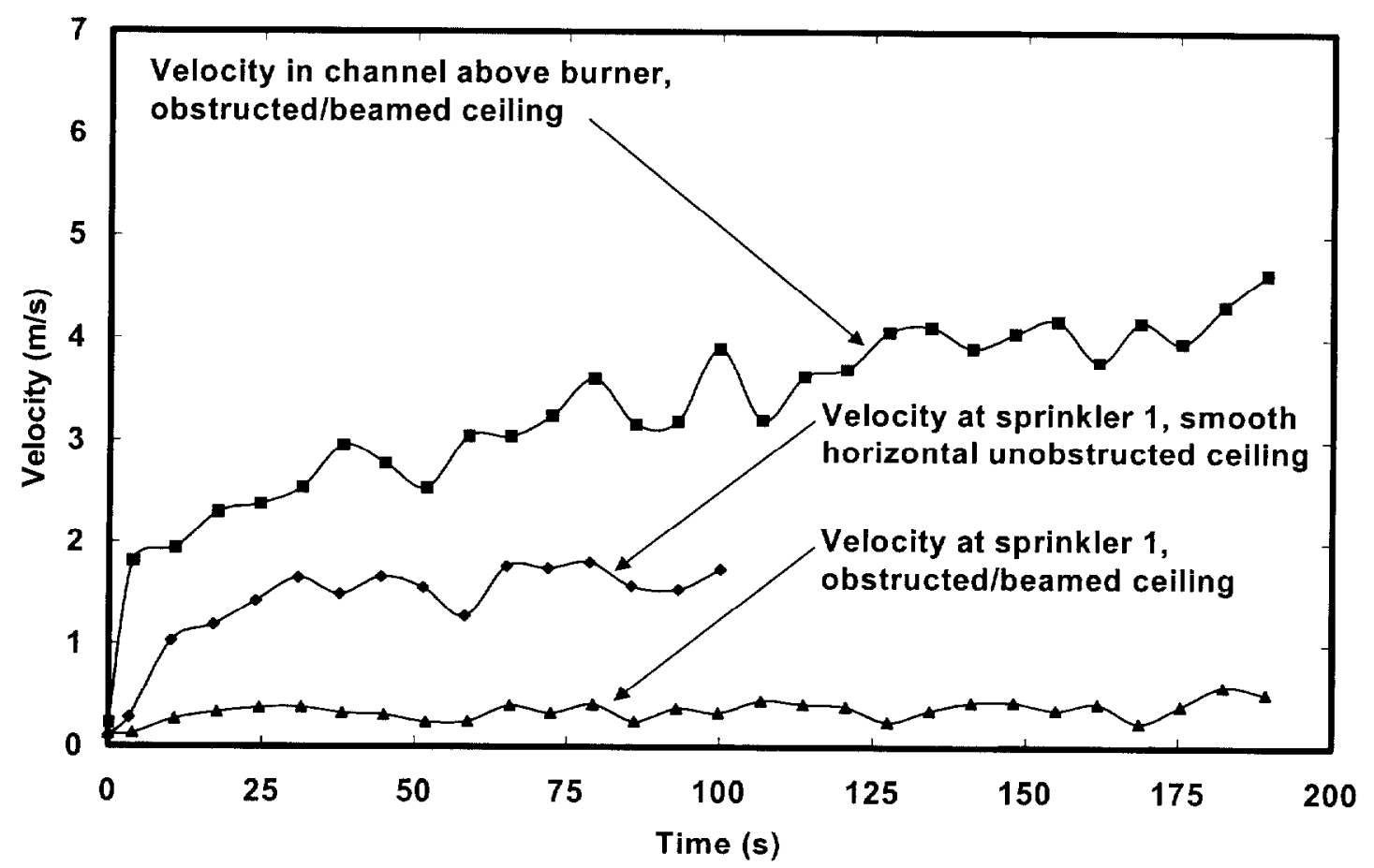

Figure 14 - Ceiling jet velocity comparisons for corner burner position, slow fire growth. 


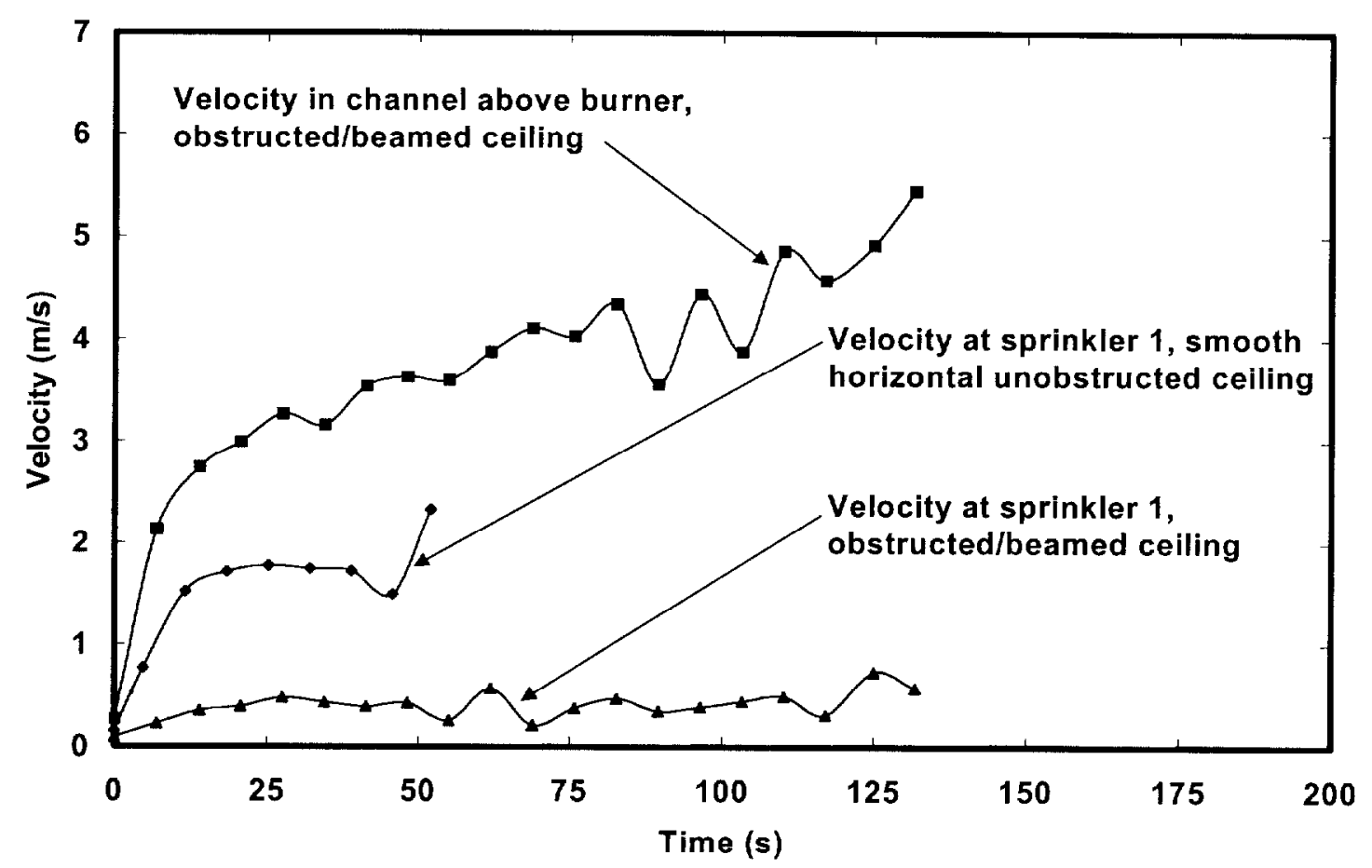

Figure 15 - Ceiling jet velocity comparisons for corner burner position, medium fire growth.

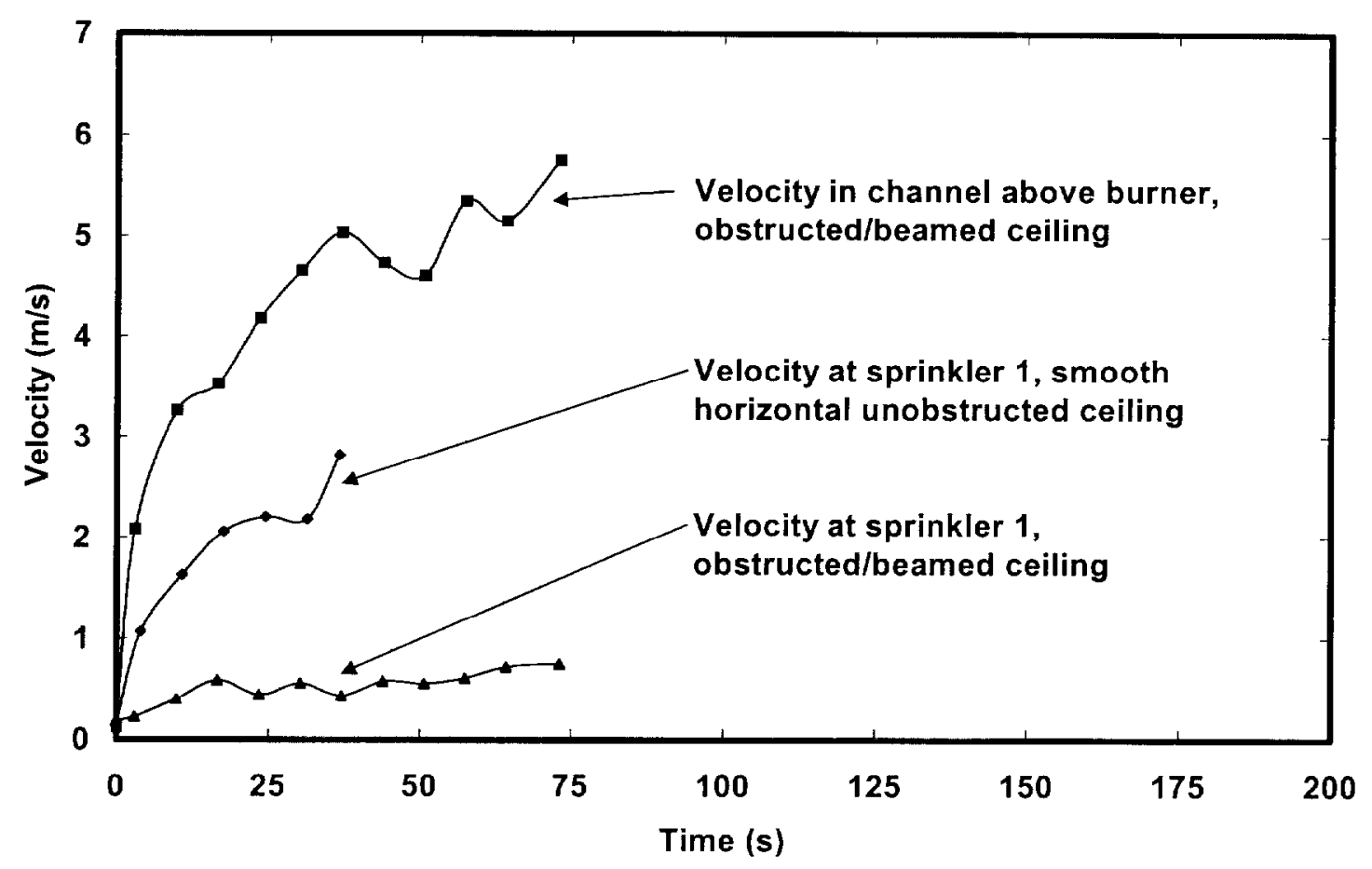

Figure 16 - Ceiling jet velocity comparisons for corner burner position, fast fire growth. 


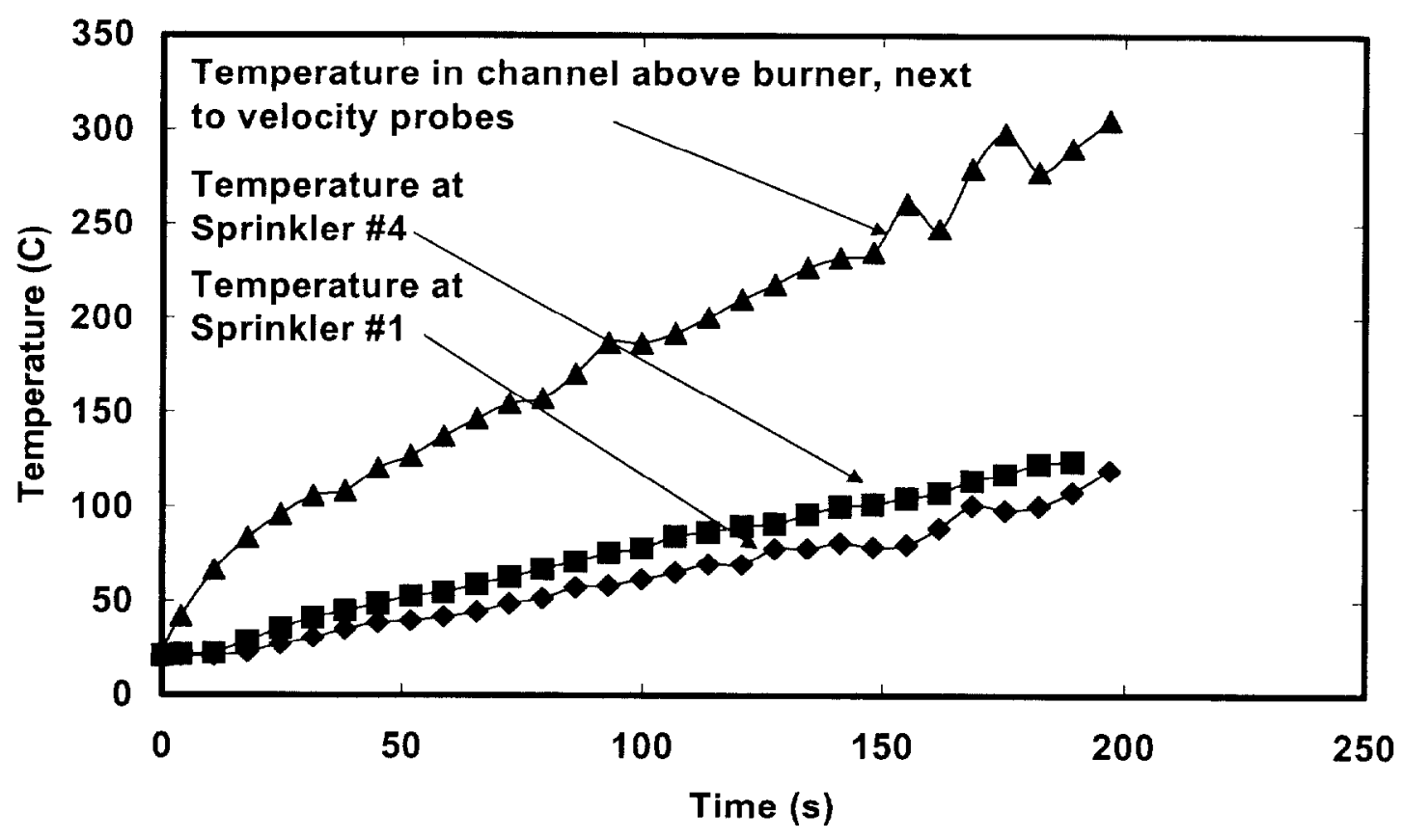

Figure 17 - Ceiling jet temperature comparisons at sprinklers 1 and 4 and in the channel above the burner, obstructed/beamed ceiling, burner in corner, slow fire growth.

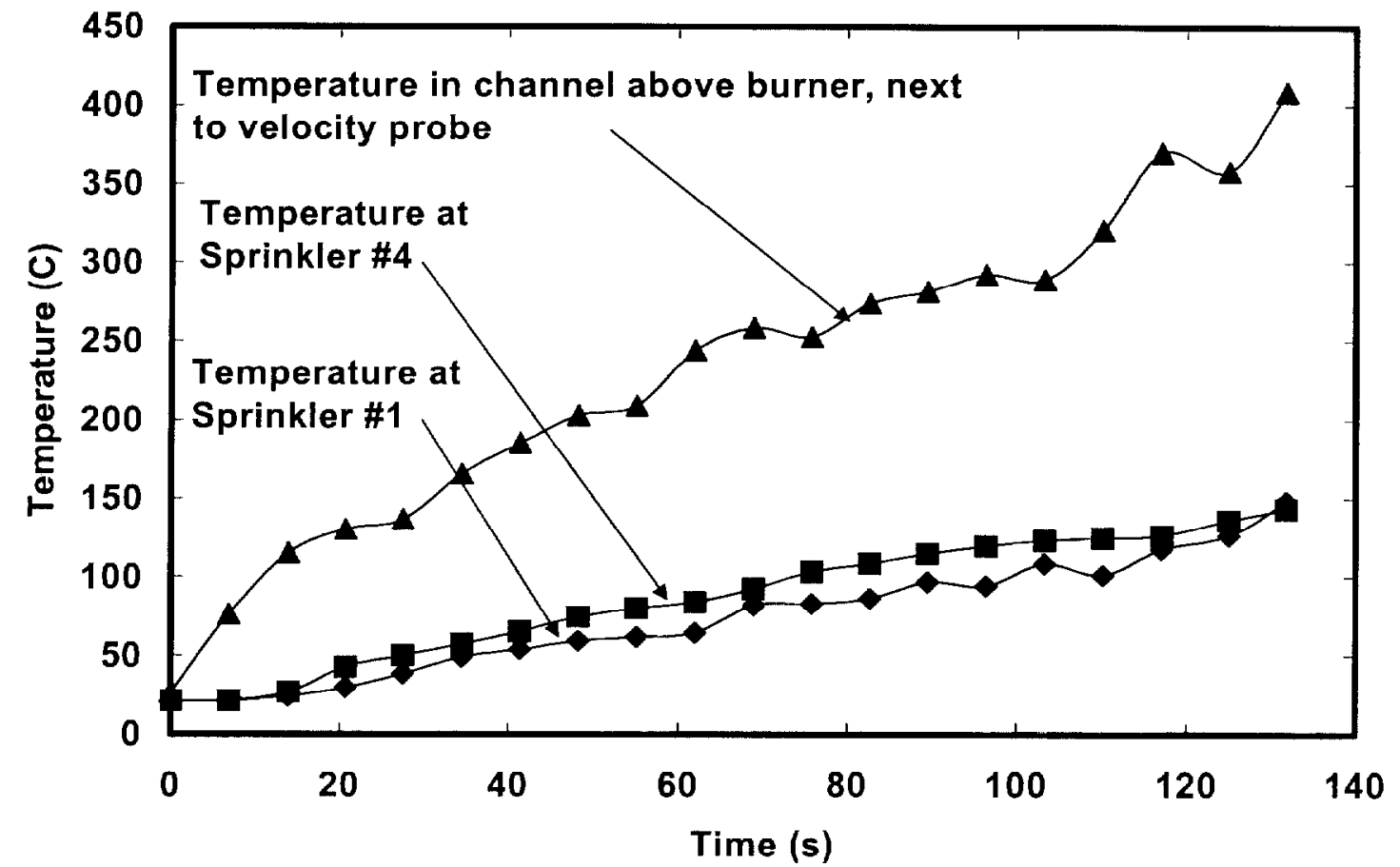

Figure 18 - Ceiling jet temperature comparisons at sprinklers 1 and 4 and in the channel above the burner, obstructed/beamed ceiling, burner in corner, medium fire growth. 


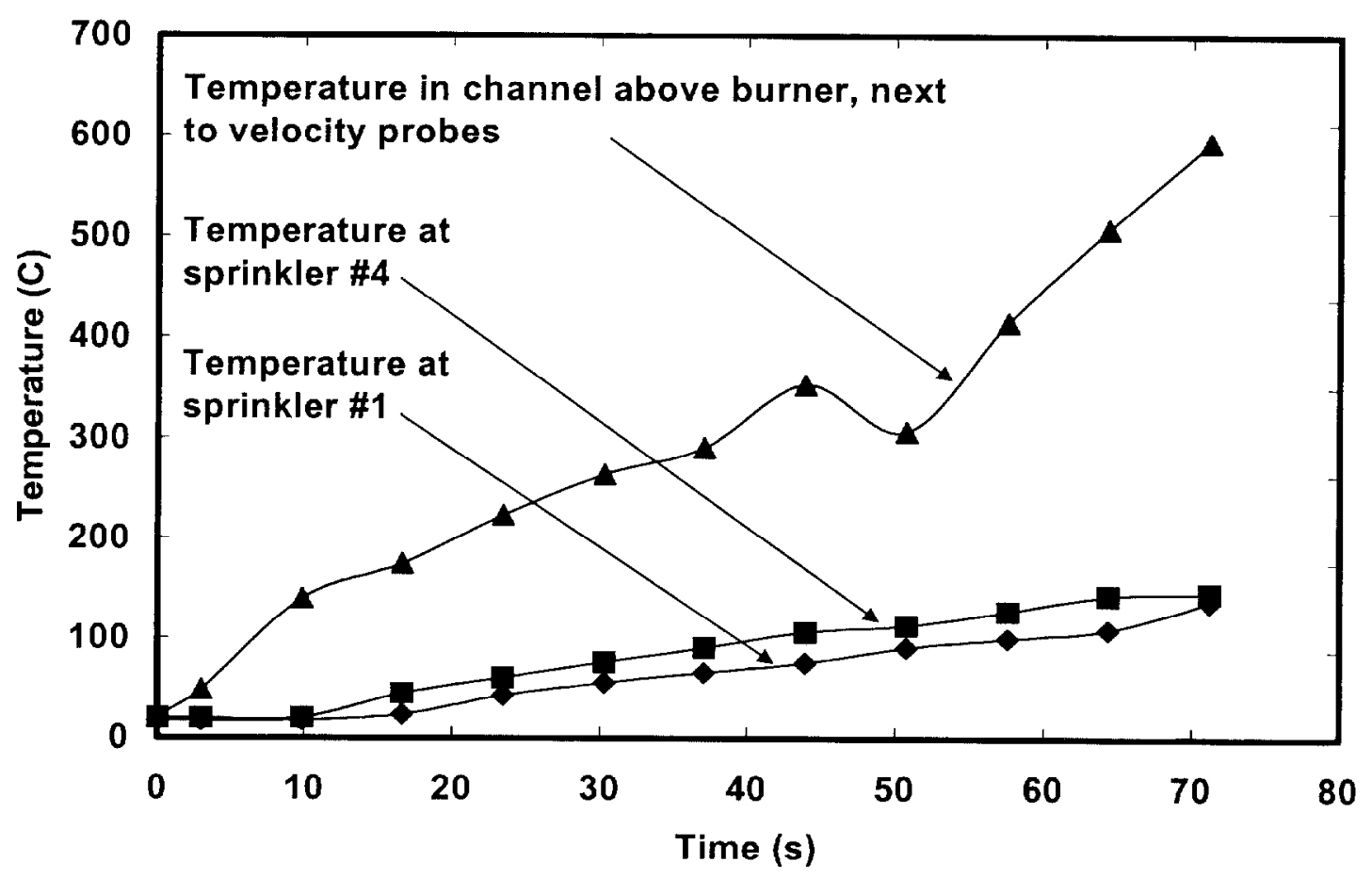

Figure 19 - Ceiling jet temperatures comparisons at sprinklers 1 and 4 and in the channel above the burner, obstructed/beamed ceiling, burner in corner, fast fire growth. 


\section{Appendix A: Determination of the Response Time Index (RTI) and Conductivity (C) Factor}

The attached is Underwriters Laboratory's report and test data obtained during their investigation of the Response Time Index and Conductivity Factor for the quick response residential sprinkler used in these experiments.

As requested, we have prepared a summary of the Response Time Index, (RTI) and Conductivity Factor, (C) generated on the sample automatic sprinklers submitted. The tests were conducted in accordance with the Standard for the Requirements and Methods of Test for Sprinklers, ISO 6128-1.

\section{$\underline{\mathrm{T}} \underline{\mathrm{S}} \underline{\mathrm{T}} \quad \underline{\mathrm{R}} \underline{\mathrm{E}} \underline{\mathrm{O}} \underline{\mathrm{R}} \underline{\mathrm{D}}$}

\section{SAMPLES:}

Representative standard orifice automatic sprinklers utilizing a $3.0 \mathrm{~mm}$ glass bulb in the $68^{\circ} \mathrm{C}\left(155^{\circ} \mathrm{F}\right)$ temperature rating were provided by the National Institute of Standards and Technology.

\section{DETERMINATION OF CONDUCTIVITY FACTOR (C):}

\section{METHOD}

Sample sprinklers were fitted with 1 to 1.5 wraps of PTFE sealant tape and screwed into a test fixture mount. The test fixture mount and sprinkler were then conditioned for at least 30 minutes at $20 \pm 0.5^{\circ} \mathrm{C}$.

Following the conditioning, the inlet of each sprinkler was filled with water and connected to a source of air pressure at $4 \pm 1$ psi. The sample was then plunged into the oven test section which was preheated to a temperature of $128^{\circ} \mathrm{C}$. 
The temperature of the sprinkler mount was monitored during each test and maintained at a temperature of $20 \pm 0.5^{\circ} \mathrm{C}$ by flowing cooling water through tubing around the mount.

It was planned to repeat the plunge test at various air velocities in the test section such that the sprinkler would not operate at one velocity and operate within 15 minutes at a higher velocity where the square root of the ratio of the high velocity to the low velocity did not exceed 1.1 .

\section{RESULTS}

The results of the prolonged plunge test are shown in Table 1 below. Since the sprinklers operated at zero velocity, a conductivity factor of 0.25 was established for the sprinklers in accordance with Note 3 to Paragraph 7.6.2.2.1 of ISO 6182-1.

\section{TABLE 1 - PROLONGED PLUNGE TEST RESULTS}

\begin{tabular}{ccc} 
Sample No. & Plunge Temperature, ${ }^{\circ} \mathrm{C}$ & Operating Time sec \\
\hline 1 & 128 & 804 \\
2 & 128 & 252 \\
3 & 128 & 724
\end{tabular}

\section{DYNAMIC HEATING - DETERMINATION OF RESPONSE TMME INDEX (RTI):}

\section{METHOD}

The Response Time Index, (RTI) of the representative sprinklers was determined using the Sensitivity Oven Heat Test method. Twenty samples rated $155^{\circ} \mathrm{F}$ were subjected to this test.

Each sample was fitted with 1 to 1.5 wraps of PTFE sealant tape and screwed into a test a test fixture mount. The test fixture mount and sprinkler were then conditioned for at least 30 minutes at $20 \pm 0.5^{\circ} \mathrm{C}$.

Following the conditioning, the inlet of each sprinkler was filled with water and connected to a source of air pressure at $4 \pm 1$ psi. The sprinkler was quickly plunged into the oven and its operating time was automatically recorded.

The test was repeated so that 10 samples were tested with the frame arms (1) perpendicular to the air flow, and (2) offset 25 degrees from the parallel position to the air flow.

The RTI of each sample was calculated using the following parameters: 
Oven Velocity, $\mathrm{m} / \mathrm{s}$ : $\quad 1.765$

Operation Bath Temperature, ${ }^{\circ} \mathrm{C}$ : $\quad 67.53$

Room Ambient Temperature, ${ }^{\circ} \mathrm{C}$ : $\quad 23.89$

Oven Air Temperature, ${ }^{\circ} \mathrm{C}$ : $\quad 135$

Mount Temperature, ${ }^{\circ} \mathrm{C}$ : $\quad 20$

Conductivity Factor, (C): $\quad 0.25$

\section{RESULTS}

The results of the Dynamic Heating Test are contained in Tables 2 and 3.

TABLE 2 - PLUNGE OVEN RESULT'

Frame Arms Perpendicular to Air Flow

$\begin{array}{ccc}\text { Sample No. } & \text { Response Time, sec } & \text { RTI, }\left(\mathrm{m}^{*} \mathrm{~s}\right)^{1 / 2} \\ 1 & 24.46 & 61.42 \\ 2 & 21.43 & 53.82 \\ 3 & 19.91 & 49.99 \\ 4 & 25.66 & 64.44 \\ 5 & 19.15 & 48.08 \\ 6 & 22.03 & 55.31 \\ 7 & 19.97 & 50.14 \\ 8 & 25.42 & 63.83 \\ 9 & 23.71 & 59.54 \\ 10 & 20.39 & 51.20\end{array}$

Average $\mathrm{RTI}=55.78\left(\mathrm{~m}^{*} \mathrm{~s}\right)^{1 / 2}$

TABLE 3 - PLUNGE OVEN RESULTS

Frame Arms Offset 25 Degrees From the Parallel Position to the Air Flow

\begin{tabular}{|c|c|c|}
\hline Sample No. & Response Time, sec & $\underline{\mathrm{RTI}} .\left(\mathrm{m}^{*} \mathrm{~s}\right)^{1 / 2}$ \\
\hline 1 & 27.93 & 70.88 \\
\hline 2 & 23.68 & 60.10 \\
\hline 3 & 22.68 & 57.58 \\
\hline 4 & 17.39 & 44.13 \\
\hline 5 & 30.74 & 78.03 \\
\hline 6 & 21.11 & 53.57 \\
\hline 7 & 31.14 & 79.04 \\
\hline 8 & 17.97 & 45.61 \\
\hline 9 & 23.65 & 60.03 \\
\hline
\end{tabular}


Average $\mathrm{RTI}=64.29\left(\mathrm{~m}^{*} \mathrm{~s}\right)^{1 / 2}$

\section{$\underline{S} \underline{U} \underline{M} \underline{M} \underline{A} \underline{R} \underline{Y}$}

This report provides test data which was obtained under an investigation concerning the Response Time Index and the Conductivity Factor of automatic sprinklers.

In no event shall UL be responsible to anyone for whatever use or nonuse is made of the information contained in this Report, and in no event shall UL, its employees, or its agent incur any obligation or liability for damages, including, but not limited to, consequential damage arising out of or in connection with the use, or inability to use, the information contained in this Report.

This serves to terminate our work under Assignment 96NK9527.

Report by:

Jennifer Owers

Engineer
Reviewed by:

William M. Carey

Senior Staff Engineer 


\section{$9 \quad$ Appendix B - Data Set}

Data from all 45 experiments is given in this appendix. For all 45 experiments, the following data are listed. All thermocouple data at sprinklers 1, 2, 3, and 4 . All ceiling jet velocity data at sprinklers 1 and 2. For the obstructed/beamed ceiling in which the burner was placed in the corner, data from the two velocity probes located within the ceiling joists above the burner is also given. For the smooth horizontal unobstructed ceiling experiments the distance labels at the top of each column represents the distance below the ceiling the instrument was located. For the horizontal obstructed/beamed ceiling experiments the gypsum board on the ceiling was removed exposing the ceiling joists. Since none of the original instrumentation was moved, the distance labels at the top of each column represent the distance below the ceiling as if the gypsum board ceiling was still in place. 
Experiment Number 1

Thermocouples located at sprinkler number 1

\begin{tabular}{|c|c|c|c|c|c|c|c|c|c|c|c|c|}
\hline Time & $0 \mathrm{~mm}$ & $25 \mathrm{~mm}$ & $50 \mathrm{~mm}$ & $75 \mathrm{~mm}$ & $100 \mathrm{~mm}$ & $125 \mathrm{~mm}$ & $150 \mathrm{~mm}$ & $250 \mathrm{~mm}$ & $350 \mathrm{~mm}$ & $450 \mathrm{~mm}$ & $550 \mathrm{~mm}$ & $900 \mathrm{~mm}$ \\
\hline 0.0 & 16.6 & 16.8 & 16.8 & 16.8 & 16.8 & 16.8 & 16.8 & 16.7 & 16.6 & 16.5 & 16.4 & 16.1 \\
\hline 6.1 & 16.7 & 17.0 & 18.6 & 19.1 & 19.8 & 19.8 & $1 \mathrm{~B} .6$ & 20.9 & 18.7 & 17.4 & 16.6 & 16.3 \\
\hline 13.0 & 22.3 & 39.7 & 46.8 & 46.7 & 44.6 & 45.2 & 42.4 & 37.8 & 27.5 & 18.2 & 17.0 & 16.7 \\
\hline 19.7 & 28.7 & 58.6 & 67.4 & 66.3 & 64.5 & 66.1 & 61.4 & 55.6 & 38.8 & 32.4 & 30.4 & 18.4 \\
\hline 26.6 & 35.0 & 70.2 & 78.6 & 78.6 & 76.9 & 77.7 & 74.4 & 68.5 & 60.5 & 55.7 & 31.0 & 17.8 \\
\hline 33.4 & 40.8 & 82.5 & 93.1 & 88.8 & 89.0 & 87.4 & 87.1 & 80.1 & 74.6 & 67.8 & 46.9 & 19.7 \\
\hline 40.3 & 49.2 & 107.3 & 127.5 & 126.7 & 126.9 & 123.6 & 118.6 & 107.6 & 96.3 & 89.6 & 69.7 & 24.0 \\
\hline 47.2 & 56.8 & 117.6 & 131.9 & 130.0 & 135.0 & 133.7 & 135.1 & 122.8 & 114.5 & 105.0 & 78.3 & 37.2 \\
\hline 53.9 & 62.1 & 129.8 & 147.7 & 135.1 & 127.2 & 132.0 & 101.4 & 135.7 & 122.5 & 116.0 & 93.5 & 47.2 \\
\hline 60.9 & 61.6 & 113.5 & 125.0 & 111.7 & 112.3 & 119.2 & 108.2 & 124.5 & 117.8 & 107.5 & 95.2 & 51.3 \\
\hline \multicolumn{13}{|c|}{ Thermocouples located at sprinkler number 2} \\
\hline Time & $0 \mathrm{~mm}$ & $25 \mathrm{~mm}$ & $50 \mathrm{~mm}$ & $75 \mathrm{~mm}$ & $100 \mathrm{~mm}$ & $125 \mathrm{~mm}$ & $150 \mathrm{~mm}$ & $250 \mathrm{~mm}$ & $350 \mathrm{~mm}$ & $450 \mathrm{~mm}$ & $550 \mathrm{~mm}$ & $900 \mathrm{~mm}$ \\
\hline 0.0 & $16 . l$ & 16.8 & 16.8 & 16.9 & 16.9 & 16.9 & 16.8 & 16.8 & 16.8 & 16.7 & 16.6 & 16.1 \\
\hline 6.1 & 17.7 & 23.7 & 24.1 & 24.1 & 24.9 & 25.0 & 24.7 & 22.2 & 19.6 & 18.0 & 16.8 & 16.3 \\
\hline 13.0 & 24.0 & 490 & 50.7 & 50.9 & 50.1 & 49.0 & 45.7 & 35.9 & 27.5 & 20.2 & 17.3 & 16.8 \\
\hline 19.7 & 33.0 & 72.8 & 74.7 & 74.5 & 73.7 & 73.4 & 69.9 & 59.5 & 46.6 & 36.4 & 31.6 & 21.1 \\
\hline 26.6 & 38.8 & 85.5 & 89.8 & 89.4 & 90.5 & 90.9 & 91.3 & 81.5 & 66.3 & 49.5 & 32.2 & 18.4 \\
\hline 33.4 & 41.6 & 94.4 & 100.5 & 100.0 & 103.1 & 100.3 & 102.9 & 92.9 & 86.4 & 71.7 & 58.9 & 24.7 \\
\hline 40.3 & 48.2 & 116.7 & 121.3 & 123.8 & 127.5 & 125.8 & 125.9 & 113.9 & 97.7 & 89.1 & 75.8 & 29.3 \\
\hline 47.2 & 54.6 & 125.4 & 133.0 & 131.8 & 135.4 & 130.5 & 132.3 & 121.9 & 111.0 & 95.1 & 68.9 & 45.2 \\
\hline 53.9 & 57.8 & 105.9 & 105.1 & 71.7 & 116.0 & 63.4 & 109.4 & 125.9 & 126.4 & $\uparrow 14.0$ & 98.1 & 54.6 \\
\hline 60.9 & 50.3 & 100.0 & 96.3 & 98.0 & 102.2 & 104.7 & 101.6 & 109.7 & 115.8 & 104.7 & 97.5 & 51.8 \\
\hline \multicolumn{13}{|c|}{ Thermocouples located at sprinkler number 3} \\
\hline Time & $0 \mathrm{~mm}$ & $25 \mathrm{~mm}$ & $50 \mathrm{mrn}$ & $75 \mathrm{~mm}$ & $100 \mathrm{~mm}$ & $125 \mathrm{~mm}$ & $150 \mathrm{~mm}$ & $250 \mathrm{~mm}$ & $350 \mathrm{~mm}$ & $450 \mathrm{~mm}$ & $550 \mathrm{~mm}$ & $900 \mathrm{~mm}$ \\
\hline 0.0 & 16.6 & 16.7 & 16.9 & 16.9 & 16.9 & 16.9 & 16.9 & 16.8 & 16.6 & 16.3 & 16.2 & 16.1 \\
\hline 6.1 & 16.6 & 16.8 & 17.0 & 17.0 & 16.9 & 16.9 & 16.8 & 16.8 & 16.6 & 16.4 & 16.3 & 16.4 \\
\hline 13.0 & 16.9 & 22.3 & 24.9 & 24.1 & 21.8 & 21.6 & 21.2 & 19.9 & 18.2 & 16.9 & 16.7 & 16.5 \\
\hline 19.7 & 18.3 & 30.9 & 38.6 & 39.0 & 36.1 & 36.4 & 34.3 & 28.8 & 24.9 & 20.7 & 17.4 & 16.8 \\
\hline 26.6 & 20.1 & 46.2 & 58.1 & 59.4 & 55.7 & 57.4 & 54.0 & 49.2 & 44.2 & 35.5 & 29.1 & 21.9 \\
\hline 33.4 & 22.2 & 51.3 & 63.3 & 62.9 & 61.4 & 63.2 & 60.3 & 54.5 & 49.1 & 42.4 & 38.8 & 27.7 \\
\hline 40.3 & 25.6 & 68.8 & 79.3 & 79.0 & 76.0 & 76.7 & 73.0 & 67.6 & 59.6 & 50.2 & 48.3 & 38.7 \\
\hline 47.2 & 27.6 & 75.7 & 95.4 & 95.2 & 91.5 & 92.1 & 87.7 & 76.5 & 66.2 & 61.0 & 60.4 & 49.0 \\
\hline 53.9 & 29.8 & 82.3 & 103.5 & 103.5 & 101.2 & 99.8 & 96.3 & 84.2 & 77.5 & 72.4 & 72.2 & 58.7 \\
\hline 60.9 & 29.6 & 79.2 & 105.5 & 105.3 & 101.9 & 102.4 & 100.2 & 89.0 & 83.5 & 82.9 & 82.3 & 59.9 \\
\hline \multicolumn{13}{|c|}{ Thermocouples localed at sprinkler number 4} \\
\hline Time & $0 \mathrm{~mm}$ & $25 \mathrm{~mm}$ & $50 \mathrm{~mm}$ & $75 \mathrm{~mm}$ & $100 \mathrm{~mm}$ & $125 \mathrm{~mm}$ & $150 \mathrm{~mm}$ & $250 \mathrm{~mm}$ & $350 \mathrm{~mm}$ & $450 \mathrm{~mm}$ & $550 \mathrm{~mm}$ & $900 \mathrm{~mm}$ \\
\hline 0.0 & 16.6 & 16.7 & 16.8 & 16.8 & 16.7 & 16.7 & 16.6 & 16.5 & 16.4 & 16.3 & 16.3 & 16.1 \\
\hline 6.1 & 16.8 & 16.7 & 16.9 & 16.9 & 16.8 & 16.8 & 16.7 & 16.6 & 16.5 & 16.5 & 16.4 & 16.5 \\
\hline 13.0 & 20.2 & 27.7 & 30.5 & 29.1 & 24.0 & 22.7 & 21.7 & 20.6 & 18.6 & 16.7 & 16.7 & 17.0 \\
\hline 19.7 & 21.6 & 40.3 & 40.6 & 39.9 & 38.4 & 39.5 & 38.9 & 30.6 & 25.8 & 22.2 & 22.1 & 19.2 \\
\hline 26.6 & 26.2 & 52.6 & 55.3 & 55.4 & 53.9 & 55.9 & 56.2 & 49.5 & 48.0 & 38.8 & 35.8 & 20.2 \\
\hline 33.4 & 28,9 & 61.4 & 68.0 & 67.6 & 66.3 & 68.2 & 69.5 & 63.1 & 57.4 & 49.7 & 46.3 & 21.3 \\
\hline 40.3 & 36.0 & 81.3 & 84.9 & 84.2 & 81.7 & 83.5 & 83.1 & 76.1 & 69.3 & 60.3 & 60.2 & 27.8 \\
\hline 47.2 & 44.9 & 93.7 & 95.7 & 94.7 & 90.8 & 92.3 & 92.5 & 84.0 & 79.7 & 76.2 & 72.8 & 38.6 \\
\hline 53.9 & 44.5 & 101.1 & 108.5 & 107.9 & 104.2 & 405.1 & 105.5 & 97.1 & 94.9 & 90.3 & 86.0 & 47.5 \\
\hline 60.9 & 44.7 & 108.5 & 109.5 & 110.1 & 108.8 & 111.5 & 111.8 & 104.7 & 102.4 & 98.7 & 90.1 & 49.3 \\
\hline \multicolumn{6}{|c|}{ Velocity probes at sprinkler number 1} & \multicolumn{7}{|c|}{ Velocily probes at sprinkler number 2} \\
\hline Time & $25 \mathrm{~mm}$ & $75 \mathrm{~mm}$ & $125 \mathrm{~mm}$ & $250 \mathrm{~mm}$ & & Time & $25 \mathrm{~mm}$ & $75 \mathrm{~mm}$ & $125 \mathrm{~mm}$ & $250 \mathrm{~mm}$ & & \\
\hline 0.0 & 0.0 & 0.0 & 0.0 & 0.0 & & 0.0 & 0.0 & 0.0 & 0.0 & 0.0 & & \\
\hline 6.1 & -1.5 & -1.4 & -1.4 & -0.5 & & 6.1 & 1.2 & 1.1 & 0.9 & 0.4 & & \\
\hline 13.0 & -1.3 & -1.2 & -1.0 & -0.2 & & 13.0 & 1.1 & 1.2 & 1.2 & 0.4 & & \\
\hline 19.7 & -1.5 & -1.8 & -1.7 & -0.9 & & 19.7 & 1.6 & 1.6 & 1.3 & 0.4 & & \\
\hline 26.6 & -1.8 & -1.7 & -1.4 & -0.5 & & 26.6 & 1.3 & 1.6 & 1.4 & 0.9 & & \\
\hline 33.4 & -1.6 & -1.4 & -1.4 & -0.7 & & 33.4 & 1.1 & 1.2 & 1.1 & 0.3 & & \\
\hline 40.3 & -1.2 & -1.6 & -1.6 & -0.8 & & 40.3 & 1.5 & 1.5 & 1.7 & 0.7 & & \\
\hline 47.2 & -1.2 & -1.5 & -1.7 & -0.8 & & 17.2 & 1.3 & 1.4 & 1.4 & 0.8 & & \\
\hline 53.9 & -0.8 & -0.6 & -0.4 & -0.5 & & 53.9 & 0.5 & 0.6 & 0.5 & 0.6 & & \\
\hline 60.9 & 0.2 & 0.2 & 0.1 & -0.6 & & 60.9 & 0.4 & 0.4 & 0.5 & 0.5 & & \\
\hline
\end{tabular}


Experiment Number 2

Thermocouples located at sprinkler number 1

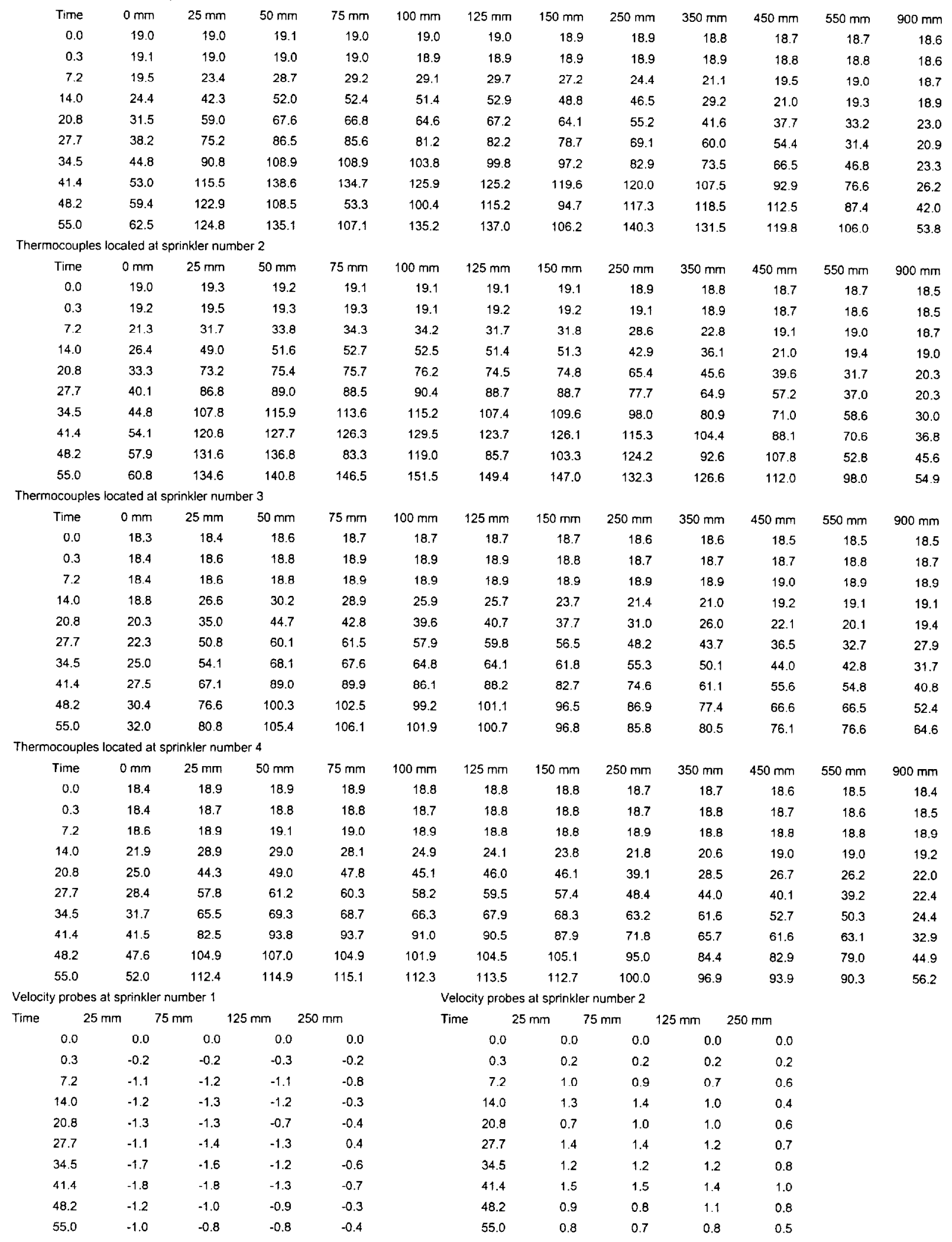


Experiment Number 3

Thermocouples located at sprinkler number 1

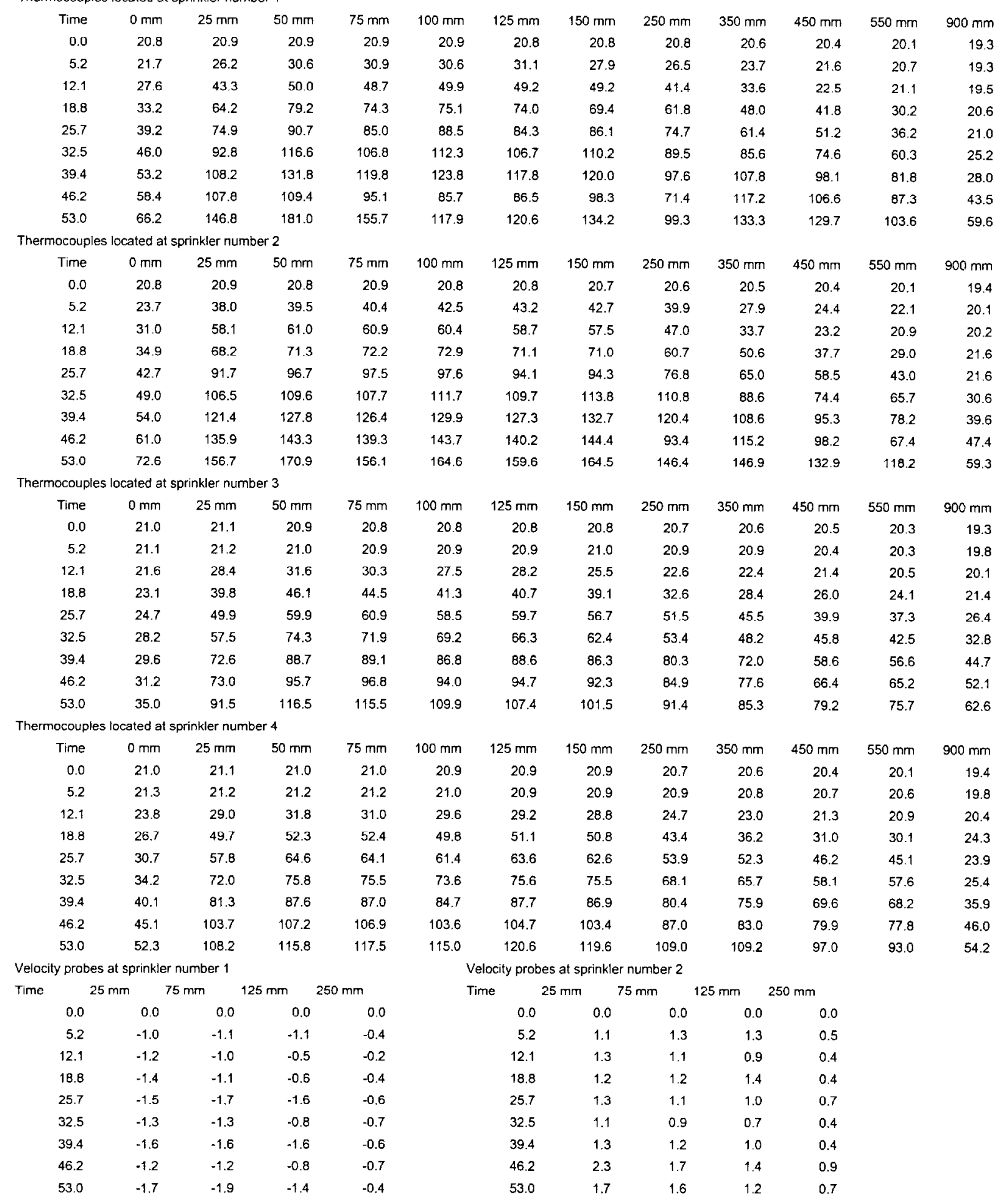




\section{Experiment Number 4}

Thermocouples localed at sprinkler number 1

\begin{tabular}{|c|c|c|c|c|c|c|c|c|c|c|c|c|}
\hline Time & $0 \mathrm{~mm}$ & $25 \mathrm{~mm}$ & $50 \mathrm{~mm}$ & $75 \mathrm{~mm}$ & $100 \mathrm{~mm}$ & $125 \mathrm{~mm}$ & $150 \mathrm{~mm}$ & $250 \mathrm{~mm}$ & $350 \mathrm{~mm}$ & $450 \mathrm{~mm}$ & $550 \mathrm{~mm}$ & $900 \mathrm{~mm}$ \\
\hline 0.0 & 21.0 & 21.1 & 21.3 & 21.5 & 21.3 & 21.2 & 21.2 & 21.3 & 21.0 & 21.0 & 20.9 & 20.2 \\
\hline 6.8 & 21.1 & 21.0 & 21.3 & 21.5 & 21.2 & 21.1 & 21.2 & 21.4 & 21.2 & 21.1 & 21.1 & 20.3 \\
\hline 13.5 & 22.5 & 23.1 & 24.2 & 25.3 & 26.2 & 25.3 & 24.9 & 28.0 & 27.3 & 25.2 & 23.3 & 20.8 \\
\hline 20.3 & 44.4 & 43.2 & 46.9 & 46.6 & 43.9 & 43.5 & 37.3 & 29.1 & 24.1 & 23.0 & 22.3 & 21.0 \\
\hline 27.2 & 55.3 & 56.2 & 60.1 & 61.3 & 61.3 & 60.9 & 52.8 & 47.9 & 29.3 & 23.8 & 22.5 & 21.5 \\
\hline 33.9 & 69.6 & 70.8 & 75.9 & 75.2 & 72.7 & 73.0 & 64.2 & 56.1 & 40.2 & 33.9 & 32.0 & 24.6 \\
\hline 40.8 & 76.7 & 76.5 & 79.1 & 78.7 & 77.3 & 77.2 & 73.2 & 71.7 & 63.6 & 50.4 & 41.8 & 29.6 \\
\hline 47.6 & 94.3 & 95.1 & 102.5 & 104.3 & 102.5 & 106.2 & 95.2 & 80.7 & 65.9 & 62.5 & 53.4 & 34.5 \\
\hline 54.3 & 102.9 & 103.4 & 108.4 & 108.9 & 107.5 & 108.7 & 102.0 & 93.3 & 76.2 & 68.7 & 64.1 & 41.4 \\
\hline 61.3 & 111.3 & 112.1 & 118.8 & 121.5 & 118.1 & 121.8 & 116.1 & 112.3 & 91.9 & 80.5 & 75.1 & 55.6 \\
\hline 68.0 & 149.5 & 152.3 & 154.5 & 167.1 & 160.4 & 160.6 & 143.8 & 126.4 & 101.9 & 87.6 & 81.4 & 62.9 \\
\hline \multicolumn{13}{|c|}{ Thermocouples located at sprinkler number 2} \\
\hline Time & $0 \mathrm{~mm}$ & $25 \mathrm{~mm}$ & $50 \mathrm{~mm}$ & $75 \mathrm{~mm}$ & $100 \mathrm{~mm}$ & $125 \mathrm{~mm}$ & $150 \mathrm{~mm}$ & $250 \mathrm{~mm}$ & $350 \mathrm{~mm}$ & $450 \mathrm{~mm}$ & $550 \mathrm{~mm}$ & $900 \mathrm{~mm}$ \\
\hline 0.0 & 21.1 & 21.3 & 21.3 & 21.3 & 21.2 & 21.3 & 21.2 & 21.0 & 21.0 & 21.0 & 20.9 & 20.1 \\
\hline 6.8 & 21.1 & 21.3 & 21.3 & 21.3 & 21.3 & 21.3 & 21.2 & 21.2 & 21.0 & 21.0 & 21.1 & 20.4 \\
\hline 13.5 & 30.0 & 34.1 & 34.3 & 38.1 & 37.9 & 37.0 & 35.0 & 29.1 & 26.0 & 26.4 & 22.6 & 21.1 \\
\hline 20.3 & 44.9 & 50.6 & 50.9 & 51.8 & 50.7 & 48.7 & 45.9 & 30.1 & 23.4 & 22.7 & 22.0 & 21.2 \\
\hline 27.2 & 57.4 & 62.6 & 62.9 & 64.6 & 64.2 & 63.9 & 62.2 & 52.9 & 38.4 & 24.1 & 22.8 & 21.8 \\
\hline 33.9 & 70.4 & 76.2 & 76.6 & 78.6 & 77.7 & 77.0 & 75.3 & 63.9 & 48.5 & 36.3 & 33.2 & 24.5 \\
\hline 40.8 & 82.3 & 89.7 & 91.7 & 95.8 & 97.6 & 96.7 & 95.3 & 75.9 & 62.1 & 50.5 & 44.3 & 24.7 \\
\hline 47.6 & 95.8 & 103.6 & 105.3 & 108.9 & 107.7 & 104.6 & 101.9 & 83.6 & 69.2 & 62.1 & 57.0 & 30.7 \\
\hline 54.3 & 98.0 & 103.2 & 104.4 & 107.4 & 107.3 & 109.2 & 108.9 & 102.5 & 86.6 & 77.2 & 67.3 & 37.8 \\
\hline 61.3 & 106.6 & 116.1 & 117.6 & 121.2 & 122.7 & 124.8 & 123.2 & 106.2 & 95.1 & 82.7 & 69.0 & 44.6 \\
\hline 68.0 & 150.0 & 164.8 & 162.5 & 162.0 & 160.4 & 157.6 & 153.6 & 133.3 & 102.5 & 90.3 & 81.0 & 50.3 \\
\hline \multicolumn{13}{|c|}{ Thermocouples located at sprinkler number 3} \\
\hline Time & $0 \mathrm{~mm}$ & $25 \mathrm{~mm}$ & $50 \mathrm{~mm}$ & $75 \mathrm{~mm}$ & $100 \mathrm{~mm}$ & $125 \mathrm{~mm}$ & $150 \mathrm{~mm}$ & $250 \mathrm{~mm}$ & $350 \mathrm{~mm}$ & $450 \mathrm{~mm}$ & $550 \mathrm{~mm}$ & $900 \mathrm{~mm}$ \\
\hline 0.0 & 21.1 & 21.1 & 21.0 & 21.0 & 21.2 & 21.2 & 21.2 & 21.0 & 20.9 & 20.9 & 20.7 & 20.2 \\
\hline 6.8 & 21.2 & 21.5 & 21.6 & 21.8 & 21.4 & 21.3 & 20.8 & 21.0 & 20.6 & 20.8 & 21.4 & 21.1 \\
\hline 13.5 & 21.6 & 21.8 & 21.3 & 21.0 & 21.1 & 21.2 & 21.7 & 21.4 & 21.9 & 21.3 & 20.9 & 20.0 \\
\hline 20.3 & 25.5 & 23.5 & 27.2 & 26.0 & 25.1 & 26.1 & 26.9 & 27.5 & 28.4 & 28.3 & 27.6 & 22.9 \\
\hline 27.2 & 33.5 & 34.7 & 38.5 & 38.1 & 38.5 & 39.9 & 40.5 & 40.5 & 38.5 & 31.3 & 28.3 & 22.1 \\
\hline 33.9 & 36.6 & 39.6 & 39.3 & 43.4 & 42.4 & 45.0 & 44.8 & 44.7 & 46.8 & 43.0 & 42.7 & 23.9 \\
\hline 40.8 & 45.1 & 48.7 & 49.0 & 50.2 & 51.5 & 56.0 & 55.6 & 55.4 & 58.3 & 53.4 & 52.9 & 24.4 \\
\hline 47.6 & 42.5 & 46.3 & 49.2 & 54.6 & 57.1 & 61.6 & 64.1 & 67.9 & 72.7 & 66.8 & 59.4 & 33.6 \\
\hline 54.3 & 64.0 & 75.0 & 74.2 & 76.6 & 75.6 & 79.2 & 81.1 & 80.7 & 82.7 & 73.7 & 60.3 & 42.9 \\
\hline 61.3 & 55.4 & 53.9 & 64.2 & 65.5 & 70.1 & 72.0 & 74.0 & 77.7 & 83.1 & 79.6 & 74.9 & 48.4 \\
\hline 68.0 & 62.9 & 76.3 & 84.3 & 87.8 & 88.5 & 95.7 & 100.3 & 98.8 & 105.6 & 95.3 & 81.6 & 55.4 \\
\hline \multicolumn{13}{|c|}{ Thermocouples located at sprinkler number 4} \\
\hline Time & $0 \mathrm{~mm}$ & $25 \mathrm{~mm}$ & $50 \mathrm{~mm}$ & $75 \mathrm{~mm}$ & $100 \mathrm{~mm}$ & $125 \mathrm{~mm}$ & $150 \mathrm{~mm}$ & $250 \mathrm{~mm}$ & $350 \mathrm{~mm}$ & $450 \mathrm{~mm}$ & $550 \mathrm{~mm}$ & $900 \mathrm{~mm}$ \\
\hline 0.0 & 21.2 & 21.3 & 21.3 & 21.3 & 21.1 & 21.2 & 21.2 & 21.1 & 21.1 & 20.9 & 20.7 & 20.2 \\
\hline 6.8 & 21.2 & 21.3 & 21.3 & 21.3 & 21.1 & 21.2 & 21.2 & 21.2 & 21.2 & 20.9 & 20.7 & 20.2 \\
\hline 13.5 & 21.7 & 21.7 & 21.7 & 21.6 & 21.9 & 22.3 & 22.4 & 22.0 & 21.4 & 21.3 & 21.3 & 20.9 \\
\hline 20.3 & 26.0 & 30.0 & 30.1 & 31.6 & 31.7 & 32.4 & 32.9 & 32.9 & 32.2 & 28.8 & 26.6 & 22.0 \\
\hline 27.2 & 33.1 & 34.9 & 35.7 & 37.1 & 37.8 & 38.9 & 39.6 & 40.7 & 42.5 & 39.9 & 34.6 & 22.2 \\
\hline 33.9 & 41.7 & 45.1 & 44.5 & 45.8 & 46.5 & 47.4 & 48.1 & 49.6 & 51.9 & 50.9 & 50.1 & 25.5 \\
\hline 40.8 & 47.7 & 55.4 & 54.8 & 58.6 & 58.1 & 58.5 & 63.3 & 67.3 & 68.0 & 64.1 & 59.1 & 26.4 \\
\hline 47.6 & 66.3 & 66.2 & 66.7 & 69.5 & 70.3 & 73.0 & 74.7 & 73.9 & 75.7 & 72.9 & 70.7 & 36.0 \\
\hline 54.3 & 77.9 & 78.6 & 78.0 & 81.6 & 78.7 & 79.8 & 79.5 & 80.3 & 83.6 & 81.2 & 78.4 & 43.2 \\
\hline 61.3 & 67.7 & 72.1 & 72.2 & 76.4 & 79.8 & 81.8 & 82.4 & 82.9 & 89.5 & 89.9 & 91.9 & 64.0 \\
\hline 68.0 & 79.6 & 89.4 & 85.8 & 96.4 & 105.0 & 106.3 & 108.4 & 111.4 & 113.0 & 106.9 & 109.7 & 75.3 \\
\hline \multicolumn{6}{|c|}{ Velority prnbes at sprinkler number 1} & \multicolumn{7}{|c|}{ Velocity probes at sprinkler number 2} \\
\hline Time & $25 \mathrm{~mm}$ & $75 \mathrm{~mm}$ & $125 \mathrm{~mm}$ & $250 \mathrm{~mm}$ & & Time & $25 \mathrm{~mm}$ & $75 \mathrm{~mm}$ & $125 \mathrm{~mm}$ & $250 \mathrm{~mm}$ & & \\
\hline 0.0 & 0.0 & 0.0 & 0.0 & 0.0 & & 0.0 & 0.0 & 0.0 & 0.0 & 0.0 & & \\
\hline 6.8 & -0.1 & -0.1 & 0.0 & -0.1 & & 6.8 & 0.2 & 0.2 & 0.3 & 0.2 & & \\
\hline 13.5 & -0.8 & -0.8 & -0.8 & -0.5 & & 13.5 & 0.8 & 0.8 & 0.6 & 0.1 & & \\
\hline 20.3 & -1.0 & -1.0 & -0.9 & -0.3 & & 20.3 & 0.9 & 1.0 & 1.0 & 0.7 & & \\
\hline 27.2 & -0.9 & -0.8 & -0.6 & -0.3 & & 27.2 & 1.0 & 1.0 & 1.0 & 0.3 & & \\
\hline 33.9 & -0.8 & -0.9 & -0.7 & -0.4 & & 33.9 & 0.8 & 0.9 & 0.9 & 0.5 & & \\
\hline 40.8 & -0.9 & -0.9 & -0.8 & -0.5 & & 40.8 & 0.9 & 0.9 & 0.9 & 0.6 & & \\
\hline 47.6 & -0.7 & -0.8 & -0.9 & -0.6 & & 47.6 & 0.7 & 0.8 & 0.9 & 0.4 & & \\
\hline 54.3 & -0.8 & -1.0 & -0.9 & -0.7 & & 54.3 & 0.7 & 0.7 & 0.8 & 0.5 & & \\
\hline
\end{tabular}




\section{Experiment Number 4}

\begin{tabular}{|c|c|c|c|c|c|c|c|c|c|}
\hline 61.3 & -1.1 & -1.3 & -1.1 & -0.9 & 61.3 & 1.2 & 0.1 & 1.3 & 0.6 \\
\hline 68.0 & -1.1 & -1.1 & -1.0 & -0.6 & 68.0 & 1.2 & 1.3 & 1.3 & 0.2 \\
\hline \multicolumn{10}{|c|}{ Velocity probes in channels above burner } \\
\hline \multicolumn{10}{|c|}{ Time Channel 1 Channel 2} \\
\hline 0.0 & 0.0 & 0.0 & & & & & & & \\
\hline 6.8 & 2.0 & 1.0 & & & & & & & \\
\hline 13.5 & 2.1 & 0.8 & & & & & & & \\
\hline 20.3 & 2.4 & 1.3 & & & & & & & \\
\hline 27.2 & 2.6 & 1.4 & & & & & & & \\
\hline 33.9 & 3.1 & 1.3 & & & & & & & \\
\hline 40.8 & 3.2 & 1.3 & & & & & & & \\
\hline 47.6 & 3.1 & 1.5 & & & & & & & \\
\hline 54.3 & 3.0 & 1.8 & & & & & & & \\
\hline 61.3 & 3.0 & 2.2 & & & & & & & \\
\hline 68.0 & 3.9 & 2.0 & & & & & & & \\
\hline
\end{tabular}




\section{Experiment Number 5}

Thermocouples located at sprinkler number 1

\begin{tabular}{|c|c|c|c|c|c|c|c|c|c|c|c|c|}
\hline Time & $0 \mathrm{~mm}$ & $25 \mathrm{~mm}$ & $50 \mathrm{~mm}$ & $75 \mathrm{~mm}$ & $100 \mathrm{~mm}$ & $125 \mathrm{~mm}$ & $150 \mathrm{~mm}$ & $250 \mathrm{~mm}$ & $350 \mathrm{~mm}$ & $450 \mathrm{~mm}$ & $550 \mathrm{~mm}$ & $900 \mathrm{~mm}$ \\
\hline 0.0 & 20.0 & 20.1 & 20.2 & 20.2 & 20.2 & 20.0 & 20.2 & 20.0 & 20.3 & 20.2 & 20.0 & 19.9 \\
\hline 5.9 & 20.1 & 20.2 & 20.3 & 20.3 & 20.3 & 20.1 & 20.2 & 20.1 & 20.2 & 20.2 & 20.2 & 20.0 \\
\hline 12.7 & 25.4 & 26.3 & 28.8 & 29.2 & 29.3 & 30.1 & 25.7 & 25.3 & 22.7 & 21.4 & 20.8 & 20.5 \\
\hline 19.5 & 48.6 & 47.7 & 51.2 & 50.2 & 46.4 & 46.7 & 39.6 & 26.5 & 22.9 & 21.3 & 21.2 & 20.9 \\
\hline 26.4 & 61.3 & 62.2 & 66.8 & 66.7 & 64.3 & 64.8 & 55.3 & 46.2 & 27.5 & 22.3 & 22.8 & 21.2 \\
\hline 33.2 & 72.7 & 74.2 & 78.6 & 77.5 & 73.7 & 71.7 & 63.7 & 52.6 & 39.6 & 34.5 & 33.2 & 24.2 \\
\hline 40.1 & 87.1 & 86.4 & 88.9 & 87.4 & 84.6 & 85.9 & 79.5 & 71.2 & 56.1 & 44.7 & 38.7 & 27.6 \\
\hline 46.9 & 93.8 & 94.6 & 98.2 & 100.4 & 99.6 & 104.1 & 96.4 & 92.2 & 71.0 & 59.4 & 50.8 & 28.2 \\
\hline 53.6 & 110.3 & 109.5 & 114.9 & 114.7 & 110.9 & 111.8 & 104.3 & 94.4 & 84.1 & 69.3 & 59.1 & 35.1 \\
\hline 60.6 & 108.2 & 113.7 & 119.1 & 123.0 & 121.7 & 123.9 & 115.1 & 103.8 & 90.2 & 80.2 & 73.8 & 42.3 \\
\hline 67.3 & 146.3 & 150.0 & 161.0 & 161.9 & 156.9 & 157.4 & 141.3 & 129.8 & 106.2 & 89.7 & 83.4 & 62.2 \\
\hline \multicolumn{13}{|c|}{ Thermocouples located at sprinkler number 2} \\
\hline Time & $0 \mathrm{~mm}$ & $25 \mathrm{~mm}$ & $50 \mathrm{~mm}$ & $75 \mathrm{~mm}$ & $100 \mathrm{~mm}$ & $125 \mathrm{~mm}$ & $150 \mathrm{~mm}$ & $250 \mathrm{~mm}$ & $350 \mathrm{~mm}$ & $450 \mathrm{~mm}$ & $550 \mathrm{~mm}$ & $900 \mathrm{~mm}$ \\
\hline 0.0 & 20.2 & 20.3 & 20.4 & 20.3 & 20.4 & 20.4 & 20.3 & 20.2 & 20.4 & 20.3 & 20.3 & 19.9 \\
\hline 5.9 & 20.2 & 20.4 & 20.5 & 20.4 & 20.4 & 20.4 & 20.3 & 20.2 & 20.4 & 20.3 & 20.3 & 20.1 \\
\hline 12.7 & 29.6 & 35.9 & 37.0 & 39.5 & 39.7 & 39.7 & 37.4 & 31.4 & 24.4 & 23.0 & 21.8 & 20.5 \\
\hline 19.5 & 42.2 & 47.0 & 46.2 & 44.0 & 43.0 & 41.4 & 39.1 & 32.3 & 22.8 & 21.9 & 21.4 & 20.8 \\
\hline 26.4 & 52.8 & 56.6 & 57.7 & 59.6 & 60.2 & 59.6 & 58.9 & 55.0 & 35.6 & 22.4 & 21.9 & 21.4 \\
\hline 33.2 & 70.4 & 77.3 & 79.5 & 79.5 & 81.0 & 79.7 & 78.7 & 67.6 & 51.6 & 41.9 & 33.9 & 24.3 \\
\hline 40.1 & 75.8 & 84.4 & 86.6 & 89.5 & 90.5 & 90.4 & 89.1 & 76.6 & 57.4 & 50.7 & 39.8 & 25.9 \\
\hline 46.9 & 97.5 & 106.4 & 105.7 & 108.0 & 107.6 & 107.2 & 105.2 & 89.5 & 68.2 & 59.9 & 56.5 & 34.3 \\
\hline 53.6 & 103.8 & 111.2 & 112.4 & 113.3 & 115.2 & 114.5 & 114.1 & 105.1 & 85.2 & 70.5 & 64.4 & 38.4 \\
\hline 60.6 & 116.9 & 127.4 & 130.4 & 131.9 & 133.2 & 134.0 & 131.9 & 120.9 & 100.3 & 83.0 & 72.0 & 45.5 \\
\hline 67.3 & 143.2 & 154.7 & 158.7 & 162.2 & 168.5 & 170.0 & 168.4 & 149.3 & 111.7 & 94.2 & 82.8 & 52.0 \\
\hline \multicolumn{13}{|c|}{ Thermocouples located at sprinkler number 3} \\
\hline Time & $0 \mathrm{~mm}$ & $25 \mathrm{~mm}$ & $50 \mathrm{~mm}$ & $75 \mathrm{~mm}$ & $100 \mathrm{~mm}$ & $125 \mathrm{~mm}$ & $150 \mathrm{~mm}$ & $250 \mathrm{~mm}$ & $350 \mathrm{~mm}$ & $450 \mathrm{~mm}$ & $550 \mathrm{~mm}$ & $900 \mathrm{~mm}$ \\
\hline 0.0 & 20.4 & 20.5 & 20.6 & 20.7 & 20.7 & 20.8 & 20.7 & 20.7 & 20.6 & 20.4 & 20.3 & 19.0 \\
\hline 5.9 & 20.6 & 20.6 & 20.7 & 20.8 & 20.8 & 20.8 & 20.7 & 20.6 & 20.5 & 20.4 & 20.4 & 20.0 \\
\hline 12.7 & 20.7 & 20.8 & 20.8 & 20.8 & 20.8 & 20.9 & 20.9 & 20.9 & 20.7 & 20.6 & 20.5 & 20.2 \\
\hline 19.5 & 25.1 & 23.5 & 26.3 & 27.0 & 26.9 & 27.5 & 28.2 & 27.9 & 29.6 & 27.4 & 26.4 & 23.8 \\
\hline 26.4 & 27.1 & 28.5 & 31.8 & 32.3 & 32.6 & 33.4 & 34.0 & 36.3 & 37.0 & 34.4 & 30.1 & 21.6 \\
\hline 33.2 & 34.8 & 33.6 & 38.8 & 40.3 & 40.5 & 42.2 & 43.0 & 44.7 & 49.4 & 46.1 & 38.7 & 22.6 \\
\hline 40.1 & 41.2 & 47.0 & 48.6 & 50.9 & 51.8 & 55.6 & 58.6 & 58.9 & 64.1 & 56.6 & 54.3 & 25.3 \\
\hline 46.9 & 47.7 & 56.9 & 58.3 & 58.9 & 61.5 & 65.1 & 66.2 & 67.1 & 70.6 & 62.5 & 55.0 & 35.5 \\
\hline 53.6 & 63.8 & 78.1 & 71.9 & 78.4 & 75.5 & 79.4 & 80.7 & 80.8 & 84.2 & 76.0 & 63.2 & 36.9 \\
\hline 60.6 & 63.8 & 71.6 & 75.1 & 80.0 & 80.3 & 82.3 & 83.7 & 85.8 & 89.3 & 84.5 & 71.9 & 46.9 \\
\hline 67.3 & 82.5 & 97.8 & 94.0 & 99.0 & 98.4 & 103.1 & 107.4 & 104.5 & 111.0 & 99.2 & 87.3 & 55.2 \\
\hline \multicolumn{13}{|c|}{ Thermocouples located at sprinkler number 4} \\
\hline Time & $0 \mathrm{~mm}$ & $25 \mathrm{~mm}$ & $50 \mathrm{~mm}$ & $75 \mathrm{~mm}$ & $100 \mathrm{~mm}$ & $125 \mathrm{~mm}$ & $150 \mathrm{~mm}$ & $250 \mathrm{~mm}$ & $350 \mathrm{~mm}$ & $450 \mathrm{~mm}$ & $550 \mathrm{~mm}$ & $900 \mathrm{~mm}$ \\
\hline 0.0 & 20.4 & 20.4 & 20.3 & 20.6 & 20.5 & 20.5 & 20.5 & 20.3 & 20.2 & 20.0 & 19.9 & 19.8 \\
\hline 5.9 & 20.5 & 20.4 & 20.3 & 20.6 & 20.4 & 20.5 & 20.5 & 20.4 & 20.3 & 20.2 & 20.0 & 19.9 \\
\hline 12.7 & 20.7 & 20.7 & 20.5 & 20.7 & 20.5 & 20.5 & 20.6 & 21.2 & 21.0 & 20.6 & 20.4 & 20.3 \\
\hline 19.5 & 23.7 & 25.7 & 26.3 & 28.8 & 29.0 & 29.5 & 30.4 & 31.1 & 34.4 & 31.6 & 26.3 & 23.4 \\
\hline 26.4 & 31.3 & 36.5 & 37.3 & 37.7 & 37.8 & 38.5 & 39.5 & 38.7 & 42.7 & 40.3 & 37.9 & 22.2 \\
\hline 33.2 & 39.4 & 43.4 & 43.0 & 45.7 & 45.3 & 48.0 & 48.4 & 48.1 & 51.2 & 50.7 & 51.6 & 26.2 \\
\hline 40.1 & 40.2 & 46.8 & 46.1 & 51.1 & 57.9 & 59.8 & 63.5 & 65.4 & 67.8 & 62.0 & 59.5 & 28.7 \\
\hline 46.9 & 50.2 & 60.5 & 64.1 & 66.7 & 70.9 & 72.8 & 73.9 & 78.4 & 81.4 & 77.9 & 73.7 & 36.6 \\
\hline 53.6 & 60.2 & 66.5 & 68.2 & 71.2 & 72.8 & 74.4 & 76.9 & 80.9 & 85.9 & 82.9 & 82.4 & 39.1 \\
\hline 60.6 & 14.1 & 82.6 & 80.4 & $8 / .8$ & 88.1 & 89.0 & 91.4 & 92.5 & 96.0 & 94.6 & 96.2 & 58.1 \\
\hline 67.3 & 74.2 & 96.8 & 94.3 & 103.1 & 105.3 & 107.7 & 110.4 & 110.8 & 114.8 & 110.1 & 107.3 & 62.9 \\
\hline \multicolumn{6}{|c|}{ Velocity probes at sprinkler number 1} & \multicolumn{7}{|c|}{ Velority probes at sprinkler number 2} \\
\hline Time & $25 \mathrm{~mm}$ & $75 \mathrm{~mm}$ & $125 \mathrm{~mm}$ & $250 \mathrm{~mm}$ & & Time & $25 \mathrm{~mm}$ & $75 \mathrm{~mm}$ & $125 \mathrm{~mm}$ & $250 \mathrm{~mm}$ & & \\
\hline 0.0 & 0.0 & 0.0 & 0.0 & 0.0 & & 0.0 & 0.0 & 0.0 & 0.0 & 0.0 & & \\
\hline 5.9 & -0.2 & -0.1 & -0.1 & -0.1 & & 5.9 & 0.1 & 0.2 & 0.2 & 0.2 & & \\
\hline 12.7 & -0.9 & -0.9 & -0.7 & -0.2 & & 12.7 & 0.9 & 0.9 & 0.9 & 0.5 & & \\
\hline 10.5 & -0.9 & -0.9 & -0.8 & -0.5 & & 19.5 & 0.9 & 0.9 & 0.7 & 0.4 & & \\
\hline 26.4 & -1.0 & -1.0 & -0.8 & -0.4 & & 26.4 & 0.9 & 1.1 & 1.1 & 0.6 & & \\
\hline 33.2 & -0.7 & -0.8 & -0.9 & -0.6 & & 33.2 & 0.6 & 0.7 & 0.7 & 0.8 & & \\
\hline 40.1 & -0.7 & -0.9 & -0.9 & -0.7 & & 40.1 & 1.0 & 1.0 & 1.0 & 0.7 & & \\
\hline 46.9 & -1.2 & -1.2 & -1.0 & -0.4 & & 46.9 & 0.9 & 1.0 & 1.1 & 0.6 & & \\
\hline 53.6 & -0.7 & -0.9 & -1.0 & -0.6 & & 53.6 & 0.8 & 0.8 & 0.9 & 0.7 & & \\
\hline
\end{tabular}




\section{Experiment Number 5}

\begin{tabular}{|c|c|c|c|c|c|c|c|c|c|}
\hline 60.6 & -1.2 & -1.1 & -0.8 & -0.5 & 60.6 & 1.0 & 0.8 & 0.9 & 0.6 \\
\hline 67.3 & -1.3 & -1.3 & -0.8 & -0.6 & 67.3 & 1.0 & 1.2 & 1.4 & 0.8 \\
\hline \multicolumn{10}{|c|}{ Velocity probes in channels above burner } \\
\hline Time & Channel 1 & Channel 2 & & & & & & & \\
\hline 0.0 & 0.0 & 0.0 & & & & & & & \\
\hline 5.9 & 1.7 & 0.7 & & & & & & & \\
\hline 12.7 & 2.3 & 1.3 & & & & & & & \\
\hline 19.5 & 2.4 & 1.3 & & & & & & & \\
\hline 26.4 & 2.6 & 1.4 & & & & & & & \\
\hline 33.2 & 2.7 & 1.5 & & & & & & & \\
\hline 40.1 & 2.8 & 1.6 & & & & & & & \\
\hline 46.9 & 1.7 & 1.5 & & & & & & & \\
\hline 53.6 & 3.2 & 1.7 & & & & & & & \\
\hline 60.6 & 4.1 & 2.1 & & & & & & & \\
\hline 67.3 & 4.2 & 1.4 & & & & & & & \\
\hline
\end{tabular}


Experiment Number 6

Thermocouples localed at sprinkler number

\begin{tabular}{|c|c|c|c|c|c|c|c|c|c|c|c|c|}
\hline Time & $0 \mathrm{~mm}$ & $25 \mathrm{~mm}$ & $50 \mathrm{~mm}$ & $75 \mathrm{~mm}$ & $100 \mathrm{~mm}$ & $125 \mathrm{~mm}$ & $150 \mathrm{~mm}$ & $250 \mathrm{~mm}$ & $350 \mathrm{~mm}$ & $450 \mathrm{~mm}$ & $550 \mathrm{~mm}$ & $900 \mathrm{~mm}$ \\
\hline 0.0 & 17.1 & 17.6 & 17.7 & 17.7 & 17.7 & 17.7 & 17.7 & 17.7 & 17.6 & 17.6 & 17.5 & 17.4 \\
\hline 2.7 & 17.2 & 17.6 & 17.8 & 17.9 & 17.9 & 17.9 & 17.8 & 17.8 & 17.6 & 17.5 & 17.5 & 17.3 \\
\hline 9.6 & 17.1 & 18.5 & 20.7 & 21.1 & 21.5 & 22.0 & 21.0 & 21.4 & 19.3 & 17.7 & 17.6 & 17.4 \\
\hline 16.4 & 20.3 & 30.0 & 34.0 & 34.4 & 32.3 & 33.9 & 31.7 & 29.1 & 21.1 & 18.6 & 17.8 & 17.5 \\
\hline 23.3 & 23.2 & 38.3 & 42.0 & 41.8 & 40.7 & 41.4 & 40.6 & 38.8 & 31.2 & 25.2 & 20.9 & 18.9 \\
\hline 30.2 & 26.4 & 49.6 & 56.6 & 56.6 & 53.4 & 54.9 & 51.6 & 46.5 & 38.1 & 34.8 & 28.1 & 18.7 \\
\hline 36.9 & 29.3 & 55.3 & 64.9 & 64.4 & 61.8 & 59.8 & 57.2 & 53.6 & 49.2 & 45.3 & 31.9 & 18.3 \\
\hline 43.9 & 31.6 & 62.1 & 74.3 & 71.7 & 59.9 & 67.8 & 66.2 & 59.7 & 55.6 & 50.1 & 35.9 & 22.3 \\
\hline 50.6 & 34.1 & 65.1 & 73.8 & 74.4 & 73.2 & 73.1 & 72.0 & 67.0 & 62.5 & 59.2 & 47.1 & 22.4 \\
\hline 57.5 & 37.3 & 71.6 & 80.8 & 80.1 & 78.7 & 79.0 & 78.0 & 73.8 & 68.1 & 65.3 & 54.5 & 25.3 \\
\hline 64.3 & 39.6 & 77.1 & 86.8 & 86.3 & 83.1 & 84.5 & 82.8 & 81.0 & 78.0 & 73.0 & 62.4 & 35.4 \\
\hline 71.1 & 44.1 & 82.9 & 93.7 & 93.4 & 93.7 & 93.3 & 92.1 & 87.5 & 82.0 & 75.3 & 64.4 & 38.7 \\
\hline 78.1 & 44.0 & 89.2 & 101.4 & 101.4 & 100.6 & 102.5 & 101.4 & 97.2 & 91.3 & 83.4 & 73.3 & 43.3 \\
\hline 84.8 & 47.7 & 99.7 & 113.6 & 113.6 & 110.2 & 113.2 & 109.8 & 103.1 & 99.3 & 93.9 & 86.2 & 47.0 \\
\hline 91.8 & 54.3 & 105.1 & 57.7 & 70.7 & 74.0 & 89.8 & 87.2 & 106.3 & 104.5 & 102.3 & 83.0 & 50.3 \\
\hline 98.5 & 58.6 & 123.5 & 61.0 & $\uparrow 17.5$ & 116.0 & 126.6 & 106.1 & 120.1 & 121.7 & 116.2 & 95.3 & 47.1 \\
\hline \multicolumn{13}{|c|}{ mocouples located at sprinkler number 2} \\
\hline Time & $0 \mathrm{~mm}$ & $25 \mathrm{~mm}$ & $50 \mathrm{~mm}$ & $75 \mathrm{~mm}$ & $100 \mathrm{~mm}$ & $125 \mathrm{~mm}$ & $150 \mathrm{~mm}$ & $250 \mathrm{~mm}$ & $350 \mathrm{~mm}$ & $450 \mathrm{~mm}$ & $550 \mathrm{~mm}$ & $900 \mathrm{~mm}$ \\
\hline 0.0 & 17.1 & 17.7 & 17.8 & 17.8 & 17.8 & 17.8 & 17.8 & 17.8 & 17.7 & 17.7 & 17.7 & 17.5 \\
\hline 2.7 & 17.1 & 17.8 & 17.9 & 17.9 & 17.9 & 17.9 & 17.9 & 17.9 & 17.8 & 17.7 & 17.5 & 17.4 \\
\hline 9.6 & 18.1 & 25.6 & 25.5 & 24.9 & 23.9 & 24.0 & 23.9 & 23.3 & 20.2 & 17.7 & 17.7 & 17.5 \\
\hline 16.4 & 22.7 & 44.2 & 44.3 & 44.8 & 43.7 & 42.8 & 40.7 & 32.9 & 24.6 & 19.0 & 17.9 & 17.7 \\
\hline 23.3 & 25.7 & 47.6 & 48.7 & 48.5 & 48.2 & 46.9 & 44.3 & 38.5 & 31.0 & 24.5 & 22.3 & 17.9 \\
\hline 30.2 & 26.8 & 49.7 & 51.7 & 52.1 & 53.7 & 53.7 & 53.6 & 52.8 & 45.8 & 39.7 & 28.0 & 18.7 \\
\hline 36.9 & 29.7 & 62.1 & 63.2 & 63.0 & 64.2 & 62.0 & 61.8 & 57.3 & 48.0 & 43.2 & 37.2 & 18.5 \\
\hline 43.9 & 30.0 & 57.5 & 62.1 & 60.2 & 63.1 & 61.3 & 63.4 & 57.6 & 52.1 & 46.8 & 41.8 & 26.1 \\
\hline 50.6 & 33.5 & 74.6 & 78.2 & 78.2 & 80.7 & 79.4 & 79.3 & 74.2 & 61.8 & 54.7 & 45.4 & 25.6 \\
\hline 57.5 & 35.9 & 76.0 & 80.7 & 80.8 & 83.9 & 82.5 & 83.3 & 78.7 & 75.3 & 64.5 & 45.2 & 32.8 \\
\hline 64.3 & 40.4 & 92.4 & 95.3 & 96.7 & 97.6 & 95.3 & 94.5 & 86.0 & 81.8 & 75.2 & 64.3 & 36.2 \\
\hline 71.1 & 43.3 & 97.4 & 105.0 & 103.3 & 106.2 & 103.0 & 104.8 & 97.5 & B7.5 & 70.3 & 55.6 & 40.4 \\
\hline 78.1 & 48.7 & 117.0 & 119.2 & 117.4 & 118.5 & $\uparrow 15.0$ & 113.1 & 106.5 & 98.5 & 86.0 & 75.8 & 48.6 \\
\hline 84.8 & 51.6 & 117.3 & 124.4 & 121.1 & 123.3 & 116.5 & 119.2 & 109.3 & 103.5 & 92.9 & 73.9 & 54.7 \\
\hline 91.8 & 54.3 & 125.5 & 129.5 & 130.2 & 130.9 & 128.7 & 127.6 & 124.2 & 118.8 & 112.7 & 89.6 & 62.1 \\
\hline 98.5 & 56.1 & 124.0 & 68.6 & 117.9 & 131.6 & 130.4 & 131.5 & 129.7 & 126.1 & 102.9 & 88.4 & 66.9 \\
\hline \multicolumn{13}{|c|}{ mocouples located at sprinkler number 3} \\
\hline Time & $0 \mathrm{~mm}$ & $25 \mathrm{~mm}$ & $50 \mathrm{~mm}$ & $75 \mathrm{~mm}$ & $100 \mathrm{~mm}$ & $125 \mathrm{~mm}$ & $150 \mathrm{~mm}$ & $250 \mathrm{~mm}$ & $350 \mathrm{~mm}$ & $450 \mathrm{~mm}$ & $550 \mathrm{~mm}$ & $900 \mathrm{~mm}$ \\
\hline 0.0 & 16.7 & 17.3 & 17.7 & 17.7 & 17.7 & 17.7 & 17.7 & 17.6 & 17.6 & 17.5 & 17.4 & 17.4 \\
\hline 2.7 & 16.9 & 17.2 & 17.5 & 17.7 & 17.6 & 17.6 & 17.5 & 17.5 & 17.6 & 17.6 & 17.6 & 17.5 \\
\hline 9.6 & 16.7 & 17.1 & 17.4 & 17.5 & 17.6 & 17.6 & 17.6 & 17.6 & 17.6 & 17.5 & 17.5 & 17.5 \\
\hline 16.4 & 16.9 & 19.3 & 21.7 & 21.6 & 20.4 & 20.6 & 19.9 & 19.1 & 18.3 & 17.7 & 17.7 & 17.7 \\
\hline 23.3 & 17.5 & 25.9 & 28.7 & 27.4 & 26.0 & 26.1 & 24.5 & 19.8 & 17.9 & 17.8 & 17.8 & 17.8 \\
\hline 30.2 & 18.5 & 32.0 & 37.4 & 37.6 & 36.8 & 37.9 & 36.2 & 31.8 & 23.9 & 21.5 & 21.8 & 20.6 \\
\hline 36.9 & 19.7 & 40.7 & 46.8 & 47.3 & 45.1 & 45.9 & 44.0 & 39.9 & 34.6 & 30.6 & 30.2 & 22.6 \\
\hline 43.9 & 20.2 & 38.1 & 46.6 & 46.2 & 45.0 & 44.9 & 43.7 & 39.8 & 35.7 & 33.7 & 32.8 & 25.3 \\
\hline 50.6 & 21.2 & 45.9 & 54.7 & 54.6 & 53.1 & 52.9 & 51.1 & 47.4 & 43.5 & 37.8 & 36.4 & 29.9 \\
\hline 57.5 & 21.8 & 49.0 & 59.2 & 59.5 & 57.9 & 58.3 & 56.8 & 51.7 & 46.8 & 42.3 & 42.5 & 34.1 \\
\hline 64.3 & 22.7 & 52.6 & 64.9 & 65.3 & 63.8 & 64.2 & 62.5 & 56.2 & 51.3 & 47.9 & 46.9 & 39.0 \\
\hline 71.1 & 23.5 & 54.9 & 68.8 & 69.5 & 67.9 & 68.7 & 67.0 & 62.5 & 56.6 & 51.7 & 50.4 & 41.6 \\
\hline 78.1 & 25.6 & 63.1 & 77.6 & 77.3 & 75.2 & 75.5 & 73.5 & 67.8 & 58.8 & 55.9 & 56.4 & 46.5 \\
\hline 84.8 & 26.8 & 65.3 & 81.2 & 80.0 & 77.8 & 77.8 & 75.2 & 68.6 & 66.0 & 65.1 & 64.8 & 53.6 \\
\hline 91.8 & 27.3 & 71.9 & 87.7 & 87.7 & 86.1 & 86.2 & 84.0 & 76.3 & 73.3 & 70.6 & 69.9 & 58.5 \\
\hline 98.5 & 30.2 & 79.2 & 96.2 & 97.3 & 94.5 & 95.4 & 92.3 & 83.7 & 78.2 & 74.9 & 75.7 & 66.0 \\
\hline \multicolumn{13}{|c|}{ mocouples located at sprinkler number 4} \\
\hline Time & $0 \mathrm{~mm}$ & $25 \mathrm{~mm}$ & $50 \mathrm{~mm}$ & $75 \mathrm{~mm}$ & $100 \mathrm{~mm}$ & $125 \mathrm{~mm}$ & $150 \mathrm{~mm}$ & $250 \mathrm{~mm}$ & $350 \mathrm{~mm}$ & $450 \mathrm{~mm}$ & $550 \mathrm{~mm}$ & $900 \mathrm{~mm}$ \\
\hline 0.0 & 16.7 & 17.3 & 17.6 & 17.6 & 17.6 & $\uparrow 7.6$ & 17.6 & 17.6 & 17.5 & 17.5 & 17.4 & 17.3 \\
\hline 2.7 & 17.0 & 17.1 & 17.5 & 17.6 & 17.5 & 17.5 & 17.6 & 17.5 & 17.4 & 17.4 & 17.4 & 17.4 \\
\hline 9.6 & 16.8 & 17.0 & 17.6 & 17.5 & 17.5 & 17.5 & 17.5 & 17.5 & 17.6 & 17.5 & 17.4 & 17.4 \\
\hline 16.4 & 17.8 & 21.8 & 24.2 & 23.6 & 22.7 & 22.6 & 22.4 & 20.8 & 19.5 & 17.5 & 17.5 & 17.7 \\
\hline 23.3 & 19.9 & 27.3 & 28.7 & 28.0 & 26.6 & 26.1 & 25.3 & 22.1 & 22.1 & 19.5 & 18.9 & 17.7 \\
\hline 30.2 & 21.0 & 38.2 & 39.3 & 38.6 & 36.6 & 38.2 & 38.1 & 33.6 & 30.3 & 27.4 & 25.3 & 20.8 \\
\hline 36.9 & 22.0 & 39.4 & 41.9 & 42.4 & 42.1 & 43.9 & 44.7 & 41.9 & 40.5 & 34.5 & 32.7 & 20.1 \\
\hline
\end{tabular}




\section{Experiment Number 6}

\begin{tabular}{|c|c|c|c|c|c|c|c|c|c|c|c|c|}
\hline 43.9 & 23.9 & 45.0 & 46.9 & 47.6 & 46.6 & 47.9 & 48.3 & 45.5 & 44.0 & 39.2 & 37.4 & 20.4 \\
\hline 50.6 & 25.1 & 52.3 & 53.6 & 53.0 & 51.8 & 52.7 & 52.4 & 48.2 & 44.8 & 42.6 & 41.1 & 24.4 \\
\hline 57.5 & 28.9 & 59.4 & 60.4 & 60.0 & 58.6 & 59.8 & 60.4 & 57.2 & 52.4 & 47.2 & 46.0 & 30.5 \\
\hline 64.3 & 30.1 & 66.1 & 67.3 & 67.3 & 65.3 & 67.4 & 67.9 & 63.0 & 58.8 & 53.4 & 52.3 & 33.2 \\
\hline 71.1 & 36.0 & 72.6 & 74.5 & 74.9 & 73.3 & 74.9 & 74.8 & 68.4 & 64.6 & 59.6 & 58.3 & 36.6 \\
\hline 78.1 & 38.0 & 79.5 & 81.3 & 81.3 & 79.5 & 81.2 & 81.5 & 77.3 & 76.1 & 68.3 & 64.8 & 39.9 \\
\hline 84.8 & 41.0 & 85.8 & 86.6 & 86.9 & 85.4 & 87.6 & 87.3 & 83.1 & 81.2 & 72.7 & 70.8 & 44.3 \\
\hline 91.8 & 45.5 & 92.3 & 93.2 & 93.0 & 90.5 & 92.5 & 92.2 & 86.6 & 86.2 & 79.5 & 76.8 & 49.6 \\
\hline 98.5 & 48.7 & 99.9 & 102.7 & 102.5 & 99.7 & 102.8 & 103.9 & 98.1 & 93.6 & 85.4 & 83.6 & 53.7 \\
\hline \multicolumn{5}{|c|}{ Velocity probes at sprinkler number 1} & \multicolumn{6}{|c|}{ Velocily probes at sprlnkler number 2} & & \\
\hline Time & $25 \mathrm{~mm}$ & $75 \mathrm{~mm}$ & $125 \mathrm{~mm}$ & $250 \mathrm{~mm}$ & & Time & $25 \mathrm{~mm}$ & $75 \mathrm{~mm}$ & $125 \mathrm{~mm}$ & $250 \mathrm{~mm}$ & & \\
\hline 0.0 & 0.0 & 0.0 & 0.0 & 0.0 & & 0.0 & 0.0 & 0.0 & 0.0 & 0.0 & & \\
\hline 2.7 & -0.2 & -0.2 & -0.2 & 0.0 & & 2.7 & 0.2 & 0.1 & 0.1 & 0.1 & & \\
\hline 9.6 & -1.0 & -0.7 & -0.4 & -0.3 & & 9.6 & 0.9 & 0.9 & 0.8 & 0.3 & & \\
\hline 16.4 & -0.9 & -0.6 & -0.7 & -0.6 & & 16.4 & 1.0 & 0.8 & 0.7 & -0.1 & & \\
\hline 23.3 & -1.2 & -1.1 & -1.2 & -0.3 & & 23.3 & 1.1 & 1.0 & 1.0 & 0.5 & & \\
\hline 30.2 & -1.2 & -1.1 & -1.3 & -0.5 & & 30.2 & 0.8 & 0.8 & 0.7 & 0.4 & & \\
\hline 36.9 & -1.4 & -1.3 & -1.1 & -0.7 & & 36.9 & 1.4 & 1.4 & 1.2 & 0.5 & & \\
\hline 43.9 & -0.8 & -1.0 & -1.0 & -0.1 & & 43.9 & 0.7 & 0.7 & 0.6 & 0.3 & & \\
\hline 50.6 & -1.5 & -1.5 & -1.0 & -0.5 & & 50.6 & 1.2 & 1.1 & 1.1 & 0.4 & & \\
\hline 57.5 & -0.9 & -1.0 & -0.6 & -0.3 & & 57.5 & 0.9 & 0.9 & 0.9 & 0.6 & & \\
\hline 64.3 & -1.1 & -1.2 & -0.9 & -0.3 & & 64.3 & 1.1 & 1.2 & 1.0 & 0.5 & & \\
\hline 71.1 & -1.0 & -1.1 & -1.0 & -0.5 & & 71.1 & 1.4 & 1.4 & 1.4 & 0.8 & & \\
\hline 78.1 & -1.4 & -1.6 & -1.5 & -0.9 & & 78.1 & 1.2 & 1.5 & 1.4 & 0.7 & & \\
\hline 84.8 & -1.8 & -1.6 & -1.3 & -0.1 & & 84.8 & 1.1 & 1.2 & 1.3 & 0.4 & & \\
\hline 91.8 & -1.6 & -1.4 & -1.2 & .0 .9 & & 91.8 & 1.4 & 1.6 & 1.4 & 0.9 & & \\
\hline 98.5 & -1.2 & -1.2 & -1.4 & -0.7 & & 98.5 & 1.3 & 1.3 & 1.1 & 0.6 & & \\
\hline
\end{tabular}




\section{Experiment Number 7}

Thermocouples located at sprinkler number 1

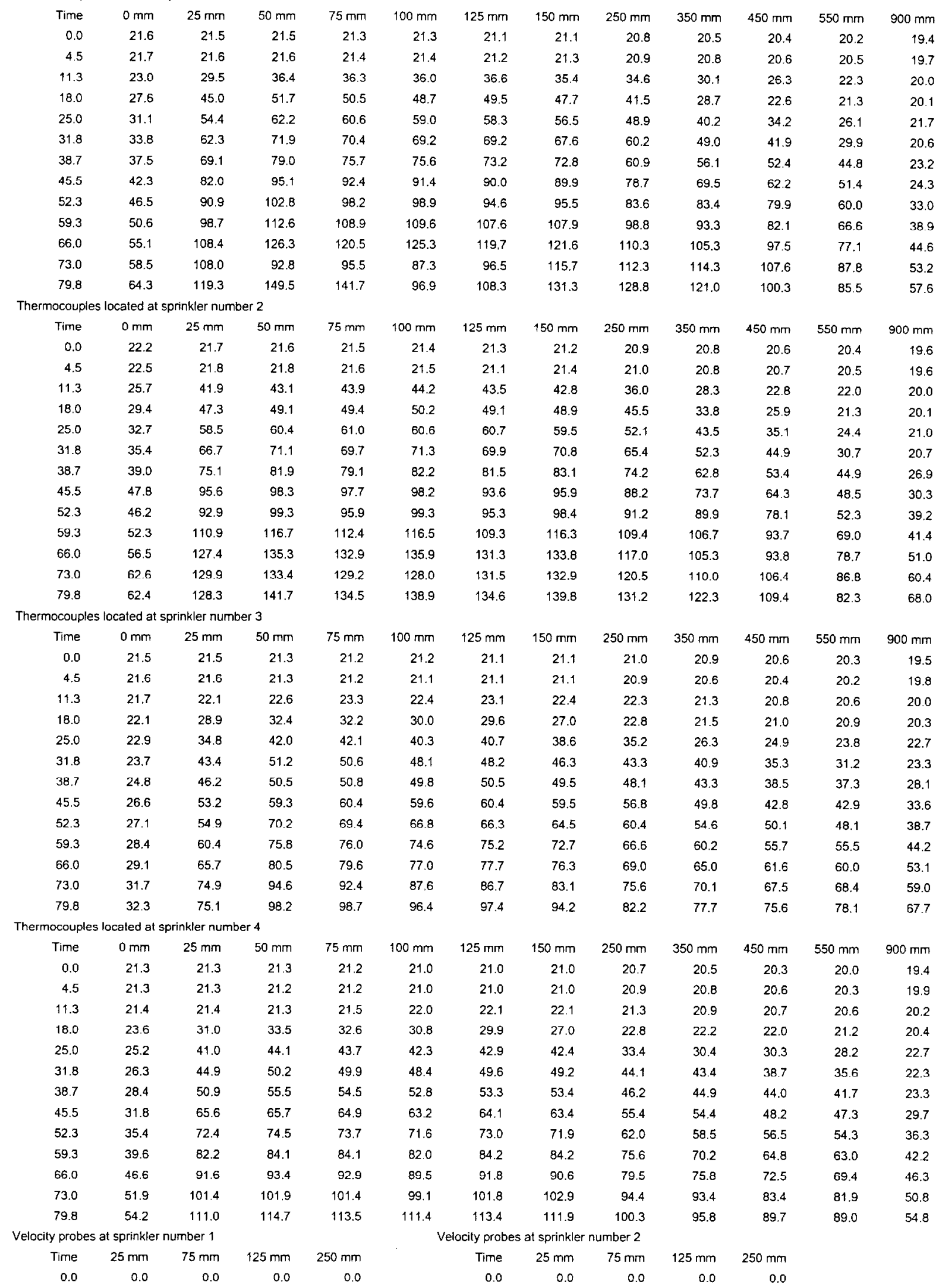


Experiment Number 7

\begin{tabular}{|c|c|c|c|c|c|c|c|c|c|}
\hline 4.5 & -0.9 & -1.0 & -0.6 & -0.5 & 4.5 & 0.8 & 0.7 & 0.7 & 0.4 \\
\hline 11.3 & -1.0 & -0.9 & -0.7 & -0.3 & 11.3 & 1.1 & 1.0 & 0.8 & 0.1 \\
\hline 18.0 & -1.1 & -0.9 & -0.6 & -0.1 & 18.0 & 0.9 & 0.8 & 0.9 & 0.5 \\
\hline 25.0 & -1.0 & -1.0 & -1.0 & -0.6 & 25.0 & 1.1 & 1.0 & 0.9 & 0.1 \\
\hline 31.8 & -1.1 & -1.0 & -1.0 & -0.1 & 31.8 & 1.1 & 1.0 & 0.9 & 0.0 \\
\hline 38.7 & -1.2 & -1.2 & -1.0 & -0.5 & 38.7 & 1.5 & 1.4 & 1.3 & 0.5 \\
\hline 45.5 & -1.3 & -1.2 & -0.6 & -0.3 & 45.5 & 1.1 & 1.0 & 1.0 & 0.5 \\
\hline 52.3 & -1.0 & -1.1 & -0.6 & -0.2 & 52.3 & 1.4 & 1.4 & 1.4 & 0.6 \\
\hline 59.3 & -1.4 & -1.5 & -1.3 & -0.9 & 59.3 & 1.5 & 1.6 & 1.2 & 0.5 \\
\hline 66.0 & -1.7 & -1.8 & -1.4 & -1.0 & 66.0 & 1.5 & 1.5 & 1.5 & 0.9 \\
\hline 73.0 & -1.3 & -1.1 & -0.7 & -0.4 & 73.0 & 1.8 & 1.8 & 1.7 & 0.8 \\
\hline 79.8 & -1.2 & -1.3 & -1.0 & -0.8 & 79.8 & 0.6 & 1.0 & 1.2 & 0.7 \\
\hline
\end{tabular}


Experiment Number 8

Thermocouples located at sprinkler number 1

\begin{tabular}{|c|c|c|c|c|c|c|c|c|c|c|c|c|}
\hline Time & $0 \mathrm{~mm}$ & $25 \mathrm{~mm}$ & $50 \mathrm{~mm}$ & $75 \mathrm{~mm}$ & $100 \mathrm{~mm}$ & $125 \mathrm{~mm}$ & $150 \mathrm{~mm}$ & $250 \mathrm{~mm}$ & $350 \mathrm{~mm}$ & $450 \mathrm{~mm}$ & $550 \mathrm{~mm}$ & $900 \mathrm{~mm}$ \\
\hline 0.0 & 21.8 & 21.6 & 21.6 & 21.6 & 21.6 & 21.5 & 21.6 & 21.4 & 21.4 & 21.3 & 21.2 & 20.7 \\
\hline 5.7 & 22.1 & 21.7 & 21.8 & 21.8 & 21.5 & 21.5 & 21.5 & 21.4 & 21.3 & 21.2 & 21.0 & 20.5 \\
\hline 12.5 & 23.4 & 27.8 & 33.2 & 31.5 & 31.7 & 31.6 & 30.3 & 27.8 & 26.6 & 24.1 & 21.5 & 20.9 \\
\hline 19.4 & 27.8 & 42.3 & 48.0 & 45.6 & 45.1 & 44.1 & 42.0 & 35.8 & 30.7 & 24.3 & 21.8 & 21.1 \\
\hline 26.2 & 30.6 & 49.0 & 54.1 & 51.9 & 52.9 & 51.3 & 51.9 & 50.0 & 43.1 & 36.5 & 29.6 & 22.6 \\
\hline 33.0 & 33.4 & 55.8 & 64.8 & 60.9 & 64.1 & 60.1 & 62.3 & 57.8 & 52.3 & 47.3 & 32.5 & 21.7 \\
\hline 39.9 & 36.2 & 65.4 & 78.7 & 72.2 & 76.5 & 71.1 & 73.1 & 61.7 & 56.9 & 52.5 & 44.4 & 23.5 \\
\hline 46.7 & 39.2 & 70.2 & 82.4 & 79.7 & 81.6 & 76.3 & 82.1 & 67.3 & 66.6 & 61.0 & 45.9 & 26.1 \\
\hline 53.6 & 42.2 & 75.2 & 88.1 & 83.7 & 87.6 & 84.0 & 89.2 & 79.3 & 82.1 & 73.1 & 54.0 & 26.4 \\
\hline 60.4 & 45.6 & 86.0 & 100.3 & 94.5 & 99.3 & 94.2 & 96.7 & 85.2 & 85.0 & 79.8 & 673 & 36.7 \\
\hline 67.1 & 50.2 & 96.1 & 111.5 & 105.9 & 109.0 & 103.4 & 106.0 & 91.3 & 93.7 & 82.4 & 71.4 & 43.3 \\
\hline 74.1 & 55.2 & 105.1 & 119.7 & 114.9 & 117.3 & 112.0 & 115.0 & 101.0 & 99.5 & 89.5 & 74.6 & 48.9 \\
\hline 80.9 & 60.0 & 116.5 & 74.5 & 62.5 & 78.6 & 57.0 & 59.0 & 76.7 & 109.5 & 101.3 & 84.1 & 51.8 \\
\hline 87.8 & 61.4 & 119.5 & 52.0 & 119.5 & 126.6 & 56.8 & 115.9 & 110.4 & 109.3 & 108.7 & 84.1 & 54.7 \\
\hline \multicolumn{13}{|c|}{ Thermocouples located at sprinkler number 2} \\
\hline Time & $0 \mathrm{~mm}$ & $25 \mathrm{~mm}$ & $50 \mathrm{~mm}$ & $75 \mathrm{~mm}$ & $100 \mathrm{~mm}$ & $125 \mathrm{~mm}$ & $150 \mathrm{~mm}$ & $250 \mathrm{~mm}$ & $350 \mathrm{~mm}$ & $450 \mathrm{~mm}$ & $550 \mathrm{~mm}$ & $900 \mathrm{~mm}$ \\
\hline 0.0 & 22.0 & 21.8 & 21.7 & 21.7 & 21.7 & 21.6 & 21.6 & 21.5 & 21.4 & 21.3 & 21.2 & 20.5 \\
\hline 5.7 & 22.1 & 21.8 & 21.8 & 21.7 & 21.9 & 21.8 & 21.7 & 21.6 & 21.5 & 21.5 & 21.4 & 21.1 \\
\hline 12.5 & 25.4 & 39.2 & 39.3 & 40.0 & 39.6 & 39.4 & 38.6 & 32.1 & 25.1 & 22.6 & 21.6 & 21.2 \\
\hline 19.4 & 30.0 & 47.1 & 47.7 & 48.2 & 48.2 & 47.6 & 47.1 & 42.9 & 29.7 & 22.5 & 21.7 & 21.2 \\
\hline 26.2 & 33.2 & 55.4 & 59.3 & 57.9 & 59.3 & 58.7 & 59.5 & 57.1 & 44.9 & 35.8 & 28.3 & 21.8 \\
\hline 33.0 & 39.2 & 71.8 & 73.9 & 73.3 & 74.4 & 71.9 & 70.4 & 58.8 & 49.5 & 44.6 & 31.1 & 21.8 \\
\hline 39.9 & 39.4 & 73.0 & 76.9 & 75.3 & 77.1 & 75.9 & 77.0 & 70.4 & 62.2 & 54.5 & 41.7 & 22.6 \\
\hline 46.7 & 43.8 & 78.4 & 83.0 & 81.6 & 83.4 & 83.4 & 85.9 & 80.5 & 67.6 & 62.2 & 50.9 & 28.2 \\
\hline 53.6 & 47.3 & 85.9 & 90.7 & 88.9 & 90.9 & 89.7 & 90.0 & 84.9 & 76.7 & 66.3 & 52.3 & 36.2 \\
\hline 60.4 & 51.3 & 99.2 & 106.5 & 105.3 & 108.0 & 105.3 & 105.0 & 98.3 & 88.5 & 80.8 & 58.7 & 39.6 \\
\hline 67.1 & 56.7 & 1070 & 113.1 & 108.9 & 115.0 & 109.7 & 113.5 & 101.5 & 95.0 & 83.6 & 65.3 & 46.2 \\
\hline 74.1 & 62.4 & 115.8 & 122.3 & 118.9 & 121.7 & 118.6 & 120.3 & 109.3 & 102.0 & 92.4 & 79.0 & 49.6 \\
\hline 80.9 & 63.0 & 129.9 & 136.6 & 133.9 & 136.3 & 133.7 & $\uparrow 31.8$ & 120.8 & 110.9 & 95.9 & 80.1 & 60.5 \\
\hline 87.8 & 63.5 & 121.1 & 132.4 & 130.0 & 131.5 & 133.4 & 138.2 & 130.2 & 123.1 & 108.3 & 93.0 & 69.9 \\
\hline \multicolumn{13}{|c|}{ Thermocouples located at sprinkler number 3} \\
\hline Time & $0 \mathrm{~mm}$ & $25 \mathrm{~mm}$ & $50 \mathrm{~mm}$ & $75 \mathrm{~mm}$ & $100 \mathrm{~mm}$ & $125 \mathrm{~mm}$ & 150 гінгі & $250 \mathrm{~mm}$ & $350 \mathrm{~mm}$ & $450 \mathrm{~mm}$ & $550 \mathrm{~mm}$ & $900 \mathrm{~mm}$ \\
\hline 0.0 & 21.5 & 21.6 & 21.4 & 21.4 & 21.3 & 21.4 & 21.3 & 21.3 & 21.2 & 21.1 & 20.9 & 20.5 \\
\hline 5.7 & 21.4 & 21.5 & 21.4 & 21.4 & 21.3 & 21.3 & 21.3 & 21.3 & 21.3 & 21.3 & 21.3 & 21.0 \\
\hline 12.5 & 21.6 & 21.6 & 21.5 & 21.4 & 21.4 & 21.5 & 21.4 & 21.4 & 21.3 & 21.3 & 21.3 & 21.0 \\
\hline 19.4 & 21.9 & 27.5 & 30.9 & 30.4 & 28.8 & 28.7 & 27.2 & 22.8 & 22.0 & 21.5 & 21.4 & 21.0 \\
\hline 26.2 & 22.5 & 32.5 & 37.3 & 37.7 & 36.4 & 37.2 & 35.5 & 29.6 & 27.1 & 26.1 & 25.2 & 21.4 \\
\hline 33.0 & 23.4 & 38.7 & 46.3 & 45.9 & 44.5 & 45.0 & 43.7 & 39.9 & 35.1 & 30.5 & 29.1 & 24.7 \\
\hline 39.9 & 24.1 & 41.6 & 49.9 & 49.4 & 48.3 & 48.2 & 46.9 & 43.7 & 40.4 & 36.1 & 35.1 & 28.6 \\
\hline 46.7 & 25.0 & 45.7 & 59.0 & 58.7 & 56.8 & 58.2 & 56.3 & 50.4 & 46.4 & 40.1 & 39.2 & 31.5 \\
\hline 53.6 & 26.2 & 51.8 & 63.7 & 63.8 & 62.8 & 63.1 & 62.0 & 55.1 & 49.0 & 45.2 & 43.2 & 35.5 \\
\hline 60.4 & 27.2 & 60.5 & 70.2 & 70.2 & 68.5 & 69.2 & 68.2 & 61.0 & 54.1 & 51.1 & 49.2 & 41.6 \\
\hline 67.1 & 27.8 & 57.0 & 77.2 & 77.0 & 72.7 & 73.4 & 71.4 & 66.0 & 60.3 & 58.1 & 57.1 & 47.6 \\
\hline 74.1 & 28.7 & 65.5 & 80.5 & 80.0 & 77.5 & 70.0 & 76.2 & 74.8 & 64.8 & 61.5 & 61.0 & 51.4 \\
\hline 80.9 & 30.6 & 74.3 & 93.3 & 92.4 & 89.6 & 88.4 & 84.9 & 76.2 & 70.4 & 67.4 & 68.4 & 61.8 \\
\hline 87.8 & 32.7 & 77.1 & 101,4 & 101.0 & 97.8 & 97.6 & 94.6 & 79.6 & 74.3 & 73.4 & 73.3 & 68.9 \\
\hline \multicolumn{13}{|c|}{ Thermocouples located at sprinkler number 4} \\
\hline Time & $0 \mathrm{~mm}$ & $25 \mathrm{~mm}$ & $50 \mathrm{~mm}$ & $75 \mathrm{mrn}$ & $100 \mathrm{~mm}$ & $125 \mathrm{~mm}$ & $150 \mathrm{~mm}$ & $250 \mathrm{~mm}$ & $350 \mathrm{~mm}$ & $450 \mathrm{~mm}$ & $550 \mathrm{~mm}$ & $900 \mathrm{~mm}$ \\
\hline 0.0 & 21.3 & 21.4 & 21.4 & 21.4 & 21.3 & 21.3 & 21.3 & 21.2 & 21.1 & 21.0 & 21.0 & 20.6 \\
\hline 5.7 & 21.5 & 21.4 & 21.5 & 21.4 & 21.2 & 21.2 & 21.2 & 21.1 & 21.0 & 21.0 & 20.9 & 20.5 \\
\hline 12.5 & 21.4 & 22.7 & 23.6 & 23.3 & 23.1 & 23.5 & 23.7 & 22.6 & 21.4 & 21.1 & 20.9 & 20.5 \\
\hline 19.4 & 23.9 & 29.8 & 31.8 & 31.4 & 30.5 & 30.6 & 30.5 & 24.5 & 21.7 & 21.2 & 21.2 & 20.8 \\
\hline 26.2 & 24.2 & 38.5 & 40.3 & 39.9 & 38.7 & 39.5 & 38.5 & 30.5 & 28.2 & 25.5 & 24.2 & 22.9 \\
\hline 33.0 & 25.7 & 42.1 & 46.5 & 46.5 & 45.7 & 47.0 & 47.1 & 42.4 & 41.0 & 34.7 & 31.5 & 23.6 \\
\hline 39.9 & 27.1 & 49.0 & 51.3 & 51.0 & 49.7 & 50.8 & 50.6 & 47.0 & 45.4 & 39.7 & 39.0 & 23.3 \\
\hline 46.7 & 28.9 & 54.5 & 55.9 & 56.0 & 54.5 & 56.1 & 56.7 & 53.2 & 51.6 & 45.8 & 43.7 & 26.3 \\
\hline 53.6 & 32.9 & 64.6 & 64.6 & 63.7 & 61.3 & 62.3 & 61.7 & 56.1 & 54.5 & 53.1 & 50.1 & 32.3 \\
\hline 60.4 & 39.7 & 72.7 & 73.3 & 72.2 & 69.5 & 71.3 & 71.3 & 66.8 & 66.9 & 60.2 & 58.3 & 36.7 \\
\hline 67.1 & 38.1 & 82.4 & 83.7 & 83.3 & 81.7 & 83.5 & 83.2 & 74.3 & 69.8 & 64.9 & 63.7 & 41.0 \\
\hline 74.1 & 45.5 & 85.2 & 86.0 & 85.6 & 83.8 & 84.9 & 83.7 & 77.9 & 76.8 & 74.0 & 72.5 & 45.6 \\
\hline 80.9 & 50.5 & 98.2 & 98.6 & 98.4 & 96.9 & 99.0 & 99.0 & 91.2 & 85.8 & 78.5 & 76.3 & 49.6 \\
\hline
\end{tabular}




\section{Experiment Number 8}

$\begin{array}{ccrrr}87.8 & 54.6 & 107.3 & 109.8 & 109.3 \\ \text { Velocity probes at sprinkler number } 1 & & \\ \text { Time } & 25 \mathrm{~mm} & 75 \mathrm{~mm} & 125 \mathrm{~mm} & 250 \mathrm{~mm} \\ 0.0 & 0.0 & 0.0 & 0.0 & 0.0 \\ 5.7 & -1.0 & -1.0 & -0.9 & -0.6 \\ 12.5 & -1.1 & -1.0 & -0.6 & -0.3 \\ 19.4 & -1.2 & -1.1 & -0.8 & -0.7 \\ 26.2 & -0.8 & -1.0 & -1.1 & -0.4 \\ 33.0 & -1.0 & -0.9 & -0.6 & -0.4 \\ 39.9 & -1.1 & -1.0 & -0.6 & -0.5 \\ 46.7 & -1.4 & -1.4 & -1.1 & -0.9 \\ 53.6 & -1.3 & -1.2 & -0.7 & -0.3 \\ 60.4 & -0.9 & -1.2 & -1.4 & -0.9 \\ 67.1 & -1.8 & -1.6 & -1.3 & -1.1 \\ 74.1 & -1.3 & -1.2 & -0.8 & -0.8 \\ 80.9 & -1.7 & -1.6 & -1.3 & -0.5 \\ 87.8 & -1.1 & -1.0 & -0.4 & -0.5\end{array}$

\begin{tabular}{|c|c|c|c|c|}
\hline 107.8 & 106.8 & 97.1 & 93.4 & 89.7 \\
\hline \multicolumn{5}{|c|}{ Velocity probes at sprinkler number 2} \\
\hline Time & $25 \mathrm{~mm}$ & $75 \mathrm{~mm}$ & $125 \mathrm{~mm}$ & $250 \mathrm{~mm}$ \\
\hline 0.0 & 0.0 & 0.0 & 0.0 & 0.0 \\
\hline 5.7 & 0.8 & 0.7 & 0.5 & 0.1 \\
\hline 12.5 & 1.0 & 1.1 & 0.8 & 0.2 \\
\hline 19.4 & 1.3 & 1.4 & 1.2 & 0.6 \\
\hline 26.2 & 1.4 & 1.4 & 1.0 & 0.4 \\
\hline 33.0 & 0.9 & 1.0 & 1.0 & 0.4 \\
\hline 39.9 & 1.1 & 1.1 & 1.1 & 0.1 \\
\hline 46.7 & 0.8 & 0.7 & 0.6 & -0.1 \\
\hline 53.6 & 1.0 & 1.1 & 0.9 & -0.1 \\
\hline 60.4 & 1.0 & 1.1 & 0.9 & 0.4 \\
\hline 67.1 & 1.3 & 1.2 & 1.1 & 0.5 \\
\hline 74.1 & 1.2 & 1.1 & 1.0 & 0.4 \\
\hline 80.9 & 1.3 & 1.3 & 1.2 & 0.4 \\
\hline 87.8 & 1.1 & 1.2 & 0.9 & 0.5 \\
\hline
\end{tabular}


Experiment Number 9

Thermocouples located at sprinkler number 1

\begin{tabular}{|c|c|c|c|c|c|c|c|c|c|c|c|c|}
\hline Time & $0 \mathrm{~mm}$ & $25 \mathrm{~mm}$ & $50 \mathrm{~mm}$ & $75 \mathrm{~mm}$ & $100 \mathrm{~mm}$ & $125 \mathrm{~mm}$ & $150 \mathrm{~mm}$ & $250 \mathrm{~mm}$ & $350 \mathrm{~mm}$ & $450 \mathrm{~mm}$ & $550 \mathrm{~mm}$ & $900 \mathrm{~mm}$ \\
\hline 0.0 & 21.3 & 21.1 & 21.2 & 20.8 & 20.7 & 20.0 & 20.3 & 19.6 & 19.6 & 19.4 & 19.1 & 18.2 \\
\hline 1.3 & 21.4 & 21.2 & 21.2 & 20.8 & 20.7 & 20.0 & 20.3 & 19.7 & 19.7 & 19.3 & 19.2 & 18.5 \\
\hline 8.2 & 21.5 & 21.3 & 21.2 & 20.9 & 20.8 & 20.1 & 20.5 & 19.9 & 19.8 & 19.4 & 19.3 & 18.5 \\
\hline 15.0 & 21.9 & 22.0 & 22.1 & 22.1 & 22.0 & 22.1 & 21.9 & 21.8 & 21.0 & 20.6 & 20.0 & 18.8 \\
\hline 21.9 & 30.0 & 30.1 & 32.0 & 31.9 & 30.5 & 29.3 & 26.4 & 23.5 & 22.4 & 21.8 & 20.8 & 18.9 \\
\hline 28.7 & 37.4 & 37.2 & 39.8 & 40.4 & 39.3 & 39.7 & 36.0 & 31.9 & 23.5 & 20.8 & 20.5 & 19.0 \\
\hline 35.4 & 42.9 & 43.0 & 45.9 & 46.1 & 44.1 & 44.0 & 40.3 & 36.0 & 29.7 & 23.9 & 22.9 & 21.0 \\
\hline 42.4 & 49.4 & 49.1 & 50.8 & 50.8 & 49.2 & 48.9 & 45.7 & 42.3 & 34.1 & 28.2 & 27.3 & 21.7 \\
\hline 49.1 & 55.9 & 55.5 & 58.1 & 58.4 & 56.7 & 56.9 & 53.4 & 50.5 & 36.3 & 32.9 & 31.0 & 24.6 \\
\hline 56.0 & 59.2 & 60.0 & 62.1 & 63.7 & 62.4 & 63.5 & 59.5 & 58.6 & 43.7 & 38.2 & 36.1 & 27.1 \\
\hline 62.8 & 67.8 & 67.2 & 68.8 & 70.4 & 68.3 & 69.0 & 64.6 & 65.1 & 56.2 & 45.4 & 39.7 & 29.6 \\
\hline 69.5 & 74.1 & 74.5 & 78.5 & 79.0 & 77.3 & 76.2 & 70.9 & 65.7 & 57.3 & 50.2 & 47.2 & 35.4 \\
\hline 76.5 & 81.2 & 81.1 & 84.2 & 85.4 & 83.4 & 85.2 & 81.3 & 78.6 & 65.2 & 54.4 & 50.3 & 42.1 \\
\hline 83.2 & 83.2 & 85.1 & 88.6 & 89.9 & 88.2 & 89.8 & 84.5 & 83.9 & 73.4 & 61.8 & 56.7 & 48.2 \\
\hline 90.2 & 84.4 & 87.5 & 91.8 & 93.6 & 92,0 & 94.4 & 90.0 & 91.6 & 86.7 & 65.6 & 60.6 & 47.3 \\
\hline 96.9 & 90.0 & 94.8 & 99.8 & 103.1 & 101.7 & 103.8 & 98.5 & 95.1 & 83.8 & 71.3 & 65.8 & 50.8 \\
\hline 103.6 & 97.1 & 100.2 & 107.4 & 111.0 & 109.2 & 112.7 & 106.8 & 108.9 & 99.0 & 82.3 & 68.2 & 58.4 \\
\hline 110.5 & 109.1 & 110.0 & 115.4 & 116.3 & 112.9 & 115.0 & 108.4 & 106.0 & 97.4 & 84.4 & 76.2 & 61.4 \\
\hline 117.3 & $10 \mathrm{~B} .8$ & 110.3 & 116.3 & 118.2 & 117.2 & 120.7 & 113.3 & 109.3 & 100.1 & 90.5 & B1.7 & 63.1 \\
\hline \multicolumn{13}{|c|}{ Thermocouples located at sprinkler number 2} \\
\hline Time & $0 \mathrm{~mm}$ & $25 \mathrm{~mm}$ & $50 \mathrm{~mm}$ & $75 \mathrm{~mm}$ & $100 \mathrm{~mm}$ & $125 \mathrm{~mm}$ & $150 \mathrm{~mm}$ & $250 \mathrm{~mm}$ & $350 \mathrm{~mm}$ & $450 \mathrm{~mm}$ & $550 \mathrm{~mm}$ & $900 \mathrm{~mm}$ \\
\hline 0.0 & 21.7 & 21.6 & 21.2 & 21.0 & 20.8 & 20.5 & 20.4 & 19.8 & 19.5 & 19.2 & 18.9 & 18.3 \\
\hline 1.3 & 22.0 & 21.9 & 21.8 & 21.4 & 21,3 & 20.8 & 20.5 & 20.0 & 19.7 & 19.5 & 19.3 & 18.5 \\
\hline 8.2 & 22.1 & 22.0 & 21.8 & 21.3 & 21.4 & 21.1 & 21.2 & 20.1 & 20.0 & 19.5 & 19.5 & 18.5 \\
\hline 15.0 & 22.6 & 23.2 & 23.7 & 23.6 & 23.9 & 24.2 & 24.4 & 24.9 & 22.6 & 21.0 & 20.1 & 18.9 \\
\hline 21.9 & 30.3 & 33.8 & 33.6 & 33.9 & 33.4 & 33.1 & 32.4 & 27.6 & 22.4 & 21.0 & 20.1 & 18.8 \\
\hline 28.7 & 37.7 & 41.7 & 42.4 & 43.5 & 4.32 & 42.1 & 40.3 & 33.1 & 22.9 & 20.8 & 20.0 & 19.1 \\
\hline 35.4 & 42.6 & 45.3 & 45.6 & 46.4 & 47.2 & 47.4 & 46.1 & 33.2 & 27.0 & 24.7 & 23.2 & 20.9 \\
\hline 42.4 & 49.7 & 53.5 & 53.4 & 52.9 & 53.3 & 52.5 & 50.0 & 43.9 & 36.4 & 29.1 & 27.8 & 21.1 \\
\hline 49.1 & 56.2 & 60.5 & 60.7 & 61.4 & 61.6 & 61.1 & 59.3 & 48.4 & 39.7 & 34.7 & 33.1 & 22.4 \\
\hline 56.0 & 61.5 & 66.6 & 67.6 & 68.8 & 69.1 & 69.1 & 68.3 & 55.1 & 41.6 & 40.4 & 37.7 & 27.0 \\
\hline 62.8 & 72.7 & 76.5 & 76.3 & 77.7 & 77.9 & 76.5 & 74.9 & 57.1 & 47.7 & 45.1 & 41.1 & 30.0 \\
\hline 69.5 & 72.6 & 78.1 & 79.4 & 79.9 & 80.9 & 80.8 & 80.3 & 70.4 & 55.9 & 51.7 & 47.7 & 34.7 \\
\hline 76.5 & 84.0 & 90.7 & 92.2 & 93.8 & 94.0 & 93.8 & 91.5 & 79.6 & 65.2 & 56.9 & 48.5 & 38.2 \\
\hline 83.2 & 91.1 & 98.8 & 99.0 & 101.3 & 101.6 & 101.3 & 98.0 & 82.5 & 66.2 & 62.4 & 58.6 & 43.1 \\
\hline 90.2 & 96.6 & 103.1 & 103.2 & 103.7 & 103.2 & 102.4 & 100.7 & 88.4 & 75.4 & 67.5 & 63.0 & 46.4 \\
\hline 96.9 & 104.2 & 112.2 & $110 . r$ & 111.0 & 111.6 & 110.1 & 107.4 & 94.7 & 83.9 & 74.7 & 69.1 & 51.6 \\
\hline 103.6 & 116.9 & 125.7 & 127.3 & 129.3 & 128.3 & 128.1 & 125.7 & 100.1 & 89.4 & 80.2 & 72.7 & 55.3 \\
\hline 110.5 & 119.6 & 124.6 & $\uparrow 25.0$ & 125.5 & 124.9 & 122.8 & 121.1 & 1065 & 92.7 & 83.1 & 74.5 & 60.0 \\
\hline 117.3 & 122.8 & 129.5 & 131.5 & 132.6 & 133.0 & 132.5 & 129.4 & 113.0 & 100.9 & 91.0 & 77.1 & 64.3 \\
\hline \multicolumn{13}{|c|}{ Thermocouples localed at sprinkler number 3} \\
\hline Time & $0 \mathrm{~mm}$ & $25 \mathrm{~mm}$ & $50 \mathrm{~mm}$ & $75 \mathrm{~mm}$ & $100 \mathrm{~mm}$ & $125 \mathrm{~mm}$ & $150 \mathrm{~mm}$ & $250 \mathrm{~mm}$ & $350 \mathrm{~mm}$ & $450 \mathrm{~mm}$ & $550 \mathrm{~mm}$ & $900 \mathrm{~mm}$ \\
\hline 0.0 & 21.6 & 21.6 & 21.3 & 21.0 & 20.8 & 20.6 & 20.5 & 19.8 & 19.5 & 19.4 & 19.0 & 18.4 \\
\hline 1.3 & 21.0 & 20.1 & 20.9 & 21.1 & 21.2 & 21.2 & 21.0 & 20.0 & 19.6 & 19.7 & 18.6 & 18.6 \\
\hline B. 2 & 21.7 & 22.3 & 21.9 & 21.4 & 20.7 & 20.5 & 20.7 & 20.1 & 20.3 & 19.8 & 19.5 & 18.2 \\
\hline 15.0 & 21.9 & 22.4 & 21.8 & 21.2 & 21.1 & 21.2 & 21.5 & 20.6 & 20.6 & 20.1 & 19.2 & 18.4 \\
\hline 21.9 & 22.6 & 23.7 & 23.5 & 23.8 & 23.1 & 23.6 & 23.9 & 23.2 & 23.8 & 22.7 & 23.2 & 19.2 \\
\hline 28.7 & 25.7 & 27.2 & 26.2 & 27.7 & 27.1 & 27.9 & 27.7 & 27.4 & 27.9 & 24.2 & 21.7 & 19.6 \\
\hline 35.4 & 27.0 & 25.8 & 28.6 & 30.0 & 30.4 & 30.3 & 29.9 & 32.6 & 32.5 & 29.7 & 28.3 & 20.2 \\
\hline 42.4 & 30.4 & 29.0 & 32.6 & 34.7 & 35.0 & 36.0 & 35.5 & 35.0 & 36.0 & 35.1 & 29.3 & 21.6 \\
\hline 49.1 & 36.7 & 41.5 & 39.3 & 41.6 & 40.0 & 41.2 & 40.7 & 41.3 & 43.6 & 41.1 & 40.3 & 22.7 \\
\hline 56.0 & 39.4 & 43.5 & 42.5 & 43.4 & 42.5 & 43.7 & 44.4 & 44.9 & 48.3 & 46.1 & 41.9 & 24.2 \\
\hline 62.8 & 43.7 & 50.5 & 49.2 & 50.1 & 48.4 & 49.6 & 50.5 & 51.5 & 54.8 & 50.3 & 46.8 & 29.1 \\
\hline 69.5 & 51.2 & 59.8 & 55.2 & 56.9 & 55.8 & 58.2 & 59.0 & 58.8 & 62.4 & 53.8 & 46.4 & 30.8 \\
\hline 76.5 & 58.9 & 62.3 & 64.3 & 62.4 & 63.5 & 66.1 & 66.8 & 65.5 & 66.5 & 58.9 & 50.9 & 38.3 \\
\hline 83.2 & 55.9 & 65.1 & 65.0 & 65.1 & 66.3 & 68.8 & 71.1 & 71.5 & 71.7 & 64.7 & 52.9 & 42.2 \\
\hline 90.2 & 54.8 & 61.1 & 64.8 & 67.3 & 68.5 & 70.8 & 71.3 & 72.2 & 71.8 & 68.7 & 67.4 & 43.0 \\
\hline 96.9 & 56.6 & 56.3 & 69.4 & 72.2 & 72.6 & 73.9 & 75.1 & 74.8 & 77.4 & 76.4 & 75.1 & $4 B .5$ \\
\hline 103.6 & 60.9 & 66.5 & 70.9 & 72.9 & 74.3 & 78.3 & 79.6 & 80.8 & 82.6 & 79.5 & 78.5 & 53.4 \\
\hline 110.5 & 63.5 & 67.1 & 71.7 & 75.6 & 80.7 & 85.3 & 88.3 & 90.4 & 91.8 & 87.7 & 84.2 & 55.7 \\
\hline 117.3 & 77.3 & 87.7 & 85.8 & 91.1 & 88.5 & 92.6 & 92.9 & 94.3 & 96.8 & 90.7 & 86.3 & 61.8 \\
\hline
\end{tabular}




\section{Experiment Number 9}

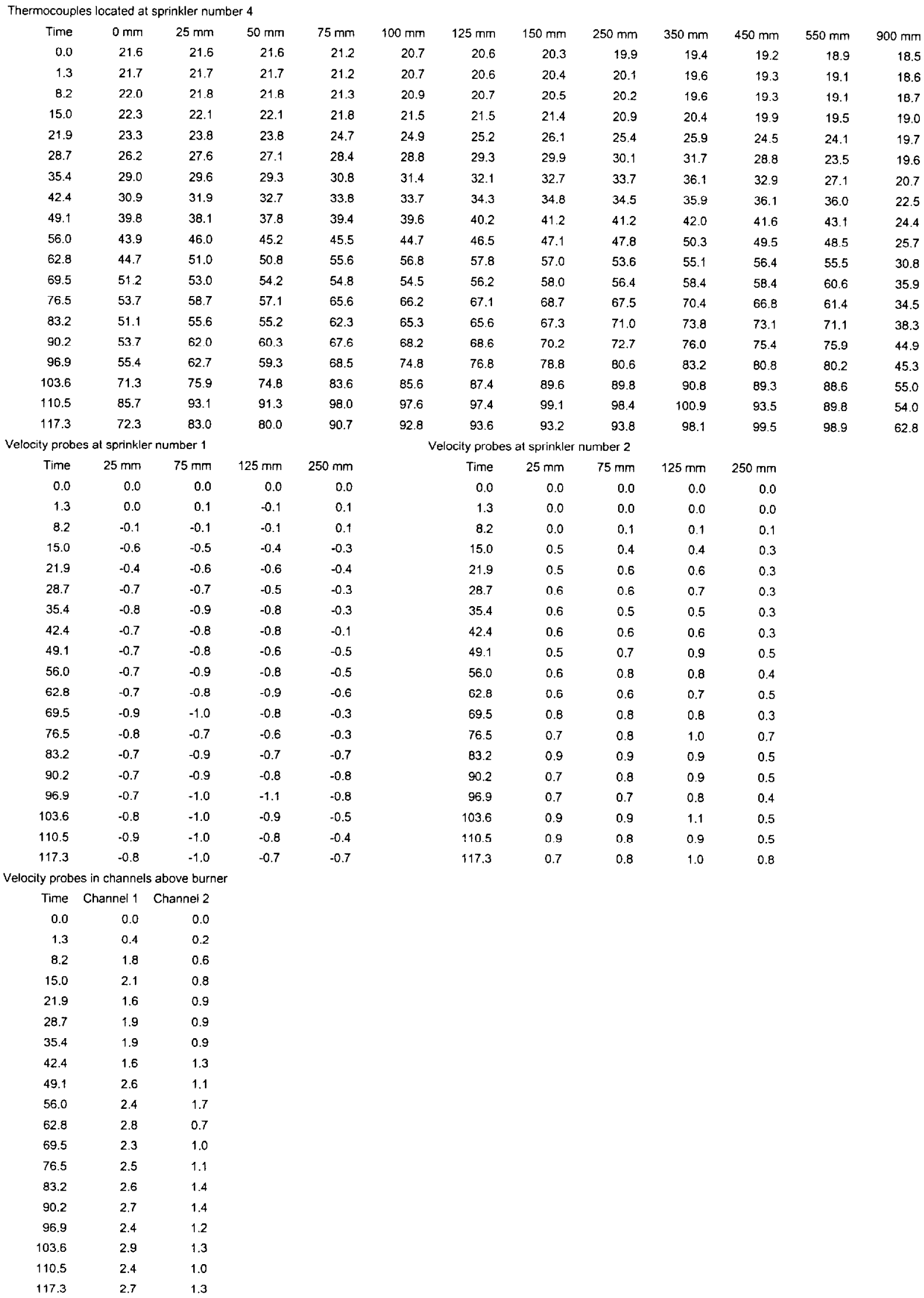


Experiment Number 10

Thermocouples located at sprinkler number 1

\begin{tabular}{|c|c|c|c|c|c|c|c|c|c|c|c|c|}
\hline Time & $0 \mathrm{~mm}$ & $25 \mathrm{~mm}$ & $50 \mathrm{~mm}$ & $75 \mathrm{~mm}$ & $100 \mathrm{~mm}$ & $125 \mathrm{~mm}$ & $150 \mathrm{~mm}$ & $250 \mathrm{~mm}$ & $350 \mathrm{~mm}$ & $450 \mathrm{~mm}$ & $550 \mathrm{~mm}$ & $900 \mathrm{~mm}$ \\
\hline 0.0 & 22.8 & 22.6 & 22.4 & 22.2 & 22.0 & 21.6 & 21.5 & 20.7 & 20.3 & 20.0 & 19.8 & 18.9 \\
\hline 6.3 & 22.9 & 22.6 & 22.4 & 22.2 & 22.0 & 21.8 & 21.7 & 20.9 & 20.8 & 20.4 & 20.2 & 19.5 \\
\hline 13.2 & 23.3 & 231 & 22.9 & 22.6 & 22.4 & 21.9 & 21.9 & 21.2 & 21.0 & 20.7 & 20.4 & 19.6 \\
\hline 19.9 & 26.5 & 26.8 & 27.8 & 27.7 & 26.5 & 26.0 & 24.8 & 25.7 & 24.9 & 22.5 & 21.5 & 19.9 \\
\hline 26.8 & 35.4 & 35.4 & 37.6 & 37.5 & 35.9 & 35.9 & 31.6 & 24.8 & 22.7 & 21.7 & 21.1 & 19.7 \\
\hline 33.6 & 40.2 & 40.8 & 43.0 & 44.5 & 43.2 & 43.5 & 40.1 & 36.0 & 22.7 & 21.8 & 21.5 & 20.1 \\
\hline 40.3 & 47.9 & 47.7 & 50.1 & 50.1 & 48.5 & 48.1 & 44.9 & 38.8 & 30.9 & 26.3 & 25.9 & 22.8 \\
\hline 47.3 & 52.3 & 53.2 & 55.8 & 56.1 & 54.5 & 55.0 & 51.0 & 42.3 & 35.5 & 32.0 & 30.7 & 23.6 \\
\hline 54.0 & 57.9 & 58.3 & 60.7 & 60.9 & 59.0 & 58.5 & 55.0 & 48.9 & 39.5 & 36.6 & 35.2 & 24.4 \\
\hline 60.9 & 59.6 & 61.2 & 64.5 & 66.1 & 65.4 & 65.8 & 62.6 & 53.9 & 45.0 & 40.3 & 37.3 & 27.6 \\
\hline 67.6 & 66.6 & 69.2 & 74.3 & 75.5 & 74.5 & 74.3 & 69.1 & 64.6 & 53.2 & 47.8 & 44.6 & 28.1 \\
\hline 74.4 & 76.1 & 77.7 & 82.0 & 82.9 & 80.2 & 79.1 & 73.0 & 63.4 & 58.2 & 53.2 & 49.5 & 38.4 \\
\hline 81.3 & 82.3 & 84.1 & 89.1 & 89.3 & 87.8 & 88.4 & 81.2 & 78.3 & 67.2 & 60.1 & 55.5 & 39.1 \\
\hline 88.0 & 89.9 & 91.4 & 96.4 & 97.1 & 95.1 & 96.4 & 90.3 & 82.1 & 71.6 & 65.0 & 56.6 & 42.0 \\
\hline 95.0 & 95.1 & 974 & 101.7 & 103.0 & 101.2 & 101.6 & 95.4 & 90.5 & 76.1 & 69.1 & 61.7 & 49.6 \\
\hline 101.7 & 107.0 & 110.2 & 115.5 & 116.6 & 112.1 & 112.9 & 104.4 & 94.9 & 78.8 & 73.6 & 68.0 & 54.8 \\
\hline 108.5 & 108.8 & 111.0 & $\uparrow 16.2$ & 117.7 & 114.5 & 115.0 & 109.3 & 101.6 & 92.5 & 81.8 & 74.6 & 54.6 \\
\hline 115.4 & 111.3 & 115.9 & 120.9 & 122.2 & 119.1 & 120.1 & 113,3 & 114.0 & 103.7 & 90.8 & 80.9 & 62.7 \\
\hline 122.2 & 114.9 & 118.5 & 124.5 & 126.5 & 126.2 & 129.8 & 121.3 & $\uparrow 16.3$ & 97.9 & 90.4 & 83.2 & 65.0 \\
\hline 120.1 & 115.7 & 119.3 & 123.5 & 126.1 & 122.7 & 124.8 & 120.4 & 117.4 & 105.8 & 94.6 & 85.4 & 70.4 \\
\hline \multicolumn{13}{|c|}{ Thermocouples located at sprinkler number 2} \\
\hline Time & $0 \mathrm{~mm}$ & $25 \mathrm{~mm}$ & $50 \mathrm{~mm}$ & $75 \mathrm{~mm}$ & $100 \mathrm{mr}$ & $125 \mathrm{~mm}$ & $150 \mathrm{~mm}$ & $250 \mathrm{~mm}$ & $350 \mathrm{~mm}$ & $450 \mathrm{~mm}$ & $550 \mathrm{~mm}$ & $900 \mathrm{~mm}$ \\
\hline 0.0 & 22.9 & 23.2 & 22.1 & 22.9 & 21.7 & 21.9 & 21.3 & 20.8 & 20.3 & 20.0 & 19.8 & 19.2 \\
\hline 6.3 & 23.2 & 23.3 & 22.5 & 22.9 & 22.1 & 22.0 & 21.7 & 21.2 & 20.7 & 20.5 & 20.3 & 19.5 \\
\hline 13.2 & 23.6 & 23.9 & 23.5 & 23.8 & 23.6 & 23.5 & 23.3 & 21.8 & 21.1 & 20.8 & 20.6 & 19.6 \\
\hline 19.9 & 27.9 & 29.9 & 29.8 & 29.7 & 29.6 & 28.7 & 28.1 & 25.7 & 23.6 & 22.1 & 21.0 & 19.6 \\
\hline 26.8 & 35.1 & 37.8 & 38.0 & 37.6 & 37.2 & 36.0 & 34.5 & 26.2 & 22.6 & 21.4 & 20.9 & 19.8 \\
\hline 33.6 & 39.8 & 42.3 & 43.3 & 43.0 & 43.5 & 43.1 & 42.1 & 33.8 & 27.4 & 21.8 & 21.6 & 20.5 \\
\hline 40.3 & 43.4 & 46.2 & 46.5 & 45.4 & 46.3 & 44.9 & 43.8 & 38.5 & 29.3 & 26.7 & 26.2 & 22.7 \\
\hline 47.3 & 50.0 & 53.9 & 54.2 & 53.2 & 52.9 & 52.0 & 50.7 & 42.2 & 34.4 & 30.7 & 29.2 & 21.7 \\
\hline 54.0 & 56.7 & 62.1 & 62.5 & 62.8 & 64.1 & 63.6 & 62.6 & 57.5 & 45.1 & 36.9 & 34.0 & 24.0 \\
\hline 60.9 & 58.3 & 61.0 & 63.5 & 62.6 & 64.3 & 65.5 & 65.9 & 63.2 & 49.7 & 43.4 & 39.5 & 29.2 \\
\hline 67.6 & 67.6 & 72.1 & 73.7 & 73.4 & 75.8 & 76.0 & 75.1 & 72.0 & 55.6 & 48.7 & 45.0 & 32.5 \\
\hline 74.4 & 75.0 & 81.2 & 83.0 & 82.6 & 85.0 & 84.9 & 83.2 & 75.0 & 63.3 & 54.3 & 49.1 & 35.5 \\
\hline 81.3 & 85.3 & 90.7 & 93.0 & 93.2 & 94.5 & 94.2 & 93.3 & 76.9 & 64.8 & 55.8 & 52.4 & 39.8 \\
\hline 88.0 & 87.8 & 90.9 & 91.5 & 91.3 & 93.4 & 94.8 & 94.7 & 86.6 & 79.6 & 68.4 & 59.9 & 44.8 \\
\hline 95.0 & 94.6 & 102.2 & 103.3 & 104.7 & 107.4 & 107.0 & 106.3 & 98.3 & 79.7 & 71.3 & 59.3 & 47.7 \\
\hline 101.7 & 103.6 & 113.9 & 117.7 & 115.9 & 118.0 & 115.8 & 114.3 & 101.2 & 89.8 & 82.2 & 70.8 & 52.3 \\
\hline 108.5 & 117.5 & 127.2 & 128.3 & 127.2 & 128.6 & 128.0 & 124.5 & 106.4 & 90.8 & 82.8 & 77.7 & 57.9 \\
\hline 115.4 & 122.0 & 129.8 & 131.0 & 130.0 & 130.7 & 128.3 & 126.1 & 109.2 & 96.9 & 89.6 & 82.0 & 62.4 \\
\hline 122.2 & 120.2 & 125.8 & 125.4 & 125.6 & 126.6 & 127.0 & 126.3 & 117.9 & 111.2 & 101.2 & 88.0 & 67.2 \\
\hline 129.1 & 132.9 & 141.7 & 142.2 & 139.9 & 142.1 & 139.2 & 137.5 & 124.6 & 105.2 & 96.8 & 84.7 & 69.5 \\
\hline \multicolumn{13}{|c|}{ Thermocouples located at sprinkler number 3} \\
\hline Time & $0 \mathrm{~mm}$ & $25 \mathrm{~mm}$ & $50 \mathrm{~mm}$ & $75 \mathrm{~mm}$ & $100 \mathrm{~mm}$ & $125 \mathrm{~mm}$ & $150 \mathrm{~mm}$ & $250 \mathrm{~mm}$ & $350 \mathrm{~mm}$ & $450 \mathrm{~mm}$ & $550 \mathrm{~mm}$ & $900 \mathrm{~mm}$ \\
\hline 0.0 & 22.9 & 22.8 & 22.4 & 22.1 & 21.8 & 21.5 & 21.3 & 20.8 & 20.4 & 20.2 & 20.0 & 19.4 \\
\hline 6.3 & 22.8 & 22.9 & 22.9 & 22.7 & 22.5 & 22.0 & 21.5 & 21.0 & 20.8 & 20.5 & 20.1 & 19.5 \\
\hline 13.2 & 23.0 & 22.9 & 22.9 & 22.6 & 22.4 & 22.0 & 21.7 & 21.1 & 21.0 & 20.7 & 20.3 & 19.7 \\
\hline 19.9 & 23.1 & 23.1 & 23.1 & 23.0 & 22.9 & 22.8 & 22.7 & 22.6 & 22.5 & 21.9 & 22.1 & 20.1 \\
\hline 26.8 & 24.7 & 26.1 & 25.1 & 26.4 & 26.0 & 26.9 & 26.5 & 26.2 & 26.4 & 25.6 & 25.6 & 20.1 \\
\hline 33.6 & 26.9 & 28.9 & 28.9 & 29.8 & 29.7 & 29.6 & 29.5 & 30.4 & 30.9 & 29.2 & 26.6 & 20.1 \\
\hline 40.3 & 31.5 & 34.0 & 33.9 & 34.1 & 34.5 & 34.2 & 34.8 & 33.6 & 33.9 & 33.5 & 32.5 & 21.8 \\
\hline 47.3 & 30.0 & 31.3 & 33.2 & 34.2 & 34.6 & 36.1 & 36.9 & 38.6 & 40.8 & 34.6 & 34.1 & 23.1 \\
\hline 54.0 & 42.7 & 46.6 & 45.6 & 46.3 & 44.8 & 45.5 & 47.0 & 44.9 & 44.9 & 39.0 & 33.6 & 23.0 \\
\hline 60.9 & 41.2 & 47.6 & 46.3 & 47.5 & 47.1 & 50.4 & 51.2 & 51.5 & 51.7 & 39.9 & 35.4 & 29.7 \\
\hline 67.6 & 46.6 & 52.6 & 51.5 & 55.9 & 55.1 & 57.5 & 56.9 & 56.6 & 59.0 & 52.6 & 51.4 & 33.2 \\
\hline 74.4 & 48.5 & 57.2 & 55.7 & 56.6 & 58.1 & 60.6 & 61.1 & 61.2 & 62.6 & 58.8 & 18.0 & 34.7 \\
\hline 81.3 & 53.3 & 60.2 & 63.5 & 64.5 & 64.1 & 65.9 & 68.0 & 67.3 & 69.0 & 63.2 & 54.2 & 39.5 \\
\hline 88.0 & 50.5 & 54.4 & 59.3 & 60.5 & 61.8 & 65.3 & 66.1 & 68.4 & 73.1 & 68.8 & 65.5 & 42.8 \\
\hline 95.0 & 61.6 & 72.2 & 70.4 & 75.1 & 72.7 & 76.1 & 77.9 & 77.8 & 79.7 & 71.8 & 65.4 & 48.4 \\
\hline 101.7 & 64.9 & 75.7 & 76.5 & 79.8 & 78.3 & 80.2 & 81.2 & 84.5 & 89.2 & 80.1 & 65.2 & 50.4 \\
\hline 108.5 & 75.7 & 91.1 & 85.1 & 88.2 & 86.7 & 88.4 & 88.9 & 91.0 & 90.0 & 77.4 & 70.4 & 56.8 \\
\hline
\end{tabular}


Experiment Number 10

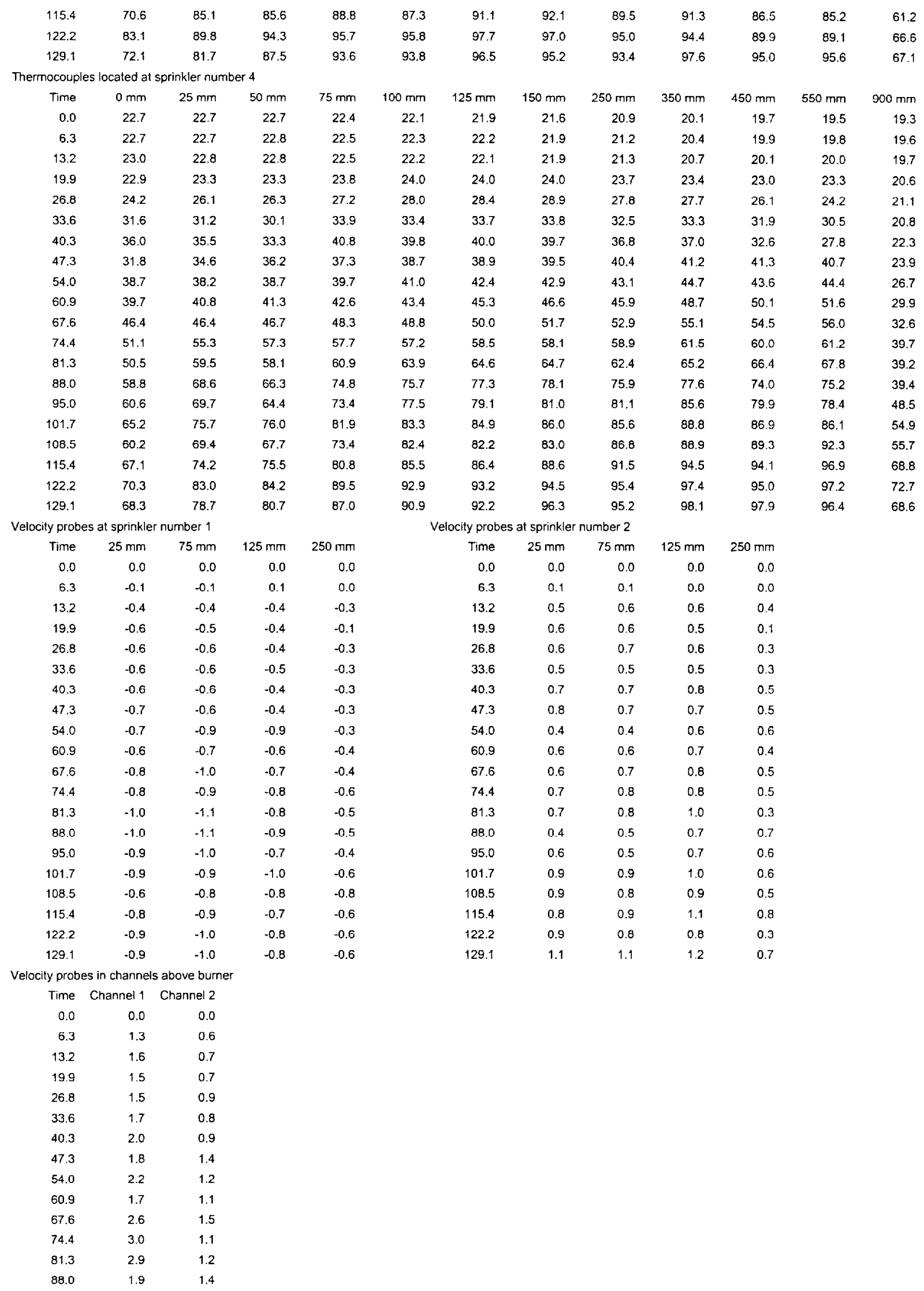


Experiment Number 10

$\begin{array}{rll}95.0 & 2.9 & 1.7 \\ 101.7 & 3.3 & 1.7 \\ 108.5 & 3.2 & 1.4 \\ 115.4 & 3.0 & 1.7 \\ 122.2 & 2.8 & 1.4 \\ 129.1 & 3.0 & 1.5\end{array}$


Experiment Number 11

Thermocouples located at sprinkler number 1

\begin{tabular}{|c|c|c|c|c|c|c|c|c|c|c|c|c|}
\hline Time & $0 \mathrm{~mm}$ & $25 \mathrm{~mm}$ & $50 \mathrm{~mm}$ & $75 \mathrm{~mm}$ & $100 \mathrm{~mm}$ & $125 \mathrm{~mm}$ & $150 \mathrm{~mm}$ & $250 \mathrm{~mm}$ & $350 \mathrm{~mm}$ & $450 \mathrm{~mm}$ & $550 \mathrm{~mm}$ & $900 \mathrm{~mm}$ \\
\hline 0.0 & 18.2 & 17.8 & 17.6 & 17.6 & 17.5 & 17.4 & 17.4 & 17.2 & 17.0 & 16.8 & 16.7 & 16.3 \\
\hline 5.3 & 18.3 & 17.8 & 17.7 & 17.7 & 17.7 & 17.6 & 17.6 & 17.5 & 17.4 & 17.3 & 17.0 & 16.7 \\
\hline 12.2 & 20.5 & 31.1 & 38.6 & 37.3 & 35.3 & 35.7 & 31.5 & 27.9 & 23.0 & 19.2 & 17.5 & 16.8 \\
\hline 19.0 & 24.8 & 40.8 & 46.0 & 46.3 & 43.2 & 43.7 & 40.5 & 35.3 & 25.2 & 19.2 & 17.6 & 16.9 \\
\hline 25.9 & 27.4 & 48.6 & 55.6 & 56.1 & 53.4 & 53.4 & 48.4 & 41.7 & 36.9 & 30.5 & 24.1 & 19.3 \\
\hline 32.7 & 28.8 & 51.4 & 55.9 & 56.2 & 54.3 & 54.9 & 53.1 & 49.4 & 43.5 & 39.6 & 28.5 & 18.0 \\
\hline 39.5 & 31.3 & 57.0 & 65.5 & 65.2 & 63.2 & 62.0 & 61.5 & 52.5 & 49.4 & 48.1 & 38.8 & 18.8 \\
\hline 46.4 & 34.0 & 62.6 & 69.8 & 70.8 & 70.8 & 71.4 & 69.4 & 66.3 & 59.1 & 53.5 & 41.6 & 21.9 \\
\hline 53.2 & 36.2 & 68.3 & 75.6 & 74.9 & 72.9 & 73.2 & 73.2 & 71.2 & 68.3 & 60.5 & 50.1 & 24.6 \\
\hline 59.9 & 37.9 & 70.4 & 77.4 & 77.4 & 76.1 & 77.1 & 76.1 & 75.0 & 73.0 & 68.5 & 59.4 & 31.4 \\
\hline 66.9 & 41.2 & 79.4 & 88.3 & 87.0 & 85.2 & 85.2 & 84.7 & 81.3 & 78.5 & 71.5 & 56.9 & 33.7 \\
\hline 73.7 & 42.9 & 84.2 & 95.7 & 94.9 & 91.0 & 91.2 & 88.8 & 83.5 & 82.1 & 77.4 & 64.8 & 36.8 \\
\hline B0.6 & 45.9 & 90.8 & 102.3 & 101.8 & 98.1 & 99.4 & 96.5 & 91.3 & 90.2 & 81.0 & 68.3 & 36.9 \\
\hline 87.4 & 50.7 & 98.3 & 110.5 & 108.5 & 105.7 & 105.3 & 104.5 & 94.5 & 89.0 & 83.9 & 73.9 & 41.6 \\
\hline 94.2 & 51.8 & 101.4 & 116.1 & 119.2 & 119.4 & 118.0 & 112.8 & 109.9 & 100.7 & 92.2 & 78.0 & 43.0 \\
\hline 101.1 & 55.0 & 109.7 & 120.9 & 120.8 & 117.3 & 118.5 & 115.5 & 108.8 & 101.7 & 91.4 & 82.5 & 47.7 \\
\hline 107.8 & 53.0 & 62.5 & 114.1 & 102.6 & 86.2 & 103.0 & 90.4 & 102.4 & 100.3 & 99.0 & 84.5 & 51.4 \\
\hline \multicolumn{13}{|c|}{ ermocouples located at sprinkler number 2} \\
\hline Time & $0 \mathrm{~mm}$ & $25 \mathrm{~mm}$ & $50 \mathrm{~mm}$ & $75 \mathrm{~mm}$ & $100 \mathrm{~mm}$ & $125 \mathrm{~mm}$ & $150 \mathrm{~mm}$ & $250 \mathrm{~mm}$ & $350 \mathrm{~mm}$ & $450 \mathrm{~mm}$ & $550 \mathrm{~mm}$ & $900 \mathrm{~mm}$ \\
\hline 0.0 & 18.1 & 17.6 & 17.3 & 17.2 & 17.2 & 17.2 & 17.1 & 17.0 & 16.9 & 16.8 & 16.7 & 16.4 \\
\hline 5.3 & 18.2 & 17.7 & 17.5 & 17.6 & 18.4 & 18.4 & 18.9 & 20.8 & 19.1 & 18,0 & 17.1 & 16.9 \\
\hline 12.2 & 22.3 & 37.1 & 38.2 & 38.2 & 38.5 & 38.1 & 36.7 & 30.9 & 25.7 & 18.5 & 17.2 & 17.0 \\
\hline 19.0 & 25.1 & 45.2 & 45.5 & 44.9 & 44.9 & 43.3 & 42.1 & 34.2 & 24.6 & 20.5 & 18.3 & 17.1 \\
\hline 25.9 & 26.9 & 47.5 & 48.9 & 49.0 & 49.3 & 48.5 & 47.4 & 41.6 & 35.2 & 28.6 & 22.7 & 18.0 \\
\hline 32.7 & 27.5 & 51.2 & 53.9 & 53.5 & 55.5 & 54.5 & 55.6 & 52.3 & 45.1 & 40.7 & 28.7 & 17.7 \\
\hline 39.5 & 31.1 & 59.1 & 62.1 & 60.7 & 62.6 & 59.8 & 61.0 & 55.0 & 53.4 & 48.5 & 40.8 & 21.3 \\
\hline 46.4 & 32.4 & 66.7 & 70.8 & 69.8 & 72.1 & 71.4 & 71.4 & 66.2 & 58.8 & 55.9 & 47.5 & 25.3 \\
\hline 53.2 & 36.1 & 76.1 & 80.3 & 80.8 & 82.7 & 80.3 & 80.9 & 77.5 & 69.2 & 61.3 & 42.1 & 31.2 \\
\hline 59.9 & 38.3 & 78.1 & 85.6 & 82.1 & 86.6 & 82.0 & 85.3 & 80.6 & 77.1 & 69.2 & 48.2 & 32.6 \\
\hline 66.9 & 41.4 & 89.9 & 92.7 & 93.1 & 94.7 & 93.9 & 94.3 & 90.0 & 82.8 & 74.7 & 53.8 & 36.0 \\
\hline 73.7 & 42.4 & 94.1 & 99.2 & 98.9 & 102.4 & 97.3 & 101.1 & 99.0 & 91.8 & 84.5 & 69.0 & 44.8 \\
\hline 80.6 & 45.7 & 95.5 & 102.5 & 102.3 & 104.9 & 103.0 & 103.9 & 98.1 & 94.8 & 84.5 & 67.8 & 49.5 \\
\hline 87.4 & 46.7 & 101.3 & 107.7 & 107.1 & 111.6 & 110.3 & 110.6 & 104.9 & 96.5 & 90.3 & 76.1 & 51.6 \\
\hline 94.2 & 49.6 & 107.4 & 111.9 & 112.7 & 115.0 & 113.2 & 112.8 & 108.6 & 102.1 & 92.3 & 78.6 & 59.1 \\
\hline 101.1 & 53.9 & 119.2 & 123.6 & 121.5 & 123.9 & 123.1 & 121.3 & 113.6 & 108.4 & 92.2 & 75.7 & 62.4 \\
\hline 107.8 & 54.5 & 112.1 & 119.3 & 115.0 & 127.0 & 123.7 & 123.6 & 115.4 & 105.5 & 96.2 & 82.2 & 63.8 \\
\hline \multicolumn{13}{|c|}{ ermocouples located at sprinkler number 3} \\
\hline Time & $0 \mathrm{~mm}$ & $25 \mathrm{~mm}$ & $50 \mathrm{~mm}$ & $75 \mathrm{~mm}$ & $100 \mathrm{~mm}$ & $125 \mathrm{~mm}$ & $150 \mathrm{~mm}$ & $250 \mathrm{~mm}$ & $350 \mathrm{~mm}$ & $450 \mathrm{~mm}$ & $550 \mathrm{~mm}$ & $900 \mathrm{~m} m$ \\
\hline 0.0 & 17.4 & 17.3 & 17.2 & 17.2 & 17.1 & 17.0 & 17.0 & 16.9 & 16.7 & 16.6 & 16.6 & 16.5 \\
\hline 5.3 & 17.4 & 17.4 & 17.5 & 17.5 & 17.5 & 17.4 & 17.4 & 17.2 & 17.1 & 17.0 & 16.9 & 16.7 \\
\hline 12.2 & 17.5 & 18.6 & 20.0 & 20.5 & 19.5 & 20.1 & 19.3 & 19.1 & 18.2 & 17.2 & 17.0 & 15.7 \\
\hline 19.0 & 17.9 & 24.1 & 27.3 & 26.8 & 25.3 & 24.4 & 23.1 & 19.6 & 18.3 & 17.5 & 17.3 & 16.9 \\
\hline 25.9 & 18.5 & 28.6 & 32.2 & 31.9 & 31.1 & 31.3 & 30.3 & 28.1 & 23.6 & 21.6 & 20.9 & 19.2 \\
\hline 32.7 & 19.3 & 37.0 & 44.4 & 45.3 & 43.4 & 44.1 & 41.8 & 38.0 & 33.1 & 28.0 & 28.2 & 19.5 \\
\hline 39.5 & 19.9 & 40.0 & 43.8 & 42.1 & 40.2 & 39.1 & 37.4 & 34.4 & 32.8 & 31.2 & 29.9 & 23.4 \\
\hline 46.4 & 20.3 & 40.2 & 49.2 & 49.6 & 48.2 & 48.3 & 46.5 & 39.8 & 36.6 & 35.8 & 34.3 & 27.3 \\
\hline 53.2 & 21.4 & 44.4 & 56.1 & 55.9 & 53.3 & 53.3 & 52.0 & 46.5 & 40.5 & 39.4 & 39.4 & 28.9 \\
\hline 59.9 & 22.3 & 48.7 & 60.3 & 58.9 & 56.8 & 56.9 & 54.5 & 49.2 & 47.1 & 43.9 & 42.9 & 36.2 \\
\hline 66.9 & 23.1 & 49.3 & 60.4 & 61.2 & 60.0 & 60.7 & 59.2 & 51.7 & 50.2 & 41.0 & 46.2 & 37.7 \\
\hline 73.7 & 24.4 & 52.2 & 66.6 & 66.4 & 64.7 & 64.1 & 61.7 & 55.6 & 53.9 & 50.7 & 49.7 & 42.0 \\
\hline 80.6 & 25.5 & 55.6 & 72.4 & 72.4 & 70.2 & 70.6 & 68.6 & 624 & 61.6 & 58.1 & 55.7 & 45.9 \\
\hline 87.4 & 26.4 & 65.9 & 79.2 & 79.1 & 77.3 & 77.5 & 74.6 & 65.2 & 60.7 & 60.0 & 59.0 & 49.4 \\
\hline 94.2 & 26.5 & 64.1 & 80.2 & 81.0 & 79.5 & 79.7 & 76.3 & 68.0 & 63.7 & 64.1 & 64.5 & 55.3 \\
\hline 101.1 & 29.0 & 68.7 & 80.7 & 79.7 & 78.8 & 79.3 & 76.5 & 69.4 & 68.7 & 67.9 & 68.1 & 61.2 \\
\hline 107.8 & 28.5 & 71.2 & 90.0 & 89.2 & 87.5 & 87.6 & 85.8 & 80.2 & 77.8 & 73.1 & 73.0 & 62.1 \\
\hline \multicolumn{13}{|c|}{ ermocouples located at sprinkler number 4} \\
\hline Time & $0 \mathrm{~mm}$ & $25 \mathrm{~mm}$ & $50 \mathrm{~mm}$ & $75 \mathrm{~mm}$ & $100 \mathrm{~mm}$ & $125 \mathrm{~mm}$ & $150 \mathrm{~mm}$ & $250 \mathrm{~mm}$ & $350 \mathrm{~mm}$ & $450 \mathrm{~mm}$ & $550 \mathrm{~mm}$ & $900 \mathrm{~mm}$ \\
\hline 0.0 & 17.4 & 17.3 & 17.3 & 17.3 & 17.2 & 17.1 & 17.1 & 16.9 & 16.8 & 16.7 & 16.6 & 16.4 \\
\hline 5.3 & 17.5 & 17.5 & 17.6 & 17.6 & 17.4 & 17.4 & 17.3 & 17.2 & 17.0 & 16.9 & 16.7 & 16.6 \\
\hline 12.2 & 18.3 & 21.6 & 24.1 & 23.9 & 22.8 & 23.1 & 22.9 & 21.4 & 19.0 & 17.2 & 17.0 & 16.9 \\
\hline 19.0 & 18.8 & 26.4 & 27.1 & 26.9 & 25.7 & 26.1 & 25.1 & 19.0 & 17.6 & 17.1 & 17.1 & 17.1 \\
\hline
\end{tabular}




\section{Experiment Number 11}

\begin{tabular}{|c|c|c|c|c|c|c|c|c|c|c|c|c|}
\hline 25.9 & 20.2 & 33.5 & 35.6 & 35.6 & 34.8 & 36.4 & 36.8 & 32.6 & 29.1 & 25.3 & 24.8 & 20.3 \\
\hline 32.7 & 21.9 & 40.0 & 42.8 & 42.7 & 42.1 & 43.8 & 43.9 & 39.7 & 38.8 & 32.1 & 30.7 & 19.7 \\
\hline 39.5 & 24.9 & 44.0 & 47.7 & 47.2 & 46.1 & 46.7 & 46.5 & 42.7 & 40.8 & 36.1 & 34.6 & 19.6 \\
\hline 46.4 & 25.9 & 50.6 & 52.3 & 51.4 & 49.8 & 50.7 & 49.9 & 44.9 & 42.2 & 39.9 & 38.6 & 21.4 \\
\hline 53.2 & 29.7 & 54.6 & 56.5 & 56.5 & 55.9 & 56.7 & 56.7 & 51.6 & 48.5 & 43.2 & 45.0 & 27.4 \\
\hline 59.9 & 29.3 & 57.7 & 61.4 & 62.2 & 61.6 & 63.0 & 63.1 & 55.5 & 54.3 & 52.1 & 51.0 & 30.8 \\
\hline 66.9 & 33.2 & 63.4 & 65.5 & 65.6 & 64.2 & 64.5 & 63.7 & 58.5 & 56.7 & 53.9 & 52.6 & 34.0 \\
\hline 73.7 & 37.1 & 68.0 & 68.0 & 68.6 & 67.7 & 68.1 & 67.7 & 61.9 & 59.9 & 59.0 & 56.7 & 36.6 \\
\hline 80.6 & 38.2 & 75.6 & 77.3 & 77.8 & 76.2 & 77.8 & 78.6 & 74.6 & 72.2 & 64.1 & 63.0 & 39.6 \\
\hline 87.4 & 44.3 & 84.8 & 84.1 & 82.7 & 78.1 & 79.0 & 78.7 & 72.5 & 70.4 & 66.8 & 64.9 & 41.8 \\
\hline 94.2 & 38.8 & 79.9 & 84.2 & 84.9 & 84.1 & 86.0 & 86.2 & 78.9 & 76.6 & 72.6 & 70.8 & 45.0 \\
\hline 101.1 & 43.8 & 93.7 & 95.4 & 95.0 & 92.2 & 92.2 & 90.6 & 80.5 & 80.0 & 76.4 & 74.7 & 48.9 \\
\hline 107.8 & 47.4 & 97.0 & 98.6 & 98.6 & 96.7 & 98.2 & 97.5 & 86.3 & 83.6 & 80.0 & 80.1 & 52.4 \\
\hline \multicolumn{5}{|c|}{ Velocity probes at sprinkler number 1} & \multicolumn{6}{|c|}{ Velocity probes at sprinkler number 2} & & \\
\hline Time & $25 \mathrm{~mm}$ & $75 \mathrm{~mm}$ & $125 \mathrm{~mm}$ & $250 \mathrm{~mm}$ & & Time & $25 \mathrm{~mm}$ & $75 \mathrm{~mm}$ & $125 \mathrm{~mm}$ & $250 \mathrm{~mm}$ & & \\
\hline 0.0 & 0.0 & 0.0 & 0.0 & 0.0 & & 0.0 & 0.0 & 0.0 & 0.0 & 0.0 & & \\
\hline 5.3 & -0.9 & 0.9 & -0.9 & -0.3 & & 5.3 & 1.0 & 0.8 & 0.7 & 0.2 & & \\
\hline 12.2 & -0.9 & -0.9 & -0.6 & -0.2 & & 12.2 & 0.8 & 0.9 & 0.9 & 0.3 & & \\
\hline 19.0 & -1.1 & -1.0 & -0.4 & -0.2 & & 19.0 & 1.2 & 0.9 & 0.6 & 0.1 & & \\
\hline 25.9 & -0.7 & -0.6 & -0.5 & -0.3 & & 25.9 & 0.9 & 0.9 & 0.9 & 0.7 & & \\
\hline 32.7 & -1.0 & -1.0 & -0.9 & -0.3 & & 32.7 & 1.1 & 1.0 & 0.9 & 0.7 & & \\
\hline 39.5 & -1.4 & -1.3 & -0.7 & -0.6 & & 39.5 & 0.9 & 0.9 & 0.9 & 0.4 & & \\
\hline 46.4 & -0.8 & -0.9 & -1.1 & -0.4 & & 46.4 & 1.1 & 1.1 & 0.8 & -0.2 & & \\
\hline 53.2 & -0.9 & -0.9 & -0.6 & -0.2 & & 53.2 & 1.2 & 1.2 & 0.8 & 0.4 & & \\
\hline 59.9 & -1.5 & -1.2 & -0.8 & -0.5 & & 59.9 & 0.9 & 0.8 & 0.9 & 0.3 & & \\
\hline 66.9 & -1.2 & -1.2 & -1.3 & -0.4 & & 66.9 & 1.2 & 1.1 & 0.8 & 0.4 & & \\
\hline 73.7 & -1.0 & -1.1 & -0.8 & -0.6 & & 73.7 & 1.2 & 1.1 & 0.9 & 0.3 & & \\
\hline 80.6 & -1.4 & -1.5 & -1.5 & -0.8 & & 80.6 & 1.1 & 1.4 & 1.4 & 0.5 & & \\
\hline 87.4 & -1.1 & -1.1 & -0.9 & -0.9 & & 87.4 & 1.5 & 1.3 & 1.2 & 0.7 & & \\
\hline 94.2 & -1.0 & -1.0 & -0.8 & -0.5 & & 94.2 & 1.3 & 1.5 & 1.5 & 0.8 & & \\
\hline 101.1 & -1.6 & -1.6 & -1.1 & -0.5 & & 101.1 & 1.9 & 2.0 & 1.6 & 0.6 & & \\
\hline 107.8 & -1.4 & -1.4 & -1.2 & -0.8 & & 107.8 & 1.2 & 1.2 & 1.0 & 0.6 & & \\
\hline
\end{tabular}


Experiment Number 12

Thermocouples located at sprinkler number 1

\begin{tabular}{|c|c|c|c|c|c|c|c|c|c|c|c|c|}
\hline Time & $0 \mathrm{~mm}$ & $25 \mathrm{~mm}$ & $50 \mathrm{~mm}$ & $75 \mathrm{~mm}$ & $100 \mathrm{~mm}$ & $125 \mathrm{~mm}$ & $150 \mathrm{~mm}$ & $250 \mathrm{~mm}$ & $350 \mathrm{~mm}$ & $450 \mathrm{~mm}$ & $550 \mathrm{~mm}$ & $900 \mathrm{~mm}$ \\
\hline 0.0 & 21.0 & 20.6 & 20.7 & 20.6 & 20.4 & 20.4 & 20.5 & 20.3 & 20.2 & 20.3 & 20.1 & 19.8 \\
\hline 1.2 & 21.7 & 20.9 & 21.6 & 20.9 & 20.7 & 20.7 & 20.9 & 20.5 & 20.3 & 20.7 & 20.1 & 20.0 \\
\hline 8.1 & 21.8 & 22.2 & 25.2 & 25.2 & 24.6 & 25.0 & 23.9 & 23.8 & 22.3 & 22.0 & 20.3 & 20.0 \\
\hline 14.9 & 23.3 & 30.0 & 35.1 & 35.0 & 32.1 & 33.4 & 30.8 & 28.6 & 24.0 & 21.4 & 20.4 & 20.0 \\
\hline 21.9 & 25.4 & 37.1 & 43.1 & 42.5 & 40.6 & 40.4 & 37.5 & 34.4 & 28.2 & 21.3 & 20.6 & 20.1 \\
\hline 28.6 & 28.0 & 44.2 & 48.9 & 47.8 & 45.9 & 45.8 & 44.1 & 40.2 & 33.8 & 27.8 & 25.3 & 21.8 \\
\hline 35.4 & 29.0 & 47.5 & 54.0 & 53.0 & 50.4 & 50.1 & 48.1 & 42.8 & 38.2 & 33.4 & 26.4 & 20.7 \\
\hline 42.3 & 30.6 & 48.7 & 55.6 & 53.7 & 54.1 & 53.7 & 53.2 & 51.6 & 47.8 & 44.5 & 38.4 & 22.8 \\
\hline 49.1 & 31.2 & 51.5 & 58.9 & 57.1 & 56.7 & 55.9 & 55.3 & 53.4 & 51.5 & 48.0 & 41.2 & 23.0 \\
\hline 56.1 & 33.9 & 59.1 & 66.7 & 67.1 & 65.2 & 66.7 & 64.7 & 64.3 & 60.3 & 52.7 & 38.4 & 23.9 \\
\hline 62.9 & 35.3 & 62.2 & 69.6 & 67.9 & 67.3 & 66.8 & 66.1 & 63.5 & 59.4 & 54.8 & 42.4 & 28.4 \\
\hline 69.7 & 37.3 & 68.0 & 76.6 & 76.0 & 73.2 & 73.7 & 72.2 & 69.7 & 66.4 & 63.6 & 49.5 & 32.3 \\
\hline 76.6 & 39.0 & 69.3 & 79.5 & 78.5 & 77.7 & 77.8 & 76.4 & 74.2 & 70.5 & 64.1 & 49.8 & 34.2 \\
\hline 83.4 & 40.7 & 74.4 & 86.1 & 85.0 & 83.0 & 81.7 & 78.9 & 76.8 & 73.2 & 71.6 & 63.0 & 38.5 \\
\hline 90.1 & 42.8 & 78.7 & 87.9 & 86.8 & 87.6 & 87.2 & 84.5 & 79.0 & 77.5 & 69.9 & 57.4 & 39.9 \\
\hline 97.0 & 45.6 & 87.8 & 101.3 & 100.0 & 97.2 & 97.1 & 94.9 & 88.4 & 80.0 & 75.2 & 58.4 & 40.0 \\
\hline 103.9 & 48.7 & 94.1 & 104.2 & 101.9 & 100.4 & 100.1 & 97.9 & 91.9 & 85.6 & 77.7 & 64.3 & 41.7 \\
\hline 110.7 & 51.5 & 99.7 & 113.1 & 109.3 & 108.0 & 106.8 & 103.3 & 96.4 & 85.9 & B0.6 & 68.7 & 43.9 \\
\hline 117.5 & 53.5 & 100.0 & 110.7 & 108.1 & 109.8 & 109.1 & 108.4 & 104.7 & 96.0 & 92.4 & 78.5 & 44.6 \\
\hline 124.3 & 56.7 & 94.1 & 105.4 & 106.0 & 75.0 & 106.4 & 92.4 & 93.8 & 96.2 & 89.5 & 69.8 & 47.6 \\
\hline 131.2 & 57.2 & 100.1 & 119.0 & 120.5 & 111.5 & 123.4 & 109.0 & 114.9 & 105.7 & 99.7 & 80.1 & 53.3 \\
\hline 138.1 & 62.1 & 107.6 & 124.5 & 126.9 & 117.9 & 124.7 & 115.7 & 120.6 & 110.9 & 99.5 & 82.3 & 57.4 \\
\hline \multicolumn{13}{|c|}{ mocouples located at sprinkler number 2} \\
\hline Time & $0 \mathrm{~mm}$ & $25 \mathrm{~mm}$ & $50 \mathrm{~mm}$ & $75 \mathrm{~mm}$ & $100 \mathrm{~mm}$ & $125 \mathrm{~mm}$ & $150 \mathrm{~mm}$ & $250 \mathrm{~mm}$ & $350 \mathrm{~mm}$ & $450 \mathrm{~mm}$ & $550 \mathrm{~mm}$ & $900 \mathrm{~mm}$ \\
\hline 0.0 & 20.6 & 20.6 & 20.6 & 20.5 & 20.5 & 20.4 & 20.4 & 20.3 & 20.3 & 20.2 & 20.1 & 19.9 \\
\hline 1.2 & 20.8 & 20.5 & 20.6 & 20.6 & 20.6 & 20.5 & 20.5 & 20.4 & 20.4 & 20.3 & 20.2 & 19.9 \\
\hline 8.1 & 21.2 & 25.7 & 26.4 & 26.2 & 26.4 & 25.0 & 23.6 & 24.0 & 23.0 & 20.8 & 20.4 & 200 \\
\hline 14.9 & 23.8 & 39.1 & 39.5 & 39.4 & 39.9 & 39.8 & 38.3 & 32.9 & 28.5 & 23.4 & 20.7 & 20.2 \\
\hline 21.9 & 25.6 & 41.4 & 42.7 & 42.2 & 42.8 & 41.7 & 40.9 & 38.5 & 33.1 & 23.6 & 20.7 & 20.2 \\
\hline 28.6 & 26.7 & 45.0 & 45.8 & 45.1 & 45.9 & 44.9 & 45.3 & 42.7 & 37.5 & 31.3 & 27.0 & 20.6 \\
\hline 35.4 & 29.4 & 54.7 & 56.9 & 55.8 & 57.2 & 57.4 & 56.5 & 49.5 & 41.0 & 34.7 & 26.7 & 20.6 \\
\hline 42.3 & 30.5 & 53.9 & 57.0 & 54.8 & 58.4 & 57.2 & 58.5 & 56.2 & 49.0 & 44.0 & 39.0 & 20.8 \\
\hline 49.1 & 31.8 & 58.6 & 61.6 & 59.3 & 61.2 & 59.6 & 61.2 & 56.5 & 50.2 & 45.2 & 41.3 & 26.0 \\
\hline 56.1 & 34.3 & 65.7 & 67.9 & 66.1 & 67.6 & 65.8 & 67.7 & 64.2 & 57.2 & 48.7 & 42.0 & 26.8 \\
\hline 62.9 & 35.0 & 64.6 & 69.1 & 66.8 & 70.1 & 67.4 & 70.7 & 63.9 & 60.3 & 51.9 & 42.6 & 32.9 \\
\hline 69.7 & 37.0 & 72.8 & 76.5 & 74.5 & 76.9 & 74.6 & 76.8 & 71.9 & 67.4 & 53.6 & 41.1 & 35.0 \\
\hline 76.6 & 38.2 & 75.7 & 80.0 & 76.6 & 81.9 & 78.9 & 81.5 & 78.0 & 74.6 & 68.6 & 54.0 & 35.4 \\
\hline 83.4 & 40.0 & 80.9 & 84.0 & 82.0 & 84.3 & 82.5 & 83.8 & 81.0 & 77.6 & 72.8 & 58.0 & 41.2 \\
\hline 90.1 & 43.0 & 93.8 & 98.3 & 96.3 & 98.8 & 94.5 & 97.4 & 89.7 & 81.4 & 71.8 & 57.1 & 42.9 \\
\hline 97.0 & 44.2 & 94.0 & 98.9 & 96.5 & 99.0 & 97.5 & 98.7 & 95.9 & 89.9 & 76.3 & 65.1 & 48.8 \\
\hline 103.9 & 45.0 & 94.5 & 99.4 & 97.9 & 99.8 & 98.0 & 99.8 & 94.5 & 94.1 & 78.1 & 59.6 & 53.7 \\
\hline 110.7 & 47.8 & 100.4 & 105.5 & 105.4 & 106.5 & 104.4 & 106.1 & 104.5 & 97.1 & 88.3 & 64.9 & 54.1 \\
\hline 117.5 & 49.8 & 111.0 & 116.2 & 112.4 & 117.9 & 114.4 & 117.0 & 107.2 & 109.1 & 99.1 & 81.4 & 56.1 \\
\hline 124.3 & 50.5 & 108.0 & 114.4 & 111.0 & 115.0 & 111.8 & 115.2 & 105.4 & 100.2 & 87.6 & 76.4 & 61.6 \\
\hline 131.2 & 54.1 & 109.6 & 113.5 & 91,9 & 100.0 & 110.8 & 116.8 & 107.2 & 104.6 & 87.4 & 91.1 & 63.7 \\
\hline 138.1 & 57.9 & 119.2 & 135.7 & 128.6 & 135.1 & 134.0 & 132.5 & 119.1 & 110.7 & 84.0 & 90.0 & 67.1 \\
\hline \multicolumn{13}{|c|}{ rmocouples located at sprinkler number 3} \\
\hline Time & $0 \mathrm{~mm}$ & $25 \mathrm{~mm}$ & $50 \mathrm{~mm}$ & $75 \mathrm{~mm}$ & $100 \mathrm{~mm}$ & $125 \mathrm{~mm}$ & $150 \mathrm{~mm}$ & $250 \mathrm{~mm}$ & $350 \mathrm{~mm}$ & $450 \mathrm{~mm}$ & $550 \mathrm{~mm}$ & $900 \mathrm{~mm}$ \\
\hline 0.0 & 19.6 & 19.9 & 20.1 & 20.2 & 20.2 & 20.2 & 20.2 & 20.1 & 20.1 & 20.1 & 20.0 & 19.8 \\
\hline 1.2 & 19.6 & 20.0 & 20.2 & 20.2 & 20.2 & 20.2 & 20.2 & 20.2 & 20.2 & 20.2 & 20.1 & 20.0 \\
\hline 8.1 & 19.5 & 19.9 & 20.2 & 20.2 & 20.2 & 202 & 20.2 & 20.1 & 20.2 & 20.2 & 20.2 & 20.0 \\
\hline 14.9 & 19.6 & 21.1 & 23.4 & 24.0 & 23.0 & 23.6 & 22.9 & 22.0 & 21.4 & 20.2 & 20.2 & 20.1 \\
\hline 21.9 & 20.0 & 25.5 & 28.4 & 27.7 & 26.1 & 25.9 & 24.7 & 21.0 & 20.5 & 20.2 & 20.2 & 20.2 \\
\hline 28.6 & 20.3 & 27.3 & 31.7 & 32.1 & 31.2 & 31.3 & 29.8 & 27.8 & 25.2 & 23.9 & 23.8 & 21.5 \\
\hline 35.4 & 20.8 & 31.2 & 37.0 & 37.3 & 36.7 & 37.6 & 36.4 & 33.2 & 27.1 & 25.5 & 25.4 & 20.9 \\
\hline 42.3 & 21.4 & 35.2 & 41.1 & 41.6 & 40.1 & 40.6 & 39.5 & 35.9 & 32.5 & 29.9 & 29.6 & 24.5 \\
\hline 49.1 & 22.2 & 37.2 & 44.3 & 45.3 & 44.3 & 44.8 & 43.4 & 39.8 & 35.7 & 34.1 & 32.5 & 24.8 \\
\hline 56.1 & 22.7 & 40.5 & 48.9 & 48.9 & 47.8 & 48.0 & 46.6 & 42.2 & 37.8 & 35.5 & 35.7 & 29.0 \\
\hline 62.9 & 23.1 & 41.4 & 51.4 & 51.4 & 50.5 & 51.0 & 50.2 & 46.4 & 40.7 & 39.5 & 37.7 & 32.0 \\
\hline 69.7 & 23.5 & 46.5 & 55.4 & 55.2 & 53.6 & 53.7 & 52.3 & 47.5 & 44.2 & 42.9 & 42.5 & 34.2 \\
\hline 6.6 & 24.3 & 46.1 & 58.5 & 58.7 & 57.4 & 57.2 & 55.7 & 49.2 & 46.8 & 45.5 & 45.2 & 35.7 \\
\hline
\end{tabular}


Experiment Number 12

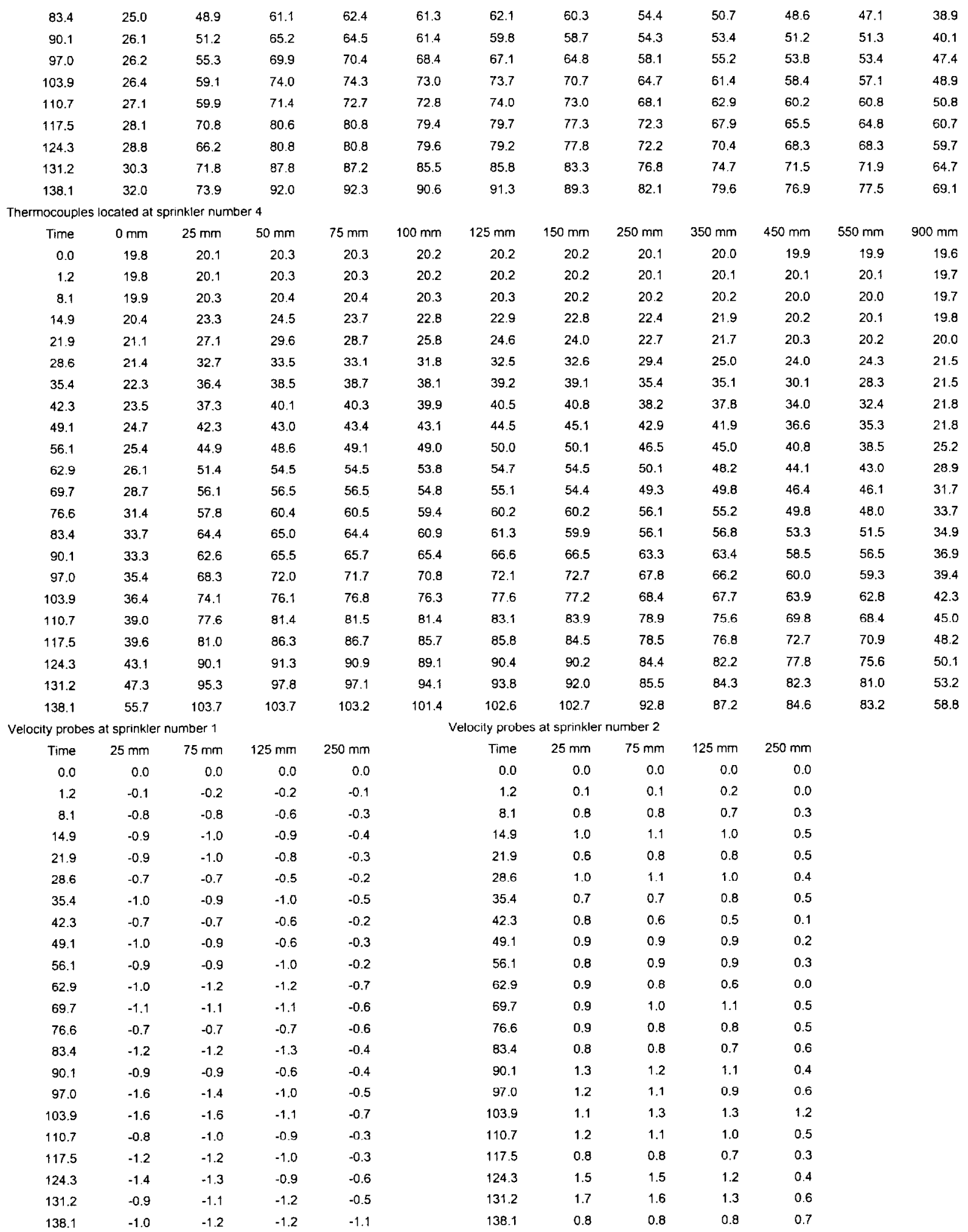


Experiment Number 13

Thermocouples located at sprinkler number 1

\begin{tabular}{|c|c|c|c|c|c|c|c|c|c|c|c|c|}
\hline Time & $0 \mathrm{~mm}$ & $25 \mathrm{~mm}$ & $50 \mathrm{~mm}$ & $75 \mathrm{~mm}$ & $100 \mathrm{~mm}$ & $125 \mathrm{~mm}$ & $150 \mathrm{~mm}$ & $250 \mathrm{~mm}$ & $350 \mathrm{~mm}$ & $450 \mathrm{~mm}$ & $550 \mathrm{~mm}$ & $900 \mathrm{~mm}$ \\
\hline 0.0 & 22.0 & 21.7 & 21.9 & 21.5 & 21.6 & 21.2 & 21.4 & 20.8 & 20.8 & 20.5 & 20.3 & 19.7 \\
\hline 5.2 & 21.9 & 21.7 & 21.8 & 21.4 & 21.5 & 21.2 & 21.4 & 20.9 & 21.1 & 20.8 & 20.5 & 19.9 \\
\hline 12.2 & 22.8 & 25.5 & 29.3 & 28.4 & 28.5 & 28.0 & 26.5 & 24.6 & 23.0 & 21.2 & 21.0 & 20.1 \\
\hline 18.9 & 24.8 & 34.0 & 40.1 & 37.9 & 37.8 & 36.3 & 34.7 & 29.2 & 25.3 & 22.5 & 21.1 & 20.1 \\
\hline 25.9 & 27.0 & 37.8 & 42.7 & 40.6 & 41.7 & 40.6 & 40.5 & 34.8 & 29.7 & 26.6 & 25.3 & 20.6 \\
\hline 32.6 & 28.4 & 43.8 & 51.3 & 49.7 & $4 \mathrm{~B} .5$ & 47.6 & 46.3 & 39.7 & 36.1 & 31.4 & 24.0 & 20.3 \\
\hline 39.4 & 30.1 & 46.4 & 52.8 & 49.2 & 50.7 & 49.2 & 50.3 & 43.1 & 40.4 & 36.9 & 30.1 & 20.5 \\
\hline 46.3 & 31.9 & 50.7 & 59.7 & 55.6 & 57.4 & 53.2 & 55.0 & 47.7 & 44.7 & 41.8 & 34.5 & 22.7 \\
\hline 53.1 & 33.8 & 53.6 & 61.2 & 56.8 & 60.1 & 55.9 & 59.5 & 51.9 & 53.7 & 45.9 & 41.1 & 23.7 \\
\hline 60.1 & 35.5 & 59,4 & 68.3 & 67.3 & 68.8 & 66.6 & 67.2 & 57.6 & 57.4 & 54.2 & 45.7 & 24.6 \\
\hline 66.8 & 37.9 & 63.2 & 72.8 & 69.8 & 72.2 & 69.9 & 71.9 & 64.9 & 64.1 & 57.2 & 45.9 & 31.9 \\
\hline 73.6 & 38.9 & 70.0 & 81.3 & 76.3 & 78.9 & 74.9 & 75.1 & 66.7 & 65.5 & 60.2 & 49.1 & 35.6 \\
\hline$B 0.5$ & 42.9 & 78.8 & 89.2 & 84.0 & 86.7 & 82.6 & 84.2 & 67.3 & 67.7 & 60.6 & 50.7 & 39.0 \\
\hline 87.3 & 44.9 & 80.8 & 94.6 & 88.1 & 92.7 & 85.0 & 88.3 & 77.0 & 76.6 & 66.2 & 56.4 & 40.5 \\
\hline 94.1 & 46.6 & 86.1 & 101.1 & 96.3 & 98.0 & 92.7 & 96.7 & 80.0 & 81.0 & 73.4 & 56.6 & 43.5 \\
\hline 101.0 & 47.6 & 84.5 & 99.6 & 97.0 & 102.0 & 96.3 & 101.0 & 84.8 & 85.6 & 75.0 & 62.0 & 44.3 \\
\hline 107.8 & 49.9 & 91.0 & 105.2 & 99.9 & 102.9 & 98.3 & 100.4 & 85.6 & 90.2 & 83.4 & 65.5 & 45.9 \\
\hline 114.7 & 51.1 & 92.9 & 104.4 & 100.4 & 105.4 & 100.1 & 104.4 & 90.0 & 92.7 & 83.2 & 70.7 & 47.6 \\
\hline 121.5 & 53.1 & 93.6 & 84.1 & 80.4 & 102.9 & 57.7 & 93.1 & 76.1 & 92.8 & 84.9 & 74.5 & 50.5 \\
\hline 128.2 & 56.4 & 94.8 & 111.1 & 99.1 & 112.5 & 98.0 & 97.1 & 91.1 & 102.6 & 92.2 & 78.8 & 54.3 \\
\hline \multicolumn{13}{|c|}{ mocouples located at sprinkler number 2} \\
\hline Time & $0 \mathrm{~mm}$ & $25 \mathrm{~mm}$ & $50 \mathrm{~mm}$ & $75 \mathrm{~mm}$ & $100 \mathrm{~mm}$ & $125 \mathrm{~mm}$ & $150 \mathrm{~mm}$ & $250 \mathrm{~mm}$ & $350 \mathrm{~mm}$ & $450 \mathrm{~mm}$ & $550 \mathrm{~mm}$ & $900 \mathrm{~mm}$ \\
\hline 0.0 & 22.7 & 22.1 & 21.9 & 21.6 & 21.6 & 21.4 & 21.3 & 21.0 & 20.7 & 20.5 & 20.3 & 19.7 \\
\hline 5.2 & 22.5 & 22.2 & 22.1 & 21.8 & 21.6 & 21.4 & 21.6 & 21.2 & 21.0 & 20.9 & 20.8 & 20.1 \\
\hline 12.2 & 24.4 & 30.5 & 31.5 & 31.4 & 31.1 & 30.4 & 29.9 & 25.0 & 24.2 & 23.3 & 22.4 & 20.3 \\
\hline 18.9 & 26.9 & 39.4 & 40.1 & 40.1 & 39.7 & 37.8 & 37.8 & 29.2 & 23.8 & 22.7 & 21.8 & 20.3 \\
\hline 25.9 & 29.2 & 45.8 & 48.0 & 47.8 & 47.2 & 45.7 & 45.1 & 39.2 & 290 & 258 & 247 & 20.6 \\
\hline 32.6 & 29.3 & 47.3 & 49.0 & 49.6 & 49.7 & 48.9 & 48.5 & 44.5 & 39.3 & 33.2 & 24.1 & 20.6 \\
\hline 39.4 & 32.5 & 50.6 & 51.8 & 50.1 & 51.7 & 49.6 & 50.2 & 47.3 & 43.7 & 40.0 & 31.3 & 20.7 \\
\hline 46.3 & 33.2 & 54.8 & 58.0 & 57.0 & 57.5 & 56.8 & 57.5 & 53.4 & 50.7 & 45.5 & 38.5 & 23.3 \\
\hline 53.1 & 35.0 & 59.1 & 62.6 & 62.8 & 63.2 & 62.9 & 62.5 & 59.1 & 53.0 & 48.1 & 43.6 & 26.7 \\
\hline 60.1 & 38.9 & 70.2 & 74.0 & 71.1 & 74.0 & 71.3 & 72.8 & 67.5 & 62.7 & 54.0 & 46.2 & 29.4 \\
\hline 66.8 & 41.6 & 72.7 & 78.0 & 77.3 & 78.9 & 75.1 & 79.1 & 73.4 & 67.9 & 60.7 & 47.9 & 33.7 \\
\hline 73.6 & 42.8 & 79.6 & 84.4 & 83.0 & 84.9 & 81.5 & 84.5 & 79.8 & 74.1 & 63.4 & 51.3 & 35.8 \\
\hline 80.5 & 45.2 & 82.8 & 89.9 & 89.0 & 92.3 & 91.3 & 92.8 & 83.1 & 80.9 & 70.3 & 48.7 & 40.9 \\
\hline 87.3 & 46.5 & 84.8 & 90.2 & 90.1 & 90.6 & 90.0 & 91.0 & 86.1 & 81.0 & 67.9 & 56.0 & 41.8 \\
\hline 94.1 & 47.8 & 86.5 & 92.9 & 91.2 & 92.5 & 92.2 & 93.5 & 86.1 & 81.8 & 71.0 & 59.8 & 45.9 \\
\hline 101.0 & 51.4 & 95.3 & 100.1 & 99.2 & 101.0 & 99.2 & 99.6 & 93.2 & 88.5 & 82.6 & 65.0 & 51.2 \\
\hline 107.8 & 53.7 & 99.6 & 104.1 & 101.3 & 103.2 & 100.0 & 102.4 & 96.4 & 92.5 & 84.1 & 70.6 & 53.4 \\
\hline 114.7 & 55.1 & 101.4 & 105.2 & 103.8 & 105.7 & 103.3 & 106.4 & 103.0 & 98.2 & 88.0 & 75.0 & 57.2 \\
\hline 121.5 & 57.1 & 107.0 & 111.2 & $\$ 10.1$ & 111.9 & 111.6 & 114.4 & 113.9 & 106.6 & 92.0 & 77.4 & 59.7 \\
\hline 128.2 & 57.9 & 106.5 & 115.0 & 113.3 & 114.8 & 112.8 & 117.8 & 112.4 & 106.8 & 101.9 & 94.0 & 62.1 \\
\hline \multicolumn{13}{|c|}{ mocouples located at sprinkler number 3} \\
\hline Time & 0 тाгт & $25 \mathrm{~mm}$ & $50 \mathrm{~mm}$ & $75 \mathrm{~mm}$ & $100 \mathrm{~mm}$ & $125 \mathrm{~mm}$ & $150 \mathrm{~mm}$ & $250 \mathrm{~mm}$ & $350 \mathrm{~mm}$ & $450 \mathrm{~mm}$ & $550 \mathrm{~mm}$ & $900 \mathrm{~mm}$ \\
\hline 0.0 & 21.7 & 21.7 & 21.5 & 21.4 & 21.3 & 21.2 & 21.2 & 20.9 & 20.7 & 20.5 & 20.4 & 19.8 \\
\hline 5.2 & 21.7 & 21.7 & 21.5 & 21.4 & 21.3 & 21.3 & 21.2 & 21.1 & 21.0 & 20.8 & 20.6 & 20.1 \\
\hline 12.2 & 21.8 & 21.9 & 21.6 & 21.4 & 21.3 & 21.3 & 21.3 & 21.2 & 21.0 & 20.9 & 20.8 & 20.2 \\
\hline 18.9 & 22.0 & 24.4 & 25.6 & 24.9 & 24.0 & 23.7 & 23.2 & 23.2 & 21.8 & 21.2 & 21.0 & 20.5 \\
\hline 25.9 & 22.2 & 26.8 & 29.9 & 29.4 & 28.1 & 27.7 & 26.5 & 23.9 & 22.1 & 21.2 & 21.1 & 20.6 \\
\hline 32.6 & 22.4 & 29.0 & 33.3 & 33.6 & 32.4 & 33.0 & 31.8 & 30.6 & 27.7 & 24.4 & 24.2 & 22.2 \\
\hline 39.4 & 22.7 & 33.0 & 38.3 & 37.9 & 36.6 & 36.8 & 35.8 & 33.9 & 32.3 & 29.6 & 27.9 & 22.1 \\
\hline 46.3 & 23.0 & 36.8 & 41.8 & 41.6 & 40.8 & 41.1 & 40.4 & 37.9 & 35.8 & 31.8 & 30.6 & 25.3 \\
\hline 53.1 & 24.2 & 40.9 & 46.4 & 46.7 & 46.1 & 46.7 & 45.8 & 43.6 & 40.9 & 34.4 & 33.8 & 27.0 \\
\hline 60.1 & 24.4 & 41.5 & 52.2 & 52.3 & 50.9 & 50.5 & 49.0 & 44.9 & 41.5 & 37.9 & 37.7 & 30.7 \\
\hline 66.8 & 24.7 & 45.0 & 54.0 & 54.5 & 53.4 & 53.8 & 52.5 & 49.6 & 46.4 & 42.2 & 40.9 & 33.2 \\
\hline 73.6 & 25.3 & 47.5 & 58.6 & 58.6 & 57.4 & 58.2 & 56.8 & 52.6 & 47.8 & 44.3 & 43.4 & 37.3 \\
\hline 80.5 & 26.1 & 51.3 & 61.6 & 61.6 & 60.4 & 60.6 & 60.0 & 54.9 & 50.9 & 48.4 & 46.6 & 39.3 \\
\hline 87.3 & 26.9 & 53.3 & 66.6 & 66.1 & 63.8 & 63.4 & 50.8 & 54.8 & 51.5 & 49.9 & 49.3 & 42.9 \\
\hline 94.1 & 26.9 & 54.2 & 67.7 & 68.7 & 67.8 & 68.7 & 67.5 & 63.3 & 57.9 & 53.8 & 53.3 & 47.3 \\
\hline 101.0 & 28.9 & 64.8 & 71.4 & 71.8 & 70.1 & 69.2 & 67.6 & 60.5 & 59.7 & 58.6 & 57.5 & 47.6 \\
\hline $10 / .8$ & 28.9 & 65.9 & 76.9 & 75.3 & 73.7 & 73.6 & 71.0 & 64.7 & 61.8 & 60.6 & 61.5 & 53.2 \\
\hline
\end{tabular}




\section{Experiment Number 13}

\begin{tabular}{|c|c|c|c|c|c|c|c|c|c|c|c|c|}
\hline 114.7 & 30.2 & 71.5 & 79.8 & 79.3 & 77.3 & 75.9 & 73.5 & 67.4 & 66.8 & 64.8 & 65.0 & 55.4 \\
\hline 121.5 & 29.9 & 66.0 & 82.2 & 82.2 & 81.5 & 82.5 & 80.6 & 73.2 & 70.3 & 67.9 & 69.0 & 62.6 \\
\hline 128.2 & 31.7 & 71.7 & 90.1 & 90.0 & 87.4 & 86.7 & 82.9 & 74.9 & 72.7 & 72.1 & 71.0 & 60.6 \\
\hline \multicolumn{13}{|c|}{ Thermocouples located at sprinkler number 4} \\
\hline Time & $0 \mathrm{~mm}$ & $25 \mathrm{~mm}$ & $50 \mathrm{~mm}$ & $75 \mathrm{~mm}$ & $100 \mathrm{~mm}$ & $125 \mathrm{~mm}$ & $150 \mathrm{~mm}$ & $250 \mathrm{~mm}$ & $350 \mathrm{~mm}$ & $450 \mathrm{~mm}$ & $550 \mathrm{~mm}$ & $900 \mathrm{rnm}$ \\
\hline 0.0 & 21.5 & 21.5 & 21.5 & 21.5 & 21.2 & 21.2 & 21.1 & 20.9 & 20.6 & 20.3 & 20.1 & 19.6 \\
\hline 5.2 & 21.4 & 21.5 & 21.4 & 21.3 & 21.1 & 21.1 & 21.2 & 21.0 & 21.0 & 20.7 & 20.5 & 20.0 \\
\hline 12.2 & 21.5 & 21.6 & 21.5 & 21.4 & 21.2 & 21.2 & 21.2 & 21.0 & 20.9 & 20.7 & 20.5 & 20.1 \\
\hline 18,9 & 23.4 & 27.7 & 28.4 & 26.9 & 25.2 & 25.0 & 24.4 & 23.3 & 22.6 & 21.1 & 20.8 & 20.2 \\
\hline 25.9 & 23.3 & 30.1 & 31.6 & 31.3 & 30.1 & 30.4 & 30.3 & 26.8 & 25.4 & 22.8 & 21.9 & 20.5 \\
\hline 32.6 & 23.3 & 33.9 & 35.8 & 36.0 & 35.3 & 36.5 & 36.5 & 33.2 & 32.0 & 28.1 & 26.5 & 22.8 \\
\hline 39.4 & 24.6 & 36.2 & 40.1 & 39.9 & 39.2 & 39.7 & 39.6 & 36.1 & 35.3 & 31.4 & 29.4 & 21.7 \\
\hline 46.3 & 26.0 & 39.3 & 43.3 & 43.1 & 42.0 & 42.6 & 42.7 & 40.1 & 38.3 & 34.6 & 33.8 & 21.6 \\
\hline 53.1 & 26.4 & 44.6 & 45.8 & 46.1 & 45.7 & 47.1 & 47.5 & 44.3 & 42.9 & 37.9 & 36.6 & 25.0 \\
\hline 60.1 & 27.5 & 49.7 & 50.3 & 49.9 & 49.0 & 49.8 & 49.1 & 44.4 & 43.5 & 42.7 & 41.4 & 26.6 \\
\hline 66.8 & 28.8 & 52.5 & 54.4 & 54.2 & 53.4 & 54.9 & 54.8 & 49.4 & 47.3 & 46.0 & 45.1 & 30.6 \\
\hline 73.6 & 33.0 & 56.7 & 56.4 & 55.7 & 54.4 & 54.7 & 54.1 & 48.9 & 50.0 & 49.7 & 48.5 & 33.4 \\
\hline 80.5 & 33.9 & 62.9 & 62.6 & 62.5 & 61.0 & 62.3 & 62.5 & 58.7 & 59.6 & 53.0 & 51.3 & 34.9 \\
\hline 87.3 & 39.8 & 70.2 & 70.1 & 70.0 & 68.2 & 69.4 & 69.0 & 61.0 & 60.4 & 56.8 & 55.7 & 36.5 \\
\hline 94.1 & 36.0 & 74.3 & 74.5 & 74.6 & 72.8 & 73.8 & 73.0 & 66.9 & 62.5 & 60.7 & 60.0 & 38.2 \\
\hline 101.0 & 39.0 & 79.5 & 80.9 & 80.4 & 78.4 & 79.9 & 79.6 & 71.8 & 68.7 & 66.0 & 65.2 & 41.2 \\
\hline 107.8 & 38.4 & 78.2 & 79.3 & 80.0 & 78.7 & 80.4 & 80.2 & 73.1 & 71.8 & 69.0 & 66.7 & 44.5 \\
\hline 114.7 & 43.8 & 84.9 & 85.6 & 84.7 & 82.3 & 83.2 & 83.0 & 76.7 & 75.6 & 72.1 & 70.6 & 47.8 \\
\hline 121.5 & 43.8 & 87.4 & 87.9 & 87.6 & 86.6 & 88.0 & 87.0 & BO. 2 & 79.2 & 76.8 & 73.8 & 50.8 \\
\hline 128.2 & 43.0 & 89.9 & 94.3 & 93.4 & 91.8 & 92.4 & 91.6 & 86.6 & 83.9 & 79.0 & 78.0 & 53.0 \\
\hline \multicolumn{5}{|c|}{ Velocity probes at sprinkler number 1} & \multicolumn{6}{|c|}{ Velocity probes at sprinkler number 2} & & \\
\hline Time & $25 \mathrm{~mm}$ & $75 \mathrm{~mm}$ & $125 \mathrm{~mm}$ & $250 \mathrm{~mm}$ & & Time & $25 \mathrm{~mm}$ & $75 \mathrm{~mm}$ & $125 \mathrm{~mm}$ & $250 \mathrm{~mm}$ & & \\
\hline 0.0 & 0.0 & 0.0 & 0.0 & 0.0 & & 0.0 & 0.0 & 0.0 & 0.0 & 0.0 & & \\
\hline 5.2 & -0.7 & -0.8 & .0 .6 & -0.3 & & 5.2 & 0.7 & 0.7 & 0.7 & 0.6 & & \\
\hline 12.2 & -0.7 & -0.7 & -0.7 & -0.4 & & 12.2 & 1.1 & 1.0 & 0.6 & 0.3 & & \\
\hline 18.9 & -0.9 & -0.9 & -0.7 & -0.1 & & 18.9 & 0.9 & 0.9 & 0.6 & 0.4 & & \\
\hline 25.9 & -0.7 & -0.7 & -0.4 & -0.1 & & 25.9 & 0.6 & 0.7 & 0.4 & 0.1 & & \\
\hline 32.6 & -0.6 & -0.6 & -0.4 & -0.1 & & 32.6 & 0.8 & 0.7 & 0.5 & 0.1 & & \\
\hline 39.4 & -0.8 & -0.7 & 0.1 & -0.2 & & 39.4 & 0.9 & 0.9 & 0.8 & 0.4 & & \\
\hline 46.3 & -1.0 & -1.0 & 0.3 & -0.2 & & 46.3 & 0.7 & 0.7 & 0.8 & 0.3 & & \\
\hline 53.1 & -0.9 & -0.8 & -0.5 & -0.2 & & 53.1 & 0.9 & 0.9 & 0.9 & 0.5 & & \\
\hline 60.1 & -0.9 & -0.9 & -0.9 & -0.4 & & 60.1 & 0.8 & 0.8 & 0.8 & 0.5 & & \\
\hline 66.8 & -1.0 & -0.8 & -0.6 & -0.2 & & 66.8 & 1.2 & 1.1 & 0.9 & 0.6 & & \\
\hline 73.6 & -1.2 & -1.1 & -0.8 & -0.3 & & 73.6 & 0.9 & 0.9 & 0.8 & 0.6 & & \\
\hline 80.5 & -0.8 & -0.8 & -0.5 & -0.3 & & 80.5 & 1.1 & 0.9 & 0.8 & 0.5 & & \\
\hline 87.3 & -1.3 & -1.3 & -1.1 & -0.6 & & 87.3 & 1.1 & 1.0 & 0.9 & 0.8 & & \\
\hline 94.1 & -1.3 & -1.2 & -1.3 & -0.7 & & 94.1 & 1.2 & 1.2 & 0.8 & 0.3 & & \\
\hline 101.0 & -1.4 & -1.3 & -1.1 & -0.3 & & 101.0 & 1.2 & 1.1 & 1.0 & 0.5 & & \\
\hline 107.8 & -1.3 & -1.4 & -1.2 & -0.8 & & 107.8 & 1.3 & 1.2 & 1.2 & 0.9 & & \\
\hline 114.7 & -1.3 & -1.2 & -1.0 & -0.4 & & 114.7 & 0.7 & 0.8 & 1.1 & 0.8 & & \\
\hline 121.5 & -1.1 & -1.2 & -1.1 & -0.4 & & 121.5 & 1.1 & 1.1 & 1.1 & 0.6 & & \\
\hline 128.2 & -1.2 & -1.4 & -1.4 & -0.5 & & 128.2 & 1.5 & 1.4 & 1.1 & 0.6 & & \\
\hline
\end{tabular}


Experiment Number 14

Thermocouples located at sprinkler number 1

\begin{tabular}{|c|c|c|c|c|c|c|c|c|c|c|c|c|}
\hline Time & $0 \mathrm{~mm}$ & $25 \mathrm{~mm}$ & $50 \mathrm{~mm}$ & $75 \mathrm{~mm}$ & $100 \mathrm{~mm}$ & $125 \mathrm{~mm}$ & $150 \mathrm{~mm}$ & $250 \mathrm{~mm}$ & $350 \mathrm{~mm}$ & $450 \mathrm{~mm}$ & $550 \mathrm{~mm}$ & $900 \mathrm{~mm}$ \\
\hline 0.0 & 21.8 & 21.5 & 21.4 & 21.1 & 21.1 & 20.5 & 20.8 & 20.3 & 20.2 & 19.9 & 19.8 & 19.2 \\
\hline 5.4 & 22.6 & 22.4 & 22.4 & 21.9 & 21.8 & 21.1 & 21.4 & 20.9 & 21.0 & 20.7 & 20.5 & 19.6 \\
\hline 12.1 & 22.8 & 22.6 & 22.6 & 22.1 & 21.9 & 21.3 & 21.6 & 21.0 & 21.1 & 20.8 & 20.7 & 19.8 \\
\hline 19.0 & 24.7 & 25.3 & 26.2 & 26.8 & 26.4 & 27.0 & 25.6 & 25.7 & 24.7 & 23.8 & 21.9 & 19.9 \\
\hline 25.7 & 31.7 & 32.0 & 33.7 & 34.0 & 32.4 & 32.1 & 29.8 & 25.3 & 22.1 & 21.7 & 21.2 & 19.8 \\
\hline 32.5 & 36.1 & 36.3 & 37.6 & 37.8 & 37.6 & 37.6 & 35.2 & 31.0 & 23.6 & 21.5 & 21.3 & 20.2 \\
\hline 39.4 & 38.6 & 38.7 & 40.3 & 41.0 & 40.7 & 40.8 & 38.4 & 36.3 & 32.0 & 24.7 & 23.8 & 22.2 \\
\hline 46.1 & 37.7 & 39.6 & 40.7 & 41.5 & 40.7 & 41.6 & 41.1 & 41.5 & 35.0 & 28.0 & 26.9 & 22.0 \\
\hline 53.1 & 43.9 & 45.0 & 46.6 & 47.1 & 46.2 & 47.1 & 44.6 & 44.0 & 36.5 & 31.6 & 30.5 & 23.1 \\
\hline 59.8 & 50.2 & 50.3 & 51.3 & 51.9 & 51.2 & 51.9 & 49.6 & 47.8 & 39.5 & 35.7 & 33.8 & 25.5 \\
\hline 66.6 & 53.2 & 53.9 & 55.8 & 57.0 & 56.5 & 57.4 & 53.8 & 51.3 & 43.8 & 39.0 & 36.2 & 28.4 \\
\hline 73.5 & 57.3 & 57.6 & 59.5 & 59.8 & 57.9 & 58.7 & 55.7 & 50.5 & 43.8 & 41.0 & 38.5 & 31.7 \\
\hline 80.3 & 59.8 & 60.4 & 62.6 & 63.1 & 62.2 & 61.7 & 58.3 & 55.2 & 51.6 & 48.8 & 43.6 & 33.1 \\
\hline 87.0 & 64.3 & 64.7 & 67.0 & 66.8 & 63.8 & 65.1 & 62.5 & 58.1 & 52.3 & 47.7 & 44.1 & 36.5 \\
\hline 33.9 & 68.4 & 68.7 & 71.4 & 72.2 & 70.8 & 71.6 & 67.8 & 64.4 & 56.2 & 50.9 & 47.0 & 39.4 \\
\hline 100.7 & 68.8 & 70.4 & 73.0 & 73.2 & 72.6 & 73.7 & 70.5 & 68.9 & 59.6 & 54.0 & 49.4 & 40.8 \\
\hline 107.6 & 76.1 & 76.6 & 80.1 & 81.2 & 78.4 & 79.0 & 74.5 & 72.2 & 66.4 & 59.9 & 53.5 & 44.0 \\
\hline 114.3 & $7 B .2$ & 79.0 & 81.0 & 81.1 & 79.5 & 80.1 & 75.9 & 73.9 & 65.6 & 59.3 & 56.3 & 44.9 \\
\hline 121.1 & 85.7 & 87.1 & 92.5 & 94.1 & 90.6 & 91.7 & 83.9 & 78.6 & 71.1 & 63.8 & 58.1 & 49.1 \\
\hline 128.0 & 83.8 & 87.6 & 91.9 & 93.8 & 92.9 & 93.2 & 86.0 & 81.8 & 73.7 & 67.8 & 62.9 & 49.6 \\
\hline 134.8 & 90.7 & 91.5 & 95.0 & 96.1 & 94.4 & 94.4 & 89.3 & 84.9 & 77.8 & 70.6 & 64.5 & 54.6 \\
\hline 141.7 & 87.7 & 91.6 & 95.3 & 96.5 & 96.3 & 96.8 & 92.8 & 88.7 & 83.2 & 78.6 & 70.7 & 53.2 \\
\hline 148.5 & 92.7 & 95.4 & 99.0 & 100.4 & 98.5 & 99.8 & 94.4 & 89.1 & 81.1 & 75.2 & 70.1 & 61.3 \\
\hline 155.2 & 102.8 & 105.2 & 109.7 & 111.5 & 109.4 & 111.1 & 103.8 & 96.9 & 84.6 & 77.8 & 72.0 & 62.6 \\
\hline 162.1 & 100.7 & 103.1 & 105.9 & 107.2 & 104.7 & 107.8 & 105.5 & 105.1 & 89.2 & 81.3 & 76.3 & 64.3 \\
\hline 168.8 & 114.2 & 114.9 & 119.0 & 120.4 & $\uparrow 16.1$ & 116.5 & 111.5 & 106.6 & 92.6 & 81.7 & 77.4 & 64.1 \\
\hline 175.7 & 116.7 & $\uparrow 18.4$ & 123.3 & 124.4 & 120.9 & 120.9 & 114.9 & 108.7 & 98.4 & 88.6 & 80.2 & 65.8 \\
\hline 182.5 & 116.7 & $\$ 19.5$ & 123.1 & 124.9 & 122.2 & 125.3 & 119.1 & 120.4 & 108.0 & 92.0 & 85.0 & 71.7 \\
\hline 189.3 & 124.2 & 126.9 & 132.9 & 133.3 & 130.4 & 130.7 & 125.6 & 120.7 & 108.0 & 98.0 & 87.5 & 71.8 \\
\hline \multicolumn{13}{|c|}{ Thermocouples located at sprinkler number 2} \\
\hline Time & $0 \mathrm{~mm}$ & $25 \mathrm{~mm}$ & $50 \mathrm{~mm}$ & $75 \mathrm{~mm}$ & $100 \mathrm{~mm}$ & $125 \mathrm{~mm}$ & $150 \mathrm{~mm}$ & $250 \mathrm{~mm}$ & $350 \mathrm{~mm}$ & $450 \mathrm{~mm}$ & $550 \mathrm{~mm}$ & $900 \mathrm{~mm}$ \\
\hline 0.0 & 22.3 & 22.1 & 21.8 & 21.4 & 21.4 & 21.1 & 21.1 & 20.6 & 20.4 & 20.1 & 19.9 & 19.2 \\
\hline 5.4 & 23.0 & 22.7 & 22.6 & 22.3 & 22.2 & 21.8 & 21.6 & 21.0 & 20.7 & 20.5 & 20.4 & 19.4 \\
\hline 12.1 & 23.3 & 23.1 & 23.2 & 23.3 & 23.5 & 23.4 & 23.2 & 22.3 & 21.4 & 20.8 & 20.5 & 19.8 \\
\hline 19.0 & 28.5 & 31.3 & 31.1 & 30.7 & 29.8 & 29.0 & 28.1 & 26.9 & 23.8 & 21.8 & 20.9 & 20.1 \\
\hline 25.7 & 33.3 & 36.1 & 36.0 & 37.1 & 37.2 & 36.7 & 35.5 & 29.7 & 23.7 & 21.2 & 20.9 & 20.0 \\
\hline 32.5 & 37.7 & 40.7 & 40.8 & 41.3 & 40.8 & 40.2 & 39.1 & 33.7 & 27.8 & 22.3 & 21.7 & 20.3 \\
\hline 39.4 & 40.7 & 43.3 & 44.2 & 45.8 & 45.5 & 44.9 & 44.4 & 38.4 & 29.2 & 26.4 & 25.7 & 22.2 \\
\hline 46.1 & 44.3 & 46.8 & 47.0 & 47.3 & 47.2 & 46.6 & 46.0 & 40.4 & 33.7 & 31.4 & 29.2 & 21.7 \\
\hline 53,1 & 46.7 & 49.0 & 49.7 & 50.9 & 50.8 & 50.5 & 49.3 & 42.7 & 35.2 & 33.4 & 31.4 & 22.2 \\
\hline 59.8 & 51.5 & 54.5 & 54.4 & 55.1 & 54.5 & 53.8 & 53.4 & 46.3 & 40.7 & 37.7 & 35.1 & 23.4 \\
\hline 66.6 & 55.6 & 58.2 & 58.0 & 59.5 & 60.3 & 61.1 & 59.8 & 49.0 & 41.8 & 39.1 & 37.0 & 24.7 \\
\hline 73.5 & 58.1 & 61.7 & 62.2 & 62.6 & 62.7 & 62.7 & 61.3 & 55.9 & 47.5 & 42.5 & 40.8 & 28.0 \\
\hline$B 0.3$ & 62.0 & 65.7 & 66.3 & 66.8 & 67.0 & 67.1 & 66.3 & 57.5 & 49.5 & 46.1 & 43.2 & 31.4 \\
\hline 87.0 & 71.2 & 75.8 & 75.8 & 77.0 & 76.8 & 75.3 & 73.2 & 61.9 & 51.3 & 48.2 & 45.9 & 32.9 \\
\hline 93.9 & 70.9 & 74.2 & 74.4 & 75.8 & 76.1 & 75.7 & 75.0 & 70.1 & 58.1 & 52.9 & 49.4 & 36.4 \\
\hline 100.7 & 76.8 & $B 1.9$ & 82.3 & 83.7 & 83.3 & 82.5 & 81.0 & 72.2 & 59.1 & 55.6 & 52.9 & 41.3 \\
\hline 107.6 & 80.5 & 85.5 & 85.3 & 86.4 & 85.5 & 84.5 & 82.6 & 68.2 & 62.6 & 60.4 & 53.5 & 44.1 \\
\hline 114.3 & 85.0 & 91.2 & 91.2 & 93.4 & 91.8 & 89.0 & 86.9 & 76.0 & 67.8 & 62.0 & 55.3 & 45.8 \\
\hline 121.1 & 89.5 & 95.1 & 95.4 & 96.7 & 94.6 & 93.6 & 90.6 & 77.6 & 72.0 & 62.1 & 57.2 & 47.9 \\
\hline 128.0 & 89.6 & 93.5 & 94.5 & 94.8 & 947 & 94.6 & 93.2 & 88.2 & 78.6 & 68.8 & 61.4 & 49.7 \\
\hline 134.8 & 94.4 & 100.5 & 101.3 & 102.7 & 103.3 & 103.1 & 102.0 & 92.9 & 76.7 & 73.0 & 64.4 & 51.2 \\
\hline 141.7 & 102.4 & 108.2 & 109.3 & 109.3 & 108.0 & 104.2 & 101.2 & 85.6 & 85.1 & 76.0 & 69.2 & 52.4 \\
\hline 148.5 & 100.9 & 105.2 & 106.0 & 107.2 & 107.5 & 106.9 & 105.4 & 88.8 & 85.8 & 80.1 & 73.4 & 55.4 \\
\hline 155.2 & 110.2 & 118.1 & 117.8 & 119.2 & 118.1 & 116.1 & 112.3 & 91.6 & 90.3 & 81.8 & 77.0 & 60,3 \\
\hline 162.1 & 116.7 & 124.9 & 125.2 & 127.8 & 126.6 & 125.3 & 121.1 & 103.5 & 90.5 & 80.0 & 74.1 & 62.8 \\
\hline 168.8 & 116.8 & 121.4 & 123.3 & 125.4 & 126.6 & 127.1 & 125.2 & 112.0 & 96.8 & 88.3 & 81.8 & 65.5 \\
\hline 175.7 & 121.0 & 126.7 & 126.8 & 128.8 & 128.6 & 127.3 & 125.5 & 109.6 & 98.0 & 91.9 & 86.7 & 68.5 \\
\hline 182.5 & 123.2 & 128.8 & 128.9 & 129.4 & 128.9 & 128.1 & 126.3 & 117.6 & 104.5 & 97.3 & 89.8 & 71.8 \\
\hline 189.3 & 130.0 & 136.4 & 137.5 & 139.7 & 140.9 & 140.5 & 138.6 & 122.3 & 113.7 & 101.7 & 92.0 & 72.9 \\
\hline
\end{tabular}

Thermocouples located at sprinkler number 3 
Experiment Number 14

\begin{tabular}{|c|c|c|c|c|c|c|c|c|c|c|c|c|}
\hline Time & $0 \mathrm{~mm}$ & $25 \mathrm{~mm}$ & $50 \mathrm{~mm}$ & $75 \mathrm{~mm}$ & $100 \mathrm{~mm}$ & $125 \mathrm{~mm}$ & $150 \mathrm{~mm}$ & $250 \mathrm{~mm}$ & $350 \mathrm{~mm}$ & $450 \mathrm{~mm}$ & $550 \mathrm{~mm}$ & $900 \mathrm{~mm}$ \\
\hline 0.0 & 22.4 & 22.4 & 22.0 & 21.6 & 21.3 & 21.1 & 21.2 & 20.7 & 20.5 & 20.2 & 20.0 & 19.4 \\
\hline 5.4 & 22.5 & 21.8 & 22.0 & 21.9 & 22.2 & 22.4 & 22.0 & 20.9 & 20.6 & 20.2 & 19.4 & 19.9 \\
\hline 12.1 & 22.9 & 22.5 & 22.1 & 22.1 & 22.2 & 22.4 & 21.9 & 21.1 & 20.8 & 20.4 & 19.6 & 20.1 \\
\hline 19.0 & 23.1 & 23.0 & 22.5 & 22.9 & 23.3 & 23.5 & 23.1 & 22.5 & 22.3 & 21.8 & 21.4 & 20.5 \\
\hline 25.7 & 26.6 & 28.7 & 26.1 & 27.2 & 27.2 & 28.2 & 28.0 & 27.0 & 25.1 & 23.4 & 22.3 & 20.7 \\
\hline 32.5 & 28.2 & 29.4 & 29.1 & 29.8 & 29.8 & 29.0 & 28.5 & 29.6 & 30.3 & 28.4 & 27.1 & 20.8 \\
\hline 39.4 & 30.1 & 32.3 & 32.0 & 32.2 & 32.8 & 33.1 & 33.0 & 31.7 & 32.4 & 30.0 & 26.4 & 21.2 \\
\hline 46.1 & 29.0 & 27.4 & 32.2 & 32.9 & 32.9 & 32.5 & 32.4 & 33.8 & 35.6 & 33.9 & 31.4 & 22.6 \\
\hline 53.1 & 32.9 & 32.9 & 37.1 & 38.4 & 38.7 & 38.4 & 38.0 & 36.2 & 37.0 & 35.8 & 34.6 & 21.8 \\
\hline 59.8 & 38.9 & 41.5 & 41.2 & 42.6 & 42.0 & 42.4 & 42.4 & 41.4 & 42.2 & 38.9 & 34.8 & 25.6 \\
\hline 66.6 & 32.9 & $34 ?$ & 37.6 & 36.7 & 37.5 & 37.8 & 39.5 & 43.4 & 46.1 & 42.9 & 37.0 & 27.1 \\
\hline 73.5 & 36.1 & 41.7 & 43.1 & 43.6 & 44.7 & 47.6 & 48.8 & 49.0 & 48.3 & 44.8 & 41.1 & 26.9 \\
\hline 80.3 & 39.6 & 42.2 & 45.2 & 46.7 & 47.6 & 48.3 & 49.1 & 49.6 & 51.0 & 46.4 & 43.6 & 32.4 \\
\hline 87.0 & 45.0 & 50.6 & 54.5 & 55.0 & 53.4 & 53.7 & 53.2 & 51.5 & 52.4 & 50.6 & 48.2 & 35.8 \\
\hline 93.9 & 41.6 & 46.7 & 50.2 & 50.7 & 51.9 & 53.0 & 54.1 & 54.8 & 57.3 & 54.0 & 52.5 & 36.1 \\
\hline 100.7 & 44.4 & 48.8 & 50.0 & 51.4 & 54.6 & 56.1 & 56.9 & 56.8 & 58.7 & 57.3 & 55.8 & 37.7 \\
\hline 107.6 & 50.9 & 57.8 & 60.3 & 61.1 & 61.2 & 62.8 & 62.5 & 62.2 & 62.4 & 59.1 & 57.5 & 41.2 \\
\hline 114.3 & 48.9 & 58.7 & 59.3 & 61.2 & 61.1 & 63.1 & 63.6 & 64.2 & 65.8 & 64.7 & 58.9 & 43.4 \\
\hline 121.1 & 50.7 & 56.9 & 59.9 & 61.9 & 64.0 & 67.0 & 68.0 & 68.9 & 70.9 & 65.5 & 61.7 & 45.4 \\
\hline 128.0 & 57.7 & 67.2 & 67.0 & 71.4 & 69.7 & 71.4 & 71.0 & 71.0 & 74.0 & 70.0 & 68.5 & 48.1 \\
\hline 134.8 & 62.9 & 71.6 & $/ 1.1$ & 73.6 & 72.6 & 75.2 & 75.3 & 74.0 & 78.5 & 72.6 & 66.3 & 47.8 \\
\hline 141.7 & 60.1 & 68.1 & 70.6 & 74.4 & 75.9 & 78.6 & 78.5 & 78.1 & 79.6 & 72.8 & 68.2 & 52.9 \\
\hline 148.5 & 56.3 & 61.4 & 68.9 & 70.8 & 74.6 & 77.2 & 76.9 & 76.5 & 75.7 & 74.7 & 73.7 & 53.6 \\
\hline 155.2 & 63.9 & 73.2 & 72.8 & 79.9 & 77.6 & 83.5 & B1.0 & 79.7 & 82.7 & 79.1 & 81.3 & 55.0 \\
\hline 162.1 & 66.7 & 75.1 & 77.2 & 83.3 & 82.2 & 84.1 & 84.5 & 85.2 & 90.2 & 83.0 & 79.2 & 58.8 \\
\hline 168.8 & 67.8 & 71.7 & 75.6 & 79.0 & 80.8 & 84.9 & 85.1 & 85.5 & 91.5 & 88.8 & 85.8 & 60.6 \\
\hline 175.7 & 76.7 & 88.8 & 87.9 & 94.3 & 91.4 & 94.3 & 94.2 & 93.7 & 100.5 & 93.6 & 86.6 & 66.3 \\
\hline 182.5 & 75.9 & 85.0 & B7.8 & 93.4 & 93.9 & 98.0 & 98.0 & 98.1 & 102.1 & 94.5 & 84.9 & 67.2 \\
\hline 189.3 & 84.3 & 96.1 & 93.6 & 99.6 & 98.0 & 102.2 & 101.4 & 101.5 & 104.1 & 93.2 & 84.5 & 68.1 \\
\hline
\end{tabular}

Thermocouples located at sprinkler number 4

Time $\quad 0 \mathrm{~mm} \quad 25 \mathrm{~mm} \quad 50 \mathrm{~mm} \quad 75 \mathrm{~mm} \quad 100 \mathrm{~mm} \quad 125 \mathrm{~mm} \quad 150 \mathrm{~mm} \quad 250 \mathrm{~mm} \quad 350 \mathrm{~mm} \quad 450 \mathrm{~mm} \quad 550 \mathrm{~mm} \quad 900 \mathrm{~mm}$

\begin{tabular}{llllllllllll}
0.0 & 22.0 & 21.9 & 21.9 & 21.6 & 21.1 & 21.0 & 20.8 & 20.4 & 20.0 & 19.8 & 19.5 \\
\hline
\end{tabular}

$\begin{array}{lllllllllllll}5.4 & 22.5 & 22.4 & 22.4 & 22.0 & 21.6 & 21.5 & 21.4 & 21.0 & 20.7 & 20.4 & 20.3 & 19.9\end{array}$

$\begin{array}{lllllllllllll}12.1 & 22.8 & 22.7 & 22.7 & 22.4 & 21.9 & 21.8 & 21.5 & 21.2 & 20.8 & 20.4 & 20.2 & 19.9\end{array}$

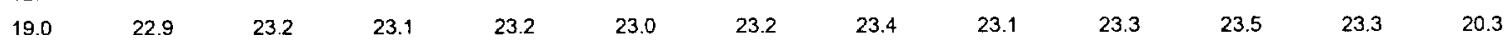

\begin{tabular}{llllllllllll}
25.7 & 24.9 & 26.4 & 25.7 & 28.3 & 28.3 & 28.5 & 28.5 & 28.1 & 28.6 & 26.2 & 22.6 \\
\hline
\end{tabular}

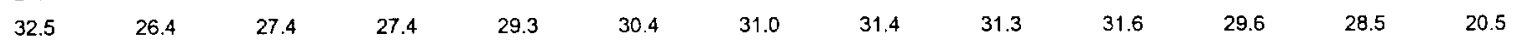

$\begin{array}{llllllllllll}39.4 & 33.2 & 32.5 & 31.7 & 35.7 & 36.1 & 36.6 & 36.3 & 33.9 & 33.8 & 31.9 & 26.6\end{array}$

$\begin{array}{llllllllllll}46.1 & 31.2 & 31.2 & 31.4 & 31.5 & 33.4 & 33.5 & 34.0 & 34.1 & 35.2 & 36.1 & 34.2\end{array}$

$\begin{array}{llllllllllll}5.1 & 34.5 & 36.8 & 36.0 & 40.3 & 10.2 & 40.9 & 40.8 & 40.2 & 41.4 & 39.7 & 37.6\end{array}$

$\begin{array}{lllllllllllll}59.8 & 36.9 & 37.7 & 37.0 & 39.2 & 39.9 & 40.4 & 40.6 & 40.5 & 42.3 & 42.0 & 42.8 & 25.9\end{array}$

$\begin{array}{lllllllllllll}66.6 & 45.5 & 43.2 & 41.3 & 48.1 & 49.0 & 49.8 & 50.8 & 47.9 & 45.7 & 42.6 & 43.1 & 27.3\end{array}$

$\begin{array}{llllllllllllll}73.5 & 45.2 & 42.2 & 41.7 & 49.6 & 50.6 & 51.3 & 51.7 & 50.1 & 49.3 & 45.7 & 42.6 & 29.5\end{array}$

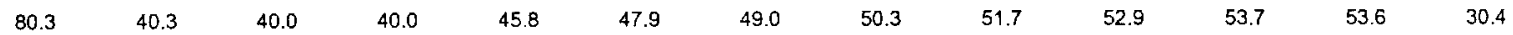

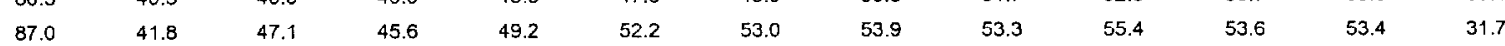

$\begin{array}{lllllllllllll}93.9 & 47.5 & 47.4 & 47.2 & 57.9 & 58.5 & 59.7 & 59.8 & 59.7 & 60.7 & 60.0 & 56.4 & 37.2\end{array}$

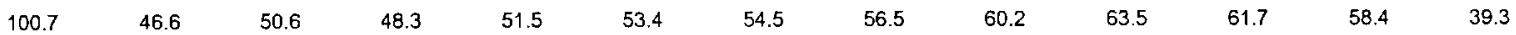

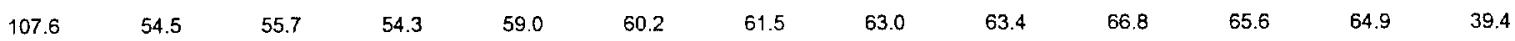

$\begin{array}{lllllllllllll}114.3 & 48.4 & 51.8 & 55.4 & 56.3 & 60.3 & 60.7 & 62.2 & 61.7 & 65.7 & 66.8 & 65.7 & 46.5\end{array}$

$\begin{array}{lllllllllllll}121.1 & 50.3 & 57.1 & 57.6 & 60.0 & 62.0 & 62.7 & 64.5 & 65.8 & 65.0 & 65.9 & 67.1 & 45.8\end{array}$

$\begin{array}{lllllllllllll}128.0 & 50.6 & 57.6 & 58.3 & 61.3 & 65.5 & 66.0 & 69.4 & 68.6 & 73.9 & 73.2 & 72.3 & 53.1\end{array}$

$\begin{array}{llllllllllll}134.8 & 55.3 & 60.3 & 60.7 & 65.1 & 66.4 & 69.3 & 69.9 & 70.9 & 74.8 & 74.1 & 71.5\end{array}$

$\begin{array}{llllllllllllll}141.7 & 62.0 & 72.1 & 70.6 & 74.2 & 76.3 & 77.6 & 79.1 & 78.1 & 79.8 & 79.1 & 79.2 & 55.8\end{array}$

$\begin{array}{llllllllllllll}148.5 & 58.7 & 63.0 & 62.4 & 70.1 & 74.9 & 75.2 & 76.8 & 81.0 & 82.7 & 82.3 & 83.5 & 59.9\end{array}$

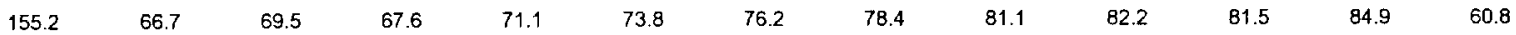

$\begin{array}{lllllllllllll}162.1 & 65.7 & 69.0 & 71.6 & 72.4 & 74.5 & 76.8 & 79.4 & 81.0 & 85.3 & 84.4 & 85.3 & 66.4\end{array}$

$\begin{array}{lllllllllllll}168.8 & 65.9 & 81.7 & 75.2 & 86.9 & 89.5 & 92.3 & 94.4 & 92.9 & 94.4 & 01.9 & 90.9 & 61.9\end{array}$

$\begin{array}{lllllllllllll}175.7 & 66.9 & B 5.5 & 81.1 & 89.7 & 94.3 & 95.1 & 96.5 & 97.3 & 98.7 & 95.5 & 94.3 & 65.0\end{array}$

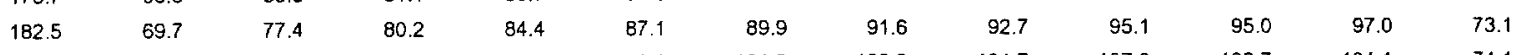

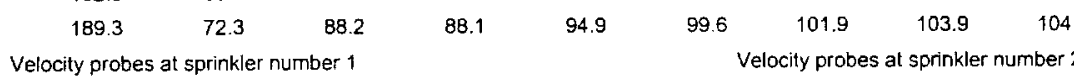

Time $\quad 25 \mathrm{~mm} \quad 75 \mathrm{~mm} \quad 125 \mathrm{~mm}-250 \mathrm{~mm}$

Time $\quad 25 \mathrm{~mm} \quad 75 \mathrm{~mm} \quad 125 \mathrm{~mm} \quad 250 \mathrm{~mm}$ 
Experiment Number 14

\begin{tabular}{|c|c|c|c|c|c|c|c|c|c|}
\hline 0.0 & 0.0 & 0.0 & 0.0 & 0.0 & 0.0 & 0.0 & 0.0 & 0.0 & 0.0 \\
\hline 5.4 & 0.1 & 0.0 & 0.1 & 0.1 & 5.4 & 0.1 & -0.1 & 0.1 & 0.1 \\
\hline 12.1 & -0.2 & -0.3 & -0.3 & -0.2 & 12.1 & 0.4 & 0.5 & 0.5 & 0.4 \\
\hline 19.0 & -0.6 & -0.4 & -0.3 & -0.1 & 19.0 & 0.6 & 0.6 & 0.6 & 0.3 \\
\hline 25.7 & -0.7 & -0.7 & -0.6 & -0.3 & 25.7 & 0.5 & 0.6 & 0.6 & 0.3 \\
\hline 32.5 & -0.5 & -0.5 & -0.4 & -0.2 & 32.5 & 0.4 & 0.5 & 0.5 & 0.2 \\
\hline 39.4 & -0.3 & -0.4 & -0.4 & -0.3 & 39.4 & 0.5 & 0.5 & 0.4 & 0.1 \\
\hline 46.1 & -0.6 & -0.6 & -0.5 & -0.3 & 46.1 & 0.6 & 0.6 & 0.6 & 0.1 \\
\hline 53.1 & -0.5 & -0.5 & -0.5 & -0.3 & 53.1 & 0.6 & 0.6 & 0.6 & 0.2 \\
\hline 59.8 & -0.5 & -0.5 & -0.4 & -0.3 & 59.8 & 0.5 & 0.7 & 0.7 & 0.4 \\
\hline 66.6 & -0.6 & -0.7 & -0.6 & -0.4 & 66.6 & 0.5 & 0.5 & 0.5 & 0.4 \\
\hline 73.5 & -0.6 & +0.7 & -0.7 & -0.4 & 73.5 & 0.6 & 0.5 & 0.5 & 0.2 \\
\hline 80.3 & -0.6 & -0.6 & -0.5 & -0.3 & 80.3 & 0.5 & 0.5 & 0.6 & 0.3 \\
\hline 87.0 & -0.6 & -0.6 & -0.7 & -0.3 & 87.0 & 0.6 & 0.5 & 0.7 & 0.4 \\
\hline 93.9 & -0.7 & -0.7 & -0.7 & -0.5 & 93.9 & 0.8 & 0.7 & 0.8 & 0.2 \\
\hline 100.7 & -0.7 & -0.8 & -0.5 & -0.4 & 100.7 & 0.7 & 0.6 & 0.6 & 0.1 \\
\hline 107.6 & -0.6 & -0.7 & -0.7 & -0.4 & 107.6 & 0.7 & 0.6 & 0.6 & 0.2 \\
\hline 114.3 & -0.8 & -0.8 & -0.7 & -0.3 & 114.3 & 0.8 & 0.7 & 0.8 & 0.6 \\
\hline 121.1 & -0.7 & -0.7 & -0.3 & -0.4 & 121.1 & 0.7 & 0.6 & 0.7 & 0.2 \\
\hline 128.0 & -0.7 & -1.0 & -0.9 & -0.5 & 128.0 & 0.9 & 0.8 & 0.9 & 0.4 \\
\hline 134.8 & -0.7 & -0.8 & -0.7 & -0.5 & 134.8 & 0.7 & 0.7 & 0.9 & 0.5 \\
\hline 141.7 & -0.6 & -0.8 & -0.9 & -0.5 & 141.7 & 0.7 & 0.6 & 0.7 & 0.1 \\
\hline 148.5 & -0.8 & -0.8 & -0.5 & -0.3 & 148.5 & 0.8 & 0.7 & 0.7 & 0.2 \\
\hline 155.2 & -0.6 & -0.8 & -0.8 & -0.7 & 155.2 & 0.8 & 0.8 & 0.9 & 0.4 \\
\hline 162.1 & -1.0 & -1.0 & -0.9 & -0.6 & 162.1 & 1.1 & 1.1 & 1.2 & 0.4 \\
\hline 168.8 & -0.8 & -0.9 & -0.9 & -0.6 & 168.8 & 0.9 & 1.0 & 1.1 & 0.5 \\
\hline 175.7 & -0.9 & -0.9 & -0.8 & -0.7 & 175.7 & 1.1 & 1.1 & 1.3 & 0.7 \\
\hline 182.5 & -1.0 & -0.9 & -0.6 & -0.5 & 182.5 & 0.7 & 0.7 & 0.9 & 0.4 \\
\hline 189.3 & -1.1 & -1.2 & -1.2 & -0.9 & 189.3 & 1.4 & 1.8 & 1.5 & 1.0 \\
\hline
\end{tabular}
Velocity probes in channels above burner

Time Channel 1 Channel 2

$\begin{array}{lll}0.0 & 0.0 & 0.0\end{array}$

$\begin{array}{lll}5.4 & 1.5 & 0.6\end{array}$

$\begin{array}{lll}12.1 & 1.5 & 0.7\end{array}$

$\begin{array}{lll}19.0 & 1.5 & 0.7\end{array}$

$\begin{array}{lll}25.7 & 1.7 & 0.5\end{array}$

$\begin{array}{lll}32.5 & 2.0 & 0.6\end{array}$

$\begin{array}{lll}39.4 & 1.8 & 1.0\end{array}$

$\begin{array}{lll}46.1 & 2.2 & 0.7\end{array}$

$\begin{array}{lll}53.1 & 2.3 & 0.9\end{array}$

$\begin{array}{lll}59.8 & 2.0 & 1.0\end{array}$

$\begin{array}{lll}66.6 & 2.1 & 1.2\end{array}$

$\begin{array}{lll}73.5 & 2.4 & 0.9\end{array}$

$\begin{array}{lll}80.3 & 2.2 & 0.7\end{array}$

$\begin{array}{lll}87.0 & 2.4 & 1.2\end{array}$

$\begin{array}{lll}93.9 & 2.3 & 1.3\end{array}$

$\begin{array}{lll}100.7 & 2.7 & 1.2\end{array}$

$\begin{array}{lll}107.6 & 2.4 & 0.7\end{array}$

$114.3 \quad 2.1 \quad 1.1$

$\begin{array}{lll}121.1 & 2.6 & 0.8\end{array}$

$128.0 \quad 2.4 \quad 1.2$

$\begin{array}{lll}134.8 & 2.8 & 0.9\end{array}$

$\begin{array}{lll}141.7 & 2.3 & 1.4\end{array}$

$\begin{array}{lll}148.5 & 2.3 & 1.5\end{array}$

$\begin{array}{lll}155.2 & 2.5 & 1.4\end{array}$

$162.1 \quad 2.3 \quad 1.3$

$\begin{array}{lll}168.8 & 2.6 & 1.5\end{array}$

$\begin{array}{lll}175.7 & 2.1 & 1.2\end{array}$

$\begin{array}{lll}182.5 & 3.0 & 1.7\end{array}$

$\begin{array}{lll}189.3 & 2.5 & 1.0\end{array}$ 
Experiment Number 15

Thermocouples located at sprinkler number 1

\begin{tabular}{|c|c|c|c|c|c|c|c|c|c|c|c|c|}
\hline & & & & & & & & & & & & \\
\hline Time & $0 \mathrm{~mm}$ & $25 \mathrm{~mm}$ & $50 \mathrm{~mm}$ & $75 \mathrm{~mm}$ & $100 \mathrm{~mm}$ & $125 \mathrm{~mm}$ & $150 \mathrm{~mm}$ & $250 \mathrm{~mm}$ & $350 \mathrm{~mm}$ & $450 \mathrm{~mm}$ & $550 \mathrm{~mm}$ & $900 \mathrm{~mm}$ \\
\hline 0.0 & 23.1 & 22.6 & 22.3 & 21.9 & 21.7 & 21.2 & 21.3 & 20.4 & 20.3 & 19.9 & 19.8 & 18.9 \\
\hline 4.7 & 23.5 & 23.0 & 22.9 & 22.4 & 22.4 & 21.7 & 22.0 & 21.1 & 20.9 & 20.5 & 20.4 & 19.6 \\
\hline 11.5 & 23.9 & 23.4 & 23.2 & 22.7 & 22.6 & 21.9 & 22.1 & 21.3 & 21.0 & 20.6 & 20.5 & 19.8 \\
\hline 18.5 & 25.2 & 25.3 & 26.1 & 26.5 & 26.9 & 27.0 & 25.9 & 26.7 & 24.3 & 22.2 & 21.4 & 19.9 \\
\hline 25.2 & 31.6 & 31.4 & 32.3 & 32.1 & 30.8 & 30.5 & 28.4 & 25.1 & 22.8 & 21.6 & 21.1 & 19.9 \\
\hline 32.1 & 34.8 & 35.0 & 37.0 & 37.3 & 37.0 & 36.9 & 34.3 & 29.8 & 23.5 & 21.5 & 21.1 & 20.1 \\
\hline 38.8 & 37.1 & 37.3 & 38.6 & 38.4 & 37.8 & 37.5 & 35.6 & 33.4 & 27.4 & 24.9 & 24.2 & 21.9 \\
\hline 45.6 & 38.5 & 38.5 & 39.9 & 40.4 & 39.6 & 39.5 & 37.9 & 33.8 & 28.8 & 25.9 & 26.0 & 21.7 \\
\hline 52.5 & 44.8 & 45.4 & 47.1 & 47.3 & 46.1 & 46.7 & 43.6 & 40.4 & 33.8 & 29.6 & 28.3 & 22.5 \\
\hline 59.3 & 47.3 & 48.0 & 49.4 & 49.7 & 48.2 & 48.4 & 45.4 & 40.2 & 36.1 & 32.4 & 30.9 & 22.9 \\
\hline 66.2 & 49.3 & 50.1 & 51.7 & 52.3 & 51.0 & 50.6 & 48.8 & 45.0 & 37.4 & 35.1 & 33.3 & 25.3 \\
\hline 72.9 & 51.7 & 53.2 & 55.3 & 56.2 & 55.3 & 56.1 & 53.5 & 51.4 & 42.0 & 38.8 & 36.4 & 25.1 \\
\hline 79.7 & 54.3 & 55.1 & 57.5 & 58.6 & 57.7 & 58.4 & 56.5 & 52.5 & 44.8 & 42.6 & 39.3 & 29.9 \\
\hline 86.6 & 62.7 & 62.9 & 65.2 & 65.3 & 63.0 & 62.3 & 59.0 & 54.2 & 48.2 & 44.3 & 41.5 & 31.5 \\
\hline 93.4 & 64.1 & 65.1 & 67.7 & 68.3 & 66.4 & 66.4 & 62.7 & 56.7 & 49.7 & 47.3 & 44.8 & 33.1 \\
\hline 100.1 & 68.8 & 68.9 & 72.6 & 72.9 & 69.7 & 70.0 & 65.4 & 59.5 & 51.5 & 49.3 & 45.6 & 35.5 \\
\hline 107.0 & 71.3 & 71.5 & 74.1 & 74.3 & 71.8 & 72.5 & 68.4 & 62.0 & 54.5 & 50.9 & 48.5 & 35.3 \\
\hline 113.8 & 75.9 & 76.6 & 79.3 & 80.4 & 78.8 & 79.6 & 75.1 & 67.8 & 59.0 & 54.5 & 50.2 & 39.0 \\
\hline 120.7 & 78.0 & 78.7 & 81.8 & 81.5 & 79.9 & 80.2 & 75.4 & 68.8 & 60.2 & 56.4 & 53.9 & 42.4 \\
\hline 127.5 & 78.6 & 80.3 & 83.0 & 83.2 & 82.3 & 82.7 & 78.4 & 76.3 & 67.6 & 62.6 & 58.4 & 44.8 \\
\hline 134.2 & 79.4 & 80.8 & 86.0 & 87.6 & 89.3 & 91.5 & 86.1 & 84.4 & 74.2 & 64.7 & 58.9 & 47.3 \\
\hline $14 \uparrow .1$ & 86.8 & 88.2 & 92.6 & 93.6 & 91.6 & 92.5 & 87.9 & 85.9 & 77.4 & 67.8 & 62.8 & 51.8 \\
\hline 147.8 & 95.6 & 96.7 & 101.0 & 102.0 & 99.0 & 99.4 & 92.3 & 81.3 & 75.0 & 70.3 & 65.5 & 53.9 \\
\hline 154.8 & 98.7 & 99.9 & 103.2 & 103.6 & 99.1 & 99.5 & 93.8 & 87.2 & 78.9 & 72.2 & 68.9 & 56.3 \\
\hline 161.6 & 101.0 & 103.2 & 107.9 & 108.8 & 105.3 & 105.5 & 99.7 & 94.2 & 87.2 & 78.0 & 70.2 & 58.1 \\
\hline 168.3 & 106.5 & 108.3 & 112.8 & 113.9 & 110.3 & 109.8 & 101.7 & 93.4 & 86.9 & 78.9 & 74.5 & 64.1 \\
\hline 175.2 & 109.1 & 112.5 & 116.9 & 117.2 & 115.0 & 116.2 & 109.4 & 102.9 & 92.2 & 79.3 & 75.2 & 65.0 \\
\hline 181.9 & 109.5 & 112.5 & 117.0 & 119.1 & 116.4 & 117.6 & 112.6 & 113.1 & 99.2 & 89.2 & 78.2 & 68.4 \\
\hline 188.9 & 112.9 & 115.9 & 121.4 & 124.1 & 121.7 & 123.0 & 116.6 & 110.6 & 96.3 & 88.2 & 81.0 & 67.8 \\
\hline 195.6 & 123.3 & 127.3 & 132.9 & 132.0 & 128.6 & 125.6 & 118.9 & 109.0 & 99.5 & 89.9 & 85.2 & 71.9 \\
\hline \multicolumn{13}{|c|}{ Thermocouples located at sprinkler number 2} \\
\hline Iime & umm & $25 \mathrm{~mm}$ & $50 \mathrm{~mm}$ & $75 \mathrm{~mm}$ & $100 \mathrm{~mm}$ & $125 \mathrm{~mm}$ & $150 \mathrm{~mm}$ & $250 \mathrm{~mm}$ & $350 \mathrm{~mm}$ & 450 гікти & $550 \mathrm{rmm}$ & $900 \mathrm{~mm}$ \\
\hline 0.0 & 23.4 & 23.0 & 22.6 & 22.1 & 22.0 & 21.5 & 21.4 & 20.5 & 20.4 & 20.0 & 19.7 & 19.0 \\
\hline 4.7 & 24.1 & 23.8 & 23.4 & 22.7 & 22.6 & 22.0 & 22.0 & 21.0 & 210 & 20.7 & 20.5 & 19.6 \\
\hline 11.5 & 24.3 & 24.0 & 23.6 & 22.9 & 22.9 & 22.4 & 22.3 & 21.2 & 21.3 & 21.0 & 20.7 & 19.7 \\
\hline 18.5 & 27.4 & 29.0 & 29.3 & 29.6 & 29.5 & 29.2 & 28.6 & 26.5 & 24.5 & 22.4 & 21.5 & 20.0 \\
\hline 25.2 & 31.2 & 33.6 & 33.9 & 33.7 & 33.9 & 33.2 & 32.2 & 24.7 & 22.7 & 21.6 & 21.0 & 19.9 \\
\hline 32.1 & 34.0 & 36.2 & 36.8 & 36.1 & 37.0 & 36.5 & 35.8 & 32.7 & 29.4 & 23.7 & 21.7 & 20.1 \\
\hline 38.8 & 38.5 & 40.6 & 41.1 & 40.2 & 10.5 & 39.3 & 38.2 & 29.7 & 27.4 & 25.8 & 24.5 & 21.6 \\
\hline 45.6 & 40.2 & 42.5 & 43.2 & 42.1 & 43.4 & 43.0 & 42.4 & 36.8 & 31.8 & 28.2 & 27.1 & 21.5 \\
\hline 52.5 & 44.4 & 46.2 & 46.4 & 45.4 & 45.9 & 44.9 & 44.4 & 39.6 & 34.1 & 31.3 & 29.7 & 21.8 \\
\hline 59.3 & 48.5 & 53.0 & 53.6 & 53.3 & 54.0 & 53.6 & 52.1 & 45.2 & 37.6 & 34.0 & 33.1 & 23.5 \\
\hline 66.2 & 52.5 & 55.4 & 55.2 & 53.9 & 54.4 & 53.4 & 51.9 & 42.0 & 39.2 & 36.7 & 34.6 & 26.8 \\
\hline 72.9 & 55.9 & 58.4 & 59.0 & 57.8 & 59.3 & 58.4 & 57.2 & 51.3 & 43.8 & 39.5 & 36.0 & 26.9 \\
\hline 79.7 & 58.7 & 62.6 & 63.0 & 62.6 & 63.3 & 63.2 & 61.7 & 57.3 & 49.2 & 42.4 & 39.1 & 29.6 \\
\hline 86.6 & 62.0 & 64.9 & 65.8 & 65.9 & 66.8 & 66.6 & 65.3 & 56.5 & 50.2 & 45.2 & 42.3 & 32.0 \\
\hline 93.4 & 67.0 & 70.5 & 70.7 & 70.2 & 71.2 & 69.6 & 68.0 & 57.1 & 52.2 & 48.6 & 45.5 & 32.6 \\
\hline 100.1 & 66.1 & 68.3 & 68.3 & 66.5 & 68.4 & 67.7 & 66.6 & 60.5 & 58.8 & 51.3 & 47.4 & 37.3 \\
\hline 107.0 & 69.9 & 75.3 & 77.3 & 79.0 & 80.9 & 81.0 & 80.1 & 67.6 & 60.1 & 54.8 & 51.1 & 39.9 \\
\hline 113.8 & 72.0 & 75.7 & 76.2 & 74.9 & 76.4 & 75.7 & 75.9 & 70.8 & 65.9 & 57.0 & 54.3 & 41.6 \\
\hline 120.7 & 80.9 & 86.1 & 86.7 & 87.5 & 89.0 & 87.9 & 85.7 & 75.7 & 64.1 & 58.7 & 53.8 & 44.6 \\
\hline 127.5 & 78.7 & 81.9 & 84.0 & 83.5 & 86.4 & 87.5 & 87.4 & 85.8 & 75.9 & 63.9 & 55.3 & 45.9 \\
\hline 134.2 & 82.8 & 89.2 & 90.3 & 90.9 & 92.4 & 93.2 & 92.1 & 84.5 & 76.1 & 69.5 & 62.1 & 47.6 \\
\hline 141.1 & 85.8 & 92.1 & 93.7 & 93.4 & 95.6 & 96.5 & 94.9 & 87.3 & 81.6 & 72.1 & 66.9 & 48.8 \\
\hline 147.8 & 94.4 & 100.7 & 100.8 & 101.3 & 101.6 & 100.4 & 98.2 & 90.3 & 83.1 & 77.4 & 72.7 & 51.6 \\
\hline 154.8 & 100.3 & 107.3 & 109.0 & 109.2 & 110.2 & 110.2 & 100.9 & 89.2 & 82.4 & 76.5 & 70.3 & 55.5 \\
\hline 161.6 & 99.1 & 103.2 & 104.2 & 102.9 & 106.0 & 105.9 & 105.5 & 98.1 & 88.8 & 79.1 & 73.1 & 57.4 \\
\hline 168.3 & 105.1 & 112.0 & 113.1 & 114.2 & 116.3 & 115.9 & 113.7 & 99.0 & 90.2 & 82.4 & 75.3 & 62.7 \\
\hline 175.2 & 113.2 & 119.8 & 121.7 & 122.0 & 123.9 & 123.5 & 121.4 & 108.8 & 94.0 & 82.1 & 74.1 & 63.3 \\
\hline 181.9 & 114.8 & 119.7 & 120.7 & 118.8 & 120.6 & 119.7 & 118.0 & 108.4 & 101.3 & 97.6 & 86.5 & 66.4 \\
\hline 188.9 & 115.9 & 124.2 & 126.7 & 127.4 & 129.9 & 128.8 & 126.4 & 116.4 & 107.4 & 99.2 & 84.1 & 68.0 \\
\hline
\end{tabular}




\section{Experiment Number 15}

\begin{tabular}{|c|c|c|c|c|c|c|c|c|c|c|c|c|}
\hline 195.6 & 128.7 & 134.3 & 135.8 & 135.5 & 137.9 & 136.6 & 134.2 & 126.5 & 114.4 & 102.3 & 84.4 & 71.3 \\
\hline \multicolumn{13}{|c|}{ Thermocouples located at sprinkler number 3} \\
\hline Time & $0 \mathrm{~mm}$ & $25 \mathrm{~mm}$ & $50 \mathrm{~mm}$ & $75 \mathrm{~mm}$ & $100 \mathrm{~mm}$ & $125 \mathrm{~mm}$ & $150 \mathrm{~mm}$ & $250 \mathrm{~mm}$ & $350 \mathrm{~mm}$ & $450 \mathrm{~mm}$ & $550 \mathrm{~mm}$ & $900 \mathrm{~mm}$ \\
\hline 0.0 & 23.8 & 23.5 & 23.1 & 22.6 & 22.2 & 21.8 & 21.5 & 20.8 & 20.3 & 20.0 & 19.6 & 19.1 \\
\hline 4.7 & 24.0 & 23.9 & 23.8 & 23.3 & 22.7 & 22.3 & 22.1 & 21.5 & 21.3 & 21.0 & 20.5 & 19.8 \\
\hline 11.5 & 24.1 & 24.0 & 23.9 & 23.4 & 22.8 & 22.3 & 22.0 & 21.6 & 21.4 & 21.0 & 20.7 & 20.0 \\
\hline 18.5 & 24.2 & 24.2 & 24.2 & 23.9 & 23.6 & 23.4 & 23.5 & 22.7 & 22.4 & 21.6 & 21.2 & 20.2 \\
\hline 25.2 & 25.0 & 25.6 & 25.6 & 26.2 & 25.7 & 25.9 & 25.6 & 25.1 & 25.5 & 24.3 & 24.3 & 20.3 \\
\hline 32.1 & 28.0 & 29.6 & 28.2 & 28.7 & 28.4 & 28.8 & 29.2 & 29.4 & 29.8 & 25.4 & 21.8 & 20.2 \\
\hline 38.8 & 29.1 & 30.3 & 30.6 & 30.7 & 30.2 & 30.4 & 30.8 & 30.9 & 31.1 & 27.4 & 24.3 & 20.8 \\
\hline 45.6 & 29.0 & 30.3 & 31.3 & 31.5 & 31.9 & 32.2 & 32.7 & 33.0 & 34.1 & 31.6 & 28.6 & 21.9 \\
\hline 52.5 & 30.0 & 31.5 & 32.3 & 32.6 & 33.1 & 33.5 & 33.8 & 35.2 & 35.7 & 34.1 & 32.1 & 21.9 \\
\hline 59.3 & 35.8 & 39.3 & 37.4 & 37.6 & 37.2 & 38.3 & 38.4 & 38.8 & 38.8 & 37.0 & 36.9 & 23.9 \\
\hline 66.2 & 33.9 & 35.8 & 35.9 & 36.6 & 37.8 & 38.9 & 39.0 & 40.1 & 41.6 & 39.0 & 37.1 & 27.4 \\
\hline 72.9 & 34.0 & 38.6 & 38.7 & 40.8 & 40.1 & 42.1 & 42.8 & 42.7 & 44.4 & 42.5 & 42.1 & 29.2 \\
\hline 79.7 & 38.9 & 45.1 & 43.9 & 46.2 & 44.9 & 44.9 & 45.0 & 45.6 & 48.7 & 45.9 & 44.9 & 29.4 \\
\hline 86.6 & 42.3 & 45.7 & 43.2 & 45.6 & 45.5 & 47.1 & 46.8 & 46.8 & 49.3 & 47.6 & 47.5 & 32.8 \\
\hline 93.4 & 42.8 & 46.9 & 45.8 & 48.0 & 47.8 & 49.7 & 50.2 & 51.7 & 52.8 & 50.8 & 49.0 & 33.9 \\
\hline 100.1 & 41.8 & 44.8 & 47.5 & 49.0 & 50.2 & 52.5 & 53.4 & 55.0 & 57.7 & 53.0 & 47.9 & 36.7 \\
\hline 107.0 & 49.2 & 56.2 & 54.8 & 56.6 & 56.0 & 58.2 & 58.3 & 58.1 & 61.3 & 56.6 & 51.7 & 37.5 \\
\hline 113.8 & 52.9 & 59.4 & 59.2 & 60.2 & 59.6 & 62.0 & 62.2 & 62.4 & 62.9 & 56.8 & 52.6 & 40.5 \\
\hline 120.7 & 51.5 & 56.4 & 58.3 & 60.0 & 59.8 & 61.8 & 62.6 & 62.9 & 65.3 & 61.9 & 58.1 & 42.5 \\
\hline 127.5 & 55.4 & 61.9 & 62.1 & 63.1 & 62.9 & 64.9 & 65.6 & 65.1 & 67.0 & 64.2 & 62.1 & 44.4 \\
\hline 134.2 & 57.6 & 64.3 & 65.0 & 65.8 & 66.6 & 70.9 & 70.4 & 67.4 & 67.8 & 65.8 & 67.4 & 46.7 \\
\hline 141.1 & 58.1 & 66.3 & 65.4 & 67.0 & 67.2 & 70.3 & 71.0 & 71.7 & 72.5 & 68.9 & 67.8 & 49.8 \\
\hline 147.8 & 62.8 & 72.1 & 72.7 & 73.4 & 72.9 & 75.1 & 76.1 & 75.7 & 76.1 & 69.1 & 63.6 & 51.8 \\
\hline 154.8 & 60.1 & 63.5 & 65.9 & 67.4 & 70.5 & 73.2 & 74.9 & 75.9 & 78.0 & 74.9 & 74.2 & 52.6 \\
\hline 161.6 & 63.3 & 70.5 & 73.2 & 75.4 & 75.6 & 78.1 & 80.4 & 80.0 & 84.4 & 78.9 & 77.3 & 54.2 \\
\hline 168.3 & 70.8 & 80.2 & 78.7 & 81.0 & 80.3 & 83.9 & 85.2 & 82.8 & 85.1 & 79.1 & 78.1 & 57.6 \\
\hline 175.2 & 69.6 & 82.4 & 81.5 & 84.3 & 82.6 & 85.5 & 87.1 & 89.6 & 90.1 & 81.0 & 78.8 & 60.4 \\
\hline 181.9 & 75.6 & 85.2 & 86.7 & 88.6 & 88.5 & 91.3 & 92.8 & 92.5 & 94.7 & 88.5 & 82.9 & 64.7 \\
\hline 188.9 & 72.5 & 81.8 & 85.9 & 89.4 & 88.7 & 91.2 & 91.9 & 92.9 & 97.3 & 93.7 & 85.7 & 67.0 \\
\hline 195.6 & 82.1 & 93.4 & 94.2 & 101.1 & 98.0 & 102.3 & 100.5 & 100.7 & 102.9 & 98.1 & 93.9 & 68.9 \\
\hline \multicolumn{13}{|c|}{ Thermocouples located at sprinkler number 4} \\
\hline Time & $0 \mathrm{~mm}$ & $25 \mathrm{~mm}$ & $50 \mathrm{~mm}$ & $75 \mathrm{~mm}$ & $100 \mathrm{~mm}$ & $125 \mathrm{~mm}$ & $150 \mathrm{~mm}$ & $250 \mathrm{~mm}$ & $350 \mathrm{~mm}$ & $450 \mathrm{~mm}$ & $550 \mathrm{~mm}$ & $900 \mathrm{~mm}$ \\
\hline 0.0 & 23.2 & 23.0 & 22.9 & 22.4 & 21.9 & 21.6 & 21.3 & 20.6 & 19.9 & 19.6 & 19.3 & 19.1 \\
\hline 4.7 & 23.8 & 23.6 & 23.6 & 22.9 & 22.4 & 22.1 & 21.9 & 21.3 & 20.6 & 20.2 & 20.0 & 19.6 \\
\hline 11.5 & 23.9 & 23.7 & 23.6 & 23.2 & 22.9 & 22.6 & 22.1 & 21.4 & 20.7 & 20.3 & 20.1 & 19.7 \\
\hline 18.5 & 23.9 & 24.0 & 23.9 & 23.8 & 23.6 & 23.7 & 23.8 & 23.5 & 23.7 & 23.1 & 21.6 & 20.2 \\
\hline 25.2 & 26.2 & 26.9 & 26.5 & 27.4 & 27.9 & 28.4 & 28.9 & 27.1 & 26.9 & 25.8 & 24.4 & 20.4 \\
\hline 32.1 & 26.6 & 28.0 & 27.9 & 28.4 & 28.5 & 28.8 & 28.8 & 29.0 & 30.1 & 28.3 & 26.0 & 20.5 \\
\hline 38.8 & 31.1 & 30.7 & 29.7 & 31.8 & 31.7 & 32.4 & 33.0 & 32.2 & 32.6 & 31.1 & 28.6 & 21.5 \\
\hline 45.6 & 32.7 & 32.2 & 31.2 & 37.2 & 36.5 & 36.8 & 36.6 & 35.1 & 34.9 & 33.3 & 29.4 & 21.5 \\
\hline 52.5 & 36.3 & 35.8 & 35.2 & 36.9 & 37.1 & 37.7 & 38.0 & 37.5 & 38.4 & 36.8 & 36.8 & 22.9 \\
\hline 59.3 & 34.7 & 35.7 & 36.4 & 37.7 & 37.4 & 38.4 & 38.9 & 40.2 & 42.3 & 41.2 & 39.7 & 22.1 \\
\hline 66.2 & 38.0 & 38.3 & 37.2 & 40.3 & 40.7 & 41.1 & 42.0 & 42.2 & 43.0 & 42.5 & 40.0 & 24.2 \\
\hline 72.9 & 41.9 & 41.6 & 41.7 & 46.7 & 46.7 & 47.4 & 47.6 & 46.0 & 46.0 & 44.2 & 43.7 & 26.3 \\
\hline 79.7 & 39.9 & 40.9 & 41.6 & 42.3 & 42.3 & 43.1 & 44.4 & 45.1 & 46.9 & 47.4 & 46.5 & 30.8 \\
\hline 86.6 & 37.0 & 41.2 & 41.3 & 44.2 & 45.7 & 46.5 & 47.7 & 48.1 & 49.0 & 49.7 & 50.1 & 32.1 \\
\hline 93.4 & 39.0 & 45.8 & 46.1 & 50.0 & 51.6 & 52.3 & 52.3 & 52.0 & 51.7 & 51.1 & 51.8 & 34.2 \\
\hline 100.1 & 51.7 & 56.0 & 56.6 & 55.5 & 55.1 & 55.6 & 55.4 & 55.0 & 56.2 & 55.9 & 56.6 & 34.7 \\
\hline 107.0 & 49.0 & 52.3 & 51.4 & 53.1 & 53.4 & 53.9 & 54.9 & 55.2 & 57.8 & 60.3 & 60.7 & 36.9 \\
\hline 113.8 & 49.2 & 51.0 & 52.7 & 52.9 & 52.7 & 53.9 & 54.7 & 57.4 & 59.5 & 60.2 & 62.0 & 40.4 \\
\hline 120.7 & 60.7 & 56.7 & 56.5 & 64.0 & 64.2 & 65.8 & 67.4 & 65.3 & 66.2 & 63.4 & 62.2 & 42.3 \\
\hline 127.5 & 56.9 & 58.8 & 58.5 & 61.0 & 63.4 & 65.3 & 65.9 & 65.4 & 67.2 & 66.1 & 67.0 & 46.9 \\
\hline 134.2 & 60.0 & 61.1 & 60.6 & 64.7 & 66.7 & 67.9 & 69.3 & 69.3 & 73.0 & 72.7 & 72.1 & 45.1 \\
\hline 141.1 & 60.1 & 60.2 & 59.1 & 61.2 & 63.5 & 64.4 & 65.7 & 68.4 & 70.8 & 71.9 & 73.3 & 58.3 \\
\hline 147.8 & 54.1 & 57.7 & 57.3 & 64.7 & 67.8 & 70.4 & 72.7 & 73.7 & 76.7 & 75.1 & 76.5 & 59.4 \\
\hline 154.8 & 66.7 & 74.0 & 70.2 & 77.6 & 79.3 & 79.4 & 80.9 & 81.0 & 83.5 & 80.2 & 79.3 & 58.5 \\
\hline 161.6 & 60.8 & 63.4 & 65.1 & 67.4 & 68.9 & 70.4 & 71.2 & 74.5 & 77.5 & 77.7 & 81.0 & 62.8 \\
\hline 168.3 & 67.8 & 73.4 & 71.8 & 78.2 & 81.5 & 82.6 & 83.9 & 84.6 & 87.6 & 87.2 & 89.2 & 62.5 \\
\hline 175.2 & 72.9 & 80.9 & 79.9 & 84.0 & 86.0 & 86.8 & 88.1 & 86.8 & 89.8 & 92.1 & 93.1 & 61.3 \\
\hline 181.9 & 66.5 & 75.3 & 73.1 & 15.4 & 80.7 & 82.3 & 84.1 & 84.0 & 86.7 & 86.7 & 92.5 & 71.5 \\
\hline
\end{tabular}




\section{Experiment Number 15}

\begin{tabular}{|c|c|c|c|c|c|c|c|c|c|c|c|c|}
\hline 188.9 & 66.2 & 75.9 & 77.9 & 81.6 & 86.2 & 87.2 & 88.6 & 89.6 & 91.2 & 88.0 & 92.7 & 75.1 \\
\hline 195.6 & 77.9 & 88.2 & 88.3 & 93.9 & 98.9 & 100.8 & 101.9 & 101.4 & 103.2 & 101.8 & 101.0 & 69.1 \\
\hline \multicolumn{5}{|c|}{ Velocity probes at sprinkler number 1} & \multicolumn{6}{|c|}{ Velocity probes at sprinkler number 2} & & \\
\hline Time & $25 \mathrm{~mm}$ & $75 \mathrm{~mm}$ & $125 \mathrm{~mm}$ & $250 \mathrm{~mm}$ & & Time & $25 \mathrm{~mm}$ & $75 \mathrm{~mm}$ & $125 \mathrm{~mm}$ & $250 \mathrm{~mm}$ & & \\
\hline 0.0 & 0.0 & 0.0 & 0.0 & 0.0 & & 0.0 & 0.0 & 0.0 & 0.0 & 0.0 & & \\
\hline 4.7 & -0.1 & 0.1 & -0.1 & -0.1 & & 4.7 & -0.1 & -0.1 & 0.0 & -0.1 & & \\
\hline 11.5 & -0.3 & -0.3 & -0.3 & -0.3 & & 11.5 & 0.3 & 0.4 & 0.4 & 0.2 & & \\
\hline 18.5 & -0.5 & -0.4 & -0.4 & -0.1 & & 18.5 & 0.5 & 0.5 & 0.4 & -0.1 & & \\
\hline 25.2 & -0.5 & -0.5 & -0.5 & -0.3 & & 25.2 & 0.5 & 0.4 & 0.4 & 0.2 & & \\
\hline 32.1 & .0 .4 & -0.3 & -0.3 & -0.1 & & 32.1 & 0.6 & 0.5 & 0.5 & 0.1 & & \\
\hline 38.8 & -0.4 & -0.4 & -0.4 & -0.2 & & 38.8 & 0.3 & 0.3 & 0.3 & 0.2 & & \\
\hline 45.6 & -0.5 & -0.5 & -0.5 & -0.3 & & 45.6 & 0.5 & 0.5 & 0.4 & 0.1 & & \\
\hline 52.5 & -0.6 & -0.7 & -0.7 & -0.4 & & 52.5 & 0.5 & 0.5 & 0.5 & 0.3 & & \\
\hline 59.3 & -0.6 & -0.6 & -0.6 & -0.3 & & 59.3 & 0.5 & 0.5 & 0.6 & 0.3 & & \\
\hline 66.2 & -0.5 & -0.6 & -0.5 & -0.4 & & 66.2 & 0.7 & 0.7 & 0.6 & 0.2 & & \\
\hline 72.9 & -0.5 & -0.6 & -0.4 & -0.3 & & 72.9 & 0.6 & 0.6 & 0.7 & 0.4 & & \\
\hline 79.7 & -0.7 & -0.7 & -0.6 & -0.4 & & 79.7 & 0.6 & 0.5 & 0.5 & 0.3 & & \\
\hline 86.6 & -0.7 & -0.7 & -0.4 & -0.3 & & 86.6 & 0.6 & 0.7 & 0.7 & 0.3 & & \\
\hline 93.4 & -0.7 & .0 .7 & -0.5 & -0.2 & & 93.4 & 0.8 & 0.9 & 0.9 & 0.6 & & \\
\hline 100.1 & -0.7 & -0.7 & -0.7 & -0.4 & & 100.1 & 0.6 & 0.7 & 0.8 & 0.6 & & \\
\hline 107.0 & -0.8 & -0.9 & -0.8 & -0.5 & & 107.0 & 0.5 & 0.5 & 0.6 & 0.4 & & \\
\hline 113.8 & -0.7 & -0.7 & -0.4 & -0.4 & & 113.8 & 0.7 & 0.7 & 0.7 & 0.3 & & \\
\hline 120.7 & -0.6 & -0.6 & -0.4 & -0.3 & & 120.7 & 0.5 & 0.5 & 0.7 & 0.6 & & \\
\hline 127.5 & -0.7 & -0.8 & -0.7 & -0.5 & & 127.5 & 0.5 & 0.4 & 0.6 & 0.2 & & \\
\hline 134.2 & -0.4 & -0.5 & -0.4 & -0.5 & & 134.2 & 0.6 & 0.7 & 0.8 & 0.4 & & \\
\hline 141.1 & -0.8 & -0.9 & -0.8 & -0.4 & & 141.1 & 0.6 & 0.5 & 0.5 & 0.1 & & \\
\hline 147.8 & -0.8 & -0.9 & -0.6 & -0.4 & & 147.8 & 0.9 & 0.9 & 1.0 & 0.1 & & \\
\hline 154.8 & -0.8 & -0.9 & -0.8 & -0.5 & & 154.8 & 0.7 & 0.7 & 0.9 & 0.4 & & \\
\hline 161.6 & -0.9 & -0.9 & -0.8 & .0 .4 & & 161.6 & 0.8 & 0.8 & 0.9 & 0.4 & & \\
\hline 168.3 & -1.1 & -1.0 & -0.8 & -0.6 & & 168.3 & 0.8 & 0.9 & 0.9 & 0.4 & & \\
\hline 175.2 & -0.8 & -0.8 & -0.7 & -0.5 & & 175.2 & 0.5 & 0.6 & 0.8 & 0.7 & & \\
\hline 181.9 & -0.8 & -0.9 & -0.7 & -0.6 & & 181.9 & 0.6 & 0.7 & 0.9 & 0.5 & & \\
\hline 188.9 & -0.7 & -0.7 & -0.7 & -0.7 & & 188.9 & 0.8 & 0.7 & 1.0 & 0.6 & & \\
\hline 195.6 & -0.7 & -1.0 & -0.8 & -0.7 & & 195.6 & 0.8 & 0.8 & 1.0 & 0.6 & & \\
\hline \multicolumn{12}{|c|}{ Velocity probes in channels above burner } & \\
\hline Time & Channel 1 & Channel 2 & & & & & & & & & & \\
\hline 0.0 & 0.0 & 0.0 & & & & & & & & & & \\
\hline 4.7 & 1.1 & 0.6 & & & & & & & & & & \\
\hline 11.5 & 1.1 & 0.5 & & & & & & & & & & \\
\hline 18.5 & 1.3 & 0.5 & & & & & & & & & & \\
\hline 25.2 & 1.7 & 0.6 & & & & & & & & & & \\
\hline 32.1 & 1.4 & 0.8 & & & & & & & & & & \\
\hline 38.8 & 1.6 & 1.0 & & & & & & & & & & \\
\hline 45.6 & 1.6 & 1.0 & & & & & & & & & & \\
\hline 52.5 & 2.1 & 1.0 & & & & & & & & & & \\
\hline 59.3 & 1.8 & 1.0 & & & & & & & & & & \\
\hline 66.2 & 1.8 & 1.0 & & & & & & & & & & \\
\hline 72.9 & 2.3 & 0.8 & & & & & & & & & & \\
\hline 79.7 & 2.3 & 0.8 & & & & & & & & & & \\
\hline 86.6 & 2.0 & 1.1 & & & & & & & & & & \\
\hline 93.4 & 1.8 & 1.5 & & & & & & & & & & \\
\hline 100.1 & 2.0 & 0.8 & & & & & & & & & & \\
\hline 107.0 & 2.0 & 1.1 & & & & & & & & & & \\
\hline 113.8 & 2.6 & 0.7 & & & & & & & & & & \\
\hline 120.7 & 3.0 & 1.2 & & & & & & & & & & \\
\hline 127.5 & 2.2 & 1.2 & & & & & & & & & & \\
\hline 134.2 & 1.9 & 1.2 & & & & & & & & & & \\
\hline 141.1 & 2.6 & 1.2 & & & & & & & & & & \\
\hline 147.8 & 2.2 & 1.7 & & & & & & & & & & \\
\hline 154.8 & 2.5 & 1.3 & & & & & & & & & & \\
\hline 161.6 & 2.4 & 1.6 & & & & & & & & & & \\
\hline 168.3 & 2.8 & 1.1 & & & & & & & & & & \\
\hline 175.2 & 2.7 & 1.3 & & & & & & & & & & \\
\hline
\end{tabular}




\section{Experiment Number 15}

$\begin{array}{lll}181.9 & 2.3 & 1.1 \\ 188.9 & 3.1 & 1.2 \\ 195.6 & 2.9 & 1.1\end{array}$




\section{Experiment Number 16}

Thermocouples located at sprinkler number

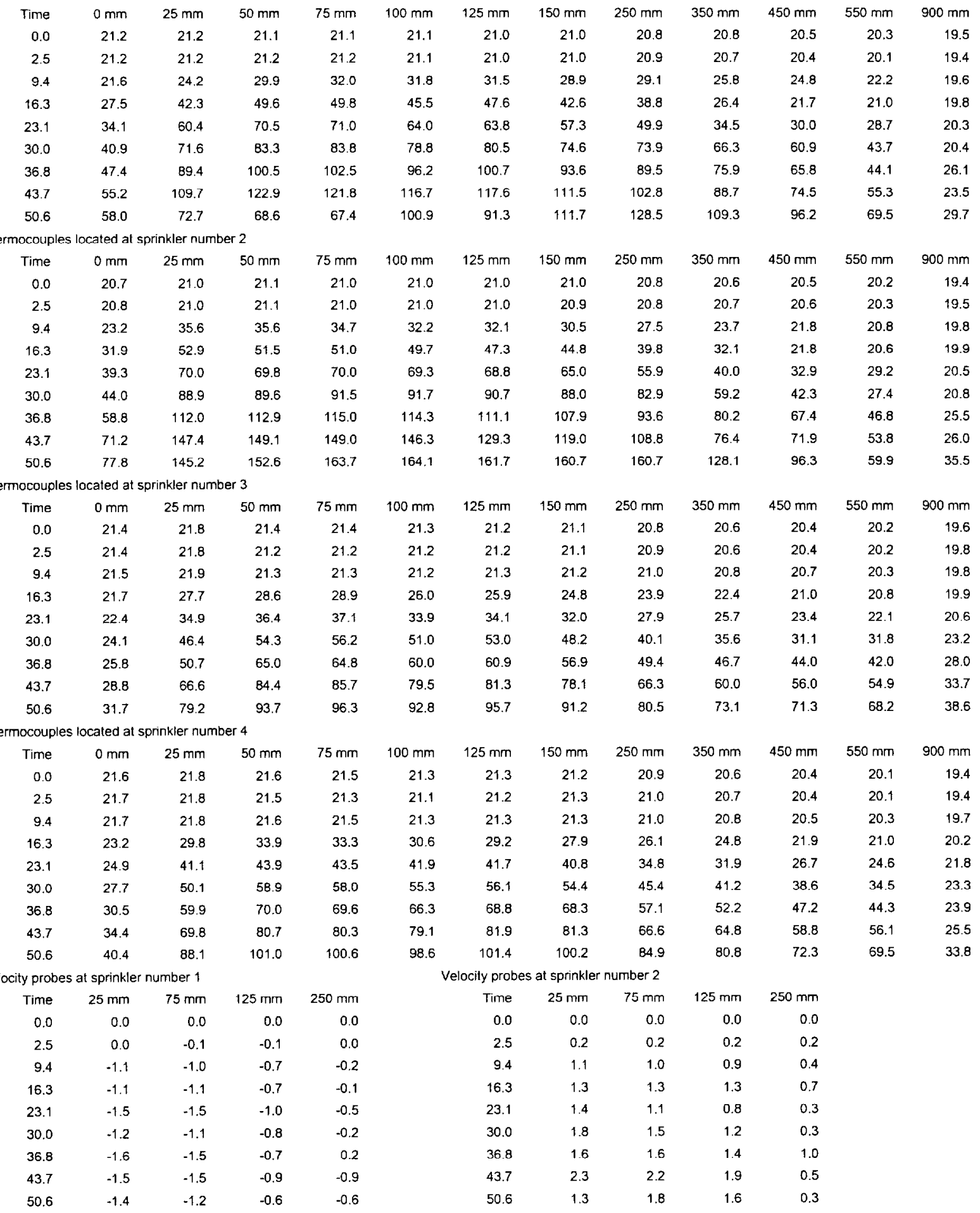




\section{Experiment Number 17}

\begin{tabular}{|c|c|c|c|c|c|c|c|c|c|c|c|c|}
\hline Time & $0 \mathrm{~mm}$ & $25 \mathrm{~mm}$ & $50 \mathrm{~mm}$ & $75 \mathrm{~mm}$ & $100 \mathrm{~mm}$ & $125 \mathrm{~mm}$ & $150 \mathrm{~mm}$ & $250 \mathrm{~mm}$ & $350 \mathrm{~mm}$ & $450 \mathrm{~mm}$ & $550 \mathrm{~mm}$ & $900 \mathrm{~mm}$ \\
\hline 0.0 & 20.4 & 20.3 & 20.3 & 19.8 & 19.9 & 19.4 & 19.7 & 19.0 & 18.9 & 18.6 & 18.4 & 17.8 \\
\hline 0.3 & 20.7 & 20.6 & 20.5 & 20.2 & 20.3 & 19.9 & 20.0 & 19.0 & 18.8 & 18.4 & 18.2 & 18.0 \\
\hline 7.2 & 20.7 & 22.0 & 23.6 & 24.9 & 26.0 & 25.7 & 24.8 & 27.3 & 23.5 & 21.9 & 21.8 & 17.9 \\
\hline 14.1 & 29.4 & 48.2 & 54.8 & 53.6 & 49.1 & 50.8 & 45.3 & 33.3 & 26.1 & 20.2 & 19.2 & 18.1 \\
\hline 20.8 & 33.7 & 56.1 & 61.5 & 61.5 & 57.4 & 58.5 & 54.7 & 52.0 & 44.4 & 37.9 & 25.6 & 18.4 \\
\hline 27.7 & 40.5 & 76.2 & 83.0 & 83.6 & 80.0 & 82.9 & 78.0 & 68.7 & 56.2 & 42.0 & 25.7 & 18.7 \\
\hline 34.5 & 52.0 & 100.7 & 113.4 & 113.6 & 109.5 & 111.3 & 104.3 & 96.8 & 85.5 & 69.7 & 51.7 & 23.5 \\
\hline 41.4 & 60.6 & 116.7 & 130.3 & 132.7 & 126.7 & 128.1 & 121.9 & 117.6 & 104.6 & 78.0 & 56.8 & 21.5 \\
\hline \multicolumn{13}{|c|}{ Thermocouples located at sprinkler number 2} \\
\hline Time & $0 \mathrm{~mm}$ & $25 \mathrm{~mm}$ & $50 \mathrm{~mm}$ & $75 \mathrm{~mm}$ & $100 \mathrm{~mm}$ & $125 \mathrm{~mm}$ & $150 \mathrm{~mm}$ & $250 \mathrm{~mm}$ & $350 \mathrm{~mm}$ & $450 \mathrm{~mm}$ & $550 \mathrm{~mm}$ & $900 \mathrm{~mm}$ \\
\hline 0.0 & 20.7 & 20.2 & 20.1 & 19.6 & 19.6 & 19.3 & 19.4 & 19.0 & 18.7 & 18.4 & 18.2 & 17.7 \\
\hline 0.3 & 20.8 & 20.6 & 20.4 & 19.8 & 20.2 & 19.7 & 20.2 & 19.6 & 19.4 & 19.2 & 18.9 & 17.9 \\
\hline 7.2 & 22.6 & 28.5 & 29.8 & 31.0 & 30.8 & 32.7 & 32.7 & 31.1 & 28.8 & 25.8 & 21.3 & 18.2 \\
\hline 14.1 & 31.6 & 57.4 & 58.3 & 59.6 & 59.3 & 59.5 & 55.4 & 49.1 & 30.0 & 20.6 & 19.7 & 18.5 \\
\hline 20.8 & 40.3 & 71.0 & 71.8 & 72.2 & 71.8 & 70.6 & 69.0 & 63.0 & 49.3 & 38.7 & 33.3 & 18.8 \\
\hline 27.7 & 48.4 & 90.4 & 92.7 & 95.2 & 94.4 & 91.3 & 90.6 & 81.9 & 61.4 & 51.5 & 32.1 & 19.3 \\
\hline 34.5 & 55.9 & 110.7 & 110.8 & 113.6 & 112.6 & 111.4 & 109.0 & 93.9 & 79.8 & 74.0 & 61.7 & 25.5 \\
\hline 41.4 & 64.6 & 123.1 & 126.9 & 129.4 & 130.2 & 129.0 & 125.5 & 112.4 & 93.9 & 78.7 & 64.3 & 27.3 \\
\hline \multicolumn{13}{|c|}{ Thermocouples located at sprinkler number 3} \\
\hline Time & $0 \mathrm{~mm}$ & $25 \mathrm{~mm}$ & $50 \mathrm{~mm}$ & $75 \mathrm{~mm}$ & $100 \mathrm{~mm}$ & $125 \mathrm{~mm}$ & $150 \mathrm{~mm}$ & $250 \mathrm{~mm}$ & $350 \mathrm{~mm}$ & $450 \mathrm{~mm}$ & $550 \mathrm{~mm}$ & $900 \mathrm{~mm}$ \\
\hline 0.0 & 20.5 & 20.7 & 19.8 & 19.6 & 19.4 & 19.3 & 19.2 & 18.9 & 18.7 & 18.5 & 18.3 & 17.9 \\
\hline 0.3 & 20.9 & 21.1 & 20.2 & 20.0 & 19.9 & 19.8 & 19.7 & 19.3 & 19.0 & 18.7 & 18.6 & 18.2 \\
\hline 7.2 & 20.6 & 21.0 & 20.3 & 20.1 & 20.0 & 20.0 & 19.9 & 19.6 & 19.3 & 19.0 & 18.7 & 18.4 \\
\hline 14.1 & 21.0 & 27.8 & 28.4 & 29.9 & 26.7 & 27.5 & 26.4 & 23.0 & 20.2 & 19.5 & 19.2 & 18.7 \\
\hline 20.8 & 21.9 & 34.9 & 40.7 & 41.4 & 38.0 & 39.6 & 35.6 & 30.9 & 27.6 & 25.3 & 22.6 & 20.4 \\
\hline 27.7 & 23.5 & 45.2 & 52.9 & 55.5 & 50.5 & 50.5 & 46.9 & 39.0 & 35.6 & 32.1 & 30.4 & 21.3 \\
\hline 34.5 & 25.7 & 52.1 & 62.5 & 66.2 & 62.7 & 64.5 & 60.8 & 50.7 & 45.3 & 41.5 & 42.7 & 24.7 \\
\hline 41.4 & 28.1 & 63.2 & 80.4 & 84.3 & 79.5 & 80.8 & 75.4 & 65.7 & 61.2 & 56.2 & 56.7 & 36.2 \\
\hline \multicolumn{13}{|c|}{ Thermocouples located at sprinkler number 4} \\
\hline Time & $0 \mathrm{~mm}$ & $25 \mathrm{~mm}$ & $50 \mathrm{~mm}$ & $75 \mathrm{~mm}$ & $100 \mathrm{~mm}$ & $125 \mathrm{~mm}$ & $150 \mathrm{~mm}$ & $250 \mathrm{~mm}$ & $350 \mathrm{~mm}$ & $450 \mathrm{~mm}$ & $550 \mathrm{~mm}$ & $900 \mathrm{~mm}$ \\
\hline 0.0 & 20.5 & 20.3 & 20.2 & 20.0 & 19.7 & 19.7 & 19.6 & 19.1 & 18.6 & 18.5 & 18.3 & 17.8 \\
\hline 0.3 & 20.8 & 20.4 & 20.1 & 19.9 & 19.7 & 19.6 & 19.5 & 19.1 & 18.5 & 18.3 & 18.2 & 17.8 \\
\hline 7.2 & 20.6 & 20.5 & 20.4 & 20.2 & 19.8 & 19.6 & 19.5 & 19.1 & 18.5 & 18.5 & 18.4 & 18.1 \\
\hline 14.1 & 22.2 & 27.2 & 29.8 & 28.9 & 26.9 & 26.4 & 24.7 & 22.7 & 22.3 & 20.2 & 18.8 & 18.4 \\
\hline 20.8 & 24.8 & 41.9 & 45.5 & 44.8 & 43.4 & 44.5 & 44.2 & 37.2 & 35.5 & 27.0 & 24.1 & 20.9 \\
\hline 27.7 & 27.7 & 51.8 & 58.0 & 57.2 & 55.2 & 56.5 & 56.2 & 46.1 & 41.5 & 37.1 & 33.5 & 21.9 \\
\hline 34.5 & 31.6 & 65.6 & 70.3 & 69.8 & 66.6 & 67.8 & 66.9 & 57.2 & 52.2 & 49.1 & 46.3 & 22.2 \\
\hline 41.4 & 37.6 & 79.6 & 91.0 & 90.1 & 87.5 & 89.5 & 87.7 & 74.0 & 68.1 & 64.9 & 62.8 & 25.3 \\
\hline \multicolumn{5}{|c|}{ Velocity probes at sprinkler number 1} & \multicolumn{6}{|c|}{ Velocity probes at sprinkler number 2} & & \\
\hline Time & $25 \mathrm{~mm}$ & $75 \mathrm{~mm}$ & $125 \mathrm{~mm}$ & $250 \mathrm{~mm}$ & & Time & $25 \mathrm{~mm}$ & $75 \mathrm{~mm}$ & $125 \mathrm{~mm}$ & $250 \mathrm{~mm}$ & & \\
\hline 0.0 & 0.0 & 0.0 & 0.0 & 0.0 & & 0.0 & 0.0 & 0.0 & 0.0 & 0.0 & & \\
\hline 0.3 & -0.1 & 0.1 & -0.1 & -0.1 & & 0.3 & -0.1 & -0.1 & -0.1 & -0.1 & & \\
\hline 7.2 & -1.3 & -1.5 & -1.4 & -0.4 & & 7.2 & 0.9 & 0.8 & 0.8 & 0.6 & & \\
\hline 14.1 & -0.9 & -0.9 & -0.8 & -0.8 & & 14.1 & 1.2 & 1.3 & 1.0 & 0.5 & & \\
\hline 20.8 & -1.5 & -1.4 & $\cdot 1.2$ & -0.5 & & 20.8 & 1.7 & 1.7 & 1.3 & 0.7 & & \\
\hline 27.7 & -1.2 & -1.1 & -0.7 & -0.1 & & 27.7 & 1.5 & 1.4 & 1.2 & 0.7 & & \\
\hline 34.5 & -1.3 & -1.5 & -1.6 & -0.7 & & 34.5 & 1.8 & 1.5 & 1.2 & 0.4 & & \\
\hline 41.4 & -1.7 & -1.8 & -1.8 & -1.2 & & 41.4 & 2.0 & 2.0 & 1.5 & 0.5 & & \\
\hline
\end{tabular}




\section{Experiment Number 18}

Thermocouples located at sprinkler number 1

\begin{tabular}{|c|c|c|c|c|c|c|c|c|c|c|c|c|}
\hline Time & $0 \mathrm{~mm}$ & $25 \mathrm{~mm}$ & $50 \mathrm{~mm}$ & $75 \mathrm{~mm}$ & $100 \mathrm{~mm}$ & $125 \mathrm{~mm}$ & $150 \mathrm{~mm}$ & $250 \mathrm{~mm}$ & $350 \mathrm{~mm}$ & $450 \mathrm{~mm}$ & $550 \mathrm{~mm}$ & $900 \mathrm{~mm}$ \\
\hline 0.0 & 19.9 & 19.6 & 19.7 & 19.3 & 19.4 & 19.0 & 19.3 & 18.6 & 18.6 & 18.3 & 17.9 & 17.2 \\
\hline 0.3 & 20.0 & 19.9 & 20.0 & 19.8 & 19.7 & 19.3 & 19.4 & 18.8 & 18.5 & 18.2 & 17.9 & 17.3 \\
\hline 7.2 & 20.1 & 20.3 & 21.3 & 22.1 & 23.3 & 24.9 & 23.8 & 25.5 & 23.4 & 20.6 & 18.5 & 17.5 \\
\hline 14.1 & 26.4 & 42.0 & 51.6 & 53.9 & 46.9 & 48.7 & 44.0 & 36.2 & 21.6 & 19.3 & 18.3 & 17.7 \\
\hline 20.8 & 35.1 & 62.6 & 69.5 & 70.4 & 64.9 & 65.9 & 59.6 & 55.3 & 42.1 & 34.3 & 26.4 & $\uparrow 8.1$ \\
\hline 27.8 & 40.8 & 79.2 & 88.1 & 88.1 & 81.5 & 83.2 & 78.9 & 73.8 & 55.1 & 38.9 & 24.4 & 18.3 \\
\hline 34.5 & 55.4 & 108.2 & 119.6 & 120.2 & 112.4 & 112.8 & 104.1 & 87.7 & 75.2 & 62.0 & 36.0 & 21.3 \\
\hline 41.5 & 58.5 & 114.6 & 127.1 & 128.5 & 120.4 & 122.2 & 115.5 & 100.4 & 96.6 & 80.4 & 52.9 & 20.9 \\
\hline \multicolumn{13}{|c|}{ mocouples located at sprinkler number 2} \\
\hline Time & $0 \mathrm{~mm}$ & $25 \mathrm{~mm}$ & $50 \mathrm{~mm}$ & $75 \mathrm{~mm}$ & $100 \mathrm{~mm}$ & $125 \mathrm{~mm}$ & $150 \mathrm{~mm}$ & $250 \mathrm{~mm}$ & $350 \mathrm{~mm}$ & $450 \mathrm{~mm}$ & $550 \mathrm{~mm}$ & $900 \mathrm{~mm}$ \\
\hline 0.0 & 20.3 & 19.7 & 19.6 & 19.2 & 19.2 & 18.8 & 19.0 & 18.6 & 18.3 & 18.1 & 17.8 & 17.3 \\
\hline 0.3 & 20.4 & 20.0 & 19.9 & 19.3 & 19.7 & 19.2 & 19.5 & 18.9 & 18.6 & 18.3 & 18.0 & 17.6 \\
\hline 7.2 & 23.0 & 33.4 & 32.9 & 33.3 & 32.0 & 31.8 & 29.9 & 26.8 & 22.8 & 19.7 & 18.4 & 17.6 \\
\hline 14.1 & 31.1 & 57.1 & 55.9 & 57.4 & 57.8 & 56.5 & 54.0 & 51.1 & 31.4 & 20.9 & 18.5 & 17.9 \\
\hline 20.8 & 39.5 & 68.7 & 69.0 & 69.9 & 67.0 & 67.0 & 65.1 & 61.0 & 50.7 & 35.7 & 31.2 & 18.4 \\
\hline 27.8 & 49.6 & 94.3 & 96.2 & 98.4 & 97.0 & 95.0 & 93.0 & 85.9 & 70.2 & 58.0 & 33.0 & 18.9 \\
\hline 34.5 & 59.7 & 118.0 & 116.7 & 119.6 & 118.1 & 117.8 & 115.3 & 97.4 & 84.2 & 65.6 & 46.0 & 26.0 \\
\hline $4 \uparrow .5$ & 68.2 & 64.4 & 112.7 & 57.9 & 110.7 & 100.5 & 98.8 & 109.1 & 97.6 & 89.4 & 71.8 & 24.6 \\
\hline \multicolumn{13}{|c|}{ rmocouples located al sprinkler number 3} \\
\hline Time & $0 \mathrm{~mm}$ & $25 \mathrm{~mm}$ & $50 \mathrm{~mm}$ & $75 \mathrm{~mm}$ & $100 \mathrm{~mm}$ & $125 \mathrm{~mm}$ & $150 \mathrm{~mm}$ & $250 \mathrm{~mm}$ & $350 \mathrm{~mm}$ & $450 \mathrm{~mm}$ & $550 \mathrm{~mm}$ & $900 \mathrm{~mm}$ \\
\hline 0.0 & 19.8 & 20.1 & 19.2 & 19.0 & 18.9 & 18.9 & $18 . B$ & 18.5 & 18.3 & 18.0 & 17.8 & 17.4 \\
\hline 0.3 & 20.1 & 20.4 & 19.5 & 19.4 & 19.3 & 19.3 & 19.3 & 19.1 & 18.8 & 18.5 & 18.1 & 18.0 \\
\hline 7.2 & 19.9 & 20.3 & 19.6 & 19.5 & 19.4 & 19.3 & 19.3 & 19.1 & 18.9 & 18.7 & 18.2 & 18.1 \\
\hline 14.1 & 20.2 & 27.3 & 28.2 & 30.9 & 26.7 & 27.1 & 24.8 & 22.5 & 22.2 & 20.3 & 19.4 & 18.2 \\
\hline 20.8 & 21.1 & 33.3 & 38.3 & 39.8 & 36.1 & 36.3 & 33.6 & 28.4 & 26.5 & 21.9 & 21.2 & 18.8 \\
\hline 27.8 & 22.3 & 40.9 & 47.0 & 48.7 & 45.3 & 45.7 & 44.7 & 39.6 & 36.0 & 31.7 & 32.0 & 21.7 \\
\hline 34.5 & 24.1 & 50.3 & 61.4 & 64.0 & 61.5 & 62.4 & 59.7 & 52.7 & 16.8 & 12.0 & 41.5 & 26.8 \\
\hline 41.5 & 28.1 & 63.1 & 85.2 & 86.9 & B1.8 & 83.6 & 78.2 & 68.4 & 62.4 & 55.5 & 56.1 & 33.5 \\
\hline \multicolumn{13}{|c|}{ rmocouples located at sprinkler number 4} \\
\hline Time & $0 \mathrm{~mm}$ & $25 \mathrm{~mm}$ & $50 \mathrm{~mm}$ & $75 \mathrm{~mm}$ & $100 \mathrm{~mm}$ & $125 \mathrm{~mm}$ & $150 \mathrm{~mm}$ & $250 \mathrm{~mm}$ & $350 \mathrm{~mm}$ & $450 \mathrm{~mm}$ & $550 \mathrm{~mm}$ & $900 \mathrm{~mm}$ \\
\hline 0.0 & 19.7 & 19.6 & 19.4 & 19.3 & 19.1 & 19.0 & 19.0 & 18.7 & 18.3 & 18.0 & 17.8 & 17.3 \\
\hline 0.3 & 19.9 & 19.8 & 19.6 & 19.4 & 19.1 & 19.0 & 19.0 & 18.8 & 18.3 & 18.0 & 17.8 & 17.3 \\
\hline 7.2 & 19.9 & 19.9 & 19.7 & 19.7 & 19.4 & 19.3 & 19.2 & 18.7 & 18.1 & 17.9 & 17.8 & 17.6 \\
\hline 14.1 & 23.2 & 29.5 & 32.7 & 32.4 & 29.7 & 27.9 & 26.0 & 22.8 & 21.4 & 20.5 & 18.7 & 17.9 \\
\hline 20.8 & 24.4 & 44.3 & 46.7 & 45.7 & 42.8 & 43.2 & 42.1 & 34.6 & 31.0 & 26.6 & 26.1 & 21.0 \\
\hline 27.8 & 27.9 & 51.3 & 54.5 & 54.1 & 52.7 & 54.8 & 54.5 & 45.5 & 44.1 & 38.0 & 33.1 & 22.1 \\
\hline 34.5 & 32.2 & 73.1 & 76.7 & 75.0 & 70.0 & 74.5 & 71.3 & 59.8 & 53.6 & 49.2 & 45.8 & 22.0 \\
\hline 41.5 & 38.2 & 82.5 & 89.3 & 88.4 & 85.4 & 87.6 & 86.1 & 70.7 & 66.1 & 63.8 & 62.7 & 23.8 \\
\hline \multicolumn{6}{|c|}{ city probes at sprinkler number 1} & \multicolumn{7}{|c|}{ Velocity probes at sprinkler number 2} \\
\hline Time & $25 \mathrm{~mm}$ & $75 \mathrm{~mm}$ & $125 \mathrm{~mm}$ & $250 \mathrm{~mm}$ & & Time & $25 \mathrm{~mm}$ & $75 \mathrm{~mm}$ & $125 \mathrm{~mm}$ & $250 \mathrm{~mm}$ & & \\
\hline 0.0 & 0.0 & 0.0 & 0.0 & 0.0 & & 0.0 & 0.0 & 0.0 & 0.0 & 0.0 & & \\
\hline 0.3 & -0.1 & 0.0 & -0.1 & 0.0 & & 0.3 & 0.1 & -0.1 & 0.1 & 0.1 & & \\
\hline 7.2 & -0.9 & -1.0 & -0.9 & -0.3 & & 7.2 & 0.9 & 0.8 & 0.8 & 0.4 & & \\
\hline 14.1 & -1.5 & -1.2 & -0.5 & -0.1 & & 14.1 & 1.7 & 1.7 & 1.5 & 1.1 & & \\
\hline 20.8 & -1.6 & -1.6 & -1.6 & -1.0 & & 20.8 & 1.7 & 1.7 & 1.6 & 0.9 & & \\
\hline 27.8 & -1.9 & -1.9 & -1.3 & 0.0 & & 27.8 & 1.6 & 1.3 & 1.1 & 0.6 & & \\
\hline 34.5 & -0.9 & -1.2 & -1.2 & -0.5 & & 34.5 & 2.0 & 1.7 & 1.2 & -0.4 & & \\
\hline 41.5 & -0.6 & -0.9 & -1.1 & -0.8 & & 41.5 & 1.0 & 1.2 & 1.4 & 0.9 & & \\
\hline
\end{tabular}


Experiment Number 19

Thermocouples located at sprinkler number 1

\begin{tabular}{|c|c|c|c|c|c|c|c|c|c|c|c|c|}
\hline Time & $0 \mathrm{~mm}$ & $25 \mathrm{~mm}$ & $50 \mathrm{~mm}$ & $75 \mathrm{~mm}$ & $100 \mathrm{~mm}$ & $125 \mathrm{~mm}$ & $150 \mathrm{~mm}$ & $250 \mathrm{~mm}$ & $350 \mathrm{~mm}$ & $450 \mathrm{~mm}$ & $550 \mathrm{~mm}$ & $900 \mathrm{~mm}$ \\
\hline 0.0 & 20.4 & 20.4 & 20.5 & 20.6 & 20.6 & 20.5 & 20.6 & 20.5 & 20.6 & 20.6 & 20.4 & 19.8 \\
\hline 6.1 & 20.4 & 20.5 & 20.5 & 20.5 & 20.6 & 20.5 & 20.6 & 20.6 & 20.6 & 20.6 & 20.4 & 19.9 \\
\hline 12.0 & 20.8 & 20.8 & 20.9 & 20.8 & 20.9 & 20.8 & 20.8 & 20.8 & 20.9 & 20.8 & 20.9 & 20.2 \\
\hline 19.6 & 27.1 & 28.4 & 30.7 & 31.6 & 31.4 & 31.7 & 30.0 & 31.5 & 26.7 & 23.4 & 22.3 & 20.6 \\
\hline 26.5 & 40.6 & 40.0 & 42.8 & 44.5 & 43.5 & 45.0 & 43.4 & 40.5 & 33.9 & 26.8 & 21.8 & 20.9 \\
\hline 33.3 & 54.5 & 55.5 & 60.2 & 61.7 & 58.8 & 59.5 & 57.5 & 57.1 & 46.6 & 38.0 & 29.3 & 23.7 \\
\hline 40.2 & 64.8 & 68.4 & 75.0 & 76.2 & 73.9 & 74.3 & 71.8 & 63.5 & 47.3 & 36.2 & 32.1 & 25.9 \\
\hline 47.0 & 86.1 & 88.0 & 94.7 & 96.8 & 94.1 & 94.4 & 88.1 & 79.6 & 63.4 & 43.3 & 39.0 & 27.3 \\
\hline 53.7 & 92.8 & 96.2 & 101.0 & 100.7 & 96.9 & 94.6 & 92.7 & 79.4 & 68.6 & 58.7 & 51.0 & 28.8 \\
\hline 60.6 & 98.7 & 103.1 & 108.9 & 111.0 & 107.3 & 106.6 & 103.2 & 88.7 & 78.2 & 65.9 & 59.4 & 32.9 \\
\hline 67.4 & 122.7 & 130.3 & 142.6 & 144.8 & 138.1 & 139.9 & 133.7 & 115.0 & 90.0 & 77.1 & 72.1 & 43.7 \\
\hline 74.3 & 137.9 & 149.9 & 163.4 & 167.8 & 164.2 & 164.0 & 152.5 & 137.2 & 98.2 & 87.3 & 83.0 & 53.3 \\
\hline B1.0 & 161.9 & 169.5 & 180.5 & 181.2 & 173.5 & 170.2 & 167.1 & 143.2 & 111.6 & 102.6 & 93.6 & 56.9 \\
\hline \multicolumn{13}{|c|}{ ermocouples localed at sprinkler number 2} \\
\hline Time & $0 \mathrm{~mm}$ & $25 \mathrm{~mm}$ & $50 \mathrm{~mm}$ & $75 \mathrm{~mm}$ & $100 \mathrm{~mm}$ & $125 \mathrm{~mm}$ & $150 \mathrm{~mm}$ & $250 \mathrm{~mm}$ & $350 \mathrm{~mm}$ & $450 \mathrm{~mm}$ & $550 \mathrm{~mm}$ & $900 \mathrm{~mm}$ \\
\hline 0.0 & 20.6 & 20.6 & 20.7 & 20.7 & 20.7 & 20.7 & 20.7 & 20.6 & 20.6 & 20.6 & 20.4 & 20.0 \\
\hline 6.1 & 20.7 & 20.7 & 20.7 & 20.7 & 20.7 & 20.7 & 20.7 & 20.6 & 20.7 & 20.7 & 20.7 & 20.1 \\
\hline 12.9 & 20.8 & 20.9 & 21.0 & 21.0 & 21.3 & 21.5 & 21.7 & 21.0 & 20.8 & 20.8 & 20.9 & 20.3 \\
\hline 19.6 & 28.9 & 29.1 & 35.0 & 35.4 & 37.7 & 37.5 & 36.9 & 24.7 & 23.6 & 23.3 & 21.9 & 20.5 \\
\hline 26.5 & 41.6 & 41.2 & 49.1 & 48.3 & 50.5 & 50.4 & 48.6 & 40.2 & 31.4 & 24.3 & 21.8 & 21.0 \\
\hline 33.3 & 56.0 & 57.9 & 64.5 & 63.5 & 66.6 & 65.4 & 65.2 & 56.3 & 42.4 & 31.5 & 26.2 & 22.3 \\
\hline 40.2 & 70.3 & 67.8 & 78.0 & 74.6 & 79.9 & 75.1 & 77.0 & 51.7 & 49.8 & 40.7 & 33.4 & 25.3 \\
\hline 47.0 & 87.3 & 86.8 & 97.9 & 96.8 & 100.0 & 99.3 & 98.8 & 76.2 & 57.4 & 48.3 & 43.6 & 26.2 \\
\hline 53.7 & 91.7 & 90.6 & 99.3 & 99.0 & 101.5 & 101.7 & 101.1 & 89.1 & 72.2 & 57.3 & 54.4 & 35.6 \\
\hline 60.6 & 105.8 & 101.8 & 113.1 & 110.4 & 112.3 & 109.9 & 110.1 & 90.1 & 79.2 & 65.5 & 58.8 & 38.7 \\
\hline 67.4 & 124.6 & 127.0 & 141.7 & 139.0 & 145.9 & 142.6 & 140.0 & 111.5 & 94.1 & 78.1 & 62.3 & 46.2 \\
\hline 74.3 & 136.5 & 131.5 & 147.5 & 145.5 & 152.5 & 154.2 & 153.8 & 143.9 & 128.2 & 97.3 & 85.0 & 52.1 \\
\hline 81.0 & 163.1 & 161.7 & 184.7 & 182.1 & 189.1 & 186.7 & 185.3 & 163.5 & 140.8 & 114.4 & 101.2 & 64.0 \\
\hline \multicolumn{13}{|c|}{ ermocouples located at sprinkler number 3} \\
\hline Time & $0 \mathrm{~mm}$ & $25 \mathrm{~mm}$ & $50 \mathrm{~mm}$ & $75 \mathrm{~mm}$ & $100 \mathrm{~mm}$ & $125 \mathrm{~mm}$ & $150 \mathrm{~mm}$ & $250 \mathrm{~mm}$ & $350 \mathrm{~mm}$ & $450 \mathrm{~mm}$ & $550 \mathrm{~mm}$ & $900 \mathrm{~mm}$ \\
\hline 0.0 & 20.6 & 20.7 & 20.7 & 20.7 & 20.7 & 20.7 & 20.7 & 20.7 & 20.6 & 20.5 & 20.5 & 19.9 \\
\hline 6.1 & 20.6 & 20.6 & 20.6 & 20.7 & 20.7 & 20.7 & 20.7 & 20.7 & 20.6 & 20.5 & 20.5 & 20.0 \\
\hline 12.9 & 20.7 & 20.9 & 20.7 & 20.7 & 20.9 & 21.4 & 21.7 & 21.5 & 21.7 & 21.1 & 20.8 & 20.2 \\
\hline 19.6 & 32.8 & 33.3 & 33.5 & 32.0 & 32.1 & 32.4 & 32.9 & 32.2 & 28.7 & 24.9 & 23.5 & 20.9 \\
\hline 26.5 & 42.3 & 44.9 & 46.0 & 43.7 & 43.4 & 43.8 & 44.9 & 43.1 & 36.5 & 23.1 & 21.7 & 20.6 \\
\hline 33.3 & 45.1 & 49.5 & 51.8 & 51.6 & 51.7 & 52.7 & 52.9 & 51.8 & 49.6 & 37.7 & 28.5 & 21.5 \\
\hline 40.2 & 53.4 & 62.5 & 62.3 & 63.7 & 63.8 & 67.7 & 67.3 & 62.1 & 61.5 & 52.7 & 41.6 & 22.6 \\
\hline 47.0 & 67.7 & 75.3 & 74.1 & 74.2 & 74.1 & 76.7 & 77.2 & 75.1 & 74.5 & 55.7 & 46.1 & 29.4 \\
\hline 53.7 & 72.5 & 84.6 & 80.3 & 84.0 & 82.5 & 88.5 & 87.3 & 88.7 & 90.0 & 61.5 & 46.2 & 34.1 \\
\hline 60.6 & 81.6 & 93.5 & 91.2 & 93.0 & 91.2 & 94.4 & 93.2 & 93.1 & 93.2 & 75.4 & 60.8 & 40.0 \\
\hline 67.4 & 84.7 & 95.0 & 94.8 & 97.5 & 96.3 & 103.1 & 101.3 & 104.2 & 101.7 & 86.7 & 73.3 & 47.8 \\
\hline 74.3 & 91.0 & 107.6 & 103.6 & 111.1 & 114.0 & 121.6 & 121.0 & 126.0 & 129.1 & 107.3 & 83.1 & 59.8 \\
\hline 81.0 & 120.9 & 145.6 & 141.8 & 142.2 & 143.2 & 151.1 & 153.2 & 150.8 & 136.7 & 103.5 & 84.6 & 68.9 \\
\hline \multicolumn{13}{|c|}{ ermocouples located at sprinkler number 4} \\
\hline Time & $0 \mathrm{~mm}$ & $25 \mathrm{~mm}$ & $50 \mathrm{~mm}$ & $75 \mathrm{~mm}$ & $100 \mathrm{~mm}$ & $125 \mathrm{~mm}$ & $150 \mathrm{~mm}$ & $250 \mathrm{~mm}$ & $350 \mathrm{~mm}$ & $450 \mathrm{~mm}$ & $550 \mathrm{~mm}$ & $900 \mathrm{~mm}$ \\
\hline 0.0 & 20.6 & 20.8 & 20.8 & 20.9 & 20.7 & 20.7 & 20.7 & 20.5 & 20.5 & 20.4 & 20.2 & 19.7 \\
\hline 6.1 & 20.9 & 20.8 & 20.8 & 20.9 & 20.7 & 20.8 & 20.7 & 20.6 & 20.6 & 20.4 & 20.3 & 19.7 \\
\hline 12.9 & 22.4 & 22.9 & 22.8 & 23.8 & 23.4 & 23.6 & 23.5 & 23.0 & 23.9 & 22.6 & 22.4 & 20.3 \\
\hline 19.6 & 32.2 & 33.2 & 32.2 & 35.6 & 35.0 & 35.5 & 35.6 & 32.4 & 31.8 & 28.3 & 23.9 & 21.2 \\
\hline 26.5 & 46.2 & 43.6 & 37.9 & 48.6 & 47.1 & 47.4 & 46.8 & 43.0 & 35.5 & 25.2 & 21.8 & 21.4 \\
\hline 33.3 & 52.3 & 49.1 & 45.3 & 55.3 & 533 & 55.0 & 55.6 & 53.1 & 52.7 & 44.8 & 35.6 & 24.6 \\
\hline 40.2 & 55.0 & 53.1 & 52.6 & 60.5 & 61.8 & 64.0 & 66.8 & 64.3 & 65.5 & 61.2 & 41.7 & 25.2 \\
\hline 47.0 & 66.8 & 66.9 & 60.9 & 83.4 & 82,4 & 84.8 & 85.2 & 83.0 & 83.1 & 68.8 & 52.1 & 25.1 \\
\hline 53.7 & 79.9 & 73.6 & 71.7 & 86.3 & 88.0 & 89.8 & 91.3 & 87.1 & 83.5 & 73.9 & 60.6 & 29.5 \\
\hline 60.6 & 93.0 & 87.6 & 80.3 & 96.0 & 95.5 & 96.3 & 95.8 & 91.7 & 93.3 & 87.8 & 84.1 & 37.2 \\
\hline 67.4 & 77.1 & 74.8 & 75.5 & 89.3 & 92.3 & 94.1 & 94.0 & 95.3 & 99.3 & 00.3 & 93.3 & 42.4 \\
\hline 74.3 & 92.0 & 86.4 & 86.2 & 100.9 & 106.1 & 112.7 & 114.8 & 116.8 & 117.6 & 110.1 & 98.4 & 52.0 \\
\hline 81.0 & 93.8 & 101.6 & 102.1 & 111.2 & 117.7 & 121.8 & 129.8 & 137.9 & 142.9 & 134.7 & 125.7 & 58.2 \\
\hline \multicolumn{5}{|c|}{ locity probes at sprinkler number 1} & \multicolumn{6}{|c|}{ Velocity probes at sprinkler number 2} & & \\
\hline Time & $25 \mathrm{~mm}$ & $75 \mathrm{~mm}$ & $125 \mathrm{~mm}$ & $250 \mathrm{~mm}$ & & Time & $25 \mathrm{~mm}$ & $75 \mathrm{~mm}$ & $125 \mathrm{~mm}$ & $250 \mathrm{~mm}$ & & \\
\hline 0.0 & 0.0 & 0.0 & 0.0 & 0.0 & & 0.0 & 0.0 & 0.0 & 0.0 & 0.0 & & \\
\hline
\end{tabular}




\section{Experiment Number 19}

$\begin{array}{rrrr}6.1 & 0.0 & 0.1 & 0.0 \\ 12.9 & -0.3 & -0.4 & -0.4 \\ 19.6 & -0.4 & -0.5 & -0.5 \\ 26.5 & -0.5 & -0.5 & -0.5 \\ 33.3 & -0.0 & -0.8 & -0.8 \\ 40.2 & -0.7 & -0.6 & -0.5 \\ 47.0 & -0.7 & -0.6 & -0.5 \\ 53.7 & -0.5 & -0.5 & -0.4 \\ 60.6 & -1.0 & -0.9 & -0.6 \\ 67.4 & -1.0 & -0.9 & -0.5 \\ 74.3 & -0.9 & -0.9 & -0.5 \\ 81.0 & -0.6 & -0.7 & -0.5\end{array}$

0.0
-0.4
-0.5
-0.4
-0.4
-0.4
-0.4
-0.4
-0.6
-0.5
-0.8
-0.7

Velocity probes in channels above burner

Time Channel 1 Channel 2

$\begin{array}{lll}0.0 & 0.0 & 0.0\end{array}$

$\begin{array}{lll}6.1 & 2.6 & 1.5\end{array}$

$\begin{array}{lll}12.9 & 2.9 & 1.5\end{array}$

$\begin{array}{lll}19.6 & 2.8 & 2.1\end{array}$

$\begin{array}{lll}26.5 & 3.1 & 1.8\end{array}$

$\begin{array}{lll}33.3 & 2.8 & 1.8\end{array}$

$\begin{array}{lll}40.2 & 3.4 & 2.1\end{array}$

$\begin{array}{lll}47.0 & 3.3 & 1.9\end{array}$

$\begin{array}{lll}53.7 & 3.1 & 2.2\end{array}$

$\begin{array}{lll}60.6 & 3.9 & 2.5\end{array}$

$\begin{array}{lll}67.4 & 4.2 & 3.1\end{array}$

$\begin{array}{lll}74.3 & 3.7 & 3.3\end{array}$

$\begin{array}{lll}81.0 & 4.6 & 2.7\end{array}$ 
Experiment Number 20

Thermocouples located at sprinkler number 1

\begin{tabular}{|c|c|c|c|c|c|c|c|c|c|c|c|c|}
\hline Time & $0 \mathrm{~mm}$ & $25 \mathrm{~mm}$ & $50 \mathrm{~mm}$ & $75 \mathrm{~mm}$ & $100 \mathrm{~mm}$ & $125 \mathrm{~mm}$ & $150 \mathrm{~mm}$ & $250 \mathrm{~mm}$ & $350 \mathrm{~mm}$ & $450 \mathrm{~mm}$ & $550 \mathrm{~mm}$ & $900 \mathrm{~mm}$ \\
\hline 0.0 & 25.0 & 24.7 & 24.8 & 24.4 & 24.4 & 23.8 & 23.9 & 23.2 & 22.9 & 22.5 & 22.3 & 21.1 \\
\hline 6.8 & 26.1 & 25.7 & 25.8 & 25.2 & 25.3 & 24.7 & 25.2 & 24.2 & 23.4 & 22.9 & 22.7 & 21.7 \\
\hline 13.6 & 28.8 & 20.5 & 29.8 & 30.1 & 29.6 & 29.2 & 27.9 & 28.1 & 26.5 & 24.4 & 23.7 & 22.1 \\
\hline 20.3 & 34.8 & 35.7 & 36.8 & 36.6 & 35.9 & 34.4 & 33.9 & 30.5 & 28.1 & 24.5 & 24.0 & 22.7 \\
\hline 27.2 & 43.4 & 46.0 & 49.3 & 51.0 & 50.1 & 51.7 & 48.3 & 46.7 & 42.0 & 26.9 & 25.2 & 23.7 \\
\hline 33.9 & 60.2 & 61.4 & 65.6 & 67.4 & 64.5 & 63.6 & 60.5 & 54.6 & 50.1 & 37.5 & 32.5 & 27.0 \\
\hline 40.8 & 80.0 & 82.1 & 87.6 & 88.0 & 86.2 & 85.4 & 81.9 & 75.6 & 55.2 & 40.8 & 38.9 & 27.9 \\
\hline 47.6 & 89.3 & 94.3 & 101.0 & 101.2 & 98.5 & 96.4 & 94.1 & 84.7 & 76.2 & 53.1 & 49.0 & 30.7 \\
\hline 54.3 & 104.3 & 108.5 & $\uparrow 14.1$ & 115.8 & 113.1 & 113.1 & 108.7 & 102.9 & 93.0 & 67.9 & 62.0 & 32.4 \\
\hline 61.3 & 111.8 & 116.9 & 124.0 & 125.3 & 121.9 & 120.3 & 114.9 & 104.4 & 90.8 & 76.6 & 72.2 & 40.8 \\
\hline 68.0 & 122.6 & 131.5 & 137.6 & 138.8 & 134.8 & 134.0 & 128.1 & 120.7 & 98.5 & 86.2 & 79.3 & 57.3 \\
\hline 74.9 & 140.0 & 149.9 & 158.2 & 160.9 & 158.5 & 158.4 & 152.3 & 145.4 & 109.4 & 94.9 & 87.0 & 61.2 \\
\hline \multicolumn{13}{|c|}{ Thermocouples located at sprinkler number 2} \\
\hline Time & $0 \mathrm{~mm}$ & $25 \mathrm{~mm}$ & $50 \mathrm{~mm}$ & $75 \mathrm{~mm}$ & $100 \mathrm{~mm}$ & $125 \mathrm{~mm}$ & $150 \mathrm{~mm}$ & $250 \mathrm{~mm}$ & $350 \mathrm{~mm}$ & $450 \mathrm{~mm}$ & $550 \mathrm{~mm}$ & $900 \mathrm{~mm}$ \\
\hline 0.0 & 27.3 & 26.5 & 26.0 & 25.6 & 25.3 & 24.8 & 24.5 & 23.8 & 22.9 & 22.4 & 22.2 & 21.2 \\
\hline 6.8 & 27.4 & 26.9 & 26.5 & 26.1 & 25.7 & 25.1 & 24.8 & 23.8 & 22.9 & 22.6 & 22.4 & 22.0 \\
\hline 13.6 & 29.6 & 30.0 & 30.7 & 30.0 & 30.9 & 30.4 & 29.6 & 28.4 & 26.7 & 24.4 & 23.2 & 22.1 \\
\hline 20.3 & 40.3 & 44.0 & 44.7 & 43.0 & 45.6 & 44.3 & 42.4 & 36.7 & 26.0 & 24.1 & 23.3 & 22.3 \\
\hline 27.2 & 53.9 & 58.5 & 60.7 & 58.6 & 61.4 & 62.3 & 60.7 & 53.2 & 36.5 & 26.9 & 24.5 & 23.0 \\
\hline 33.9 & 60.0 & 62.8 & 67.5 & 63.1 & 69.0 & 68.4 & 68.5 & 57.8 & 52.2 & 35.5 & 32.7 & 27.5 \\
\hline 40.8 & 82.7 & 89.0 & 93.1 & 90.4 & 96.3 & 95.1 & 92.4 & 74.1 & 53.3 & 44.7 & 38.8 & 26.3 \\
\hline 47.6 & 89.0 & 93.4 & 96.3 & 96.8 & 101.4 & 102.5 & 101.3 & 90.4 & 70.4 & 56.8 & 51.7 & 31.2 \\
\hline 54.3 & 96.3 & 100.1 & 102.8 & 99.7 & 104.2 & 104.2 & 105.1 & 97.7 & 77.2 & 64.7 & 60.1 & 39.7 \\
\hline 61.3 & 103.1 & 110.3 & 114.1 & 113.0 & 117.9 & 119.6 & 119.9 & 110.0 & 94.7 & 78.0 & 65.9 & 47.7 \\
\hline 68.0 & 134.0 & 144.8 & 150.8 & 150.4 & 156.9 & 156.6 & 153.3 & 129.9 & 102.6 & 85.5 & 75.7 & 48.0 \\
\hline 74.9 & 152.2 & 164.8 & 167.5 & 162.0 & 168.6 & 166.3 & 164.6 & 157.5 & 130.3 & 102.0 & 95.6 & 59.9 \\
\hline \multicolumn{13}{|c|}{ Thermocouples located at sprinkler number 3} \\
\hline Time & $0 \mathrm{~mm}$ & $25 \mathrm{~mm}$ & $50 \mathrm{~mm}$ & $75 \mathrm{~mm}$ & $100 \mathrm{~mm}$ & $125 \mathrm{~mm}$ & $150 \mathrm{~mm}$ & $250 \mathrm{~mm}$ & $350 \mathrm{~mm}$ & $450 \mathrm{~mm}$ & $550 \mathrm{~mm}$ & $900 \mathrm{~mm}$ \\
\hline 0.0 & 27.2 & 26.8 & 26.1 & 25.6 & 25.0 & 24.7 & 24.4 & 23.8 & 23.2 & 22.7 & 22.2 & 21.4 \\
\hline 6.8 & 27.2 & 27.2 & 26.3 & 25.9 & 25.3 & 24.6 & 24.3 & 24.1 & 23.6 & 23.4 & 23.1 & 22.0 \\
\hline 13.6 & 27.8 & 27.3 & 27.8 & 27.5 & 27.4 & 27.4 & 27.6 & 27.1 & 27.0 & 25.6 & 25.3 & 22.4 \\
\hline 20.3 & 36.1 & 38.3 & 37.8 & 38.1 & 37.6 & 38.2 & 39.5 & 37.0 & 36.1 & 29.9 & 25.8 & 22.3 \\
\hline 27.2 & 47.0 & 50.8 & 49.3 & 49.8 & 50.4 & 53.3 & 54.7 & 50.4 & 47.1 & 28.1 & 24.3 & 22.4 \\
\hline 33.9 & 52.9 & 60.5 & 56.7 & 58.0 & 57.4 & 59.0 & 61.0 & 59.6 & 59.8 & 44.8 & 35.2 & 23.8 \\
\hline 40.8 & 62.4 & 70.3 & 67.2 & 69.3 & 70.5 & 73.1 & 73.6 & 72.2 & 69.2 & 52.2 & 41.9 & 25.7 \\
\hline 47.6 & 69.4 & 85.1 & 77.8 & 81.5 & 80.2 & 84.2 & 85.6 & 89.7 & 79.0 & 59.7 & 44.9 & 35.0 \\
\hline 54.3 & 83.6 & 99.1 & 89.4 & 93.0 & 93.4 & 97.4 & 99.1 & 97.8 & 95.4 & 73.0 & 58.2 & 40.0 \\
\hline 61.3 & 90.6 & 108.4 & 99.1 & 101.6 & 102.7 & 106.1 & 107.6 & 101.1 & 97.9 & 80.0 & 63.8 & 47.0 \\
\hline 68.0 & 88.9 & 106.0 & 102.0 & 107.3 & 108.0 & 112.6 & 114.9 & 118.1 & 123.6 & 99.5 & 69.7 & 57.9 \\
\hline 74.9 & 103.9 & 131.2 & 121.7 & 130.3 & 129.3 & 135.2 & 138.1 & 143.3 & 152.8 & 125.7 & 90.6 & 66.3 \\
\hline \multicolumn{13}{|c|}{ Thermocouples located at sprinkler number 4} \\
\hline Time & $0 \mathrm{~mm}$ & $25 \mathrm{~mm}$ & $50 \mathrm{~mm}$ & $75 \mathrm{~mm}$ & $100 \mathrm{~mm}$ & $125 \mathrm{~mm}$ & $150 \mathrm{~mm}$ & $250 \mathrm{~mm}$ & $350 \mathrm{~mm}$ & $450 \mathrm{~mm}$ & $550 \mathrm{~mm}$ & $900 \mathrm{~mm}$ \\
\hline 0.0 & 26.4 & 25.7 & 25.6 & 24.8 & 24.3 & 24.1 & 23.7 & 23.1 & 22.6 & 22.4 & 22.1 & 21.6 \\
\hline 6.8 & 27.3 & 26.6 & 26.7 & 25.0 & 24.7 & 24.5 & 24.3 & 23.8 & 23.4 & 22.9 & 22.6 & 22.3 \\
\hline 13.6 & 29.5 & 29.3 & 28.7 & 29.5 & 29.1 & 29.4 & 29.2 & 28.8 & 28.9 & 27.5 & 26.6 & 23.0 \\
\hline 20.3 & 37.1 & 35.5 & 34.9 & 38.6 & 38.5 & 39.7 & 40.6 & 40.9 & 40.4 & 30.6 & 26.2 & 23.5 \\
\hline 27.2 & 48.5 & 46.5 & 43.3 & 53.4 & 51.7 & 53.0 & 53.6 & 51.7 & 53.3 & 32.7 & 27.5 & 24.6 \\
\hline 33.9 & 59.0 & 56.1 & 51.6 & 63.1 & 62.5 & 63.8 & 64.5 & 60.6 & 60.8 & 53.8 & 39.9 & 25.7 \\
\hline 40.8 & 69.7 & 68.3 & 65.8 & 72.5 & 71.5 & 71.3 & 70.9 & 70.5 & 74.2 & 66.9 & 51.4 & 27.3 \\
\hline 47.6 & 75.1 & 73.5 & 71.0 & 82.6 & 83.1 & 84.9 & 85.5 & 83.5 & 76.5 & 70.5 & 59.4 & 29.6 \\
\hline 54.3 & 94.6 & 87.2 & 76.4 & 97.5 & 96.4 & 97.0 & 97.1 & 91.2 & 86.7 & 72.3 & 65.3 & 387 \\
\hline 61.3 & 95.6 & 95.4 & 89.9 & 101.1 & 101.0 & 103.4 & 106.3 & 99.8 & 98.8 & 91.2 & 87.6 & 4.5 .2 \\
\hline 68.0 & 118.2 & 115.3 & 108.3 & 120.7 & 120.8 & 123.8 & 124.1 & 119.4 & 115.0 & 106.2 & 100.1 & 52.7 \\
\hline 74.9 & 131.0 & 124.4 & 112.2 & 141.1 & 140.6 & 142.8 & 143.0 & 133.4 & 133.1 & 122.7 & $\uparrow 16.9$ & 62.2 \\
\hline \multicolumn{6}{|c|}{ Velocity probes at sprinkler number 1} & \multicolumn{7}{|c|}{ Velocity probes at sprinkler number 2} \\
\hline Time & $25 \mathrm{~mm}$ & $75 \mathrm{~mm}$ & 125 гा!и & $250 \mathrm{~mm}$ & & Time & $25 \mathrm{~mm}$ & $75 \mathrm{~mm}$ & $125 \mathrm{~mm}$ & $250 \mathrm{~mm}$ & & \\
\hline 0.0 & 0.0 & 0.0 & 0.0 & 0.0 & & 0.0 & 0.0 & 0.0 & 0.0 & 0.0 & & \\
\hline 6.8 & -0.2 & -0.2 & -0.2 & -0.1 & & 6.8 & 0.2 & 0.1 & 0.1 & -0.1 & & \\
\hline 13.6 & -0.4 & -0.3 & -0.4 & -0.4 & & 13.6 & 0.3 & 0.3 & 0.3 & 0.2 & & \\
\hline 20.3 & -0.3 & -0.5 & -0.5 & -0.5 & & 20.3 & 0.5 & 0.6 & 0.5 & 0.2 & & \\
\hline 27.2 & -0.4 & -0.4 & -0.2 & -0.3 & & $2 / .2$ & 0.4 & 0.3 & 0.5 & 0.3 & & \\
\hline
\end{tabular}


Experiment Number 20

\begin{tabular}{|c|c|c|c|c|c|c|c|c|c|}
\hline 33.9 & -0.6 & -0.7 & -0.4 & -0.2 & 33.9 & 0.7 & 0.7 & 0.7 & 0.2 \\
\hline 40.8 & -0.7 & -0.6 & -0.3 & -0.3 & 40.8 & 0.6 & 0.7 & 0.6 & 0.3 \\
\hline 47.6 & -0.8 & -0.8 & -0.5 & -0.6 & 47.6 & 0.6 & 0.7 & 0.7 & 0.2 \\
\hline 54.3 & -0.5 & -0.6 & -0.5 & -0.5 & 54.3 & 0.8 & 0.7 & 0.7 & 0.3 \\
\hline 61.3 & -0.6 & -0.7 & -0.4 & -0.5 & 61.3 & 0.8 & 0.7 & 0.8 & 0.5 \\
\hline 68.0 & -1.2 & -1.0 & -0.7 & -0.6 & 68.0 & 0.9 & 0.9 & 1.2 & 0.7 \\
\hline 74.9 & -0.9 & -1.0 & -0.8 & -0.7 & 74.9 & 0.9 & 0.8 & 0.9 & 0.4 \\
\hline \multicolumn{10}{|c|}{ Velocity probes in channels above bumer } \\
\hline Time & Channel 1 & Channel 2 & & & & & & & \\
\hline 0.0 & 0.0 & 0.0 & & & & & & & \\
\hline 6.8 & 2.2 & 1.1 & & & & & & & \\
\hline 13.6 & 2.4 & 1.8 & & & & & & & \\
\hline 20.3 & 3.3 & 2.0 & & & & & & & \\
\hline 27.2 & 3.8 & 2.1 & & & & & & & \\
\hline 33.9 & 3.2 & 2.8 & & & & & & & \\
\hline 40.8 & 2.6 & 2.2 & & & & & & & \\
\hline 47.6 & 3.0 & 2.5 & & & & & & & \\
\hline 54.3 & 3.9 & 2.5 & & & & & & & \\
\hline 61.3 & 4.0 & 3.0 & & & & & & & \\
\hline 68.0 & 4.6 & 2.4 & & & & & & & \\
\hline 74.9 & 4.1 & 3.4 & & & & & & & \\
\hline
\end{tabular}




\section{Experiment Number 21}

Thermocouples located at sprinkler number 1

\begin{tabular}{|c|c|c|c|c|c|c|c|c|c|c|c|c|}
\hline Time & $0 \mathrm{~mm}$ & $25 \mathrm{~mm}$ & $50 \mathrm{~mm}$ & $75 \mathrm{~mm}$ & $100 \mathrm{~mm}$ & $125 \mathrm{~mm}$ & $150 \mathrm{~mm}$ & $250 \mathrm{~mm}$ & $350 \mathrm{~mm}$ & $450 \mathrm{~mm}$ & $550 \mathrm{~mm}$ & $900 \mathrm{~mm}$ \\
\hline 0.0 & 21.3 & 21.2 & 21.3 & 21.0 & 20.9 & 20.6 & 20.6 & 20.1 & 19.9 & 19.6 & 19.3 & 17.8 \\
\hline 1.7 & 21.4 & 21.2 & 21.1 & 20.9 & 20.8 & 20.5 & 20.5 & 20.0 & 19.9 & 19.6 & 19.2 & 18.1 \\
\hline 8.5 & 21.4 & 21.8 & 23.8 & 25.4 & 25.4 & 26.0 & 24.8 & 26.1 & 22.7 & 20.9 & 19.6 & 18.3 \\
\hline 15.4 & 25.1 & 34.8 & 38.3 & 38.9 & 35.1 & 35.9 & 33.6 & 31.7 & 22.3 & 20.1 & 19.5 & 18.2 \\
\hline 22.2 & 28.2 & 41.1 & 43.5 & 44.0 & 42.4 & 43.6 & 41.5 & 39.1 & 32.8 & 29.0 & 25.1 & 18.6 \\
\hline 29.0 & 33.1 & 56.2 & 62.3 & 61.6 & 55.5 & 56.2 & 50.6 & 44.9 & 39.4 & 30.8 & 22.1 & 18.4 \\
\hline 36.0 & 37.2 & 63.3 & 68.4 & 69.5 & 66.9 & 67.3 & 63.7 & 59.8 & 52.4 & 44.2 & 24.9 & 19.4 \\
\hline 42.7 & 39.4 & 70.9 & 77.7 & 77.1 & 72.9 & 13.1 & 71.7 & 67.9 & 56.9 & 49.2 & 33.1 & 20.5 \\
\hline 49.6 & 41.8 & 76.4 & 86.2 & 87.8 & 86.2 & 86.2 & 82.8 & 75.2 & 70.3 & 62.2 & 43.5 & 20.7 \\
\hline 56.4 & 46.6 & R9 6 & 99.2 & 100.3 & 96.1 & 97.6 & 93.2 & 86.2 & 79.7 & 70.8 & 57.7 & 30.3 \\
\hline 63.2 & 51.1 & 99.1 & 110.7 & 111.5 & 106.3 & 105.3 & 104.3 & 101.0 & 93.6 & 74.3 & 55.5 & 31.9 \\
\hline 70.2 & 56.3 & 105.8 & 116.0 & 116.4 & 112.2 & 113.4 & 109.7 & 99.5 & 96.1 & 74.0 & 62.8 & 38.4 \\
\hline \multicolumn{13}{|c|}{ rmocouples located at sprinkler number 2} \\
\hline Time & $0 \mathrm{~mm}$ & $25 \mathrm{~mm}$ & $50 \mathrm{~mm}$ & $75 \mathrm{~mm}$ & $100 \mathrm{~mm}$ & $125 \mathrm{~mm}$ & $150 \mathrm{~mm}$ & $250 \mathrm{~mm}$ & $350 \mathrm{~mm}$ & $450 \mathrm{~mm}$ & $550 \mathrm{~mm}$ & $900 \mathrm{~mm}$ \\
\hline 0.0 & 21.1 & 21.5 & 21.4 & 21.0 & 21.0 & 20.6 & 20.6 & 20.1 & 19.9 & 19.6 & 19.3 & 18.0 \\
\hline 1.7 & 21.4 & 21.9 & 21.7 & 22.8 & 21.3 & 20.7 & 20.5 & 20.3 & 20.1 & 19.8 & 19.4 & 18.3 \\
\hline 8.5 & 22.4 & 28.3 & 28.4 & 29.5 & 28.4 & 27.7 & 27.6 & 29.0 & 25.4 & 23.8 & 20.0 & 18.7 \\
\hline 15.4 & 29.9 & 50.5 & 49.2 & 49.2 & 47.7 & 46.7 & 43.7 & 35.3 & 22.8 & 20.7 & 19.5 & 18.2 \\
\hline 22.2 & 31.3 & 50.6 & 50.5 & 49.9 & 48.1 & 45.9 & 44.1 & 39.7 & 31.3 & 27.3 & 24.4 & 18.5 \\
\hline 29.0 & 33.7 & 57.4 & 57.9 & 38.7 & 58.1 & 57.4 & 56.0 & 52.0 & 42.7 & 32.0 & 21.4 & 18.5 \\
\hline 36.0 & 39.1 & 67.7 & 68.5 & 70.5 & 69.1 & 68.0 & 67.6 & 66.1 & 59.4 & 49.1 & 36.6 & 20.0 \\
\hline 42.7 & 44.2 & 84.9 & 86.5 & 87.4 & 86.2 & 83.9 & 79.5 & 73.2 & 63.1 & 52.0 & 39.1 & 22.6 \\
\hline 49.6 & 48.8 & 88.9 & 90.3 & 92.7 & 91.4 & 88.9 & 88.5 & 76.6 & 67.4 & 57.8 & 43.6 & 23.5 \\
\hline 56.4 & 53.6 & 100.2 & 101.5 & 104.5 & 105.8 & 104.7 & 101.6 & 88.1 & 78.2 & 61.1 & 44.9 & 30.2 \\
\hline 63.2 & 58.5 & $\$ 10.2$ & 113.3 & 115.3 & 113.6 & 111.0 & 110.7 & 98.7 & 91.7 & 67.3 & 55.2 & 32.4 \\
\hline 70.2 & 61.7 & 113.6 & 115.6 & 117.5 & 116.8 & 114.3 & 113.6 & 106.8 & 100.6 & 81.5 & 63.7 & 36.0 \\
\hline \multicolumn{13}{|c|}{ rmocouples located at sprinkler number 3} \\
\hline Time & $0 \mathrm{~mm}$ & $25 \mathrm{~mm}$ & $50 \mathrm{~mm}$ & $75 \mathrm{~mm}$ & $100 \mathrm{~mm}$ & $125 \mathrm{~mm}$ & $150 \mathrm{~mm}$ & $250 \mathrm{~mm}$ & $350 \mathrm{~mm}$ & $450 \mathrm{~mm}$ & $550 \mathrm{~mm}$ & $900 \mathrm{~mm}$ \\
\hline 0.0 & 20.7 & 21.0 & 20.6 & 20.6 & 20.5 & 20.5 & 20.4 & 20.2 & 20.0 & 19.6 & 19.1 & 18.1 \\
\hline 1.7 & 20.7 & 21.1 & 20.6 & 20.5 & 20.3 & 20.2 & 20.2 & 20.0 & 19.9 & 19.7 & 19.3 & 18.4 \\
\hline 8.5 & 20.6 & 21.0 & 20.5 & 20.5 & 20.5 & 20.5 & 20.5 & 20.3 & 20.1 & 19.8 & 19.4 & 18.5 \\
\hline 15.4 & 20.8 & 21.7 & 21.7 & 22.7 & 21.8 & 22.4 & 22.1 & 21.7 & 21.5 & 20.6 & 20.1 & 18.8 \\
\hline 22.2 & 21.1 & 28.6 & 30.8 & 31.3 & 28.4 & 27.9 & 26.0 & 22.3 & 21.1 & 20.5 & 19.9 & 19.0 \\
\hline 290 & 21.7 & 33.1 & 37.2 & 38.7 & 36.2 & 36.8 & 34.7 & 29.8 & 26.7 & 25.7 & 26.0 & 22.2 \\
\hline 36.0 & 22.5 & 37.2 & 42.8 & 43.4 & 40.4 & 40.0 & 38.5 & 34.7 & 33.0 & 31.4 & 31.8 & 22.2 \\
\hline 42.7 & 23.2 & 40.5 & 48.6 & 49.1 & 46.7 & 47.1 & 45.1 & 40.3 & 37.5 & 35.6 & 35.4 & 25.2 \\
\hline 49.6 & 24.9 & 48.0 & 61.7 & 62.9 & 58.1 & 58.1 & 55.0 & 49.1 & 44.4 & 42.3 & 42.0 & 30.7 \\
\hline 56.4 & 25.7 & 54.6 & 65.0 & 66.2 & 63.6 & 63.6 & 61.4 & 54.7 & 51.4 & 48.6 & 49.7 & 35.6 \\
\hline 63.2 & 27.1 & 55.6 & 73.2 & 74.0 & 69.2 & 69.2 & 65.3 & 59.9 & 56.9 & 55.5 & 55.3 & 38.8 \\
\hline 70.2 & 28.0 & 60.3 & 81.0 & 82.1 & 77.4 & 78.9 & 75.2 & 68.8 & 65.6 & 63.3 & 63.8 & 45.7 \\
\hline \multicolumn{13}{|c|}{ rmocouples located at sprinkler number 4} \\
\hline Time & $0 \mathrm{~mm}$ & $25 \mathrm{~mm}$ & $50 \mathrm{~mm}$ & $75 \mathrm{~mm}$ & $100 \mathrm{~mm}$ & $125 \mathrm{~mm}$ & $150 \mathrm{~mm}$ & $250 \mathrm{~mm}$ & $350 \mathrm{~mm}$ & $450 \mathrm{~mm}$ & $550 \mathrm{~mm}$ & $900 \mathrm{~mm}$ \\
\hline 0.0 & 20.4 & 20.7 & 20.7 & 20.7 & 20.5 & 20.4 & 20.4 & 20.0 & 19.8 & 19.2 & 18.9 & 18.1 \\
\hline 1.7 & 20.5 & 20.6 & 20.6 & 20.5 & 20.3 & 20.3 & 20.2 & 19.9 & 19.7 & 19.4 & 19.2 & 18.4 \\
\hline 8.5 & 20.5 & 20.7 & 20.6 & 20.5 & 20.3 & 20.3 & 20.3 & 20.0 & 19.8 & 19.5 & 19.2 & 18.3 \\
\hline 15.4 & 21.2 & 25.8 & 29.1 & 28.4 & 27.1 & 26.9 & 25.8 & 23.3 & 22.4 & 20.3 & 19.8 & 18.6 \\
\hline 22.2 & 22.2 & 32.3 & 35.0 & 34.5 & 33.0 & 33.3 & 32.7 & 28.4 & 26.3 & 22.4 & 21.8 & 19.3 \\
\hline 29.0 & 23.5 & 38.5 & 41.3 & 40.4 & 38.8 & 39.7 & 39.7 & 34.5 & 30.6 & 29.2 & 26.2 & 21.5 \\
\hline 36.0 & 24.9 & 42.3 & 46.8 & 46.3 & 44.6 & 45.4 & 45.6 & 40.1 & 36.0 & 34.4 & 31.6 & 21.0 \\
\hline 42.7 & 27.2 & 55.2 & 56.1 & 55.7 & 54.0 & 55.1 & 54.8 & 45.5 & 40.8 & 41.0 & 38.8 & 21.2 \\
\hline 49.6 & 28.1 & 54.4 & 58.1 & 58.2 & 57.5 & 58.9 & 57.9 & 50.7 & 48.3 & 46.8 & 46.5 & 247 \\
\hline 56.4 & 31.9 & 58.8 & 67.6 & 67.2 & 65.1 & 66.2 & 65.2 & 54.1 & 52.8 & 52.1 & 52.0 & 29.0 \\
\hline 63.2 & 35.7 & 80.5 & 81.3 & 79.7 & 76.4 & 76.5 & 75.8 & 61.0 & 59.3 & 59.0 & 58.0 & 33.3 \\
\hline 70.2 & 37.5 & 80.7 & 86.4 & 84.4 & 81.6 & 82.3 & 81.1 & 68.4 & 67.6 & 67.9 & 66.7 & 37.3 \\
\hline \multicolumn{5}{|c|}{ city probes at sprinkler number 1} & \multicolumn{6}{|c|}{ Velocily probes at sprinkler number 2} & & \\
\hline Time & $25 \mathrm{~mm}$ & $75 \mathrm{~mm}$ & $125 \mathrm{~mm}$ & 250 гит & & Time & $25 \mathrm{~mm}$ & $75 \mathrm{~mm}$ & $125 \mathrm{~mm}$ & $250 \mathrm{~mm}$ & & \\
\hline 0.0 & 0.0 & 0.0 & 0.0 & 0.0 & & 0.0 & 0.0 & 0.0 & 0.0 & 0.0 & & \\
\hline 1.7 & -0.1 & -0.1 & -0.1 & 0.0 & & 1.7 & 0.1 & 0.1 & 0.1 & 0.0 & & \\
\hline B.5 & -0.6 & -0.9 & -0.9 & 0.0 & & 8.5 & 1.2 & 1.1 & 0.7 & 0.2 & & \\
\hline 15.4 & -1.1 & -1.1 & -0.9 & -0.6 & & 15.4 & 1.3 & 1.1 & 0.7 & 0.5 & & \\
\hline 22.2 & -0.7 & -0.9 & -0.6 & -0.3 & & 22.2 & 0.7 & 0.8 & 0.9 & 0.1 & & \\
\hline
\end{tabular}


Experiment Number 21

$\begin{array}{llllllllll}29.0 & -1.3 & -1.2 & -0.8 & -0.6 & 29.0 & 1.3 & 1.2 & 1.0 & 0.8 \\ 36.0 & -1.4 & -1.6 & -1.2 & -0.8 & 36.0 & 1.3 & 1.4 & 1.4 & 0.8 \\ 42.7 & -0.8 & -1.0 & -0.9 & -0.4 & 42.7 & 1.4 & 1.5 & 1.3 & 0.5 \\ 49.6 & -1.2 & -1.0 & -0.6 & -0.2 & 49.6 & 1.5 & 1.7 & 1.6 & 1.1 \\ 56.4 & -1.0 & -1.1 & -1.2 & -0.8 & 56.4 & 1.4 & 1.2 & 1.0 & 0.7 \\ 63.2 & -1.2 & -1.3 & -1.3 & -0.1 & 63.2 & 1.7 & 1.3 & 1.1 & 0.8 \\ 70.2 & -1.7 & -1.8 & -1.4 & -1.0 & 70.2 & 1.8 & 1.6 & 1.5 & 0.8\end{array}$




\section{Experiment Number 22}

Thermocouples located at sprinkler number 1

\begin{tabular}{|c|c|c|c|c|c|c|c|c|c|c|c|c|}
\hline Time & $0 \mathrm{~mm}$ & $25 \mathrm{~mm}$ & $50 \mathrm{~mm}$ & $75 \mathrm{~mm}$ & $100 \mathrm{~mm}$ & $125 \mathrm{~mm}$ & $150 \mathrm{~mm}$ & $250 \mathrm{~mm}$ & $350 \mathrm{~mm}$ & $450 \mathrm{~mm}$ & $550 \mathrm{~mm}$ & $900 \mathrm{~mm}$ \\
\hline 0.0 & 21.7 & 21.7 & 22.0 & 21.7 & 21.8 & 21.4 & 21.6 & 21.2 & 21.2 & 20.7 & 20.2 & 18.4 \\
\hline 2.8 & 21.7 & 21.6 & 21.8 & 21.6 & 21.6 & 21.3 & 21.5 & 21.0 & 21.0 & 20.8 & 20.4 & 19.1 \\
\hline 9.6 & 21.9 & 22.8 & 25.3 & 26.4 & 25.9 & 26.3 & 24.9 & 27.1 & 25.4 & 22.7 & 20.9 & 19.3 \\
\hline 16.5 & 25.8 & 35.9 & 39.7 & 39.7 & 36.6 & 36.9 & 34.6 & 30.7 & 22.9 & 21.5 & 21.0 & 19.2 \\
\hline 23.3 & 29.0 & 44.2 & 48.1 & 48.8 & 46.4 & 47.0 & 44.4 & 38.9 & 30.3 & 27.0 & 22.7 & 19.7 \\
\hline 30.0 & 33.3 & 54.4 & 58.8 & 58.8 & 55.7 & 57.5 & 55.4 & 43.8 & 37.7 & 32.8 & 24.4 & 19.6 \\
\hline 37.0 & 37.0 & 64.6 & 71.0 & 72.9 & 69.7 & 72.1 & 68.5 & 66.9 & 53.4 & 44.2 & 33.0 & 20,6 \\
\hline 43.8 & 41.6 & 75.0 & 83.0 & 82.6 & 79.3 & 78.7 & 76.5 & 76.1 & 69.4 & 56.0 & 35.7 & 20.8 \\
\hline 50.7 & 44.1 & 80.4 & 88.5 & 89.0 & 85.4 & 86.7 & 84.2 & 79.1 & 73.2 & 62.5 & 46.5 & 21.5 \\
\hline 57.5 & 50.0 & 92.3 & 101.3 & 103.3 & 98.4 & 100.3 & 94.7 & 92.2 & 81.7 & 70.8 & 50.6 & 25.1 \\
\hline 64.3 & 54.2 & 104.3 & 115.8 & 117.1 & 112.3 & 113.2 & 107.0 & 103.5 & 92.7 & 81.3 & 59.0 & 31.7 \\
\hline 71.2 & 56.7 & 106.6 & 118.0 & 121.9 & 116.9 & 120.5 & 116.1 & 109.9 & 100.9 & 86.9 & 67.4 & 38.5 \\
\hline 78.0 & 60.6 & 83.9 & 114.1 & 113.2 & 59.3 & 120.4 & 95.1 & 117.1 & 106.7 & 90.6 & 71.2 & 42.9 \\
\hline \multicolumn{13}{|c|}{ mocouples localed at sprinkler number 2} \\
\hline Time & $\mathrm{amm}$ & $25 \mathrm{~mm}$ & $50 \mathrm{~mm}$ & $75 \mathrm{~mm}$ & $100 \mathrm{~mm}$ & $125 \mathrm{~mm}$ & $150 \mathrm{~mm}$ & $250 \mathrm{~mm}$ & $350 \mathrm{~mm}$ & $450 \mathrm{~mm}$ & $550 \mathrm{~mm}$ & $900 \mathrm{~mm}$ \\
\hline 0.0 & 19.9 & 22.0 & 22.1 & 22.3 & 22.0 & 21.8 & $2 \uparrow .8$ & 21.4 & 21.3 & 20.9 & 20.6 & 18.6 \\
\hline 2.8 & 19.8 & 21.4 & 21.5 & 21.7 & 21.7 & 21.6 & 21.6 & 21.0 & 21.0 & 20.9 & 20.6 & 18.6 \\
\hline 9.6 & 21.5 & 29.6 & 29.8 & 30.9 & 30.4 & 30.8 & 30.0 & 30.4 & 26.6 & 25.1 & 21.5 & 18.6 \\
\hline 16.5 & 26.2 & 43.0 & 43.4 & 44.3 & 44.3 & 43.8 & 43.7 & 37.5 & 31.3 & 24.9 & 20.8 & 18.3 \\
\hline 23.3 & 29.5 & 48.4 & 49.2 & 50.6 & 50.2 & 49.6 & 48.9 & 45.4 & 37.0 & 29.2 & 25.7 & 18.7 \\
\hline 30.0 & 34.8 & 64.4 & 64.1 & 63.8 & 62.6 & 61.3 & 60.3 & 55.5 & 44.8 & 33.8 & 21.9 & 18.8 \\
\hline 37.0 & 38.6 & 73.4 & 74.8 & 73.4 & 70.5 & 67.5 & 66.7 & 57.9 & 50.7 & 44.9 & 37.1 & 21.7 \\
\hline 43.8 & 42.2 & 77.2 & 77.9 & 79.5 & 78.5 & 77.1 & 75.1 & 70.1 & 59.6 & 49.6 & 39.8 & 23.2 \\
\hline 50.7 & 48.6 & 88.7 & 89.9 & 92.3 & 91.4 & 89.6 & 87.7 & 79.5 & 67.0 & 57.4 & 48.7 & 26.8 \\
\hline 57.5 & 49.8 & 102.3 & 105.3 & 109.6 & 109.7 & 106.8 & 105.5 & 101.4 & 82.0 & 57.1 & 45.7 & 30.5 \\
\hline 64.3 & 56.0 & 108.4 & 109.9 & 111.7 & 111.3 & 110.9 & 110.0 & 96.1 & 80.6 & 60.7 & 53.7 & 32.2 \\
\hline 71.2 & 63.7 & 123.9 & 127.1 & $\uparrow 30.3$ & 129.8 & 127.6 & 125.7 & 113.2 & 98.2 & 83.3 & 64.2 & 38.2 \\
\hline 78.0 & 61.7 & 102.7 & 105.2 & 90.4 & 98.9 & 86.5 & 66.2 & 102.6 & 100.9 & 90.3 & 66.3 & 44.1 \\
\hline \multicolumn{13}{|c|}{ mocouples located at sprinkler number 3} \\
\hline Time & $0 \mathrm{~mm}$ & $25 \mathrm{~mm}$ & $50 \mathrm{~mm}$ & $75 \mathrm{~mm}$ & $100 \mathrm{~mm}$ & $125 \mathrm{~mm}$ & $150 \mathrm{~mm}$ & $250 \mathrm{~mm}$ & $350 \mathrm{~mm}$ & $450 \mathrm{~mm}$ & $550 \mathrm{~mm}$ & $900 \mathrm{~mm}$ \\
\hline 0.0 & 20.9 & 21.6 & 21.6 & 21.6 & 21.5 & 21.6 & 21.5 & 21.3 & 21.1 & 20.8 & 20.5 & 18.6 \\
\hline 2.8 & 21.0 & 21.5 & 21.3 & 21.4 & 21.3 & 21.4 & 21.3 & 21.2 & 20.8 & 20.5 & 20.3 & 18.8 \\
\hline 9.6 & 21.0 & 21.5 & 21.3 & 21.3 & 21.2 & 21.3 & 21.3 & 21.1 & 20.9 & 20.6 & 20.3 & 18.3 \\
\hline 16.5 & 20.9 & 22.0 & 22.3 & 23.5 & 22.6 & 23.0 & 22.7 & 22.2 & 22.0 & 21.2 & 20.9 & 19.2 \\
\hline 23.3 & 21.6 & 29.8 & 33.6 & 34.3 & 31.2 & 31.1 & 28.6 & 22.7 & 22.1 & 21.7 & 21.5 & 20.2 \\
\hline 30.0 & 22.2 & 32.9 & 38.8 & 39.9 & 37.5 & 38.2 & 36.1 & 32.2 & 31.3 & 28.4 & 27.4 & 22.2 \\
\hline 37.0 & 22.7 & 35.7 & 42.1 & 43.4 & 40.5 & 41.4 & 39.9 & 36.1 & 34.5 & 33.2 & 33.1 & 23.8 \\
\hline 43.8 & 23.7 & 40.5 & 50.9 & 51.5 & 48.3 & 49.0 & 46.3 & 41.2 & 38.3 & 36.1 & 36.2 & 24.8 \\
\hline 50.7 & 24.9 & 47.0 & 59.5 & 59.5 & 55.6 & 55.7 & 53.5 & 48.0 & 45.3 & 42.8 & 42.7 & 31.1 \\
\hline 57.5 & 25.9 & 52.3 & 69.2 & 68.8 & 64.5 & 65.3 & 61.6 & 53.3 & 50.8 & 49.2 & 49.0 & 35.5 \\
\hline 64.3 & 26.9 & 57.3 & 76.2 & 76.9 & 71.4 & 71.5 & 68.1 & 59.0 & 56.7 & 55.3 & 55.9 & 38.4 \\
\hline 71.2 & 28.4 & 67.9 & 82.4 & 84.3 & 79.7 & 81.0 & 76.9 & 67.9 & 64.1 & 62.0 & 62.4 & 43.9 \\
\hline 78.0 & 29.6 & 69.1 & 85.7 & 87.8 & 82.7 & 83.4 & 81.7 & 73.9 & 72.7 & 71.4 & 70.7 & 50.9 \\
\hline \multicolumn{13}{|c|}{ mocouples located at sprinkler number 4} \\
\hline Time & $0 \mathrm{~mm}$ & $25 \mathrm{~mm}$ & $50 \mathrm{~mm}$ & $75 \mathrm{~mm}$ & $100 \mathrm{~mm}$ & $125 \mathrm{~mm}$ & $150 \mathrm{~mm}$ & $250 \mathrm{~mm}$ & $350 \mathrm{~mm}$ & $450 \mathrm{~mm}$ & $550 \mathrm{~mm}$ & $900 \mathrm{~mm}$ \\
\hline 0.0 & 20.7 & 21.1 & 21.3 & 21.4 & 21.3 & 21.4 & 21.3 & 21.1 & 21.0 & 20.6 & 20.1 & 18.4 \\
\hline 2.8 & 20.7 & 21.0 & 21.1 & 21.1 & 21.1 & 21.2 & 21.2 & 21.0 & 21.0 & 20.6 & 20.2 & 18.8 \\
\hline 9.6 & 20.8 & 21.1 & 21.2 & 21.2 & 21.2 & 21.2 & 21.1 & 21.0 & 20.8 & 20.7 & 20.4 & 19.0 \\
\hline 16.5 & 21.8 & 25.9 & 28.9 & 28.8 & 27.4 & 27.3 & 26.8 & 24.9 & 24.3 & 21.7 & 20.9 & 19.5 \\
\hline 23.3 & 22.6 & 33.9 & 34.5 & 33.9 & 32.1 & 32.4 & 31.6 & 27.5 & 24.8 & 22.2 & 21.3 & 20.4 \\
\hline 30.0 & 23.4 & 38.9 & 40.0 & 40.0 & 38.9 & 40.1 & 39.6 & 34.6 & 32.2 & 29.3 & 27.4 & 22.0 \\
\hline 37.0 & 25.3 & 44.3 & 49.2 & 48.9 & 46.9 & 47.9 & 47.2 & 39.5 & 37.6 & 34.9 & 32.9 & 21.5 \\
\hline 43.8 & 26.6 & 53.9 & 58.0 & 57.7 & 55.9 & 56.6 & 55.9 & 47.3 & 43.3 & 39.5 & 38.6 & 22.4 \\
\hline 50.7 & 28.7 & 56.4 & 61.8 & 62.0 & 61.2 & 62.3 & 61.4 & 52.4 & 49.1 & 48.1 & 46.6 & 25.3 \\
\hline 57.5 & 32.9 & 70.9 & 73.6 & 72.8 & 69.7 & 70.3 & 68.7 & 58.5 & 54.1 & 52.1 & 51.7 & 29.8 \\
\hline 64.3 & 33.6 & 74.6 & 80.0 & 79.1 & 76.5 & 77.4 & 76.8 & 68.8 & 65.6 & 61.4 & 60.3 & 33.4 \\
\hline 71.2 & 36.5 & 77.2 & 85.9 & 85.9 & 85.0 & 87.3 & 86.5 & 78.5 & 72.7 & 69.2 & 67.3 & 37.3 \\
\hline 78.0 & 37.8 & 86.3 & 93.7 & 93.8 & 91.0 & 92.1 & 90.8 & 81.0 & 77.5 & 74.9 & 74.4 & 41.9 \\
\hline \multicolumn{5}{|c|}{ city probes at sprinkler number 1} & \multicolumn{6}{|c|}{ Velocity probes al sprinkler number 2} & & \\
\hline Time & $25 \mathrm{~mm}$ & $75 \mathrm{~mm}$ & $125 \mathrm{~mm}$ & $250 \mathrm{~mm}$ & & Time & $25 \mathrm{~mm}$ & $75 \mathrm{~mm}$ & $125 \mathrm{~mm}$ & $250 \mathrm{~mm}$ & & \\
\hline 0.0 & 0.0 & 0.0 & 0.0 & 0.0 & & 0.0 & 0.0 & 0.0 & 0.0 & 0.0 & & \\
\hline
\end{tabular}


Experiment Number 22

\begin{tabular}{|c|c|c|c|c|c|c|c|c|c|}
\hline 2.8 & -0.1 & -0.1 & 0.0 & .01 & 2.8 & 0.1 & 0.1 & 0.1 & 0.1 \\
\hline 9.6 & -1.0 & -1.1 & -1.1 & -0.2 & 9.6 & 0.9 & 0.7 & 0.6 & 0.3 \\
\hline 16.5 & -1.2 & -1.4 & -1.2 & -0.3 & 16.5 & 1.1 & 0.9 & 0.9 & 0.4 \\
\hline 23.3 & -0.9 & -0.9 & -0.8 & 0.0 & 23.3 & 1.6 & 1.4 & 1.2 & 0.4 \\
\hline 30.0 & -1.0 & -1.1 & -1.1 & -0.6 & 30.0 & 1.1 & 1.0 & 0.9 & 0.6 \\
\hline 37.0 & -1.1 & -1.1 & -0.6 & -0.4 & 37.0 & 1.6 & 1.7 & 1.4 & 0.4 \\
\hline 43.8 & -1.4 & -1.4 & -1.1 & -0.8 & 43.8 & 1.5 & 1.5 & 1.0 & 0.7 \\
\hline 50.7 & -1.5 & -1.5 & -1.0 & -0.8 & 50.7 & 1.5 & 1.6 & 1.4 & 0.5 \\
\hline 57.5 & -1.5 & -1.7 & -1.1 & -0.5 & 57.5 & 1.8 & 1.7 & 1.4 & 0.6 \\
\hline 64.3 & -1.6 & -1.6 & -1.3 & -0.9 & 64.3 & 1.6 & 1.5 & 1.2 & 0.4 \\
\hline 71.2 & -1.2 & -1.1 & -0.8 & -0.5 & 71.2 & 1.1 & 1.1 & 0.7 & -0.3 \\
\hline 78.0 & -1.5 & -1.3 & -0.8 & -0.5 & 78.0 & 1.0 & 1.2 & 1.1 & 0.6 \\
\hline
\end{tabular}




\section{Experiment Number 23}

Thermocouples located at sprinkler number 1

\begin{tabular}{|c|c|c|c|c|c|c|c|c|c|c|c|c|}
\hline Time & $0 \mathrm{~mm}$ & $25 \mathrm{~mm}$ & $50 \mathrm{~mm}$ & $75 \mathrm{~mm}$ & $100 \mathrm{~mm}$ & $125 \mathrm{~mm}$ & $150 \mathrm{~mm}$ & $250 \mathrm{~mm}$ & $350 \mathrm{~mm}$ & $450 \mathrm{~mm}$ & $550 \mathrm{~mm}$ & $900 \mathrm{~mm}$ \\
\hline 0.0 & 21.8 & 21.9 & 22.1 & 21.7 & 21.8 & 21.2 & 21.5 & 20.6 & 21.0 & 20.8 & 20.3 & 18.6 \\
\hline 1.7 & 21.7 & 21.7 & 21.8 & 21.5 & 21.5 & 21.1 & 21.3 & 20.5 & 20.8 & 20.6 & 20.1 & 19.1 \\
\hline 8.5 & 22.1 & 23.4 & 26.3 & 27.2 & 26.6 & 27.3 & 25.7 & 28.0 & 26.6 & 24.1 & 20.9 & 19.3 \\
\hline 15.4 & 26.0 & 38.2 & 46.1 & 47.6 & 43.0 & 43.9 & 39.2 & 28.5 & 23.1 & 21.9 & 21.1 & 19.1 \\
\hline 22.2 & 28.8 & 44.8 & 51.0 & 52.6 & 49.2 & 51.0 & 48.0 & 41.3 & 32.9 & 28.8 & 22.3 & 19.3 \\
\hline 29.0 & 34.0 & 56.4 & 62.4 & 62.8 & 59.6 & 59.6 & 57.3 & 51.9 & 44.5 & 27.8 & 21.6 & 19.4 \\
\hline 35.9 & 37.7 & 66.5 & 74.5 & 75.5 & 72.1 & 74.0 & 68.4 & 65.4 & 54.6 & 41.1 & 30.1 & 20.5 \\
\hline 42.7 & 42.3 & 73.5 & 19.1 & 78.1 & 75.6 & 74.3 & 71.9 & 68.1 & 61.9 & 49.0 & 34.6 & 20.8 \\
\hline 49.7 & 45.5 & 81.2 & 88.8 & 90.6 & 88.0 & 89.7 & 85.8 & 78.5 & 70.3 & 63.2 & 49.0 & 21.8 \\
\hline 56.4 & 48.0 & 90.9 & 102.2 & 104.6 & 99.5 & 101.7 & 98.5 & 97.8 & 85.9 & 72.2 & 56.5 & 26.4 \\
\hline 63.2 & 54.1 & 101.5 & 111.8 & 114.0 & 108.7 & 110.7 & 107.2 & 98.4 & 89.3 & 74.1 & 57.6 & 32.8 \\
\hline 70.2 & 58.6 & 110.3 & 122.0 & 125.2 & 121.2 & 123.5 & 118.3 & 112.3 & 103.4 & 88.9 & 69.5 & 38.5 \\
\hline 76.9 & 58.3 & 43.5 & 109.8 & 124.4 & 103.7 & 115.9 & 109.4 & 113.9 & 107.8 & 95.2 & 74.6 & 41.8 \\
\hline \multicolumn{13}{|c|}{ mocouples located at sprinkler number 2} \\
\hline Time & $0 \mathrm{~mm}$ & $25 \mathrm{~mm}$ & $50 \mathrm{~mm}$ & $75 \mathrm{~mm}$ & $100 \mathrm{~mm}$ & $125 \mathrm{~mm}$ & $150 \mathrm{~mm}$ & $250 \mathrm{~mm}$ & $350 \mathrm{~mm}$ & $450 \mathrm{~mm}$ & $550 \mathrm{~mm}$ & $900 \mathrm{~mm}$ \\
\hline 0.0 & 20.1 & 22.3 & 22.4 & 22.2 & 22.2 & 21.5 & 21.7 & 21.3 & 21.0 & 20.6 & 20.3 & 19.0 \\
\hline 1.7 & 20.1 & 21.9 & 22.1 & 22.2 & 22.1 & 21.3 & 21.6 & 21.1 & 20.9 & 20.6 & 20.2 & 19.0 \\
\hline 8.5 & 20.9 & 26.9 & 27.2 & 27.9 & 27.8 & 28.4 & 27.2 & 29.3 & 26.1 & 23.7 & 21.2 & 19.5 \\
\hline 15.4 & 26.9 & 45.5 & 45.7 & 47.1 & 46.0 & 45.3 & 42.9 & 36.5 & 26.6 & 21.6 & 21.0 & 19.3 \\
\hline 22.2 & 31.0 & $\$ 1.1$ & 50.7 & 50.6 & 49.2 & 47.9 & 47.4 & 41.9 & 28.4 & 25.5 & 24.1 & 19.3 \\
\hline 29.0 & 32.2 & 57.3 & 57.6 & 59.2 & 58.5 & 59.2 & 58.5 & 54.4 & 44.3 & 38.5 & 26.3 & 19.4 \\
\hline 35.9 & 37.5 & 70.3 & 70.6 & 71.2 & 71.0 & 70.4 & 68.3 & 62.4 & 52.6 & 46.0 & 34.9 & 21.5 \\
\hline 42.7 & 43.8 & 80.1 & 81.1 & 82.2 & 81.6 & 79.8 & 77.8 & 64.9 & 60.2 & 47.9 & 39.9 & 23.8 \\
\hline 49.7 & 46.8 & 87.8 & 89.4 & 91.0 & 90.9 & 89.3 & 88.2 & 80.7 & 71.4 & 60.0 & 43.6 & 28.0 \\
\hline 56.4 & 51.6 & 98.1 & 99.2 & 102.0 & 100.1 & 100.2 & 101.4 & 97.3 & 82.4 & 67.3 & 58.1 & 31.7 \\
\hline 63.2 & 56.7 & 105.7 & 109.0 & 111.5 & 113.4 & 113.9 & 112.1 & 109.3 & 96.3 & B1.1 & 66.4 & 33.5 \\
\hline 70.2 & 59.0 & 114.5 & 117.0 & 119.5 & 119.9 & 119.3 & 117.0 & 112.0 & 105.2 & 99.9 & 80.7 & 38.4 \\
\hline 76.9 & 64.9 & 126.5 & 129.7 & 133.7 & 132.8 & 130.2 & 127.1 & 118.4 & 114.0 & 104.0 & 78.9 & 45.7 \\
\hline \multicolumn{13}{|c|}{ mocouples located at sprinkler number 3} \\
\hline Time & $0 \mathrm{~mm}$ & $25 \mathrm{~mm}$ & $50 \mathrm{~mm}$ & $75 \mathrm{~mm}$ & $100 \mathrm{~mm}$ & $125 \mathrm{~mm}$ & $150 \mathrm{~mm}$ & $250 \mathrm{~mm}$ & $350 \mathrm{~mm}$ & $450 \mathrm{~mm}$ & $550 \mathrm{~mm}$ & $900 \mathrm{~mm}$ \\
\hline 0.0 & 20.9 & 21.4 & 21.5 & 21.5 & 21.4 & 21.4 & 21.3 & 21.1 & 20.8 & 20.4 & 20.1 & 19.0 \\
\hline 1.7 & 20.9 & 21.3 & 21.2 & 21.3 & 21.2 & 21.3 & 21.3 & 21.1 & 20.7 & 20.3 & 20.0 & 19.1 \\
\hline 8.5 & 20.9 & 21.4 & 21.3 & 21.3 & 21.2 & 21.2 & 21.2 & 21.1 & 20.9 & 20.5 & 20.2 & 19.2 \\
\hline 15.4 & 21.0 & 22.4 & 22.6 & 24.0 & 22.8 & 23.5 & 23.0 & 22.6 & 22.7 & 21.3 & 20.9 & 19.6 \\
\hline 22.2 & 21.5 & 29.8 & 31.7 & 33.1 & 29.9 & 30.0 & 28.2 & 23.1 & 22.0 & 21.3 & 21.0 & 19.9 \\
\hline 29.0 & 22.0 & 33.4 & 38.6 & 39.5 & 37.0 & 37.4 & 36.0 & 31.4 & 30.2 & 28.6 & 27.5 & 21.8 \\
\hline 35.9 & 22.6 & 34.8 & 42.2 & 42.4 & 39.7 & 39.8 & 38.7 & 35.3 & 34.0 & 33.2 & 33.2 & 24.9 \\
\hline 42.7 & 23.1 & 40.4 & 51.8 & 52.0 & 49.0 & 49.7 & 46.9 & 42.6 & 40.4 & 36.1 & 35.7 & 26.5 \\
\hline 49.7 & 24.3 & 45.5 & 57.2 & 58.9 & 56.4 & 57.7 & 55.2 & 50.1 & 46.5 & 43.1 & 42.6 & 31.4 \\
\hline 56.4 & 25.8 & 53.6 & 65.5 & 66.4 & 63.6 & 64.7 & 61.8 & 56.6 & 53.9 & 50.2 & 50.0 & 36.7 \\
\hline 63.2 & 26.5 & 59.2 & 71.2 & 74.0 & 70.6 & 72.5 & 69.3 & 63.8 & 59.0 & 56.7 & 57.0 & 41.8 \\
\hline 70.2 & 28.8 & 62.3 & 82.4 & 83.2 & 79.4 & 80.5 & 77.6 & 71.4 & 67.3 & 65.0 & 63.3 & 47.7 \\
\hline 76.9 & 29.9 & 67.0 & 90.2 & 92.1 & 88.2 & 90.4 & 87.0 & 80.3 & 75.4 & 72.6 & 71.1 & 51.3 \\
\hline \multicolumn{13}{|c|}{ mocouples localed at sprinkler number 4} \\
\hline Time & $0 \mathrm{~mm}$ & $25 \mathrm{~mm}$ & $50 \mathrm{~mm}$ & $75 \mathrm{~mm}$ & $100 \mathrm{~mm}$ & $125 \mathrm{~mm}$ & $150 \mathrm{~mm}$ & $250 \mathrm{~mm}$ & $350 \mathrm{~mm}$ & $450 \mathrm{~mm}$ & $550 \mathrm{~mm}$ & $900 \mathrm{~mm}$ \\
\hline 0.0 & 20.8 & 21.2 & 21.5 & 21.5 & 21.3 & 21.4 & 21.4 & 21.2 & 20.9 & 20.3 & 19.9 & 18.7 \\
\hline 1.7 & 20.7 & 21.1 & 21.3 & 21.2 & 21.1 & 21.1 & 21.2 & 21.0 & 21.0 & 20.5 & 20.2 & 19.4 \\
\hline 8.5 & 20.8 & 21.1 & 21.2 & 21.2 & 21.2 & 21.2 & 21.1 & 21.0 & 20.9 & 20.6 & 20.1 & 19.6 \\
\hline 15.4 & 21.8 & 27.3 & 30.2 & 29.7 & 28.7 & 28.3 & 26.9 & 24.3 & 22.7 & 21.0 & 20.8 & 19.7 \\
\hline 22.2 & 22.2 & 33.3 & 35.7 & 35.1 & 34.1 & 33.8 & 33.1 & 27.7 & 26.4 & 23.3 & 22.4 & 20.3 \\
\hline 29.0 & 23.5 & 37.3 & 41.3 & 41.4 & 40.4 & 40.8 & 40.5 & 35.1 & 32.0 & 30.6 & 28.9 & 21.1 \\
\hline 35.9 & 25.7 & 44.1 & 50.5 & 50.1 & 48.2 & 48.4 & 48.2 & 40.5 & 38.3 & 36.1 & 33.2 & 21.4 \\
\hline 42.7 & 27.0 & 55.6 & 56.9 & 56.3 & 53.9 & 54.9 & 54.7 & 49.2 & 45.7 & 42.1 & 40.1 & 22.3 \\
\hline 49.7 & 28.3 & 56.1 & 59.0 & 59.2 & 59.2 & 60.5 & 60.4 & 56.2 & 52.9 & 48.2 & 48.6 & 25.1 \\
\hline 56.4 & 31.6 & 65.4 & 70.9 & 69.7 & 67.5 & 68.2 & 67.1 & 62.0 & 59.1 & 55.0 & 53.5 & 28.7 \\
\hline 63.2 & 35.1 & 71.9 & 77.1 & 76.8 & 74.9 & 76.1 & 75.1 & 65.1 & 63.3 & 61.8 & 61.0 & 33.9 \\
\hline 70.2 & 38.2 & 82.3 & 86.8 & 84.7 & 81.6 & 82.9 & 82.0 & 74.1 & 72.2 & 69.3 & 68.7 & 37.9 \\
\hline 76.9 & 39.6 & 95.0 & 97.1 & 95.3 & 93.0 & 93.1 & 92.2 & 77.8 & 77.6 & 77.0 & 75.1 & 41.2 \\
\hline
\end{tabular}

Velocily probes at sprinkler number 1

$\begin{array}{rrrrrrrrrr}\text { Time } & 25 \mathrm{~mm} & 75 \mathrm{~mm} & 125 \mathrm{~mm} & 250 \mathrm{~mm} & \text { Time } & 25 \mathrm{~mm} & 75 \mathrm{~mm} & 125 \mathrm{~mm} & 250 \mathrm{~mm} \\ 0.0 & 0.0 & 0.0 & 0.0 & 0.0 & 0.0 & 0.0 & 0.0 & 0.0 & 0.0\end{array}$


Experiment Number 23

$\begin{array}{rrrrr}1.7 & -0.1 & -0.1 & -0.2 & -0.1 \\ 8.5 & -0.5 & -0.6 & -0.6 & -0.1 \\ 15.4 & -1.1 & -1.0 & -0.7 & 0.2 \\ 22.2 & -1.4 & -1.5 & -1.1 & -0.6 \\ 29.0 & -1.1 & -1.3 & -0.9 & 0.4 \\ 35.9 & -1.4 & -1.4 & -1.0 & -1.0 \\ 42.7 & -1.4 & -1.4 & -1.3 & -0.3 \\ 49.7 & -1.0 & -1.1 & -0.6 & -0.3 \\ 56.4 & -0.8 & -0.8 & -0.6 & -0.7 \\ 63.2 & -1.3 & -1.2 & -0.9 & -0.7 \\ 70.2 & -0.7 & -0.6 & -0.5 & -0.8 \\ 76.9 & -1.3 & -1.2 & -0.8 & -0.6\end{array}$

$\begin{array}{rrrrr}1.7 & 0.1 & 0.1 & 0.0 & 0.0 \\ 8.5 & 0.8 & 0.8 & 0.5 & 0.2 \\ 15.4 & 0.8 & 0.9 & 0.9 & 0.5 \\ 22.2 & 1.0 & 0.8 & 0.6 & 0.4 \\ 29.0 & 1.2 & 1.3 & 1.1 & -0.2 \\ 35.9 & 1.7 & 1.6 & 1.4 & 0.8 \\ 42.7 & 1.5 & 1.4 & 1.1 & -0.2 \\ 49.7 & 1.5 & 1.4 & 1.3 & 0.8 \\ 56.4 & 1.3 & 1.5 & 1.7 & 1.2 \\ 63.2 & 1.5 & 1.4 & 1.1 & 0.0 \\ 70.2 & 1.7 & 1.6 & 1.0 & 0.5 \\ 76.9 & 2.0 & 2.1 & 1.6 & -0.3\end{array}$


Experiment Number 24

Thermocouples located at sprinkler number 1

\begin{tabular}{|c|c|c|c|c|c|c|c|c|c|c|c|c|}
\hline & & & & & & & & & & & & \\
\hline Time & $0 \mathrm{~mm}$ & $25 \mathrm{~mm}$ & $50 \mathrm{~mm}$ & $75 \mathrm{~mm}$ & $100 \mathrm{~mm}$ & $125 \mathrm{~mm}$ & $150 \mathrm{~mm}$ & $250 \mathrm{~mm}$ & $350 \mathrm{~mm}$ & $450 \mathrm{~mm}$ & $550 \mathrm{~mm}$ & $900 \mathrm{~mm}$ \\
\hline 0.0 & 23.0 & 22.8 & 22.8 & 22.6 & 22.6 & 22.1 & 22.3 & 21.8 & 21.8 & 21.5 & 21.3 & 20.5 \\
\hline 2.3 & 23.6 & 23.5 & 23.6 & 23.5 & 23.4 & 22.9 & 23.1 & 22.4 & 22.0 & 21.6 & 21.3 & 20.7 \\
\hline 9.2 & 23.7 & 23.7 & 23.8 & 23.6 & 23.5 & 22.9 & 23.2 & 22.5 & 21.9 & 21.7 & 21.5 & 21.0 \\
\hline 16.0 & 24.1 & 24.1 & 24.4 & 24.1 & 24.4 & 24.0 & 24.2 & 24.1 & 23.0 & 22.5 & 22.2 & 21.1 \\
\hline 22.9 & 28.3 & 28.0 & 28.3 & 29.0 & 28.7 & 29.2 & 28.1 & 26.5 & 23.8 & 23.1 & 22.5 & 21.2 \\
\hline 29.7 & 32.8 & 33.4 & 34.1 & 34.0 & 34.4 & 33.4 & 33.0 & 31.5 & 28.6 & 23.5 & 22.8 & 21.8 \\
\hline 36.4 & 35.8 & 37.0 & 38.7 & 40.1 & 39.2 & 38.4 & 39.0 & 38.2 & 31.0 & 25.8 & 24.2 & 22.7 \\
\hline 43.3 & 40.7 & 42.9 & 44.5 & 45.5 & 45.0 & 44.6 & 43.6 & 40.5 & 35.3 & 29.1 & 27.2 & 24.0 \\
\hline 50.0 & 47.1 & 49.7 & 52.4 & 53.1 & 51.4 & 48.9 & 49.2 & 42.0 & 42.4 & 34.4 & 28.6 & 24.8 \\
\hline 57.1 & 56.6 & 586 & 61.8 & 62.6 & 60.8 & 58.7 & 59.8 & 54.9 & 44.3 & 37.9 & 33.6 & 23.8 \\
\hline 63.8 & 60.1 & 64.7 & 68.2 & 69.0 & 67.0 & 67.3 & 65.8 & 57.2 & 54.1 & 43.1 & 40.0 & 25.2 \\
\hline 70.6 & 63.9 & 68.6 & 74.1 & 76.2 & 74.4 & 74.2 & 73.7 & 64.8 & 55.5 & 45.8 & 43.8 & 27.0 \\
\hline 77.5 & 75.2 & 79.1 & 83.5 & 85.0 & 83.0 & 81.7 & 80.0 & 75.6 & 64.4 & 50.9 & 48.0 & 33.1 \\
\hline 84.3 & 80.2 & 85.8 & 92.1 & 92.8 & 91.0 & 90.4 & 89.5 & 86.3 & 68.5 & 54.3 & 52.0 & 40.3 \\
\hline 91.2 & 86.2 & 90.9 & 95.5 & 96.2 & 94.3 & 94.8 & 92.4 & 90.1 & 77.2 & 61.0 & 57.7 & 45.8 \\
\hline 97.9 & 89.3 & 95.9 & 100.8 & 102.0 & 99.7 & 100.8 & 96.8 & 86.8 & 74.0 & 64.2 & 61.4 & 47.4 \\
\hline 104.7 & 94.2 & 100.1 & 105.7 & 107.1 & 104.9 & 104.7 & 103.1 & 93.0 & 78.5 & 70.4 & 64.8 & 50.1 \\
\hline 111.6 & 96.8 & 105.8 & 111.2 & 113.5 & 111.3 & 112.7 & 109.4 & 96.3 & 88.9 & 74.7 & 70.0 & 52.8 \\
\hline 118.4 & 102.2 & 107.7 & 111.5 & 113.0 & 110.8 & 113.3 & 111.8 & 107.1 & 94.2 & 79.8 & 74.3 & 57.9 \\
\hline$\{25.3$ & 94.6 & 107.0 & 114.3 & 115.2 & 112.5 & 112.0 & 111.0 & 107.0 & 98.3 & 83.3 & 77.6 & 62.5 \\
\hline 132.1 & 117.0 & 121.5 & 125.4 & 127.5 & 124.8 & 125.4 & 121.7 & 109.7 & 101.9 & 86.4 & 79.2 & 66.0 \\
\hline 138.8 & 127.8 & 130.2 & 136.9 & 139.7 & 135.3 & 130.5 & 132.1 & 122.0 & 106.0 & 91.2 & 85.4 & 69.9 \\
\hline 145.7 & 131.0 & 143.2 & 153.8 & 155.7 & 153.9 & 155.0 & 149.6 & 144.5 & 119.6 & 103.7 & 91.7 & 73.9 \\
\hline \multicolumn{13}{|c|}{ Thermocouples localed at sprinkler number 2} \\
\hline Time & $0 \mathrm{~mm}$ & $25 \mathrm{~mm}$ & $50 \mathrm{~mm}$ & $75 \mathrm{~mm}$ & $100 \mathrm{~mm}$ & $125 \mathrm{~mm}$ & $150 \mathrm{~mm}$ & $250 \mathrm{~mm}$ & $350 \mathrm{~mm}$ & $450 \mathrm{~mm}$ & $550 \mathrm{~mm}$ & $900 \mathrm{~mm}$ \\
\hline 0.0 & 24.1 & 24.1 & 23.7 & 23.7 & 23.4 & 23.2 & 23.0 & 22.2 & 21.7 & 21.4 & 21.2 & 20.5 \\
\hline 2.3 & 23.7 & 23.9 & 22.9 & 23.3 & 22.8 & 22.9 & 22.6 & 22.2 & 22.1 & 21.9 & 21.7 & 21.0 \\
\hline 9.2 & 24.0 & 24.2 & 23.3 & 23.4 & 22.9 & 22.9 & 22.5 & 22.3 & 22.0 & 21.8 & 21.6 & 21.0 \\
\hline 16.0 & 24.5 & 24.5 & 24.5 & 23.9 & 24.7 & 23.6 & 23.7 & 22.5 & 22.3 & 22.1 & 21.8 & 21.0 \\
\hline 22.9 & 27.3 & 27.5 & 28.4 & 27.2 & 28.2 & 26.9 & 27.2 & 23.3 & 22.5 & 22.4 & 22.2 & 21.1 \\
\hline 29.7 & 31.0 & 31.8 & 33.9 & 31,9 & 35.7 & 34.8 & 35.9 & 34.9 & 25.9 & 22.8 & 22.4 & 21.3 \\
\hline 36.4 & 42.5 & 45.6 & 45.9 & 44.8 & 46.2 & 45.6 & 44.3 & 35.8 & 31.7 & 24.9 & 23.5 & 21.9 \\
\hline 43.3 & 45.7 & 47.9 & 48.5 & 44.9 & 49.3 & 47.4 & 48.2 & 41.4 & 34.6 & 29.4 & 26.9 & 24.8 \\
\hline 50.0 & 50.7 & 54.1 & 56.4 & 55.1 & 59.2 & 58.6 & 58.2 & 42.9 & 38.9 & 32.9 & 30.9 & 24.5 \\
\hline 57.1 & 57.3 & 59.6 & 61.2 & 57.7 & 63.6 & 64.0 & 64.0 & 52.8 & 47.9 & 40.0 & 35.9 & 25.4 \\
\hline 63.8 & 64.6 & 68.2 & 70.3 & 66.9 & 72.1 & 69.5 & 69.6 & 59.3 & 56.0 & 44.3 & 40.7 & 30.0 \\
\hline 70.6 & 71.5 & 76.7 & 76.8 & 74.6 & 78.4 & 77.1 & 77.5 & 60.5 & 56.0 & 44.9 & 42.7 & 33.9 \\
\hline 77.5 & 77.5 & 82.3 & 84.2 & 83.5 & 87.4 & 88.2 & 87.6 & 73.5 & 66.4 & 53.9 & 46.9 & 35.7 \\
\hline 84.3 & 86.3 & 91.4 & 91.5 & 89.4 & 92.6 & 92.1 & 90.9 & 73.4 & 70.6 & 57.3 & 51.7 & 39.1 \\
\hline 91.2 & 93.0 & 100.9 & 102.5 & 103.1 & 106.8 & 107.1 & 106.4 & 92.3 & 75.4 & 64.5 & 59.5 & 45.3 \\
\hline 97.9 & 104.7 & 112.8 & 113.0 & 107.3 & 113.1 & 110.1 & 110.2 & 83.8 & 79.6 & 70.0 & 64.2 & 48.3 \\
\hline 104.7 & 107.2 & 114.3 & 115.5 & 115.7 & 118.7 & 117.5 & 116.9 & 106.4 & 86.6 & 74.1 & 67.2 & 52.9 \\
\hline 111.6 & 107.7 & 117.6 & 118.7 & 116.1 & 118.3 & 113.6 & 114.6 & 100.9 & 92.2 & 77.9 & 68.6 & 56.0 \\
\hline 118.4 & 115.5 & 123.1 & 123.6 & 120.6 & 124.6 & 122.5 & 122.6 & 110.7 & 106.3 & 84.7 & 76.8 & 57.6 \\
\hline 125.3 & 113.2 & 118.7 & 120.7 & 120.0 & 124.2 & 123.8 & 123.2 & 106.3 & 103.9 & 92.0 & 82.9 & 62.4 \\
\hline 132.1 & 125.6 & 134.0 & 137.2 & 138.0 & 140.2 & 140.8 & 139.0 & 133.5 & 115.8 & 92.0 & 85.4 & 63.6 \\
\hline 138.8 & 133.8 & 146.3 & 147.9 & 147.5 & 152.4 & 145.3 & 150.0 & 121.1 & 112.1 & 96.6 & 88.8 & 69.0 \\
\hline 145.7 & 146.1 & 157.6 & 157.3 & 158.0 & 146.9 & 124.7 & 128.2 & 132.1 & 125.0 & 105.7 & 98.5 & 74.7 \\
\hline \multicolumn{13}{|c|}{ Thermocouples located at sprinkler number 3} \\
\hline Time & $0 \mathrm{~mm}$ & $25 \mathrm{~mm}$ & $50 \mathrm{~mm}$ & $75 \mathrm{~mm}$ & $100 \mathrm{~mm}$ & $125 \mathrm{~mm}$ & $150 \mathrm{~mm}$ & $250 \mathrm{~mm}$ & $350 \mathrm{~mm}$ & $450 \mathrm{~mm}$ & $550 \mathrm{~mm}$ & $900 \mathrm{~mm}$ \\
\hline 0.0 & 24.0 & 23.9 & 23.5 & 23.3 & 23.2 & 23.1 & 23.0 & 22.3 & 219 & 21.5 & 21.3 & 20.7 \\
\hline 2.3 & 24.6 & 24.4 & 23.8 & 23.4 & 23.2 & 23.0 & 22.8 & 22.5 & 22.2 & 22.0 & 21.8 & 20.9 \\
\hline 9.2 & 24.3 & 24.1 & 23.8 & 23.5 & 23.4 & 23.2 & 23.0 & 22.6 & 22.2 & 21.9 & 21.7 & 20.9 \\
\hline 16.0 & 24.3 & 24.2 & 24.3 & 24.1 & 24.1 & 24.1 & 24.2 & 23.6 & 23.3 & 22.5 & 22.1 & 21.0 \\
\hline 22.9 & 27.0 & 28.1 & 28.0 & 27.5 & 27.4 & 27.8 & 28.6 & 26.9 & 26.4 & 25.6 & 24.9 & 21.2 \\
\hline 29.7 & 33.4 & 35.1 & 35.1 & 33.9 & 33.4 & 33.7 & 34.6 & 33.0 & 29.0 & 25.1 & 22.7 & 21.1 \\
\hline 36.4 & 38.5 & 40.2 & 41.2 & 39.6 & 40.0 & 40.6 & 40.9 & 39.0 & 34.9 & 28.2 & 23.9 & 21.4 \\
\hline 43.3 & 41.6 & 43.9 & 45.4 & 44.1 & 44.6 & 45.4 & 46.1 & 43.5 & 41.3 & 33.6 & 26.9 & 22.2 \\
\hline 50.0 & 45.0 & 47.9 & 49.2 & 48.4 & 49.0 & 49.8 & 50.4 & 48.3 & 47.4 & 39.2 & 31.7 & 23.3 \\
\hline 57.1 & 47.8 & 53.2 & 52.3 & 53.1 & 52.9 & 55.0 & 54.8 & 55.4 & 49.3 & 38.7 & 34.4 & 26.7 \\
\hline 63.8 & 46.7 & 53.0 & 55.0 & 56.8 & 37.1 & 59.0 & 59.2 & 60.7 & 62.6 & 45.1 & 37.3 & 28.3 \\
\hline
\end{tabular}


Experiment Number 24

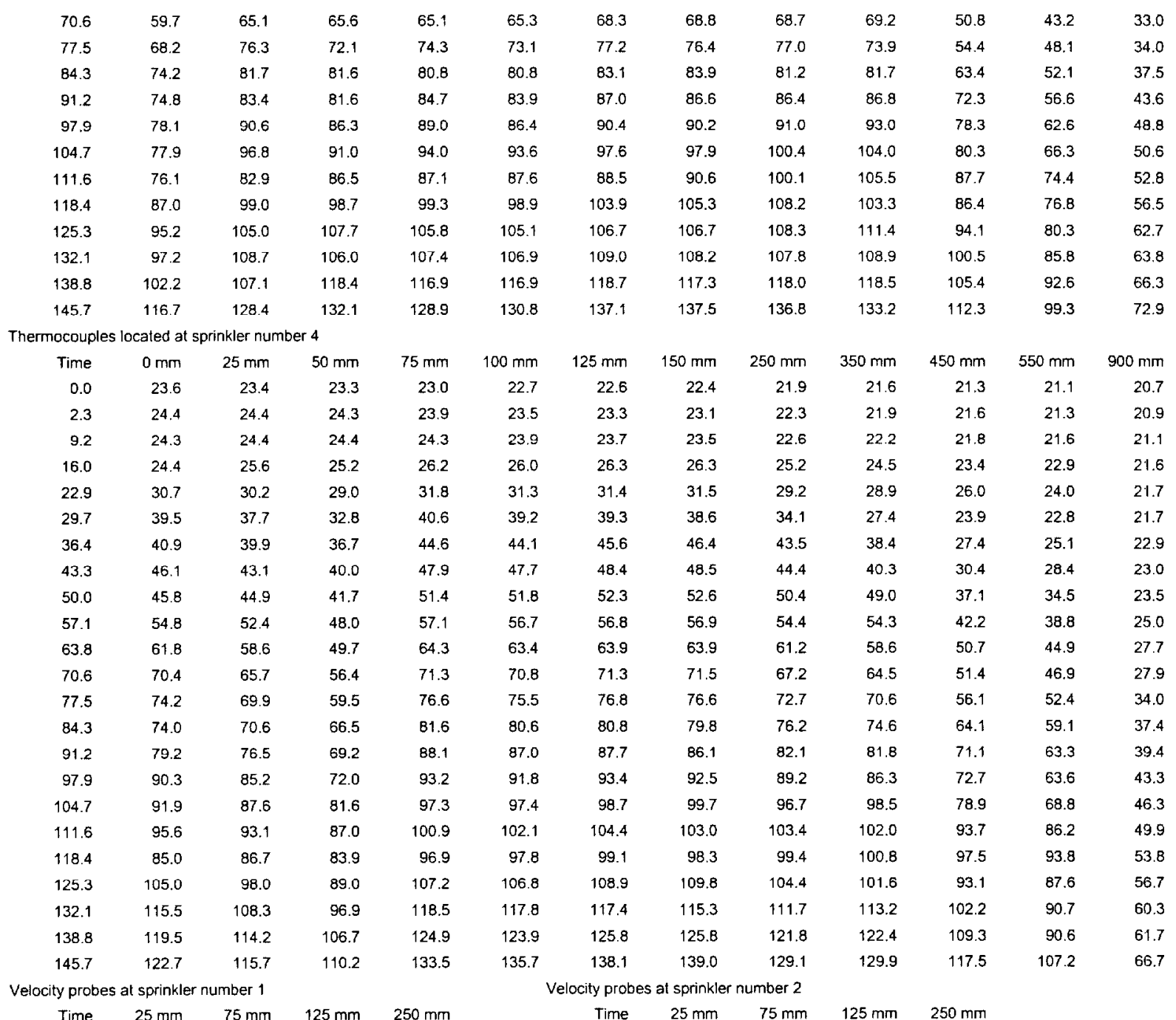

\begin{tabular}{|c|c|c|c|c|c|c|c|c|c|}
\hline Time & $25 \mathrm{~mm}$ & $75 \mathrm{~mm}$ & $125 \mathrm{~mm}$ & $250 \mathrm{~mm}$ & Time & $25 \mathrm{~mm}$ & $75 \mathrm{~mm}$ & $125 \mathrm{~mm}$ & $250 \mathrm{~mm}$ \\
\hline 0.0 & 0.0 & 0.0 & 0.0 & 0.0 & 0.0 & 0.0 & 0.0 & 0.0 & 0.0 \\
\hline 2.3 & -0.1 & 0.0 & -0.1 & 0.1 & 2.3 & -0.1 & -0.1 & -0.1 & -0.1 \\
\hline 9.2 & -0.1 & 0.0 & -0.1 & -0.1 & 9.2 & 0.1 & -0.1 & -0.1 & -0.1 \\
\hline 16.0 & -0.4 & -0.3 & -0.3 & -0.1 & 16.0 & 0.2 & 0.2 & 0.2 & 0.1 \\
\hline 22.9 & -0.3 & -0.3 & -0.4 & -0.3 & 22.9 & 0.2 & 0.2 & 0.3 & 0.2 \\
\hline 29.7 & -0.3 & -0.3 & -0.3 & -0.3 & 29.7 & 0.3 & 0.2 & 0.3 & 0.1 \\
\hline 36.4 & -0.3 & -0.3 & -0.4 & -0.3 & 36.4 & 0.2 & 0.2 & 0.3 & 0.2 \\
\hline 43.3 & -0.4 & -0.4 & -0.3 & -0.2 & 43.3 & 0.4 & 0.4 & 0.4 & 0.2 \\
\hline 50.0 & -0.4 & -0.4 & -0.4 & -0.4 & 50.0 & 0.5 & 0.4 & 0.6 & 0.2 \\
\hline 57.1 & -0.5 & -0.5 & -0.4 & -0.4 & 57.1 & 0.4 & 0.4 & 0.5 & 0.4 \\
\hline 63.8 & -0.4 & -0.6 & -0.5 & -0.4 & 63.8 & 0.5 & 0.4 & 0.5 & 0.2 \\
\hline 70.6 & -0.5 & -0.6 & -0.5 & -0.4 & 70.6 & 0.6 & 0.6 & 0.5 & 0.3 \\
\hline 77.5 & -0.5 & -0.5 & -0.4 & -0.6 & 77.5 & 0.4 & 0.3 & 0.5 & 0.2 \\
\hline 84.3 & -0.5 & -0.5 & -0.6 & -0.3 & 84.3 & 0.5 & 0.5 & 0.7 & 0.3 \\
\hline 91.2 & -0.5 & -0.6 & -0.4 & -0.4 & 91.2 & 0.8 & 0.5 & 0.6 & 0.6 \\
\hline 97.9 & -0.5 & -0.7 & -0.4 & -0.5 & 97.9 & 0.6 & 0.6 & 0.7 & 0.3 \\
\hline 104.7 & -0.5 & -0.5 & -0.5 & -0.5 & 104.7 & 0.4 & 0.5 & 0.7 & 0.6 \\
\hline 111.6 & -0.4 & -0.5 & -0.2 & -0.5 & 111.6 & 0.6 & 0.5 & 0.7 & 0.4 \\
\hline 118.4 & -0.3 & -0.5 & -0.4 & -0.5 & 118.4 & 0.7 & 0.5 & 0.8 & 0.4 \\
\hline 125.3 & -0.7 & -0.7 & -0.6 & -0.5 & 125.3 & 0.5 & 0.5 & 0.8 & 0.3 \\
\hline 132.1 & -0.6 & -0.7 & -0.7 & -0.6 & 132.1 & 0.6 & 0.6 & 0.8 & 0.3 \\
\hline 138.8 & -0.8 & -0.9 & -0.8 & -0.8 & 138.8 & 0.8 & 0.5 & 0.8 & 0.3 \\
\hline 145.7 & -0.7 & -0.9 & -0.6 & -0.5 & 145.7 & 0.7 & 0.6 & 0.8 & 0.3 \\
\hline
\end{tabular}

Velocity probes in channels above burner 
Experiment Number 24

$\begin{array}{rrr}\text { Time } & \text { Channel 1 } & \text { Channel 2 } \\ 0.0 & 0.0 & 0.0 \\ 2.3 & 1.1 & 0.9 \\ 9.2 & 1.8 & 1.0 \\ 16.0 & 2.1 & 1.2 \\ 22.9 & 1.9 & 1.3 \\ 29.7 & 1.8 & 1.2 \\ 36.4 & 2.8 & 1.5 \\ 43.3 & 2.5 & 1.9 \\ 50.0 & 2.5 & 1.9 \\ 57.1 & 2.6 & 1.8 \\ 63.8 & 3.4 & 1.7 \\ 70.6 & 3.1 & 1.7 \\ 77.5 & 2.8 & 1.8 \\ 84.3 & 2.8 & 1.9 \\ 91.2 & 3.3 & 1.9 \\ 97.9 & 2.6 & 2.2 \\ 104.7 & 3.0 & 1.9 \\ 111.6 & 3.2 & 1.9 \\ 118.4 & 2.8 & 2.3 \\ 125.3 & 3.4 & 2.2 \\ 132.1 & 2.8 & 2.6 \\ 138.8 & 3.2 & 2.2 \\ 145.7 & 3.2 & 2.7\end{array}$


Experiment Number 25

Thermocouples located at sprinkler number 1

Time $\quad 0 \mathrm{~mm} \quad 25 \mathrm{~mm} \quad 50 \mathrm{~mm} \quad 75 \mathrm{~mm} \quad 100 \mathrm{~mm} \quad 125 \mathrm{~mm} \quad 150 \mathrm{~mm} \quad 250 \mathrm{~mm} \quad 350 \mathrm{~mm} \quad 450 \mathrm{~mm} \quad 550 \mathrm{~mm} \quad 900 \mathrm{~mm}$

$\begin{array}{llllllllllll}0.0 & 27.0 & 26.8 & 26.9 & 26.4 & 26.2 & 25.4 & 25.5 & 24.7 & 24.4 & 24.0 & 23.6\end{array}$

$\begin{array}{lllllllllllll}2.9 & 27.1 & 26.9 & 27.0 & 26.5 & 26.3 & 25.6 & 25.9 & 25.0 & 25.1 & 24.8 & 24.3 & 23.1\end{array}$

$\begin{array}{llllllllllll}9.8 & 27.6 & 27.1 & 27.0 & 26.5 & 26.4 & 25.7 & 26.1 & 25.1 & 25.2 & 24.9 & 24.5\end{array}$

$\begin{array}{lllllllllllll}16.6 & 29.3 & 29.6 & 30.2 & 30.3 & 30.0 & 29.8 & 29.3 & 28.7 & 26.6 & 25.7 & 25.1 & 23.5\end{array}$

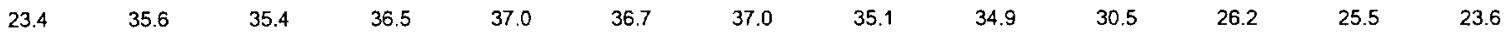

$\begin{array}{llllllllllll}30.3 & 42.2 & 42.1 & 44.0 & 43.6 & 43.2 & 43.6 & 41.5 & 41.4 & 38.0 & 31.1 & 26.0\end{array}$

$\begin{array}{lllllllllllll}37.0 & 45.6 & 48.0 & 51.6 & 52.5 & 51.3 & 51.1 & 49.1 & 48.5 & 44.5 & 34.9 & 30.7 & 26.7\end{array}$

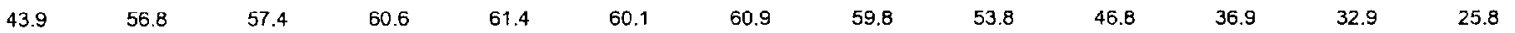

$\begin{array}{lllllllllllll}50.7 & 65.9 & 67.4 & 70.3 & 71.6 & 70.9 & 72.0 & 68.9 & 65.5 & 60.1 & 44.5 & 38.8 & 26.5\end{array}$

$\begin{array}{llllllllllll}574 & 70.3 & 76.3 & 81.0 & 82.2 & 79.4 & 77.8 & 76.4 & 67.3 & 58.2 & 48.0 & 44.2\end{array}$

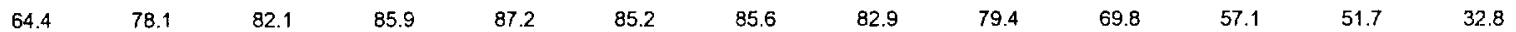

$\begin{array}{lllllllllllll}71.1 & 83.2 & 88.7 & 93.3 & 94.4 & 91.8 & 90.9 & 89.0 & 76.8 & 65.3 & 60.0 & 57.9 & 36.8\end{array}$

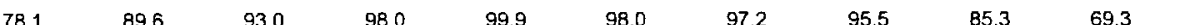

$\begin{array}{llllllllllll}84.8 & 94.6 & 100.9 & 108.2 & 109.9 & 106.4 & 101.5 & 101.6 & 82.4 & 74.8 & 67.0 & 64.0\end{array}$

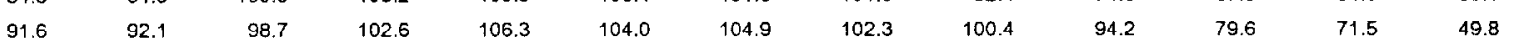

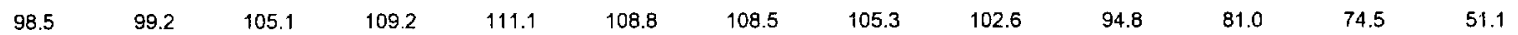

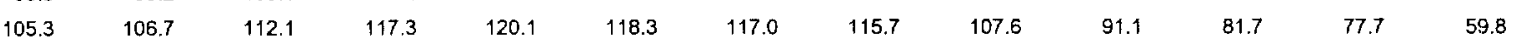

$\begin{array}{lllllllllllll}112.2 & 114.6 & 119.7 & 125.7 & 126.2 & 124.0 & 125.5 & 123.1 & 115.7 & 107.8 & 89.6 & 81.9 & 61.6\end{array}$

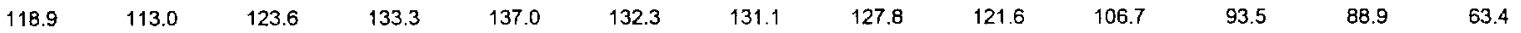

$\begin{array}{lllllllllllll}125.7 & 128.2 & 134.0 & 140.8 & 142.6 & 138.5 & 140.5 & 136.6 & 133.8 & 113.0 & 97.0 & 92.3 & 68.3\end{array}$

Thermocouples located at sprinkler number 2

\begin{tabular}{|c|c|c|c|c|c|c|c|c|c|c|c|c|}
\hline Time & $0 \mathrm{~mm}$ & $25 \mathrm{~mm}$ & $50 \mathrm{~mm}$ & $75 \mathrm{~mm}$ & $100 \mathrm{~mm}$ & $125 \mathrm{~mm}$ & $150 \mathrm{~mm}$ & $250 \mathrm{~mm}$ & $350 \mathrm{~mm}$ & $450 \mathrm{~mm}$ & $550 \mathrm{~mm}$ & $900 \mathrm{~mm}$ \\
\hline 0.0 & 28.5 & 28.2 & 27.6 & 27.1 & 26.7 & 26.4 & 26.2 & 25.2 & 24.6 & 24.3 & 23.9 & 22.9 \\
\hline 2.9 & 29.1 & 28.4 & 28.7 & 27.7 & 27.8 & 27.1 & 26.9 & 25.4 & 24.9 & 24.6 & 24.0 & 23.2 \\
\hline 9.8 & 29.3 & 28.7 & 28.7 & 27.6 & 28.2 & 27.0 & 27.0 & 25.6 & 25.2 & 25.0 & 24.5 & 23.5 \\
\hline 16.6 & 31.8 & 31.5 & 33.1 & 30.5 & 32.4 & 31.0 & 30.6 & 27.5 & 26.4 & 25.4 & 24.8 & 23.5 \\
\hline 23.4 & 37.6 & 36.6 & 38.6 & 38.1 & 40.4 & 41.6 & 41.3 & 37.8 & 28.8 & 25.8 & 25.0 & 23.6 \\
\hline 30.3 & 46.1 & 47.0 & 48.8 & 47.4 & 51.0 & 50.1 & 49.6 & 39.3 & 31.5 & 30.6 & 28.0 & 24.4 \\
\hline 37.0 & 48.0 & 48.8 & 51.1 & 48.0 & 53.3 & 51.6 & 53.3 & 50.8 & 44.2 & 32.5 & 31.3 & 26.9 \\
\hline 43.9 & 55.2 & 55.9 & 61.5 & 57.2 & 62.5 & 61.2 & 61.6 & 58.6 & 46.4 & 36.8 & 34.6 & 26.5 \\
\hline 50.7 & 64.5 & 67.5 & 69.4 & 67.7 & 71.2 & 71.8 & 72.1 & 63.3 & 50.8 & 45.5 & 42.1 & 29.8 \\
\hline 57.4 & 68.8 & 71.5 & 75.4 & 74.0 & 78.8 & 79.1 & 78.6 & 63.0 & 53.5 & 47.8 & 44.2 & 3.0 \\
\hline 64.4 & 70.7 & 70.4 & 76.4 & 71.1 & 78.1 & 74.8 & 77.0 & 75.3 & 67.9 & 54.4 & 50.1 & 37.2 \\
\hline 71.1 & 85.3 & 93.3 & 95.1 & 93.3 & 95.4 & 95.6 & 95.2 & 76.7 & 68.2 & 57.7 & 52.5 & 40.4 \\
\hline 78.1 & 94.3 & 103.9 & 105.3 & 102.9 & 107.4 & 107.3 & 105.2 & 77.8 & 75.7 & 66.0 & 58.0 & 45.9 \\
\hline 84.8 & 99.4 & 104.2 & 107.0 & 102.4 & 109.2 & 106.6 & 107.9 & 93.5 & 86.5 & 74.8 & 66.2 & 49.3 \\
\hline 91.6 & 100.6 & 103.8 & 111.7 & 107.8 & 114.9 & 112.4 & 115.4 & 106.2 & 95.3 & 79.2 & 74.0 & 54.4 \\
\hline 98.5 & 100.8 & 101.5 & 111.4 & 103.0 & 113.9 & 113.1 & 116.4 & 110.4 & 93.4 & 82.4 & 74.4 & 60.4 \\
\hline 105.3 & 112.0 & 117.7 & 121.4 & 117.1 & 121.8 & 118.7 & 119.5 & 103.5 & 94.8 & 85.0 & 77.3 & 62.2 \\
\hline 112.2 & 116.6 & 124.3 & 125.7 & 127.2 & 131.0 & 129.6 & 128.9 & 121.1 & 110.7 & 94.8 & 83.9 & 63.3 \\
\hline 118.9 & 130.1 & 138.0 & 140.8 & 136.9 & 143.5 & 138.7 & 136.7 & 107.8 & 107.3 & 94.5 & 88.2 & 64.8 \\
\hline 125.7 & 133.8 & 119.8 & 69.8 & 58.2 & 120.5 & 113.3 & 135.4 & 107.7 & 119.9 & 100.7 & 96.3 & 69.7 \\
\hline \multicolumn{13}{|c|}{ Iocouples located at sprinkler number 3} \\
\hline Time & $0 \mathrm{~mm}$ & $25 \mathrm{~mm}$ & $50 \mathrm{~mm}$ & 75 เाแ! & $100 \mathrm{~mm}$ & $125 \mathrm{~mm}$ & $150 \mathrm{~mm}$ & $250 \mathrm{~mm}$ & $350 \mathrm{~mm}$ & $450 \mathrm{~mm}$ & $550 \mathrm{~mm}$ & $900 \mathrm{~mm}$ \\
\hline 0.0 & 28.5 & 28.5 & 28.1 & 27.5 & 27.0 & 26.6 & 26.2 & 25.1 & 24.5 & 24.2 & 23.8 & 23.1 \\
\hline 2.9 & 28.6 & 28.8 & 28.5 & 27.7 & 27.2 & 26.9 & 26.5 & 25.6 & 24.7 & 24.3 & 24.0 & 23.2 \\
\hline 9.8 & 29.3 & 28.6 & 28.7 & 27.7 & 27.2 & 26.8 & 26.5 & 25.6 & 24.9 & 24.5 & 24.4 & 23.6 \\
\hline 16.6 & 29.9 & 30.9 & 30.2 & 30.2 & 29.7 & 29.8 & 30.2 & 29.4 & 29.5 & 28.3 & 27.8 & 23.8 \\
\hline 23.4 & 34.6 & 37.0 & 36.0 & 36.3 & 35.8 & 36.6 & 37.3 & 36.0 & 34.6 & 29.4 & 25.5 & 23.5 \\
\hline 30.3 & 41.9 & 43.2 & 43.1 & 43.6 & 43.5 & 44.1 & 45.2 & 43.2 & 39.8 & 33.7 & 26.1 & 24.0 \\
\hline 37.0 & 47.4 & 48.7 & 48.7 & 49.3 & 49.1 & 500 & 50.3 & 49.5 & 46.1 & 36.1 & 30.3 & 25.3 \\
\hline 43.9 & 51.9 & 58.5 & 54.6 & 56.5 & 56.4 & 58.3 & 58.5 & 56.7 & 55.0 & 41.2 & 35.3 & 26.1 \\
\hline 50.7 & 55.7 & 62.5 & 58.2 & 60.3 & 60.2 & 62.6 & 62.4 & 61.4 & 60.5 & 46.1 & 38.9 & 29.3 \\
\hline 57.4 & 59.3 & 67.7 & 63.5 & 65.9 & 65.6 & 68.2 & 68.8 & 68.9 & 69.2 & 54.1 & 44.6 & 32.0 \\
\hline 64.4 & 66.8 & 76.3 & 71.4 & 74.5 & 73.4 & 77.5 & 76.4 & 75.3 & 73.2 & 56.8 & 50.5 & 34.1 \\
\hline 71.1 & 71.7 & 77.5 & 77.1 & 76.9 & 76.1 & 78.1 & 79.5 & 79.6 & 82.2 & 64.1 & 53.4 & 41.3 \\
\hline 78.1 & 78.5 & 85.2 & 84.8 & 85.2 & 86.6 & B9. 8 & 90.4 & 90.0 & 89.7 & 73.2 & 59.0 & 45.5 \\
\hline 84.8 & 83.9 & 94.2 & 88.6 & 90.6 & 91.8 & 94.9 & 96.5 & 98.8 & 100.4 & 70.6 & 59.0 & 49.2 \\
\hline 91.6 & 83.2 & 90.1 & 92.0 & 92.2 & 94.4 & 97.7 & 99.9 & 103.6 & 106.6 & 86.3 & 67.7 & 54.9 \\
\hline 98.5 & 90.1 & 97.2 & 97.7 & 99.3 & 101.0 & 102.9 & 103.5 & 104.0 & 101.9 & B7.2 & 73.8 & 58.0 \\
\hline 105.3 & 90.4 & 101.8 & 99.2 & 105.1 & 105.3 & 109.6 & 110.7 & 12.4 & 114.0 & 88.3 & 74.3 & 60.0 \\
\hline
\end{tabular}




\section{Experiment Number 25}

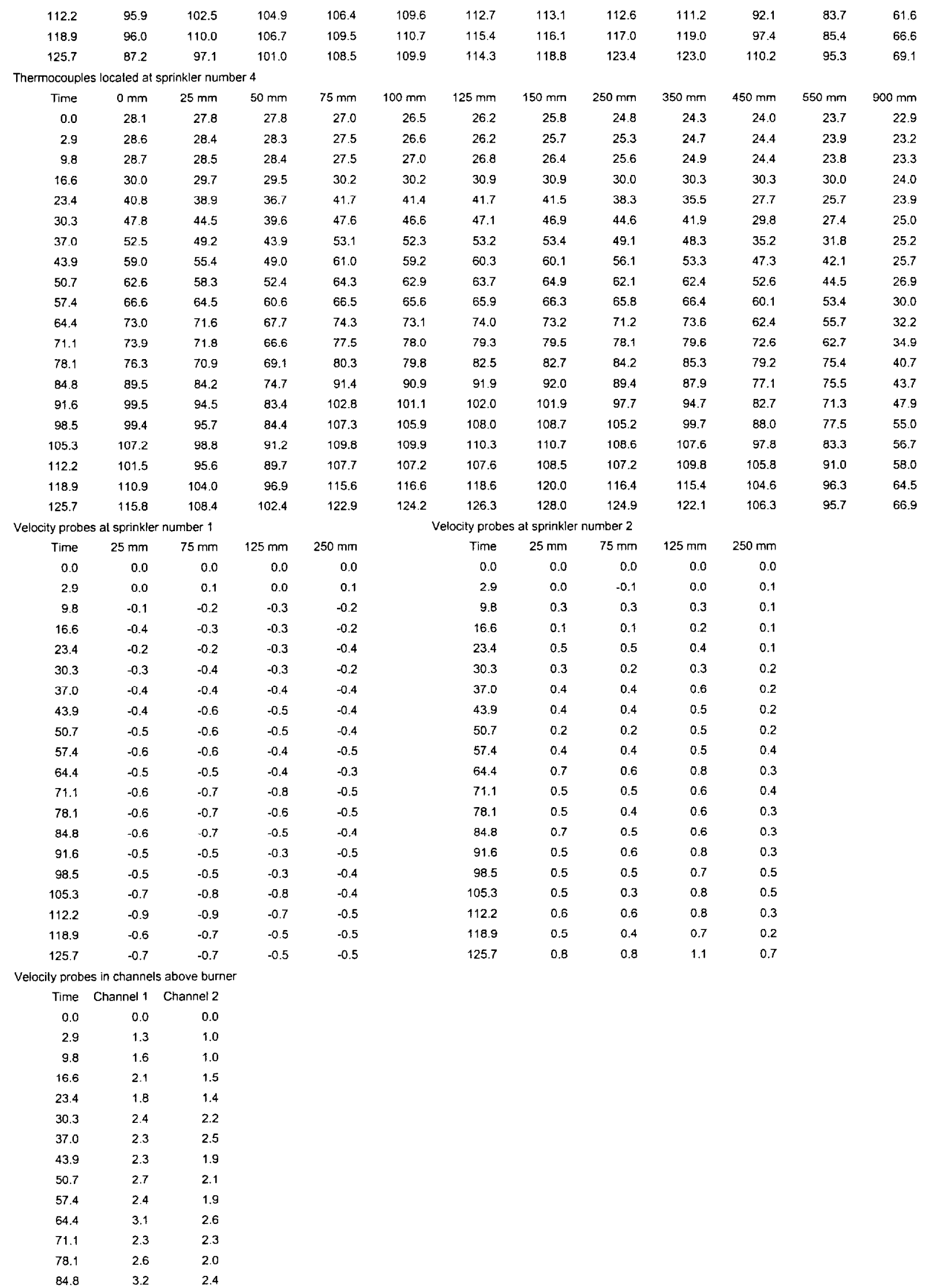


Experiment Number 25

$\begin{array}{rll}91.6 & 2.9 & 2.1 \\ 98.5 & 3.4 & 2.2 \\ 105.3 & 2.3 & 2.1 \\ 112.2 & 3.3 & 2.7 \\ 118.9 & 4.0 & 2.3 \\ 125.7 & 3.3 & 2.6\end{array}$


Experiment Number 26

Thermocouples located at sprinkler number 1

\begin{tabular}{|c|c|c|c|c|c|c|c|c|c|c|c|c|}
\hline Time & $0 \mathrm{~mm}$ & $25 \mathrm{~mm}$ & $50 \mathrm{~mm}$ & $75 \mathrm{~mm}$ & $100 \mathrm{~mm}$ & $125 \mathrm{~mm}$ & $150 \mathrm{~mm}$ & $250 \mathrm{~mm}$ & $350 \mathrm{~mm}$ & $450 \mathrm{~mm}$ & $550 \mathrm{~mm}$ & $900 \mathrm{~mm}$ \\
\hline 0.0 & 21.5 & 21.3 & 21.3 & 21.2 & 21.1 & 20.9 & 20.8 & 20.3 & 20.0 & 19.7 & 19.2 & 17.5 \\
\hline 2.7 & 21.6 & 21.3 & 21.2 & 21.1 & 21.0 & 20.8 & 20.7 & 20.3 & 20.2 & 20.1 & 19.8 & 18.4 \\
\hline 9.5 & 21.6 & 22.4 & 24.0 & 24.4 & 24.0 & 24.2 & 22.6 & 22.8 & 21.8 & 21.3 & 20.5 & 18.7 \\
\hline 16.3 & 23.3 & 29.3 & 32.8 & 33.6 & 31.8 & 32.0 & 29.4 & 27.6 & 22.4 & 20.6 & 20.2 & 18.3 \\
\hline 23.3 & 26.2 & 36.9 & 38.5 & 37.9 & 34.5 & 33.9 & 32.6 & 28.7 & 21.7 & 20.4 & 20.0 & 18.2 \\
\hline 30.0 & 26.9 & 37.6 & 39.7 & 40.0 & 37.9 & 38.3 & 36.7 & 35.9 & 32.9 & 28.1 & 22.4 & 18.3 \\
\hline 37.0 & 29.0 & 43.4 & 46.3 & 46.5 & 44.6 & 45.2 & 43.3 & 37.9 & 34.0 & 28.8 & 23.4 & 18.2 \\
\hline 43.7 & 31.5 & 49.1 & 52.0 & 52.6 & 50.5 & 50.3 & 48.2 & 44.3 & 40.4 & 31.0 & 23.8 & 19.1 \\
\hline 50.5 & 32.8 & 51.3 & 54.6 & 55.8 & 53.9 & 55.2 & 52.2 & 53.1 & 49.1 & 42.7 & 30.3 & 19.0 \\
\hline 57.4 & 34.9 & 55.4 & 59.9 & 60.8 & 59.3 & 60.0 & 58.4 & 55.6 & 54.2 & 47.1 & 34.8 & 19.7 \\
\hline 64.3 & 36.7 & 60.1 & 65.2 & 66.1 & 64.7 & 65.1 & 63.0 & 63.7 & 56.7 & 50.9 & 40.1 & 22.4 \\
\hline 71.2 & 39.0 & 66.3 & 72.9 & 74.4 & 71.3 & 72.1 & 69.7 & 68.7 & 63.7 & 54.9 & 42.3 & 24.8 \\
\hline 77.9 & 40.2 & 70.0 & 75.2 & 76.6 & 73.9 & 75.0 & 73.3 & 70.7 & 67.6 & 59.8 & 49.2 & 27.3 \\
\hline 84.7 & 43.9 & 75.8 & 80.8 & 80.9 & 77.3 & 78.9 & 76.9 & 73.4 & 69.0 & 57.0 & 49.4 & 29.4 \\
\hline 91.7 & 46.5 & 81.4 & 80.2 & 89.4 & 85.2 & 88.6 & 86.0 & 82.8 & 75.1 & 63.5 & 50.8 & 32.0 \\
\hline 98.5 & 46.7 & 80.1 & 86.4 & 88.7 & 87.0 & $8 B .8$ & 86.2 & 84.5 & 80.8 & 72.8 & 61.0 & 35.5 \\
\hline 105.3 & 49.8 & 88.9 & 97.6 & 100.5 & 96.5 & 100.2 & 94.6 & 90.0 & 79.5 & 75.6 & 63.7 & 36.5 \\
\hline 112.2 & 52.4 & 93.5 & 102.2 & 103.6 & 100.0 & 100.5 & 96.7 & 95.6 & 87.2 & 81.2 & 68.5 & 39.9 \\
\hline 119.0 & 55.3 & 96.2 & 103.9 & 105.7 & 103.1 & 103.9 & 101.6 & 101.0 & 98.4 & 96.9 & 86.7 & 41.6 \\
\hline 125.9 & $b / .1$ & 102.2 & 111.7 & 113.4 & 108.5 & 110.7 & 104.5 & 102.6 & 99.0 & 93.7 & 71.0 & 45.0 \\
\hline 132.7 & 55.2 & 86.9 & 52.3 & 67.7 & 70.3 & 86.4 & 86.3 & 101.9 & 100.5 & 69.2 & 82.8 & 45.9 \\
\hline 139.5 & 57.6 & 84.2 & 109.0 & 112.2 & 89.5 & 107.4 & 96.4 & 103.6 & 99.2 & 86.6 & 73.0 & 48.9 \\
\hline \multicolumn{13}{|c|}{ Thermocouples located at sprinkler number 2} \\
\hline Time & $0 \mathrm{~mm}$ & $25 \mathrm{~mm}$ & $50 \mathrm{~mm}$ & $75 \mathrm{~mm}$ & $100 \mathrm{~mm}$ & $125 \mathrm{~mm}$ & $150 \mathrm{~mm}$ & $250 \mathrm{~mm}$ & $350 \mathrm{~mm}$ & $450 \mathrm{~mm}$ & $550 \mathrm{~mm}$ & $900 \mathrm{~mm}$ \\
\hline 0.0 & 19.2 & 21.2 & 21.2 & 21.1 & 21.0 & 20.8 & 20.7 & 20.4 & 20.1 & 19.8 & 19.4 & 17.5 \\
\hline 2.7 & 19.4 & 21.4 & 21.3 & 21.3 & 21.0 & 20.8 & 20.7 & 20.3 & 20.0 & 19.6 & 19.3 & 18.1 \\
\hline 9.5 & 19.8 & 24.3 & 24.9 & 25.7 & 25.6 & 25.6 & 25.1 & 26.0 & 22.6 & 20.7 & 19.9 & 18.2 \\
\hline 16.3 & 22.5 & 33.1 & 33.4 & 33.8 & 33.6 & 33.1 & 31.6 & 29.3 & 24.2 & 20.6 & 19.7 & 17.9 \\
\hline 23.3 & 24.2 & 39.0 & 39.1 & 40.1 & 40.1 & 39.7 & 38.9 & 35.4 & 26.6 & 20.8 & 19.6 & 18.0 \\
\hline 30.0 & 25.9 & 40.2 & 41.4 & 42.8 & 42.5 & 42.5 & 41.3 & 35.5 & 32.0 & 27.5 & 22.0 & 18.8 \\
\hline 37.0 & 28.6 & 48.0 & 48.7 & 49.5 & 49.1 & 48.7 & 48.0 & 45.5 & 39.5 & 33.2 & 23.0 & 19.0 \\
\hline 43.7 & 30.0 & 50.9 & 50.9 & 50.6 & 50.4 & 49.1 & 49.2 & 47.7 & 42.6 & 36.7 & 27.7 & 21.1 \\
\hline 50.5 & 31.4 & 54.7 & 56.0 & 58.8 & 60.1 & 61.0 & 60.5 & 54.1 & 42.9 & 34.5 & 27.2 & 21.3 \\
\hline 57.4 & 34.0 & 60.6 & 60.8 & 61.7 & 61.5 & 60.3 & 60.2 & 56.3 & 53.4 & 43.5 & 34.1 & 21.8 \\
\hline 64.3 & 36.6 & 65.6 & 66.8 & 68.2 & 68.1 & 68.2 & 67.4 & 62.8 & 58.6 & 52.3 & 39.3 & 25.2 \\
\hline 71.2 & 39.4 & 71.4 & 72.1 & 74.3 & 74.5 & 72.7 & 70.1 & 64.3 & 58.4 & 52.1 & 44.5 & 26.6 \\
\hline 77.9 & 39.2 & 72.2 & 73.9 & 74.4 & 75.0 & 75.4 & 75.6 & 73.8 & 68.9 & 62.3 & 54.2 & 28.8 \\
\hline 84.7 & 43.5 & 81.0 & 81.5 & $\mathrm{~B} 2.7$ & 81.9 & 81.7 & 81.2 & 75.9 & 69.0 & 60.7 & 53.4 & 32.3 \\
\hline 91.7 & 47.1 & 83.7 & 84.8 & 85.3 & 84.6 & 83.3 & 83.6 & 81.0 & 73.7 & 67.4 & 57.0 & 34.1 \\
\hline 98.5 & 47.1 & 87.0 & 88.2 & 89.5 & 88.9 & 87.8 & 87.7 & 85.7 & 78.3 & 66.7 & 55.0 & 37.7 \\
\hline 105.3 & 53.2 & 100.5 & 101.7 & 102.4 & 101.2 & 100.2 & 99.0 & 93.7 & $B 2.2$ & 71.3 & 59.7 & 39.9 \\
\hline 112.2 & 54.1 & 102.5 & 103.6 & 105.1 & 104.9 & 104.0 & 101.3 & 96.3 & 89.7 & 83.1 & 71.9 & 41.9 \\
\hline 119.0 & 51.9 & 98.1 & 100.7 & 103.4 & 103.0 & 104.5 & 104.5 & 103.2 & 98.8 & 90.2 & 73.4 & 45.8 \\
\hline 125.9 & 57.1 & 105.6 & 107.2 & 110.2 & 110.9 & 110.8 & 110.7 & 107.1 & 93.6 & 81.9 & 76.3 & 49.1 \\
\hline 132.7 & 61.9 & 116.1 & 117.9 & 120.8 & 121.4 & 121.0 & 119.0 & 107.3 & 104.1 & 94.6 & 75.9 & 53.2 \\
\hline 139.5 & 63.5 & 120.3 & 121.0 & 122.5 & 121.9 & 121.9 & 120.7 & 115.3 & 107.6 & $\uparrow 00.2$ & 79.6 & 55.4 \\
\hline \multicolumn{13}{|c|}{ Thermocouples located at sprinkler number 3} \\
\hline Time & $0 \mathrm{~mm}$ & $25 \mathrm{~mm}$ & $50 \mathrm{~mm}$ & $75 \mathrm{~mm}$ & $100 \mathrm{~mm}$ & $125 \mathrm{~mm}$ & $150 \mathrm{~mm}$ & $250 \mathrm{~mm}$ & $350 \mathrm{~mm}$ & $450 \mathrm{~mm}$ & $550 \mathrm{~mm}$ & $900 \mathrm{~mm}$ \\
\hline 0.0 & 20.6 & 21.1 & 20.6 & 20.6 & 20.5 & 20.5 & 20.5 & 20.3 & 20.1 & 19.7 & 19.3 & 17.7 \\
\hline 2.7 & 20.8 & 21.2 & 20.5 & 20.5 & 20.4 & 20.4 & 20.4 & 20.2 & 20.1 & 19.7 & 19.4 & 18.0 \\
\hline 9.5 & 20.6 & 21.1 & 20.4 & 20.4 & 20.4 & 20.4 & 20.4 & 20.2 & 20.1 & 19.7 & 19.4 & 18.0 \\
\hline 16.3 & 20.7 & 21.1 & 20.5 & 20.5 & 20.4 & 20.5 & 20.6 & 20.4 & 20.3 & 20.0 & 19.8 & 18.7 \\
\hline 23.3 & 20.9 & 24.9 & 25.8 & 26.7 & 24.8 & 24.5 & 23.4 & 21.7 & 21.2 & 20.6 & 20.1 & 19.3 \\
\hline 30.0 & 21.2 & 26.7 & 30.5 & 30.6 & 28.5 & 28.6 & 26.9 & 24.5 & 23.1 & 21.7 & 21.2 & 19.8 \\
\hline 37.0 & 21.5 & 30.3 & 34.8 & 35.2 & 32.8 & 32.7 & 31.2 & 28.6 & 26.8 & 25.1 & 24.8 & 20.1 \\
\hline 43.7 & 21.9 & 32.4 & 36.5 & 37.1 & 35.2 & 34.9 & 34.1 & 31.3 & 29.9 & 28.1 & 28.1 & 21.2 \\
\hline 50.5 & 22.0 & 33.4 & 38.8 & 38.9 & 37.5 & 37.1 & 36.0 & 32.8 & 32.0 & 30.4 & 30.0 & 23.6 \\
\hline 57.4 & 23.2 & 38.9 & 46.1 & 45.7 & 43.1 & 43.1 & 41.5 & 37.7 & 35.0 & 33.4 & 33.7 & 27.0 \\
\hline 64.3 & 23.2 & 40.5 & 47.0 & 47.7 & 45.0 & 45.0 & 43.3 & 39.3 & 37.8 & 37.3 & 37.2 & 28.4 \\
\hline 71.2 & 23.7 & 42.1 & 50.6 & 51.4 & 48.7 & 49.2 & 47.5 & 43.0 & 40.8 & 40.0 & 40.0 & 31.5 \\
\hline 77.9 & 24.3 & 43.8 & 55.0 & 55,3 & 52.8 & 53.1 & 51.2 & 46.5 & 44.8 & 43.1 & 42.6 & 33.8 \\
\hline
\end{tabular}


Experiment Number 26

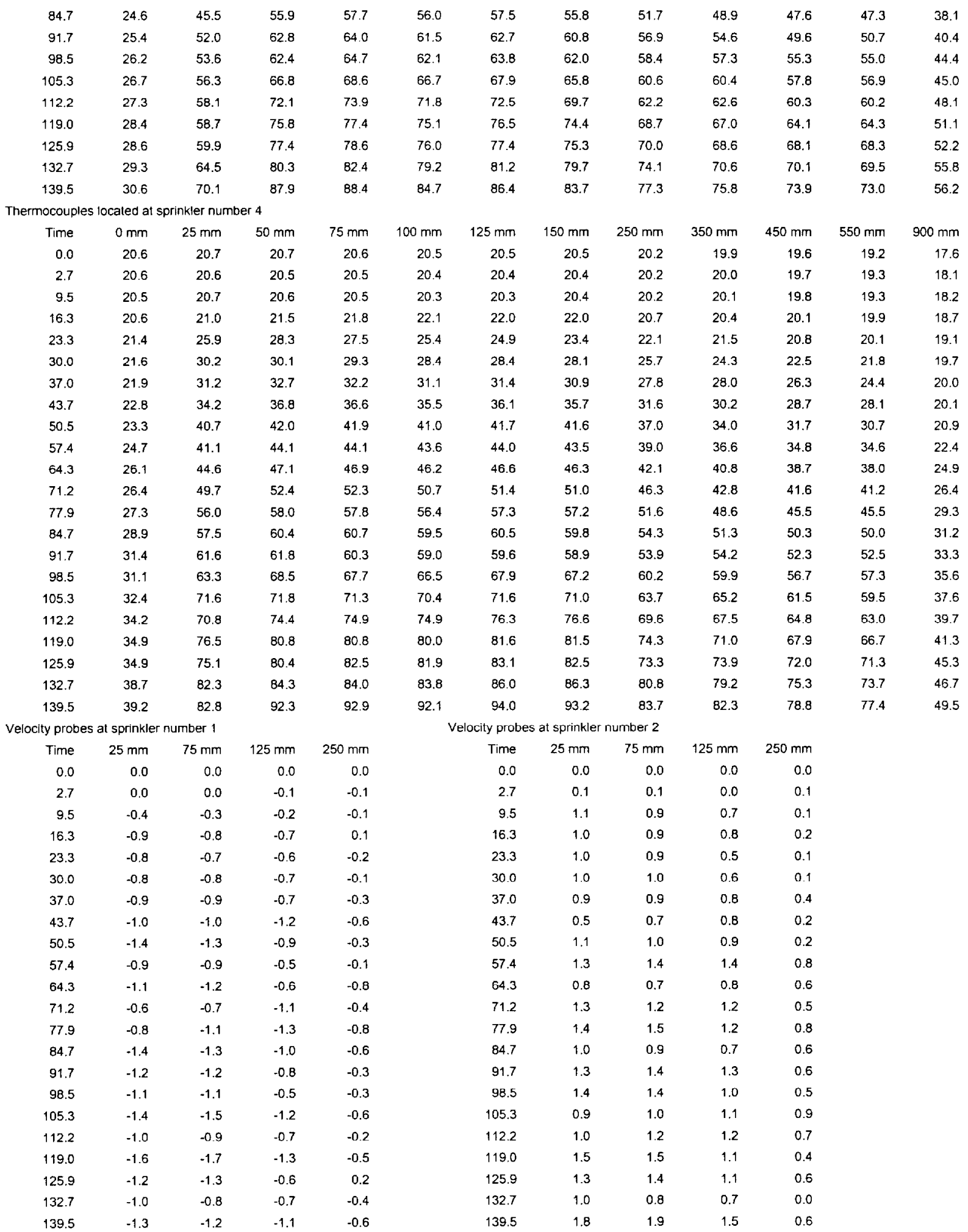


Experiment Number 27

Thermocouples located at sprinkler number 1

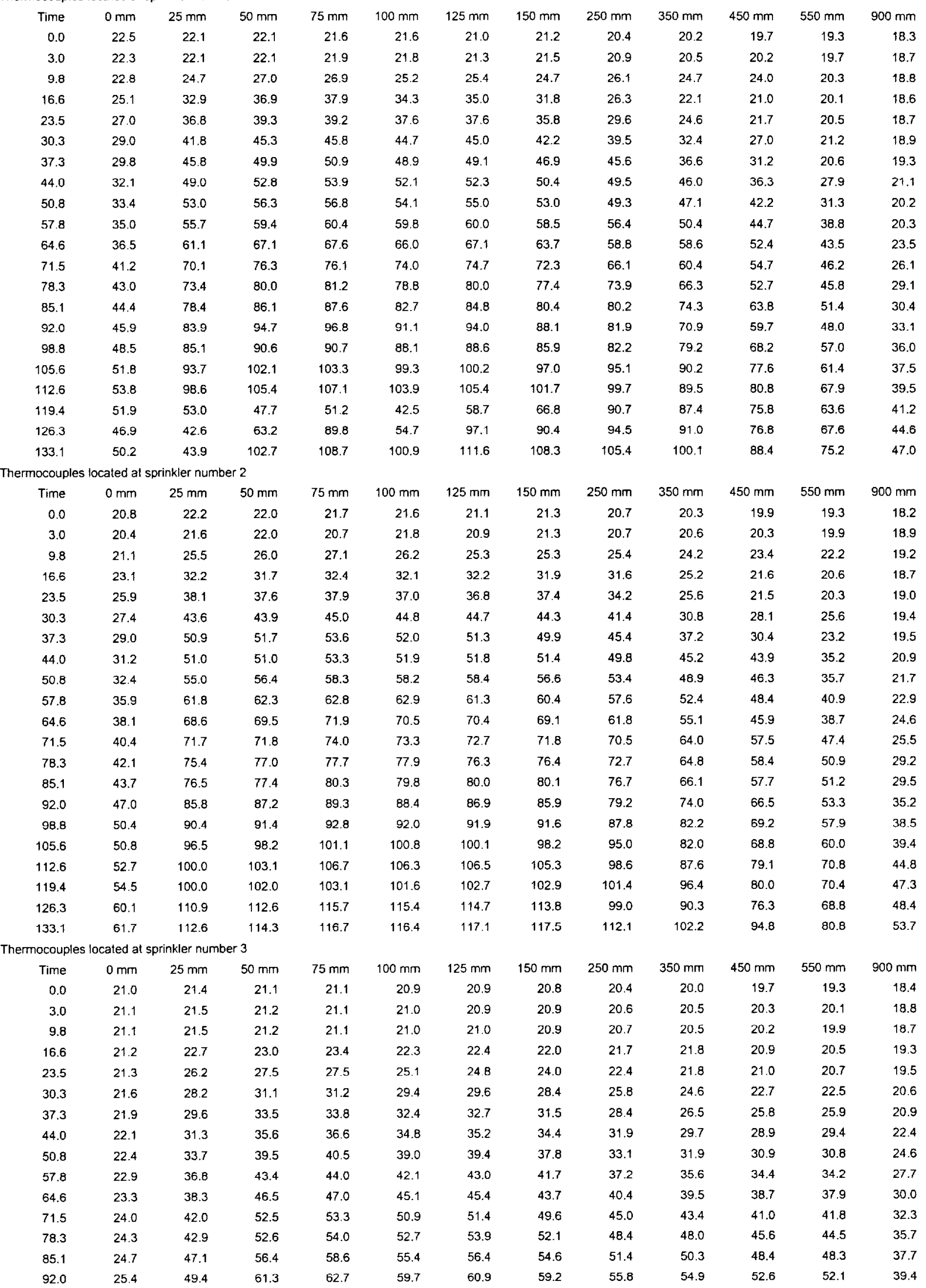


Experiment Number 27

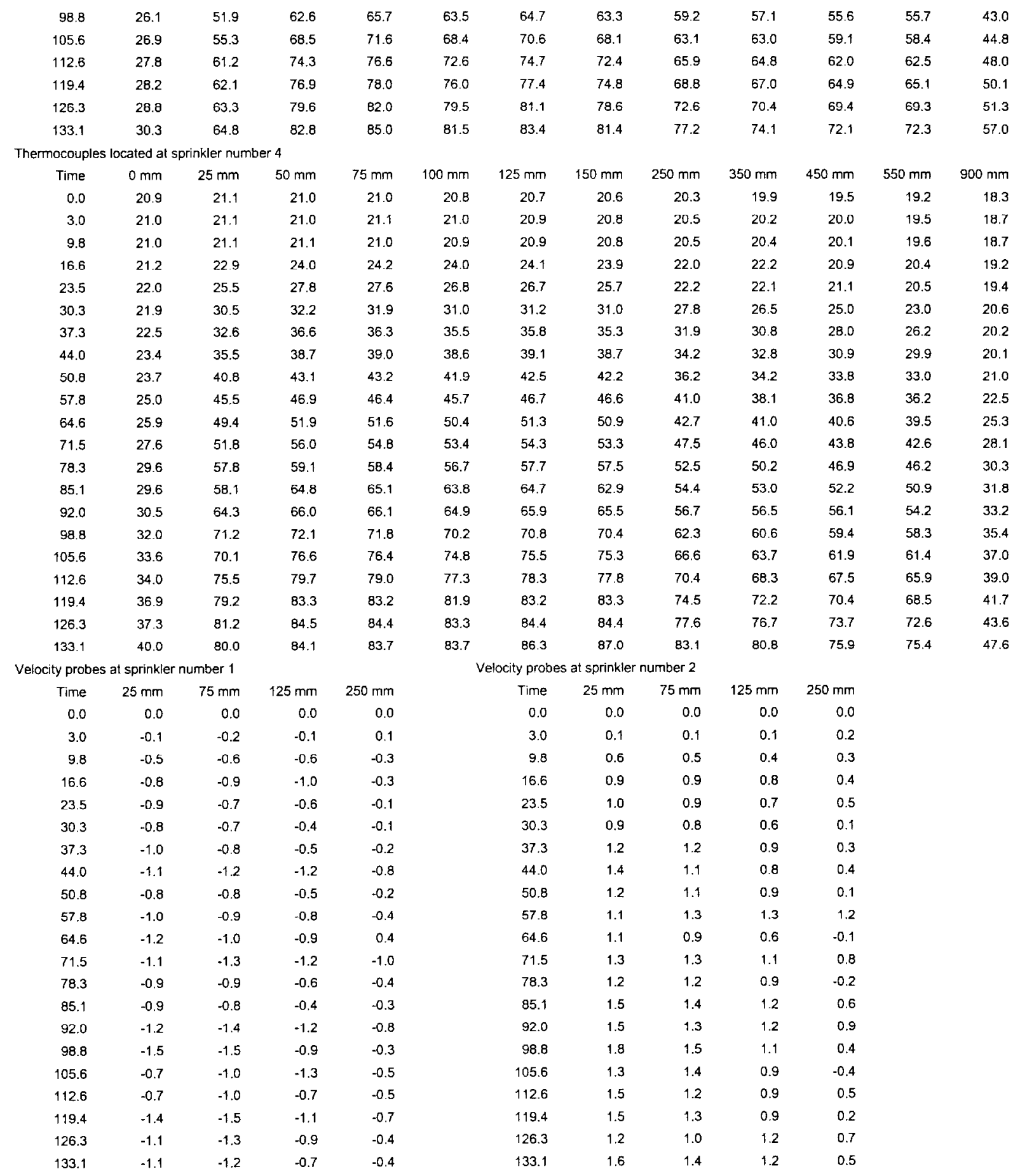


Experiment Number 28

Thermocouples located at sprinkler number 1

\begin{tabular}{|c|c|c|c|c|c|c|c|c|c|c|c|c|}
\hline Time & $0 \mathrm{~mm}$ & $25 \mathrm{~mm}$ & $50 \mathrm{~mm}$ & $75 \mathrm{~mm}$ & $100 \mathrm{~mm}$ & $125 \mathrm{~mm}$ & $150 \mathrm{~mm}$ & $250 \mathrm{~mm}$ & $350 \mathrm{~mm}$ & $450 \mathrm{~mm}$ & $550 \mathrm{~mm}$ & $900 \mathrm{~mm}$ \\
\hline 0.0 & 22.7 & 22.3 & 22.4 & 21.9 & 21.8 & 21.2 & 21.4 & 20.5 & 20.5 & 20.2 & 19.9 & 19.0 \\
\hline 4.6 & 22.8 & 22.8 & 23.0 & 22.6 & 22.5 & 22.0 & 22.1 & 20.8 & 21.0 & 20.8 & 20.4 & 19.3 \\
\hline 11.5 & 23.5 & 26.2 & 28.7 & 29.0 & 27.0 & 27.8 & 25.6 & 25.2 & 25.1 & 24.2 & 22.4 & 10.5 \\
\hline 18.2 & 25.5 & 33.1 & 36.6 & 36.6 & 32.7 & 33.3 & 31.6 & 26.7 & 22.5 & 21.2 & 20.7 & 19.3 \\
\hline 25.2 & 27.3 & 38.9 & 42.3 & 42.6 & 39.9 & 40.8 & 38.0 & 35.1 & 25.5 & 23.0 & 21.5 & 19.7 \\
\hline 32.0 & 29.3 & 43.9 & 47.6 & 47.2 & 44.5 & 45.2 & 41.3 & 37.3 & 30.4 & 26.4 & 21.4 & 19.8 \\
\hline 38.9 & 29.8 & 45.9 & 49.4 & 50.3 & 48.5 & 49.7 & 46.7 & 44.6 & 42.6 & 38.0 & 24.4 & 20.1 \\
\hline 45.6 & 31.7 & 48.9 & 52.1 & 52.3 & 50.7 & 51.0 & 49.9 & 47.4 & 42.8 & 36.7 & 27.8 & 21.2 \\
\hline 52.5 & 34.6 & 55.5 & 59.6 & 59.7 & 57.2 & 57.9 & 55.2 & 52.3 & 45.9 & 37.0 & 29.0 & 21.1 \\
\hline 59.4 & 36.9 & 59.9 & 65.1 & 65.4 & 62.9 & 62.8 & 60.9 & 55.9 & 51.0 & 43.6 & 35.2 & 21.5 \\
\hline 66.2 & 39.1 & 63.9 & 69.0 & 70.9 & 70.1 & 70.8 & 67.6 & 66.6 & 60.9 & 49.6 & 41.0 & 24.7 \\
\hline 73.1 & 42.0 & 71.5 & 77.5 & 78.2 & 73.7 & 74.6 & 71.4 & 66.6 & 61.8 & 52.7 & 43.6 & 27.2 \\
\hline 79.9 & 42.8 & 73.1 & 80.6 & 82.0 & 79.4 & 81.6 & 78.5 & 76.1 & 67.2 & 60.7 & 46.9 & 29.7 \\
\hline 86.7 & 45.2 & 78.2 & 84.5 & 84.9 & 82.6 & 83.3 & 82.0 & 78.5 & 70.9 & 62.1 & 46.9 & 31.8 \\
\hline 93.6 & 47.3 & 84.5 & 91.2 & 92.1 & 89.3 & 90.4 & 85.9 & 81.7 & 75.8 & 63.6 & 50.6 & 34.2 \\
\hline 100.4 & 49.1 & 84.6 & 91.3 & 93.8 & 94.1 & 96.8 & 91.6 & 90.9 & 84.2 & 73.7 & 54.0 & 36.0 \\
\hline 107.2 & 51.8 & 90.1 & 97.6 & 98.5 & 95.1 & 96.2 & 93.9 & 87.6 & 84.4 & 75.3 & 64.7 & 37.9 \\
\hline 114.1 & 53.1 & 94.1 & 103.7 & 104.5 & 100.7 & 102.7 & 99.0 & 97.1 & 89.5 & 76.3 & 64.9 & 40.5 \\
\hline 120.9 & 56.1 & 100.0 & 108.7 & 111.0 & 106.8 & 109.5 & 106.6 & 100.4 & 94.6 & 78.4 & 67.6 & 43.0 \\
\hline $12 / .8$ & 58.9 & 60.3 & 44.9 & 50.5 & 72.7 & 53.4 & 54.2 & 89.7 & 88.0 & 86.1 & 67.3 & 43.6 \\
\hline \multicolumn{13}{|c|}{ nocouples located at sprinkler number 2} \\
\hline Time & $0 \mathrm{~mm}$ & $25 \mathrm{~mm}$ & $50 \mathrm{~mm}$ & $75 \mathrm{~mm}$ & $100 \mathrm{~mm}$ & $125 \mathrm{~mm}$ & $150 \mathrm{~mm}$ & $250 \mathrm{~mm}$ & $350 \mathrm{~mm}$ & $450 \mathrm{~mm}$ & $550 \mathrm{~mm}$ & $900 \mathrm{~mm}$ \\
\hline 0.0 & 21.1 & 22.4 & 22.2 & 22.0 & 22.0 & 20.4 & 21.7 & 21.3 & 20.7 & 20.3 & 19.9 & 19.2 \\
\hline 4.6 & 20.9 & 22.0 & 22.5 & 23.5 & 21.4 & 23.2 & 21.8 & 21.9 & 21.6 & 21.2 & 20.7 & 19.5 \\
\hline 11.5 & 22.7 & 30.7 & 29.9 & 30.7 & 30.1 & 26.0 & 27.6 & 25.9 & 23.9 & 22.7 & 22.0 & 19.8 \\
\hline 18.2 & 24.5 & 37.1 & 36.3 & 34.8 & 35.6 & 37.5 & 34.4 & 32.7 & 25.5 & 21.6 & 20.7 & 19.7 \\
\hline 25.2 & 26.9 & 41.6 & 41.0 & 42.2 & 41.5 & 37.4 & 39.8 & 35.2 & 28.0 & 23.6 & 22.2 & 19.9 \\
\hline 32.0 & 28.5 & 43.1 & 43.3 & 45.8 & 42.9 & 41.5 & 43.1 & 41.4 & 35.3 & 28.2 & 25.7 & 19.9 \\
\hline 38.9 & 29.1 & 47.1 & 46.5 & 49.2 & 45.8 & 45.0 & 45.3 & 41.4 & 37.8 & 29.9 & 22.2 & 20.0 \\
\hline 45.6 & 29.4 & 47.4 & 48.6 & 50.9 & 49.2 & 53.1 & 49.8 & 48.7 & 45.2 & 39.5 & 30.1 & 21.4 \\
\hline 52.5 & 33.9 & 57.5 & 57.4 & 58.4 & 59.5 & 55.5 & 58.1 & 53.9 & 45.1 & 41.9 & 31.6 & 23.2 \\
\hline 59.4 & 38.3 & 68.0 & 68.0 & 67.0 & 67.1 & 69.5 & 64.3 & 56.5 & 51.2 & 44.3 & 36.5 & 23.4 \\
\hline 66.2 & 37.3 & 66.5 & 65.7 & 68.3 & 67.6 & 64.0 & 67.2 & 62.0 & 55.4 & 48.4 & 40.5 & 28.4 \\
\hline 73.1 & 39.9 & 75.2 & 75.9 & 78.4 & 76.6 & 80.7 & 75.3 & 72.8 & 66.3 & 57.9 & 48.3 & 28.2 \\
\hline 79.9 & 43.2 & 77.6 & 78.6 & 80.5 & 81.8 & 84.8 & 80.7 & 76.9 & 73.9 & 67.2 & 46.7 & 29.6 \\
\hline 86.7 & 44.6 & 81.8 & 82.1 & 85.4 & 85.1 & 81.1 & 84.5 & 81.3 & 73.9 & 59.4 & 48.6 & 30.9 \\
\hline 93.6 & 45.3 & 84.6 & 86.1 & 85.2 & 87.7 & 90.0 & 87.2 & 82.3 & 74.7 & 71.4 & 57.6 & 34.1 \\
\hline 100.4 & 49.0 & 88.0 & 90.1 & 93.7 & 92.9 & 91.0 & 91.9 & 85.1 & 78.9 & 69.8 & 60.2 & 40.6 \\
\hline 107.2 & 52.2 & 98.2 & 99.8 & 101.3 & 100.9 & 102.0 & 98.2 & 90.1 & 80.1 & 73.3 & 63.7 & 40.7 \\
\hline 114.1 & 54.3 & 101.0 & 103.0 & 105.5 & 104.7 & 107.9 & 103.3 & 99.1 & 90.0 & 78.9 & 67.3 & 45.4 \\
\hline 120.9 & 57.4 & 109.0 & 110.7 & 109.8 & $1 \uparrow 1.5$ & 108.5 & 108.3 & 101.2 & 94.7 & 85.4 & 69.2 & 48.3 \\
\hline 127.8 & 60.0 & 109.4 & 111.8 & 109.9 & 73.3 & 78.1 & 95.0 & 100.0 & 94.1 & 82.3 & 68.6 & 49.1 \\
\hline \multicolumn{13}{|c|}{ nocouples located at sprinkler number 3} \\
\hline Time & $0 \mathrm{~mm}$ & $25 \mathrm{~mm}$ & $50 \mathrm{~mm}$ & $75 \mathrm{~mm}$ & יוזוז & $125 \mathrm{~mm}$ & $150 \mathrm{~mm}$ & $250 \mathrm{~mm}$ & $350 \mathrm{~mm}$ & $450 \mathrm{~mm}$ & $550 \mathrm{~mm}$ & $900 \mathrm{~mm}$ \\
\hline 0.0 & 21.3 & 21.6 & 21.3 & 21.3 & 21.2 & 21.1 & 21.0 & 20.8 & 20.6 & 20.3 & 20.0 & 19.2 \\
\hline 4.6 & 21.4 & 21.8 & 21.5 & 21.5 & 21.4 & 21.4 & 21.4 & 21.2 & 21.1 & 20.9 & 20.8 & 19.5 \\
\hline 11.5 & 21.4 & 21.8 & 21.6 & 21.7 & 21.6 & 21.6 & 21.6 & 21.3 & 21.1 & 20.9 & 20.7 & 19.6 \\
\hline 18.2 & 21.5 & 23.3 & 24.0 & 24.4 & 23.5 & 23.7 & 23.8 & 23.0 & 22.6 & 21.4 & 21.0 & 20.0 \\
\hline 25.2 & 21.6 & 26.7 & 27.8 & 29.0 & 27.3 & 27.3 & 26.7 & 23.0 & 22.0 & 21.3 & 21.1 & 20.4 \\
\hline 32.0 & 22.0 & 28.4 & 31.2 & 31.5 & 29.9 & 29.7 & 28.7 & 27.0 & 26.2 & 24.5 & 23.8 & 21.9 \\
\hline 38.9 & 22.1 & 29.0 & 32.4 & 33.3 & 32.0 & 32.3 & 31.4 & 30.1 & 28.2 & 26.7 & 26.5 & 21.8 \\
\hline 45.6 & 22.6 & 32.2 & 36.5 & 37.6 & 35.8 & 36.3 & 35.2 & 32.7 & 31.2 & 29.7 & 29.7 & 23.5 \\
\hline 52.5 & 22.8 & 32.7 & 38.9 & 40.1 & 39.1 & 39.6 & 38.3 & 35.6 & 33.5 & 31.7 & 31.9 & 25.4 \\
\hline 59.4 & 23.3 & 37.2 & 42.5 & 43.1 & 41.4 & 42.1 & 41.5 & 38.9 & 38.2 & 35.6 & 35.2 & 29.1 \\
\hline 66.2 & 23.7 & 40.5 & 46.9 & 48.2 & 46.8 & 47.5 & 46.1 & 42.4 & 41.9 & 39.2 & 38.4 & 31.4 \\
\hline 73.1 & 24.3 & 40.8 & 52.7 & 53.7 & 51.5 & 52.3 & 50.5 & 46.7 & 45.6 & 42.6 & 41.8 & 32.8 \\
\hline 79.9 & 24.6 & 42.9 & 53.0 & 55.3 & 53.7 & 54.5 & 53.3 & 48.9 & 46.5 & 44.9 & 45.0 & 34.9 \\
\hline 86.7 & 25.2 & 46.3 & 58.0 & 59.8 & 57.5 & 59.3 & 57.5 & 53.5 & 50.6 & 49.1 & 49.2 & 38.4 \\
\hline 93.6 & 26.1 & 54.6 & 62.6 & 64.5 & 61.3 & 62.5 & 61.4 & 56.7 & 53.5 & 52.5 & 52.4 & 41.3 \\
\hline 100.4 & 27.1 & 53.3 & 67.4 & 67.8 & 65.6 & 66.3 & 64.7 & 61.0 & 58.9 & 56.1 & 56.1 & 43.8 \\
\hline 107.2 & 27.6 & 55.5 & 68.9 & $/ 1.8$ & 68.8 & 70.6 & 68.8 & 63.4 & 59.8 & 59.0 & 58.6 & 46.8 \\
\hline
\end{tabular}


Experiment Number 28

\begin{tabular}{|c|c|c|c|c|c|c|c|c|c|c|c|c|}
\hline 114.1 & 28.0 & 56.6 & 70.3 & 72.9 & 70.7 & 72.2 & 70.5 & 66.2 & 63.5 & 63.1 & 62.0 & 49.4 \\
\hline 120.9 & 29.5 & 64.8 & 77.0 & 79.3 & 76.6 & 77.9 & 75.4 & 69.8 & 68.3 & 66.2 & 66.1 & 51.8 \\
\hline 127.8 & 29.7 & 68.3 & 81.5 & 84.1 & 79.8 & 81.5 & 78.7 & 73.2 & 72.1 & 68.8 & 68.7 & 53.8 \\
\hline \multicolumn{13}{|c|}{ Thermocouples located al sprinkler number 4} \\
\hline Time & $0 \mathrm{~mm}$ & $25 \mathrm{~mm}$ & $50 \mathrm{~mm}$ & $75 \mathrm{~mm}$ & $100 \mathrm{~mm}$ & $125 \mathrm{~mm}$ & $150 \mathrm{~mm}$ & $250 \mathrm{~mm}$ & $350 \mathrm{~mm}$ & $450 \mathrm{~mm}$ & $550 \mathrm{~mm}$ & $900 \mathrm{~mm}$ \\
\hline 0.0 & 21.2 & 21.3 & 21.4 & 21.4 & 21.2 & 21.2 & 21.1 & 20.7 & 20.4 & 20.1 & 19.8 & 19.1 \\
\hline 4.6 & 21.3 & 21.4 & 21.5 & 21.6 & 21.4 & 21.4 & 21.3 & 21.1 & 20.9 & 20.7 & 20.4 & 19.8 \\
\hline 11.5 & 21.2 & 21.6 & 21.6 & 21.5 & 21.3 & 21.3 & 21.4 & 21.1 & 21.1 & 20.7 & 20.2 & 19.8 \\
\hline 18.2 & 21.8 & 24.6 & 26.5 & 26.4 & 25.6 & 25.6 & 25.3 & 23.5 & 23.0 & 21.2 & 21.0 & 20.0 \\
\hline 25.2 & 22.2 & 26.8 & 29.2 & 29.0 & 28.4 & 28.9 & 28.1 & 24.4 & 22.6 & 21.6 & 21.1 & 20.2 \\
\hline 32.0 & 22.7 & 29.6 & 32.2 & 32.2 & 31.4 & 31.8 & 31.4 & 27.8 & 26.5 & 25.3 & 24.3 & 21.8 \\
\hline 38.9 & 23.8 & 31.6 & 35.3 & $35 ?$ & 34.3 & 34.9 & 34.7 & 32.3 & 30.3 & 28.8 & 27.1 & 21.1 \\
\hline 45.6 & 24.7 & 36.2 & 41.0 & 40.2 & 38.8 & 39.2 & 38.3 & 33.9 & 32.1 & 31.5 & 30.9 & 21.1 \\
\hline 52.5 & 24.2 & 40.7 & 42.1 & 41.8 & 41.7 & 42.4 & 42.0 & 38.6 & 35.3 & 34.5 & 33.6 & 23.3 \\
\hline 59.4 & 25.3 & 43.4 & 47.1 & 46.5 & 45.3 & 46.5 & 46.3 & 40.7 & 37.6 & 37.0 & 37.1 & 24.8 \\
\hline 66.2 & 26.6 & 44.4 & 49.1 & 48.4 & 46.2 & 46.8 & 45.8 & 41.4 & 41.1 & 41.1 & 40.0 & 26.9 \\
\hline 73.1 & 27.7 & 51.7 & 54.9 & 54.6 & 52.8 & 53.4 & 53.3 & 48.0 & 44.7 & 43.8 & 43.3 & 28.7 \\
\hline 79.9 & 28.1 & 57.3 & 58.9 & 58.9 & 57.8 & 58.7 & 59.0 & 54.2 & 49.9 & 47.2 & 47.2 & 30.8 \\
\hline 86.7 & 29.5 & 58.1 & 63.0 & 62.3 & 60.4 & 61.4 & 61.0 & 56.7 & 54.9 & 52.7 & 51.7 & 32.6 \\
\hline 93.6 & 30.5 & 64.3 & 66.7 & 66.3 & 65.4 & 66.7 & 66.2 & 59.3 & 58.6 & 56.5 & 55.1 & 34.6 \\
\hline 100.4 & 31.8 & 70.2 & 71.3 & 71.8 & 70.8 & 72.4 & 72.1 & 65.7 & 61.7 & 60.4 & 58.8 & 37.0 \\
\hline 107.2 & 32.8 & 67.1 & 72.7 & 73.0 & 72.1 & 72.7 & 72.5 & 66.9 & 63.7 & 62.6 & 61.5 & 39.6 \\
\hline 114.1 & 33.8 & 74.3 & 76.0 & 75.1 & 74.7 & 75.3 & 75.2 & 69.5 & 68.8 & 67.6 & 66.0 & 41.2 \\
\hline 1209 & 35.6 & 76.8 & 81.5 & 81.0 & 79.7 & 81.4 & 81.0 & 74.4 & 73.7 & 71.3 & 70.4 & 42.3 \\
\hline 127.8 & 40.4 & 86.5 & 85.1 & 85.0 & 84.3 & 85.8 & 85.8 & 80.1 & 78.2 & 74.9 & 73.1 & 44.8 \\
\hline \multicolumn{5}{|c|}{ Velocity probes at sprinkler number 1} & \multicolumn{6}{|c|}{ Velocity probes al sprinkler number 2} & & \\
\hline Time & $25 \mathrm{~mm}$ & $75 \mathrm{~mm}$ & $125 \mathrm{~mm}$ & $250 \mathrm{~mm}$ & & Time & $25 \mathrm{~mm}$ & $75 \mathrm{~mm}$ & $125 \mathrm{~mm}$ & $250 \mathrm{~mm}$ & & \\
\hline 0.0 & 0.0 & 0.0 & 0.0 & 0.0 & & 0.0 & 0.0 & 0.0 & 0.0 & 0.0 & & \\
\hline 4.6 & -0.5 & -0.6 & -0.8 & -0.3 & & 4.6 & 0.7 & 0.8 & 0.9 & 0.5 & & \\
\hline 11.5 & -0.8 & -0.8 & -0.4 & 0.1 & & 11.5 & 0.7 & 0.6 & 0.5 & 0.3 & & \\
\hline 18.2 & -0.7 & -0.8 & -0.6 & -0.2 & & 18.2 & 0.8 & 0.9 & 0.8 & 0.3 & & \\
\hline 25.2 & -1.0 & -1.0 & -1.0 & -0.4 & & 25.2 & 0.7 & 0.7 & 0.8 & 0.4 & & \\
\hline 32.0 & -0.4 & -0.6 & -0.5 & 0.0 & & 32.0 & 1.1 & 1.1 & 0.9 & 0.3 & & \\
\hline 38.9 & -0.9 & -0.9 & -0.8 & -0.2 & & 38.9 & 0.8 & 0.7 & 0.5 & 0.2 & & \\
\hline 45.6 & -1.3 & -1.2 & -0.9 & 0.4 & & 45.6 & 1.3 & 1.4 & 1.0 & 0.5 & & \\
\hline 52.5 & -0.9 & -0.9 & -0.6 & -0.4 & & 52.5 & 1.3 & 0.9 & 0.7 & 0.0 & & \\
\hline 59.4 & -1.5 & -1.3 & -0.6 & -0.4 & & 59.4 & 0.9 & 0.7 & 0.5 & 0.4 & & \\
\hline 66.2 & -1.3 & -1.4 & -1.2 & -0.7 & & 66.2 & 1.3 & 1.4 & 1.5 & 0.8 & & \\
\hline 73.1 & -1.1 & -1.1 & -0.6 & -0.6 & & 73.1 & 1.2 & 1.1 & 1.0 & 0.4 & & \\
\hline 79.9 & -0.9 & -1.1 & -1.0 & -0.6 & & 79.9 & 1.4 & 1.3 & 1.2 & 0.6 & & \\
\hline 86.7 & -1.1 & -1.0 & -0.7 & 0.6 & & 86.7 & 1.2 & 1.3 & 1.1 & 0.7 & & \\
\hline 93.6 & -1.5 & -1.7 & -1.7 & -1.2 & & 93.6 & 1.5 & 1.4 & 1.2 & 0.4 & & \\
\hline 100.4 & -0.9 & -0.9 & -0.7 & -0.4 & & 100.4 & 1.0 & 0.9 & 0.9 & 0.1 & & \\
\hline 107.2 & -1.3 & -1.3 & -0.9 & -0.8 & & 107.2 & 0.8 & 0.9 & 0.9 & 0.1 & & \\
\hline 114.1 & -0.7 & -0.7 & -0.4 & -0.3 & & 114.1 & 1.5 & 1.6 & 1.7 & 0.6 & & \\
\hline 120.9 & -1.4 & -1.2 & -1.0 & -0.6 & & 120.9 & 1.9 & 1.6 & 1.3 & 0.6 & & \\
\hline 127.8 & -1.0 & -1.3 & -1.5 & -0.5 & & 127.8 & 1.4 & 1.2 & 0.9 & 0.4 & & \\
\hline
\end{tabular}


Experiment Number 29

\begin{tabular}{|c|c|c|c|c|c|c|c|c|c|c|c|c|}
\hline \multicolumn{13}{|c|}{ Thermocouples located at sprinkler number 1} \\
\hline Time & $0 \mathrm{~mm}$ & $25 \mathrm{~mm}$ & $50 \mathrm{~mm}$ & $75 \mathrm{~mm}$ & $100 \mathrm{~mm}$ & $125 \mathrm{~mm}$ & $150 \mathrm{~mm}$ & $250 \mathrm{~mm}$ & $350 \mathrm{~mm}$ & $450 \mathrm{~mm}$ & $550 \mathrm{~mm}$ & $900 \mathrm{~mm}$ \\
\hline 0.0 & 25.6 & 25.5 & 25.6 & 25.2 & 25.1 & 24.3 & 24.6 & 23.7 & 23.8 & 23.6 & 23.4 & 22.4 \\
\hline 0.7 & 26.0 & 26.0 & 26.1 & 25.6 & 25.4 & 24.5 & 24.9 & 24.0 & 24.3 & 24.2 & 23.8 & 22.7 \\
\hline 7.4 & 25.9 & 26.0 & 26.2 & 25.8 & 25.7 & 24.8 & 25.1 & 23.9 & 24.2 & 24.0 & 23.8 & 22.7 \\
\hline 14.3 & 26.1 & 26.2 & 26.4 & 26.1 & 26.0 & 25.0 & 25.3 & 24.1 & 24.2 & 24.0 & 23.8 & 22.9 \\
\hline 21.1 & 27.3 & 27.3 & 27.6 & 27.6 & 27.5 & 27.1 & 26.9 & 26.3 & 24.9 & 24.4 & 24.1 & 23.1 \\
\hline 28.0 & 27.5 & 27.8 & 28.0 & 27.7 & 27.9 & 27.3 & 27.2 & 25.9 & 24.8 & 24.5 & 24.3 & 23.1 \\
\hline 34.7 & 29.1 & 29.7 & 30.1 & 30.2 & 29.9 & 29.3 & 28.8 & 26.8 & 25.1 & 24.7 & 24.4 & 23.3 \\
\hline 41.5 & 33.5 & 33.5 & 34.3 & 34.3 & 33.9 & 32.9 & 31.8 & 29.2 & 27.2 & 26.5 & 25.2 & 23.9 \\
\hline 48.5 & 34.7 & 35.1 & 35.7 & 35.9 & 36.1 & 34.6 & 34.4 & 32.2 & 28.0 & 27.4 & 26.5 & 24.9 \\
\hline 55.2 & 356 & 36.2 & 36.9 & 36.9 & 36.7 & 35.7 & 36.1 & 33.5 & 29.3 & 28.3 & 27.2 & 25.3 \\
\hline 62.2 & 35.4 & 36.0 & 37.6 & 37.2 & 37.7 & 35.9 & 37.4 & 37.2 & 34.4 & 33.5 & 30.4 & 25.8 \\
\hline 68.9 & 36.1 & 37.6 & 39.7 & 40.3 & 39.9 & 39.0 & 38.9 & 37.4 & 39.0 & 37.0 & 32.3 & 25.2 \\
\hline 75.7 & 43.2 & 45.3 & 48.8 & 49.6 & 47.9 & 46.3 & 45.4 & 43.1 & 42.1 & 35.4 & 32.9 & 24.5 \\
\hline 82.6 & 43.5 & 46.0 & 49.4 & 50.4 & 49.5 & 47.4 & 49.2 & 46.0 & 44.6 & 37.5 & 34.5 & 25.6 \\
\hline 89.3 & 48.8 & 51.1 & 53.8 & 54.2 & 52.7 & 51.6 & 51.8 & 48.1 & 47.7 & 39.0 & 35.8 & 26.0 \\
\hline 96.3 & 54.1 & 55.6 & 58.5 & 59.5 & 58.0 & 58.3 & 56.6 & 49.0 & 48.0 & 42.0 & 38.7 & 27.2 \\
\hline 103.0 & 58.5 & 60.5 & 63.0 & 63.8 & 62.3 & 62.2 & 60.8 & 56.4 & 52.3 & 44.6 & 40.2 & 33.2 \\
\hline 109.8 & 60.8 & 62.1 & 64.2 & 65.8 & 64.3 & 63.1 & 64.2 & 56.3 & 49.8 & 44.6 & 42.9 & 36.5 \\
\hline 116.6 & 61.6 & 64.3 & 67.6 & 69.3 & 68.4 & 68.5 & 67.7 & 62.8 & 54.9 & 46.3 & 43.0 & 36.2 \\
\hline 123.4 & 57.9 & 62.6 & $6 / .6$ & 61.1 & 66.7 & 66.5 & 65.7 & 61.6 & 62.6 & 53.6 & 47.7 & 38.1 \\
\hline 130.2 & 66.1 & 70.8 & 75.0 & 75.2 & 73.1 & 71.0 & 69.2 & 62.2 & 64.1 & 56.5 & 49.6 & 39.4 \\
\hline 137.1 & 69.7 & 72.3 & 74.0 & 74.3 & 72.7 & 72.7 & 71.4 & 69.2 & 66.9 & 53.4 & 50.5 & 41.7 \\
\hline 143.9 & 70.0 & 75.4 & 78.5 & 79.7 & 77.0 & 76.9 & 75.0 & 65.0 & 66.6 & 56.2 & 52.1 & 42.1 \\
\hline 150.8 & 73.8 & 78.2 & 81.0 & 81.2 & 80.5 & 80.7 & 78.3 & 71.7 & 68.3 & 63.7 & 56.4 & 46.5 \\
\hline 157.6 & 76.0 & 82.0 & 86.5 & 87.6 & 85.6 & 84.9 & 84.0 & 77.1 & 70.9 & 60.7 & 57.7 & 47.7 \\
\hline 164.3 & 80.3 & 84.2 & 88.0 & 88.8 & 87.0 & 84.1 & 86.7 & 72.3 & 67.9 & 62.0 & 57.6 & $4 B .7$ \\
\hline 171.2 & 82.5 & 87.0 & 91.5 & 92.8 & 91.8 & 90.1 & 89.2 & 84.4 & 76.6 & 63.8 & 61.0 & 51.3 \\
\hline 178.0 & 87.6 & 91.7 & 94.5 & 93.5 & 92.2 & 88.8 & 92.2 & 83.2 & 72.2 & 65.7 & 62.8 & 55.3 \\
\hline 184.9 & 82.0 & 91.5 & 94.8 & 94.4 & 94.4 & 94.9 & 95.2 & 91.1 & 84.5 & 73.2 & 67.1 & 54.5 \\
\hline 191.7 & 90.4 & 95.0 & 100.4 & 101.4 & 99.1 & 98.9 & 99.1 & 94.0 & 77.3 & 70.0 & 67.5 & 56.3 \\
\hline 198.4 & 91.1 & 99.7 & 106.7 & 106.8 & 105.7 & 100.1 & 102.7 & 95.1 & 85.5 & 76.1 & 72.3 & 57.5 \\
\hline 205.3 & 92.6 & 101.4 & 106.4 & 107.8 & 106.3 & 104.4 & 104.1 & 101.2 & 95.8 & 79.5 & 73.8 & 61.6 \\
\hline 212.1 & 101.4 & 105.4 & 112.7 & 115.4 & 112.2 & 112.6 & 110.7 & 106.5 & 92.6 & 78.9 & 75.1 & 63.0 \\
\hline 2190 & 103.6 & 110.8 & 118.4 & 120.7 & 117.2 & 118.2 & 115.5 & 111.7 & 99.0 & 83.5 & 79.6 & 65.1 \\
\hline 225.8 & 107.1 & 114.4 & 121.9 & 123.7 & 122.5 & 121.9 & 118.1 & 112.8 & 105.7 & 88.2 & 82.7 & 67.2 \\
\hline 232.5 & 114.4 & 117.5 & 122.5 & 126.2 & 125.9 & 125.9 & 122.4 & 108.4 & 97.8 & 87.9 & 84.0 & 67.7 \\
\hline \multicolumn{13}{|c|}{ Thermocouples located at sprinkler number 2} \\
\hline Time & $\mathrm{D} \mathrm{mm}$ & $25 \mathrm{~mm}$ & $50 \mathrm{~mm}$ & $75 \mathrm{~mm}$ & $100 \mathrm{~mm}$ & $125 \mathrm{~mm}$ & $150 \mathrm{~mm}$ & $250 \mathrm{~mm}$ & $350 \mathrm{~mm}$ & $450 \mathrm{~mm}$ & $550 \mathrm{~mm}$ & $900 \mathrm{~mm}$ \\
\hline 0.0 & 26.7 & 26.3 & 26.1 & 25.7 & 25.5 & 25.2 & 25.1 & 24.2 & 23.9 & 23.6 & 23.3 & 22.7 \\
\hline 0.7 & 27.2 & 26.9 & 27.1 & 26.1 & 26.4 & 25.7 & 25.7 & 24.5 & 24.3 & 24.0 & 23.7 & 22.9 \\
\hline 7.4 & 26.9 & 26.8 & 26.9 & 26.1 & 26.1 & 25.5 & 25.4 & 24.4 & 24.2 & 23.9 & 23.7 & 22.9 \\
\hline 14.3 & 27.3 & 27.0 & 27.3 & 26.3 & 26.4 & 25.7 & 25.6 & 24.5 & 24.4 & 24.0 & 23.7 & 22.9 \\
\hline 21.1 & 27.8 & 27.7 & 28.3 & 27.5 & 28.5 & 27.8 & 28.0 & 25.5 & 24.9 & 24.4 & 23.9 & 23.0 \\
\hline 28.0 & 29.9 & 28.7 & 29.6 & 27.8 & 29.9 & 27.5 & 28.5 & 25.4 & 25.0 & 24.4 & 23.8 & 23.0 \\
\hline 34.7 & 30.9 & 29.6 & 31.1 & 29.1 & 31.8 & 30.5 & 31.3 & 28.2 & 25.4 & 24.8 & 24.1 & 23.2 \\
\hline 41.5 & 33.5 & 33.3 & 35.7 & 33.0 & 35.9 & 34.0 & 35.2 & 27.6 & 27.5 & 26.4 & 25.1 & 23.6 \\
\hline 48.5 & 36.1 & 35.4 & 37.5 & 36.1 & 38.3 & 37.3 & 37.5 & 30.1 & 28.3 & 27.4 & 26.8 & 24.1 \\
\hline 55.2 & 36.6 & 35.5 & 37.9 & 35.0 & 38.8 & 38.0 & 38.4 & 34.1 & 33.1 & 28.9 & 28.0 & 24.4 \\
\hline 62.2 & 41.1 & 41.5 & 43.8 & 40.7 & 44.2 & 42.2 & 43.1 & 35.0 & 31.5 & 30.0 & 28.9 & 24.9 \\
\hline 68.9 & 43.2 & 44.5 & 45.4 & 43.4 & 46.5 & 46.0 & 45.2 & 39.0 & 33.9 & 32.6 & 31.5 & 25.2 \\
\hline 75.7 & 41.0 & 42.2 & 44.8 & 42.5 & 46.0 & 46.6 & 47.2 & 44.5 & 41.0 & 36.0 & 33.7 & 25.3 \\
\hline 82.6 & 44.5 & 46.2 & 48.7 & 46.4 & 49.9 & 48.1 & 48.3 & 44.8 & 44.9 & 37.4 & 34.7 & 28.7 \\
\hline 89.3 & 51.0 & 54.5 & 56.4 & 54.6 & 56.6 & 56.7 & 56.0 & 51.0 & 49.1 & 43.3 & 37.8 & 28.8 \\
\hline 96.3 & 56.0 & 58.2 & 59.4 & 57.9 & 60.9 & 60.0 & 59.9 & 50.1 & 45.6 & 41.3 & 37.6 & 31.7 \\
\hline 103.0 & 56.9 & 59.0 & 61.2 & 60.1 & 63.2 & 63.1 & 63.3 & 57.3 & 49.1 & 42.5 & 39.9 & 34.7 \\
\hline 109.8 & 59.2 & 61.5 & 63.8 & 60.6 & 66.1 & 67.1 & 66.7 & 54.4 & 53.3 & 45.9 & 42.4 & 36.4 \\
\hline 116.6 & 66.4 & 69.1 & 70.3 & 67.2 & 71.0 & 68.7 & 69.6 & 55.1 & 56.2 & 49.3 & 45.0 & 37.5 \\
\hline 123.4 & 67.0 & 68.1 & 71.7 & 68.8 & 74.0 & 71.2 & 72.3 & 60.9 & 58.9 & 52.8 & 48.2 & 38.1 \\
\hline 130.2 & 73.4 & 77.9 & 79.5 & 77.4 & 81.5 & 81.0 & 81.2 & 73.0 & 65.1 & 55.7 & 51.5 & 40.8 \\
\hline 137.1 & 71.4 & 73.9 & 75.2 & 72.6 & 77.2 & 78.2 & 78.7 & 76.1 & 70.0 & 57.7 & 53.9 & 42.6 \\
\hline 143.9 & 76.4 & 80.8 & 82.3 & 79.5 & 82.9 & 79.8 & 81.1 & 70.3 & 66.1 & 58.3 & 54.1 & 43.2 \\
\hline
\end{tabular}


Experiment Number 29

\begin{tabular}{|c|c|c|c|c|c|c|c|c|c|c|c|c|}
\hline & & & & & & & & & \multirow[b]{2}{*}{70.1} & \multirow[b]{2}{*}{60.7} & \multirow[b]{2}{*}{57.2} & \multirow[b]{2}{*}{45.6} \\
\hline \multirow{2}{*}{$\begin{array}{l}150.8 \\
157.6\end{array}$} & 82.3 & 88.4 & 89.5 & 86.0 & 89.9 & 87.1 & 86.8 & 77.9 & & & & \\
\hline & 83.3 & 87.3 & 89.2 & 88.3 & 91.8 & 91.0 & 90.2 & 83.0 & 76.2 & 62.9 & 58.7 & 49.0 \\
\hline 164.3 & B6.5 & 90.6 & 92.7 & 92.8 & 94.7 & 95.4 & 94.6 & 88.0 & 79.3 & 66.9 & 60.8 & 50.3 \\
\hline 171.2 & 90.9 & 95.9 & 96.8 & 97.0 & 98.6 & 99.6 & 99.2 & 85.0 & 77.9 & 68.6 & 64.3 & 50.8 \\
\hline 178.0 & 89.1 & 92.2 & 94.0 & 93.0 & 97.0 & 97.9 & 98.0 & 90.3 & 79.8 & 69.7 & 65.9 & 53.5 \\
\hline 184.9 & 94.2 & 98.8 & 102.7 & 101.4 & 104.3 & 104.5 & 103.9 & 97.8 & 86.2 & 75.2 & 70.8 & 54.8 \\
\hline 191.7 & 97.3 & 102.6 & 105.7 & 102.9 & 106.3 & 104.3 & 104.7 & 95.8 & 87.0 & 77.7 & 70.8 & 56.8 \\
\hline 198.4 & 102.8 & 108.0 & 109.7 & 107.5 & 111.5 & 109.2 & 110.6 & 100.2 & 92.6 & 79.2 & 74.7 & 60.1 \\
\hline 205.3 & 109.8 & 118.3 & 119.6 & 118.3 & 119.6 & 119.3 & 117.7 & 99.7 & 97.7 & 81.3 & 76.6 & 61.1 \\
\hline 212.1 & 111.0 & 115.9 & 1117.8 & 116.7 & 119.4 & 118.6 & 117.1 & 104.7 & 99.1 & 82.4 & 78.7 & 63.6 \\
\hline 219.0 & 105.9 & 113.6 & 119.6 & 119.2 & 122.8 & 123.9 & 122.9 & 118.1 & 109.0 & 86.3 & 81.0 & 65.3 \\
\hline 225.8 & 116.0 & 123.7 & 125.3 & 123.4 & 129.0 & 128.4 & 127.8 & 117.2 & 104.2 & 89.3 & 82.0 & 67.4 \\
\hline 232.5 & 121.6 & 128.9 & 133.3 & 131.0 & 135.9 & 131.5 & 131.8 & 115.1 & 106.0 & 96.5 & 85.3 & 70.3 \\
\hline \multicolumn{13}{|c|}{ Thermocouples located at sprinkler number 3} \\
\hline Time & $0 \mathrm{~mm}$ & $25 \mathrm{~mm}$ & $50 \mathrm{~mm}$ & $75 \mathrm{~mm}$ & $100 \mathrm{~mm}$ & $125 \mathrm{~mm}$ & $150 \mathrm{~mm}$ & $250 \mathrm{~mm}$ & $350 \mathrm{~mm}$ & $450 \mathrm{~mm}$ & $550 \mathrm{~mm}$ & $900 \mathrm{~mm}$ \\
\hline 0.0 & 26.6 & 26.5 & 26.1 & 26.0 & 25.9 & 25.5 & 25.3 & 24.4 & 23.8 & 23.5 & 23.2 & 22.8 \\
\hline 0.7 & 26.9 & 26.8 & 26.4 & 26.3 & 26.4 & 25.9 & 25.6 & 24.5 & 24.1 & 23.7 & 23.5 & 23.1 \\
\hline 7.4 & 26.7 & 26.7 & 26.4 & 26.3 & 26.5 & 26.0 & 25.6 & 24.6 & 24.2 & 23.9 & 23.6 & 23.1 \\
\hline 14.3 & 26.6 & 26.7 & 26.6 & 26.4 & 26.6 & 26.3 & 25.9 & 24.8 & 24.3 & 24.0 & 23.8 & 23.0 \\
\hline 21.1 & 27.1 & 27.5 & 27.3 & 27.1 & 27.4 & 27.2 & 27.2 & 26.3 & 25.7 & 24.5 & 24.2 & 23.3 \\
\hline 28.0 & 28.6 & 28.9 & 28.8 & 28.8 & 28.7 & 28.6 & 28.8 & 28.1 & 27.9 & 25.9 & 24.5 & 23.3 \\
\hline 34.7 & 30.6 & 31.4 & 31.3 & 31.4 & 31.1 & 30.8 & 30.8 & 29.5 & 28.4 & 25.9 & 24.2 & 23.1 \\
\hline 41.5 & 34.7 & 34.2 & 34.5 & 34.2 & 34.2 & 34.2 & 34.6 & 32.1 & 28.2 & 25.0 & 24.4 & 23.3 \\
\hline 48.5 & 37.2 & 37.1 & 37.7 & 37.7 & 37.7 & 37.6 & 37.8 & 36.4 & 33.1 & 26.9 & 25.7 & 23.5 \\
\hline 55.2 & 36.6 & 38.1 & 37.9 & 38.6 & 39.0 & 39.5 & 40.2 & 39.3 & 36.5 & 28.6 & 27.1 & 23.7 \\
\hline 62.2 & 39.7 & 40.6 & 40.2 & 40.6 & 40.9 & 41.4 & $4 \uparrow .4$ & 40.5 & 38.0 & 30.6 & 29.4 & 24.3 \\
\hline 68.9 & 38.5 & 43.0 & 40.8 & 41.6 & 42.8 & 43.9 & 44.3 & 42.7 & 38.4 & 32.0 & 30.5 & 25.3 \\
\hline 75.7 & 40.5 & 44.4 & 43.7 & 44.3 & 45.5 & 46.3 & 46.3 & 45.9 & 43.1 & 34.4 & 32.7 & 26.8 \\
\hline 82.6 & 43.4 & 46.6 & 46.2 & 46.9 & 47.4 & 18.2 & 48.0 & 47.9 & 47.2 & 41.2 & 38.1 & 27.9 \\
\hline 89.3 & 43.0 & 48.0 & 46.7 & 47.8 & 47.9 & 48.1 & 48.1 & 51.3 & 51.4 & 42.4 & 39.5 & 28.8 \\
\hline 96.3 & 44.0 & 53.9 & 51.0 & 50.8 & 49.9 & 51.0 & 51.5 & 52.8 & 52.6 & 45.6 & 39.4 & 30.3 \\
\hline 103.0 & 50.3 & 54.9 & 53.4 & 54.6 & 54.1 & 55.7 & 56.3 & 56.7 & 56.0 & 50.4 & 44.2 & 32.3 \\
\hline 109.8 & 50.4 & 56.0 & 54.5 & 56.1 & 57.8 & 58.9 & 60.3 & 58.9 & 56.6 & 50.5 & 45.9 & 33.4 \\
\hline 116.6 & 51.8 & 59.0 & 56.9 & 59.6 & 60.4 & 61.3 & 62.0 & 50.5 & 59.6 & 53.1 & 50.2 & 34.7 \\
\hline 123.4 & 54.9 & 63.5 & 61.1 & 62.4 & 63.0 & 64.4 & 64.9 & 64.9 & 64.8 & 56.0 & 48.0 & 36.5 \\
\hline 130.2 & 57.1 & 63.0 & 62.6 & 63.2 & 64.0 & 65.3 & 66.3 & 66.2 & 65.4 & 58.4 & 48.4 & 38.8 \\
\hline 137.1 & 58.3 & 68.3 & 66.1 & 67.6 & 68.0 & 69.7 & 70.4 & 70.3 & 70.1 & 59.9 & 53.1 & 42.6 \\
\hline 143.9 & 61.8 & 70.8 & 67.5 & 69.6 & 70.7 & 72.8 & 73.9 & 73.9 & 74.0 & 64.0 & 54.3 & 44.2 \\
\hline 150.8 & 64.0 & 74.8 & 70.5 & 72.7 & 74.0 & 77.0 & 77.8 & 77.8 & 78.6 & 65.8 & 56.6 & 46.2 \\
\hline 157.6 & 71.3 & 83.7 & 77.4 & 79.4 & 79.4 & 81.7 & 83.1 & 82.4 & 80.6 & 66.7 & 58.3 & 46.8 \\
\hline 164.3 & 73.2 & 86.3 & 80.5 & 82.0 & 82.0 & 81.1 & 85.2 & 82.4 & 82.2 & 72.1 & 64.4 & 49.6 \\
\hline 171.2 & 74.1 & 84.2 & 79.9 & 82.4 & 83.7 & 86.3 & 86.4 & 86.0 & 86.2 & 74.4 & 63.7 & 51.4 \\
\hline 178.0 & 63.9 & 70.6 & 70.8 & 72.8 & 73.9 & 77.5 & 78.8 & 84.4 & 84.4 & 74.5 & 68.2 & 51.2 \\
\hline 184.9 & 74.7 & 87.1 & 81.5 & 85.6 & 84.4 & 87.7 & 88.3 & 87.9 & 89.5 & 82.9 & 73.4 & 54.2 \\
\hline 191.7 & 77.9 & 91.7 & 85.9 & 89.6 & 88.9 & 93.1 & 94.2 & 93.6 & 96.3 & 84.7 & 73.4 & 55.6 \\
\hline 198.4 & 79.7 & 90.5 & 88.4 & 89.0 & 88.2 & 91.1 & 93.1 & 94.2 & 96.9 & 88.5 & 75.1 & 60.0 \\
\hline 205.3 & 77.9 & 97.0 & 92.0 & 92.8 & 93.9 & 96.3 & 97.1 & 97.3 & 100.0 & 90.2 & 78.2 & 61.1 \\
\hline 212.1 & 89.6 & 101.5 & 97.7 & 99.0 & 100.5 & 104.4 & 106.0 & 102.6 & 105.7 & 90.5 & 80.6 & 62.6 \\
\hline 219.0 & 92.9 & 108.2 & 101.2 & 105.1 & 104.2 & 107.9 & 106.9 & 107.0 & 109.5 & 97.3 & 81.1 & 65.1 \\
\hline 225.8 & 98.2 & 114.2 & t06.5 & 110.1 & 110.0 & 114.7 & 115.3 & 115.1 & 114.0 & 96.2 & 83.6 & 66.7 \\
\hline 232.5 & 94.5 & 110.1 & 105.0 & 108.6 & 107.4 & 110.9 & 111.1 & 112.8 & 118.5 & 103.7 & 94.4 & 68.6 \\
\hline nermocoupl & cated at & nkler num & & & & & & & & & & \\
\hline Time & $0 \mathrm{~mm}$ & $25 \mathrm{~mm}$ & $50 \mathrm{~mm}$ & $75 \mathrm{~mm}$ & $100 \mathrm{~mm}$ & $125 \mathrm{~mm}$ & $150 \mathrm{~mm}$ & $250 \mathrm{~mm}$ & $350 \mathrm{~mm}$ & $450 \mathrm{~mm}$ & $550 \mathrm{~mm}$ & $900 \mathrm{~mm}$ \\
\hline 0.0 & 26.7 & 26.5 & 26.5 & 25.8 & 25.4 & 25.1 & 24.7 & 24.1 & 23.6 & 23.3 & 23.1 & 22.6 \\
\hline 0.7 & 27.1 & 27.0 & 26.9 & 26.1 & 25.7 & 25.5 & 25.1 & 24.5 & 24.2 & 23.9 & 23.6 & 22.8 \\
\hline 7.4 & 27.1 & 27.0 & 27.0 & 26.5 & 26.2 & 26.0 & 25.3 & 24.4 & 24.2 & 23.8 & 23.5 & 22.9 \\
\hline 14.3 & 27.0 & 27.0 & 27.0 & 26.4 & 26.0 & 25.8 & 25.4 & 24.6 & 24.2 & 23.9 & 23.6 & 23.0 \\
\hline 21.1 & 27.0 & 27.1 & 27.2 & 27.1 & 27,1 & 27.3 & 27.6 & 26.9 & 26.4 & 24.8 & 24.1 & 23.3 \\
\hline 28.0 & 29.4 & 29.4 & 29.2 & 29.7 & 29.6 & 29.8 & 29.7 & 28.4 & 28.4 & 27.6 & 25.6 & 23.5 \\
\hline 34.7 & 32.8 & 32.2 & 30.8 & 33.4 & 33.0 & 33.1 & 32.7 & 30.3 & 27.2 & 25.1 & 24.4 & 23.5 \\
\hline 41.5 & 36.2 & 35.2 & 32.6 & 37.4 & 36.5 & 36.6 & 36.5 & 34.5 & 32.1 & 25.8 & 25.2 & 23.6 \\
\hline 48.5 & 39.4 & 37.7 & 35.2 & 39.8 & 39.1 & 39.5 & 39.7 & 38.3 & 35.1 & 26.8 & 26.4 & 23.7 \\
\hline 55.2 & 39.3 & 38.1 & 35.8 & 40.6 & 40.1 & 40.3 & 40.8 & 39.5 & 36.7 & 29.2 & 26.4 & 24.0 \\
\hline
\end{tabular}


Experiment Number 29

\begin{tabular}{|c|c|c|c|c|c|c|c|c|c|c|c|c|}
\hline 62.2 & 42.6 & 40.9 & 37.0 & 44.6 & 43.8 & 44.0 & 43.3 & 40.8 & 36.7 & 32.0 & 30.3 & 24.7 \\
\hline 68.9 & 43.8 & 42.5 & 40.5 & 44.3 & 43.9 & 44.3 & 44.1 & 42.5 & 41.6 & 34.6 & 32.7 & 24.6 \\
\hline 75.7 & 46.3 & 44.5 & 40.9 & 47.7 & 47.2 & 47.7 & 47.5 & 45.4 & 43.8 & 35.3 & 33.7 & 26.2 \\
\hline 82.6 & 48.3 & 45.9 & 42.6 & 50.3 & 49.8 & 50.1 & 50.4 & 48.8 & 46.7 & 38.5 & 36.5 & 27.1 \\
\hline 89.3 & 48.9 & 47.8 & 44.4 & 52.7 & 51.9 & 52.2 & 52.5 & 50.5 & 49.5 & 43.5 & 39.3 & 27.5 \\
\hline 96.3 & 52.4 & 50.9 & 49.6 & 54.1 & 53.9 & 54.1 & 54.8 & 53.5 & 53.7 & 49.8 & 46.4 & 29.2 \\
\hline 103.0 & 56.1 & 53.5 & 49.7 & 58.6 & 57.9 & 58.3 & 58.3 & 56.3 & 54.8 & 49.7 & 46.0 & 31.3 \\
\hline 109.8 & 58.1 & 56.7 & 52.3 & 60.6 & 60.9 & 61.2 & 60.6 & 58.6 & 58.1 & 50.1 & 45.6 & 33.3 \\
\hline 116.6 & 58.6 & 56.8 & 52.6 & 61.6 & 61.9 & 63.0 & 63.7 & 61.9 & 61.9 & 52.6 & 46.5 & 34.2 \\
\hline 123.4 & 59.7 & 59.0 & 53.5 & 66.2 & 66.5 & 67.5 & 67.9 & 65.9 & 65.0 & 58.1 & 48.9 & 35.8 \\
\hline 130.2 & 69.8 & 66.0 & 59.8 & 71.9 & 71.1 & 71.8 & 71.8 & 67.5 & 64.9 & 54.7 & 51.4 & 37.3 \\
\hline 137.1 & 71.0 & 66.0 & 59.2 & 73.2 & 727 & 73.7 & 74.0 & 70.5 & 68.4 & 58.1 & 53.2 & 39.4 \\
\hline 143.9 & 72.8 & 71.6 & 67.1 & 74.3 & 73.4 & 73.3 & 73.7 & 72.0 & 70.0 & 60.0 & 56.4 & 41.5 \\
\hline 150.8 & 70.6 & 69.3 & 64.6 & 78.1 & 76.8 & 77.6 & 76.8 & 74.8 & 74.2 & 68.5 & 58.5 & 43.7 \\
\hline 157.6 & 70.4 & 68.1 & 66.0 & 73.1 & 73.5 & 74.7 & 75.5 & 75.0 & 77.0 & 74.1 & 73.3 & 44.7 \\
\hline 164.3 & 76.6 & 72.6 & 69.6 & 81.2 & 82.1 & 82.3 & 82.3 & 80.9 & 80.7 & 71.0 & 63.0 & 46.2 \\
\hline 171.2 & 82.0 & 78.1 & 70.8 & 83.9 & 84.4 & 85.9 & 86.0 & 83.2 & 83.6 & 71.4 & 63.8 & 48.5 \\
\hline 178.0 & 77.6 & 79.0 & 75.4 & 84.6 & 86.8 & 87.1 & 87.6 & 86.7 & 88.7 & 84.4 & 81.7 & 50.6 \\
\hline 184.9 & 78.1 & 74.6 & 73.7 & 81.5 & 81.5 & 82.5 & 84.5 & 86.8 & 88.1 & 85.0 & 76.6 & 51.3 \\
\hline 191.7 & $B 0.8$ & 82.5 & 76.2 & 91.4 & 91.3 & 93.6 & 94.7 & 92.5 & 92.8 & 89.6 & 80.2 & 52.3 \\
\hline 198.4 & 82.2 & 85.1 & 79.9 & 95.3 & 95.3 & 97.3 & 99.1 & 98.1 & 98.7 & 92.6 & B1.7 & 53.4 \\
\hline 205.3 & 95.7 & 91.8 & 88.6 & 99.4 & 99.4 & 100.6 & 102.2 & 98.8 & 100.5 & 91.5 & 77.1 & 56.0 \\
\hline $2 \nmid 2.1$ & 96.9 & 91.8 & 84.6 & 101.9 & $10 \uparrow .6$ & 102.9 & 105.1 & 103.0 & 104.6 & 94.7 & 80.7 & 59.4 \\
\hline 219.0 & 100.0 & 95.0 & 92 3 & 104.3 & 103.9 & 105.4 & 106.2 & 105.2 & 105.8 & 97.6 & B4.6 & 60.8 \\
\hline 225.8 & 110.5 & 103.1 & 89.4 & 114.0 & 112.1 & 112.6 & 111.0 & 107.4 & 102.9 & 89.6 & 86.0 & 62.8 \\
\hline 232.5 & 110.2 & 109.5 & 104.3 & 115.1 & 114.2 & 114.9 & 114.7 & 111.0 & 111.2 & 103.0 & 90.8 & 65.3 \\
\hline
\end{tabular}

\begin{tabular}{|c|c|c|c|c|c|c|c|c|c|}
\hline & & & & & & & \\
\hline Time & $25 \mathrm{~mm}$ & $75 \mathrm{~mm}$ & $125 \mathrm{~mm}$ & $250 \mathrm{~mm}$ & Time & $25 \mathrm{~mm}$ & $75 \mathrm{~mm}$ & $125 \mathrm{~mm}$ & $250 \mathrm{~mm}$ \\
\hline 0.0 & 0.0 & 0.0 & 0.0 & 0.0 & 0.0 & 0.0 & 0.0 & 0.0 & 0.0 \\
\hline 0.7 & 0.0 & 0.0 & 0.0 & 0.0 & 0.7 & -0.1 & 0.0 & 0.0 & -0.1 \\
\hline 7.4 & -0.1 & 0.0 & 0.0 & 0.0 & 7.4 & -0.1 & 0.0 & 0.0 & -0.1 \\
\hline 14.3 & -0.2 & -0.2 & -0.2 & -0.1 & 14.3 & 0.1 & 0.1 & 0.1 & 0.1 \\
\hline 21.1 & -0.1 & -0.1 & -0.2 & 0.0 & 21.1 & 0.1 & 0.0 & 0.0 & -0.1 \\
\hline 28.0 & -0.2 & -0.2 & -0.2 & 0.0 & 28.0 & 0.1 & 0.1 & 0.2 & 0.1 \\
\hline 34.7 & -0.4 & -0.3 & -0.2 & -0.1 & 34.7 & 0.3 & 0.2 & 0.2 & 0.1 \\
\hline 41.5 & -0.3 & -0.3 & -0.2 & -0.1 & 41.5 & 0.2 & 0.2 & 0.3 & 0.2 \\
\hline 48.5 & -0.3 & -0.3 & -0.3 & -0.2 & 48.5 & 0.2 & 0.2 & 0.2 & 0.1 \\
\hline 55.2 & -0.3 & -0.3 & -0.2 & -0.3 & 55.2 & 0.2 & 0.3 & 0.3 & -0.1 \\
\hline 62.2 & -0.2 & -0.3 & -0.2 & -0.3 & 62.2 & 0.3 & 0.3 & 0.3 & 0.2 \\
\hline 68.9 & -0.3 & -0.3 & -0.4 & -0.3 & 68.9 & 0.1 & 0.2 & 0.3 & 0.3 \\
\hline 75.7 & -0.3 & -0.3 & -0.2 & -0.2 & 75.7 & -0.1 & 0.2 & 0.4 & 0.3 \\
\hline 82.6 & -0.5 & -0.5 & -0.3 & -0.3 & 82.6 & 0.3 & 0.4 & 0.4 & 0.3 \\
\hline 89.3 & -0.5 & -0.6 & -0.5 & -0.3 & 89.3 & 0.4 & 0.3 & 0.3 & 0.2 \\
\hline 96.3 & -0.4 & -0.4 & -0.5 & -0.4 & 96.3 & 0.4 & 0.6 & 0.7 & 0.4 \\
\hline 103.0 & -0.4 & -0.4 & -0.3 & -0.5 & 103.0 & 0.3 & 0.3 & 0.5 & 0.4 \\
\hline 109.8 & -0.3 & -0.5 & .0 .3 & -0.3 & 109.8 & 0.5 & 0.4 & 0.4 & 0.3 \\
\hline 116.6 & -0.4 & -0.4 & -0.4 & -0.3 & 116.6 & 0.6 & 0.5 & 0.5 & 0.3 \\
\hline 123.4 & -0.5 & -0.4 & -0.2 & -0.2 & 123.4 & 0.5 & 0.5 & 0.5 & 0.2 \\
\hline 130.2 & -0.5 & .0 .5 & -0.3 & -0.4 & 130.2 & 0.3 & 0.2 & 0.5 & 0.3 \\
\hline 137.1 & -0.2 & -0.4 & -0.3 & -0.3 & 137.1 & 0.5 & 0.5 & 0.6 & 0.3 \\
\hline 143.9 & -0.5 & -0.5 & -0.2 & -0.3 & 143.9 & 0.5 & 0.4 & 0.5 & 0.2 \\
\hline 150.8 & -0.4 & -0.5 & -0.4 & -0.5 & 150.8 & 0.6 & 0.6 & 0.8 & 0.4 \\
\hline 157.6 & -0.3 & -0.4 & -0.4 & .0 .3 & 157.6 & 0.4 & 0.4 & 0.6 & 0.4 \\
\hline 164.3 & -0.5 & -0.5 & -0.5 & -0.5 & 164.3 & 0.5 & 0.7 & 0.8 & 0.5 \\
\hline 171.2 & -0.5 & -0.5 & -0.4 & -0.3 & 171.2 & 0.5 & 0.5 & 0.6 & 0.3 \\
\hline 178.0 & -0.4 & -0.6 & -0.3 & -0.5 & 178.0 & 0.6 & 0.6 & 0.7 & 0.3 \\
\hline 184.9 & -0.5 & -0.5 & -0.4 & -0.4 & 184.9 & 0.5 & 0.5 & 0.7 & 0.4 \\
\hline 191.7 & -0.5 & -0.4 & -0.2 & -0.4 & 191.7 & 0.4 & 0.3 & 0.5 & 0.2 \\
\hline 198.4 & -0.5 & -0.5 & -0.4 & -0.5 & 198.4 & 0.5 & 0.5 & 0.7 & 0.4 \\
\hline 205.3 & -0.4 & -0.6 & -0.5 & -0.5 & 205.3 & 0.6 & 0.4 & 0.6 & 0.2 \\
\hline 212.1 & -0.4 & -0.5 & -0.4 & -0.4 & 212.1 & 0.7 & 0.5 & 0.7 & 0.6 \\
\hline 219.0 & -0.7 & -0.6 & -0.5 & -0.5 & 219.0 & 0.5 & 0.5 & 0.8 & 0.5 \\
\hline 225.8 & -0.5 & -0.6 & -0.4 & -0.4 & 225.8 & 0.6 & 0.5 & 0.8 & 0.3 \\
\hline
\end{tabular}




\section{Experiment Number 29}

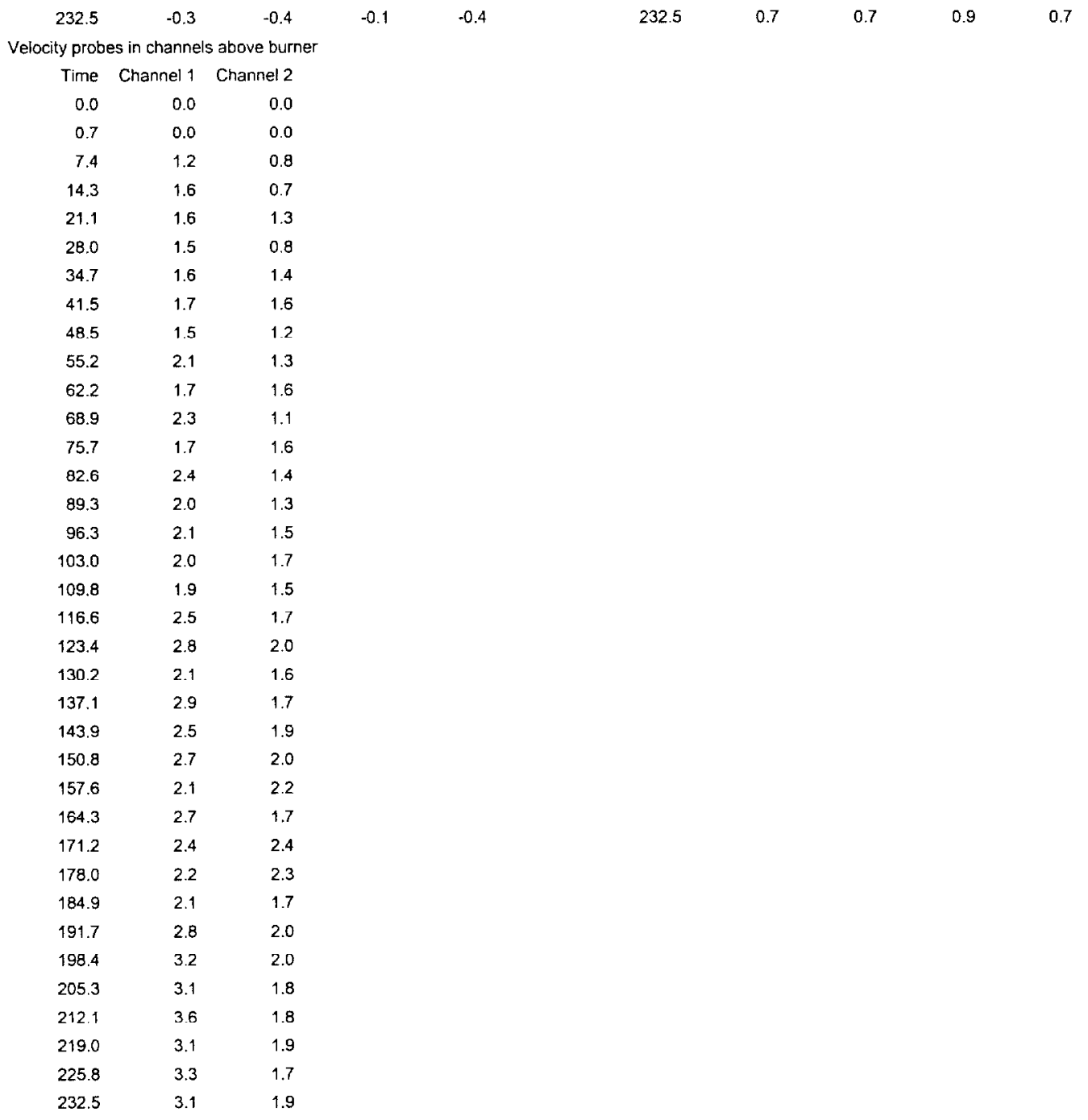


Experiment Number 30

\begin{tabular}{|c|c|c|c|c|c|c|c|c|c|c|c|c|}
\hline \multicolumn{13}{|c|}{ Thermocouples located at sprinkler number 1} \\
\hline Time & $0 \mathrm{~mm}$ & $25 \mathrm{~mm}$ & $50 \mathrm{~mm}$ & $75 \mathrm{~mm}$ & $100 \mathrm{~mm}$ & $125 \mathrm{~mm}$ & $150 \mathrm{~mm}$ & $250 \mathrm{~mm}$ & $350 \mathrm{~mm}$ & $450 \mathrm{~mm}$ & $550 \mathrm{~mm}$ & $900 \mathrm{~mm}$ \\
\hline 0.0 & 26.3 & 26.2 & 26.4 & 26.0 & 25.8 & 24.8 & 25.1 & 24.0 & 24.0 & 23.7 & 23.5 & 22.6 \\
\hline 1.4 & 27.0 & 26.9 & 27.1 & 26.6 & 26.5 & 25.2 & 25.9 & 24.4 & 24.9 & 24.6 & 24.4 & 23.4 \\
\hline 8.4 & 27.0 & 26.7 & 26.8 & 26.4 & 26.3 & 25.4 & 25.8 & 24.6 & 24.0 & 24.6 & 24.5 & 23.5 \\
\hline 15.1 & 28.0 & 28.1 & 28.3 & 28.4 & 28.5 & 27.8 & 28.0 & 26.7 & 25.4 & 24.9 & 24.6 & 23.6 \\
\hline 22.0 & 30.2 & 30.2 & 30.5 & 30.8 & 30.5 & 29.9 & 29.9 & 26.5 & 25.4 & 24.9 & 24.6 & 23.6 \\
\hline 28.8 & 35.0 & 34.7 & 36.0 & 35.9 & 35.5 & 33.9 & 33.3 & 30.5 & 25.9 & 25.2 & 24.9 & 23.7 \\
\hline 35.5 & 38.8 & 39.5 & 41.0 & 41.4 & 40.7 & 40.3 & 39.0 & 36.1 & 29.2 & 27.6 & 25.7 & 24.5 \\
\hline 42.4 & 41.5 & 42.2 & 43.3 & 43.3 & 42.1 & 41.0 & 41.2 & 39.1 & 33.4 & 29.8 & 28.4 & 26.7 \\
\hline 49.1 & 42.2 & 44.0 & 45.1 & 45.3 & 44.8 & 44.2 & 44.5 & 42.6 & 40.4 & 33.5 & 31.3 & 26.1 \\
\hline 56.1 & 42.6 & 45.4 & 47.2 & 49.4 & 49.3 & 49.7 & 48.0 & 47.5 & 46.1 & 40.7 & 33.2 & 25.3 \\
\hline 62.8 & 48.7 & 51.8 & 54.6 & 55.3 & 54.0 & 53.1 & 52.7 & 51.6 & 48.4 & 40.2 & 36.1 & 26.9 \\
\hline 69.6 & 53.8 & 56.4 & 58.3 & 59.1 & 58.6 & 57.0 & 57.7 & 52.0 & 53.8 & 40.9 & 38.6 & 28.3 \\
\hline 76.5 & 61.3 & 62.7 & 64.7 & 66.2 & 64.7 & 65.8 & 64.1 & 57.3 & 51.7 & 43.0 & 41.4 & 34.9 \\
\hline 83.3 & 60.5 & 62.4 & 64.8 & 65.7 & 65.4 & 65.2 & 64.6 & 65.3 & 54.6 & 47.3 & 44.4 & 35.6 \\
\hline 90.0 & 61.6 & 65.0 & 68.2 & 60.2 & 68.0 & 68.2 & 66.9 & 65.1 & 60.4 & 50.3 & 46.9 & 38.1 \\
\hline 96.9 & 71.2 & 73.6 & 76.5 & 78.5 & 76.3 & 77.7 & 74.8 & 70.5 & 59.3 & 53.5 & 49.9 & 39.0 \\
\hline 103.7 & 72.7 & 74.9 & 78.8 & 80.5 & 78.8 & 80.3 & 76.8 & 69.0 & 59.6 & 54.9 & 52.4 & 41.2 \\
\hline 110.6 & 74.5 & 76.8 & 80.0 & 80.7 & 79.8 & 77.9 & 78.4 & 62.4 & 60.4 & 57.0 & 55.2 & 43.6 \\
\hline 117.3 & 77.7 & 80.0 & 82.8 & 83.5 & 81.1 & 79.7 & 79.9 & 70.6 & 62.7 & 60.1 & 56.1 & 45.8 \\
\hline 124.1 & 82.7 & 85.1 & 89.0 & 89.6 & 87.6 & 84.4 & 84.9 & 72.8 & 66.0 & 59.4 & 56.7 & 46.6 \\
\hline 131.0 & 85.5 & 89.0 & 92.5 & 93.5 & 91.5 & 90.8 & 89.7 & 84.5 & 77.3 & 64.3 & 60.8 & 47.2 \\
\hline 137.8 & 87.2 & 91.5 & 95.6 & 95.8 & 93.6 & 92.8 & 92.0 & 78.5 & 71.2 & 65.0 & 63.0 & 51.6 \\
\hline 144.7 & 93.1 & 95.6 & 99.4 & 101.3 & 99.3 & 101.3 & 98.4 & 96.7 & 82.0 & 66.9 & 63.2 & 54.0 \\
\hline 151.5 & 94.3 & 97.9 & 101.7 & 102.8 & 100.1 & 100.4 & 100.5 & 92.7 & 89.3 & 77.7 & 69.3 & 58.4 \\
\hline 158.2 & 94.0 & 96.4 & 99.1 & 99.7 & 101.9 & 98.5 & 101.0 & 94.0 & 82.2 & 72.6 & 68.6 & 57.3 \\
\hline 165.1 & 99.3 & 104.4 & 109.1 & $10 \mathrm{~B} .1$ & 107.0 & 96.8 & 105.4 & 90.0 & 87.2 & 80.8 & 71.1 & 56.7 \\
\hline 171.8 & 101.8 & 106.5 & 111.6 & 113.1 & 110.7 & 111.8 & 110.2 & 101.0 & 90.1 & 81.8 & 76.4 & 61.2 \\
\hline 178.7 & 105.8 & 112.9 & 117.6 & 119.9 & 118.4 & 119.6 & 113.6 & 106.5 & 98.6 & 85.8 & 80.2 & 66.0 \\
\hline 185.5 & 115.1 & 116.6 & 120.0 & 123.0 & 119.5 & 119.3 & 116.4 & 109.5 & 93.6 & 85.7 & 82.5 & 65.8 \\
\hline 192.3 & 117.0 & 119.2 & 122.7 & 126.0 & 124.1 & 122.7 & 120.1 & 112.4 & 100.5 & 90.3 & 86.0 & 70.1 \\
\hline 199.2 & 121.9 & 129.4 & 135.1 & 138.4 & 134.0 & 134.4 & 128.3 & 121.8 & 102.7 & 91.5 & 86.7 & 70.0 \\
\hline 205.9 & 122.9 & 125.0 & 130.0 & 133.4 & 130.7 & 131.1 & 128.2 & 121.6 & 110.6 & 94.7 & 89.3 & 72.4 \\
\hline 212.9 & 123.0 & 127.1 & 131.5 & 133.5 & 129.4 & 129.0 & 125.8 & 117.0 & 103.8 & 96.8 & 93.4 & 79.3 \\
\hline 219.6 & 121.9 & 126.2 & 133.1 & 136.0 & 131.4 & 131.5 & 128.2 & 122.6 & 106.6 & 96.4 & 92.9 & 78.7 \\
\hline 226.4 & 120.4 & 127.2 & 134.8 & 136.8 & 133.9 & 131.0 & 131.5 & 122.1 & 107.7 & 99.4 & 95.3 & 80.4 \\
\hline 233.3 & 130.2 & 139.7 & 145.3 & 147.3 & 143.7 & 143.8 & 139.7 & 133.1 & 107.9 & 101.0 & 97.1 & 80.1 \\
\hline \multicolumn{13}{|c|}{ Thermocouples located at sprinkler number 2} \\
\hline Time & $0 \mathrm{~mm}$ & $25 \mathrm{~mm}$ & $50 \mathrm{~mm}$ & $75 \mathrm{~mm}$ & $100 \mathrm{~mm}$ & $125 \mathrm{~mm}$ & $150 \mathrm{~mm}$ & $250 \mathrm{~mm}$ & $350 \mathrm{~mm}$ & $450 \mathrm{~mm}$ & $550 \mathrm{~mm}$ & $900 \mathrm{~mm}$ \\
\hline 0.0 & 27.8 & 27.0 & 26.8 & 26.1 & 26.2 & 25.7 & 25.7 & 21.7 & 21.2 & 23.7 & 23.4 & 22.6 \\
\hline 1.4 & 28.2 & 27.4 & 27.9 & 26.8 & 27.0 & 26.2 & 26.4 & 25.2 & 24.9 & 24.5 & 24.2 & 23.5 \\
\hline 8.4 & 28.6 & 27.4 & 27.8 & 26.8 & 26.9 & 26.1 & 26.3 & 25.3 & 24.9 & 24.6 & 24.3 & 23.6 \\
\hline 15.1 & 29.7 & 29.2 & 29.4 & 28.2 & 29.3 & 29.1 & 29.0 & 28.3 & 25.8 & 25.3 & 24.7 & 23.7 \\
\hline 22.0 & 31.5 & 31.6 & 33.4 & 31.0 & 33.5 & 32.0 & 32.4 & 26.9 & 25.9 & 25.2 & 24.8 & 23.7 \\
\hline 28.8 & 34.0 & 33.8 & 35.6 & 33.3 & 35.9 & 36.4 & 36.1 & 35.1 & 31.0 & 26.2 & 25.5 & 24.0 \\
\hline 35.5 & 37.4 & 39.8 & 41.3 & 40.0 & 42.4 & 42.2 & 41.6 & 37.5 & 32.1 & 28.0 & 26.5 & 24.5 \\
\hline 42.4 & 43.0 & 45.7 & 47.2 & 46.2 & 48.3 & 47.8 & 46.9 & 40.7 & 35.3 & 29.6 & 28.3 & 25.0 \\
\hline 49.1 & 46.5 & 46.7 & 48.8 & 47.9 & 51.0 & 51.5 & 51.2 & 43.8 & 40.4 & 32.9 & 31.1 & 24.9 \\
\hline 56.1 & 48.7 & 50.2 & 53.1 & 52.7 & 54.7 & 55.6 & 54.7 & 48.1 & 42.8 & 36.4 & 34.3 & 26.9 \\
\hline 62.8 & 54.0 & 56.1 & 58.5 & 57.9 & 59.9 & 59.5 & 59.1 & 55.8 & 49.1 & 40.9 & 37.8 & 27.2 \\
\hline 69.6 & 57.1 & 56.2 & 59.0 & 56.7 & 61.3 & 60.8 & 61.1 & 58.1 & 51.2 & 44.1 & 40.8 & 29.4 \\
\hline 76.5 & 61.4 & 64.5 & 68.0 & 67.6 & 70.9 & 70.8 & 69.5 & 59.7 & 53.7 & 45.9 & 42.5 & 34.1 \\
\hline 83.3 & 66.0 & 69.0 & 72.0 & 70.1 & 72.8 & 72.6 & 71.4 & 56.8 & 53.3 & 49.9 & 44.9 & 36.8 \\
\hline 90.0 & 70.5 & 74.1 & 75.4 & 72.1 & 75.4 & 74.0 & 73.6 & 59.7 & 52.8 & 49.0 & 46.1 & 38.7 \\
\hline 96.9 & 73.7 & 77.3 & 79.1 & 76.7 & 80.6 & 77.4 & 79.0 & 64.2 & 61.8 & 55.1 & 49.8 & 40.1 \\
\hline 103.7 & 78.4 & 82.3 & 84.9 & 84.0 & 86.4 & 85.4 & 84.5 & 65.0 & 60.7 & 55.0 & 50.7 & 43.2 \\
\hline 110.6 & 81.3 & 80.6 & 84.9 & 79.5 & 84.7 & 81.3 & 83.0 & 75.2 & 69.4 & 59.8 & 55.5 & 44.6 \\
\hline 117.3 & 82.3 & 86.7 & 88.4 & 87.5 & 90.0 & 89.5 & 89.4 & 78.8 & 71.6 & 62.6 & 58.1 & 45.6 \\
\hline 124.1 & 89.3 & 92.0 & 93.9 & 90.9 & 95.7 & 94.3 & 93.4 & 76.0 & 73.7 & 67.6 & 63.2 & 46.3 \\
\hline 131.0 & 90.8 & 92.8 & 95.7 & 92.9 & 96.2 & 95.5 & 93.9 & 82.4 & 75.7 & 68.8 & 63.9 & 51.7 \\
\hline 137.8 & 86.0 & 91.3 & 94.6 & 91.1 & 95.6 & 96.0 & 95.6 & 92.0 & 83.0 & 71.0 & 65.6 & 51.8 \\
\hline 144.7 & 92.7 & 94.2 & 97.6 & 94.8 & 99.5 & 99.5 & 99.3 & 90.9 & 84.2 & 73.1 & 68.9 & 53.6 \\
\hline
\end{tabular}


Experiment Number 30

\begin{tabular}{|c|c|c|c|c|c|c|c|c|c|c|c|}
\hline 151.5 & 93.7 & 100.3 & 103.0 & 101.3 & 105.7 & 104.6 & 105.0 & 95.2 & 90.2 & 78.5 & 71.7 \\
\hline 158.2 & 98.5 & 103.6 & 105.9 & 104.5 & 108.1 & 107.6 & 108.5 & 100.4 & 96.7 & 82.6 & 73.9 \\
\hline 165.1 & 105.2 & 110.5 & 112.9 & 113.1 & 115.0 & 113.5 & 113.4 & 101.4 & 93.7 & 83.6 & 75.0 \\
\hline 171.8 & 109.9 & 116.7 & 120.8 & 120.6 & 123.6 & 122.3 & 121.2 & 103.3 & 101.0 & 85.0 & 77.4 \\
\hline 178.7 & 113.6 & 120.6 & 123.5 & 123.4 & 127.4 & 126.2 & 126.0 & 113.1 & 103.5 & 86.8 & 80.7 \\
\hline 185.5 & 122.3 & 129.5 & 131.4 & 130.9 & 133.4 & 131.7 & 131.1 & 113.0 & 104.1 & 91.8 & 84.7 \\
\hline 192.3 & 117.3 & 120.7 & 125.1 & 123.4 & 128.9 & 128.4 & 129.8 & 121.7 & 106.3 & 97.6 & 89.2 \\
\hline 199.2 & 124.0 & 130.7 & 132.7 & 131.0 & 134.0 & 134.7 & 133.3 & 120.4 & 116.4 & 103.3 & 91.0 \\
\hline 205.9 & 130.0 & 137.7 & 140.0 & 138.7 & 143.6 & 139.6 & 140.1 & 116.9 & 109.2 & 98.5 & 93.6 \\
\hline 212.9 & 130.0 & 137.6 & 140.1 & 139.3 & 141.6 & 139.8 & 138.4 & 118.9 & 110.8 & 101.2 & 93.6 \\
\hline 219.6 & 131.0 & 138.1 & 141.8 & 141.4 & 146.9 & 146.1 & 146.7 & 126.0 & 105.8 & 99.2 & 95.3 \\
\hline 226.4 & 129.8 & 136.7 & 140.6 & 137.2 & 142.1 & 139.5 & 139.9 & 126.5 & 117.8 & 101.1 & 96.6 \\
\hline 233.3 & 136.4 & 123.1 & B2.1 & 63.0 & 93.6 & 114.9 & 127.9 & 114.1 & 110.7 & 101.4 & 97.9 \\
\hline
\end{tabular}

Thermocouples located at sprinkler number 3

\begin{tabular}{|c|c|c|c|c|c|c|c|c|c|c|c|c|}
\hline Time & $0 \mathrm{~mm}$ & $25 \mathrm{~mm}$ & $50 \mathrm{~mm}$ & $75 \mathrm{~mm}$ & $100 \mathrm{~mm}$ & $125 \mathrm{~mm}$ & $150 \mathrm{~mm}$ & $250 \mathrm{~mm}$ & $350 \mathrm{~mm}$ & $450 \mathrm{~mm}$ & $550 \mathrm{~mm}$ & $900 \mathrm{~mm}$ \\
\hline 0.0 & 27.9 & 27.9 & 27.5 & 26.9 & 26.4 & 25.9 & 25.5 & 24.7 & 24.2 & 23.8 & 23.4 & 22.8 \\
\hline 1.4 & 27.8 & 28.2 & 28.1 & 27.8 & 27.3 & 26.8 & 20.4 & 25.4 & 24.9 & 24.5 & 24.1 & 23.3 \\
\hline 8.4 & 27.7 & 28.6 & 28.2 & 27.8 & 27.4 & 26.9 & 26.6 & 25.5 & 25.0 & 24.6 & 24.3 & 23.5 \\
\hline 15.1 & 28.8 & 28.8 & 28.7 & 28.8 & 28.5 & 28.7 & 28.0 & 26.7 & 26.7 & 25.6 & 25.1 & 24.0 \\
\hline 22.0 & 30.8 & 30.7 & 30.7 & 31.1 & 31.1 & 31.3 & 31.7 & 30.8 & 30.5 & 28.9 & 25.7 & 23.9 \\
\hline 28.8 & 35.8 & 36.1 & 36.6 & 36.3 & 36.5 & 36.4 & 36.8 & 34.3 & 30.9 & 26.2 & 25.1 & 23.6 \\
\hline 35.5 & 38.9 & 40.6 & 40.6 & 40.5 & 40.6 & 40.9 & 41.2 & 39.2 & 36.2 & 21.8 & 26.2 & 24.1 \\
\hline 42.4 & 41.6 & 42.3 & 42.7 & 42.8 & 43.0 & 43.2 & 43.4 & 42.2 & 41.1 & 31.1 & 28.7 & 24.4 \\
\hline 49.1 & 40.1 & 42.9 & 42.7 & $43 B$ & 44.3 & 45.7 & 46.4 & 45.4 & 45.9 & 35.7 & 30.9 & 24.8 \\
\hline 56.1 & 45.7 & 48.4 & 47.6 & 48.0 & 47.9 & 49.4 & 49.7 & 49.8 & 46.8 & 37.6 & 34.0 & 26.5 \\
\hline 62.8 & 46.9 & 54.2 & 50.3 & 51.7 & 52.2 & 54.1 & 54.9 & 53.6 & 47.8 & 40.3 & 36.8 & 28.2 \\
\hline 69.6 & 48.5 & 55.6 & 53.1 & 54.8 & 55.4 & 56.8 & 57.2 & 56.9 & 55.8 & 43.8 & 39.4 & 30.3 \\
\hline 76.5 & 52.6 & 56.9 & 56.3 & 57.1 & 57.5 & 58.9 & 59.6 & 58.5 & 58.1 & 47.4 & 44.2 & 31.2 \\
\hline 83.3 & 54.0 & 59.7 & 58.3 & 58.7 & 59.3 & 60.6 & 61.5 & 61.5 & 62.2 & 50.0 & 45.6 & 34.9 \\
\hline 90.0 & 60.7 & 64.0 & 63.2 & 63.6 & 63.9 & 65.3 & 66.2 & 64.6 & 65.0 & 54.9 & 49.2 & 36.2 \\
\hline 96.9 & 59.7 & 64.5 & 63.1 & 64.8 & 65.5 & 67.2 & 67.4 & 67.5 & 67.9 & 61.3 & 53.4 & 37.2 \\
\hline 103.7 & 59.2 & 68.3 & 63.7 & 67.1 & 66.6 & 68.6 & 68.3 & 69.6 & 71.9 & 64.3 & 54.9 & 40.0 \\
\hline 110.6 & 62.7 & 68.6 & 67.5 & 68.7 & 69.7 & 72.2 & 73.1 & 74.2 & 74.5 & 63.7 & 56.6 & 41.6 \\
\hline 117.3 & 65.1 & 76.7 & 71.5 & 72.9 & 72.9 & 15.1 & 15.5 & 76.8 & 77.8 & 67.1 & 60.8 & 47.2 \\
\hline 124.1 & 62.2 & 68.7 & 69.4 & 70.8 & 73.1 & 77.0 & 78.5 & 78.4 & 76.2 & 65.3 & 59.6 & 50.4 \\
\hline 131.0 & 75.2 & 790 & 790 & 80.5 & 80.3 & 82.9 & 84.2 & 83.3 & 82.3 & 71.6 & 63.2 & 50.9 \\
\hline 137.8 & 75.4 & 86.6 & 80.2 & 84.0 & 84.1 & 86.7 & 86.6 & 86.8 & 84.4 & 69.8 & 63.2 & 53.5 \\
\hline$\uparrow 44.7$ & 77.5 & 85.7 & 81.9 & 84.9 & 86.4 & 88.8 & 88.6 & 89.1 & 89.6 & 74.7 & 64.7 & 52.7 \\
\hline 151.5 & 79.8 & 90.5 & 85.4 & 87.4 & 89.6 & 93.1 & 93.9 & 94.1 & 91.3 & 77.2 & 69.4 & 57.9 \\
\hline 158.2 & 88.6 & 97.5 & 95.1 & 96.2 & 95.8 & 98.9 & 98.9 & 98.8 & 99.1 & 85.8 & 72.2 & 57.8 \\
\hline 165.1 & 88.8 & 97.9 & $9 \mathrm{G.1}$ & 96.5 & 96.5 & 99.1 & 100.4 & 100.1 & 102.8 & 89.2 & 76.9 & 60.9 \\
\hline 171.8 & 92.2 & 97.0 & 96.4 & 99.0 & 100.4 & 102.5 & 103.9 & 104.1 & 102.3 & 87.4 & 76.2 & 62.0 \\
\hline 178.7 & 95.7 & 107.7 & 101.9 & 107.0 & 106.0 & 109.4 & 109.4 & 110.1 & 109.6 & 95.4 & 80.6 & 62.1 \\
\hline 185.5 & 76.3 & 85.1 & 88.7 & 93.2 & 95.2 & 98.4 & 99.5 & 106.3 & 109.5 & 103.4 & 91.1 & 66.8 \\
\hline 192.3 & 83.1 & 93.3 & 93.7 & 96.2 & 97.7 & 101.5 & 103.4 & 110.6 & 113.8 & 104.0 & 88.3 & 68.7 \\
\hline 199.2 & 97.2 & 114.2 & 107.4 & 112.5 & 112.2 & 116.5 & 115.5 & 116.0 & 118.0 & 105.1 & 93.1 & 70.7 \\
\hline 205.9 & 101.8 & 113.8 & 112.6 & 116.7 & 118.6 & 122.0 & 124.0 & 122.4 & 116.3 & 101.3 & 91.0 & 73.3 \\
\hline 2129 & 102.9 & 115.7 & 113.0 & 115.1 & 115.3 & 118.1 & 118.3 & 118.6 & 120.6 & 112.2 & 93.3 & 75.0 \\
\hline 219.6 & 96.7 & 103.4 & 103.9 & 109.3 & 110.6 & 115.5 & 118.7 & 122.7 & 126.2 & 113.5 & 99.2 & 75.0 \\
\hline 226.4 & 98.1 & 107.0 & 106.3 & 112.5 & 115.4 & 121.4 & 122.3 & 125.4 & 129.0 & 109.1 & 95.3 & 77.7 \\
\hline 233.3 & 102.0 & 121.2 & 114.0 & 120.9 & 119.1 & 125.3 & 125.2 & 127.3 & 129.6 & 113.6 & 96.0 & 80.0 \\
\hline \multicolumn{13}{|c|}{ rmocouples located at sprinkier number 4} \\
\hline Time & $0 \mathrm{~mm}$ & $25 \mathrm{~mm}$ & $50 \mathrm{~mm}$ & $75 \mathrm{~mm}$ & $100 \mathrm{~mm}$ & $125 \mathrm{~mm}$ & $150 \mathrm{~mm}$ & $250 \mathrm{~mm}$ & $350 \mathrm{~mm}$ & $450 \mathrm{~mm}$ & $550 \mathrm{~mm}$ & $900 \mathrm{~mm}$ \\
\hline 0.0 & 27.4 & 27.1 & 27.1 & 26.3 & 25.7 & 25.3 & 25.0 & 24.4 & 23.9 & 23.5 & 23.3 & 22.8 \\
\hline 1.4 & 27.7 & 27.6 & 27.6 & 26.9 & 26.2 & 25.9 & 25.5 & 25.1 & 24.9 & 24.5 & 24.4 & 23.3 \\
\hline 8.4 & 27.6 & 27.6 & 27.6 & 26.9 & 26.3 & 25.9 & 25.5 & 25.1 & 24.8 & 24.6 & 24.4 & 23.5 \\
\hline 15.1 & 28.8 & 28.6 & 28.2 & 28.9 & 28.6 & 28.8 & 28.9 & 27.7 & 27.5 & 26.8 & 24.9 & 23.8 \\
\hline 22.0 & 32.8 & 32.3 & 31.1 & 33.9 & 33.3 & 33.5 & 33.0 & 31.0 & 28.9 & 27.2 & $24 . a$ & 23.9 \\
\hline 28.8 & 37.4 & 37.5 & 34.1 & 39.9 & 38.7 & 39.1 & 39.1 & 36.5 & 32.2 & 25.4 & 24.6 & 24.1 \\
\hline 35.5 & 43.4 & 40.8 & 36.4 & 44.1 & 43.2 & 43.4 & 43.3 & 40.8 & 36.1 & 27.5 & 27.0 & 24.6 \\
\hline 42.4 & 45.4 & 42.8 & 38.9 & 45.7 & 45.2 & 45.2 & 45.1 & 43.1 & 39.3 & 30.4 & 28.8 & 24.6 \\
\hline 49.1 & 46.4 & 45.1 & 41.7 & 48.8 & 48.3 & 48.6 & 48.7 & 47.4 & 44.8 & 34.7 & 33.5 & 24.7 \\
\hline 56.1 & 49.8 & 47.5 & 44.4 & 52.1 & 51.9 & 52.8 & 53.0 & 50.9 & 50.5 & 39.2 & 35.9 & 25.6 \\
\hline
\end{tabular}


Experiment Number 30

\begin{tabular}{|c|c|c|c|c|c|c|c|c|c|c|c|c|}
\hline 62.8 & 51.3 & 48.5 & 47.5 & 54.9 & 55.4 & 55.9 & 56.3 & 55.0 & 51.4 & 44.6 & 39.3 & 26.6 \\
\hline 69.6 & 52.6 & 52.4 & 50.7 & 55.6 & 55.0 & 55.7 & 56.7 & 54.2 & 53.7 & 48.0 & 42.0 & 29.0 \\
\hline 76.5 & 58.5 & 54.4 & 50.7 & 59.6 & 59.0 & 59.4 & 59.6 & 58.2 & 58.6 & 49.1 & 44.1 & 29.3 \\
\hline 83.3 & 58.5 & 56.1 & 52.5 & 59.8 & 59.8 & 60.5 & 60.9 & 61.0 & 59.1 & 52.8 & 51.7 & 32.2 \\
\hline 90.0 & 64.4 & 62.7 & 59.9 & 65.8 & 66.0 & 66.5 & 66.6 & 64.7 & 59.4 & 53.4 & 51.3 & 35.1 \\
\hline 96.9 & 67.4 & 64.4 & 61.2 & 69.8 & 69.4 & 70.1 & 69.8 & 65.7 & 61,1 & 51.8 & 50.3 & 37.3 \\
\hline 103.7 & 65.7 & 66.4 & 61.5 & 74.2 & 74.2 & 75.2 & 75.4 & 71.1 & 68.9 & 56.6 & 53.8 & 40.6 \\
\hline 110.6 & 70.1 & 68.1 & 63.5 & 74.4 & 74.0 & 74.5 & 74.1 & 72.9 & 72.4 & 64.2 & 57.7 & 42.1 \\
\hline 117.3 & 77.6 & 73.2 & 63.4 & 81.4 & 80.2 & 80.1 & 78.5 & 74.2 & 70.3 & 60.9 & 58.8 & 43.2 \\
\hline 124.1 & 77.8 & 74.2 & 67.8 & 79.5 & 79.2 & 19.8 & 80.3 & 78.6 & 78.2 & 71.6 & 60.9 & 44.9 \\
\hline 131.0 & 81.7 & 77.9 & 73.6 & 84.2 & 84.2 & 84.9 & 84.3 & 81.6 & 81.1 & 67.2 & 62.6 & 45.7 \\
\hline 137.8 & 81.2 & 77.2 & 730 & 86.1 & 85.6 & 86.9 & 87.8 & 83.2 & 81.5 & 70.8 & 67.8 & 48.5 \\
\hline 144.7 & 91.0 & 82.0 & 76.1 & 92.1 & 90.9 & 91.9 & 91.8 & 87.5 & 87.7 & 80.1 & 75.2 & 51.2 \\
\hline 151.5 & 85.4 & 83.9 & 79.8 & 91.1 & 91.0 & 92.4 & 92.5 & 89.9 & 91.6 & 89.4 & 80.4 & 52.6 \\
\hline 158.2 & 94.5 & 90.5 & 83.6 & 97.1 & 95.6 & 97.6 & 97.7 & 93.7 & 93.9 & 87.4 & 77.9 & 54.3 \\
\hline 165.1 & 100.8 & 94.7 & 80.8 & 105.7 & 104.3 & 105.8 & 103.9 & 96.8 & 91.1 & 87.2 & 82.5 & 56.0 \\
\hline 171.8 & 101.4 & 96.3 & 87.3 & 104.0 & 103.7 & 105.1 & 104.9 & 101.6 & 100.5 & 93.1 & 84.5 & 58.1 \\
\hline 178.7 & 104.2 & 100.0 & 91.6 & 106.0 & 105.4 & 105.8 & 106.5 & 101.3 & 97.2 & 88.6 & 84.0 & 60.4 \\
\hline 185.5 & 105.2 & 98.0 & 87.0 & 108.6 & 108.3 & 110.3 & 110.5 & 104.1 & 101.1 & 89.2 & 85.6 & 62.8 \\
\hline 192.3 & 103.4 & 100.9 & 95.0 & 107.0 & 107.2 & 107.2 & 108.5 & 107.5 & 107.9 & 100.2 & 93.8 & 64.5 \\
\hline 199.2 & 108.9 & 101.9 & 96.0 & 113.6 & 110.2 & 110.3 & 110.2 & 108.5 & 112.2 & 109.9 & 101.6 & 66.1 \\
\hline 205.9 & 100.4 & 101.4 & 100.2 & 108.3 & 110.1 & 112.5 & 113.8 & 114.3 & 115.5 & 106.2 & 97.6 & 68.5 \\
\hline 212.9 & 115.6 & 108.7 & 104.1 & 118.0 & 116.5 & 118.7 & 119.0 & 116.5 & 118.1 & 112.6 & 106.9 & 71.0 \\
\hline 219.6 & 1141 & $\uparrow 15.4$ & 108.9 & 120.3 & 118.8 & 120.9 & 119.6 & 116.2 & 116.6 & 110.1 & 101.3 & 74.4 \\
\hline 226.4 & 108.3 & 104.6 & 101.7 & 112.9 & 111.2 & 112.7 & 113.2 & 116.5 & 119.0 & 115.5 & 112.8 & 76.2 \\
\hline 233.3 & 111.7 & 106.8 & 99.5 & 125.2 & 126.2 & 128.6 & 129.7 & 126.1 & 125.8 & 115.7 & 111.7 & 76.6 \\
\hline
\end{tabular}

\begin{tabular}{|c|c|c|c|c|c|c|c|c|c|}
\hline Time & $25 \mathrm{~mm}$ & $75 \mathrm{~mm}$ & $125 \mathrm{~mm}$ & $250 \mathrm{~mm}$ & Time & $25 \mathrm{~mm}$ & $75 \mathrm{~mm}$ & $125 \mathrm{~mm}$ & $250 \mathrm{~mm}$ \\
\hline 0.0 & 0.0 & 0.0 & 0.0 & 0.0 & 0.0 & 0.0 & 0.0 & 0.0 & 0.0 \\
\hline 1.4 & 0.1 & 0.0 & -0.1 & 0.0 & 1.4 & -0.1 & 0.1 & 0.1 & -0.1 \\
\hline 8.4 & -0.1 & -0.2 & -0.1 & 0.0 & 8.4 & 0.1 & 0.2 & 0.1 & -0.1 \\
\hline 15.1 & -0.3 & -0.2 & -0.2 & 0.0 & 15.1 & 0.2 & 0.2 & 0.2 & -0.1 \\
\hline 22.0 & -0.3 & -0.3 & -0.2 & -0.2 & 22.0 & -0.1 & 0.2 & 0.3 & 0.2 \\
\hline 28.8 & -0.3 & -0.3 & -0.2 & -0.2 & 28.8 & 0.3 & 0.3 & 0.3 & 0.1 \\
\hline 35.5 & -0.1 & -0.2 & -0.1 & -0.2 & 35.5 & 0.4 & 0.3 & 0.4 & 0.2 \\
\hline 42.4 & -0.3 & -0.3 & -0.2 & -0.3 & 42.4 & 0.3 & 0.3 & 0.4 & 0.3 \\
\hline 49.1 & -0.3 & -0.4 & -0.4 & -0.4 & 49.1 & 0.3 & 0.3 & 0.3 & 0.3 \\
\hline 56.1 & -0.5 & -0.5 & -0.2 & -0.4 & 56.1 & 0.4 & 0.3 & 0.4 & 0.2 \\
\hline 62.8 & -0.4 & -0.2 & -0.3 & -0.2 & 52.8 & 0.3 & 0.3 & 0.3 & 0.1 \\
\hline 69.6 & -0.5 & -0.5 & -0.6 & -0.3 & 69.6 & 0.5 & 0.5 & 0.4 & 0.1 \\
\hline 76.5 & -0.6 & -0.5 & -0.4 & -0.3 & 76.5 & 0.6 & 0.6 & 0.5 & 0.1 \\
\hline 83.3 & -0.4 & -0.5 & -0.4 & -0.4 & 83.3 & 0.3 & 0.2 & 0.4 & 0.2 \\
\hline 90.0 & -0.4 & -0.5 & -0.3 & -0.3 & 90.0 & 0.4 & 0.4 & 0.6 & 0.4 \\
\hline 96.9 & -0.3 & -0.3 & -0.3 & -0.3 & 96.9 & 0.6 & 0.6 & 0.6 & 0.2 \\
\hline 103.7 & -0.5 & -0.6 & -0.4 & -0.3 & 103.7 & 0.5 & 0.4 & 0.6 & 0.4 \\
\hline 110.6 & -0.4 & -0.4 & -0.3 & -0.4 & 110.6 & 0.5 & 0.5 & 0.6 & 0.4 \\
\hline 117.3 & -0.7 & -0.7 & -0.5 & -0.3 & 117.3 & 0.6 & 0.5 & 0.6 & 0.4 \\
\hline 124.1 & -0.6 & .0 .6 & -0.6 & -0.5 & 124.1 & 0.7 & 0.6 & 0.6 & 0.4 \\
\hline 131.0 & -0.5 & -0.6 & -0.4 & -0.3 & 131.0 & 0.5 & 0.6 & 0.7 & 0.5 \\
\hline 137.8 & -0.4 & -0.5 & -0.4 & -0.4 & 137.8 & 0.5 & 0.6 & 0.7 & 0.4 \\
\hline 144.7 & -0.4 & -0.4 & -0.3 & -0.6 & 144.7 & 0.4 & 0.3 & 0.5 & 0.5 \\
\hline 151.5 & -0.5 & -0.6 & -0.5 & -0.4 & 151.5 & 0.5 & 0.5 & 0.7 & 0.5 \\
\hline 158.2 & -0.4 & 0.5 & -0.5 & -0.5 & 158.2 & 0.6 & 0.4 & 0.6 & 0.4 \\
\hline 165.1 & -0.4 & -0.5 & -0.4 & -0.4 & 165.1 & 0.5 & 0.6 & 0.8 & 0.6 \\
\hline 171.8 & -0.7 & -0.7 & -0.8 & -0.5 & 171.8 & 0.7 & 0.8 & 0.9 & 0.3 \\
\hline 178.7 & -0.7 & -0.7 & -0.5 & -0.5 & 178.7 & 0.5 & 0.4 & 0.7 & -0.2 \\
\hline 185.5 & -0.4 & -0.6 & -0.3 & -0.4 & 185.5 & 0.4 & 0.1 & 0.7 & 0.3 \\
\hline 192.3 & -0.6 & -0.6 & -0.5 & -0.5 & 192.3 & 0.9 & 0.7 & 0.8 & 0.3 \\
\hline 199.2 & -0.7 & -0.7 & -0.6 & -0.6 & 199.2 & 0.7 & 0.4 & 0.6 & 0.2 \\
\hline 205.9 & -0.6 & -0.7 & -0.9 & -0.5 & 205.9 & 0.7 & 0.8 & 1.0 & 0.6 \\
\hline 212.9 & -0.5 & -0.7 & -0.5 & -0.6 & 212.9 & 0.7 & 0.6 & 0.9 & 0.3 \\
\hline 219.6 & -0.7 & -0.8 & -0.7 & -0.6 & 219.6 & 0.6 & 0.6 & 0.8 & 0.4 \\
\hline 226.4 & -0.5 & -0.5 & -0.4 & -0.6 & 226.4 & 0.6 & 0.5 & 0.8 & 0.4 \\
\hline
\end{tabular}




\section{Experiment Number 30}

$\begin{array}{rrc}233.3 & -0.5 & -0.6 \\ \text { Velocity probes } & \text { in } & \text { channels above burner } \\ \text { Time } & \text { Channel } 1 & \text { Channel } 2 \\ 0.0 & 0.0 & 0.0 \\ 1.4 & 1.4 & 1.1 \\ 8.4 & 1.5 & 0.9 \\ 15.1 & 1.8 & 1.0 \\ 22.0 & 1.8 & 1.4 \\ 28.8 & 1.7 & 1.4 \\ 35.5 & 2.6 & 1.7 \\ 42.4 & 2.2 & 1.5 \\ 49.1 & 2.3 & 1.7 \\ 56.1 & 2.1 & 1.6 \\ 62.8 & 2.5 & 1.7 \\ 69.6 & 2.3 & 1.5 \\ 76.5 & 2.3 & 1.9 \\ 83.3 & 2.9 & 1.6 \\ 90.0 & 2.6 & 1.7 \\ 96.9 & 2.8 & 1.8 \\ 103.7 & 3.0 & 1.8 \\ 110.6 & 2.9 & 2.1 \\ 117.3 & 3.0 & 1.6 \\ 124.1 & 2.4 & 1.9 \\ 131.0 & 2.7 & 2.0 \\ 137.8 & 2.2 & 2.1 \\ 144.7 & 3.0 & 1.9 \\ 151.5 & 3.2 & 2.0 \\ 158.2 & 3.2 & 1.7 \\ 165.1 & 2.6 & 1.7 \\ 171.8 & 2.2 & 2.5 \\ 178.7 & 2.9 & 2.6 \\ 185.5 & 2.9 & 2.1 \\ 192.3 & 2.5 & 2.3 \\ 199.2 & 3.4 & 2.1 \\ 205.9 & 3.2 & 2.0 \\ 212.9 & 3.6 & 1.7 \\ 219.6 & 3.4 & 2.4 \\ 226.4 & 2.6 & 2.1 \\ 233.3 & 3.7 & 2.7\end{array}$


Experiment Number 31

Thermoccuples located at sprinkler number 1

$\begin{array}{rrrr}\text { Time } & 0 \mathrm{~mm} & 25 \mathrm{~mm} & 50 \mathrm{~mm} \\ 0.0 & 20.2 & 20.6 & 20.8 \\ 2.3 & 20.1 & 20.6 & 20.7 \\ 9.2 & 21.0 & 27.7 & 26.1 \\ 16.1 & 35.2 & 59.9 & 57.3 \\ 22.8 & 48.8 & 80.9 & 78.0 \\ 29.9 & 61.6 & 105.8 & 98.8 \\ 36.7 & 71.3 & 126.6 & 125.2\end{array}$

$\begin{array}{rrrrrrrrr}75 \mathrm{~mm} & 100 \mathrm{~mm} & 125 \mathrm{~mm} & 150 \mathrm{~mm} & 250 \mathrm{~mm} & 350 \mathrm{~mm} & 450 \mathrm{~mm} & 550 \mathrm{~mm} & 900 \mathrm{~mm} \\ 20.8 & 20.8 & 20.8 & 20.8 & 20.8 & 20.8 & 20.8 & 20.7 & 20.5 \\ 20.8 & 20.8 & 20.8 & 20.8 & 20.8 & 20.7 & 20.6 & 20.6 & 20.5 \\ 28.7 & 29.6 & 29.3 & 27.8 & 28.2 & 25.1 & 21.1 & 20.9 & 20.7 \\ 58.8 & 56.4 & 54.8 & 51.7 & 38.5 & 31.6 & 21.7 & 21.2 & 20.9 \\ 81.9 & 78.6 & 77.0 & 73.0 & 53.5 & 28.8 & 21.9 & 21.6 & 21.3 \\ 98.2 & 88.7 & 87.1 & 87.3 & 67.6 & 34.7 & 22.5 & 22.3 & 22.3 \\ 123.5 & 119.8 & 116.3 & 108.6 & 78.5 & 46.6 & 26.5 & 23.4 & 23.5\end{array}$

Thermocouples located at sprinkler number 2

Time $\quad 0 \mathrm{~mm} \quad 25 \mathrm{~mm} \quad 50 \mathrm{~mm}$

$00 \quad 200 \quad 207 \quad 207$

$\begin{array}{llll}2.3 & 19.9 & 20.4 & 20.4\end{array}$

$\begin{array}{llll}9.2 & 20.0 & 20.6 & 20.6\end{array}$

$\begin{array}{llll}16.1 & 20.1 & 20.7 & 20.7\end{array}$

$\begin{array}{llll}22.8 & 25.3 & 47.6 & 49.1\end{array}$

$\begin{array}{llll}29.9 & 30.2 & 60.5 & 63.3\end{array}$

$\begin{array}{llll}36.7 & 32.3 & 71.0 & 75.6\end{array}$

Thermocouples located at sprinkler number 3$$
\text { Time } 0 \mathrm{~mm} \quad 25 \mathrm{~mm}
$$

$\begin{array}{rr}75 \mathrm{~mm} & 100 \mathrm{~mm} \\ 20.9 & 20.8\end{array}$

$20.7 \quad 20.6$

$20.8 \quad 20.8$

$20.9 \quad 20.8$

$49.5 \quad 50.1$

65.7

74.4

66.9

20.9

$150 \mathrm{~mm} \quad 250 \mathrm{~mm}$

20.8

20.9

46.5

65.2

72.7

$75 \mathrm{~mm} \quad 100 \mathrm{~mm} \quad 125 \mathrm{~mm}$

20.8

20.6

20.7

20.7

46.9

65.5

20.8

20.6

20.7

20.7

35.4

$350 \mathrm{~mm}$

$\begin{array}{rrrr}20.8 & 20.8 & 20.8 & 20.8 \\ 20.6 & 20.7 & 20.7 & 20.7 \\ 20.7 & 20.7 & 20.8 & 20.7 \\ 20.7 & 20.7 & 20.7 & 20.8 \\ 26.3 & 24.1 & 22.9 & 20.8 \\ 46.3 & 31.7 & 26.1 & 24.9 \\ 53.8 & 45.4 & 44.6 & 23.7\end{array}$

Tmm $\quad 25 \mathrm{~mm} \quad 50 \mathrm{~mm}$

$20.7 \quad 20.7$

$20.6 \quad 20.6$

20.8

$150 \mathrm{~mm} \quad 250 \mathrm{~mm}$

$350 \mathrm{~mm}$

$450 \mathrm{~mm} \quad 550 \mathrm{~mm} \quad 900 \mathrm{~mm}$

$\begin{array}{llll}2.3 & 20.1 & 20.6 & 20.6\end{array}$

$\begin{array}{llll}9.2 & 20.3 & 20.7 & 20.6\end{array}$

$20.7 \quad 20.7$

$20.6 \quad 20.7$

$26.9 \quad 26.7$

26.9

$\begin{array}{llll}22.8 & 21.3 & 26.3 & 26.9\end{array}$

$\begin{array}{llll}29.9 & 23.4 & 35.5 & 38.8\end{array}$

38.4

38.5

20.8

20.8

20.8

20.7

$20.8 \quad 20.5$

20.7

$20.8 \quad 20.8$

$20.8 \quad 20.8$

$27.8 \quad 25.9$

$40.0 \quad 34.9$

20.8

20.8

20.7

$\begin{array}{lll}20.8 & 20.7 & 20.7\end{array}$

$20.8 \quad 20.7$

$24.4 \quad 22.5$

20.4

$\begin{array}{ccc}36.7 & 24.6 & 43.0 \\ & \end{array}$

Time $\quad 0 \mathrm{~mm} \quad 25 \mathrm{~mm} \quad 50 \mathrm{~mm}$

$\begin{array}{llll}0.0 & 20.3 & 20.9 & 21.0\end{array}$

$\begin{array}{llll}2.3 & 20.1 & 20.9 & 21.0\end{array}$

$\begin{array}{llll}9.2 & 20.3 & 21.0 & 21.0\end{array}$

$\begin{array}{llll}16.1 & 24.7 & 37.0 & 35.6\end{array}$

$\begin{array}{llll}22.8 & 32.1 & 51.6 & 49.6\end{array}$

$\begin{array}{llll}29.9 & 34.8 & 61.9 & 60.5\end{array}$

$\begin{array}{llll}36.7 & 40.8 & 73.5 & 73.1\end{array}$

Velocity probes at sprinkler number 1

$\begin{array}{rrrrr}\text { Time } & 25 \mathrm{~mm} & 75 \mathrm{~mm} & 125 \mathrm{~mm} & 250 \mathrm{~mm} \\ 0.0 & 0.0 & 0.0 & 0.0 & 0.0 \\ 2.3 & 0.1 & 0.1 & 0.1 & 0.1 \\ 9.2 & 1.2 & 1.2 & 0.8 & -0.2 \\ 16.1 & 1.7 & 1.5 & 1.3 & 0.5 \\ 22.8 & 1.9 & 1.8 & 1.3 & 0.7 \\ 29.9 & 2.2 & 2.0 & 1.8 & 0.7 \\ 36.7 & 2.8 & 2.4 & 1.7 & 0.5\end{array}$

$75 \mathrm{~mm}$
21.0
21.0
21.0
35.2
50.1
60.0
72.3

$250 \mathrm{~mm}$
0.0
0.1
-0.2
0.5
0.7
0.7
0.5

\begin{tabular}{|c|c|c|c|c|c|c|c|}
\hline $100 \mathrm{~mm}$ & $125 \mathrm{~mm}$ & $150 \mathrm{~mm}$ & $250 \mathrm{~mm}$ & $350 \mathrm{~mm}$ & $450 \mathrm{~mm}$ & $550 \mathrm{~mm}$ & $900 \mathrm{~mm}$ \\
\hline 20.8 & 20.9 & 20.9 & 20.7 & 20.8 & 20.7 & 20.8 & 20.3 \\
\hline 20.9 & 20.9 & 20.8 & 20.6 & 20.7 & 20.6 & 20.8 & 20.3 \\
\hline 20.8 & 20.9 & 20.9 & 20.7 & 20.8 & 20.8 & 20.8 & 20.4 \\
\hline 33.3 & 34.2 & 33.4 & 24.3 & 23.7 & 22.7 & 21.5 & 20.8 \\
\hline 44.6 & 45.9 & 44.8 & 28.7 & 25.6 & 23.7 & 22.8 & 21.2 \\
\hline 54.7 & 54.6 & 53.2 & 40.3 & 34.2 & 25.1 & 23.5 & 21.6 \\
\hline 66.1 & 65.9 & 61.4 & 47.0 & 46.1 & 35.8 & 23.3 & 22.0 \\
\hline \multicolumn{8}{|c|}{ Velocity probes at sprinkler number 2} \\
\hline & Time & $25 \mathrm{~mm}$ & $75 \mathrm{~mm}$ & $125 \mathrm{~mm}$ & $250 \mathrm{~mm}$ & & \\
\hline & 0.0 & 0.0 & 0.0 & 0.0 & 0.0 & & \\
\hline & 2.3 & 0.0 & 0.0 & 0.0 & 0.0 & & \\
\hline & 9.2 & 0.0 & 0.0 & 0.0 & 0.0 & & \\
\hline & 16.1 & 1.0 & 1.0 & 0.9 & 0.7 & & \\
\hline & 22.8 & 1.0 & 1.0 & 0.9 & 0.7 & & \\
\hline & 29.9 & 0.8 & 0.9 & 0.9 & 0.7 & & \\
\hline & 36.7 & 0.7 & 0.7 & 0.7 & 0.6 & & \\
\hline
\end{tabular}


Experiment Number 32

\begin{tabular}{|c|c|c|c|c|c|c|c|c|c|c|c|c|}
\hline \multicolumn{13}{|c|}{ Thermocouples localed at sprinkler number 1} \\
\hline Time & $0 \mathrm{~mm}$ & $25 \mathrm{~mm}$ & $50 \mathrm{~mm}$ & $75 \mathrm{~mm}$ & $100 \mathrm{~mm}$ & $125 \mathrm{~mm}$ & $150 \mathrm{~mm}$ & $250 \mathrm{~mm}$ & $350 \mathrm{~mm}$ & $450 \mathrm{~mm}$ & $550 \mathrm{~mm}$ & $900 \mathrm{~mm}$ \\
\hline 0.0 & 21.9 & 21.5 & 20.9 & 20.4 & 20.2 & 19.9 & 19.7 & 19.1 & 18.8 & 18.7 & 18.4 & 18.0 \\
\hline 5.2 & 22.0 & 21.8 & 21.0 & 20.6 & 20.3 & 20.2 & 20.1 & 19.4 & 18.9 & 18.8 & 18.5 & 18.1 \\
\hline 12.1 & 43.8 & 76.8 & 71.8 & 75.0 & 72.2 & 69.5 & 64.4 & 41.4 & 21.9 & 20.2 & 19.0 & 18.6 \\
\hline 18.8 & 54.1 & 88.3 & 85.8 & 84.9 & 80.8 & 79.2 & 74.6 & 51.3 & 24.2 & 24.4 & 19.7 & 19.3 \\
\hline 25.7 & 71.4 & 131.1 & 124.7 & 125.3 & 120.7 & 113.8 & 103.1 & 63.3 & 25.0 & 22.2 & 20.5 & 20.2 \\
\hline 32.5 & 63.8 & 96.9 & 74.0 & 63.3 & 62.6 & 107.9 & 81.8 & 69.4 & 28.4 & 23.3 & 21.7 & 22.4 \\
\hline \multicolumn{13}{|c|}{ Thermocouples located at sprinkler number 2} \\
\hline Time & $0 \mathrm{~mm}$ & $25 \mathrm{~mm}$ & $50 \mathrm{~mm}$ & $75 \mathrm{~mm}$ & $100 \mathrm{~mm}$ & $125 \mathrm{~mm}$ & $150 \mathrm{~mm}$ & $250 \mathrm{~mm}$ & $350 \mathrm{~mm}$ & $450 \mathrm{~mm}$ & $550 \mathrm{~mm}$ & $900 \mathrm{~mm}$ \\
\hline 0.0 & 20.6 & 20.2 & 20.2 & 19.7 & 19.8 & 19.5 & 19.6 & 18.9 & 18.7 & 18.5 & 18.3 & 17.6 \\
\hline 5.2 & 20.6 & 20.3 & 20.5 & 20.0 & 20.4 & 19.9 & 20.2 & 19.5 & 19.0 & 18.7 & 18.6 & 18.0 \\
\hline 12.1 & 20.7 & 20.3 & 20.5 & 20.0 & 20.5 & 19.8 & 20.3 & 19.7 & 19.1 & 18.9 & 18.6 & 18.0 \\
\hline 18.8 & 26.8 & 51.3 & 52.8 & 52.6 & 53.1 & 51.0 & 49.7 & 39.7 & 27.5 & 25.6 & 23.5 & 18.8 \\
\hline 25.7 & 31.6 & 67.2 & 71.0 & 69.6 & 71.0 & 69.0 & 70.0 & 62.9 & 45.8 & 36.0 & 33.1 & 22.7 \\
\hline 32.5 & 35.1 & 74.7 & 83.0 & 80.9 & 85.6 & 80.5 & B3.1 & 74.4 & 54.8 & 52.7 & 51.0 & 20.9 \\
\hline \multicolumn{13}{|c|}{ Thermocouples located at sprinkler number 3} \\
\hline Time & $0 \mathrm{~mm}$ & $25 \mathrm{~mm}$ & $50 \mathrm{~mm}$ & $75 \mathrm{~mm}$ & $100 \mathrm{~mm}$ & $125 \mathrm{~mm}$ & $150 \mathrm{~mm}$ & $250 \mathrm{~mm}$ & $350 \mathrm{~mm}$ & $450 \mathrm{~mm}$ & $550 \mathrm{~mm}$ & $900 \mathrm{rnm}$ \\
\hline 0.0 & 20.2 & 20.4 & 19.9 & 19.8 & 19.6 & 19.5 & 19.4 & 19.0 & 18.6 & 18.4 & 18.3 & 17.8 \\
\hline 5.2 & 20.3 & 20.5 & 20.1 & 20.1 & 20.1 & 20.1 & 20.1 & 19.8 & 19.5 & 19.1 & 18.9 & 18.1 \\
\hline 12.1 & 20.3 & 20.6 & 20.2 & 20.1 & 20.1 & 20.1 & 20.1 & 19.8 & 19.6 & 19.2 & 19.0 & 18.1 \\
\hline 18.8 & 21.6 & 29.3 & 30.5 & 29.3 & 29.5 & 29.7 & 30.7 & 27.1 & 24.3 & 22.3 & 20.1 & 18.6 \\
\hline 25.7 & 23.3 & 37.5 & 40.2 & 41.1 & 41.3 & 42.5 & 43.6 & 39.8 & 35.2 & 31.3 & 29.5 & 19.6 \\
\hline 32.5 & 25.7 & 46.9 & 516 & 51.9 & 50.1 & 50.1 & 51.5 & 44.6 & 42.4 & 39.0 & 38.7 & 25.4 \\
\hline \multicolumn{13}{|c|}{ Thermocouples located al sprinkler number 4} \\
\hline Time & $0 \mathrm{~mm}$ & $25 \mathrm{~mm}$ & $50 \mathrm{~mm}$ & $75 \mathrm{~mm}$ & $100 \mathrm{~mm}$ & $125 \mathrm{~mm}$ & $150 \mathrm{~mm}$ & $250 \mathrm{~mm}$ & $350 \mathrm{~mm}$ & $450 \mathrm{~mm}$ & $550 \mathrm{~mm}$ & $900 \mathrm{~mm}$ \\
\hline 0.0 & 20.3 & 20.2 & 20.0 & 19.9 & 19.6 & 19.5 & 19.4 & 19.0 & 18.5 & 18.3 & 18.1 & 17.9 \\
\hline 5.2 & 20.3 & 20.4 & 20.3 & 20.2 & 19.9 & 19.8 & 19.7 & 19.4 & 18.7 & 18.5 & 48.2 & 18.1 \\
\hline 12.1 & 28.3 & 42.9 & 40.8 & 39.4 & 33.0 & 32.8 & 30.7 & 24.6 & 24.1 & 21.0 & 19.3 & 18.6 \\
\hline 18.8 & 33.1 & 53.0 & 52.4 & 52.1 & 47.2 & 47.3 & 45.8 & 30.3 & 29.2 & 25.5 & 23.5 & 19.3 \\
\hline 25.7 & 36.4 & 61.4 & 60.3 & 59.7 & 55.2 & 54.8 & 52.4 & 39.0 & 39.4 & 25.9 & 21.1 & 20.0 \\
\hline 32.5 & 40.0 & 77.6 & 76.5 & 76.6 & 70.6 & 70.2 & 68.9 & 51.5 & 50.9 & 38.7 & 30.2 & 20.6 \\
\hline \multicolumn{6}{|c|}{ Velocity probes at sprinkler number 1} & \multicolumn{7}{|c|}{ Velocity probes at sprinkler number 2} \\
\hline Time & $25 \mathrm{~mm}$ & $75 \mathrm{~mm}$ & $125 \mathrm{~mm}$ & $250 \mathrm{~mm}$ & & Time & $25 \mathrm{~mm}$ & $75 \mathrm{~mm}$ & $125 \mathrm{~mm}$ & $250 \mathrm{~mm}$ & & \\
\hline 0.0 & 0.0 & 0.0 & 0.0 & 0.0 & & 0.0 & 0.0 & 0.0 & 0.0 & 0.0 & & \\
\hline 5.2 & 1.5 & 1.4 & 1.1 & 0.6 & & 5.2 & .0 .1 & -0.1 & 0.1 & 0.1 & & \\
\hline 12.1 & 1.6 & 1.6 & 1.2 & 0.4 & & 12.1 & 1.0 & 1.0 & 0.8 & 0.8 & & \\
\hline 18.8 & 2.4 & 2.3 & 2.1 & 0.4 & & 18.8 & 1.0 & 1.0 & 1.0 & 0.6 & & \\
\hline 25.7 & 2.4 & 2.1 & 1.4 & 0.5 & & 25.7 & 0.9 & 0.9 & 0.7 & 0.5 & & \\
\hline 32.5 & 2.2 & 1.9 & 1.8 & 0.9 & & 32.5 & 0.9 & 1.0 & 0.9 & 0.6 & & \\
\hline
\end{tabular}


Experiment Number 33

Thermocouples located at sprinkler number 1

\begin{tabular}{|c|c|c|c|c|c|c|c|c|c|c|c|c|}
\hline Time & $0 \mathrm{~mm}$ & $25 \mathrm{~mm}$ & $50 \mathrm{~mm}$ & $75 \mathrm{~mm}$ & $100 \mathrm{~mm}$ & $125 \mathrm{~mm}$ & $150 \mathrm{~mm}$ & $250 \mathrm{~mm}$ & $350 \mathrm{~mm}$ & $450 \mathrm{~mm}$ & $550 \mathrm{~mm}$ & $900 \mathrm{~mm}$ \\
\hline 0.0 & 21.7 & 21.6 & 21.0 & 20.7 & 20.3 & 20.1 & 19.8 & 19.3 & 19.0 & 18.7 & 18.6 & 18.2 \\
\hline 4.2 & 22.0 & 21.9 & 21.2 & 20.7 & 20.6 & 20.3 & 20.3 & 19.6 & 19.2 & 19.1 & 18.9 & 18.5 \\
\hline 11.1 & 40.3 & 74.0 & 67.0 & 72.4 & 70.8 & 67.8 & 62.7 & 37.1 & 20.0 & 19.6 & 19.4 & 19.0 \\
\hline 17.8 & 57.5 & 100.9 & 98.2 & 99.0 & 94.1 & 91.7 & 83.5 & 53.6 & 25.9 & 20.7 & 20.2 & 19.8 \\
\hline 24.8 & 64.8 & 116.2 & 110.8 & 112.8 & 105.4 & 101.8 & 93.9 & 56.9 & 26.3 & 21.7 & 20.7 & 20.7 \\
\hline 31.5 & 85.9 & 165.2 & 148.7 & 95.5 & 133.2 & 136.0 & 94.1 & 61.2 & 29.6 & 23.9 & 23.0 & 22.4 \\
\hline \multicolumn{13}{|c|}{ Thermocouples located at sprinkler number 2} \\
\hline Time & $0 \mathrm{~mm}$ & $25 \mathrm{~mm}$ & $50 \mathrm{~mm}$ & $75 \mathrm{~mm}$ & $100 \mathrm{~mm}$ & $125 \mathrm{~mm}$ & $150 \mathrm{~mm}$ & $250 \mathrm{~mm}$ & $350 \mathrm{~mm}$ & $450 \mathrm{~mm}$ & $550 \mathrm{~mm}$ & $900 \mathrm{~mm}$ \\
\hline 0.0 & 20.5 & 20.0 & 20.2 & 19.6 & 19.9 & 19.3 & 19.6 & 18.9 & 18.7 & $\uparrow 8.5$ & 18.3 & 18.1 \\
\hline 4.2 & 20.5 & 20.2 & 20.5 & 20.1 & 20.5 & 19.9 & 20.3 & 19.7 & 19.5 & 19.3 & 18.9 & 18.3 \\
\hline 11.1 & 20.5 & 20.3 & 20.6 & 20.3 & 20.5 & 20.1 & 20.3 & 19.6 & 19.4 & 19.3 & 19.0 & 18.5 \\
\hline 17.8 & 25.4 & 51.8 & 54.6 & 55.7 & 56.1 & 53.5 & 51.4 & 42.6 & 23.9 & 23.5 & 22.6 & 18.7 \\
\hline 24.8 & 29.8 & 60.6 & 66.4 & 65.8 & 68.3 & 65.9 & 68.0 & 63.2 & 47.2 & 35.1 & 34.3 & 25.4 \\
\hline 31.5 & 33.7 & 74.0 & 80.9 & 80.8 & 83.1 & 80.3 & 82.6 & 72.4 & 63.3 & 53.7 & 50.8 & 21.3 \\
\hline \multicolumn{13}{|c|}{ Thermocouples localed at sprinkler number 3} \\
\hline Time & $0 \mathrm{~mm}$ & $25 \mathrm{~mm}$ & $50 \mathrm{~mm}$ & $75 \mathrm{~mm}$ & $100 \mathrm{~mm}$ & $125 \mathrm{~mm}$ & $150 \mathrm{~mm}$ & $250 \mathrm{~mm}$ & $350 \mathrm{~mm}$ & $450 \mathrm{~mm}$ & $550 \mathrm{~mm}$ & $900 \mathrm{~mm}$ \\
\hline 0.0 & 20.1 & 20.3 & 19.7 & 19.5 & 19.3 & 19.2 & 19.1 & 18.8 & 18.7 & 18.6 & 18.6 & 18.3 \\
\hline 4.2 & 20.3 & 20.4 & 20.1 & 20.1 & 20.0 & 20.0 & 19.9 & 19.6 & 19.2 & 18.7 & 18.6 & 18.5 \\
\hline 11.1 & 20.3 & 20.5 & 20.2 & 20.1 & 20.1 & 20.1 & 20.1 & 19.9 & 19.6 & 18.8 & 18.6 & 18.5 \\
\hline 17.8 & 21.7 & 29.6 & 31.6 & 30.6 & 31.4 & 31.3 & 32.7 & 28.3 & 26.3 & 23.7 & 20.9 & 18.6 \\
\hline 24.8 & 23.6 & 39.3 & 42.0 & 42.1 & 42.0 & 42.7 & 44.1 & 38.7 & 36.8 & 32.3 & 30.8 & 20.8 \\
\hline 31.5 & 26.2 & 51.5 & 55.1 & 55.4 & 53.4 & 54.2 & 55.9 & 46.1 & 43.2 & 39.4 & 39.6 & 26.7 \\
\hline \multicolumn{13}{|c|}{ Thermocouples located at sprinkler number 4} \\
\hline Time & $0 \mathrm{~mm}$ & $25 \mathrm{~mm}$ & $50 \mathrm{~mm}$ & $75 \mathrm{~mm}$ & $100 \mathrm{~mm}$ & $125 \mathrm{~mm}$ & $150 \mathrm{~mm}$ & $250 \mathrm{~mm}$ & $350 \mathrm{~mm}$ & $450 \mathrm{~mm}$ & $550 \mathrm{~mm}$ & $900 \mathrm{~mm}$ \\
\hline 0.0 & 20.1 & 20.0 & 19.9 & 19.8 & 19.6 & 19.5 & 19.4 & 19.0 & 18.7 & 18.5 & 18.4 & 18.3 \\
\hline 4.2 & 20.4 & 20.6 & 20.5 & 20.5 & 20.2 & 20.2 & 20.2 & 19.6 & $19 . \uparrow$ & 18.7 & 18.5 & 18.4 \\
\hline 11.1 & 26.6 & 40.2 & 38.8 & 38.1 & 34.3 & 34.6 & 34.1 & 25.4 & 22.9 & 20.6 & 19.3 & 18.7 \\
\hline 17.8 & 32.0 & 54.4 & 53.4 & 53.4 & 48.9 & 49.1 & 47.0 & 31.5 & 29.6 & 25.9 & 23.7 & 19.4 \\
\hline 24.8 & 36.0 & 64.5 & 64.5 & 64.0 & 59.7 & 61.2 & 59.8 & 44.4 & 42.4 & 29.9 & 22.2 & 20.1 \\
\hline 31.5 & 42.4 & 83.2 & B2.4 & 80.3 & 74.8 & 76.0 & 74.6 & 57.3 & 53.5 & 42.7 & 31.5 & 21.0 \\
\hline \multicolumn{6}{|c|}{ Velocity probes at sprinkler number 1} & \multicolumn{7}{|c|}{ Velocity probes at sprinkler number 2} \\
\hline Time & $25 \mathrm{~mm}$ & $75 \mathrm{~mm}$ & $125 \mathrm{~mm}$ & $250 \mathrm{~mm}$ & & Time & $25 \mathrm{~mm}$ & $75 \mathrm{~mm}$ & $125 \mathrm{~mm}$ & $250 \mathrm{~mm}$ & & \\
\hline 0.0 & 0.0 & 0.0 & 0.0 & 0.0 & & 0.0 & 0.0 & 0.0 & 0.0 & 0.0 & & \\
\hline 4.2 & 1.6 & 1.3 & 1.0 & 0.2 & & 4.2 & -0.1 & 0.1 & -0.1 & 0.1 & & \\
\hline 11.1 & 2.0 & 1.7 & 1.5 & 0.4 & & 11.1 & 1.0 & 1.1 & 1.0 & 0.9 & & \\
\hline 17.8 & 2.1 & 2.2 & 2.0 & 0.4 & & 17.8 & 0.8 & 0.8 & 0.8 & 0.6 & & \\
\hline 24.8 & 2.3 & 2.2 & 1.0 & 0.2 & & 24.8 & 0.9 & 0.9 & 0.8 & 0.6 & & \\
\hline 31.5 & 2.1 & 2.1 & 1.6 & 1.3 & & 31.5 & 0.9 & 0.9 & 0.8 & 0.8 & & \\
\hline
\end{tabular}


Experiment Number 34

Thermocouples located at sprinkler number 1

\begin{tabular}{|c|c|c|c|c|c|c|c|c|c|c|c|c|}
\hline Time & $0 \mathrm{~mm}$ & $25 \mathrm{~mm}$ & $50 \mathrm{~mm}$ & $75 \mathrm{~mm}$ & $100 \mathrm{~mm}$ & $125 \mathrm{~mm}$ & $150 \mathrm{~mm}$ & $250 \mathrm{~mm}$ & $350 \mathrm{~mm}$ & $450 \mathrm{~mm}$ & $550 \mathrm{~mm}$ & $900 \mathrm{~mm}$ \\
\hline 0.0 & 19.2 & 19.3 & 19.4 & 19.5 & 19.4 & 19.3 & 19.4 & 19.3 & 19.0 & 18.7 & 18.2 & 16.3 \\
\hline 4.9 & 19.4 & 19.3 & 19.3 & 19.4 & 19.3 & 19.3 & 19.3 & 19.3 & 19.1 & 18.7 & 18.3 & 16.6 \\
\hline 11.7 & 20.0 & 20.0 & 19.9 & 19.8 & 20.2 & 10.9 & 19.0 & 19.8 & 20.1 & 19.6 & 19.3 & 17.2 \\
\hline 18.5 & 30.4 & 27.0 & 32.4 & 28.6 & 33.5 & 30.0 & 31.1 & 23.0 & 22.2 & 21.1 & 20.3 & 17.6 \\
\hline 25.4 & 52.3 & 49.4 & 57.1 & 51.7 & 58.2 & 55.8 & 56.5 & 37.2 & 29.6 & 24.1 & 21.3 & 18.9 \\
\hline 32.1 & 68.4 & 65.3 & 74.0 & 70.3 & 73.8 & 71.5 & 69.8 & 58.6 & 40.0 & 24.2 & 22.5 & 18.2 \\
\hline 39.0 & 78.7 & 75.1 & 82.5 & 74.7 & 87.9 & 87.5 & 90.6 & 84.0 & 60.5 & 25.8 & 23.5 & 18.5 \\
\hline 45.8 & 74.0 & 74.7 & 75.8 & 66.4 & 79.2 & 30.3 & 85.5 & 83.5 & 18.6 & 63.2 & $2 \pi . t$ & 19.2 \\
\hline 52.6 & 87.4 & 89.5 & 104.8 & 89.8 & 107.9 & 105.0 & 113.3 & 103.6 & 94.1 & 65.1 & 28.7 & 20.9 \\
\hline 59.5 & 109.3 & 103.5 & 116.8 & 102.8 & 116.5 & 114.3 & 117.8 & 106.6 & 100.7 & 75.7 & 45.4 & 23.9 \\
\hline 66.2 & 126.2 & 126.2 & 140.4 & 131.9 & 145.3 & 138.6 & 144.6 & 143.4 & 141.9 & 100.6 & 51.8 & 25.8 \\
\hline 73.1 & 170.1 & 162.5 & 179.2 & 166.7 & 183.2 & 180.9 & 186.8 & 167.5 & 140.6 & 93.1 & 62.0 & 28.4 \\
\hline Thermocouple & cated at & inkler num & & & & & & & & & & \\
\hline Time & $0 \mathrm{~mm}$ & $25 \mathrm{~mm}$ & $50 \mathrm{~mm}$ & $75 \mathrm{~mm}$ & $100 \mathrm{~mm}$ & $125 \mathrm{~mm}$ & $150 \mathrm{~mm}$ & $250 \mathrm{~mm}$ & $350 \mathrm{~mm}$ & $450 \mathrm{~mm}$ & $550 \mathrm{~mm}$ & $900 \mathrm{~mm}$ \\
\hline 0.0 & 19.3 & 19.4 & 19.4 & 19.6 & 19.5 & 19.7 & 19.4 & 19.5 & 10.3 & 19.0 & 18.5 & 16.2 \\
\hline 4.9 & 19.1 & 19.4 & 19.5 & 19.6 & 19.5 & 19.6 & 19.4 & 19.5 & 19.1 & 18.7 & 18.3 & 16.3 \\
\hline 11.7 & 19.3 & 19.5 & 19.5 & 19.6 & 19.5 & 19.6 & 19.5 & 19.5 & 19.1 & 18.7 & 18.3 & 16.5 \\
\hline 18.5 & 19.4 & 19.6 & 19.6 & 19.6 & 19.6 & 19.7 & 19.6 & 19.5 & 19.1 & 18.6 & 18.2 & 16.6 \\
\hline 25.4 & 19.7 & 20.1 & 20.9 & 21.0 & 21.4 & 20.5 & 20,9 & 19.8 & 19.9 & 19.9 & 19.2 & 17.8 \\
\hline 32.1 & 26.0 & 27.8 & 29.5 & 29.8 & 31.6 & 26.1 & 31.1 & 24.5 & 27.0 & 25.5 & 23.0 & 19.2 \\
\hline 39.0 & 41.0 & 38.2 & 45.2 & 40.3 & 45.5 & 34.5 & 43.8 & 29.5 & 30.7 & 24.5 & 22.3 & 19.6 \\
\hline $45 B$ & 53.6 & 51.1 & 57.9 & 51.1 & 59.3 & 40.3 & 57.8 & 38.3 & 42.1 & 31.9 & 29.4 & 23.3 \\
\hline 52.6 & 58.8 & 51.3 & 62.6 & 53.1 & 65.1 & 43.8 & 64.5 & 43.5 & 54.1 & 44.7 & 36.2 & 25.5 \\
\hline 59.5 & 69.5 & 62.0 & 73.2 & 65.1 & 75.9 & 55.1 & 74.2 & 49.0 & 59.3 & 47.9 & 43.1 & 27.3 \\
\hline 66.2 & 79.4 & 72.4 & 83.8 & 77.7 & 84.7 & 63.8 & 82.3 & 55.2 & 63.8 & 55.0 & 49.5 & 38.4 \\
\hline 73.1 & 89.4 & 88.6 & 97.9 & 95.2 & 101.4 & 74.4 & 100.3 & 63.1 & 78.9 & 64.7 & 57.1 & 44.7 \\
\hline Thermocoupl & scated at & inkler num & & & & & & & & & & \\
\hline Time & $0 \mathrm{~mm}$ & $25 \mathrm{~mm}$ & $50 \mathrm{~mm}$ & $75 \mathrm{~mm}$ & $100 \mathrm{~mm}$ & $125 \mathrm{~mm}$ & $150 \mathrm{~mm}$ & $250 \mathrm{~mm}$ & $350 \mathrm{~mm}$ & $450 \mathrm{~mm}$ & $550 \mathrm{~mm}$ & $900 \mathrm{~mm}$ \\
\hline 0.0 & 19.9 & 20.0 & 19.7 & 19.5 & 19.4 & 19.5 & 19.4 & 19.3 & 19.2 & 18.9 & 18.6 & 16.4 \\
\hline 4.9 & 19.8 & 20.0 & 19.7 & 19.4 & 19.4 & 19.4 & 19.4 & 19.3 & 19.1 & 18.8 & 18.4 & 16.7 \\
\hline 11.7 & 19.9 & 19.9 & 19.6 & 19.4 & 19.3 & 19.4 & 19.4 & 19.3 & 19.1 & 18.8 & 18.3 & 16.8 \\
\hline 18.5 & 19.6 & 19.7 & 19.6 & 19.5 & 19.4 & 19.4 & 19.4 & 19.3 & 19.2 & 18.9 & 18.4 & 16.9 \\
\hline 25.4 & 23.4 & 24.5 & 25.3 & 23.9 & 24.4 & 24.6 & 26.4 & 23.1 & 20.9 & 20.7 & 21.1 & 18.4 \\
\hline 32.1 & 36.9 & 40.3 & 41.5 & 38.0 & 38.6 & 38.8 & 41.7 & 31.5 & 25.0 & 22.2 & 21.2 & 18.4 \\
\hline 39.0 & 50.9 & 56.1 & 56.9 & 52.1 & 52.4 & 51.0 & 51.5 & 42.6 & 32.0 & 24.2 & 22.4 & 19.3 \\
\hline 45.8 & 66.0 & 69.6 & 70.9 & 64.8 & 64.7 & 63.0 & 63.8 & 49.8 & 40.8 & 34.5 & 31.1 & 23.5 \\
\hline 52.6 & 71.8 & 75.4 & 78.6 & 73.4 & 71.5 & 70.1 & 66.5 & 55.4 & 45.9 & 40.3 & 36.9 & 24.4 \\
\hline 59.5 & 78.9 & 84.8 & 88.4 & 82.8 & 83.0 & 81.3 & 82.2 & 64.1 & 52.5 & 46.5 & 42.5 & 27.0 \\
\hline 66.2 & 86.0 & 93.2 & 97.6 & 92.3 & 92.2 & 91.4 & 91.0 & 77.7 & 67.2 & 56.3 & 51.3 & 37.6 \\
\hline 73.1 & 98.8 & 107.8 & 109.8 & 103.5 & 104.6 & 104.1 & 105.4 & 91.6 & 76.5 & 65.2 & 59.4 & 47.8 \\
\hline Thermocoupl & ocated at & inkler num & & & & & & & & & & \\
\hline Time & $0 \mathrm{~mm}$ & $25 \mathrm{~mm}$ & $50 \mathrm{~mm}$ & $75 \mathrm{~mm}$ & $100 \mathrm{~mm}$ & $125 \mathrm{~mm}$ & $150 \mathrm{~mm}$ & $250 \mathrm{~mm}$ & $350 \mathrm{~mm}$ & $450 \mathrm{~mm}$ & $550 \mathrm{~mm}$ & $900 \mathrm{~mm}$ \\
\hline 0.0 & 19.5 & 19.5 & 19.6 & 19.6 & 19.4 & 19.4 & 19.4 & 19.2 & 19.0 & 18.4 & 18.0 & 16.4 \\
\hline 4.9 & 19.2 & 19.4 & 19.5 & 19.5 & 19.3 & 19.4 & 19.4 & 19.3 & 19.2 & 18.6 & 18.4 & 16.6 \\
\hline 11.7 & 19.8 & 19.9 & 19.8 & 19.9 & 19.7 & 20.4 & 21.0 & 21.7 & 21.1 & 20.4 & 19.9 & 18.0 \\
\hline 18.5 & 48.3 & 50.5 & 50.7 & 48.4 & 46.8 & 45.7 & 43.9 & 33.7 & 27.2 & 22.7 & 21.3 & 18.2 \\
\hline 25.4 & 61.2 & 60.4 & 62.6 & 60.6 & 61.9 & 63.0 & 63.6 & 52.9 & 46.1 & 24.1 & 20.4 & 17.4 \\
\hline 32.1 & 73.2 & 73.4 & 76.8 & 75.4 & 77.6 & 77.0 & 77.1 & 70.3 & 62.3 & 26.1 & 20.2 & 17.9 \\
\hline 39.0 & 87.3 & 87.6 & 92.4 & 91.8 & 94.7 & 94.1 & 94.9 & 81.0 & 65.0 & 27.7 & 20.5 & 19.4 \\
\hline 45.8 & 104.3 & 103.3 & 104.1 & 101.5 & 100.6 & 100.5 & 100.7 & 97.2 & 82.9 & 35.3 & 22.7 & 20.7 \\
\hline 52.6 & 109.8 & 110.3 & 112.8 & 110.4 & 108.5 & 110.6 & 109.0 & 103.6 & 93.1 & 63.6 & 35.1 & 23.4 \\
\hline 59.5 & 119.9 & 122.5 & 125.0 & 123.6 & 123.9 & 122.4 & 123.0 & 116.5 & 104.1 & 73.1 & 46.8 & 26.4 \\
\hline 66.2 & 138.5 & 142.7 & 149.4 & 144.1 & 149.7 & 148.2 & 146.5 & 133.1 & 125.3 & 85.3 & 52.0 & 29.7 \\
\hline 73.1 & 130.5 & 139.8 & 150.2 & 149.2 & 151.3 & 150.7 & 152.5 & 143.9 & 148.4 & 116.2 & 73.8 & 37.2 \\
\hline Velocity prob & i sprinkle & Imber 1 & & & & elocily prob & at sprinkle & umber 2 & & & & \\
\hline Time & $25 \mathrm{~mm}$ & $75 \mathrm{~mm}$ & $125 \mathrm{mrn}$ & 250 IIIIII & & Time & $25 \mathrm{~mm}$ & $75 \mathrm{~mm}$ & $125 \mathrm{~mm}$ & $250 \mathrm{~mm}$ & & \\
\hline 0.0 & 0.0 & 0.0 & 0.0 & 0.0 & & 0.0 & 0.0 & 0.0 & 0.0 & 0.0 & & \\
\hline 4.9 & 0.1 & 0.0 & 0.0 & 0.0 & & 4.9 & 0.0 & 0.1 & 0.1 & 0.1 & & \\
\hline 11.7 & 0.3 & 0.4 & 0.4 & 0.3 & & 11.7 & 0.0 & 0.1 & 0.1 & 0.1 & & \\
\hline 18.5 & 0.5 & 0.6 & 0.7 & 0.5 & & 18.5 & 0.1 & -0.1 & 0.1 & 0.1 & & \\
\hline 25.4 & 0.3 & 0.5 & 0.7 & 0.6 & & 25.4 & 0.3 & 0.4 & 0.5 & 0.6 & & \\
\hline
\end{tabular}




\section{Experiment Number 34}

$\begin{array}{llllllllll}32.1 & 0.5 & 0.7 & 0.5 & 0.4 & 32.1 & 0.5 & 0.7 & 0.6 & 0.4 \\ 39.0 & 0.2 & 0.3 & 0.5 & 0.5 & 39.0 & 0.6 & 0.6 & 0.5 & 0.4 \\ 45.8 & 0.5 & 0.7 & 0.8 & 0.4 & 45.8 & 0.5 & 0.6 & 0.6 & 0.4 \\ 52.6 & 0.5 & 0.6 & 0.7 & 0.6 & 52.6 & 0.6 & 0.6 & 0.5 & 0.4 \\ 59.5 & 0.6 & 0.6 & 0.8 & 0.6 & 59.5 & 0.6 & 0.7 & 0.7 & 0.5 \\ 66.2 & 0.6 & 0.6 & 0.8 & 0.7 & 66.2 & 0.8 & 0.8 & 0.8 & 0.6 \\ 73.1 & 0.8 & 0.8 & 1.0 & 1.0 & 73.1 & 0.9 & 0.8 & 0.7 & 0.5\end{array}$

Velocity probes in channels above burner

Time Channel 1 Channel 2

$\begin{array}{lll}0.0 & 0.0 & 0.0\end{array}$

$\begin{array}{lll}4.9 & 2.6 & 1.7\end{array}$

$11.7 \quad 3 . \quad 1.3$

$\begin{array}{lll}18.5 & 3.4 & 2.1\end{array}$

$\begin{array}{lll}25.4 & 4.5 & 2.4\end{array}$

$\begin{array}{lll}32.1 & 4.6 & 2.4\end{array}$

$\begin{array}{lll}39.0 & 4.5 & 3.1\end{array}$

$\begin{array}{lll}45.8 & 4.9 & 2.9\end{array}$

$\begin{array}{lll}52.6 & 4.3 & 2.9\end{array}$

$59.5 \quad 5.0 \quad 3.0$

$\begin{array}{lll}66.2 & 4.6 & 4.6\end{array}$

$\begin{array}{lll}73.1 & 5.7 & 4.6\end{array}$ 
Experiment Number 35

Thermocouples located at sprinkler number 1

\begin{tabular}{|c|c|c|c|c|c|c|c|c|c|c|c|c|}
\hline Time & $0 \mathrm{~mm}$ & $25 \mathrm{~mm}$ & $50 \mathrm{~mm}$ & $75 \mathrm{~mm}$ & $100 \mathrm{~mm}$ & $125 \mathrm{~mm}$ & $150 \mathrm{~mm}$ & $250 \mathrm{~mm}$ & $350 \mathrm{~mm}$ & $450 \mathrm{~mm}$ & $550 \mathrm{~mm}$ & $900 \mathrm{~mm}$ \\
\hline 0.0 & 19.9 & 19.9 & 20.0 & 20.0 & 20.0 & 19.9 & 20.0 & 20.0 & 20.0 & 20.0 & 20.0 & 19.9 \\
\hline 1.1 & 19.9 & 20.0 & 20.1 & 20.0 & 20.1 & 20.0 & 20.1 & 20.1 & 20.0 & 20.0 & 19.9 & 20.0 \\
\hline 8.0 & 20.2 & 20.3 & 20.3 & 20.2 & 20.3 & 20.2 & 20.3 & 20.2 & 20.2 & 20.2 & 20.2 & 20.2 \\
\hline 14.8 & 26.4 & 26.2 & 27.3 & 24.8 & 26.9 & 25.0 & 25.5 & 24.1 & 23.2 & 21.0 & 21.0 & 20.7 \\
\hline 21.5 & 46.7 & 48.1 & 52.2 & 45.0 & 53.5 & 48.4 & 50.0 & 42.1 & 35.9 & 23.0 & 22.4 & 21.3 \\
\hline 28.5 & 59.6 & 62.0 & 66.7 & 58.0 & 67.6 & 65.9 & 68.6 & 66.1 & 51.9 & 26.3 & 24.6 & 22.2 \\
\hline 35.2 & 74.3 & 75.7 & 82.8 & 72.2 & 84.1 & 81.9 & 86.7 & 82.2 & 70.8 & 29.2 & 26.7 & 23.0 \\
\hline 42.1 & 99.0 & 98.7 & 104.7 & 95.6 & 107.3 & 102.9 & 106.3 & 96.6 & 77.8 & 38.7 & 28.4 & 24.2 \\
\hline 48.9 & 118.0 & 118.5 & 123.5 & 111.7 & 126.2 & 127.4 & 131.6 & 118.6 & 104.9 & 58.3 & 29.5 & 24.7 \\
\hline 55.6 & 123.2 & 125.7 & 133.3 & 117.7 & 133.4 & 125.6 & 133.2 & 106.7 & 117.0 & 81.9 & 48.0 & 27.8 \\
\hline 62.5 & 115.7 & 122.8 & 127.9 & 116.6 & 134.5 & 129.9 & 141.1 & 121.2 & 123.9 & 92.8 & 54.5 & 28.0 \\
\hline 69.3 & 152.7 & 150.2 & 159.8 & 154.0 & 166.5 & 162.0 & 167.5 & 145.6 & 143.4 & 115.8 & 82.4 & 33.6 \\
\hline \multicolumn{13}{|c|}{ Thermocouples located at sprinkler number 2} \\
\hline Time & $0 \mathrm{~mm}$ & $25 \mathrm{~mm}$ & $50 \mathrm{~mm}$ & $75 \mathrm{~mm}$ & $100 \mathrm{~mm}$ & $125 \mathrm{~mm}$ & $150 \mathrm{~mm}$ & $250 \mathrm{~mm}$ & $350 \mathrm{~mm}$ & $450 \mathrm{~mm}$ & $550 \mathrm{~mm}$ & $900 \mathrm{~mm}$ \\
\hline 0.0 & 19.8 & 19.9 & 20.0 & 20.1 & 20.1 & 20.3 & 20.1 & 20.2 & 20.1 & 20.1 & 20.1 & 20.1 \\
\hline 1.1 & 19.7 & 19.8 & 19.9 & 20.0 & 20.0 & 20.1 & 20.0 & 20.3 & 20.0 & 20.0 & 20.1 & 20.0 \\
\hline 8.0 & 19.9 & 19.9 & 19.8 & 20.0 & 20.0 & 20.6 & 20.1 & 20.0 & 20.1 & 20.0 & 20.0 & 20.1 \\
\hline 14.8 & 19.8 & 19.9 & 20.0 & 20.1 & 20.1 & 20.1 & 20.1 & 20.3 & 20.1 & 20.1 & 20.1 & 20.1 \\
\hline 21.5 & 20.2 & 20.2 & 20.2 & 20.2 & 20.2 & 20.5 & 20.2 & 20.1 & 20.2 & 20.3 & 20.2 & 20.2 \\
\hline 28.5 & 26.6 & 28.6 & 31.2 & 30.7 & 33.3 & 27.3 & 32.2 & 24.4 & 27.4 & 27.1 & 26.5 & 20.3 \\
\hline 35.2 & 33.9 & 33.2 & 37.7 & 37.3 & 41.2 & 33.8 & 42.1 & 32.9 & 32.0 & 27.0 & 26.4 & 22.6 \\
\hline 42.1 & 47.8 & 434 & 51.2 & 46.2 & 55.0 & 39.4 & 54.7 & 40.8 & 45.3 & 38.5 & 28.3 & 24.8 \\
\hline 48.9 & 62.1 & 59.0 & 65.5 & 61.6 & 67.9 & 49.7 & 64.6 & 43.6 & 50.0 & 42.1 & 36.3 & 29.5 \\
\hline 55.6 & 68.6 & 62.2 & 73.6 & 66.0 & 77.1 & 53.9 & 74.2 & 50.2 & 57.1 & 51.5 & 45.3 & 32.4 \\
\hline 62.5 & 73.7 & 67.6 & 79.0 & 71.0 & BO. 9 & 56.5 & 79.9 & 56.4 & 73.3 & 66.2 & 57.1 & 38.6 \\
\hline 69.3 & 82.8 & 74.7 & 84.8 & 78.8 & B9.5 & 66.6 & 88.4 & 66.4 & 87.0 & 86.0 & 71.8 & 38.8 \\
\hline \multicolumn{13}{|c|}{ Thermocouples located at sprinkler number 3} \\
\hline Time & $0 \mathrm{~mm}$ & $25 \mathrm{~mm}$ & $50 \mathrm{~mm}$ & $75 \mathrm{~mm}$ & $100 \mathrm{~mm}$ & $125 \mathrm{~mm}$ & $150 \mathrm{~mm}$ & $250 \mathrm{~mm}$ & $350 \mathrm{~mm}$ & $450 \mathrm{~mm}$ & $550 \mathrm{~mm}$ & $900 \mathrm{~mm}$ \\
\hline 0.0 & 20.0 & 20.2 & 20.1 & 20.2 & 20.2 & 20.2 & 20.2 & 20.1 & 20.1 & 20.0 & 20.1 & 19.9 \\
\hline 1.1 & 19.9 & 20.0 & 20.0 & 20.1 & 20.1 & 20.2 & 20.2 & 20.1 & 20.0 & 19.9 & 20.0 & 19.9 \\
\hline 8.0 & 20.1 & 20.2 & 20.1 & 20.1 & 20.1 & 20.1 & 20.2 & 20.2 & 20.1 & 20.0 & 20.0 & 19.9 \\
\hline 14.8 & 20.0 & 20.1 & 20.0 & 20.1 & 20.1 & 20.1 & 20.1 & 20.1 & 20.1 & 20.1 & 20.1 & 20.0 \\
\hline 21.5 & 21.9 & 22.7 & 22.7 & 22.2 & 22.3 & 22.6 & 23.2 & 22.2 & 22.2 & 21.0 & 20.7 & 20.1 \\
\hline 28.5 & 370 & 40.7 & 41.8 & 36.8 & 35.9 & 35.9 & 36.4 & 27.1 & 24.7 & 23.4 & 22.3 & 20.3 \\
\hline 35.2 & 55.9 & 60.0 & 61.6 & 55.0 & 55.3 & 54.0 & 55.6 & 41.7 & 34.1 & 25.5 & 23.4 & 20.5 \\
\hline 42.1 & 65.4 & 68.8 & 70.2 & 66.1 & 66.2 & 66.1 & 65.9 & 54.6 & 46.9 & 36.7 & 33.6 & 25.7 \\
\hline 48.9 & 75.0 & 80.1 & 81.9 & 77.0 & 77.2 & 75.9 & 75.8 & 62.4 & 55.7 & 44.0 & 40.3 & 28.1 \\
\hline 55.6 & 84.4 & 90.2 & 92.1 & 87.8 & 88.3 & 87.2 & 88.3 & 70.4 & 58.9 & 48.6 & 46.2 & 32.9 \\
\hline 62.5 & 90.3 & 96.1 & 100.1 & 95.6 & 96.6 & 96.6 & 96.8 & 80.7 & 67.9 & 58.8 & 52.8 & 37.3 \\
\hline 69.3 & 95.0 & 102.1 & 106.3 & 102.8 & 103.4 & 103.6 & 102.6 & 88.9 & 77.6 & 69.2 & 64.9 & 44.9 \\
\hline \multicolumn{13}{|c|}{ Thermocouples located at sprinkler number 4} \\
\hline Time & $0 \mathrm{~mm}$ & $25 \mathrm{~mm}$ & $50 \mathrm{~mm}$ & $75 \mathrm{~mm}$ & $100 \mathrm{~mm}$ & $125 \mathrm{~mm}$ & $150 \mathrm{~mm}$ & $250 \mathrm{~mm}$ & $350 \mathrm{~mm}$ & $450 \mathrm{~mm}$ & $550 \mathrm{~mm}$ & $900 \mathrm{~mm}$ \\
\hline 0.0 & 20.1 & 20.2 & 20.2 & 20.2 & 20.1 & 20.1 & 20.1 & 20.0 & 20.0 & 19.9 & 20.0 & 19.7 \\
\hline 1.1 & 20.0 & 20.1 & 20.1 & 20.1 & 20.0 & 20.0 & 20.1 & 20.0 & 20.1 & 19.9 & 19.9 & 19.8 \\
\hline 8.0 & 20.2 & 20.3 & 20.3 & 20.3 & 20.1 & 20.1 & 20.0 & 20.1 & 20.1 & 20.0 & 20.1 & 20.0 \\
\hline 148 & 36.9 & 38.9 & 40.5 & 38.6 & 38.0 & 41.6 & 37.9 & 28.4 & 30.9 & 29.1 & 26.2 & 22.4 \\
\hline 21.5 & 58.1 & 60.4 & 63.9 & 61.8 & 60.7 & 66.7 & 67.2 & 46.8 & 31.7 & 22.6 & 21.6 & 21.4 \\
\hline 28.5 & 78.4 & 79.2 & 84.8 & 81.2 & 81.3 & 87.1 & 88.1 & 67.0 & 48.7 & 25.4 & 21.2 & 21.6 \\
\hline 35.2 & 89.4 & 93.7 & 98.1 & 96.3 & 96.2 & 102.8 & 102.8 & 85.5 & 68.3 & 26.3 & 21.5 & 22.2 \\
\hline 42.1 & 107.2 & 109.6 & 115.2 & 111.2 & 109.8 & 111.7 & 109.4 & 99.2 & 82.0 & 34.3 & 21.9 & 22.9 \\
\hline 48.9 & 112.0 & 115.7 & 118.1 & 117,3 & 117.0 & 122.1 & 123.7 & 111.7 & 106.2 & 70.0 & 47.5 & 25.4 \\
\hline 55.6 & 131.0 & 132.6 & 136.6 & 130.8 & 129.5 & 131.9 & 129.5 & 121.0 & 105.1 & 69.0 & 45.7 & 28.6 \\
\hline 62.5 & 139.4 & 143.7 & 148.2 & 144.1 & 141.0 & 147.4 & 144.3 & 129.9 & 124.0 & 92.4 & 61.4 & 32.2 \\
\hline 69.3 & 156.2 & 151.5 & 50.9 & 121.6 & 146.6 & 167.8 & 166.1 & 139.1 & 143.2 & 110.2 & 69.2 & 38.3 \\
\hline \multicolumn{6}{|c|}{ Velocity probes at sprinkler number 1} & \multicolumn{7}{|c|}{ Velocity probes at sprinkler number 2} \\
\hline Time & $25 \mathrm{~mm}$ & $75 \mathrm{~mm}$ & $125 \mathrm{~mm}$ & $250 \mathrm{~mm}$ & & Time & $25 \mathrm{~mm}$ & $75 \mathrm{ImH}$ & $125 \mathrm{~mm}$ & $250 \mathrm{~mm}$ & & \\
\hline 0.0 & 0.0 & 0.0 & 0.0 & 0.0 & & 0.0 & 0.0 & 0.0 & 0.0 & 0.0 & & \\
\hline 1.1 & 0.0 & 0.0 & -0.3 & -0.4 & & 1.1 & 0.1 & 0.1 & 0.0 & 0.1 & & \\
\hline 8.0 & 0.3 & 0.2 & 0.2 & 0.2 & & 8.0 & 0.1 & 0.1 & 0.0 & 0.1 & & \\
\hline 14.8 & 0.5 & 0.5 & 0.5 & 0.5 & & 14.8 & 0.1 & 0.1 & 0.0 & 0.1 & & \\
\hline 21.5 & 0.4 & 0.5 & 0.5 & 0.4 & & 21.5 & 0.6 & 0.6 & 0.6 & 0.5 & & \\
\hline
\end{tabular}


Experiment Number 35

$\begin{array}{lllll}28.5 & 0.4 & 0.5 & 0.6 & 0.7 \\ 35.2 & 0.5 & 0.4 & 0.7 & 0.6 \\ 42.1 & 0.5 & 0.4 & 0.5 & -0.3 \\ 48.9 & 0.4 & 0.4 & 0.5 & 0.4 \\ 55.6 & 0.4 & 0.5 & 0.8 & 0.5 \\ 62.5 & 0.7 & 0.7 & 1.1 & 0.8 \\ 69.3 & 0.7 & 0.6 & 0.9 & 0.7\end{array}$

Velocity probes in channels above bumer

Time Channel $\uparrow$ Channel 2

$\begin{array}{lll}0.0 & 0.0 & 0.0\end{array}$

$\begin{array}{lll}1.1 & 1.6 & 1.6\end{array}$

$\begin{array}{lll}8.0 & 3.3 & 1.5\end{array}$

$\begin{array}{lll}14.8 & 3.7 & 1.8\end{array}$

$\begin{array}{lll}21.5 & 3.9 & 1.9\end{array}$

$\begin{array}{lll}28.5 & 4.7 & 2.8\end{array}$

$35.2 \quad 5.6 \quad 3.2$

$\begin{array}{lll}42.1 & 4.5 & 3.3\end{array}$

$\begin{array}{lll}48.9 & 4.9 & 3.5\end{array}$

$\begin{array}{lll}55.6 & 5.7 & 3.6\end{array}$

$\begin{array}{lll}62.5 & 5.7 & 4.7\end{array}$

$\begin{array}{lll}69.3 & 7.0 \quad 5.9\end{array}$ 
Experiment Number 36

Thermocouples located al sprinkler number 1

\begin{tabular}{|c|c|c|c|c|c|c|c|c|c|c|c|c|}
\hline Time & $0 \mathrm{~mm}$ & $25 \mathrm{~mm}$ & $50 \mathrm{~mm}$ & $75 \mathrm{~mm}$ & $100 \mathrm{~mm}$ & $125 \mathrm{~mm}$ & $150 \mathrm{~mm}$ & $250 \mathrm{~mm}$ & $350 \mathrm{~mm}$ & $450 \mathrm{~mm}$ & $550 \mathrm{~mm}$ & $900 \mathrm{~mm}$ \\
\hline 0.0 & 22.3 & 22.2 & 21.7 & 21.4 & 21.2 & 20.9 & 20.8 & 20.0 & 19.7 & 19.3 & 18.9 & 18.0 \\
\hline 6.5 & 22.3 & 22.3 & 21.7 & 21.5 & 21.4 & 21.3 & 21.1 & 20.5 & 20.2 & 19.9 & 19.4 & 18.3 \\
\hline$\uparrow 3.5$ & 29.2 & 41.1 & 39.8 & 42.0 & 42.7 & 40.8 & 39.1 & 28.6 & 20.8 & 20.3 & 19.7 & 18.5 \\
\hline 20.3 & 41.8 & 71.4 & 65.2 & 67.9 & 64.7 & 64.4 & 61.5 & 36.6 & 21.7 & 20.0 & 19.5 & 18.7 \\
\hline 27.1 & 48.2 & 80.8 & 79.6 & 80.0 & 77.7 & 75.7 & 72.2 & 49.1 & 21.1 & 20.2 & 19.4 & 18.9 \\
\hline 34.0 & 53.2 & 88.1 & 86.2 & 87.1 & 82.7 & B1.0 & 77.8 & 57.6 & 33.1 & 21.3 & 20.0 & 19.6 \\
\hline 40.8 & 60.6 & 103.6 & 97.3 & 99.8 & 95.0 & 93.0 & 86.4 & 64.6 & 31.3 & 21.9 & 20.9 & 20.1 \\
\hline 47.7 & 60.9 & 102.8 & 102.0 & 103.9 & 105.2 & 103.4 & 99.0 & 78.5 & 60.2 & 43.0 & 31.2 & 21.1 \\
\hline 54.5 & 71.9 & 125.8 & 126.1 & 133.1 & 138.3 & 140.6 & 139.3 & 114.7 & 77.9 & 52.4 & 34.9 & 22.9 \\
\hline \multicolumn{13}{|c|}{ Thermocouples located at sprinkler number 2} \\
\hline Time & $0 \mathrm{~mm}$ & $25 \mathrm{~mm}$ & $50 \mathrm{~mm}$ & $75 \mathrm{~mm}$ & $100 \mathrm{~mm}$ & $125 \mathrm{~mm}$ & $150 \mathrm{~mm}$ & $250 \mathrm{~mm}$ & $350 \mathrm{~mm}$ & $450 \mathrm{~mm}$ & $550 \mathrm{~mm}$ & $900 \mathrm{~mm}$ \\
\hline 0.0 & 20.6 & 20.7 & 20.9 & 20.5 & 20.7 & 20.3 & 20.5 & 19.9 & 19.7 & 19.4 & 19.1 & 18.0 \\
\hline 6.5 & 20.6 & 21.0 & 21.1 & 21.0 & 21.0 & 20.5 & 20.6 & 19.8 & 19.8 & 19.6 & 19.4 & 18.4 \\
\hline 13.5 & 20.6 & 21.0 & 21.1 & 21.0 & 21.0 & 20.5 & 20.8 & 20.1 & 20.0 & 19.8 & 19.5 & 18.3 \\
\hline 20.3 & 22.6 & 35.5 & 37.8 & 36.7 & 36.9 & 33.1 & 32.3 & 28.8 & 28.0 & 27.2 & 23.1 & 19.3 \\
\hline 27.1 & 25.3 & 47.8 & 50.0 & 50.6 & 50.8 & 49.3 & 48.8 & 41.5 & 24.3 & 22.0 & 20.9 & 18.8 \\
\hline 34.0 & 27.8 & 52.8 & 56.7 & 56.3 & 57.6 & 55.6 & 56.6 & 53.0 & 36.7 & 31.8 & 29.5 & 22.1 \\
\hline 40.8 & 29.0 & 63.0 & 64.0 & 64.9 & 64.2 & 61.4 & 60.4 & 54.4 & 47.3 & 42.4 & 40.8 & 21.3 \\
\hline 47.7 & 31.6 & 67.8 & 72.2 & 72.3 & 73.4 & 69.5 & 70.8 & 63.6 & 52.9 & 49.3 & 39.6 & 22.0 \\
\hline 54.5 & 34.6 & 79.8 & 82.1 & 83.4 & 84.1 & 83.1 & 83.4 & 77.0 & 61.5 & 55.2 & 48.0 & 24.8 \\
\hline \multicolumn{13}{|c|}{ Thermocouples located at sprinkler number 3} \\
\hline Time & $0 \mathrm{~mm}$ & $25 \mathrm{~mm}$ & $50 \mathrm{~mm}$ & $75 \mathrm{~mm}$ & $100 \mathrm{~mm}$ & $125 \mathrm{~mm}$ & $150 \mathrm{~mm}$ & $250 \mathrm{~mm}$ & $350 \mathrm{~mm}$ & $450 \mathrm{~mm}$ & $550 \mathrm{~mm}$ & $900 \mathrm{~mm}$ \\
\hline 0.0 & 20.2 & 20.6 & 20.3 & 20.2 & 20.1 & 20.1 & 20.0 & 19.8 & 19.6 & 19.3 & 19.0 & 18.1 \\
\hline 6.5 & 20.3 & 20.7 & 20.6 & 20.6 & 20.5 & 20.5 & 20.4 & 20.0 & 19.9 & 19.5 & 19.2 & 18.2 \\
\hline 13.5 & 20.2 & 20.6 & 20.4 & 20.5 & 20.5 & 20.5 & 20.5 & 20.3 & 19.9 & 19.5 & 19.4 & 18.5 \\
\hline 20.3 & 20.5 & 23.8 & 24.3 & 24.4 & 24.9 & 25.5 & 26.9 & 24.8 & 23.0 & 20.4 & 20.2 & 19.2 \\
\hline 27.1 & 21.8 & 28.0 & 32.7 & 31.5 & 32.9 & 33.6 & 33.4 & 30.2 & 27.5 & 25.3 & 22.7 & 19.6 \\
\hline 34.0 & 22.9 & 32.4 & 36.1 & 36.2 & 36.0 & 36.8 & 37.3 & 34.0 & 31.5 & 29.7 & 29.3 & 21.7 \\
\hline 40.8 & 24.2 & 38.1 & 43.2 & 43.7 & 43.0 & 44.3 & 45.1 & 39.6 & 36.9 & 35.1 & 34.1 & 23.4 \\
\hline 47.7 & 25.2 & 41.3 & 47.2 & 47.8 & 46.4 & 47.6 & 47.7 & 44.1 & 42.8 & 40.8 & 40.2 & 23.6 \\
\hline 54.5 & 25.7 & 47.0 & 53.0 & 54.6 & 52.4 & 54.0 & 55.2 & 51.4 & 47.8 & 46.7 & 46.7 & 24.8 \\
\hline \multicolumn{13}{|c|}{ Thermocouples located at sprinkler number 4} \\
\hline Time & $0 \mathrm{~mm}$ & $25 \mathrm{~mm}$ & $50 \mathrm{~mm}$ & $75 \mathrm{~mm}$ & $100 \mathrm{~mm}$ & $125 \mathrm{~mm}$ & $150 \mathrm{~mm}$ & $250 \mathrm{~mm}$ & $350 \mathrm{~mm}$ & $450 \mathrm{~mm}$ & $550 \mathrm{~mm}$ & $900 \mathrm{~mm}$ \\
\hline 0.0 & 203 & 20.5 & 20.5 & 20.4 & 20.2 & 20.2 & 20.2 & 19.8 & 19.5 & 19.0 & 18.7 & 18.1 \\
\hline 6.5 & 20.4 & 20.6 & 20.6 & 20.5 & 20.3 & 20.3 & 20.3 & 19.9 & 19.5 & 19.2 & 18.9 & 18.3 \\
\hline 13.5 & 23.3 & 33.2 & 32.8 & 32.7 & 30.7 & 32.2 & 31.1 & 27.0 & 25.3 & 20.6 & 19.9 & 18.6 \\
\hline 20.3 & 26.3 & 40.5 & 40.3 & 40.5 & 37.9 & 38.4 & 36.0 & 26.4 & 22.6 & 21.8 & 20.9 & 18.8 \\
\hline 27.1 & 27.1 & 42.1 & 41.3 & 41.6 & 40.0 & 39.7 & 38.4 & 30.5 & 29.4 & 24.3 & 20.7 & 18.9 \\
\hline 34.0 & 29.9 & 52.4 & 51.9 & 51.5 & 48.3 & 48.3 & 46.8 & 36.6 & 36.1 & 26.8 & 20.5 & 19.0 \\
\hline 40.8 & 30.6 & 57.4 & 56.5 & 55.6 & 53.4 & 53.9 & 53.4 & 44.2 & 43.3 & 36.6 & 27.5 & 19.5 \\
\hline 47.7 & 36.4 & 67.8 & 68.7 & 68.3 & 65.1 & 65.7 & 65.8 & 56.2 & 48.4 & 45.7 & 39.6 & 22.6 \\
\hline 54.5 & 41.6 & 81.0 & 79.3 & 78.3 & 73.2 & 72.2 & 69.7 & 56.8 & 56.7 & 49.3 & 40.3 & 22.1 \\
\hline \multicolumn{6}{|c|}{ Velocity probes at sprinkler number 1} & \multicolumn{7}{|c|}{ Velocity probes at sprinkler number 2} \\
\hline Time & $25 \mathrm{~mm}$ & $15 \mathrm{~mm}$ & $125 \mathrm{~mm}$ & $250 \mathrm{~mm}$ & & Time & $25 \mathrm{~mm}$ & $75 \mathrm{~mm}$ & $125 \mathrm{~mm}$ & $250 \mathrm{rmm}$ & & \\
\hline 0.0 & 0.0 & 0.0 & 0.0 & 0.0 & & 0.0 & 0.0 & 0.0 & 0.0 & 0.0 & & \\
\hline 6.5 & 0.8 & 0.8 & 0.5 & 0.2 & & 6.5 & -0.1 & -0.1 & 0.0 & 0.1 & & \\
\hline 13.5 & 1.6 & 1.4 & 1.0 & 0.2 & & 13.5 & 0.4 & 0.5 & 0.6 & 0.4 & & \\
\hline 20.3 & 1.8 & 1.8 & 0.9 & 0.2 & & 20.3 & 0.8 & 0.8 & 0.9 & 0.5 & & \\
\hline 27.1 & 1.8 & 1.4 & 0.6 & 0.3 & & 27.1 & 0.8 & 0.8 & 0.7 & 0.6 & & \\
\hline 34,0 & 1.8 & 1.7 & 1.7 & 0.6 & & 34.0 & 0.7 & 0.7 & 0.7 & 0.5 & & \\
\hline 40.8 & 1.2 & 1.2 & 0.9 & 0.4 & & 40.8 & 0.8 & 0.9 & 0.8 & 0.5 & & \\
\hline 47.7 & 1.6 & 1.3 & 1.1 & 0.4 & & 47.7 & 0.8 & 0.8 & 0.8 & 0.5 & & \\
\hline 54.5 & 2.4 & 2.2 & 1.2 & -0.1 & & 54.5 & 0.8 & 0.9 & 0.8 & 0.6 & & \\
\hline
\end{tabular}


Experiment Number 37

\begin{tabular}{|c|c|c|c|c|c|c|c|c|c|c|c|c|}
\hline \multicolumn{13}{|c|}{ Thermocouples located at sprinkler number 1} \\
\hline Time & $0 \mathrm{~mm}$ & $25 \mathrm{~mm}$ & $50 \mathrm{~mm}$ & $75 \mathrm{~mm}$ & $100 \mathrm{~mm}$ & $125 \mathrm{~mm}$ & $150 \mathrm{~mm}$ & $250 \mathrm{~mm}$ & $350 \mathrm{~mm}$ & $450 \mathrm{~mm}$ & $550 \mathrm{~mm}$ & $900 \mathrm{~mm}$ \\
\hline 0.0 & 22.3 & 21.8 & 21.1 & 20.8 & 20.5 & 20.3 & 20.2 & 19.6 & 19.2 & 18.8 & 18.4 & 17.9 \\
\hline 5.7 & 22.4 & 22.1 & 21.9 & 21.8 & 21.8 & 21.6 & 21.3 & 20.5 & 20.0 & 19.4 & 18.9 & 17,8 \\
\hline 12.6 & 30.9 & 46.5 & 42.5 & 44.7 & 42.7 & 41.1 & 37.5 & 25.1 & 21.2 & 20.1 & 10.4 & 18.0 \\
\hline 19.4 & 41.5 & 71.9 & 67.4 & 68.7 & 66.0 & 63.0 & 60.6 & 43.2 & 23.0 & 19.9 & 19.4 & 18.3 \\
\hline 26.2 & 48.9 & 80.8 & 78.8 & 79.2 & 74.4 & 71.5 & 64.6 & 45.6 & 23.4 & 20.2 & 19.4 & 18.5 \\
\hline 33.2 & 50.6 & 82.0 & 83.6 & 84.3 & 84.5 & 83.7 & 81.4 & 67.3 & 38.8 & 25.2 & 21.0 & 19.3 \\
\hline 40.0 & 54.3 & 87.7 & 84.8 & 86.1 & 83.6 & 84.5 & 81.2 & 61.5 & 29.1 & 21.4 & 20.7 & 20.2 \\
\hline 46.8 & 68.6 & 115.5 & 113.0 & 110.4 & 104.1 & 103.7 & 99.7 & 19.0 & 50.0 & 32.6 & 25.5 & 21.3 \\
\hline \multicolumn{13}{|c|}{ Thermocouples located at sprinkler number 2} \\
\hline Time & $0 \mathrm{~mm}$ & $25 \mathrm{~mm}$ & $50 \mathrm{~mm}$ & $75 \mathrm{~mm}$ & $100 \mathrm{~mm}$ & $125 \mathrm{~mm}$ & $150 \mathrm{~mm}$ & $250 \mathrm{~mm}$ & $350 \mathrm{~mm}$ & $450 \mathrm{~mm}$ & $550 \mathrm{~mm}$ & $900 \mathrm{~mm}$ \\
\hline 0.0 & 20.7 & 20.5 & 20.7 & 20.1 & 20.4 & 19.7 & 20.1 & 19.4 & 19.1 & 18.8 & 18.5 & 17.7 \\
\hline 5.7 & 20.7 & 20.6 & 20.7 & 20.2 & 20.5 & 20.1 & 20.4 & 20.1 & 19.8 & 19.5 & 19.0 & 18.1 \\
\hline 12.6 & 20.7 & 20.6 & 20.7 & 20.3 & 20.5 & 20.1 & 20.4 & 20.2 & 19.7 & 19.3 & 19.0 & 18.2 \\
\hline 19.4 & 22.8 & 33.9 & 36.0 & 36.8 & 37.6 & 37.0 & 37.2 & 32.2 & 28.1 & 26.4 & 25.5 & 18.9 \\
\hline 26.2 & 25.4 & 47.0 & 48.1 & 49.3 & 49.0 & 50.1 & 50.0 & 44.3 & 27.8 & 21.4 & 20.8 & 18.6 \\
\hline 33.2 & 27.5 & 51.5 & 54.4 & 54.8 & 56.9 & 55.0 & 56.3 & 49.6 & 41.2 & 33.7 & 33.5 & 23.5 \\
\hline 40.0 & 29.0 & 53.3 & 57.0 & 58.0 & 59.1 & 57.6 & 58.7 & 56.7 & 47.7 & 44.2 & 42.7 & 21.6 \\
\hline 46.8 & 30.8 & 61.2 & 64.8 & 64.6 & 65.4 & 63.0 & 63.3 & 59.0 & 52.7 & 49.7 & 45.1 & 23.3 \\
\hline \multicolumn{13}{|c|}{ Thermocouples located at sprinkler number 3} \\
\hline Time & $0 \mathrm{~mm}$ & $25 \mathrm{~mm}$ & $50 \mathrm{~mm}$ & $75 \mathrm{~mm}$ & $100 \mathrm{~mm}$ & $125 \mathrm{~mm}$ & $150 \mathrm{~mm}$ & $250 \mathrm{~mm}$ & $350 \mathrm{~mm}$ & $450 \mathrm{~mm}$ & $550 \mathrm{~mm}$ & $900 \mathrm{~mm}$ \\
\hline 0.0 & 20.2 & 20.4 & 20.0 & 20.0 & 19.9 & 19.8 & 19.7 & 19.4 & 19.0 & 18.8 & 18.6 & 17.8 \\
\hline 5.7 & 20.3 & 20.6 & 20.1 & 20.2 & 20.1 & 20.2 & 20.1 & 19.9 & 19.6 & 19.2 & 19.0 & 17.9 \\
\hline 12.6 & 20.3 & 20.7 & 20.2 & 20.2 & 20.1 & 20.1 & 20.1 & 20.1 & 19.7 & 19.4 & 19.1 & 17.9 \\
\hline 19.4 & 20.5 & 22.2 & 22.8 & 22.7 & 22.8 & 23.4 & 24.5 & 23.8 & 23.2 & 21.8 & 19.8 & 18.3 \\
\hline 26.2 & 22.0 & 29.4 & 33.2 & 31.3 & 32.9 & 32.9 & 33.0 & 30.3 & 27.3 & 24.2 & 22.6 & 19.1 \\
\hline 33.2 & 22.4 & 31.7 & 34.9 & 35.7 & 35.2 & 35.8 & 36.2 & 34.6 & 33.8 & 30.0 & 28.4 & 22.1 \\
\hline 40.0 & 23.4 & 38.9 & 41.8 & 42.8 & 41.2 & 41.7 & 42.0 & 38.9 & 36.7 & 35.1 & 34.7 & 24.2 \\
\hline 46.8 & 24.5 & 41.8 & 48.0 & 49.3 & 48.0 & 48.9 & 48.8 & 45.2 & 44.2 & 41.5 & 40.4 & 24.0 \\
\hline \multicolumn{13}{|c|}{ Thermocouples located at sprinkler number 4} \\
\hline Time & $\mathrm{Omm}$ & $25 \mathrm{~mm}$ & $50 \mathrm{~mm}$ & $75 \mathrm{~mm}$ & $100 \mathrm{~mm}$ & $125 \mathrm{~mm}$ & $150 \mathrm{~mm}$ & $250 \mathrm{~mm}$ & $350 \mathrm{~mm}$ & $450 \mathrm{~mm}$ & $550 \mathrm{~mm}$ & $900 \mathrm{~mm}$ \\
\hline 0.0 & 20.3 & 20.4 & 20.2 & 20.1 & 19.9 & 19.9 & 19.8 & 19.5 & 19.1 & 18.7 & 18.4 & 17.8 \\
\hline 5.7 & 20.3 & 20.7 & 20.5 & 20.4 & 20.2 & 20.2 & 20.3 & 20.0 & 19.3 & 18.9 & 18.5 & 18.0 \\
\hline 12.6 & 24.3 & 32.8 & 30.7 & 30.1 & 27.8 & 28.1 & 27.9 & 25.0 & 23.2 & 19.6 & 19.3 & 18.4 \\
\hline 19.4 & 27.2 & 40.9 & 40.6 & 41.0 & 37.6 & 37.4 & 35.2 & 24.4 & 22.6 & 21.0 & 20.2 & 18.9 \\
\hline 26.2 & 28.0 & 43.1 & 42.5 & 42.3 & 40.5 & 40.8 & 39.4 & 30.0 & 26.9 & 22.7 & 21.6 & 19.3 \\
\hline 33.2 & 30.1 & 50.2 & 49.4 & 49.6 & 46.6 & 46.9 & 45.8 & 35.1 & 35.5 & 28.4 & 20.6 & 19.3 \\
\hline 40.0 & 31.7 & 55.7 & 57.2 & 56.5 & 53.6 & 53.4 & 52.2 & 44.8 & 41.3 & 36.6 & 27.1 & 19.3 \\
\hline 46.8 & 35.8 & 70.9 & 70.0 & 68.2 & 63.8 & 63.0 & 61.7 & 53.3 & 47.1 & 44.6 & 39.4 & 22.4 \\
\hline \multicolumn{5}{|c|}{ Velocity probes at sprinkler number 1} & \multicolumn{6}{|c|}{ Velocity probes at sprinkler number 2} & & \\
\hline Time & $25 \mathrm{~mm}$ & $75 \mathrm{~mm}$ & $125 \mathrm{~mm}$ & $250 \mathrm{~mm}$ & & Time & $25 \mathrm{~mm}$ & $75 \mathrm{~mm}$ & $125 \mathrm{~mm}$ & $250 \mathrm{~mm}$ & & \\
\hline 0.0 & 0.0 & 0.0 & 0.0 & 0.0 & & 0.0 & 0.0 & 0.0 & 0.0 & 0.0 & & \\
\hline 5.7 & 0.9 & 0.8 & 0.5 & 0.0 & & 5.7 & -0.1 & -0.1 & -0.1 & -0.1 & & \\
\hline 12.6 & 1.6 & 1.4 & 1.0 & -0.1 & & 12.6 & 0.4 & 0.5 & 0.5 & 0.4 & & \\
\hline 19.4 & 1.8 & 1.5 & 0.9 & 0.4 & & 19.4 & 0.8 & 0.9 & 0.8 & 0.4 & & \\
\hline 26.2 & 1.5 & 1.6 & 1.4 & 0.6 & & 26.2 & 0.7 & 0.8 & 0.8 & 0.6 & & \\
\hline 33.2 & 1.8 & 1.8 & 1.4 & 0.7 & & 33.2 & 0.6 & 0.7 & 0.6 & 0.4 & & \\
\hline 40.0 & 2.2 & 1.8 & 1.4 & 0.7 & & 40.0 & 0.9 & 0.8 & 0.7 & 0.3 & & \\
\hline 46.8 & 1.3 & 1.5 & 1.8 & 0.7 & & 46.8 & 0.8 & 0.9 & 0.8 & 0.6 & & \\
\hline
\end{tabular}




\section{Experiment Number 38}

Thermocouples located at sprinkler number 1

\begin{tabular}{|c|c|c|c|c|c|c|c|c|c|c|c|c|}
\hline Time & $0 \mathrm{~mm}$ & $25 \mathrm{~mm}$ & $50 \mathrm{~mm}$ & $75 \mathrm{~mm}$ & $100 \mathrm{~mm}$ & $125 \mathrm{~mm}$ & $150 \mathrm{~mm}$ & $250 \mathrm{~mm}$ & $350 \mathrm{~mm}$ & $450 \mathrm{~mm}$ & $550 \mathrm{~mm}$ & $900 \mathrm{~mm}$ \\
\hline 0.0 & 21.7 & 21.3 & 20.6 & 20.3 & 20.0 & 19.9 & 19.7 & 19.3 & 18.7 & 18.4 & 18.1 & 17.8 \\
\hline 1.8 & 21.8 & 21.4 & 20.8 & 20.4 & 20.2 & 20.0 & 19.7 & 19.3 & 18.9 & 18.8 & 18.6 & 18.1 \\
\hline 8.6 & 24.7 & 34.1 & 31.0 & 31.7 & 30.3 & 28.0 & 28.9 & 25.9 & 21.2 & 18.9 & 18.6 & 18.2 \\
\hline 15.5 & 35.7 & 57.7 & 54.9 & 57.1 & 54.2 & 51.2 & 46.1 & 30.5 & 22.9 & 19.2 & 18.8 & 18.4 \\
\hline 22.3 & 43.6 & 71.4 & 69.6 & 70.3 & 67.8 & 66.2 & 62.0 & 45.0 & 22.4 & 19.6 & 19.0 & 18.8 \\
\hline 29.1 & 51.6 & 84.5 & 79.9 & 79.7 & 75.4 & 72.4 & 68.0 & 38.4 & 21.9 & 19.6 & 19.2 & 19.1 \\
\hline 36.1 & 56.9 & 93.4 & 90.4 & 92.3 & 85.2 & 82.4 & 75.2 & 51.1 & 33.4 & 21.9 & 19.9 & 19.8 \\
\hline 42.8 & 58.1 & 96.4 & 96.7 & $9 / .5$ & 96.1 & 94.6 & 91.2 & 72.9 & 47.9 & 29.1 & 22.7 & 19.8 \\
\hline 49.7 & 71.7 & 130.7 & 124.8 & 127.5 & 122.9 & 121.4 & $\$ 14.1$ & 77.5 & 56.0 & 42.0 & 33.2 & 21.5 \\
\hline 56.5 & 793 & 140.6 & 131.3 & 84.0 & 64.3 & 63.2 & 84.5 & 85.3 & 74.7 & 46.2 & 37.7 & 22.5 \\
\hline Thermocoupl & caled at & inkler num & & & & & & & & & & \\
\hline Time & $0 \mathrm{~mm}$ & $25 \mathrm{~mm}$ & $50 \mathrm{~mm}$ & $75 \mathrm{~mm}$ & $100 \mathrm{~mm}$ & $125 \mathrm{~mm}$ & $150 \mathrm{~mm}$ & $250 \mathrm{~mm}$ & $350 \mathrm{~mm}$ & $450 \mathrm{~mm}$ & $550 \mathrm{~mm}$ & $900 \mathrm{~mm}$ \\
\hline 0.0 & 20.2 & 19.6 & 19.7 & 19.3 & 19.5 & 19.1 & 19.3 & 18.8 & 18.6 & 18.4 & 18.1 & 17.6 \\
\hline 1.8 & 20.3 & 20.2 & 20.2 & 19.8 & 20.1 & 19.8 & 19.9 & 19.5 & 19.3 & 19.1 & 18.9 & 17.9 \\
\hline 8.6 & 20.3 & 20.1 & 20.4 & 19.7 & 20.1 & 19.7 & 20.2 & 19.9 & 19.3 & 19.2 & 19.0 & 17.8 \\
\hline 15.5 & 20.2 & 20.0 & 20.4 & 19.6 & 20.1 & 19.5 & 20.1 & 19.7 & 19.3 & 19.2 & 19.1 & 17.9 \\
\hline 22.3 & 22.4 & 38.1 & 40.3 & 40.7 & 41.3 & 39.0 & 38.5 & 27.1 & 23.3 & 22.2 & 21.6 & 18.8 \\
\hline 29.1 & 25.6 & 49.2 & 53.0 & 51.8 & 52.9 & 50.5 & 50.3 & 43.5 & 26.1 & 21.8 & 21.1 & 18.8 \\
\hline 36.1 & 27.3 & 55.9 & 58.6 & 57.4 & 58.1 & 55.4 & 56.7 & 52.2 & 41.5 & 36.4 & 33.4 & 21.8 \\
\hline 42.8 & 28.3 & 56.1 & 58.2 & 57.4 & 58.5 & 55.8 & 57.1 & 53.2 & 47.0 & 44.3 & 37.0 & 21.3 \\
\hline 49.7 & 31.0 & 67.6 & 71.2 & 72.2 & 73.2 & 71.7 & 71.9 & 63.6 & 56.8 & 52.0 & 47.3 & 23.4 \\
\hline 56.5 & 36.2 & 88.1 & 91.0 & 93.3 & 93.5 & 92.9 & 92.9 & 85.1 & 65.1 & 57.8 & 50.9 & 31.3 \\
\hline Thermocoup & cated at & inkler num & & & & & & & & & & \\
\hline Time & $0 \mathrm{~mm}$ & $25 \mathrm{~mm}$ & $50 \mathrm{~mm}$ & $75 \mathrm{~mm}$ & $100 \mathrm{~mm}$ & $125 \mathrm{~mm}$ & $150 \mathrm{~mm}$ & $250 \mathrm{~mm}$ & $350 \mathrm{~mm}$ & $450 \mathrm{~mm}$ & $550 \mathrm{~mm}$ & $900 \mathrm{~mm}$ \\
\hline 0.0 & 19.7 & 19.9 & 19.5 & 19.4 & 19.3 & 19.2 & 19.1 & 18.8 & 18.5 & 18.2 & 18.0 & 17.8 \\
\hline 1.8 & 19.8 & 20.1 & 19.7 & 19.7 & 19.7 & 19.7 & 19.8 & 19.6 & 19.4 & 19.2 & 18.5 & 18.0 \\
\hline 8.6 & 10.9 & 20.2 & 19.9 & 19.9 & 19.9 & 19.9 & 19.8 & 19.7 & 19.6 & 19.3 & 18.7 & 18.0 \\
\hline 15.5 & 19.8 & 20.2 & 19.9 & 20.0 & 20.0 & 20.0 & 20.0 & 19.8 & 19.6 & 19.4 & 19.1 & 18.2 \\
\hline 22.3 & 20.7 & 25.4 & 27.3 & 26.3 & 27.0 & 27.1 & 27.2 & 23.9 & 22.4 & 20.5 & 19.9 & 18.7 \\
\hline 29.1 & 22.0 & 29.4 & 33.2 & 32.1 & 33.2 & 33.8 & 34.5 & 31.8 & 27.4 & 24.1 & 22.8 & 19.3 \\
\hline 36.1 & 22.8 & 33.3 & 37.2 & 37.5 & 38.0 & 38.7 & 39.3 & 35.5 & 34.8 & 32.5 & 31.4 & 23.5 \\
\hline 42.8 & 23.7 & 39.1 & 43.1 & 44.9 & 42.2 & 43.2 & 43.5 & 39.1 & 38.2 & 36.3 & 36.5 & 23.6 \\
\hline 49.7 & 24.6 & 45.7 & 50.8 & 51.7 & 49.7 & 50.3 & 50.2 & 45.2 & 43.8 & 41.8 & 41.5 & 24.6 \\
\hline 56.5 & 26.3 & 53.2 & 58.6 & 58.7 & 57.0 & 58.1 & 58.4 & 52.1 & 49.9 & 46.9 & 47.5 & 26.9 \\
\hline Thermocoup & scated at & inkler num & & & & & & & & & & \\
\hline Time & $0 \mathrm{~mm}$ & $25 \mathrm{~mm}$ & $50 \mathrm{~mm}$ & $75 \mathrm{~mm}$ & $100 \mathrm{~mm}$ & $125 \mathrm{~mm}$ & $150 \mathrm{~mm}$ & $250 \mathrm{~mm}$ & $350 \mathrm{~mm}$ & $450 \mathrm{~mm}$ & $550 \mathrm{~mm}$ & $900 \mathrm{~mm}$ \\
\hline 0.0 & 19.8 & 19.8 & 19.6 & 19.6 & 19.4 & 19.3 & 19.2 & 18.9 & 18.6 & 18.3 & 18.0 & 17.8 \\
\hline 1.8 & 19.8 & 20.0 & 20.0 & 19.9 & 19.7 & 19.6 & 19.6 & 19.2 & 18.8 & 18.5 & 18.2 & 17.8 \\
\hline 8.6 & 20.0 & 20.1 & 20.2 & 20.4 & 20.3 & 20.5 & 20.4 & 19.5 & 18.9 & 18.6 & 18.4 & 180 \\
\hline 15.5 & 23.9 & 35.3 & 33.0 & 32.3 & 29.4 & 29.5 & 28.6 & 22.9 & 22.3 & 20.2 & 19.0 & 18.3 \\
\hline 22.3 & 27.0 & 42.3 & 41.9 & 41.7 & 38.9 & 38.9 & 37.1 & 26.1 & 26.3 & 25.3 & 23.1 & 18.7 \\
\hline 29.1 & 28.1 & 44.4 & 43.1 & 42.3 & 39.3 & 39.0 & 38.3 & 31.9 & 32.2 & 26.4 & 20.6 & 19.1 \\
\hline 36.1 & 28.2 & 49.6 & 48.6 & 48.2 & 44.5 & 45.1 & 43.8 & 35.5 & 34.6 & 24.6 & 19.9 & 19.3 \\
\hline 42.8 & 29.4 & 58.9 & 58.1 & 56.9 & 53.6 & 53.5 & 52.8 & 44.1 & 42.9 & 38.3 & 37.2 & 20.0 \\
\hline 49.7 & 33.3 & 64.7 & 65.5 & 64.7 & 61.9 & 63.2 & 62.1 & 51.9 & 49.5 & 45.5 & 42.1 & 21.8 \\
\hline 56.5 & 37.1 & 75.6 & 74.5 & 74.1 & 71.1 & 72.5 & 70.9 & 55.7 & 55.0 & 49.7 & 42.4 & 21.8 \\
\hline Velocity prob & t sprinkle & Imber 1 & & & & locity probs & at sprinkle & umber 2 & & & & \\
\hline Time & $25 \mathrm{~mm}$ & $75 \mathrm{~mm}$ & $125 \mathrm{~mm}$ & $250 \mathrm{~mm}$ & & Time & $25 \mathrm{~mm}$ & $75 \mathrm{~mm}$ & $125 \mathrm{~mm}$ & $250 \mathrm{~mm}$ & & \\
\hline 0.0 & 0.0 & 0.0 & 0.0 & 0.0 & & 0.0 & 0.0 & 0.0 & 0.0 & 0.0 & & \\
\hline 1.8 & 0.5 & 0.6 & 0.6 & 0.3 & & 1.8 & -0.1 & -0.1 & -0.1 & -0.1 & & \\
\hline 8.6 & 1.3 & 1.0 & 0.7 & -0.2 & & 8.6 & -0.1 & -0.1 & -0.1 & -0.1 & & \\
\hline 15.5 & 1.5 & 1.4 & 1.4 & 0.1 & & 15.5 & 0.7 & 0.8 & 0.8 & 0.6 & & \\
\hline 22.3 & 2.0 & 1.8 & 1.6 & 0.6 & & 22.3 & 0.9 & 0.9 & 0.8 & 0.6 & & \\
\hline 29.1 & 1.6 & 1.5 & 1.3 & 0.6 & & 29.1 & 0.8 & 0.8 & 0.8 & 0.5 & & \\
\hline 36.1 & 1.8 & 1.7 & 1.4 & 0.4 & & 36.1 & 0.7 & 0.7 & 0.7 & 0.4 & & \\
\hline 42.8 & 1.5 & 1.6 & 0.7 & 0.1 & & 42.8 & 0.6 & 0.6 & 0.6 & 0.4 & & \\
\hline 49.7 & 2.2 & 2.1 & 1.8 & 0.4 & & 49.7 & 1.0 & 1.0 & 0.9 & 0.5 & & \\
\hline 56.5 & 2.0 & 2.0 & 1.6 & 0.6 & & 56.5 & 1.0 & 1.1 & 1.0 & 0.5 & & \\
\hline
\end{tabular}


Experiment Number 39

Thermocouples located at sprinkler number 1

\begin{tabular}{|c|c|c|c|c|c|c|c|c|c|c|c|c|}
\hline Time & $0 \mathrm{~mm}$ & $25 \mathrm{~mm}$ & $50 \mathrm{~mm}$ & $75 \mathrm{~mm}$ & $100 \mathrm{~mm}$ & $125 \mathrm{~mm}$ & $150 \mathrm{~mm}$ & $250 \mathrm{~mm}$ & $350 \mathrm{~mm}$ & $450 \mathrm{~mm}$ & $550 \mathrm{~mm}$ & $900 \mathrm{~mm}$ \\
\hline 0.0 & 20.0 & 19.6 & 19.8 & 19.5 & 19.8 & 19.3 & 19.3 & 18.5 & 18.1 & 17.6 & 17.2 & 15.4 \\
\hline 6.8 & 20.1 & 19.6 & 19.4 & 18.6 & 19.1 & 19.6 & 20.2 & 19.5 & 18.5 & 17.4 & 17.0 & 15.0 \\
\hline 14.1 & 21.3 & 21.3 & 22.2 & 20.6 & 20.6 & 19.5 & 20.3 & 19.5 & 20.2 & 19.9 & 18.5 & 15.5 \\
\hline 21.0 & 22.8 & 22.2 & 23.6 & 24.0 & 27.2 & 27.2 & 27.4 & 22.3 & 19.5 & 18.6 & 19.3 & 16.6 \\
\hline 27.8 & 37.2 & 31.5 & 38.3 & 31.9 & 39.6 & 36.2 & 39.0 & 27.8 & 24.3 & 20.6 & 19.1 & 17.0 \\
\hline 34.9 & 45.5 & 42.4 & 47.6 & 45.2 & 49.2 & 46.6 & 46.0 & 37.9 & 23.9 & 22.8 & 21.0 & 17.2 \\
\hline 41.8 & 52.5 & 50.6 & 56.3 & 49.0 & 55.8 & 53.3 & 55.4 & 45.7 & 30.5 & 22.5 & 19.2 & 15.3 \\
\hline 48.8 & 58.5 & 54.4 & 60.0 & 51.8 & 62.0 & 58.7 & 59.3 & 49.1 & 39.3 & 25.1 & 21.3 & 17.0 \\
\hline 55.7 & 60.2 & 56.8 & 66.5 & 55.5 & 66.9 & 61.9 & 66.1 & 60.5 & 47.2 & 29.0 & 23.6 & $\uparrow 7.3$ \\
\hline 62.6 & 65.9 & 63.7 & 73.2 & 62.8 & 75.4 & 716 & 76.7 & 68.4 & 544 & 339 & 25.3 & 18.9 \\
\hline 69.7 & 80.8 & 77.7 & 85.4 & 75.4 & 85.0 & 83.7 & 85.5 & 79.3 & 64.2 & 43.1 & 26.5 & 19.2 \\
\hline 76.5 & 76.9 & 73.0 & 84.0 & 72.7 & 83.1 & 81.7 & 86.2 & 85.6 & 67.3 & 48.5 & 33.7 & 21.8 \\
\hline 83.6 & 93.3 & 91.3 & 97.9 & 88.3 & 98.6 & 93.5 & 98.1 & 90.7 & 82.7 & 48.5 & 38.0 & 23.1 \\
\hline 90.5 & 101.4 & 94.5 & 105.7 & 90.2 & 103.4 & 97.3 & 104.9 & 103.3 & 90.8 & 60.0 & 42.7 & 26.2 \\
\hline 97.4 & 103.7 & 99.1 & 107.2 & 99.5 & 109.0 & 108.2 & 111.7 & 99.4 & 87.3 & 63.6 & 47.0 & 31.0 \\
\hline 104.3 & 102.8 & 101.7 & 110.0 & 103.3 & 112.2 & 110.5 & 115.1 & 106.7 & 96.3 & 76.1 & 54.0 & 33.8 \\
\hline 111.1 & 93.5 & 95.9 & 103.1 & 103.0 & 112.6 & 115.0 & 118.1 & 108.1 & 105.7 & 84.6 & 60.2 & 37.4 \\
\hline 118.2 & 115.7 & 114.9 & 125.9 & 120.9 & 131.3 & 131.9 & 134.9 & 122.8 & 113.5 & 81.5 & 62.8 & 40.6 \\
\hline 125.0 & 126.6 & 125.3 & 136.4 & 129.8 & 143.9 & 134.9 & 141.4 & 118.9 & 114.4 & 92.9 & 62.4 & 45.0 \\
\hline 131.8 & 148.5 & 148.0 & $15 / .8$ & 146.7 & 157.4 & 156.9 & 162.2 & 146.2 & 143.0 & 93.8 & 74.0 & 46.8 \\
\hline \multicolumn{13}{|c|}{ rmocouples located at sprinkler number 2} \\
\hline Time & $0 \mathrm{~mm}$ & $25 \mathrm{~mm}$ & $50 \mathrm{~mm}$ & $75 \mathrm{~mm}$ & $100 \mathrm{~mm}$ & $125 \mathrm{~mm}$ & $150 \mathrm{~mm}$ & $250 \mathrm{~mm}$ & $350 \mathrm{~mm}$ & $450 \mathrm{~mm}$ & $550 \mathrm{~mm}$ & $900 \mathrm{~mm}$ \\
\hline 0.0 & 19.1 & 19.2 & 19.3 & 19.2 & 19.4 & 19.0 & 19.3 & 18.8 & 18.6 & 17.9 & 17.3 & 15.3 \\
\hline 6.8 & 19.1 & 19.1 & 19.3 & 19.1 & 19.2 & 18.8 & 18.9 & 18.6 & 17.4 & 17.1 & 16.9 & 15.4 \\
\hline 14.1 & 19.0 & 19.0 & 19.2 & 19.1 & 19.2 & 18.7 & 19.0 & 18.5 & 17.4 & 17.0 & 16.7 & 15.2 \\
\hline 21.0 & 19.2 & 19.2 & 19.2 & 19.1 & 19.3 & 19.2 & 19.2 & 18.6 & 17.7 & 17.1 & 16.8 & 15.3 \\
\hline 27.8 & 19.3 & 19.3 & 19.6 & 19.3 & 19.6 & 19.1 & 19.7 & 18.9 & 18.7 & 18.4 & 17.8 & 16.0 \\
\hline 34.9 & 21.1 & 21.1 & 21.9 & 20.9 & 22.2 & 20.3 & 22.3 & 20.3 & 22.3 & 21.1 & 20.4 & 17.3 \\
\hline 41.8 & 24.0 & 23.2 & 26.3 & 24.4 & 27.9 & 22.9 & 27.6 & 23.1 & 25.3 & 23.0 & 21.4 & 18.3 \\
\hline 48.8 & 31.8 & 30.0 & 35.2 & 31.5 & 37.3 & 28.6 & 36.0 & 24.0 & 26.2 & 23.9 & 23.1 & 19.1 \\
\hline 55.7 & 39.5 & 34.7 & 40.8 & 35.8 & 41.2 & 30.3 & 40.0 & 28.7 & 30.2 & 27.5 & 25.0 & 20.4 \\
\hline 62.6 & 42.3 & 40.7 & 46.0 & 42.0 & 47.8 & 36.1 & 45.4 & 32.4 & 35.6 & 31.7 & 28.0 & 23.3 \\
\hline 69.7 & 52.2 & 47.9 & 55.7 & 48.7 & 56.6 & 39.5 & 54.3 & 37.0 & 41.1 & 34.9 & 31.8 & 27.3 \\
\hline 76.5 & 52.1 & 47.4 & 54.9 & 49.4 & 57.6 & 42.2 & 56.7 & 40.3 & 48.8 & 43.0 & 37.9 & 31.6 \\
\hline 83.6 & 54.5 & 49.7 & 56.7 & 50.8 & 62.1 & 45.9 & 61.9 & 45.6 & 56.3 & 47.1 & 42.2 & 30.1 \\
\hline 90.5 & 63.1 & 59.4 & 67.3 & 62.5 & 68.3 & 51.3 & 65.5 & 45.1 & 55.4 & 48.6 & 45.0 & 35.7 \\
\hline 97.4 & 71.2 & 63.0 & 73.2 & 66.8 & 74.6 & 55.8 & 73.0 & 52.1 & 59.5 & 50.1 & 49.5 & 40.6 \\
\hline 104.3 & 76.4 & 65.1 & 78.1 & 67.8 & 80.0 & 57.3 & 78.6 & 54.3 & 61.9 & 56.0 & 55.3 & 45.2 \\
\hline 111.1 & 74.2 & 68.8 & 78.3 & 71.0 & 80.0 & 58.8 & 80.5 & 55.2 & 68.9 & 62.2 & 57.9 & 46.6 \\
\hline 118.2 & 79.9 & 75.0 & 83.0 & 77.4 & 84.2 & 63.7 & 82.6 & 59.3 & 72.0 & 64.0 & 61.7 & 45.6 \\
\hline 125.0 & 82.1 & 77.4 & 86.8 & 82.0 & 90.7 & 66.6 & 89.7 & 62.6 & 77.8 & 72.2 & 66.6 & 55.4 \\
\hline 131.8 & 93.0 & 87.6 & 96.1 & 89.4 & 96.4 & 72.3 & 94.9 & 66.1 & 83.4 & 77.8 & 70.0 & 61.2 \\
\hline \multicolumn{13}{|c|}{ rmocouples located at sprinkler number 3} \\
\hline Time & $0 \mathrm{~mm}$ & $25 \mathrm{~mm}$ & $50 \mathrm{~mm}$ & $75 \mathrm{~mm}$ & $100 \mathrm{~mm}$ & $125 \mathrm{~mm}$ & 150 m & 250 ווזיווז & $350 \mathrm{~mm}$ & $450 \mathrm{~mm}$ & $550 \mathrm{~mm}$ & $900 \mathrm{~mm}$ \\
\hline 0.0 & 19.3 & 19.3 & 19.1 & 19.2 & 19.1 & 19.2 & 18.9 & 18.8 & 18.2 & 17.8 & 17.3 & 15.6 \\
\hline 6.8 & 19.2 & 20.5 & 20.2 & 19.0 & 19.2 & 19.0 & 19.0 & 18.3 & 19.5 & 17.6 & 17.2 & 15.8 \\
\hline 14.1 & 18.9 & 18.5 & 18.6 & 18.8 & 18.7 & 18.7 & 19.3 & 18.6 & 16.9 & 17.2 & 16.5 & 15.4 \\
\hline 21.0 & 18.9 & 19.3 & 19.0 & 19.0 & 19.0 & 19.1 & 18.7 & 18.6 & 18.1 & 18.2 & 17.7 & 16.1 \\
\hline 27.8 & 20.7 & 22.2 & 22.3 & 21.2 & 21.4 & 21.6 & 21.3 & 20.4 & 20.8 & 20.3 & 19.1 & 16.9 \\
\hline 34.9 & 28.4 & 29.8 & 30.8 & 29.1 & 28.0 & 28.0 & 27.4 & 23.3 & 20.2 & 20.3 & 18.8 & 16.8 \\
\hline 41.8 & 37.9 & 40.4 & 41.7 & 37.6 & 37.4 & 35.9 & 34.7 & 28.1 & 25.8 & 21.5 & 20.0 & 17.7 \\
\hline 48.8 & 42.5 & 44.9 & 45.2 & 43.0 & 42.4 & 41.6 & 42.4 & 33.6 & 25.0 & 24.7 & 22.5 & 20.0 \\
\hline 55.7 & 44.9 & 46.4 & 48.3 & 46.0 & 45.8 & 44.9 & 45.0 & 36.8 & 28.6 & 25.5 & 24.9 & 20.2 \\
\hline 62.6 & 51.6 & 56.9 & 59.1 & 53.8 & 54.2 & 53.3 & 51.8 & 38.9 & 34.7 & 29.4 & 28.4 & 21.6 \\
\hline 69.7 & 57.6 & 62.1 & 63.8 & 59.0 & 59.2 & 58.0 & 58.4 & 47.6 & 40.0 & 33.4 & 33.2 & 23.5 \\
\hline 76.5 & 62.1 & 65.8 & 69.5 & 65.0 & 65.4 & 63.3 & 63.0 & 52.4 & 44.1 & 37.2 & 35.4 & 25.0 \\
\hline B3.6 & 70.5 & 76.8 & 78.4 & 71.9 & 72.0 & 69.9 & 69.7 & 58.4 & 49.9 & 43.5 & 41.5 & 30.6 \\
\hline 90.5 & 71.5 & 77.5 & 81.9 & 77.9 & 77.4 & 76.6 & 77.8 & 64.4 & 50.7 & 47.9 & 43.7 & 33.8 \\
\hline 97.4 & 78.0 & 84.6 & 85.8 & 82.7 & 83.0 & 83.0 & 82.8 & 70.2 & 59.7 & 54.6 & 49.1 & 39.5 \\
\hline 104.3 & 77.1 & 83.1 & 87.7 & 84.7 & 85.4 & 85.9 & 87.9 & 76.0 & 63.2 & 56.5 & 53.2 & 39.4 \\
\hline 111.1 & 78.4 & 84.6 & 87.1 & 86.4 & 86.5 & 87.4 & 87.8 & 78.1 & 65.8 & 60.1 & 55.6 & 41.7 \\
\hline
\end{tabular}


Experiment Number 39

\begin{tabular}{|c|c|c|c|c|c|c|c|c|c|c|c|c|}
\hline 118.2 & 86.8 & 91.4 & 94.5 & 90.7 & 90.3 & 90.2 & 88.7 & 75.9 & 67.4 & 66.3 & 58.8 & 43.1 \\
\hline 125.0 & 93.8 & 95.1 & 100.9 & 96.3 & 96.2 & 96.3 & 96.7 & 83.8 & 71.9 & 69.0 & 65.5 & 50.1 \\
\hline 131.8 & 97.4 & 102.9 & 106.1 & 101.7 & 101.9 & 100.3 & 99.1 & 85.9 & 77.0 & 71.9 & 68.7 & 54.7 \\
\hline \multicolumn{13}{|c|}{ Thermocouples located at sprinkler number 4} \\
\hline Time & $0 \mathrm{~mm}$ & $25 \mathrm{~mm}$ & $50 \mathrm{~mm}$ & $75 \mathrm{~mm}$ & $100 \mathrm{~mm}$ & $125 \mathrm{~mm}$ & $150 \mathrm{~mm}$ & $250 \mathrm{~mm}$ & $350 \mathrm{~mm}$ & $450 \mathrm{~mm}$ & $550 \mathrm{~mm}$ & $900 \mathrm{~mm}$ \\
\hline 0.0 & 19.7 & 19.7 & 19.7 & 20.0 & 19.6 & 19.6 & 19.6 & 19.1 & 18.3 & 17.4 & \#\#\#\#\# & 15.2 \\
\hline 6.8 & 19.7 & 19.9 & 19.9 & 20.1 & 19.6 & 19.6 & 19.4 & 19.0 & 18.6 & 17.7 & -16.6 & 16.1 \\
\hline 14.1 & 20.4 & 21.1 & 21.4 & 21.8 & 21.1 & 21.4 & 21.8 & 22.3 & 22.0 & 20.6 & -232785.7 & 16.9 \\
\hline 21.0 & 36.3 & 37.7 & 38.7 & 39.3 & 34.7 & 33.0 & 31.1 & 26.8 & 23.0 & 21.1 & \#\#\#\#\# & 16.4 \\
\hline 27.8 & 44.1 & 44.2 & 46.1 & 47.6 & 45.5 & 45.3 & 45.3 & 39.1 & 30.7 & 19.6 & 227.4 & 15.8 \\
\hline 34.9 & 52.9 & 53.3 & 55.0 & 56.0 & 53.8 & 53.5 & 53.4 & 45.1 & 28.9 & 20.4 & -8309233.1 & 16.3 \\
\hline 41.8 & 57.4 & 58.4 & 61.3 & 63.4 & 62.3 & 62.1 & 60.9 & 53.3 & 36.4 & 20.3 & -2317474.4 & 16.8 \\
\hline 48.8 & 66.8 & 67.8 & 70.3 & 71.7 & 70.3 & 69.4 & 68.8 & 58.7 & 43.1 & 22.3 & \#\#\# & 17.3 \\
\hline 55.7 & 73.0 & 73.6 & 76.3 & 77.6 & 75.7 & 75.4 & 75.0 & 67.0 & 53.6 & 30.2 & \#\#\#\#\#\# & 18.7 \\
\hline 62.6 & 78.7 & 79.3 & 81.3 & 83.9 & 83.4 & 82.1 & 82.2 & 73.9 & 54.9 & 34.6 & \#\#\#\# & 20.1 \\
\hline 69.7 & 85.5 & 86.4 & 89.9 & 92.1 & 92.1 & 91.8 & 92.0 & 78.2 & 64.9 & 38.5 & -16763.9 & 21.2 \\
\hline 76.5 & 95.2 & 97.2 & 99.1 & 99.5 & 97.9 & 97.5 & 98.7 & 89.5 & 74.6 & 39.8 & \#\#\#\#\#\#\# & 23.5 \\
\hline 83.6 & 104.4 & 103.6 & 104.0 & 104.6 & 104.5 & 103.3 & 103.3 & 97.1 & 81.3 & 52.7 & \#\#\# & 26.1 \\
\hline 90.5 & 104.0 & 105.6 & 109.4 & 112.3 & 112.8 & 113.1 & 114.5 & 102.4 & 92.8 & 63.0 & H\#\#\# & 29.2 \\
\hline 97.4 & 111.1 & 112.9 & 116.1 & 119.1 & 117.2 & 116.8 & 116.8 & 108.1 & 89.6 & 67.3 & 47.4 & 32.1 \\
\hline 104.3 & 114.7 & 115.8 & 118.9 & 119.8 & 118.5 & 117.4 & 119.6 & 110.7 & 98.4 & 73.1 & \#\#\# & 36.0 \\
\hline 111.1 & 118.7 & 119.2 & 122.2 & 121.9 & 120.2 & 119.2 & 118.7 & 115.0 & 101.6 & 71.4 & 58.6 & 40.0 \\
\hline 118.2 & 122.6 & 125.5 & 129.8 & 132.4 & 131.0 & 129.1 & 129.6 & 119.0 & 108.9 & 85.4 & 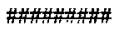 & 42.1 \\
\hline 125.0 & 132.8 & 136.4 & 139.1 & 138.7 & 137.6 & 135.8 & 134.8 & 124.7 & 123.0 & 104.9 & -241170.2 & 43.8 \\
\hline 131.8 & 141.4 & 143.8 & 147.5 & 148.0 & 147.7 & 147.6 & 148.8 & 139.2 & 135.3 & 111.3 & -37228.1 & 48.7 \\
\hline \multicolumn{5}{|c|}{ Velocity probes at sprinkler number 1} & \multicolumn{6}{|c|}{ Velocity probes at sprinkler number 2} & & \\
\hline Time & $25 \mathrm{~mm}$ & $75 \mathrm{~mm}$ & $\uparrow 25 \mathrm{~mm}$ & $250 \mathrm{~mm}$ & & Time & $25 \mathrm{~mm}$ & $75 \mathrm{~mm}$ & $125 \mathrm{~mm}$ & $250 \mathrm{~mm}$ & & \\
\hline 0.0 & 0.0 & 0.0 & 0.0 & 0.0 & & 0.0 & 0.0 & 0.0 & 0.0 & 0.0 & & \\
\hline 6.8 & 0.2 & 0.1 & 0.1 & 0.0 & & 6.8 & 0.0 & 0.1 & 0.1 & 0.0 & & \\
\hline 14.1 & 0.3 & 0.3 & 0.3 & -0.3 & & 14.1 & 0.0 & -0.1 & 0.1 & 0.0 & & \\
\hline 21.0 & 0.4 & 0.3 & 0.3 & 0.4 & & 21.0 & 0.1 & 0.1 & 0.1 & 0.1 & & \\
\hline 27.8 & 0.5 & 0.5 & 0.6 & 0.3 & & 27.8 & 0.4 & 0.4 & 0.4 & 0.3 & & \\
\hline 34.9 & 0.4 & 0.5 & 0.5 & 0.3 & & 34.9 & 0.3 & 0.4 & 0.4 & 0.3 & & \\
\hline 41.8 & 0.3 & 0.4 & 0.6 & 0.4 & & 41.8 & 0.5 & 0.5 & 0.5 & 0.2 & & \\
\hline 48.8 & 0.5 & 0.5 & 0.4 & 0.3 & & 48.8 & 0.4 & 0.3 & 0.3 & 0.2 & & \\
\hline 55.7 & 0.4 & 0.4 & 0.5 & 0.3 & & 55.7 & 0.6 & 0.5 & 0.5 & 0.4 & & \\
\hline 62.6 & 0.6 & 0.6 & 0.7 & 0.5 & & 62.6 & 0.5 & 0.5 & 0.5 & 0.3 & & \\
\hline 69.7 & 0.2 & 0.1 & 0.5 & 0.6 & & 69.7 & 0.4 & 0.4 & 0.5 & 0.4 & & \\
\hline 76.5 & 0.5 & 0.4 & 0.5 & 0.3 & & 76.5 & 0.5 & 0.6 & 0.6 & 0.5 & & \\
\hline 83.6 & 0.6 & 0.6 & 0.6 & 0.4 & & 83.6 & 0.5 & 0.5 & 0.5 & 0.4 & & \\
\hline 90.5 & 0.5 & 0.6 & 0.7 & 0.3 & & 90.5 & 0.5 & 0.6 & 0.7 & 0.4 & & \\
\hline 97.4 & 0.3 & 0.3 & 0.7 & 0.5 & & 97.4 & 0.6 & 0.6 & 0.6 & 0.4 & & \\
\hline 104.3 & 0.3 & 0.3 & 0.7 & 0.6 & & 104.3 & 0.6 & 0.6 & 0.6 & 0.6 & & \\
\hline 111.1 & 0.5 & 0.7 & 1.0 & 0.7 & & 111.1 & 0.6 & 0.5 & 0.6 & 0.4 & & \\
\hline 118.2 & 0.3 & 0.1 & 0.5 & 0.5 & & 118.2 & 0.6 & 0.4 & 0.6 & 0.5 & & \\
\hline 125.0 & 0.7 & 1.0 & 0.9 & 0.7 & & 125.0 & 0.7 & 0.6 & 0.7 & 0.5 & & \\
\hline 131.8 & 0.6 & 0.5 & 0.8 & 0.7 & & 131.8 & 0.7 & 0.7 & 0.8 & 0.7 & & \\
\hline
\end{tabular}

Velocity probes in channels above burner

$\begin{array}{rrr}\text { Time } & \text { Channel 1 } & \text { Channel 2 } \\ 0.0 & 0.0 & 0.0 \\ 6.8 & 1.9 & 1.0 \\ 14.1 & 2.7 & 1.2 \\ 21.0 & 3.0 & 1.2 \\ 27.8 & 2.8 & 1.8 \\ 34.9 & 3.2 & 2.2 \\ 41.8 & 3.1 & 2.2 \\ 48.8 & 3.3 & 1.8 \\ 55.7 & 3.2 & 1.9 \\ 62.6 & 3.6 & 2.2 \\ 69.7 & 3.7 & 2.3 \\ 76.5 & 4.4 & 2.9 \\ 83.6 & 4.1 & 3.0 \\ 90.5 & 3.5 & 2.8\end{array}$


Experiment Number 39

$\begin{array}{rrr}97.4 & 4.4 & 2.8 \\ 104.3 & 3.9 & 2.5 \\ 111.1 & 4.5 & 2.7 \\ 118.2 & 4.0 & 3.3 \\ 125.0 & 4.9 & 3.0 \\ 131.8 & 5.5 & 3.3\end{array}$


Experiment Number 40

Thermocouples located at sprinkler number 1

\begin{tabular}{|c|c|c|c|c|c|c|c|c|c|c|c|c|}
\hline Time & $0 \mathrm{~mm}$ & $25 \mathrm{~mm}$ & $50 \mathrm{~mm}$ & $75 \mathrm{~mm}$ & $100 \mathrm{~mm}$ & $125 \mathrm{~mm}$ & $150 \mathrm{~mm}$ & $250 \mathrm{~mm}$ & $350 \mathrm{~mm}$ & $450 \mathrm{~mm}$ & $550 \mathrm{~mm}$ & $900 \mathrm{~mm}$ \\
\hline 0.0 & 22.3 & 22.1 & 22.5 & 21.6 & 22.4 & 21.8 & 22.3 & 21.5 & 21.4 & 21.2 & 21.1 & 20.3 \\
\hline 6.9 & 22.4 & 22.4 & 22.5 & 21.8 & 22.5 & 22.0 & 22.5 & 21.6 & 21.6 & 21.4 & 21.4 & 20.7 \\
\hline 13.7 & 27.0 & 26.6 & 27.0 & 25.6 & 27.8 & 26.4 & 27.4 & 24.8 & 24.5 & 23.4 & 21.8 & 20.9 \\
\hline 20.4 & 36.6 & 36.0 & 38.0 & 32.1 & 38.0 & 34.6 & 36.8 & 28.4 & 24.0 & 22.5 & 22.1 & 21.1 \\
\hline 27.3 & 39.8 & 40.7 & 43.6 & 37.0 & 43.3 & 44.1 & 46.4 & 40.7 & 32.0 & 26.4 & 24.3 & 21.3 \\
\hline 34.1 & 52.4 & 51.3 & 55.6 & 48.6 & 56.1 & 53.5 & 55.3 & 49.2 & 34.6 & 25.3 & 23.2 & 21.4 \\
\hline 41.0 & 55.1 & 56.7 & 61.9 & 51.7 & 63.0 & 59.4 & 62.6 & 60.0 & 43.0 & 24.2 & 23.6 & 21.7 \\
\hline 47.7 & 60.4 & 62.2 & 66.0 & 55.4 & 65.0 & 62.4 & 67.3 & 64.3 & 45.6 & 26.6 & 24.2 & 21.7 \\
\hline 54.5 & 63.4 & 66.4 & 69.8 & 59.6 & 69.9 & 66.9 & 70.4 & 66.1 & 49.8 & 30.2 & 24.9 & 22.4 \\
\hline 61.4 & 63.2 & 67.1 & 69.8 & 62.9 & 71.7 & 70.9 & 76.9 & 74.4 & 68.2 & 44.4 & 31.2 & 24.0 \\
\hline 68.1 & 83.3 & 86.2 & 91.4 & 74.8 & 92.9 & 82.9 & 93.0 & 86.7 & 72.7 & 47.8 & 35.9 & 24.9 \\
\hline 75.1 & 89.1 & 91.1 & 95.3 & 85.8 & 99.2 & 97.1 & 102.0 & 95.0 & 82.4 & 57.3 & 41.0 & 25.2 \\
\hline 81.8 & 79.9 & 85.9 & 87.4 & 83.1 & 92.0 & 94.8 & 101.2 & 102.5 & 101.9 & 85.3 & 47.1 & 27.5 \\
\hline 88.6 & 92.5 & 101.5 & 106.3 & 91.7 & 106.3 & 101.5 & 108.2 & 103.9 & 101.4 & 73.3 & 47.7 & 30.5 \\
\hline 95.5 & 85.2 & 90.0 & 93.4 & 86.9 & 99.6 & 102.5 & 108.2 & 113.0 & 110.8 & 85.8 & 51.2 & 34.8 \\
\hline 102.3 & 114.4 & 116.7 & 122.2 & 105.3 & 123.5 & 115.1 & 126.5 & 106.0 & 102.3 & 78.8 & 55.8 & 36.7 \\
\hline 109.2 & 109.1 & 109.5 & 115.1 & 104.8 & 117.9 & 112.9 & 119.8 & 112.7 & 110.7 & 98.2 & 65.4 & 40.8 \\
\hline 115.9 & 120.0 & 123.5 & 127.4 & 121.3 & 131.2 & 128.4 & 134.3 & 124.2 & 122.7 & 95.0 & 65.3 & 44.7 \\
\hline \multicolumn{13}{|c|}{ Thermocouples localed at sprinkler number 2} \\
\hline lime & $0 \mathrm{~mm}$ & $25 \mathrm{~mm}$ & $50 \mathrm{~mm}$ & $75 \mathrm{~mm}$ & $100 \mathrm{~mm}$ & $125 \mathrm{~mm}$ & $150 \mathrm{~mm}$ & $250 \mathrm{~mm}$ & $350 \mathrm{mrn}$ & $450 \mathrm{~mm}$ & $550 \mathrm{~mm}$ & $900 \mathrm{~mm}$ \\
\hline 0.0 & 21.1 & 21.0 & 21.1 & 20.9 & 21.1 & 20.5 & 21.0 & 20.3 & 21.2 & 21.1 & 21.1 & 20.1 \\
\hline 6.9 & 21.2 & 21.2 & 21.3 & 21.1 & 21.3 & 20.7 & 21.3 & 20.4 & 21.3 & 21.2 & 20.9 & 20.2 \\
\hline 13.7 & 21.3 & 21.2 & 21.4 & 21.1 & 21.4 & 20.7 & 21.3 & 20.4 & 21.3 & 21.3 & 21.1 & 20.3 \\
\hline 20.4 & 21.3 & 21.2 & 21.3 & 21.0 & 21.2 & 20.7 & 21.2 & 20.4 & 21.3 & 21.3 & 21.2 & 20.5 \\
\hline 27.3 & 24.7 & 25.2 & 25.8 & 25.1 & 25.8 & 23.0 & 25.0 & 22.2 & 23.5 & 23.4 & 22.7 & 21.0 \\
\hline 34.1 & 29.1 & 28.3 & 29.5 & 28.4 & 30.5 & 26.1 & 29.9 & 25.0 & 26.8 & 25.6 & 24.5 & 21.4 \\
\hline 41.0 & 31.3 & 30.5 & 33.4 & 32.5 & 36.2 & 30.2 & 34.9 & 27.2 & 29.1 & 25.5 & 24.4 & 21.4 \\
\hline 47.7 & 40.0 & 37.9 & 42.8 & 39.1 & 43.7 & 33.1 & 42.6 & 31.6 & 32.8 & 27.9 & 27.2 & 23.1 \\
\hline 54.5 & 42.1 & 39.8 & 45.6 & 41.9 & 47.9 & 37.0 & 47.5 & 34.8 & 37.7 & 30.7 & 28.5 & 24.4 \\
\hline 61.4 & 46.9 & 43.3 & 49.7 & 45.2 & 50.5 & 38.8 & 49.9 & 36.5 & 39.5 & 35.6 & 32.4 & 26.1 \\
\hline 68.1 & 56.8 & 48.9 & 58.9 & 52.5 & 59.5 & 45.4 & 57.9 & 41.8 & 45.6 & 39.3 & 36.5 & 29.7 \\
\hline 75.1 & 59.7 & 56.1 & 62.4 & 59.1 & 62.8 & 48.2 & 59.9 & 42.8 & 49.7 & 43.9 & 41.1 & 31.9 \\
\hline 81.8 & 66.3 & 58.2 & 69.4 & 62.5 & 71.5 & 53.5 & 68.5 & 48.7 & 58.1 & 48.8 & 44.4 & 33.1 \\
\hline 88.6 & 69.5 & 63.3 & 71.6 & 66.6 & 72.5 & 57.2 & 72.5 & 53.5 & 60.6 & 52.9 & 48.9 & 36.3 \\
\hline 95.5 & 75.0 & 70.5 & 78.0 & 71.7 & 78.9 & 58.7 & 77.7 & 55.6 & 68.6 & 59.0 & 56.4 & 42.3 \\
\hline 102.3 & 73.1 & 69.7 & 77.0 & 68.8 & 79.5 & 58.4 & 80.0 & 58.2 & 73.6 & 64.3 & 60.0 & 43.6 \\
\hline 109.2 & 77.6 & 74.2 & 81.7 & 75.6 & 83.1 & 62.3 & 82.3 & 57.0 & 71.9 & 65.9 & 60.8 & 45.3 \\
\hline 115.9 & 76.7 & 78.0 & 82.3 & 75.0 & 85.2 & 63.2 & 85.5 & 62.4 & 76.0 & 70.0 & 62.8 & 53.7 \\
\hline \multicolumn{13}{|c|}{ Thermocouples located at sprinkler number 3} \\
\hline Time & $0 \mathrm{~mm}$ & $25 \mathrm{~mm}$ & $50 \mathrm{~mm}$ & $75 \mathrm{~mm}$ & $100 \mathrm{~mm}$ & $125 \mathrm{~mm}$ & $150 \mathrm{~mm}$ & $250 \mathrm{~mm}$ & $350 \mathrm{~mm}$ & $450 \mathrm{~mm}$ & $550 \mathrm{~mm}$ & $900 \mathrm{~mm}$ \\
\hline 0.0 & 21.7 & 21.7 & 21.6 & 21.4 & 21.4 & 21.4 & 21.3 & 21.2 & 21.1 & 21.0 & 20.9 & 20.2 \\
\hline 6.9 & 21.6 & 21.6 & 21.6 & 21.5 & 21.5 & 21.5 & 21.4 & 21.1 & 21.1 & 21.1 & 21.0 & 20.3 \\
\hline 13.7 & 21.7 & 21.6 & 21.5 & 21.4 & 21.4 & 21.4 & 21.4 & 21.2 & 21.1 & 21.0 & 21.0 & 20.3 \\
\hline 20.4 & 21.6 & 22.0 & 22.1 & 21.9 & 22.4 & 22.6 & 23.2 & 22.3 & 21.5 & 21.3 & 21.2 & 20.7 \\
\hline 27.3 & 25.5 & 26.6 & 26.4 & 26.3 & 26.6 & 27.2 & 27.9 & 25.5 & 23.9 & 22.4 & 22.1 & 21.0 \\
\hline 34.1 & 36.2 & 38.1 & 39.7 & 37.1 & 37.2 & 36.7 & 37.4 & 29.5 & 24.8 & 23.1 & 21.7 & 21.0 \\
\hline 41.0 & 44.8 & 47.2 & 47.2 & 44.8 & 44.9 & 44.5 & 43.7 & 35.1 & 28.1 & 24.9 & 23.2 & 21.8 \\
\hline 47.7 & 50.8 & 53.6 & 53.9 & 49.9 & 49.7 & 48.7 & 47.9 & 39.0 & 33.6 & 30.0 & 28.3 & 24.8 \\
\hline 54.5 & 57.1 & 60.3 & 60.5 & 55.9 & 55.1 & 53.4 & 52.2 & 41.7 & 35.5 & 31.3 & 29.2 & 24.9 \\
\hline 61.4 & 59.7 & 63.3 & 65.8 & 61.8 & 60.9 & 59.6 & 58.7 & 44.6 & 39.0 & 35.6 & 33.9 & 26.2 \\
\hline 68.1 & 64.2 & 68.5 & 70.5 & 67.0 & 66.8 & 65.7 & 65.9 & 52.9 & 44.2 & 39.8 & 36.9 & 27.2 \\
\hline 75.1 & 56.0 & 70.2 & 73.4 & 71.0 & 71.1 & 71.0 & 71.3 & 57.0 & 49.4 & 43.9 & 41.2 & 32.0 \\
\hline 81.8 & 78.0 & 82.7 & 83.2 & 78.0 & 77.5 & 76.7 & 75.6 & 65.1 & 54.5 & 50.1 & 46.9 & 34.7 \\
\hline 88.6 & 80.8 & 85.7 & 88.4 & 84.7 & 84.3 & 83.4 & 82.7 & 69.3 & 58.7 & 54.5 & 50.8 & 39.4 \\
\hline 95.5 & 82.9 & 88.0 & 91.4 & 89.0 & 88.3 & 87.9 & 86.1 & 72.8 & 64.4 & 59.1 & 55.1 & 42.4 \\
\hline 102.3 & 90.4 & 94.7 & 97.3 & 93.4 & 92.2 & 91.3 & 89.6 & 75.0 & 68.6 & 63.5 & 59.0 & 43.9 \\
\hline 109.2 & 95.7 & 100.5 & 100.5 & 95.7 & 94.9 & 93.5 & 91.8 & 77.6 & 71.2 & 66.3 & 61.3 & 44.1 \\
\hline 115.9 & 92.5 & 98.9 & 103.0 & 98.9 & 98.9 & 98.8 & 99.9 & 85.4 & 72.2 & 68.8 & 64.5 & 48.7 \\
\hline \multicolumn{13}{|c|}{ Thermocouples located at sprinkler number 4} \\
\hline Time & $0 \mathrm{~mm}$ & $25 \mathrm{~mm}$ & $50 \mathrm{~mm}$ & $75 \mathrm{~mm}$ & $100 \mathrm{~mm}$ & $125 \mathrm{~mm}$ & $150 \mathrm{~mm}$ & $250 \mathrm{~mm}$ & $350 \mathrm{~mm}$ & $450 \mathrm{~mm}$ & $550 \mathrm{~mm}$ & $900 \mathrm{~mm}$ \\
\hline 0.0 & 22.7 & 22.8 & 22.3 & 22.5 & 22.2 & 22.1 & 22.1 & 21.5 & 21.3 & 21.0 & 20.9 & 20.1 \\
\hline
\end{tabular}




\section{Experiment Number 40}

\begin{tabular}{|c|c|c|c|c|c|c|c|c|c|c|c|c|}
\hline 6.9 & 22.8 & 22.9 & 22.5 & 22.7 & 22.2 & 22.3 & 22.3 & 21.9 & 21.5 & 21.1 & 21.0 & 20.6 \\
\hline 13.7 & 30.9 & 32.6 & 34.0 & 35.5 & 33.4 & 31.9 & 33.4 & 27.5 & 29.8 & 29.3 & 28.1 & 22.8 \\
\hline 20.4 & 47.9 & 48.0 & 49.7 & 48.1 & 46.4 & 42.6 & 46.1 & 34.4 & 26.0 & 22.9 & 22.1 & 21.4 \\
\hline 27.3 & 54.8 & 56.0 & 57.7 & 56.6 & 55.4 & 54.0 & 56.7 & 44.2 & 31.9 & 22.4 & 21.4 & 21.1 \\
\hline 34.1 & 60.8 & 61.5 & 64.4 & 64.3 & 65.3 & 62.7 & 65.6 & 53.1 & 40.2 & 23.4 & 21.5 & 21.1 \\
\hline 41.0 & 71.5 & 72.2 & 74.9 & 73.2 & 71.9 & 69.4 & 72.8 & 61.3 & 39.3 & 22.7 & 21.7 & 21.3 \\
\hline 47.7 & 79.6 & 81.6 & 82.4 & 81.5 & 81.5 & 78.3 & 82.0 & 67.8 & 48.3 & 24.0 & 21.9 & 21.9 \\
\hline 54.5 & 85.7 & 86.7 & 90.6 & 88.7 & 88.4 & 87.0 & 91.9 & 77.1 & 56.9 & 30.8 & 28.4 & 22.8 \\
\hline 61.4 & 88.9 & 89.1 & 91.7 & 91.9 & 93.8 & 91.3 & 95.6 & 84.4 & 73.1 & 37.1 & 32.7 & 24.9 \\
\hline 68.1 & 100.0 & 99.0 & 100.3 & 98.8 & 100.3 & 99.1 & 100.9 & 90.6 & 75.3 & 45.0 & 33.3 & 26.0 \\
\hline 75.1 & 108.4 & 109.8 & 113.0 & 113.7 & 113.0 & 110.0 & 113.3 & 98.3 & 76.0 & 45.1 & 38.0 & 27.4 \\
\hline 81.8 & 111.2 & 1136 & 117.6 & 116.2 & 117.6 & 114.7 & 119.4 & 105.4 & 86.3 & 51.6 & 44.7 & 31.3 \\
\hline 88.6 & 124.0 & 124.2 & 127.2 & 124.1 & 124.5 & 120.5 & 124.2 & 109.9 & 89.6 & 57.0 & 47.3 & 33.7 \\
\hline 95.5 & 125.0 & 126.7 & 130.7 & 130.1 & 131.2 & 128.4 & 134.0 & 115.2 & 98.0 & 64.2 & 53.4 & 37.9 \\
\hline 102.3 & 130.9 & 131.1 & 133.1 & 132.2 & 132.3 & 130.3 & 133.9 & 118.5 & 104.5 & 67.0 & 56.2 & 40.5 \\
\hline 109.2 & 132.6 & 130.9 & 134.0 & 133.9 & 134.5 & 131.7 & 134.4 & 122.4 & 107.5 & 75.4 & 60.0 & 40.9 \\
\hline 115.9 & 125.6 & 128.0 & 44.9 & 116.9 & 125.9 & 129.9 & 136.9 & 125.0 & 113.0 & 81.0 & 63.9 & 44.8 \\
\hline \multicolumn{5}{|c|}{ Velocity probes at sprinkler number 1} & \multicolumn{6}{|c|}{ Velocity probes at sprinkler number 2} & & \\
\hline Time & $25 \mathrm{~mm}$ & $75 \mathrm{~mm}$ & $125 \mathrm{~mm}$ & $250 \mathrm{~mm}$ & & Time & $25 \mathrm{~mm}$ & $75 \mathrm{~mm}$ & $125 \mathrm{~mm}$ & $250 \mathrm{~mm}$ & & \\
\hline 0.0 & 0.0 & 0.0 & 0.0 & 0.0 & & 0.0 & 0.0 & 0.0 & 0.0 & 0.0 & & \\
\hline 6.9 & 0.2 & 0.3 & 0.3 & 0.3 & & 6.9 & 0.1 & 0.1 & -0.1 & 0.0 & & \\
\hline 13.7 & 0.4 & 0.4 & 0.4 & 0.1 & & 13.7 & 0.1 & 0.1 & 0.1 & 0.0 & & \\
\hline 20.4 & 0.4 & 0.3 & 0.4 & 0.3 & & 20.4 & 0.1 & 0.2 & 0.2 & 0.1 & & \\
\hline 27.3 & 0.4 & 0.4 & 0.4 & 0.2 & & 27.3 & 0.4 & 0.5 & 0.5 & 0.5 & & \\
\hline 34.1 & 0.4 & 0.5 & 0.6 & 0.6 & & 34.1 & 0.4 & 0.5 & 0.5 & 0.5 & & \\
\hline 41.0 & 0.4 & 0.4 & 0.4 & -0.2 & & 41.0 & 0.5 & 0.4 & 0.5 & 0.4 & & \\
\hline 47.7 & 0.3 & 0.4 & 0.5 & 0.1 & & 47.7 & 0.4 & 0.4 & 0.5 & 0.4 & & \\
\hline 54.5 & -0.2 & 0.2 & 0.5 & 0.4 & & 54.5 & 0.5 & 0.4 & 0.5 & 0.5 & & \\
\hline 61.4 & 0.5 & 0.4 & 0.5 & 0.5 & & 61.1 & 0.5 & 0.5 & 0.5 & 0.4 & & \\
\hline 68.1 & 0.1 & 0.3 & 0.6 & 0.3 & & 68.1 & 0.7 & 0.7 & 0.6 & 0.5 & & \\
\hline 75.1 & 0.1 & 0.0 & 0.4 & 0.4 & & 75.1 & 0.6 & 0.6 & 0.7 & 0.6 & & \\
\hline 81.8 & 0.3 & 0.3 & 0.5 & 0.6 & & 81.8 & 0.6 & 0.6 & 0.6 & 0.5 & & \\
\hline 88.6 & -0.3 & -0.2 & 0.4 & 0.6 & & 88.6 & 0.4 & 0.3 & 0.4 & 0.5 & & \\
\hline 95.5 & 0.4 & 0.4 & 0.6 & 0.5 & & 95.5 & 0.5 & 0.5 & 0.6 & 0.5 & & \\
\hline 102.3 & 0.5 & 0.5 & 0.7 & 0.7 & & 102.3 & 0.5 & 0.4 & 0.5 & 0.3 & & \\
\hline 109.2 & 0.4 & 0.5 & 0.7 & 0.6 & & 109.2 & 0.5 & 0.5 & 0.6 & 0.2 & & \\
\hline 115.9 & -0.4 & -0.1 & 0.4 & 0.6 & & 115.9 & 0.5 & 0.5 & 0.6 & 0.4 & & \\
\hline \multicolumn{13}{|c|}{ Velocity probes in channels above burner } \\
\hline Time & Channel 1 & Channel 2 & & & & & & & & & & \\
\hline 0.0 & 0.0 & 0.0 & & & & & & & & & & \\
\hline 6.9 & 2.2 & 1.2 & & & & & & & & & & \\
\hline 13.7 & 2.8 & 1.7 & & & & & & & & & & \\
\hline 20.4 & 2.9 & 1.3 & & & & & & & & & & \\
\hline 27.3 & 3.7 & 1.7 & & & & & & & & & & \\
\hline 34.1 & 3.1 & 1.7 & & & & & & & & & & \\
\hline 41.0 & 3.9 & 2.0 & & & & & & & & & & \\
\hline 47.7 & 3.9 & 1.8 & & & & & & & & & & \\
\hline 54.5 & 3.9 & 2.1 & & & & & & & & & & \\
\hline 61.4 & 4.1 & 2.4 & & & & & & & & & & \\
\hline 68.1 & 4.4 & 2.3 & & & & & & & & & & \\
\hline 75.1 & 3.6 & 2.9 & & & & & & & & & & \\
\hline 81.8 & 4.6 & 1.9 & & & & & & & & & & \\
\hline 88.6 & 3.6 & 2.9 & & & & & & & & & & \\
\hline 95.5 & 4.4 & 2.6 & & & & & & & & & & \\
\hline 102.3 & 3.8 & 2.7 & & & & & & & & & & \\
\hline 109.2 & 5.2 & 3.1 & & & & & & & & & & \\
\hline 115.9 & 5.1 & 3.2 & & & & & & & & & & \\
\hline
\end{tabular}


Experiment Number 41

Thermocouples located at sprinkler number 1

Time $\quad 0 \mathrm{~mm} \quad 25 \mathrm{~mm} \quad 50 \mathrm{~mm} \quad 75 \mathrm{~mm} \quad 100 \mathrm{~mm} \quad 125 \mathrm{~mm} \quad 150 \mathrm{~mm} \quad 250 \mathrm{~mm} \quad 350 \mathrm{~mm} \quad 450 \mathrm{~mm} \quad 550 \mathrm{~mm} \quad 900 \mathrm{~mm}$

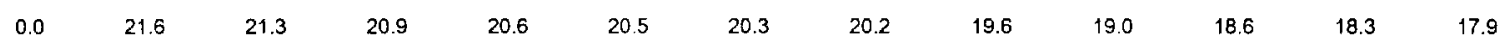

$\begin{array}{llllllllllll}4.2 & 21.5 & 21.4 & 21.0 & 20.7 & 20.5 & 20.5 & 20.4 & 19.9 & 19.5 & 19.1 & 18.7\end{array}$

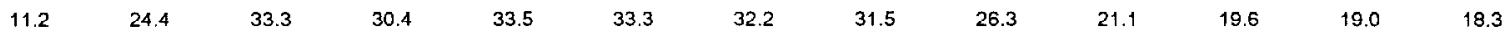

$\begin{array}{lllllllllllll}17.9 & 31.3 & 51.4 & 48.8 & 50.4 & 50.7 & 48.1 & 45.7 & 30.2 & 22.4 & 19.6 & 19.0 & 18.5\end{array}$

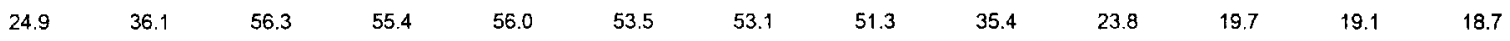

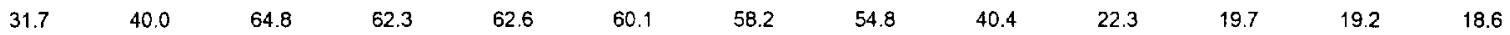

$\begin{array}{lllllllllllll}38.5 & 43.3 & 72.1 & 71.4 & 72.3 & 68.3 & 65.8 & 62.9 & 44.6 & 26.1 & 20.3 & 19.5 & 19.0\end{array}$

$\begin{array}{lllllllllllll}45.4 & 44.0 & 69.5 & 68.6 & 67.1 & 66.1 & 65.1 & 61.8 & 47.5 & 29.9 & 20.7 & 20.0 & 19.4\end{array}$

$\begin{array}{lllllllllllll}52.2 & 50.6 & 79.5 & 79.2 & 77.6 & 74.6 & 72.8 & 70.0 & 57.8 & 36.6 & 23.6 & 22.2 & 20.0\end{array}$

$\begin{array}{lllllllllllll}59.1 & 55.2 & 90.1 & 89.4 & 92.8 & 90.6 & 89.9 & 88.5 & 75.1 & 50.8 & 33.7 & 27.1 & 20.6\end{array}$

$\begin{array}{llllllllllll}65.9 & 60.4 & 101.9 & 100.0 & 103.5 & 100.9 & 97.5 & 93.4 & 74.0 & 51.2 & 33.5 & 31.4\end{array}$

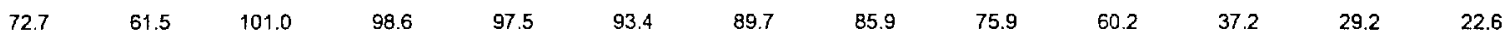

$\begin{array}{llllllllllll}79.6 & 64.4 & 108.7 & 107.7 & 109.7 & 106.5 & 103.4 & 99.3 & 82.4 & 60.1 & 49.0 & 41.4\end{array}$

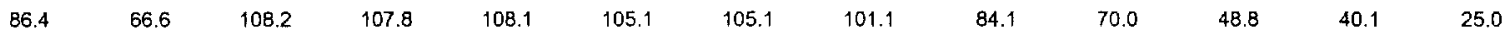

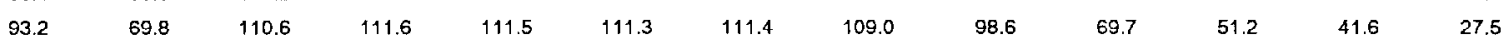

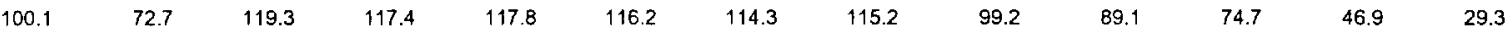

Thermocouples localed at sprinkler number 2

Time $\quad 0 \mathrm{~mm} \quad 25 \mathrm{~mm} \quad 50 \mathrm{~mm} \quad 75 \mathrm{~mm} \quad 100 \mathrm{~mm} \quad 125 \mathrm{~mm} \quad 150 \mathrm{~mm} \quad 250 \mathrm{~mm} \quad 350 \mathrm{~mm} \quad 450 \mathrm{~mm} \quad 550 \mathrm{~mm} \quad 900 \mathrm{~mm}$

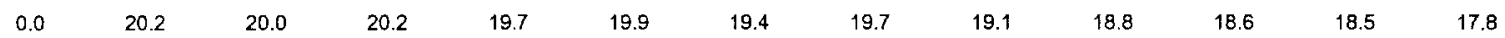

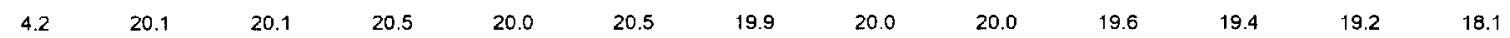

$\begin{array}{llllllllllll}11.2 & 20.3 & 20.2 & 20.5 & 19.9 & 20.5 & 19.9 & 20.1 & 20.1 & 19.5 & 19.3 & 19.2\end{array}$

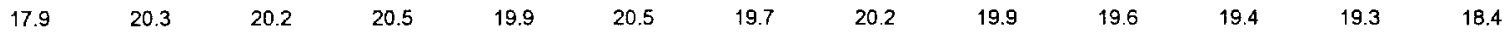

$\begin{array}{lllllllllllll}24.9 & 22.6 & 35.8 & 37.3 & 36.5 & 36.0 & 32.9 & 32.2 & 24.6 & 23.3 & 22.6 & 21.5 & 18.8\end{array}$

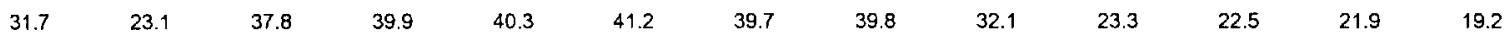

$\begin{array}{llllllllllll}38.5 & 24.6 & 43.5 & 45.6 & 45.9 & 46.9 & 45.6 & 46.7 & 43.6 & 34.1 & 28.5 & 27.7 \\ 4 & 25.7 & 48.7 & 50.3 & 22.5\end{array}$

$\begin{array}{lllllllllllll}45.4 & 25.7 & 48.7 & 50.3 & 50.2 & 50.4 & 49.5 & 49.5 & 46.9 & 36.8 & 34.6 & 33.7 & 21.2\end{array}$

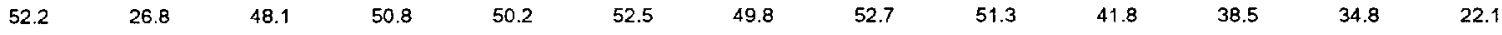

$\begin{array}{llllllllllll}59.1 & 27.7 & 54.1 & 56.4 & 57.5 & 57.4 & 55.9 & 57.0 & 56.2 & 48.4 & 43.9 & 39.6\end{array}$

$\begin{array}{llllllllllllll}65.9 & 30.7 & 65.1 & 66.7 & 67.6 & 68.9 & 68.8 & 69.8 & 66.1 & 50.0 & 46.5 & 39.6 & 23.3\end{array}$

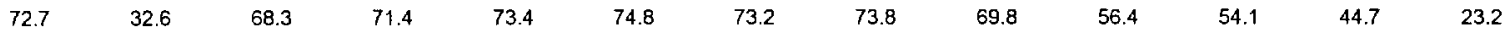

$\begin{array}{lllllllllllll}79.6 & 32.4 & 66.9 & 69.3 & 70.0 & 71.4 & 70.1 & 71.7 & 68.1 & 62.2 & 59.3 & 49.9 & 27.1\end{array}$

$\begin{array}{lllllllllllll}86.4 & 35.1 & 76.0 & 80.6 & 82.1 & 83.1 & 81.1 & 82.7 & 74.8 & 66.3 & 60.7 & 49.7 & 29.9\end{array}$

$\begin{array}{lllllllllllll}93.2 & 35.0 & 77.2 & 78.3 & 79.4 & 80.3 & 78.4 & 80.5 & 77.1 & 68.2 & 65.0 & 59.9 & 37.1\end{array}$

$\begin{array}{lllllllllllll}100.1 & 36.6 & 79.5 & 83.9 & 85.4 & 86.6 & 84.5 & 86.1 & 83.4 & 77.1 & 70.5 & 65.7 & 37.0\end{array}$

Thermocouples located at sprinkler number 3

\begin{tabular}{|c|c|c|c|c|c|c|c|c|c|c|c|c|}
\hline Time & $0 \mathrm{~mm}$ & $25 \mathrm{~mm}$ & $50 \mathrm{~mm}$ & $75 \mathrm{~mm}$ & $100 \mathrm{~mm}$ & $125 \mathrm{~mm}$ & $150 \mathrm{~mm}$ & $250 \mathrm{~mm}$ & $350 \mathrm{~mm}$ & $450 \mathrm{~mm}$ & $550 \mathrm{~mm}$ & $900 \mathrm{~mm}$ \\
\hline 0.0 & 19.7 & 20.0 & 19.6 & 19.6 & 19.5 & 19.5 & 19.4 & 19.2 & 18.9 & 18.7 & 18.5 & 17.9 \\
\hline 4.2 & 19.6 & 20.1 & 19.9 & 19.9 & 19.9 & 20.0 & 20.0 & 20.0 & 19.7 & 19.4 & 19.2 & 18.0 \\
\hline 11.2 & 20.0 & 20.3 & 19.9 & 19.9 & 19.9 & 20.0 & 20.0 & 20.0 & 19.8 & 19.4 & 19.2 & 18.0 \\
\hline 17.9 & 19.9 & 20.3 & 19.9 & 20.0 & 20.0 & 20.1 & 20.0 & 20.0 & 19.9 & 19.5 & 19.2 & 18.3 \\
\hline 24.9 & 20.6 & 24.3 & 25.1 & 24.8 & 24.7 & 24.7 & 24.8 & 22.9 & 22.0 & 20.7 & 20.0 & 19.1 \\
\hline 31.7 & 21.2 & 25.9 & 29.5 & 28.5 & 29.4 & 29.4 & 29.1 & 26.2 & 25.1 & 22.8 & 22.5 & 19.4 \\
\hline 38.5 & 22.0 & 29.7 & 32.2 & 32.3 & 31.7 & 31.6 & 31.3 & 28.6 & 28.2 & 26.8 & 25.9 & 20.3 \\
\hline 45.4 & 22.3 & 30.6 & 33.8 & 34.0 & 33.5 & 33.7 & 34.2 & 32.1 & 31.9 & 30.6 & 30.1 & 21.9 \\
\hline 52.2 & 22.9 & 35.4 & 38.8 & 40.0 & 38.1 & 38.9 & 38.8 & 34.9 & 33.8 & 33.1 & 32.7 & 21.8 \\
\hline 59.1 & 23.1 & 35.4 & 39.9 & 40.8 & 39.7 & 40.5 & 40.4 & 38.1 & 38.2 & 35.9 & 36.2 & 22.2 \\
\hline 65.9 & 23.7 & 37.6 & 42.6 & 44.2 & 42.8 & 44.1 & 44.3 & 42.0 & 41.4 & 39.7 & 39.7 & 23.4 \\
\hline 72.7 & 24.0 & 39.4 & 45.7 & 48.1 & 46.7 & 48.1 & 47.9 & 47.0 & 46.0 & 44.0 & 44.6 & 27.7 \\
\hline 79.6 & 25.9 & 44.3 & 51.4 & 52.3 & 51.9 & 53.5 & 54.5 & 51.4 & 50.6 & 48.1 & 47.8 & 26.6 \\
\hline 86.4 & 26.4 & 49.7 & 57.9 & 59.5 & 57.1 & 58.0 & 58.2 & 55.5 & 55.3 & 52.6 & 52.3 & 31.6 \\
\hline 93.2 & 28.1 & 50.8 & 61.4 & 62.3 & 61.2 & 62.3 & 62.3 & 58.3 & 56.5 & 55.6 & 55.9 & 37.0 \\
\hline 100.1 & 29.3 & 53.0 & 63.4 & 63.8 & 64.1 & 65.3 & 66.3 & 62.9 & 60.9 & 59.3 & 59.1 & 39.2 \\
\hline \multicolumn{13}{|c|}{ ocouples localed at sprinkler number 4} \\
\hline Time & $0 \mathrm{~mm}$ & $25 \mathrm{~mm}$ & $50 \mathrm{~mm}$ & $75 \mathrm{~mm}$ & $100 \mathrm{~mm}$ & $125 \mathrm{~mm}$ & $150 \mathrm{~mm}$ & $250 \mathrm{~mm}$ & $350 \mathrm{~mm}$ & $450 \mathrm{~mm}$ & $550 \mathrm{~mm}$ & $900 \mathrm{~mm}$ \\
\hline 0.0 & 19.9 & 20.0 & 19.9 & 19.9 & 19.6 & 19.6 & 19.5 & 19.1 & 18.9 & 18.5 & 18.2 & 17.9 \\
\hline 4.2 & 19.7 & 20.1 & 20.1 & 20.1 & 20.0 & 20.0 & 20.0 & 19.5 & 19.1 & 18.8 & 18.5 & 18.2 \\
\hline 11.2 & 20.1 & 20.5 & 20.5 & 20.5 & 20.3 & 20.2 & 20.1 & 19.7 & 19.4 & 19.1 & 18.9 & 18.5 \\
\hline 17.9 & 22.0 & 31.3 & 30.1 & 29.8 & 27.8 & 26.9 & 24.8 & 22.0 & 21.8 & 20.5 & 19.5 & 18.6 \\
\hline 24.9 & 23.5 & 34.9 & 34.8 & 34.7 & 32.6 & 32.6 & 31.9 & 23.5 & 23.8 & 21.2 & 20.1 & 18.7 \\
\hline 31.7 & 24.4 & 36.0 & 36.5 & 36.3 & 34.9 & 35.1 & 34.6 & 28.6 & 25.2 & 22.1 & 20.3 & 18.9 \\
\hline 38.5 & 26.8 & 41.8 & 40.4 & 39.5 & 37.0 & 36.5 & 35.1 & 31.1 & 30.6 & 25.2 & 19.8 & 18.8 \\
\hline
\end{tabular}


Experiment Number 41

\begin{tabular}{|c|c|c|c|c|c|c|c|c|c|c|c|c|}
\hline 45.4 & 27.8 & 45.0 & 45.1 & 44.5 & 42.1 & 42.3 & 41.7 & 34.6 & 32.8 & 25.5 & 19.7 & 18.9 \\
\hline 52.2 & 27.2 & 47.9 & 47.4 & 46.8 & 45.3 & 45.2 & 44.0 & 38.4 & 37.1 & 34.2 & 32.5 & 20.0 \\
\hline 59.1 & 29.1 & 53.5 & 54.2 & 54.4 & 51.9 & 52.6 & 52.3 & 44.0 & 41.8 & 37.9 & 35.4 & 21.0 \\
\hline 65.9 & 29.5 & 56.5 & 58.7 & 58.6 & 56.1 & 56.3 & 55.8 & 48.0 & 43.4 & 39.9 & 34.6 & 20.8 \\
\hline 72.7 & 31.4 & 64.4 & 64.4 & 63.6 & 61.1 & 62.1 & 60.5 & 50.1 & 48.6 & 45.8 & 39.5 & 23.1 \\
\hline 79.6 & 32.7 & 66.5 & 67.9 & 67.6 & 65.0 & 66.0 & 65.2 & 53.8 & 52.9 & 49.5 & 43.6 & 27.5 \\
\hline 86.4 & 37.5 & 75.4 & 72.9 & 71.2 & 68.0 & 67.7 & 67.7 & 59.0 & 58.3 & 54.5 & 45.9 & 27.1 \\
\hline 93.2 & 38.4 & 80.4 & 80.3 & 79.0 & 75.2 & 75.4 & 73.5 & 62.5 & 62.8 & 60.5 & 54.2 & 27.5 \\
\hline 100.1 & 38.9 & 82.5 & 85.4 & 84.4 & 80.8 & 82.4 & 80.4 & 67.6 & 66.2 & 64.9 & 56.5 & 30.4 \\
\hline \multicolumn{5}{|c|}{ Velocity probes at sprinkler number 1} & \multicolumn{6}{|c|}{ Velocity probes at sprinkler number 2} & & \\
\hline Time & $25 \mathrm{~mm}$ & $75 \mathrm{~mm}$ & $125 \mathrm{~mm}$ & $250 \mathrm{~mm}$ & & Time & $25 \mathrm{~mm}$ & $75 \mathrm{~mm}$ & $125 \mathrm{~mm}$ & $250 \mathrm{~mm}$ & & \\
\hline 0.0 & 0.0 & 0.0 & 0.0 & 0.0 & & 0.0 & 0.0 & 0.0 & 0.0 & 0.0 & & \\
\hline 4.2 & 0.4 & 0.8 & 0.9 & 0.3 & & 4.2 & 0.0 & 0.0 & 0.1 & 0.1 & & \\
\hline 11.2 & 1.3 & 1.2 & 0.7 & 0.0 & & 11.2 & 0.0 & 0.0 & 0.1 & 0.1 & & \\
\hline 17.9 & 1.3 & 1.1 & 0.9 & -0.1 & & 17.9 & 0.8 & 0.8 & 0.7 & 0.5 & & \\
\hline 24.9 & 1.2 & 1.3 & 0.7 & 0.3 & & 24.9 & 0.7 & 0.7 & 0.7 & 0.5 & & \\
\hline 31.7 & 1.6 & 1.7 & 1.3 & 0.3 & & 31.7 & 0.6 & 0.7 & 0.7 & 0.5 & & \\
\hline 38.5 & 1.8 & 1.6 & 1.3 & 0.6 & & 38.5 & 0.5 & 0.5 & 0.4 & 0.3 & & \\
\hline 45.4 & 1.9 & 1.6 & 1.1 & 0.4 & & 45.4 & 0.6 & 0.6 & 0.5 & 0.4 & & \\
\hline 52.2 & 1.5 & 1.2 & 0.7 & 0.5 & & 52.2 & 0.6 & 0.7 & 0.7 & 0.5 & & \\
\hline 59.1 & 1.5 & 1.2 & 1.3 & 0.4 & & 59.1 & 0.8 & 0.8 & 0.8 & 0.5 & & \\
\hline 65.9 & 1.8 & 2.0 & 1.6 & 0.9 & & 65.9 & 0.8 & 0.8 & 0.7 & 0.5 & & \\
\hline 72.7 & 1.6 & 1.6 & 1.2 & 0.7 & & 72.7 & 0.5 & 0.5 & 0.5 & 0.2 & & \\
\hline 79.6 & 1.8 & 1.7 & 1.3 & 0.4 & & 79.6 & 0.8 & 0.8 & 0.8 & 0.6 & & \\
\hline 86.4 & 1.7 & 1.7 & 0.7 & -0.2 & & 86.4 & 0.6 & 0.6 & 0.6 & 0.5 & & \\
\hline 93.2 & 1.5 & 1.6 & 1.2 & 0.7 & & 93.2 & 0.7 & 0.7 & 0.7 & 0.4 & & \\
\hline 100.1 & 1.7 & 1.8 & 1.5 & 1.2 & & 100.1 & 0.9 & 0.9 & 0.9 & 0.5 & & \\
\hline
\end{tabular}


Experiment Number 42

Thermocouples localed at sprinkler number 1

\begin{tabular}{|c|c|c|c|c|c|c|c|c|c|c|c|c|}
\hline Time & $0 \mathrm{~mm}$ & $25 \mathrm{~mm}$ & $50 \mathrm{~mm}$ & $75 \mathrm{~mm}$ & $100 \mathrm{~mm}$ & $125 \mathrm{~mm}$ & $150 \mathrm{~mm}$ & $250 \mathrm{~mm}$ & $350 \mathrm{~mm}$ & $450 \mathrm{~mm}$ & $550 \mathrm{~mm}$ & $900 \mathrm{~mm}$ \\
\hline 0.0 & 22.4 & 21.9 & 21.5 & 21.2 & 21.1 & 20.9 & 20.8 & 20.3 & 19.9 & 19.4 & 18.9 & 18.3 \\
\hline 2.4 & 22.3 & 21.9 & 21.8 & 21.6 & 21.5 & 21.3 & 21.3 & 20.8 & 20.4 & 20.0 & 19.6 & 18.7 \\
\hline 9.2 & 24.0 & 31.5 & 28.7 & 30.0 & 29.0 & 28.3 & 27.5 & 23.8 & 21.8 & 20.5 & 20.0 & 18.9 \\
\hline 16.2 & 29.6 & 43.2 & 40.9 & 41.6 & 38.4 & 37.1 & 35.5 & 28.7 & 21.0 & 20.4 & 19.9 & 18.9 \\
\hline 23.0 & 35.6 & 54.0 & 52.6 & 52.7 & 50.4 & 49.7 & 46.4 & 37.4 & 23.0 & 20.5 & 19.9 & 19.0 \\
\hline 29.9 & 38.8 & 58.2 & 57.9 & 57.4 & 56.0 & 53.6 & 51.3 & 34.1 & 23.7 & 20.2 & 19.7 & 19.2 \\
\hline 36.7 & 43.2 & 70.4 & 68.1 & 69.3 & 66.0 & 63.0 & 58.2 & 46.3 & 25.9 & 20.6 & 20.0 & 19.4 \\
\hline 43.5 & 45.3 & $/ 1.2$ & 70.1 & 68.7 & 66.5 & 65.0 & 61.4 & 44.7 & 23.5 & 21.4 & 20.9 & 19.6 \\
\hline 50.4 & 47.9 & 75.5 & 76.7 & 78.0 & 79.5 & 76.8 & 71.0 & 55.4 & 35.3 & 23.9 & 22.9 & 20.2 \\
\hline 57.2 & 54.5 & 910 & 88.8 & 89.9 & 88.7 & 88.0 & 83.0 & 60.8 & 42.7 & 34.8 & 26.3 & 20.8 \\
\hline 64.2 & 61.2 & 106.3 & 103.1 & 104.6 & 103.0 & 98.2 & 92.6 & 75.2 & 50.0 & 33.7 & 29.3 & 21.1 \\
\hline 70.9 & 59.8 & 97.8 & 95.2 & 96.4 & 95.4 & 95.5 & 94.8 & 85.4 & 65.3 & 39.0 & 31.5 & 22.5 \\
\hline 77.7 & 62.1 & 99.5 & 100.2 & 102.9 & 102.0 & 100.5 & 97.5 & 75.4 & 62.5 & 45.7 & 37.7 & 23.9 \\
\hline 84.6 & 70.3 & 113.9 & 112.4 & 113.6 & 108.8 & 107.6 & 103.3 & 90.2 & 60.1 & 49.9 & 39.9 & 24.1 \\
\hline \multicolumn{13}{|c|}{ mocouples located at sprinkler number 2} \\
\hline Time & $0 \mathrm{~mm}$ & $25 \mathrm{~mm}$ & $50 \mathrm{~mm}$ & $75 \mathrm{~mm}$ & $100 \mathrm{~mm}$ & $125 \mathrm{~mm}$ & $150 \mathrm{~mm}$ & $250 \mathrm{~mm}$ & $350 \mathrm{~mm}$ & $450 \mathrm{~mm}$ & $550 \mathrm{~mm}$ & $900 \mathrm{~mm}$ \\
\hline 0.0 & 20.7 & 20.5 & 20.9 & 20.4 & 20.8 & 20.2 & 20.5 & 19.8 & 19.6 & 19.3 & 19.1 & 18.1 \\
\hline 2.4 & 20.7 & 21.1 & 21.3 & 20.9 & 21.3 & 20.5 & 21.1 & 20.5 & 20.4 & 20.1 & 19.7 & 18.3 \\
\hline 9.2 & 20.8 & 21.1 & 21.3 & 20.9 & 21.2 & 20.3 & 21.0 & 20.6 & 20.5 & 20.2 & 19.8 & 18.3 \\
\hline 16.2 & 20.7 & 21.2 & 21.4 & 21.1 & 21.3 & 20.7 & 21.2 & 20.6 & 20.5 & 20.3 & 20.1 & 18.5 \\
\hline 23.0 & 22.1 & 30.8 & 31.9 & 32.0 & 32.5 & 31.5 & 30.8 & 26.7 & 24.9 & 22.7 & 22.3 & 19.7 \\
\hline 29.9 & 23.6 & 38.7 & 41.0 & 40.8 & 41.1 & 40.4 & 40.4 & 36.6 & 24.5 & 21.0 & 20.8 & 19.0 \\
\hline 36.7 & 25.0 & 45.0 & 46.4 & 45.7 & 46.9 & 43.8 & 45.3 & 40.7 & 31.1 & 27.5 & 27.9 & 22.2 \\
\hline 43.5 & 26.0 & 47.0 & 48.0 & 48.2 & 48.8 & 46.8 & 47.8 & 40.3 & 35.4 & 34.4 & 32.5 & 20.8 \\
\hline 50.4 & 26.3 & 46.9 & 49.0 & 47.7 & 49.8 & 47.4 & 49.5 & 45.4 & 40.3 & 39.2 & 35.5 & 21.6 \\
\hline 57.2 & 27.3 & 55.1 & 56.9 & 54.3 & 56.3 & 51.7 & 52.4 & 49.2 & 44.0 & 39.9 & 34.9 & 22.7 \\
\hline 64.2 & 30.7 & 63.0 & 66.5 & 68.0 & 67.9 & 65.8 & 65.5 & 57.5 & 48.0 & 43.9 & 37.9 & 23.8 \\
\hline 70.9 & 32.3 & 68.1 & 70.8 & 72.5 & 73.8 & 72.2 & 72.9 & 70.1 & 54.8 & 51.9 & 48.1 & 25.2 \\
\hline 77.7 & 32.3 & 65.1 & 69.5 & 69.4 & 72.0 & 69.5 & 71.1 & 68.5 & 61.1 & 58.3 & 47.3 & 24.1 \\
\hline 84.6 & 32.8 & 72.6 & 75.1 & 75.9 & 77.5 & 74.7 & 76.9 & 75.3 & 68.7 & 64.0 & 51.1 & 31.5 \\
\hline \multicolumn{13}{|c|}{ ermocouples located at sprinkler number 3} \\
\hline Time & $0 \mathrm{~mm}$ & $25 \mathrm{~mm}$ & $50 \mathrm{~mm}$ & $75 \mathrm{~mm}$ & $100 \mathrm{~mm}$ & $125 \mathrm{~mm}$ & $150 \mathrm{~mm}$ & $250 \mathrm{~mm}$ & $350 \mathrm{~mm}$ & $450 \mathrm{~mm}$ & $550 \mathrm{~mm}$ & $900 \mathrm{~mm}$ \\
\hline 0.0 & 20.2 & 20.5 & 20.4 & 20.4 & 20.3 & 20.4 & 20.3 & 20.1 & 19.8 & 19.5 & 19.2 & 18.2 \\
\hline 2.4 & 20.2 & 20.6 & 20.4 & 20.4 & 20.5 & 20.6 & 20.6 & 20.6 & 20.3 & 19.8 & 19.7 & 18.4 \\
\hline 9.2 & 20.3 & 20.7 & 20.6 & 20.6 & 20.5 & 20.6 & 20.6 & 20.6 & 20.5 & 20.0 & 19.9 & 18.5 \\
\hline 16.2 & 20.2 & 20.7 & 20.5 & 20.5 & 20.5 & 20.7 & 20.7 & 20.6 & 20.6 & 20.0 & 19.8 & 18.7 \\
\hline 23.0 & 20.8 & 24.3 & 24.7 & 24.6 & 24.6 & 24.5 & 24.8 & 22.6 & 21.8 & 20.5 & 20.4 & 19.0 \\
\hline 29.9 & 21.3 & 25.1 & 28.3 & 27.7 & 28.4 & 28.1 & 27.8 & 25.7 & 25.1 & 24.1 & 23.3 & 19.9 \\
\hline 36.7 & 22.0 & 28.3 & 31.5 & 31.6 & 31.8 & 32.0 & 32.2 & 29.0 & 27.7 & 25.3 & 23.9 & 20.6 \\
\hline 43.5 & 22.3 & 29.3 & 32.9 & 33.4 & 33.1 & 33.3 & 33.1 & 30.4 & 30.7 & 29.3 & 28.9 & 22.6 \\
\hline 50.4 & 22.6 & 34.0 & 37.5 & 38.2 & 36.3 & 36.8 & 36.7 & 34.9 & 34.5 & 32.1 & 32.0 & 22.1 \\
\hline 57.2 & 23.2 & 35.3 & 39.5 & 40.3 & 39.7 & 40.4 & 40.4 & 37.9 & 36.6 & 35.1 & 34.7 & 22.4 \\
\hline 64.2 & 24.1 & 38.1 & 42.1 & 43.2 & 42.7 & 43.9 & 44.4 & 42.6 & 39.4 & 38.7 & 38.3 & 23.7 \\
\hline 70.9 & 25.4 & 40.2 & 46.5 & 47.5 & 46.6 & 47.9 & 47.7 & 45.2 & 43.7 & 41.3 & 42.3 & 25.0 \\
\hline 77.7 & 25.5 & 42.7 & 49.6 & 50.2 & 50.0 & 51.3 & 52.6 & 50.3 & 47.6 & 45.1 & 46.3 & 25.2 \\
\hline 84.6 & 26.3 & 48.2 & 55.1 & 57.0 & 55.3 & 56.6 & 57.9 & 54.8 & 52.2 & 50.1 & 49.8 & 30.7 \\
\hline \multicolumn{13}{|c|}{ ermocouples located at sprinkler number 4} \\
\hline Time & $0 \mathrm{~mm}$ & $25 \mathrm{~mm}$ & $50 \mathrm{~mm}$ & $75 \mathrm{~mm}$ & $100 \mathrm{~mm}$ & $125 \mathrm{~mm}$ & $150 \mathrm{~mm}$ & $250 \mathrm{~mm}$ & $350 \mathrm{~mm}$ & $450 \mathrm{~mm}$ & $550 \mathrm{~mm}$ & $900 \mathrm{~mm}$ \\
\hline 0.0 & 20.2 & 20.6 & 20.6 & 20.5 & 20.3 & 20.2 & 20.1 & 19.8 & 19.4 & 19.1 & 18.7 & 18.3 \\
\hline 2.4 & 20.2 & 20.9 & 20.9 & 20.9 & 20.7 & 20.7 & 20.6 & 20.3 & 20.2 & 19.9 & 19.5 & 18.5 \\
\hline 9.2 & 20.4 & 21.1 & 21.1 & 21.2 & 21.1 & 21.2 & 21.2 & 20.5 & 20.1 & 19.9 & 19.5 & 18.6 \\
\hline 16.2 & 22.6 & 31.0 & 30.0 & 29.6 & 26.5 & 25.2 & 23.6 & 22.7 & 22.4 & 20.8 & 20.1 & 18.9 \\
\hline 23.0 & 24.9 & 36.1 & 35.4 & 34.7 & 32.3 & 31.6 & 29.8 & 24.1 & 21.5 & 20.9 & 20.4 & 19.1 \\
\hline 29.9 & 24.8 & 36.2 & 36.2 & 36.0 & 34.3 & 34.0 & 33.1 & 28.6 & 26.6 & 22.7 & 20.9 & 19.2 \\
\hline 36.7 & 24.4 & 38.7 & 39.0 & 38.8 & 36.9 & 37.4 & 36.7 & 32.2 & 29.6 & 25.5 & 20.4 & 19.0 \\
\hline 43.5 & 27.0 & 44.2 & 44.4 & 43.6 & 40.5 & э9.6 & 38.6 & 34.0 & 31.6 & 26.8 & 20.3 & 19.1 \\
\hline 50.4 & 26.5 & 48.8 & 48.8 & 47.7 & 45.5 & 45.7 & 44.7 & 37.4 & 35.9 & 34.2 & 33.0 & 20.3 \\
\hline 57.2 & 28.3 & 53.4 & 52.9 & 52.7 & 50.4 & 51.5 & 51.0 & 43.2 & 40.6 & 37.0 & 34.5 & 21.3 \\
\hline 64.2 & 30.5 & 55.6 & 56.9 & 56.6 & 54.8 & 54.9 & 53.7 & 43.1 & 43.1 & 38.9 & 34.2 & 21.1 \\
\hline 70.9 & 29.9 & 56.9 & 57.8 & 57.5 & 55.7 & 56.1 & 55.5 & 48.7 & 48.3 & 44.5 & 40.5 & 24.4 \\
\hline 77.7 & 33.4 & 67.1 & 61.0 & 66.6 & 64.2 & 65.2 & 64.4 & 50.1 & 51.2 & 48.0 & 42.0 & 27.2 \\
\hline
\end{tabular}


Experiment Number 42

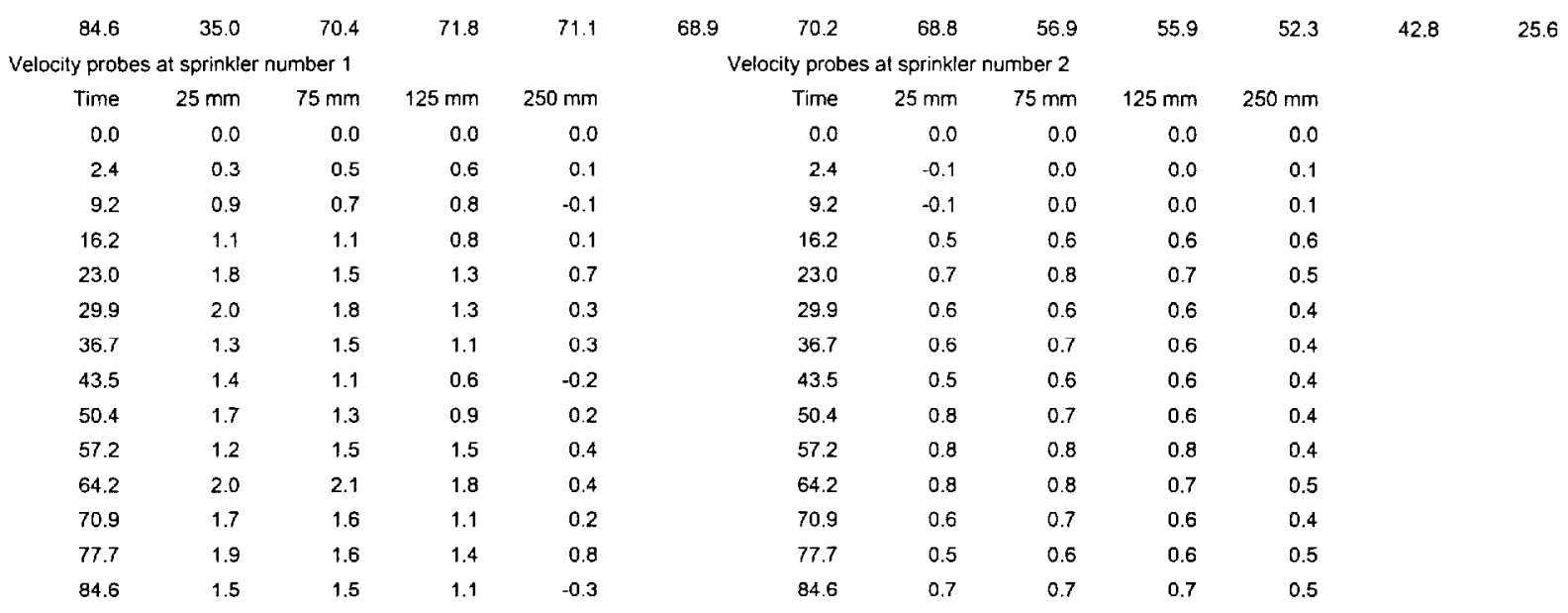


Experiment Number 43

Thermocouples located at sprinkler number 1

\begin{tabular}{|c|c|c|c|c|c|c|c|c|c|c|c|c|}
\hline Time & $0 \mathrm{~mm}$ & $25 \mathrm{~mm}$ & $50 \mathrm{~mm}$ & $75 \mathrm{~mm}$ & $100 \mathrm{~mm}$ & $125 \mathrm{~mm}$ & $150 \mathrm{~mm}$ & $250 \mathrm{~mm}$ & $350 \mathrm{~mm}$ & $450 \mathrm{~mm}$ & $550 \mathrm{~mm}$ & $900 \mathrm{~mm}$ \\
\hline 0.0 & 21.6 & 21.1 & 20.8 & 20.7 & 20.6 & 20.4 & 20.3 & 20.0 & 19.6 & 19.2 & 18.9 & 18.2 \\
\hline 3.4 & 21.6 & 21.4 & 21.2 & 21.0 & 20.5 & 20.8 & 20.8 & 20.5 & 20.2 & 19.9 & 19.4 & 18.6 \\
\hline 10.2 & 22.9 & 27.4 & 26.1 & 27.6 & 27.9 & 25.5 & 24.9 & 25.3 & 23.6 & 21.3 & 19.7 & 18.5 \\
\hline 17.2 & 28.8 & 44.2 & 42.4 & 43.4 & 43.0 & 40.8 & 39.0 & 27.0 & 21.1 & 20.2 & 19.5 & 18.4 \\
\hline 23.9 & 34.3 & 53.5 & 52.1 & 53.1 & 53.5 & 51.4 & 48.4 & 39.7 & 27.4 & 20.3 & 19.6 & 18.7 \\
\hline 30.9 & 39.4 & 61.8 & 60.0 & 58.5 & 55.6 & 52.7 & 48.3 & 35.2 & 23.9 & 20.2 & 19.6 & 18.5 \\
\hline 37.6 & 42.4 & 69.9 & 67.4 & 68.7 & 66.6 & 63.5 & 58.5 & 43.8 & 25.6 & 20.4 & 19.9 & 19.0 \\
\hline 44.4 & 45.4 & 72.7 & 71.3 & 71.6 & 66.3 & 62.5 & 60.1 & 47.1 & 22.1 & 21.1 & 20.5 & 19.7 \\
\hline 51.3 & 47.2 & 74.4 & 73.6 & 74.7 & 74.4 & 71.7 & 70.0 & 55.8 & 33.5 & 24.3 & 23.0 & 20.2 \\
\hline 58.1 & 53.8 & 87.8 & 87.0 & 884 & 860 & 83.4 & 79.9 & 65.8 & 41.8 & 32.0 & 26.9 & 20.8 \\
\hline 65.1 & 57.4 & 96.7 & 96.1 & 98.3 & 95.0 & 94.6 & 94.3 & 75.5 & 52.6 & 33.8 & 29.8 & 21.4 \\
\hline 71.9 & 61.4 & 101.2 & 98.9 & 102.4 & 100.6 & 100.7 & 98.5 & 78.8 & 53.5 & 38.3 & 34.6 & 22.1 \\
\hline 78.7 & 60.5 & 102.1 & 100.7 & 102.9 & 100.4 & 98.9 & 97.4 & 88.4 & 65.1 & 47.7 & 38.4 & 23.0 \\
\hline 85.6 & 67.7 & 111.3 & 110.4 & 112.3 & 112.4 & 110.5 & 107.6 & 89.7 & 71.1 & 51.4 & 42.2 & 23.8 \\
\hline \multicolumn{13}{|c|}{ mocouples located at sprinkler number 2} \\
\hline Time & $0 \mathrm{~mm}$ & $25 \mathrm{~mm}$ & $50 \mathrm{~mm}$ & $75 \mathrm{~mm}$ & $100 \mathrm{~mm}$ & $125 \mathrm{~mm}$ & $150 \mathrm{~mm}$ & $250 \mathrm{~mm}$ & $350 \mathrm{~mm}$ & $450 \mathrm{~mm}$ & $550 \mathrm{~mm}$ & $900 \mathrm{~mm}$ \\
\hline 0.0 & 20.2 & 20.1 & 20.3 & 20.0 & 20.2 & 19.8 & 20.1 & 19.7 & 19.4 & 19.1 & 19.0 & 18.0 \\
\hline 3.4 & 20.2 & 20.3 & 20.6 & 20.3 & 20.6 & 20.1 & 20.4 & 20.0 & 19.8 & 19.6 & 19.4 & 18.4 \\
\hline 10.2 & 20.2 & 20.4 & 20.6 & 20.4 & 20.6 & 20.2 & 20.4 & 20.1 & 19.8 & 19.6 & 19.4 & 18.4 \\
\hline 17.2 & 20.2 & 20.4 & 20.1 & 20.5 & 20.7 & 20.2 & 20.5 & 20.1 & 20.0 & 19.8 & 19.6 & 18.4 \\
\hline 23.9 & 21.3 & 31.1 & 32.6 & 32.2 & 33.4 & 31.4 & 30.9 & 25.4 & 22.6 & 23.0 & 21.8 & 19.4 \\
\hline 309 & 23.1 & 36.9 & 39.6 & 39.4 & 40.6 & 39.2 & 39.5 & 33.0 & 21.7 & 21.0 & 20.4 & 19.1 \\
\hline 37.6 & 23.7 & 40.1 & 43.3 & 43.5 & 44.7 & 42.9 & 43.7 & 40.7 & 30.5 & 26.7 & 27.2 & 22.8 \\
\hline 44,4 & 25.2 & 45.7 & 47.3 & 47.6 & 48.4 & 47.2 & 48.1 & 43.4 & 37.1 & 33.8 & 31.2 & 20.5 \\
\hline 51.3 & 26.1 & 48.0 & 51.2 & 50.6 & 52.0 & 50.9 & 51.3 & 48.2 & 41.5 & 38.0 & 32.9 & 21.6 \\
\hline 58.1 & 26.8 & 50.3 & 52.1 & 51.9 & 52.9 & 51.2 & 52.6 & 49.5 & 44.6 & 41.9 & 36.8 & 22.4 \\
\hline 65.1 & 29.0 & 61.5 & 64.7 & 64.3 & 65.4 & 62.3 & 64.0 & 59.2 & 49.4 & 45.1 & 40.1 & 24.8 \\
\hline 71.9 & 31.5 & 66.3 & 68.9 & 70.3 & 71.2 & 68.8 & 69.7 & 64.6 & 55.6 & 50.8 & 46.4 & 24.1 \\
\hline 78.7 & 31.8 & 68.6 & 72.1 & 71.5 & 73.8 & 69.9 & 72.3 & 65.4 & 58.4 & 57.7 & 47.7 & 24.9 \\
\hline 85.6 & 31.9 & 65.6 & 68.5 & 68.6 & 70.8 & 69.9 & 70.6 & 68.5 & 63.6 & 58.2 & 46.3 & 26.9 \\
\hline \multicolumn{13}{|c|}{ mocouples located at sprinkler number 3} \\
\hline Time & $0 \mathrm{~mm}$ & $25 \mathrm{~mm}$ & $50 \mathrm{~mm}$ & $75 \mathrm{~mm}$ & $100 \mathrm{~mm}$ & $125 \mathrm{~mm}$ & $150 \mathrm{~mm}$ & $250 \mathrm{~mm}$ & $350 \mathrm{~mm}$ & $450 \mathrm{~mm}$ & $550 \mathrm{~mm}$ & חזוד 900 \\
\hline 0.0 & 19.9 & 20.2 & 19.9 & 19.9 & 19.9 & 19.9 & 19.8 & 19.7 & 19.6 & 19.4 & 19.1 & 18.1 \\
\hline 3.4 & 20.0 & 20.3 & 20.0 & 20.1 & 20.1 & 20.2 & 20.2 & 20.1 & 20.0 & 19.8 & 19.5 & 18.3 \\
\hline 10.2 & 20.0 & 20.5 & 20.2 & 20.1 & 20.1 & 20.2 & 20.2 & 20.2 & 20.2 & 20.0 & 19.8 & 18.3 \\
\hline 17.2 & 19.9 & 20.4 & 20.1 & 20.2 & 20.2 & 20.2 & 20.2 & 20.1 & 20.1 & 20.0 & 19.8 & 18.4 \\
\hline 23.9 & 20.3 & 23.7 & 24.0 & 23.8 & 23.2 & 23.2 & 23.6 & 23.0 & 22.3 & 20.3 & 20.1 & 19.0 \\
\hline 30.9 & 21.0 & 25.5 & 27.7 & 26.9 & 27.8 & 28.1 & 28.7 & 25.4 & 24.8 & 23.0 & 22.6 & 19.6 \\
\hline 37.6 & 21.4 & 27.5 & 29.7 & 29.6 & 29.7 & 30.1 & 30.4 & 28.8 & 27.2 & 25.3 & 24.2 & 20.1 \\
\hline 44.4 & 22.0 & 30.0 & 32.6 & 32.8 & 32.1 & 32.9 & 32.5 & 30.6 & 30.0 & 28.7 & 28.8 & 22.0 \\
\hline 51.3 & 22.6 & 33.8 & 37.0 & 37.1 & 35.7 & 36.3 & 36.4 & 34.2 & 32.4 & 31.3 & 31.2 & 21.9 \\
\hline 58.1 & 22.6 & 35.0 & 39.3 & 40.2 & 38.7 & 39.1 & 38.8 & 36.5 & 35.2 & 34.0 & 32.7 & 22.3 \\
\hline 65.1 & 22.9 & 33.8 & 40.9 & 42.1 & 41.1 & 42.5 & 41.8 & 40.8 & 39.3 & 38.2 & 38.1 & 22.0 \\
\hline 11.9 & 24.0 & 38.3 & 44.8 & 45.9 & 44.7 & 45.6 & 45.0 & 43.9 & 42.9 & 41.5 & 42.4 & 27.7 \\
\hline 78.7 & 25.0 & 43.7 & 50.6 & 52.0 & 51.1 & 52.4 & 52.6 & 49.5 & 47.0 & 44.6 & 44.7 & 26.4 \\
\hline 85.6 & 25.6 & 47.3 & 55.1 & 57.5 & 54.5 & 56.1 & 56.4 & 54.5 & 50.6 & 48.7 & 48.4 & 29.6 \\
\hline \multicolumn{13}{|c|}{ mocouples located at sprinkler number 4} \\
\hline Time & $0 \mathrm{~mm}$ & $25 \mathrm{~mm}$ & $50 \mathrm{~mm}$ & $75 \mathrm{~mm}$ & $100 \mathrm{~mm}$ & $125 \mathrm{~mm}$ & $150 \mathrm{~mm}$ & $250 \mathrm{~mm}$ & $350 \mathrm{~mm}$ & $450 \mathrm{~mm}$ & $550 \mathrm{~mm}$ & $900 \mathrm{~mm}$ \\
\hline 0.0 & 20.0 & 20.3 & 20.2 & 20.2 & 20.0 & 20.0 & 20.0 & 19.7 & 19.4 & 19.1 & 18.9 & 18.1 \\
\hline 3.4 & 20.1 & 20.4 & 20.4 & 20.3 & 20.1 & 20.1 & $20.2^{1}$ & 20.0 & 19.9 & 19.6 & 19.4 & 18.4 \\
\hline 10.2 & 20.1 & 20.6 & 20.6 & 20.5 & 20.3 & 20.2 & 20.1 & 200 & 20.0 & 19.8 & 19.6 & 18.6 \\
\hline 17.2 & 21.1 & 28.7 & 27.7 & 27.1 & 26.0 & 26.1 & 25.7 & 23.2 & 22.3 & 20.2 & 20.0 & 18.8 \\
\hline 23.9 & 23.9 & 34.3 & 33.8 & 33.7 & 31.5 & 31.0 & 29.8 & 21.4 & 20.6 & 20.2 & 20.0 & 19.0 \\
\hline 30.9 & 24.3 & 37.4 & 37.4 & 36.5 & 34.5 & 34.8 & 34.6 & 27.1 & 25.0 & 22.1 & 21.0 & 19.1 \\
\hline 37.6 & 25.6 & 40.8 & 40.7 & 40.5 & 37.8 & 37.4 & 35.9 & 29.2 & 28.2 & 24.8 & 20.2 & 18.8 \\
\hline 44.4 & 25.7 & 42.5 & 42.3 & 41.9 & 39.8 & 39.7 & 38.2 & 31.6 & 31.1 & 23.5 & 10.9 & 18.7 \\
\hline 51.3 & 25.2 & 42.1 & 45.6 & 44.9 & 43.6 & 43.7 & 43.4 & 38.4 & 38.0 & 34.0 & 32.8 & 19.7 \\
\hline 58.1 & 27.6 & 54.1 & 54.1 & 53.4 & 50.8 & 52.1 & 51.4 & 43.9 & 39.1 & 36.3 & 34.0 & 20.8 \\
\hline 65.1 & 31.6 & 55.8 & 56.8 & 56.5 & 54.8 & 55.0 & 53.7 & 42.6 & 42.5 & 40.0 & 34.8 & 20.9 \\
\hline 71.9 & 29.9 & 61.1 & 62.2 & 62.1 & 60.5 & 61.2 & 60.3 & 49.7 & 47.8 & 46.1 & 36.1 & 21.7 \\
\hline 78.7 & 32.5 & 66.3 & 66.8 & 66.6 & 65.2 & 66.2 & 65.3 & 55.6 & 52.5 & 48.3 & 41.0 & 26.0 \\
\hline
\end{tabular}




\section{Experiment Number 43}

\begin{tabular}{|c|c|c|c|c|}
\hline 85.6 & 34.6 & 68.8 & 70.6 & 70.1 \\
\hline \multicolumn{5}{|c|}{ Velocity probes at sprinkler number 1} \\
\hline Time & $25 \mathrm{~mm}$ & $75 \mathrm{~mm}$ & $125 \mathrm{~mm}$ & $250 \mathrm{~mm}$ \\
\hline 0.0 & 0.0 & 0.0 & 0.0 & 0.0 \\
\hline 3.4 & 0.1 & 0.1 & 0.2 & 0.1 \\
\hline 10.2 & 0.9 & 0.9 & 0.3 & -0.2 \\
\hline 17.2 & 1.1 & 1.2 & 1.0 & 0.2 \\
\hline 23.9 & 1.3 & 1.0 & 0.9 & 0.1 \\
\hline 30.9 & 1.4 & 1.4 & 1.0 & 0.4 \\
\hline 37.6 & 1.4 & 1.3 & 0.7 & 0.1 \\
\hline 44.4 & 1.7 & 1.8 & 1.4 & 0.2 \\
\hline 51.3 & 1.5 & 16 & 1.1 & 0.3 \\
\hline 58.1 & 1.1 & 1.1 & 0.9 & 0.6 \\
\hline 65.1 & 1.5 & 1.5 & 1.7 & 0.6 \\
\hline 71.9 & 1.9 & 1.8 & 1.1 & 0.5 \\
\hline 78.7 & 1.7 & 1.7 & 1.2 & -0.3 \\
\hline 85.6 & 1.5 & 1.5 & 1.5 & 1.0 \\
\hline
\end{tabular}

\begin{tabular}{|c|c|c|c|c|}
\hline 69.6 & 69.2 & 58.3 & 56.0 & 53.6 \\
\hline \multicolumn{5}{|c|}{ Velocity probes at sprinkler number 2} \\
\hline Time & $25 \mathrm{~mm}$ & $75 \mathrm{~mm}$ & $125 \mathrm{~mm}$ & $250 \mathrm{~mm}$ \\
\hline 0.0 & 0.0 & 0.0 & 0.0 & 0.0 \\
\hline 3.4 & -0.1 & 0.0 & 0.1 & 0.0 \\
\hline 10.2 & -0.1 & 0.0 & 0.1 & 0.0 \\
\hline 17.2 & 0.5 & 0.5 & 0.5 & 0.5 \\
\hline 23.9 & 0.7 & 0.7 & 0.7 & 0.4 \\
\hline 30.9 & 0.6 & 0.6 & 0.6 & 0.4 \\
\hline 37.6 & 0.7 & 0.7 & 0.7 & 0.5 \\
\hline 44.4 & 0.5 & 0.5 & 0.5 & 0.4 \\
\hline 51.3 & 0.5 & 0.6 & 0.7 & 0.4 \\
\hline 58.1 & 0.7 & 0.8 & 0.8 & 0.3 \\
\hline 65.1 & 0.7 & 0.7 & 0.7 & 0.3 \\
\hline 71.9 & 0.7 & 0.7 & 0.7 & 0.4 \\
\hline 78.7 & 0.6 & 0.6 & 0.6 & 0.6 \\
\hline 85.6 & 0.6 & 0.6 & 0.7 & 0.6 \\
\hline
\end{tabular}


Experiment Number 44

Thermocouples located at sprinkler number 1

\begin{tabular}{|c|c|c|c|c|c|c|c|c|c|c|c|c|}
\hline Time & $0 \mathrm{~mm}$ & $25 \mathrm{~mm}$ & $50 \mathrm{~mm}$ & $75 \mathrm{~mm}$ & $100 \mathrm{~mm}$ & $125 \mathrm{~mm}$ & $150 \mathrm{~mm}$ & $250 \mathrm{~mm}$ & $350 \mathrm{~mm}$ & $450 \mathrm{~mm}$ & $550 \mathrm{~mm}$ & $900 \mathrm{~mm}$ \\
\hline 0.0 & 21.7 & 20.7 & 21.4 & 19.8 & 20.6 & 19.5 & 19.9 & 18.9 & 18.5 & 18.0 & 17.6 & 15.3 \\
\hline 2.0 & 21.7 & 20.6 & 21.3 & 20.2 & 20.8 & 19.9 & 19.9 & 18.8 & 18.7 & 18.5 & 18.4 & 16.4 \\
\hline 9.0 & 22.1 & 20.8 & 21.4 & 19.9 & 20.8 & 20.2 & 20.2 & 19.3 & 18.9 & 18.2 & 18.1 & 16.2 \\
\hline 15.8 & 23.4 & 22.7 & 24.0 & 22.3 & 23.4 & 22.4 & 23.0 & 20.4 & 19.9 & 19.5 & 18.7 & 16.2 \\
\hline 22.9 & 27.0 & 25.3 & 27.9 & 24.1 & 27.4 & 25.4 & 27.0 & 21.8 & 20.2 & 19.6 & 18.9 & 16.1 \\
\hline 29.7 & 31.7 & 27.9 & 32.9 & 27.0 & 32.6 & 27.9 & 31.0 & 23.3 & 23.2 & 20.7 & 19.8 & 17.1 \\
\hline 36.4 & 36.3 & 32.4 & 37.5 & 32.5 & 35.5 & 33.6 & 33.3 & 25.6 & 24.1 & 22.3 & 19.9 & 16.2 \\
\hline 13.3 & 38.8 & 35.5 & 42.6 & 37.3 & 43.2 & 40.3 & 41.0 & 35.3 & 28.1 & 21.1 & 19.7 & 16.9 \\
\hline 50.1 & 42.1 & 38.2 & 44.9 & 36.3 & 44.4 & 43.5 & 44.9 & 41.6 & 29.9 & 21.9 & 19.6 & 16.5 \\
\hline 57.1 & 44.2 & 39.7 & 47.8 & 37.8 & 48.0 & 43.9 & 47.8 & 43.6 & 30.5 & 22.9 & 21.1 & 16.6 \\
\hline 63.9 & 47.1 & 42.3 & 51.4 & 40.9 & 52.6 & 46.4 & 51.9 & 47.6 & 36.6 & 23.7 & 22.1 & 18.1 \\
\hline 70.7 & 52.5 & 46.5 & 56.2 & 44.7 & 55.6 & 51.8 & 55.0 & 51.7 & 41.1 & 25.4 & 22.1 & 18.3 \\
\hline 77.7 & 52.1 & 47.8 & 57.2 & 46.6 & 57.4 & 54.4 & 57.2 & 54.3 & 40.5 & 27.8 & 23.6 & 18.9 \\
\hline 84.5 & 60.3 & 53.4 & 64.5 & 54.1 & 65.2 & 63.0 & 64.2 & 61.0 & 39.7 & 30.3 & 26.0 & 20.7 \\
\hline 91.3 & 59.6 & 54.7 & 65.7 & 51.5 & 65.4 & 60.6 & 67.1 & 63.8 & 45.0 & 32.9 & 28.7 & 20.5 \\
\hline 98.3 & 65.7 & 63.0 & 70.0 & 61.1 & 71.7 & 67.6 & 71.0 & 67.4 & 54.2 & 38.5 & 32.4 & 22.8 \\
\hline 105.3 & 66.9 & 64.7 & 72.9 & 63.5 & 71.9 & 69.2 & 72.4 & 69.9 & 56.5 & 42.1 & 34.6 & 22.6 \\
\hline 112.3 & 68.7 & 65.1 & 73.4 & 62.1 & 75.9 & 71.2 & 77.6 & 71.4 & 61.3 & 48.0 & 37.1 & 24.8 \\
\hline 119.3 & 70.4 & 69.4 & 77.2 & 69.0 & 81.0 & 76.4 & 79.6 & 77.1 & 72.8 & 48.9 & 39.6 & 27.9 \\
\hline 126.2 & 75.4 & 74.2 & 81.9 & 72.8 & 84.3 & 80.8 & 85.3 & 74.6 & 70.7 & 54.4 & 42.4 & 29.7 \\
\hline 133.2 & 73.3 & 75.6 & 77.8 & 67.1 & 77.7 & 75.3 & 82.2 & 75.3 & 75.7 & 63.8 & 45.3 & 31.0 \\
\hline 139.9 & 89.5 & 84.0 & 94.2 & B2.9 & 94.9 & 91.4 & 96.6 & 83.5 & 75.9 & 62.3 & 46.4 & 33.2 \\
\hline 147.0 & 78.9 & 82.5 & 85.9 & 83.0 & 88.4 & 87.5 & 93.3 & 92.2 & 83.5 & 65.7 & 48.4 & 35.3 \\
\hline 154.0 & 76.9 & 78.7 & 84.1 & 77.2 & 85.7 & 86.3 & 90.2 & 89.4 & 92.6 & 84.6 & 51.8 & 36.2 \\
\hline 160.8 & 94.3 & 90.6 & 103.4 & 65.4 & 105.7 & 99.5 & 109.1 & 95.5 & 87.5 & 67.9 & 53.8 & 39.9 \\
\hline 167.7 & 107.7 & 101.8 & 110.5 & 101.9 & 109.4 & 106.9 & 109.6 & 104.0 & 95.0 & 78.0 & 61.7 & 41.0 \\
\hline 174.4 & 99.3 & 92.7 & 105.5 & 90.2 & 107.6 & 101.6 & 108.3 & 105.3 & 99.3 & 83.5 & 62.9 & 44.7 \\
\hline 181.4 & 102.0 & 97.3 & 112.4 & 98.9 & 114.8 & 110.6 & 115.3 & 109.0 & 103.7 & 79.3 & 62.3 & 46.4 \\
\hline 188.4 & 110.6 & 106.5 & 118.7 & 105.7 & 120.0 & 117.7 & 125.0 & 114.5 & 106.4 & 84.4 & 66.8 & 46.9 \\
\hline \multicolumn{13}{|c|}{ mocouples located at sprinkler number 2} \\
\hline Time & $0 \mathrm{~mm}$ & $25 \mathrm{~mm}$ & $50 \mathrm{~mm}$ & $75 \mathrm{~mm}$ & $100 \mathrm{~mm}$ & $125 \mathrm{~mm}$ & $150 \mathrm{~mm}$ & $250 \mathrm{~mm}$ & $350 \mathrm{~mm}$ & $450 \mathrm{~mm}$ & $550 \mathrm{~mm}$ & $900 \mathrm{~mm}$ \\
\hline 0.0 & 19.8 & 19.6 & 19.5 & 19.6 & 19.8 & 18.7 & 19.5 & 17.4 & 18.5 & 17.8 & 17.2 & 15.1 \\
\hline 2.0 & 19.7 & 19.3 & 21.6 & 16.2 & 19.7 & 18.8 & 18.9 & 18.6 & 15.2 & 18.2 & 17.7 & 15.8 \\
\hline 9.0 & 21.4 & 26.5 & 19.7 & 19.7 & 20.2 & 18.7 & 19.7 & 18.6 & 24.6 & 18.8 & 18.7 & 16.0 \\
\hline 15.8 & 18.9 & 15.6 & 18.5 & 22.8 & 20.0 & 19.4 & 19.9 & 17.4 & 17.3 & 18.0 & 17.8 & 15.7 \\
\hline 22.9 & 19.1 & 16.6 & 18.5 & 22.9 & 20.0 & 19.4 & 20.0 & 17.4 & 18.3 & 18.3 & 18.0 & 16.1 \\
\hline 29.7 & 21.2 & 23.6 & 22.1 & 16.7 & 20.8 & 19.1 & 20.3 & 19.2 & 19.0 & 19.2 & 18.4 & 16.9 \\
\hline 36.4 & 20.6 & 17.8 & 20.5 & 24.6 & 22.4 & 20.6 & 22.7 & 18.5 & 20.2 & 20.4 & 19.6 & 17.6 \\
\hline 43.3 & 23.1 & 24.3 & 25.7 & 19.0 & 24.1 & 20.6 & 23.1 & 20.1 & 19.9 & 21.4 & 20.4 & 18.4 \\
\hline 50.1 & 24.3 & 26.7 & 23.4 & 25.7 & 25.9 & 21.7 & 26.1 & 21.1 & 27.6 & 23.3 & 22.2 & 19.1 \\
\hline 57.1 & 28.4 & 27.1 & 27.5 & 30.9 & 30.6 & 24.9 & 30.4 & 22.6 & 27.3 & 24.5 & 23.7 & 19.5 \\
\hline 63.9 & 33.9 & 37.6 & 33.9 & 30.5 & 34.7 & 26.9 & 33.8 & 25.8 & 33.1 & 26.8 & 23.7 & 19.2 \\
\hline 70.7 & 35.4 & 35.8 & 34.4 & 37.1 & 37.0 & 29.8 & 37.0 & 26.0 & 33.2 & 27.8 & 25.0 & 22.5 \\
\hline 77.7 & 36.3 & 33.2 & 37.2 & 386 & 393 & 31.7 & 40.4 & 28.3 & 35.1 & 29.4 & 27.0 & 24.3 \\
\hline 84.5 & 41.6 & 41.8 & 43.0 & 33.9 & 42.7 & 32.6 & 41.3 & 32.6 & 36.8 & 30.7 & 29.8 & 25.6 \\
\hline 91.3 & 40.1 & 37.4 & 39.5 & 41.5 & 44.0 & 36.2 & 45.4 & 33.6 & 41.0 & 34.3 & 31.4 & 26.2 \\
\hline 98.3 & 42.1 & 41.5 & 46.9 & 37.1 & 47.7 & 35.7 & 47.6 & 34.8 & 39.3 & 35.4 & 34.5 & 28.2 \\
\hline 105.3 & 44.4 & 37.7 & 49.0 & 47.1 & 51.6 & 40.6 & 52.1 & 36.4 & 39.4 & 36.5 & 34.6 & 28.6 \\
\hline 112.3 & 51.7 & 54.7 & 52.1 & 46.7 & 53.4 & 39.0 & 51.8 & 37.8 & 47.1 & 39.8 & 37.8 & 32.6 \\
\hline 119.3 & 51.1 & 43.5 & 55.3 & 47.7 & 56.3 & 43.7 & 56.4 & 41.9 & 43.7 & 40.5 & 40.8 & 32.6 \\
\hline 126.2 & 54.9 & 55.3 & 56.2 & 56.1 & 59.5 & 44.3 & 59.7 & 41.6 & 51.9 & 45.1 & 41.7 & 34.1 \\
\hline 133.2 & 53.2 & 48.2 & 57.9 & 57.9 & 61.2 & 48.0 & 63.4 & 44.8 & 53.6 & 48.2 & 44.1 & 37.3 \\
\hline 139.9 & 62.0 & 58.6 & 63.8 & 60.9 & 66.5 & 48.6 & 66.3 & 46.1 & 57.3 & 52.6 & 47.2 & 40.6 \\
\hline 147.0 & 64.0 & 64.3 & 64.8 & 60.5 & 67.5 & 48.8 & 66.9 & 46.7 & 62.2 & 56.4 & 50.5 & 41.0 \\
\hline 154.0 & 65.7 & 67.7 & 68.6 & 64.7 & 70.4 & 53.7 & 69.0 & 49.6 & 63.4 & 57.3 & 51.1 & 44.5 \\
\hline 160.8 & 65.4 & 67.0 & 67.5 & 64.9 & 72.6 & 55.0 & 72.6 & 52.6 & 68.0 & 62.2 & 53.5 & 46.9 \\
\hline 167.7 & 66.6 & 58.2 & 70.6 & 67.5 & 74.4 & 56.7 & 73.4 & 53.6 & 62.3 & 58.1 & 53.8 & 45.4 \\
\hline 174.4 & 69.9 & 64.6 & 74.9 & 71.0 & 78.7 & 57.4 & 77.9 & 55.4 & 72.0 & 66.4 & 57.6 & 48.7 \\
\hline 181.4 & 72.0 & 67.1 & 78.3 & 67.3 & 79.5 & 61.5 & 78.6 & 57.7 & 67.2 & 63.6 & 61.7 & 50.8 \\
\hline 188.4 & 66.3 & 62.7 & 69.8 & 68.5 & 72.9 & 59.9 & 73.6 & 59.5 & 74.0 & 68.9 & 61.2 & 52.1 \\
\hline
\end{tabular}

Thermocouples located at sprinkler number 3 
Experiment Number 44

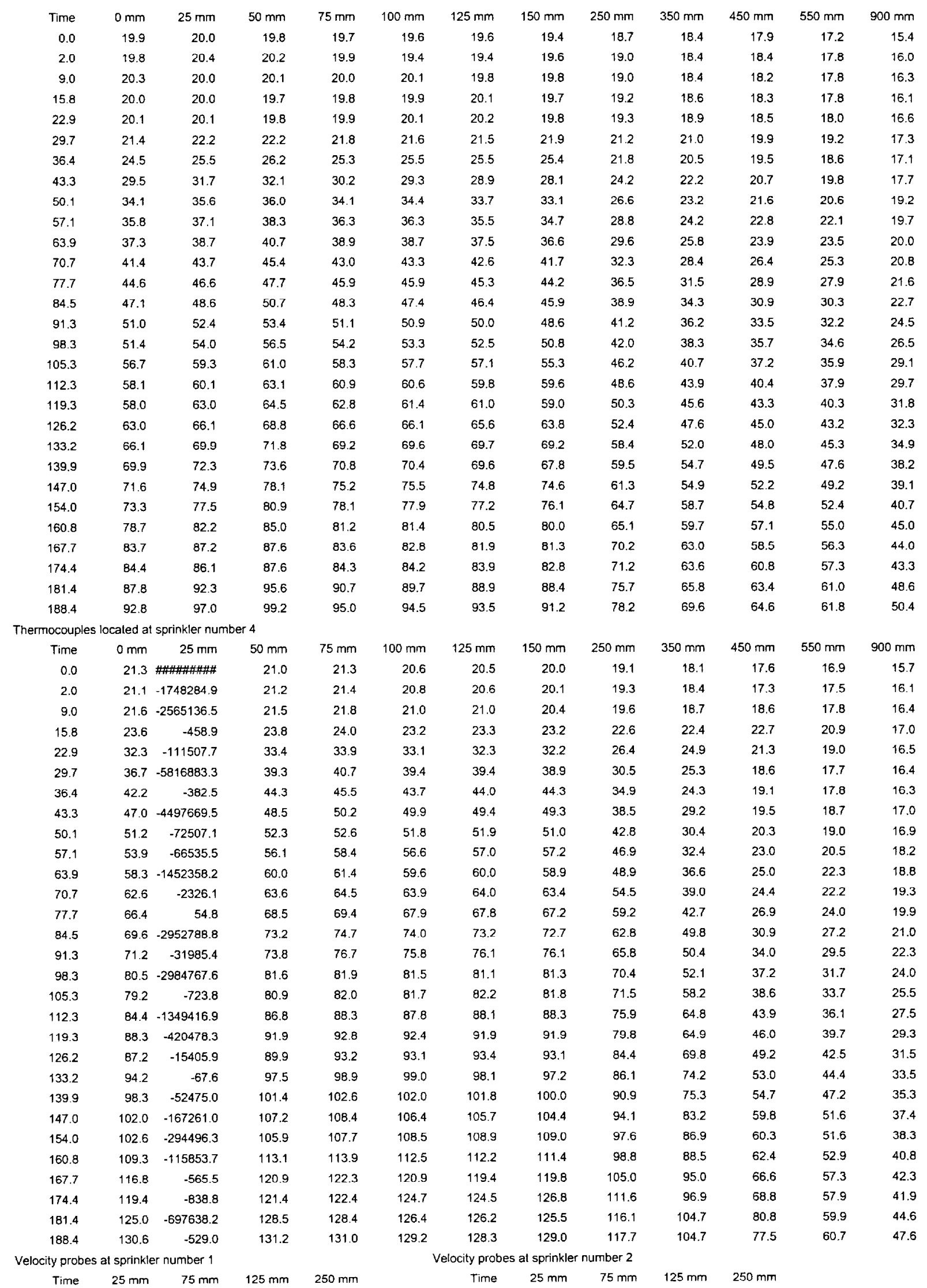


Experiment Number 44

\begin{tabular}{|c|c|c|c|c|c|c|c|c|c|}
\hline 0.0 & 0.0 & 0.0 & 0.0 & 0.0 & 0.0 & 0.0 & 0.0 & 0.0 & 0.0 \\
\hline 2.0 & 0.0 & 0.1 & 0.0 & 0.0 & 2.0 & 0.0 & 0.0 & 0.1 & 0.1 \\
\hline 9.0 & 0.2 & 0.2 & 0.3 & 0.1 & 9.0 & 0.0 & 0.0 & -0.1 & 0.1 \\
\hline 15.8 & 0.2 & 0.3 & 0.3 & 0.1 & 15.8 & 0.0 & 0.0 & -0.1 & 0.1 \\
\hline 22.9 & 0.4 & 0.4 & 0.4 & 0.3 & 22.9 & 0.1 & 0.1 & 0.2 & 0.1 \\
\hline 29.7 & 0.4 & 0.3 & 0.3 & 0.1 & 29.7 & 0.3 & 0.4 & 0.3 & 0.3 \\
\hline 36.4 & 0.3 & 0.3 & 0.3 & 0.2 & 36.4 & 0.2 & 0.2 & 0.1 & 0.2 \\
\hline 43.3 & 0.3 & 0.2 & 0.4 & 0.3 & 43.3 & 0.2 & 0.3 & 0.3 & 0.2 \\
\hline 50.1 & 0.1 & 0.2 & 0.3 & 0.2 & 50.1 & 0.3 & 0.3 & 0.3 & 0.3 \\
\hline 57.1 & 0.2 & 0.4 & 0.4 & 0.3 & 57.1 & 0.3 & 0.3 & 0.3 & 0.2 \\
\hline 63.9 & 0.4 & 0.4 & 0.4 & 0.3 & 63.9 & 0.4 & 0.4 & 0.4 & 0.3 \\
\hline 70.7 & 0.3 & 0.3 & 0.4 & 0.2 & 70.7 & 0.5 & 0.4 & 0.5 & 0.4 \\
\hline 77.7 & 0.3 & 0.4 & 0.5 & 0.4 & 77.7 & 0.3 & 0.3 & 0.4 & 0.4 \\
\hline 84.5 & 0.2 & 0.2 & 0.4 & 0.4 & 84.5 & 0.4 & 0.5 & 0.5 & 0.4 \\
\hline 91.3 & 0.4 & 0.4 & 0.5 & 0.4 & 91.3 & 0.4 & 0.4 & 0.5 & 0.4 \\
\hline 98.3 & 0.3 & 0.3 & 0.5 & 0.3 & 98.3 & 0.5 & 0.4 & 0.5 & 0.4 \\
\hline 105.3 & 0.4 & 0.5 & 0.5 & 0.5 & 105.3 & 0.5 & 0.4 & 0.4 & 0.4 \\
\hline 112.3 & 0.3 & 0.3 & 0.4 & 0.4 & 112.3 & 0.5 & 0.5 & 0.6 & 0.5 \\
\hline 119.3 & 0.2 & 0.2 & 0.4 & 0.4 & 119.3 & 0.5 & 0.4 & 0.4 & 0.4 \\
\hline 126.2 & 0.0 & 0.1 & 0.4 & 0.5 & 126.2 & 0.4 & 0.4 & 0.5 & 0.4 \\
\hline 133.2 & 0.4 & 0.4 & 0.6 & 0.4 & 133.2 & 0.5 & 0.4 & 0.5 & 0.4 \\
\hline 139.9 & 0.4 & 0.5 & 0.5 & 0.7 & 139.9 & 0.5 & 0.5 & 0.6 & 0.4 \\
\hline 147.0 & 0.3 & 0.4 & 0.6 & 0.6 & 147.0 & 0.5 & 0.5 & 0.6 & 0.5 \\
\hline 154.0 & 0.3 & 0.5 & 0.7 & 0.6 & 154.0 & 0.5 & 0.5 & 0.6 & 0.3 \\
\hline 160.8 & 0.5 & 0.5 & 0.8 & 0.6 & 160.8 & 0.6 & 0.5 & 0.5 & 0.4 \\
\hline 167.7 & 0.0 & -0.1 & 0.4 & 0.4 & 167.7 & 0.6 & 0.5 & 0.6 & 0.4 \\
\hline 174.4 & 0.3 & 0.4 & 0.6 & 0.5 & 174.4 & 0.4 & 0.3 & 0.5 & 0.4 \\
\hline 181.4 & 0.5 & 0.4 & 0.7 & 0.5 & 181.4 & 0.5 & 0.5 & 0.6 & 0.5 \\
\hline 188.4 & 0.5 & 0.4 & 0.6 & 0.5 & 188.4 & 0.6 & 0.6 & 0.6 & 0.5 \\
\hline
\end{tabular}

Velocity probes in channels above burner

Time Channel 1 Channel 2

$\begin{array}{lll}0.0 & 0.0 & 0.0\end{array}$

$\begin{array}{lll}2.0 & 1.7 & 1.1\end{array}$

$\begin{array}{rrr}9.0 & 2.0 & 1.1 \\ 15.8 & 1.9 & 0.9\end{array}$

$\begin{array}{lll}22.9 & 2.0 & 1.0\end{array}$

$\begin{array}{lll}29.7 & 2.1 & 1.0\end{array}$

$\begin{array}{lll}36.4 & 2.4 & 1.2\end{array}$

$\begin{array}{lll}43.3 & 2.7 & 1.3\end{array}$

$\begin{array}{lll}50.1 & 2.5 & 1.5\end{array}$

$\begin{array}{lll}57.1 & 2.7 & 1.3\end{array}$

$\begin{array}{lll}63.9 & 2.6 & 1.4\end{array}$

$\begin{array}{lll}70.7 & 2.9 & 1.6\end{array}$

$\begin{array}{lll}77.7 & 3.7 & 1.5\end{array}$

$\begin{array}{lll}84.5 & 3.2 & 1.9\end{array}$

$91.3 \quad 3.1 \quad 2.1$

$98.3 \quad 4.2 \quad 1.6$

$\begin{array}{lll}105.3 & 3.2 & 1.7\end{array}$

$\begin{array}{lll}112.3 & 3.8 & 1.6\end{array}$

$\begin{array}{lll}119.3 & 3.7 & 2.0\end{array}$

$126.2 \quad 4.0 \quad 1.4$

$133.2 \quad 4.2 \quad 1 . B$

$\begin{array}{lll}139.9 & 3.8 & 2.4\end{array}$

$\begin{array}{lll}147.0 & 3.9 & 2.3\end{array}$

$\begin{array}{lll}154.0 & 4.1 & 2.2\end{array}$

$\begin{array}{lll}160.8 & 3.4 & 2.8\end{array}$

$\begin{array}{lll}167.7 & 3.9 & 2.1\end{array}$

$\begin{array}{lll}174.4 & 4.0 & 2.1\end{array}$

$\begin{array}{lll}181.4 & 4.1 & 2.8\end{array}$

$\begin{array}{lll}188.4 & 4.0 & 2.6\end{array}$ 
Experiment Number 45

\begin{tabular}{|c|c|c|c|c|c|c|c|c|c|c|c|c|}
\hline Time & $0 \mathrm{~mm}$ & $25 \mathrm{~mm}$ & $50 \mathrm{~mm}$ & $75 \mathrm{~mm}$ & $100 \mathrm{~mm}$ & $125 \mathrm{~mm}$ & $150 \mathrm{~mm}$ & $250 \mathrm{~mm}$ & $350 \mathrm{~mm}$ & $450 \mathrm{~mm}$ & $550 \mathrm{~mm}$ & $900 \mathrm{~mm}$ \\
\hline 0.0 & 20.6 & 20.0 & 20.4 & 18.8 & 20.3 & 19.3 & 20.1 & 19.1 & 18.5 & 18.1 & 18.0 & 17.3 \\
\hline 5.8 & 21.4 & 21.0 & 21.2 & 19.8 & 21.2 & 20.3 & 21.2 & 20.3 & 19.4 & 19.0 & 19.0 & 18.0 \\
\hline 12.6 & 21.5 & 21.0 & 21.2 & 19.8 & 21.5 & 20.2 & 21.5 & 20.2 & 19.6 & 19.2 & 19.1 & 18.0 \\
\hline 19.6 & 22.8 & 22.9 & 23.0 & 21.0 & 23.3 & 21.9 & 23.2 & 21.1 & 20.6 & 19.3 & 19.1 & 18.2 \\
\hline 26.3 & 28.2 & 28.6 & 30.2 & 24.7 & 30.5 & 27.1 & 28.9 & 24.4 & 21.7 & 19.7 & 19.3 & 18.3 \\
\hline 33.2 & 33.0 & 33.2 & 35.7 & 28.5 & 35.3 & 32.4 & 34.8 & 28.3 & 26.1 & 23.1 & 20.3 & 18.5 \\
\hline 39.9 & 38.2 & 37.9 & 40.3 & 32.2 & 38.8 & 36.4 & 37.1 & 30.1 & 25.8 & 21.9 & 20.1 & 18.5 \\
\hline 46.7 & 40.6 & 42.2 & 45.0 & 37.7 & 44.8 & 42.2 & 44.5 & 40.7 & 27.8 & 20.7 & 19.9 & 18.4 \\
\hline 53.6 & 40.4 & 41.3 & 43.2 & 36.0 & 44.8 & 42.9 & 46.4 & 45.1 & 29.9 & 20.8 & 20.2 & 18.7 \\
\hline 60.4 & 43.4 & 44.0 & 47.3 & 41.3 & 49.3 & 46.6 & 50.0 & 48.8 & 39.9 & 22.2 & 21.3 & 18.7 \\
\hline 67.4 & 45.6 & 46.8 & 49.2 & 41.4 & 52.6 & 48.7 & 54.1 & 47.3 & 43.4 & 24.1 & 22.4 & 19.4 \\
\hline 74.1 & 51.1 & 51.1 & 55.2 & 44.9 & 56.3 & 52.2 & 56.4 & 51.2 & 46.5 & 27.6 & 23.9 & 20.4 \\
\hline 80.9 & 53.2 & 55.3 & 60.2 & 46.2 & 62.0 & 56.0 & 61.8 & 57.9 & 48.1 & 31.3 & 25.6 & 20.7 \\
\hline 87.8 & 58.6 & 61.2 & 65.4 & 52.9 & 65.3 & 59.7 & 65.6 & 56.0 & 49.4 & 34.1 & 28.0 & 21.0 \\
\hline 94.6 & 60.4 & ล2. 1 & 66.7 & 53.3 & 67.0 & 63.0 & 66.2 & 61.8 & 53.0 & 39.8 & 31.9 & 22.9 \\
\hline 101.3 & 60.0 & 60.4 & 65.8 & 52.3 & 66.7 & 59.7 & 65.6 & 61.7 & 57.4 & 41.1 & 32.5 & 23.7 \\
\hline 108.3 & 63.9 & 66.1 & 71.0 & 55.9 & 72.9 & 66.6 & 72.5 & 67.4 & 56.4 & 39.7 & 34.8 & 24.7 \\
\hline 115.0 & 72.2 & 74.5 & 79.7 & 64.6 & 80.8 & 72.7 & 78.9 & 73.3 & 61.3 & 44.1 & 36.1 & 26.7 \\
\hline 121.9 & 69.0 & 70.1 & 73.4 & 62.7 & 76.6 & 72.7 & 78.8 & 76.6 & 67.9 & 49.2 & 38.1 & 27.9 \\
\hline 128.7 & 79.0 & 82.1 & 85.9 & 72.2 & 87.4 & 81.5 & 86.7 & 78.4 & 66.4 & 51.2 & 40.6 & 29.5 \\
\hline 135.4 & 79.4 & 81.0 & 85.5 & 71.4 & 86.0 & 81.9 & 87.3 & 81.4 & 72.9 & 58.1 & 43.8 & 31.7 \\
\hline 142.3 & 74.8 & 78.8 & 82.2 & 71.6 & 85.2 & 80.2 & 87.8 & 79.4 & 79.8 & 63.0 & 45.8 & 33.9 \\
\hline 149.1 & 72.7 & 75.7 & 77.1 & 72.7 & 81.2 & 83.7 & 87.4 & 90.2 & 89.8 & 70.0 & 46.6 & 36.1 \\
\hline 156.0 & 82.0 & 82.1 & 90.7 & 78.6 & 94.8 & 91.7 & 97.1 & 89.9 & 86.3 & 69.5 & 49.3 & 37.7 \\
\hline 162.8 & 87.6 & 87.9 & 95.4 & 86.0 & 97.3 & 93.9 & 98.5 & 93.6 & 92.0 & 73.6 & 54.1 & 39.7 \\
\hline 169.5 & 97.3 & 100.3 & 103.6 & 88.8 & 104.4 & 100.2 & 105.8 & 96.2 & 89.2 & 75.5 & 58.6 & 40.1 \\
\hline 176.4 & 97.3 & 104.2 & 107.7 & 90.1 & 112.5 & 104.5 & 112.7 & 97.8 & 92.1 & 74.2 & 59.3 & 41.8 \\
\hline 183.2 & 102.4 & 104.0 & 111.1 & 90.8 & 113.7 & 108.5 & 114.5 & 101.4 & 92.4 & 77.1 & 60.0 & 43.0 \\
\hline 190.1 & 106.5 & 109.6 & 117.3 & 104.4 & 120.5 & 116.2 & 123.2 & 110.6 & 102.1 & 80.6 & 63.8 & 44.3 \\
\hline 196.9 & 114.1 & 119.8 & 124.8 & 109.4 & 125.6 & 120.4 & 125.7 & 114.4 & 104.1 & 81.3 & 64.6 & 47.8 \\
\hline \multicolumn{13}{|c|}{ Thermocouples located at sprinkler number 2} \\
\hline Time & $0 \mathrm{~mm}$ & $25 \mathrm{~mm}$ & $50 \mathrm{~mm}$ & $75 \mathrm{~mm}$ & $100 \mathrm{~mm}$ & $125 \mathrm{~mm}$ & $150 \mathrm{~mm}$ & $250 \mathrm{~mm}$ & $350 \mathrm{~mm}$ & $450 \mathrm{~mm}$ & $550 \mathrm{~mm}$ & $900 \mathrm{~mm}$ \\
\hline 0.0 & 19.5 & 19.2 & 19.3 & 18.8 & 19.2 & 18.3 & 19.0 & 17.8 & 18.4 & 18.1 & 17.8 & 17.1 \\
\hline 5.8 & 20.1 & 19.7 & 20.2 & 19.6 & 20.3 & 19.0 & 20.1 & 18.4 & 19.3 & 19.1 & 18.7 & 17.6 \\
\hline 12.6 & 20.2 & 19.8 & 20.2 & 19.5 & 20.3 & 19.0 & 20.2 & 18.5 & 19.4 & 19.2 & 18.9 & 17.5 \\
\hline 19.6 & 20.2 & 19.9 & 20.2 & 19.5 & 20.3 & 19.0 & 20.1 & 18.5 & 19.4 & 19.2 & 19.0 & 17.7 \\
\hline 26.3 & 20.3 & 20.0 & 20.2 & 19.6 & 20.2 & 19.1 & 20.1 & 18.6 & 19.4 & 19.3 & 19.0 & 18.0 \\
\hline 33.2 & 20.4 & 20.1 & 20.6 & 20.0 & 20.7 & 19.3 & 20.8 & 19.1 & 20.7 & 20.6 & 19.9 & 18.7 \\
\hline 39.9 & 23.8 & 23.4 & 24.7 & 23.3 & 25.1 & 21.6 & 24.6 & 20.1 & 22.2 & 21.5 & 21.2 & 19.4 \\
\hline 46.7 & 24.8 & 24.1 & 26.2 & 24.4 & 26.6 & 22.6 & 26.5 & 21.3 & 24.5 & 23.8 & 22.3 & 19.5 \\
\hline 53.6 & 29.7 & 27.8 & 30.8 & 28.0 & 31.2 & 25.2 & 30.4 & 21.5 & 24.4 & 22.7 & 21.5 & 19.8 \\
\hline 60.4 & 30.6 & 28.7 & 32.0 & 29.8 & 32.9 & 27.4 & 32.6 & 25.4 & 27.6 & 25.0 & 22.8 & 20.9 \\
\hline 67.4 & 35.7 & 32.9 & 36.7 & 33.0 & 36.9 & 29.2 & 36.4 & 27.0 & 28.0 & 25.2 & 24.4 & 21.1 \\
\hline 74.1 & 38.6 & 35.2 & 39.9 & 35.3 & 40.6 & 30.9 & 39.5 & 29.2 & 304 & 26.3 & 25.7 & 23.5 \\
\hline 80.9 & 38.5 & 37.7 & 40.8 & 38.4 & 42.0 & 32.9 & 41.2 & 29.7 & 31.2 & 28.4 & 28.0 & 25.5 \\
\hline 87.8 & 38.4 & 37.2 & 41.3 & 38.2 & 42.2 & 33.2 & 42.4 & 31.3 & 36.7 & 31.8 & 32.4 & 27.1 \\
\hline 94.6 & 45.4 & 41.8 & 46.9 & 42.4 & 47.6 & 35.4 & 46.4 & 32.6 & 37.9 & 33.8 & 32.4 & 27.5 \\
\hline 101.3 & 45.6 & 42.9 & 47.6 & 45.3 & 50.0 & 38.9 & 48.6 & 35.3 & 40.1 & 36.4 & 34.6 & 26.8 \\
\hline 108.3 & 46.1 & 44.3 & 49.9 & 45.5 & 51.0 & 38.6 & 50.5 & 37.1 & 43.7 & 38.9 & 37.2 & 28.5 \\
\hline 115.0 & 50.3 & 48.3 & 52.9 & 49.4 & 53.0 & 42.1 & 51.7 & 37.7 & 43.3 & 39.7 & 38.9 & 32.2 \\
\hline 121.9 & 50.3 & 49.5 & 54.2 & 49.0 & 55.6 & 41.7 & 55.6 & 38.7 & 44.9 & 41.4 & 41.1 & 32.7 \\
\hline 128.7 & 54.7 & 52.8 & 57.4 & 51.2 & 58.4 & 43.8 & 56.8 & 39.4 & 46.6 & 42.9 & 42.2 & 33.7 \\
\hline 135.4 & 57.4 & 54.9 & 58.5 & 55.3 & 59.0 & 46.7 & 58.7 & 43.1 & 51.1 & 46.0 & 43.7 & 35.5 \\
\hline 142.3 & 56.4 & 55.3 & 59.7 & 55.8 & 61.0 & 47.4 & 60.8 & 44.7 & 54.8 & 50.0 & 46.2 & 37.7 \\
\hline 149.1 & 61.7 & 60.2 & 64.0 & 62.6 & 65.7 & 52.3 & 65.5 & 48.3 & 57.2 & 50.2 & 47.6 & 38.0 \\
\hline 156.0 & 66.2 & 62.3 & 68.5 & 62.1 & 690 & 52.1 & 67.1 & 50.6 & 61.0 & 55.0 & 50.6 & 41.9 \\
\hline 162.8 & 66.1 & 62.5 & 69.0 & 61.0 & 69.6 & 51.5 & 68.6 & 51.2 & 64.1 & 58.4 & 52.3 & 44.7 \\
\hline 169.5 & 67.1 & 64.4 & 70.0 & 65.3 & 71.2 & 53.7 & 70.9 & 51.0 & 65.7 & 60.3 & 55.6 & 41.5 \\
\hline 176.4 & 71.3 & 67.4 & 73.3 & 68.8 & 74.3 & 57.4 & 73.3 & 55.5 & 67.7 & 62.8 & 56.1 & 46.3 \\
\hline 183.2 & 73.0 & 69.6 & 77.2 & 71.4 & 80.2 & 61.0 & 80.2 & 58.2 & 68.6 & 63.9 & 58.7 & 48.6 \\
\hline 190.1 & 75.1 & 72.4 & 78.5 & 71.4 & 79.6 & 61.9 & 80.9 & 59.3 & 71.0 & 65.2 & 59.5 & 49.9 \\
\hline
\end{tabular}




\section{Experiment Number 45}

\begin{tabular}{|c|c|c|c|c|c|c|c|c|c|c|c|c|}
\hline 196.9 & 79.4 & 77.7 & 84.5 & 79.2 & 85.2 & 64.9 & 83.6 & 63.2 & 75.2 & 67.1 & 61.0 & 52.1 \\
\hline \multicolumn{13}{|c|}{ Thermocouples located at sprinkler number 3} \\
\hline Time & $0 \mathrm{~mm}$ & $25 \mathrm{~mm}$ & $50 \mathrm{~mm}$ & $75 \mathrm{~mm}$ & $100 \mathrm{~mm}$ & $125 \mathrm{~mm}$ & $150 \mathrm{~mm}$ & $250 \mathrm{~mm}$ & $350 \mathrm{~mm}$ & $450 \mathrm{~mm}$ & $550 \mathrm{~mm}$ & $900 \mathrm{~mm}$ \\
\hline 0.0 & . $\quad 19.7$ & 19.8 & 19.3 & 19.3 & 19.3 & 19.2 & 19.1 & 18.8 & 18.4 & 18.2 & 18.0 & 17.2 \\
\hline 5.8 & 20.5 & 20.6 & 20.3 & 20.2 & 20.2 & 20.2 & 20.1 & 19.7 & 19.2 & 18.9 & 18.8 & 17.8 \\
\hline 12.6 & 20.4 & 20.7 & 20.4 & 20.3 & 20.3 & 20.2 & 20.2 & 19.9 & 19.5 & 19.0 & 18.9 & 17.7 \\
\hline 19.6 & 20.5 & 20.7 & 20.4 & 20.3 & 20.3 & 20.3 & 20.2 & 19.9 & 19.6 & 19.0 & 18.9 & 17.8 \\
\hline 26.3 & 21.0 & 21.6 & 22.0 & 21.5 & 21.7 & 21.8 & 22.2 & 21.4 & 21.1 & 20.5 & 20.3 & 18.6 \\
\hline 33.2 & 23.8 & 25.0 & 26.1 & 24.5 & 24.0 & 23.3 & 23.0 & 21.8 & 21.0 & 20.3 & 19.9 & 18.6 \\
\hline 39.9 & 28.3 & 30.7 & 32.3 & 29.5 & 29.5 & 28.9 & 28.2 & 23.9 & 21.5 & 20.6 & 19.9 & 18.7 \\
\hline 46.7 & 31.8 & 33.7 & 36.1 & 33.7 & 33.3 & 32.3 & 31.8 & 26.7 & 23.4 & 21.7 & 20.7 & 19.4 \\
\hline 53.6 & 34.1 & 36.4 & 38.1 & 35.8 & 35.0 & 34.0 & 32.9 & 28.5 & 25.7 & 24.1 & 22.9 & 20.6 \\
\hline 60.4 & 36.0 & 38.1 & 40.9 & 38.6 & 38.4 & 37.7 & 37.0 & 29.8 & 26.5 & 24.9 & 24.4 & 21.1 \\
\hline 67.4 & 38.2 & 40.6 & 44.0 & 40.8 & 40.1 & 38.6 & 37.6 & 30.9 & 27.4 & 25.8 & 25.5 & 22.0 \\
\hline 74.1 & 40.8 & 43.9 & 47.9 & 44.3 & 43.6 & 42.1 & 40.4 & 32.8 & 29.7 & 27.7 & 26.3 & 22.1 \\
\hline 80.9 & 43.9 & 47.2 & 50.5 & 47.0 & 46.1 & 44.9 & 43.3 & 36.4 & 32.8 & 30.6 & 29.0 & 23.2 \\
\hline 87.8 & 47.1 & 50.6 & 53.8 & 50.0 & 49.6 & 48.4 & 47.2 & 39.7 & 35.6 & 33.7 & 32.2 & 23.6 \\
\hline 94.6 & 49.4 & 51.4 & 57.6 & 53.8 & 53.4 & 52.6 & 51.1 & 43.4 & 37.8 & 35.6 & 33.9 & 24.6 \\
\hline 101.3 & 52.5 & 56.2 & 59.8 & 56.4 & 55.6 & 54.6 & 53.4 & 44.3 & 39.8 & 37.1 & 35.5 & 27.2 \\
\hline 108.3 & 56.0 & 60.0 & 63.9 & 59.7 & 59.1 & 58.1 & 57.2 & 46.4 & 41.7 & 38.9 & 36.6 & 30.0 \\
\hline 115.0 & 57.4 & 60.7 & 66.5 & 62.2 & 61.5 & 60.5 & 59.3 & 50.0 & 43.7 & 42.7 & 39.2 & 30.2 \\
\hline 121.9 & 60.8 & 64.9 & 68.8 & 64.5 & 63.9 & 63.1 & 61.7 & 52.6 & 46.4 & 43.7 & 40.5 & 31.7 \\
\hline 128.7 & 62.5 & 66.1 & 71.7 & 67.3 & 66.4 & 65.0 & 62.7 & 53.5 & 48.1 & 44.4 & 42.4 & 34.6 \\
\hline 135.4 & 64.5 & 67.8 & 75.1 & 70.4 & 69.7 & 68.5 & 66.5 & 54.3 & 50.0 & 47.6 & 45.0 & 36.3 \\
\hline 142.3 & 67.2 & 71.2 & 76.8 & 72.2 & 71.2 & 69.9 & 67.1 & 55.9 & 52.0 & 49.4 & 47.1 & 35.6 \\
\hline 149.1 & 68.7 & 72.5 & 80.3 & 75.3 & 74.7 & 74.1 & 73.3 & 61.2 & 53.7 & 51.4 & 49.2 & 37.3 \\
\hline 156.0 & 69.5 & 72.2 & 80.6 & 76.8 & 76.3 & 73.2 & 75.1 & 63.9 & 57.4 & 53.4 & 51.4 & 39.4 \\
\hline 162.8 & 75.8 & 81.6 & 86.3 & 80.7 & 79.8 & 79.0 & 77.4 & 66.0 & 58.7 & 55.6 & 53.4 & 42.1 \\
\hline 169.5 & 76.1 & B1.3 & 87.2 & 82.4 & 81.7 & 81.1 & 79.8 & 67.9 & 62.0 & 57.8 & 55.4 & 44.1 \\
\hline 176.4 & 80.6 & 86.2 & 92.0 & 85.7 & 84.6 & 83.2 & 82.4 & 71.1 & 64.6 & 59.6 & 58.7 & 46.1 \\
\hline 183.2 & 79.0 & B1.1 & 90.7 & 86.4 & 85.9 & 85.4 & 83.8 & 71.3 & 64.8 & 61.6 & 60.3 & 48.1 \\
\hline 190.1 & 82.8 & 86.7 & 95.4 & 90.4 & 89.2 & 89.2 & 86.3 & 72.3 & 67.0 & 65.1 & 61.2 & 50.1 \\
\hline 195.9 & 89.1 & 95.0 & 101.1 & 94.6 & 93.6 & 92.4 & 90.5 & 76.2 & 68.5 & 65.3 & 62.6 & 50.4 \\
\hline \multicolumn{13}{|c|}{ Thermocouples located at sprinkler number 4} \\
\hline Time & $0 \mathrm{~mm}$ & $25 \mathrm{~mm}$ & $50 \mathrm{~mm}$ & $75 \mathrm{~mm}$ & $100 \mathrm{~mm}$ & $125 \mathrm{~mm}$ & $150 \mathrm{~mm}$ & $250 \mathrm{~mm}$ & $350 \mathrm{~mm}$ & $450 \mathrm{~mm}$ & $550 \mathrm{~mm}$ & $900 \mathrm{~mm}$ \\
\hline 0.0 & 20.9 & 20.8 & 20.6 & 20.5 & 20.1 & 20.0 & 19.7 & 18.9 & 18.3 & 18.1 & 17.8 & 17.5 \\
\hline 5.8 & 21.6 & 21.4 & 21.1 & 21.0 & 20.6 & 20.4 & 20.2 & 19.3 & 19.0 & 18.8 & 18.4 & 17.9 \\
\hline 12.6 & 22.3 & 22.4 & 22.5 & 22.2 & 22.2 & 22.3 & 22.0 & 22.8 & 22.0 & 20.9 & 20.0 & 18.3 \\
\hline 19.6 & 30.5 & 28.9 & 32.9 & 30.8 & 30.5 & 29.0 & 29.9 & 25.0 & 24.4 & 22.8 & 20.8 & 18.4 \\
\hline 26.3 & 38.1 & 35.7 & 39.8 & 38.1 & 37.4 & 35.9 & 37.8 & 30.6 & 21.4 & 20.1 & 19.1 & 18.0 \\
\hline 33.2 & 42.2 & 41.3 & 44.3 & 42.9 & 42.1 & 41.7 & 43.8 & 33.5 & 22.6 & 19.6 & 18.9 & 18.1 \\
\hline 39.9 & 45.6 & 45.1 & 47.5 & 46.4 & 46.3 & 45.9 & 48.5 & 38.6 & 26.9 & 19.7 & 19.1 & 18.6 \\
\hline 46.7 & 49.2 & 48.7 & 51.6 & 50.3 & 50.1 & 49.7 & 52.5 & 42.1 & 29.1 & 21.3 & 19.6 & 18.8 \\
\hline 53.6 & 53.4 & 52.7 & 55.9 & 54.8 & 54.3 & 54.1 & 56.2 & 46.1 & 31.7 & 21.2 & 19.9 & 18.9 \\
\hline 60.4 & 56.6 & 54.8 & 58.4 & 56.4 & 56.3 & 55.6 & 56.6 & 47.5 & 36.2 & 23.8 & 20.4 & 19.2 \\
\hline 67.1 & 59.4 & 58.9 & 62.8 & 60.7 & 60.3 & 59.6 & 61.1 & $50 . ?$ & 38.8 & 27.1 & 24.4 & 20.3 \\
\hline 74.1 & 63.5 & 62.8 & 67.5 & 66.0 & 65.7 & 64.4 & 66.2 & 55.0 & 44.1 & 30.0 & 24.5 & 20.9 \\
\hline 80.9 & 67.8 & 67.0 & 71.0 & 69.2 & 68.5 & 68.2 & 70.0 & 60.4 & 48.4 & 31.8 & 26.6 & 21.5 \\
\hline 87.8 & 71.9 & 71.1 & 75.0 & 73.3 & 72.7 & 72.1 & 74.5 & 65.0 & 48.7 & 32.0 & 28.1 & 22.3 \\
\hline 94.6 & 77.0 & 75.8 & 80.8 & 78.2 & 77.8 & 76.9 & 79.1 & 68.0 & 50.4 & 35.2 & 31.8 & 23.2 \\
\hline 101.3 & 78.7 & 78.2 & 84.1 & 82.4 & 82.1 & 81.0 & 83.0 & 71.4 & 52.2 & 36.9 & 33.5 & 24.7 \\
\hline 108.3 & 86.1 & 84.9 & 90.8 & 88.2 & 87.0 & 85.3 & 87.3 & 73.9 & 52.7 & 38.3 & 34.1 & 27.0 \\
\hline 115.0 & 87.4 & 86.9 & 93.1 & 90.7 & 90.2 & 88.2 & 90.9 & 77.4 & 53.6 & 40.3 & 36.7 & 29.1 \\
\hline 121.9 & 91.3 & 90.2 & 94.2 & 91.8 & 91.8 & 90.0 & 91.4 & 78.8 & 61.5 & 42.1 & 38.6 & 30.4 \\
\hline 128.7 & 91.2 & 91.4 & 96.1 & 94.4 & 94.7 & 94.2 & 96.5 & 83.7 & 68.4 & 47.6 & 40.2 & 32.0 \\
\hline 135.4 & 95.9 & 96.3 & 102.4 & 99.7 & 99.5 & 98.3 & 100.4 & 89.4 & 71.4 & 49.7 & 44.5 & 33.3 \\
\hline 142.3 & 97.4 & 100.3 & 107.2 & 103.7 & 104.8 & 102.5 & 104.8 & 93.7 & 73.4 & 50.9 & 47.3 & 35.0 \\
\hline 149.1 & 100.7 & 101.6 & 105.3 & 103.3 & 103.7 & 102.7 & 105.2 & 95.9 & 78.1 & 52.7 & 46.9 & 36.4 \\
\hline 156.0 & 103.9 & 104.9 & 110.5 & 108.5 & 108.1 & 107.8 & 110.6 & 98.8 & 80.3 & 54.3 & 50.0 & 38.1 \\
\hline 162.8 & 108.9 & 107.7 & 114.5 & 111.3 & 111.8 & 110.0 & 113.9 & 99.6 & 85.2 & 61.1 & 50.9 & 39.1 \\
\hline 169.5 & 113.5 & 114.1 & 120.7 & 116.8 & 117.2 & 115.3 & 117.6 & 102.4 & 87.6 & 62.1 & 53.1 & 41.4 \\
\hline 176.4 & 113.4 & $\$ 17.7$ & 123.6 & 120.2 & 122.8 & 119.5 & 122.3 & 107.3 & 93.6 & 67.3 & 56.7 & 41.6 \\
\hline 183.2 & 124.9 & 123.2 & 131.9 & 129.5 & 129.8 & 127.6 & 131.6 & 115.5 & 96.3 & 65.4 & 55.5 & 44.0 \\
\hline
\end{tabular}




\section{Experiment Number 45}

\begin{tabular}{|c|c|c|c|c|c|c|c|c|c|c|c|c|}
\hline \multirow{2}{*}{$\begin{array}{l}190.1 \\
196.9\end{array}$} & \multirow{2}{*}{$\begin{array}{l}123.7 \\
121.6\end{array}$} & \multirow{2}{*}{$\begin{array}{r}124.7 \\
48.5\end{array}$} & \multirow{2}{*}{$\begin{array}{r}129.5 \\
48.9\end{array}$} & \multirow{2}{*}{$\begin{array}{l}126.9 \\
1235\end{array}$} & \multirow{2}{*}{$\begin{array}{l}129.5 \\
127.2\end{array}$} & 127.9 & 131.2 & 119.9 & 105.7 & 71.4 & 59.5 & 46.3 \\
\hline & & & & & & 126.4 & 129.4 & 120.4 & 108.5 & 63.1 & 56.9 & 47.1 \\
\hline Velocity probe & $s$ at sprinkler & number 1 & & & & ity probe & t sprinkler & mber 2 & & & & \\
\hline Time & $25 \mathrm{~mm}$ & $75 \mathrm{~mm}$ & $125 \mathrm{~mm}$ & $250 \mathrm{~mm}$ & & Time & $25 \mathrm{~mm}$ & $75 \mathrm{~mm}$ & $125 \mathrm{~mm}$ & $250 \mathrm{~mm}$ & & \\
\hline 0.0 & 0.0 & 0.0 & 0.0 & 0.0 & & 0.0 & 0.0 & 0.0 & 0.0 & 0.0 & & \\
\hline 5.8 & 0.1 & 0.1 & 0.1 & -0.4 & & 5.8 & 0.0 & 0.1 & 0.1 & 0.1 & & \\
\hline 12.6 & 0.2 & 0.2 & 0.3 & 0.1 & & 12.6 & 0.0 & 0.1 & 0.1 & 0.0 & & \\
\hline 19.6 & 0.4 & 0.3 & 0.4 & 0.1 & & 19.6 & 0.0 & 0.1 & 0.1 & 0.1 & & \\
\hline 26.3 & 0.2 & 0.4 & 0.4 & 0.3 & & 26.3 & 0.1 & 0.2 & 0.1 & 0.1 & & \\
\hline 33.2 & 0.3 & 0.3 & 0.4 & 0.1 & & 33.2 & 0.3 & 0.4 & 0.3 & 0.3 & & \\
\hline 39.9 & 0.3 & 0.3 & 0.4 & 0.3 & & 39.9 & 0.2 & 0.2 & 0.3 & 0.3 & & \\
\hline 46.7 & 0.2 & 0.3 & 0.4 & 0.4 & & 46.7 & 0.4 & 0.4 & 0.3 & 0.2 & & \\
\hline 53.6 & 0.2 & 0.3 & 0.5 & 0.4 & & 53.6 & 0.3 & 0.4 & 0.4 & 0.4 & & \\
\hline 60.4 & 0.1 & 0.3 & 0.4 & 0.3 & & 60.4 & 0.4 & 0.4 & 0.3 & 0.3 & & \\
\hline 67.4 & 0.4 & 0.4 & 0.4 & 0.3 & & 67.4 & 0.3 & 0.2 & 0.4 & 0.3 & & \\
\hline 74.1 & 0.2 & 0.3 & 0.4 & 0.4 & & 74.1 & 0.5 & 0.5 & 0.5 & 0.3 & & \\
\hline 80.9 & 0.5 & 0.5 & 0.4 & 0.3 & & 80.9 & 0.4 & 0.4 & 0.4 & 0.2 & & \\
\hline 87.8 & 0.2 & 0.2 & 0.5 & 0.3 & & 87.8 & 0.4 & 0.4 & 0.4 & 0.3 & & \\
\hline 94.6 & 0.3 & 0.2 & 0.2 & 0.3 & & 94.6 & 0.4 & 0.5 & 0.5 & 0.3 & & \\
\hline 101.3 & 0.3 & 0.3 & 0.6 & 0.4 & & 101.3 & 0.5 & 0.5 & 0.5 & 0.3 & & \\
\hline 108.3 & 0.5 & 0.4 & 0.5 & 0.4 & & 108.3 & 0.4 & 0.2 & 0.4 & 0.3 & & \\
\hline 115.0 & 0.4 & 0.5 & 0.7 & 0.5 & & 115.0 & 0.5 & 0.4 & 0.4 & 0.3 & & \\
\hline 121.9 & 0.5 & 0.4 & 0.5 & 0.4 & & 121.9 & 0.4 & 0.4 & 0.4 & 0.3 & & \\
\hline 128.7 & 0.4 & 0.3 & 0.4 & 0.3 & & 128.7 & 0.6 & 0.6 & 0.5 & 0.3 & & \\
\hline 135.4 & 0.2 & 0.2 & 0.5 & 0.5 & & 135.4 & 0.4 & 0.3 & 0.5 & 0.3 & & \\
\hline 142.3 & 0.3 & 0.3 & 0.6 & 0.4 & & 142.3 & 0.6 & 0.5 & 0.6 & 0.4 & & \\
\hline 149.1 & 0.5 & 0.5 & 0.6 & 0.4 & & 149.1 & 0.5 & 0.5 & 0.6 & 0.4 & & \\
\hline 156.0 & 0.4 & 0.2 & 0.4 & -0.2 & & 156.0 & 0.4 & 0.4 & 0.5 & 0.4 & & \\
\hline 152.8 & 0.3 & 0.3 & 0.5 & 0.4 & & 162.8 & 0.5 & 0.5 & 0.5 & 0.4 & & \\
\hline 169.5 & 0.4 & 0.3 & 0.6 & 0.6 & & 169.5 & 0.6 & 0.5 & 0.6 & 0.5 & & \\
\hline 176.4 & 0.4 & 0.3 & 0.5 & 0.4 & & 176.4 & 0.6 & 0.5 & 0.6 & 0.4 & & \\
\hline 183.2 & 0.7 & 0.6 & 0.8 & 0.4 & & 183.2 & 0.6 & 0.4 & 0.6 & 0.5 & & \\
\hline 190.1 & 0.5 & 0.4 & 0.7 & 0.5 & & 190.1 & 0.6 & 0.5 & 0.6 & 0.5 & & \\
\hline 196.9 & 0.3 & 0.4 & 0.8 & 0.5 & & 196.9 & 0.6 & 0.6 & 0.6 & 0.5 & & \\
\hline Velocity probe & s in channels & above burne & & & & & & & & & & \\
\hline Time & Channel 1 & Channel 2 & & & & & & & & & & \\
\hline 0.0 & 0.0 & 0.0 & & & & & & & & & & \\
\hline 5.8 & 1.9 & 0.8 & & & & & & & & & & \\
\hline 12.6 & 1.8 & 0.9 & & & & & & & & & & \\
\hline 19.6 & 2.6 & 1.3 & & & & & & & & & & \\
\hline 26.3 & 27 & 1.0 & & & & & & & & & & \\
\hline 33.2 & 2.9 & 1.4 & & & & & & & & & & \\
\hline 39.9 & 3.4 & 1.2 & & & & & & & & & & \\
\hline 46.7 & 2.8 & 1.2 & & & & & & & & & & \\
\hline 53.6 & 2.6 & 1.0 & & & & & & & & & & \\
\hline 60.4 & 3.3 & 1.8 & & & & & & & & & & \\
\hline 67.4 & 3.4 & 1.7 & & & & & & & & & & \\
\hline 74.1 & 3.5 & 1.4 & & & & & & & & & & \\
\hline 80.9 & 3.5 & 1.8 & & & & & & & & & & \\
\hline 87.8 & 3.0 & 1.7 & & & & & & & & & & \\
\hline 94.6 & 3.2 & 1.6 & & & & & & & & & & \\
\hline 101.3 & 3.5 & 1.8 & & & & & & & & & & \\
\hline 108.3 & 3.2 & 2.2 & & & & & & & & & & \\
\hline 115.0 & 3.4 & 1.9 & & & & & & & & & & \\
\hline 121.9 & 3.7 & 2.1 & & & & & & & & & & \\
\hline 128.7 & 4.1 & 1.6 & & & & & & & & & & \\
\hline 135.4 & 4.0 & 2.5 & & & & & & & & & & \\
\hline 142.3 & 4.0 & 2.2 & & & & & & & & & & \\
\hline 149.1 & 4.2 & 2.3 & & & & & & & & & & \\
\hline 156.0 & 4.2 & 2.6 & & & & & & & & & & \\
\hline 162.8 & 4.1 & 2.3 & & & & & & & & & & \\
\hline 169.5 & 4.3 & 2.1 & & & & & & & & & & \\
\hline 176.4 & 3.9 & 2.5 & & & & & & & & & & \\
\hline
\end{tabular}


Experiment Number 45

$183.2 \quad 4.5 \quad 2.4$

$\begin{array}{lll}190.1 & 5.2 & 2.9\end{array}$

$\begin{array}{lll}196.9 & 3.7 & 2.8\end{array}$ 
NIST-114

(REV. 6-93)

ADMAN 4.09

INSTRUCTIONS: ATTACH ORIGINAL OF THIS FORM TO ONE (1) COPY OF MANUSCRIPT AND SEND TO: WERB SECRETARY, BUILDING 820, ROOM 125 TITLE AND SUBTITLE (CITE IN FULL) U.S. DEPARTMENT OF COMMERCE NATIONAL INSTITUTE OF STANDARDS AND TECHNOLOGY MANUSCRIPT REVIEW AND APPROVAL

Effect of an Obstructed Ceiling on the Activation Time of a Residential Sprinkler

\section{CONTRACT OR GRANT NUMBER}

AUTHOR(S) (LAST NAME, FIRST INITIAL, SECOND INITIAL

Vettori, R. L.

LABORATORY AND DIVISION NAMES [FIRST NIST AUTHOR ONLY] Building and Fire Research Laboratory, Fire Safety Engineering Division SPONSORING ORGANIZATION NAME AND COMPLETE ADDRESS (STREET, CITY, STATE, ZIP)

PROPOSED FOR NIST PUBLICATION

JOURNAL OF RESEARCH (NIST JRES)

J. PHYS. \& CHEM. REF. DATA (JPCRD)

HANDBOOK INIST HB]

SPECIAL PUBLICATION INIST SPI

TECHNICAL NOTE [TN]

PROPOSED FOR NON-NIST PUBLICATION (CITE FULLY):

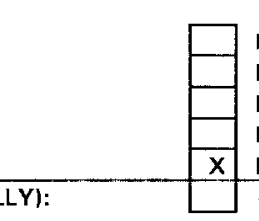

MONOGRAPH (NIST MN)

NATL. STD. REF. DATA SERIES (NIST NSRDS)

FEDERAL INFO. PROCESS. STDS. (NIST FIPS)

LIST OF PUBLICATIONS (NIST LP)

INTERAGENCY/NTERNAL REPORT [NISTIR] - U.S.
TYPE OF REPORT AND/OR PERIOD COVERED NISTIR PERFORMING ORGANIZATION (CHECK $(X)$ ONE BOX)
(ERB USE ONLY)

\begin{tabular}{l|l|}
\hline $\begin{array}{l}\text { ERB CONTROL NUMBER } \\
\text { G }\end{array}$ & DIVISION \\
\hline PUBLICATIONS REPORT NUMBER & CATEGORY CODE \\
No. NISTIR 6253 & \\
$\begin{array}{c}\text { PUBLICATION DATE } \\
\text { NOVEmber } 1998\end{array}$ & NO. PRINTED PAGES \\
\hline
\end{tabular}

NIST/JILA

\begin{tabular}{|c|c|c|c|c|c|c|c|}
\hline PUBLISHING MEDIUM: & $x$ & PAPER & DISKETTE & $\bar{x}$ & CD-ROM & WWW & OTHER \\
\hline
\end{tabular}

SUPPLEMENTARY NOTES

ABSTRACT (A 2000-CHARACTER OR LESS FACTUAL SUMMARY OF MOST SIGNIFICANT INFORMATION. IF DOCUMENT INCLUDES A SIGNIFICANT BIBLIOGRAPHY OR LITERATURE SURVEY, CITE IT HERE. SPELL OUT ACRONYMS ON FIRST REFERENCE.) (CONTINUE ON SEPARATE PAGE, IF NECESSARY.)

A series of 45 experiments were conducted to compare the effects of two different ceiling configurations on the activation times of a quick response residential pendent sprinkler. The two ceiling configurations consisted of a smooth horizontal unobstructed ceiling and a horizontal ceiling obstructed by parallel beams measuring $0.038 \mathrm{~m}$ (1.5 in) wide by $0.24 \mathrm{~m}(9.5 \mathrm{in}) \mathrm{deep}$ and spaced $0.41 \mathrm{~m}$ (16 in) on center. For each of the two ceiling configurations, the fire source, a computer controlled methane gas burner, was placed in three different locations within the fire compartment and $2.1 \mathrm{~m}(7 \mathrm{ft})$ below the ceiling. Additionally, for each burner location, the flow of methane gas to the burner was supplied in such a way as to give three different fire growth scenarios. For the smooth horizontal unobstructed ceiling three experiments were performed for every burner position and fire growth rate. For the horizontal obstructed/beamed ceiling there were two experiments for every bumer position and fire growth rate. Measurements taken include the time to sprinkler activation, temperature and velocity of the ceiling jet at the sprinkler of activation, and temperatures and ceiling jet velocitics at various other locations and elevations within the fire compartment. The horizontal obstructed/beamed ceiling increased sprinkler activation time by $57 \%$ to $137 \%$ depending on the experimental setup.

KEY WORDS IMAXIMUM OF 9; 28 CHARACTERS AND SPACES EACH; SEPARATE WITH SEMICOLONS; ALPHABETIC ORDER; CAPITALIZE ONLY PROPER NAMES| Beams, ceiling jets, corners, fire growth, residential sprinklers, sprinkler response, sprinkler systems, tenability limits, wall fires

\section{AVAILABILITY:}

$X$ UNLIMITED $\quad \square$ FOR OFFICIAL DISTRIBUTION - DO NOT RELEASE TO NTIS ORDER FROM SUPERINTENDENT OF DOCUMENTS, U.S. GPO, WASHINGTON, DC 20402 ORDER FROM NTIS, SPRINGFIELD, VA 22161 